\title{
THE ROLE OF THE INTESTINAL MICROBIOTA IN THE MODULATION OF FOOD INTAKE AND BODY WEIGHT
}

\author{
Matthew John Dalby \\ BSc (Hons) Medical Microbiology, University of Edinburgh \\ MSc (Distinction) Molecular Nutrition, University of Aberdeen
}

A thesis presented for the degree of

Doctor of Philosophy

at the

University of Aberdeen

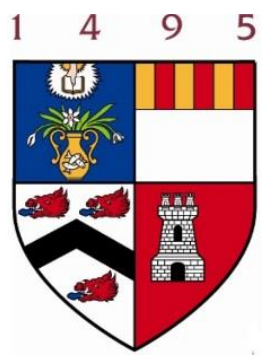

2016 


\section{DECLARATION}

I declare that this thesis has been composed by myself and not been presented or accepted in any previous application for degree. The work recorded herein was carried out by myself unless otherwise stated. All sources of information have been acknowledged by means of reference.

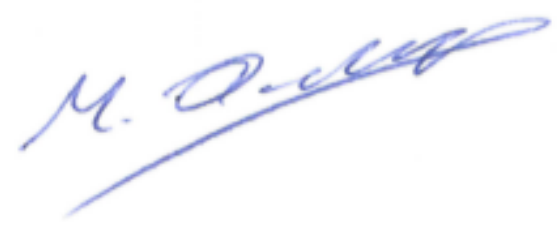

Matthew John Dalby 


\section{ACKNOWLEDGEMENTS}

I would like to express my gratitude to my supervisors, Peter Morgan and Sandy Ross, for their continuous support and guidance during my project, for being so understanding when my health has made work difficult, and for generally being the best supervisors I could have wished for. I am indebted to Lynn Thomson for her invaluable help, advice in the lab, and for always knowing where everything is; and, to Karen Garden for enabling the completion of work I would not have been able to finish. I also wish to thank Lynda Williams for her advice over the years. I am grateful to everyone associated with the Lawson Lab for being so kind, friendly, and helpful throughout my time there. I am also indebted to Alan Walker for his teaching and time in grasping the complexities Next Generation sequencing. I am grateful to Donna Wallace and all of the staff at the MRF for all of the help and work in setting up and running the mouse studies, and to Christine Grant for her aid with sample collection. I wish to thank Gill Campbell and Pauline Young at the Genomic department for all their help with sequencing and arrays, and to Claus-Dieter Mayer for help with microarray analysis. Thanks are also due to everyone at the Rowett over the past four years. I would like to thank the BBSRC and EASTBIO for funding my project and enabling me to gain so much experience. Last but not the least, I would like to thank my family for their support and belief in me, despite my being so far away for so long. Finally, to Tiffany, for making the final two years of this PhD immeasurably better.
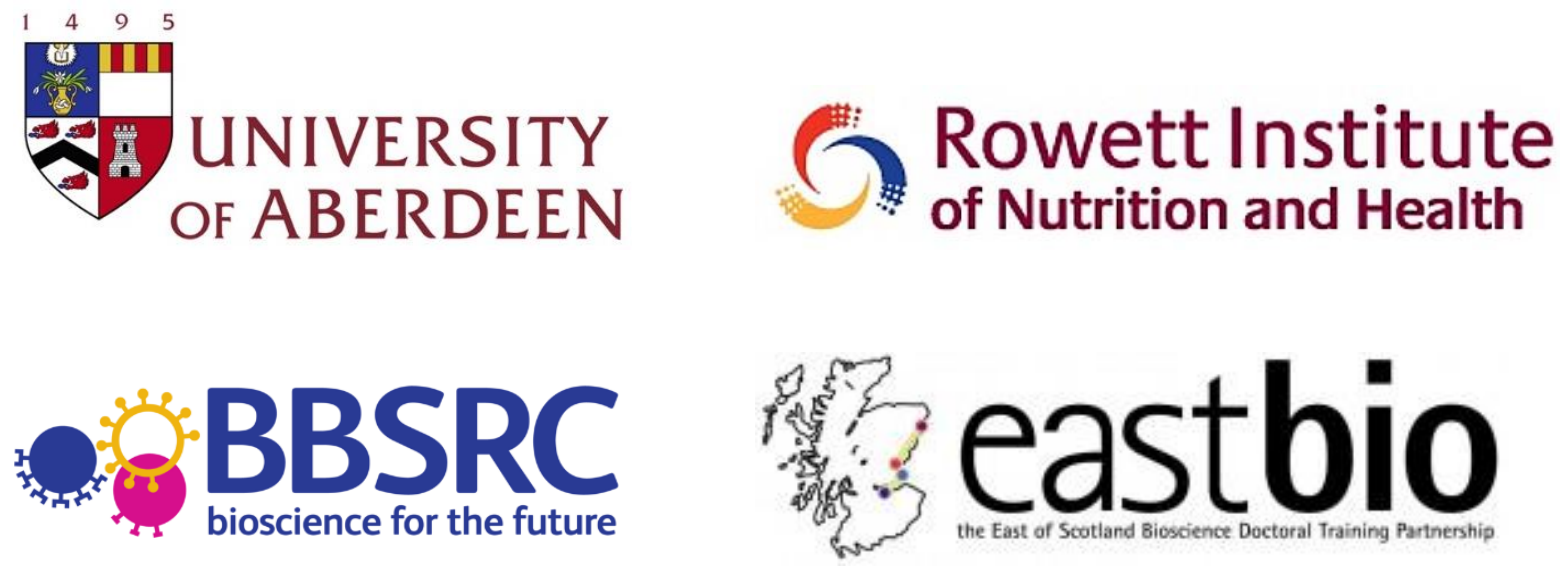


\section{SUMMARY}

The continued rise in prevalence of overweight and obesity over recent decades has focused attention on environmental factors that may be impacting on body weight. This increase has driven research into understanding mechanisms underlying energy homeostasis and the pathophysiology of obesity. Over the past decade, evidence has accumulated that suggests the intestinal microbiota, complex communities of microorganisms inhabiting the gut, have a functional role in influencing the body weight of their host. Differences in the composition of the intestinal microbiota have been observed between lean and obese mice, as well as human subjects. Transfer of microbiota from lean or obese mice into germ-free mice has been reported to transfer the obese phenotype, implicating a causal role for the microbiota. While a number of different mechanisms have been proposed to mediate these effects, the role of the gut microbiota in energy homeostasis still remains unclear.

Chronic low-grade inflammation resulting from circulating gut-derived lipopolysaccharide (LPS), which activates toll-like receptor 4 (TLR4), has been proposed as one mechanism contributing to obesity. Linked to this, inflammation in the hypothalamus, a centre of neuroregulation of energy homeostasis, has also been implicated in the development of obesity. Additionally, bacterial products from the fermentation of dietary polysaccharides in the lower intestine provides another mechanism through which the microbiota can influence bodyweight, both through the harvest of undigested energy and also the stimulation of anorectic peptide hormone released by the gut.

The aim of this thesis was to investigate some of the roles of the intestinal microbiota in shaping host food intake and body weight. This included investigation of potential immunomodulation by gut derived lipopolysaccharide (LPS) through TLR4 signalling, changes in gut microbiota composition in response to the diet, and, in particular, the role of dietary fibre in stimulating release of intestinal peptide hormones.

High-fat diet feeding in mice, in addition to causing obesity, has been reported to increase circulating LPS and infusion of low-dose LPS into mice reportedly increased body fat, while CD14 null mice were protected from both High-Fat diet and LPS-induced 
obesity. On the basis of these findings, TLR4 signalling has been implicated in the development of obesity. To investigate this, TLR4 $4^{-/}$or $\mathrm{CD}_{1} 4^{-/-}$mice and C57BL/6J controls were fed a High-Fat or Low-Fat diet. Against prediction, neither TLR4 ${ }^{-/-}$nor $\mathrm{CD}_{14} /$ - were protected against High-Fat diet-induced obesity. Body weight, body composition, and food intake remained indistinguishable between TLR4 ${ }^{-/-}$or $\mathrm{CD} 14^{-/-}$ mice and C57BL/6J controls over 8 weeks of High-Fat diet feeding. High-Fat diet increased hypothalamic expression of SerpinA3N and SOCS3 regardless of genotype, indicating diet-induced changes in the hypothalamus. However, inflammatory gene expression was not increased. Analysis of the microbiota revealed relatively small differences between the composition of the caecal microbiota in C57BL/6J fed Low or High-Fat diets, despite large increases in body weight in High-Fat fed mice.

It was hypothesised that this lack of change in the microbiota composition in the above study was due to the composition of the diets used in the experiments, namely refined High-Fat and Low-Fat diets. Previous studies reported in the literature indicate baseline control diets have typically been reported as an undefined Chow diet; this may be an important factor in microbial composition of the gut as these diets are high in fermentable dietary fibre. To investigate the relationship between diet, microbiota changes, and obesity, C57BL/6J mice were fed a Chow diet or a refined diet that was either High-Fat or Low-Fat. Relative to Chow, both High- and Low-Fat refined diets had major, yet similar, effects on the microbial composition of the gut, including changes in microbial diversity, at the phylum level with increases in the Firmicutes:Bacteroidetes ratio, and at the species level. By contrast, only High-Fat diet feeding increased obesity and glucose intolerance. This indicates that it is loss of fibre that is the main driver of shifts in microbial composition seen in mouse studies of High-Fat diet-induced obesity and that High-Fat, rather than microbial composition, drives obesity.

The addition of fermentable fibre to the diet of mice fed High-Fat diets reduced food intake and body fat. The role of colonic GLP-1 and PYY secreted by enteroendocrine Lcells in mediating this effect was investigated using GLP-1R $\mathrm{R}^{-/-}$and $\mathrm{PYY}^{-/-}$mice a fed a High-Fat diet supplemented with inulin or cellulose. Inulin supplementation reduced body fat and food intake in High-Fat diet fed C57BL/6J control mice compared to those supplemented with cellulose. In contrast, both the GLP-1R ${ }^{-/-}$and $\mathrm{PYY}^{-/-}$mice showed attenuated responses to the body fat and food intake-reducing effects of dietary inulin. 
In summary, this research questions the role of TLR4 and LPS, and also hypothalamic inflammation, in the development of diet-induced obesity in mice. These results demonstrate that the use of a well-defined control diet in studies of obesity in mice is essential and, importantly, suggests that many of the obesity-associated changes in the gut microbiota are due to the use of undefined Chow diets as an inappropriate control for the effects of refined High-Fat diets. These results also provide evidence for an essential role for both GLP-1 and PYY in mediating the food intake and bodyweightreducing effects of fermentable fibre. 


\section{TABLE OF CONTENTS}

DECLARATION .ll

ACKNOWLEDGEMENTS .III

SUMMARY IV

TABLE OF CONTENTS VII

LIST OF FIGURES $\mathrm{XVI}$

LIST OF TABLES ..XVIII

LIST OF ABBREVIATIONS. XIX

CHAPTER 1 2

INTRODUCTION. .2

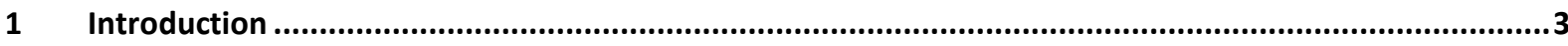

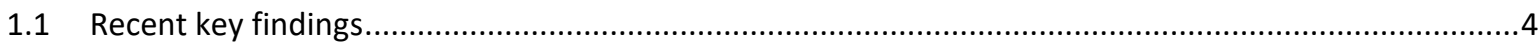

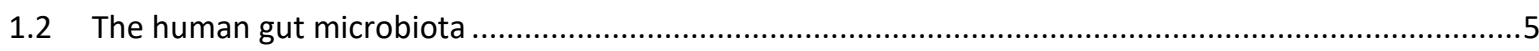

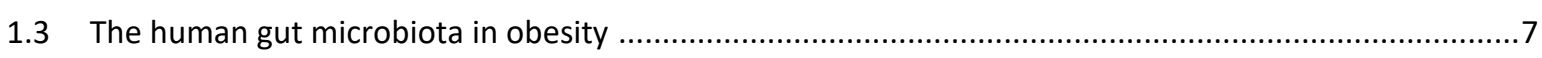

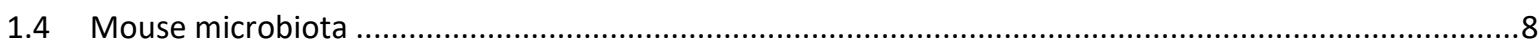

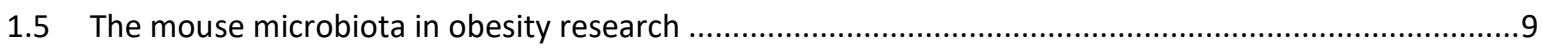

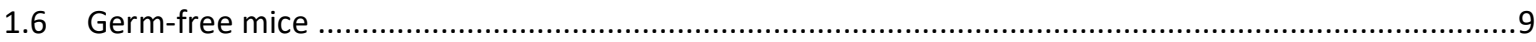

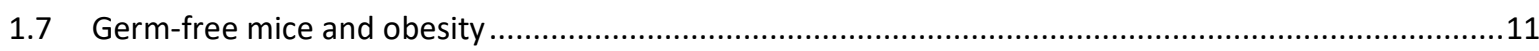

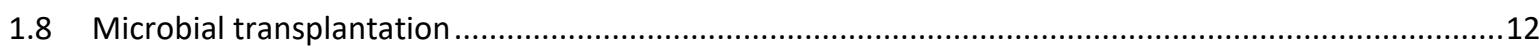

1.9 The role of individual bacterial species in obesity development .......................................................14

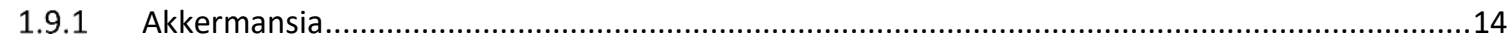

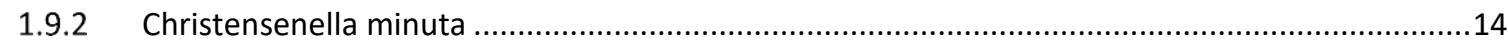

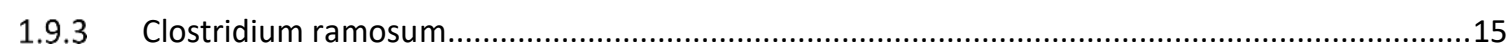

1.10 Mechanisms though which the gut microbiota can modulate obesity ............................................15

1.10.1 Immunomodulation of the host immune system ...............................................................16

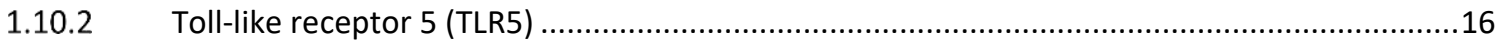

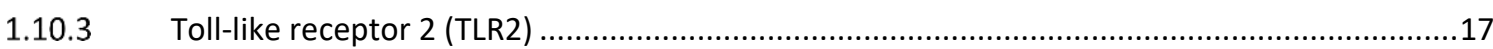




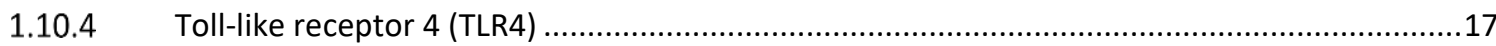

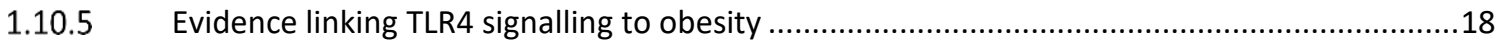

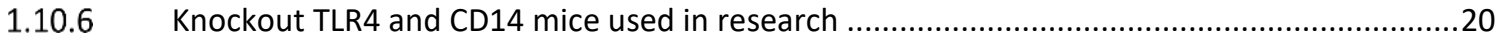

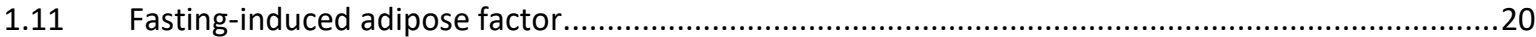

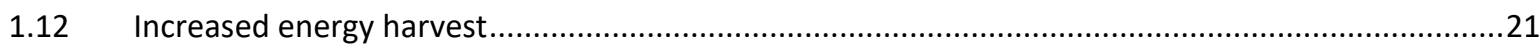

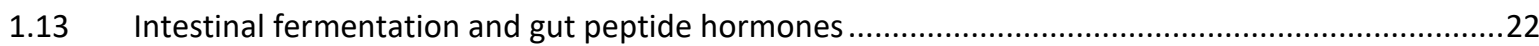

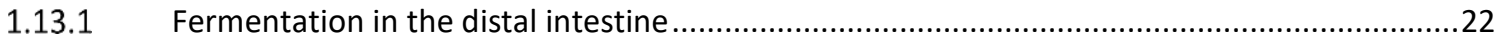

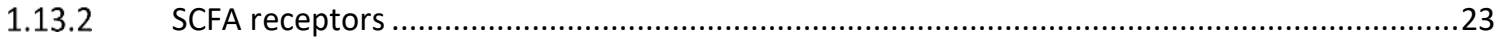

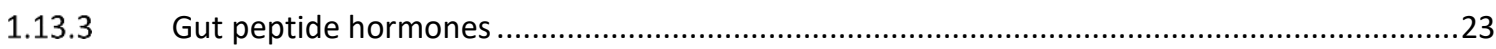

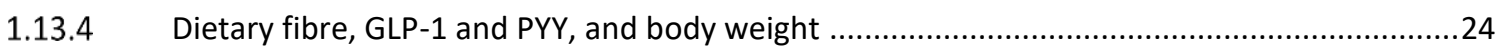

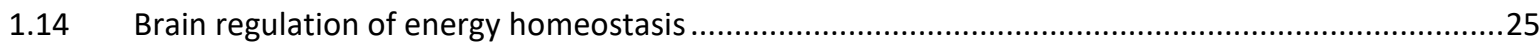

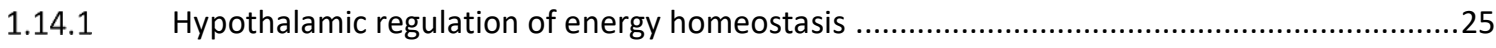

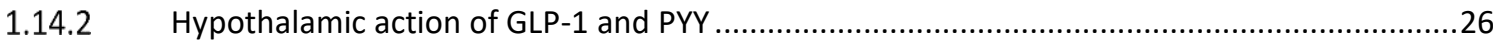

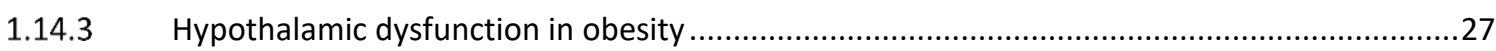

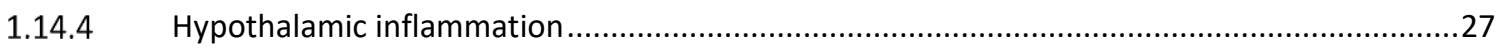

2 Summary

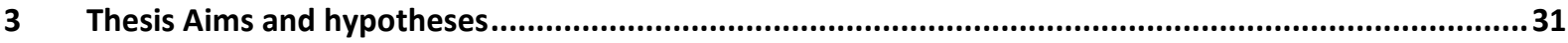

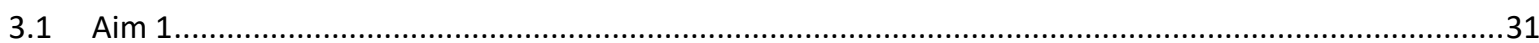

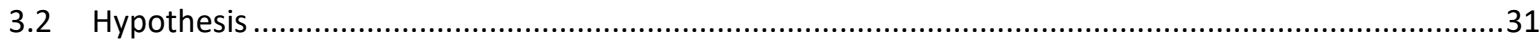

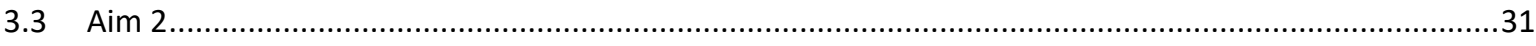

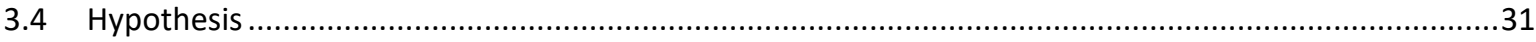

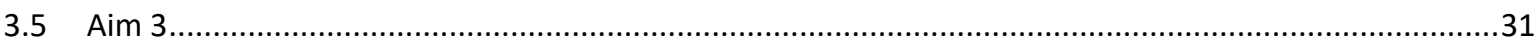

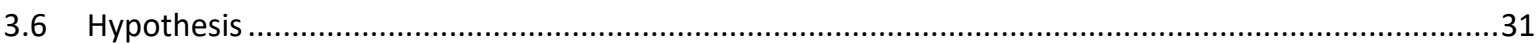

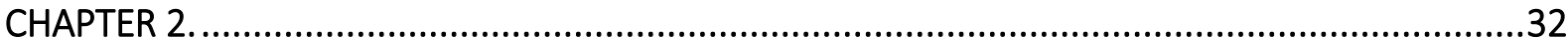

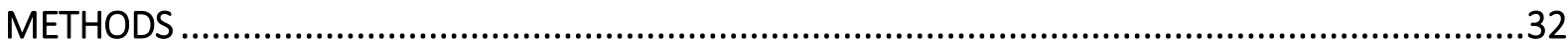

$4 \quad$ Animal Study 1: The influence of TLR4 and CD14 on diet induced obesity in mice..............................33

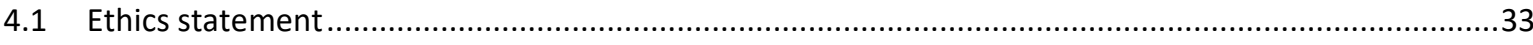

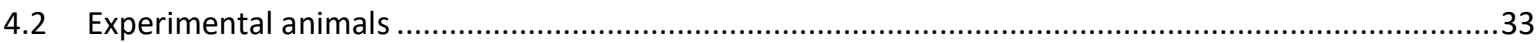

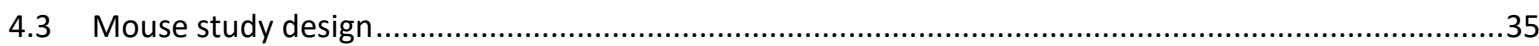

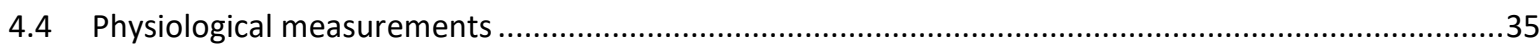

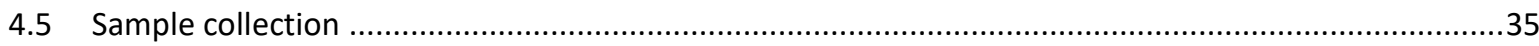

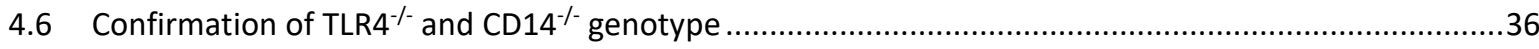

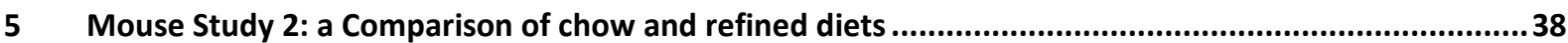

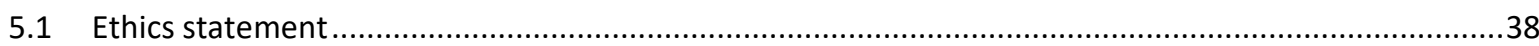

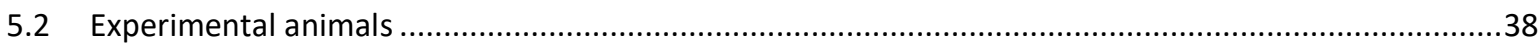




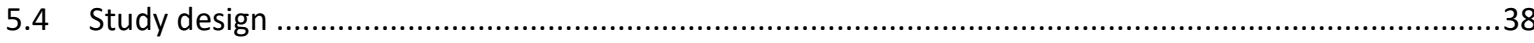

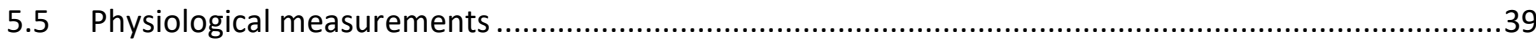

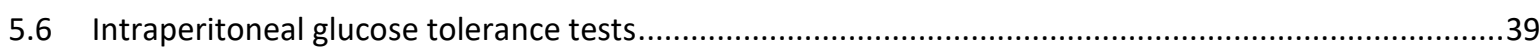

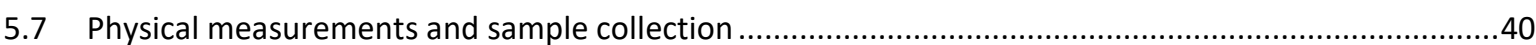

$6 \quad$ Mouse study 3: The role of GLP-1 and PYY in mediating the effects of fermentable fibre ...................41

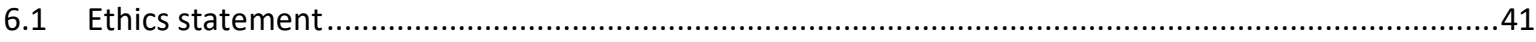

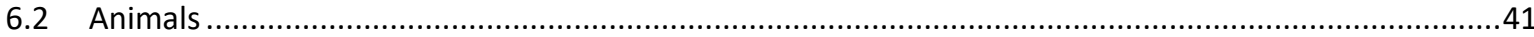

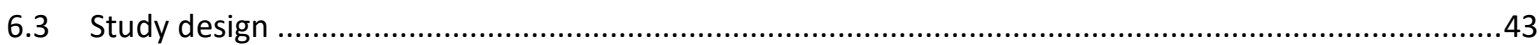

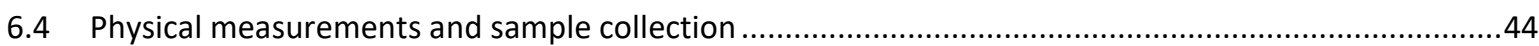

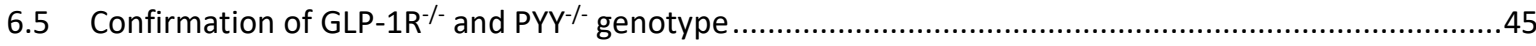

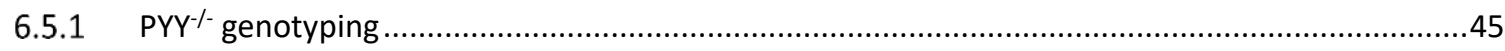

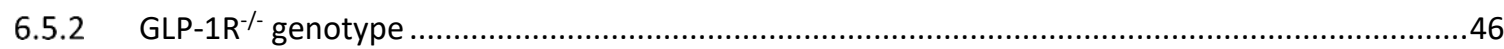

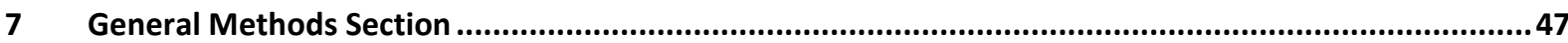

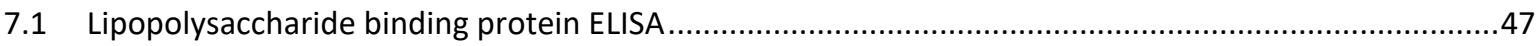

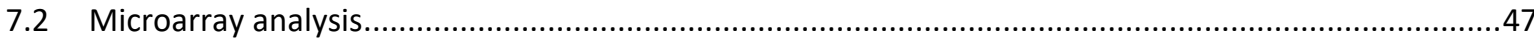

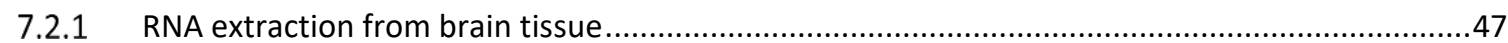

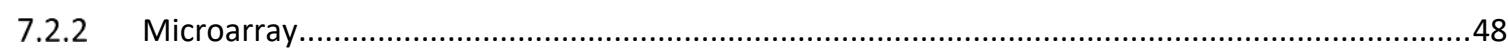

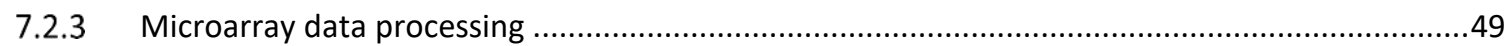

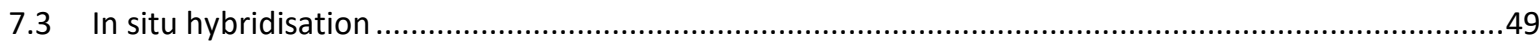

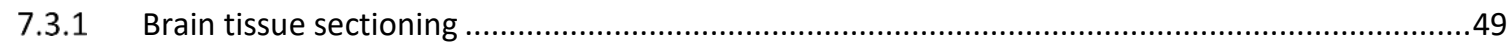

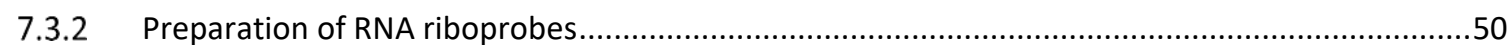

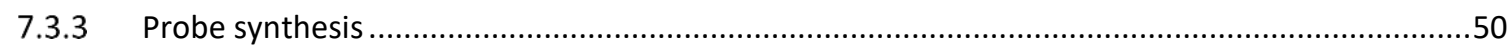

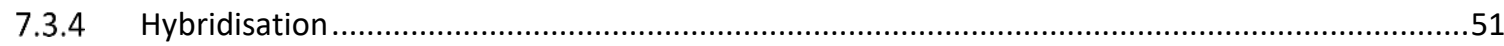

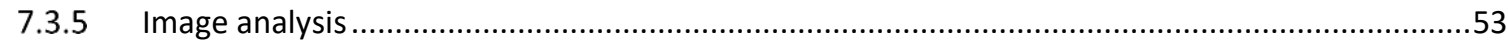

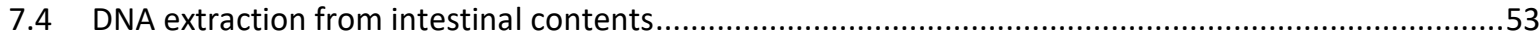

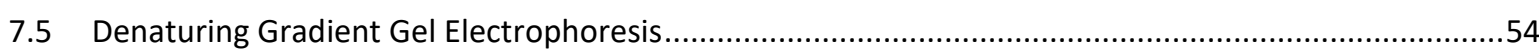

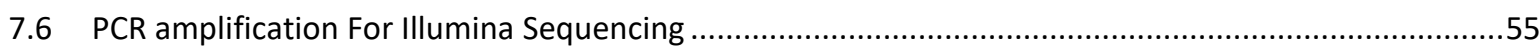

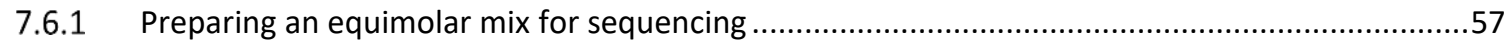

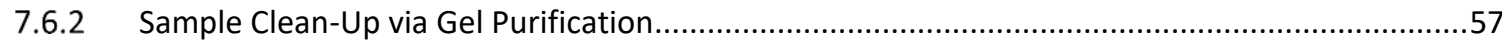

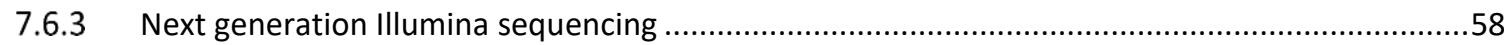

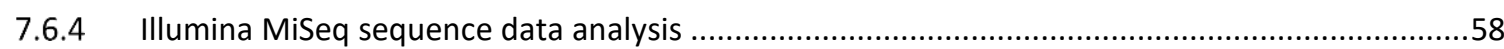

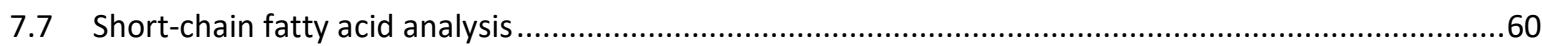

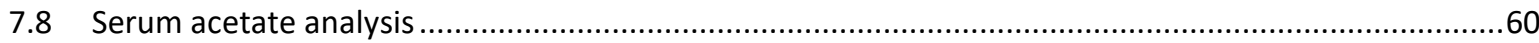

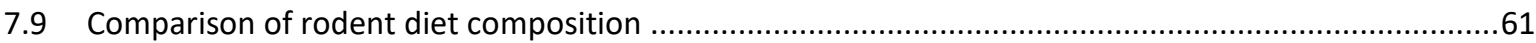

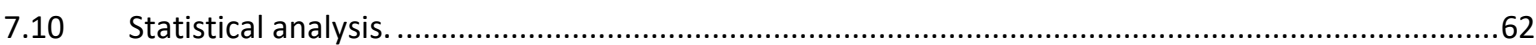


8 Introduction

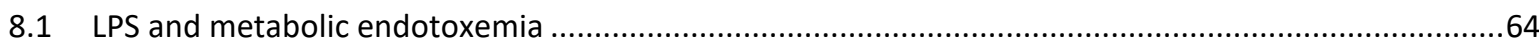

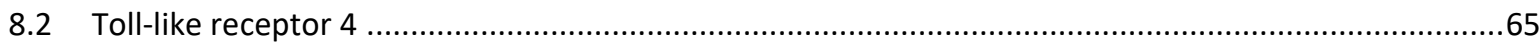

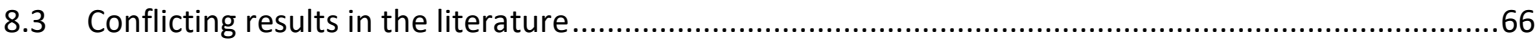

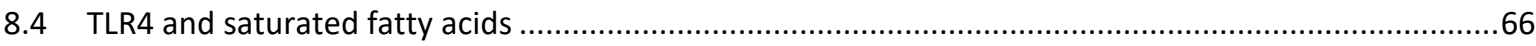

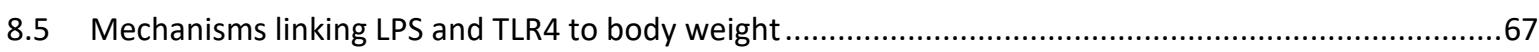

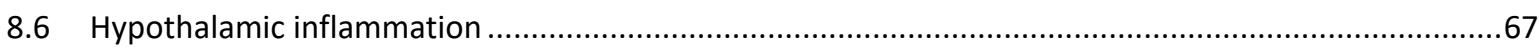

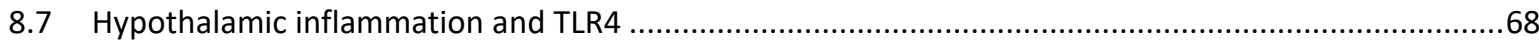

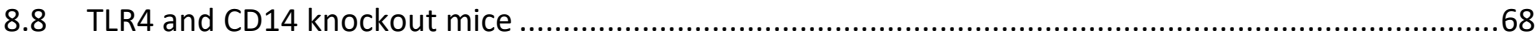

$9 \quad$ Aims

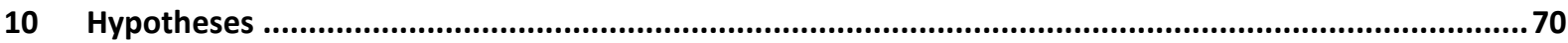

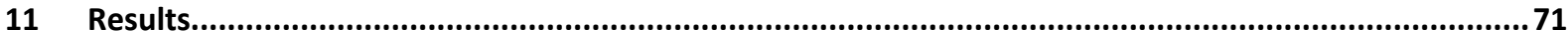

$11.1 \mathrm{TLR} 4 \%$ and $\mathrm{CD} 14 \%$ mice are not protected from Diet-Induced Obesity .......................................71

$11.2 \mathrm{TLR} 4{ }^{-/}$and $\mathrm{CD} 14 \%$ mice are not protected from increased body fat ..............................................

11.3 TLR4 $\%$ and CD14 $\%$ mice do not show any difference in food or energy intake...............................76

11.4 High-Fat diet increases lipopolysaccharide binding protein ................................................... 78

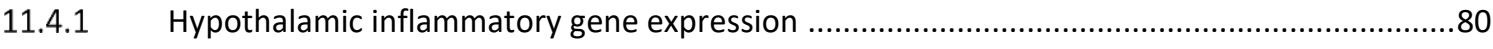

11.4.2 High-Fat diet induced change in hypothalamic gene expression ........................................80

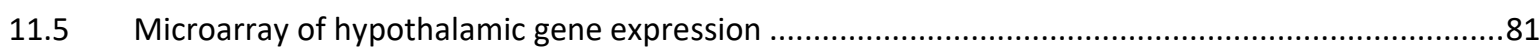

11.5.1.1 SOCS3 expression is increased in High-Fat diet feeding .................................................83

11.5.1.2 AgRP expression is not increased in High-Fat diet feeding ..........................................83

11.5.1.3 SerpinA3N expression is increased in High-Fat diet feeding ........................................83

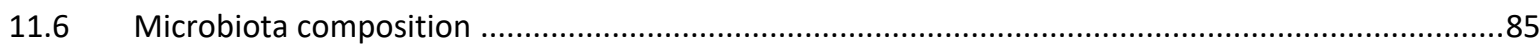

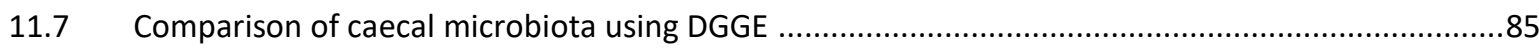

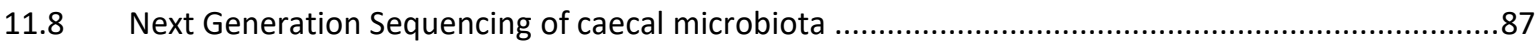

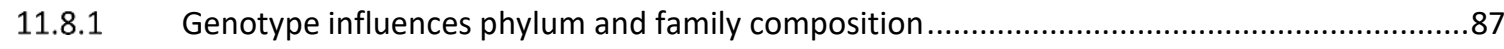

11.8.2 Genotype influences Phylum level abundance and Firmicutes to Bacteroidetes ratio ............89

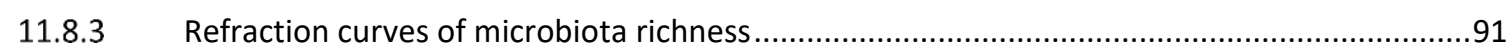

11.8.4 Genotype and not diet influences microbiota diversity ..................................................91

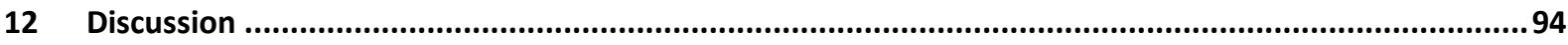

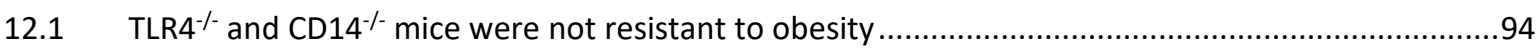

12.1.1 CD14

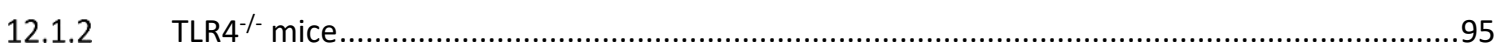

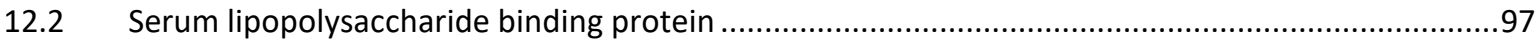

12.3 LPS infusion effects on body weight, food intake, and inflammation .........................................98

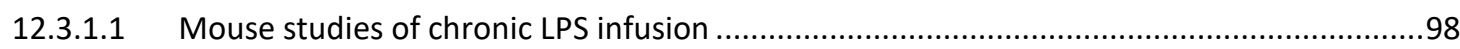


12.3.1.2 Rat studies of chronic LPS infusion

12.3.1.3 Human studies of chronic LPS exposure ..................................................................... 100

12.4 High fat diet does not increase inflammatory gene expression ................................................101

12.4.1 Microarray of hypothalamic inflammatory gene expression ...........................................101

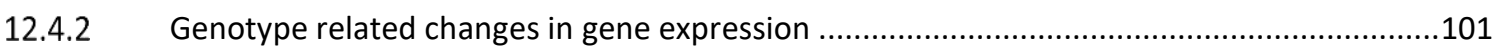

12.4.3 Serpina3n expression increased in High-Fat diet fed mice ................................................102

12.4.4 SOCS3 expression increased in High-Fat diet fed mice ...................................................103

12.4.5 Summary of hypothalamic inflammation results .........................................................103

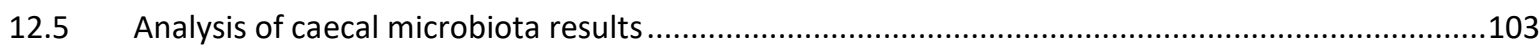

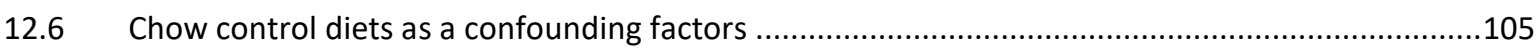

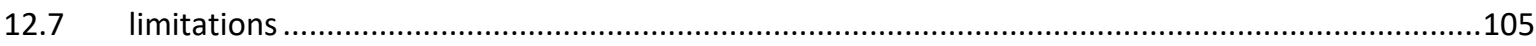

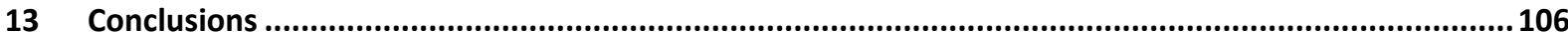

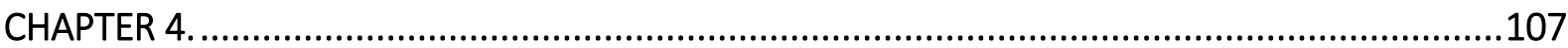

A COMPARISON OF REFINED AND CHOW CONTROL DIETS ...............................................107

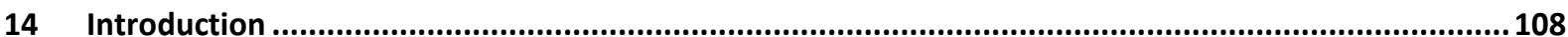

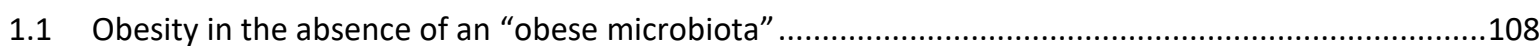

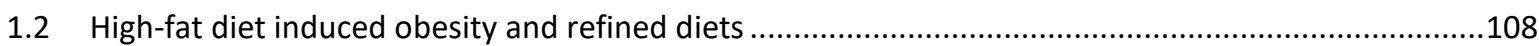

1.2.1.1 Composition of refined high-fat mouse diets.........................................................109

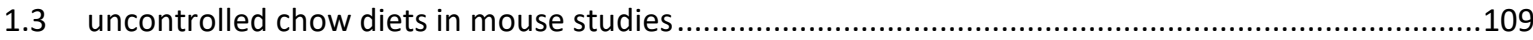

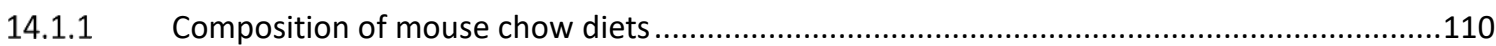

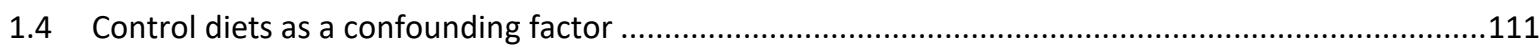

14.1.2 chow diets in mouse gut microbiota research ..........................................................112

14.1.3 Poorly matched chow diets are commonly used .....................................................113

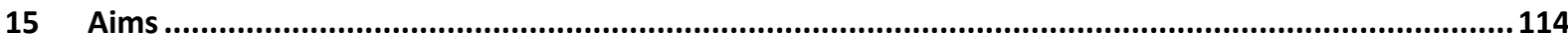

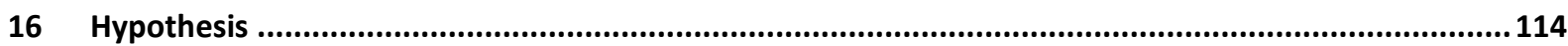

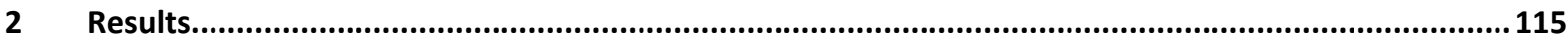

2.1 Effect of chow and refined diets on mouse body composition and metabolism ..............................115

16.1.1 Effect of chow and refined diets on body weight ..........................................................115

16.1.2 Effect of chow and refined diets on body composition ....................................................117

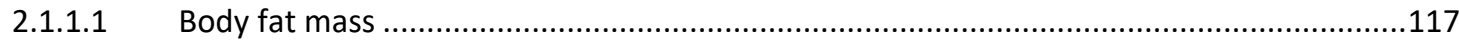

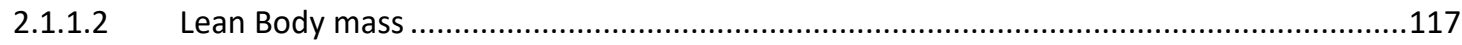

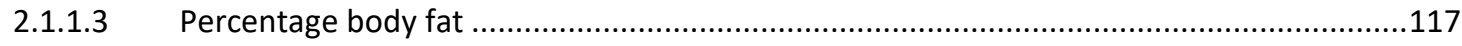

2.1.1.4 Percentage Lean body mass .................................................................................117

16.1.3 Effect of chow and refined diets on food and energy intake ............................................120

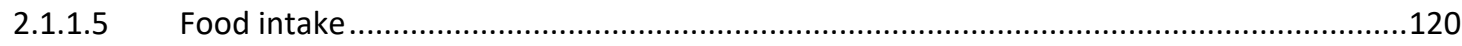


2.1.1.6 Energy intake.

16.1.4 Refined Low-Fat Diet Does Not Induce Glucose Intolerance ..........................................123

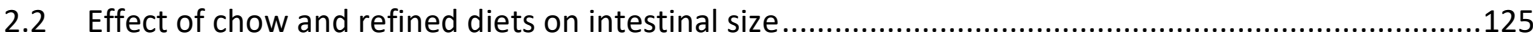

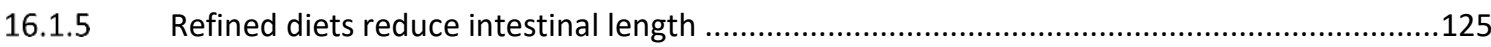

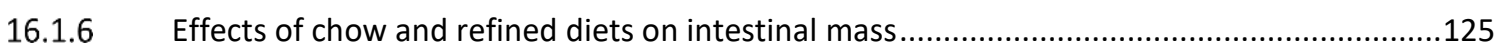

16.1.7 Gross morphological effects of diet on the caecum and colon.......................................128

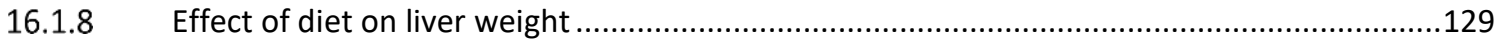

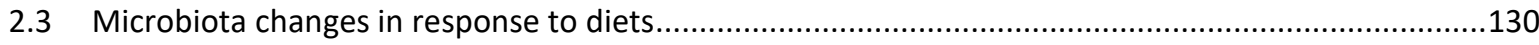

16.1.9 Microbiota from chow fed mice cluster distinctly from other groups .................................130

16.1.10 Refined diets alter phylum level composition...............................................................133

16.1.11 Phylum level changes between groups and intestinal location .......................................136

16.1.12 Refined diets reduce Firmicutes/Bacteroidetes ratio ....................................................136

16.1.13 Species richness and diversity between diet groups and intestine location........................138

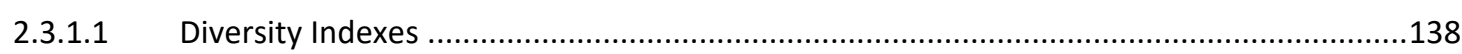

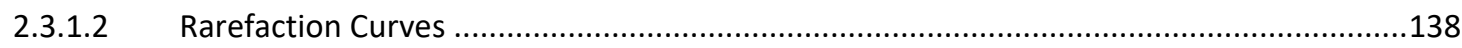

16.1.14 Microbiota profile clustering and family level composition..............................................141

16.1.15 Faecal pellet microbiota family level composition .......................................................144

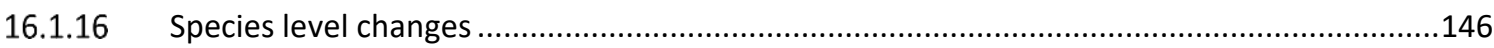

2.3.1.3 largest changes in Low-Fat and High-Fat diet groups...................................................151

2.3.1.4 Species level changes within the Family Erysipelotrichaceae ......................................153

2.3.1.5 Lactobacillaceae family and species level changes ...................................................155

2.3.1.6 Bifidobacteriaceae family and species level changes .................................................155

2.3.1.7 Species changes within the Proteobacteria phylum ......................................................158

2.3.1.8 Akkermansia muciniphila and Mucispirillum schaedleri ................................................160

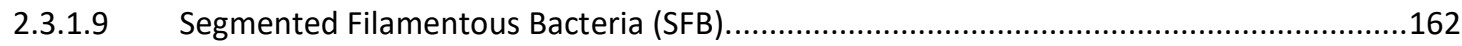

16.1.17 Short-chain fatty acid analysis of caecal contents .......................................................163

16.2 Correlations between microbiota composition and short-chain fatty acids .................................166

16.2.1 Comparison of the composition of different Chow diets....................................................168

3 Discussion 171

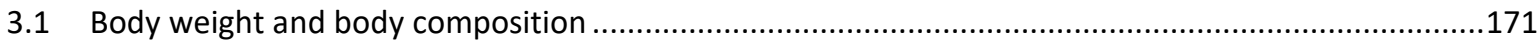

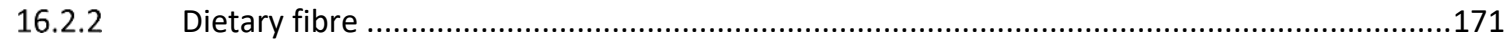

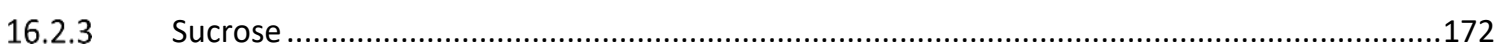

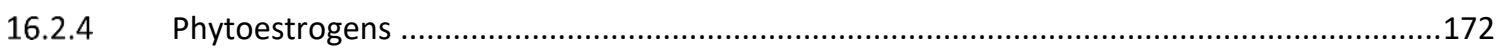

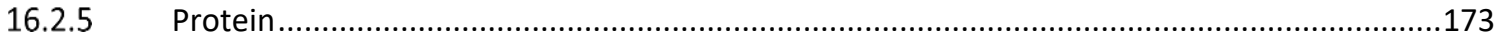

16.2.6 Summary of confounders in previous studies .............................................................173

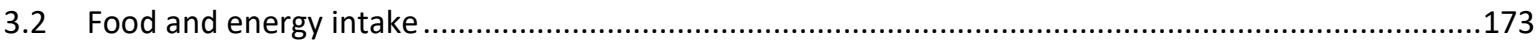

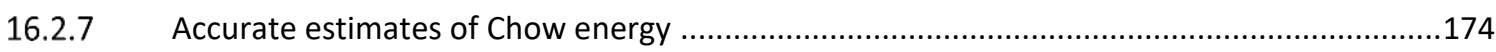

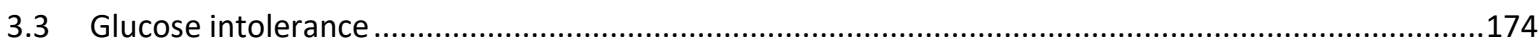


16.2.8 Ratio of Firmicutes to Bacteroidetes is not linked to obesity ..........................................175

16.2.9 Changes in microbiota diversity is not associated with obesity ........................................176

16.2.10 Microbiota changes are disassociated from obesity ..........................................................177

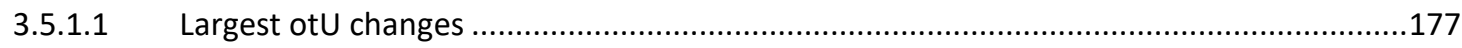

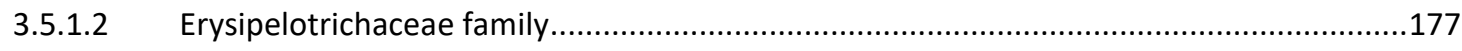

3.5.1.3 Species increased in ileum in Low-Fat and High-Fat diet groups. .................................178

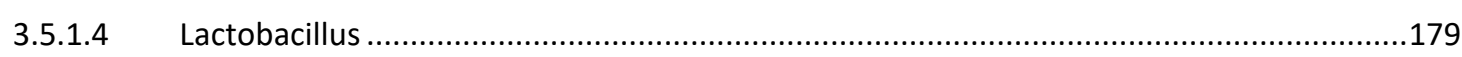

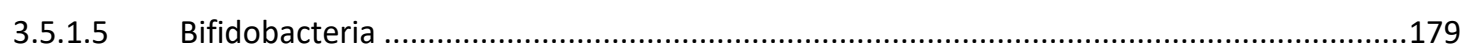

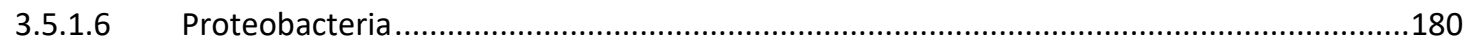

3.5.1.7 Akkermansia muciniphila and Mucispirillum schaedleri .............................................180

3.5.1.8 Segmented filamentous bacteria (SFB) ................................................................ 181

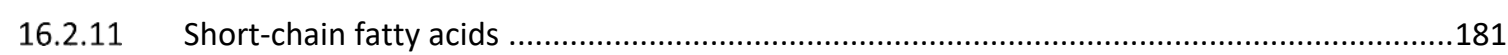

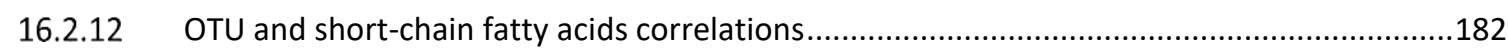

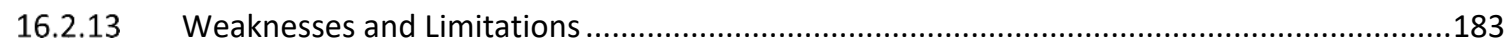

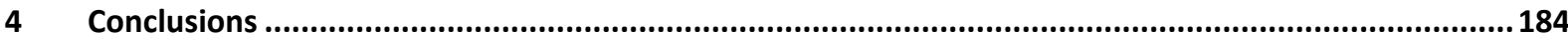

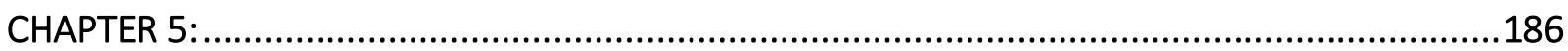

THE ROLE OF GLP-1 AND PYY IN MEDIATING THE EFFECTS OF FERMENTABLE FIBRE...............186

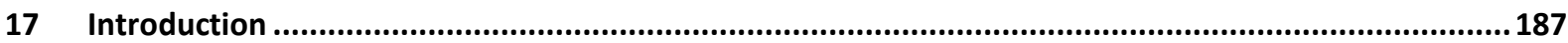

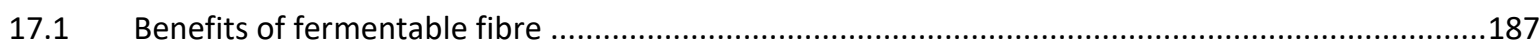

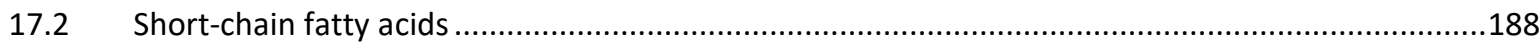

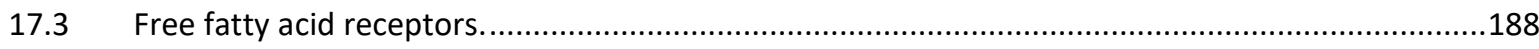

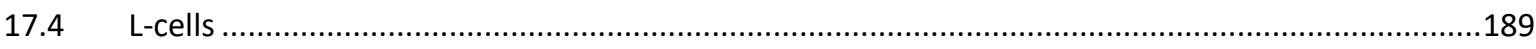

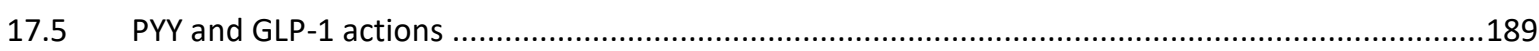

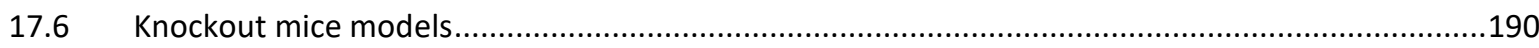

17.7 Glucagon-like peptide-1 receptor knockout mice ….........................................................191

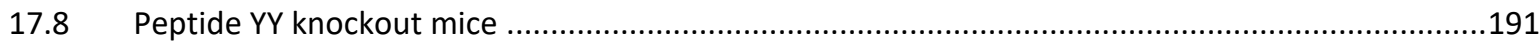

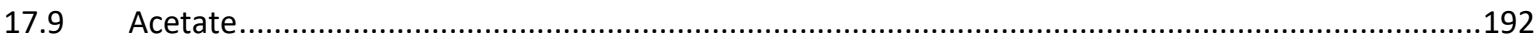

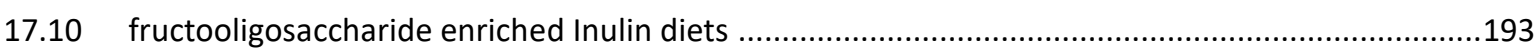

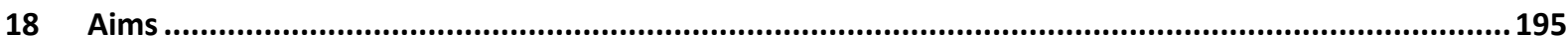

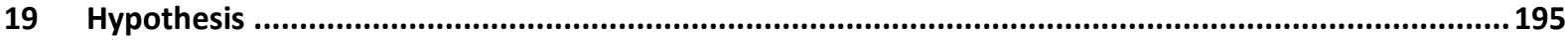

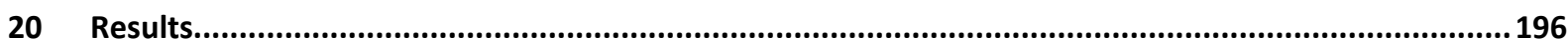

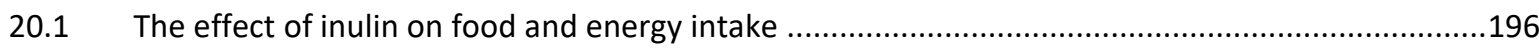

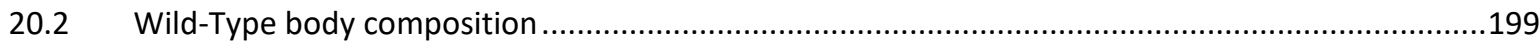




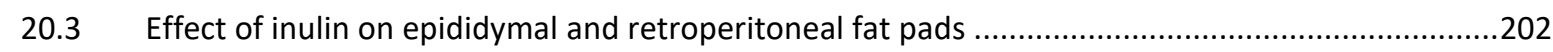

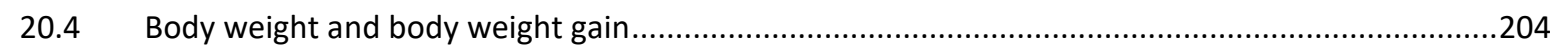

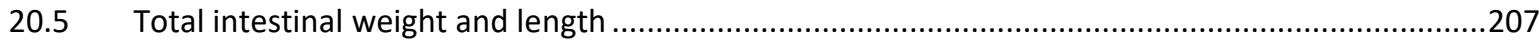

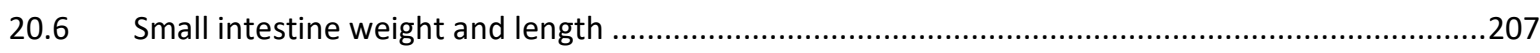

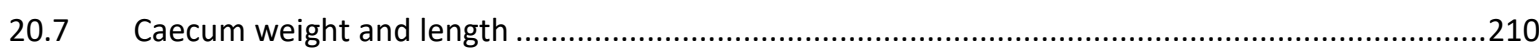

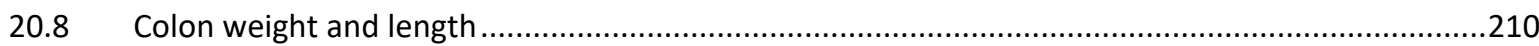

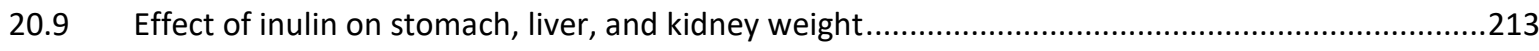

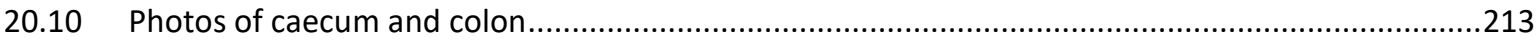

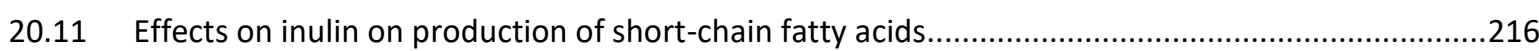

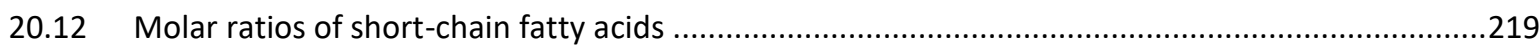

20.13 Caecal concentrations of iso-Butyrate, iso-Valerate, and Valerate .............................................219

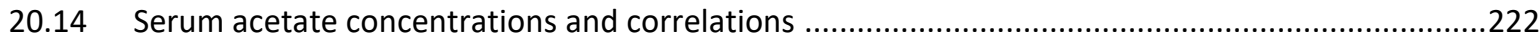

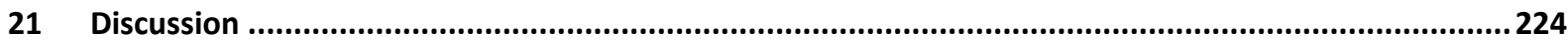

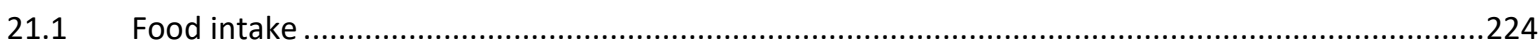

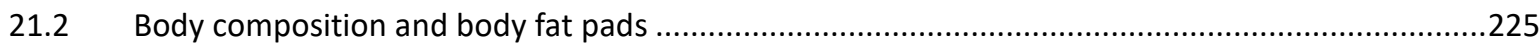

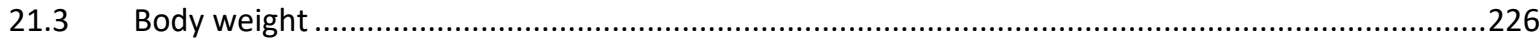

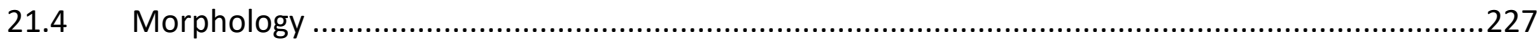

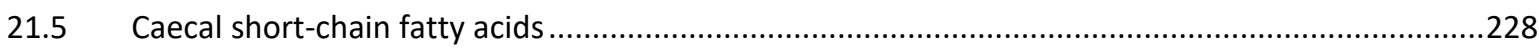

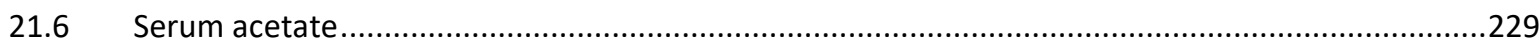

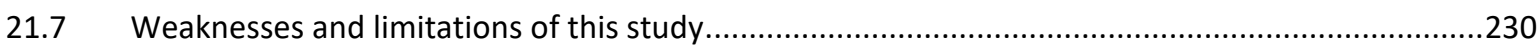

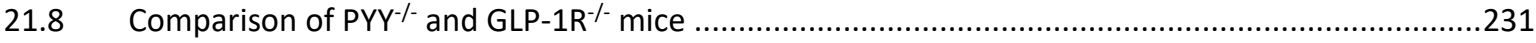

22 Conclusions

CHAPTER 6:

DISCUSSION

23 Introduction

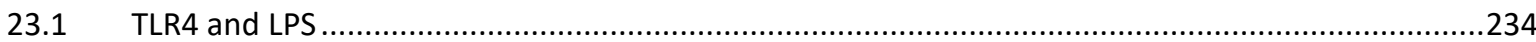

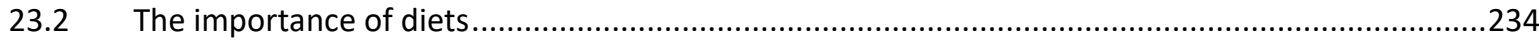

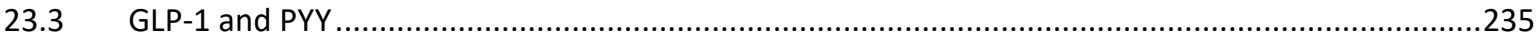

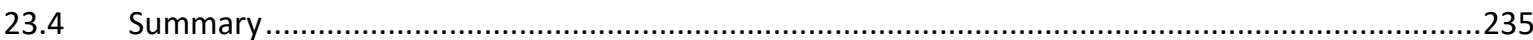

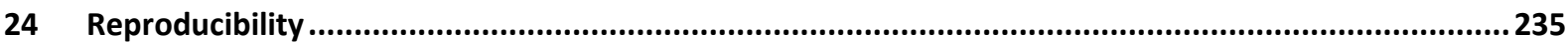

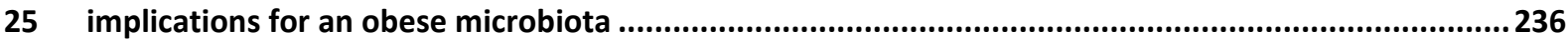

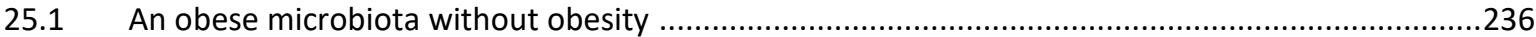

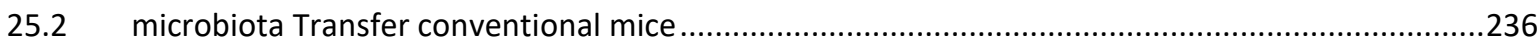

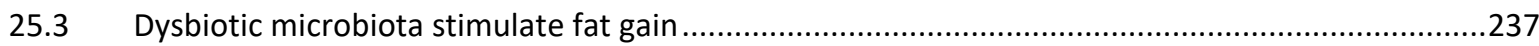

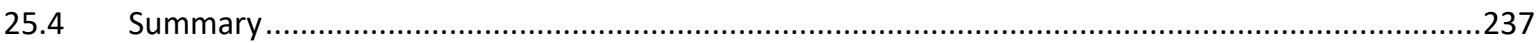


26 Conclusions

REFERENCES

APPENDIX.

.273

Page | XV 


\section{LIST OF FIGURES}

FIGURE 1. PROPOSED MECHANISMS LINKING THE MICROBIOTA TO BODY WEIGHT CHANGE..................................................29

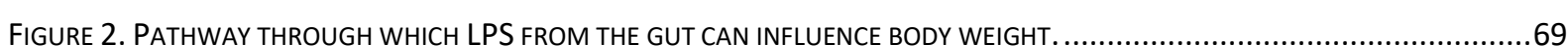

FIGURE 3. EFFECT OF DIET AND GENOTYPE ON BODY WEIGHT AND BODY WEIGHT GAIN. ......................................................72

FIGURE 4. EFFECT OF DIET AND GENOTYPE ON BODY FAT MASS AND LEAN MASS. ...........................................................

FIGURE 5. EFFECTS OF DIET AND GENOTYPE ON PERCENTAGE BODY FAT AND LEAN MASS. .................................................75

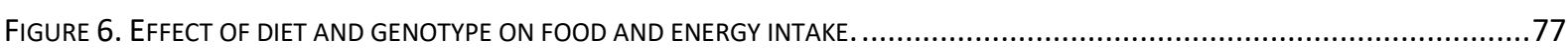

FIGURE 7. EFFECT OF DIET AND GENOTYPE ON LIPOPOLYSACCHARIDE BINDING PROTEIN. ....................................................

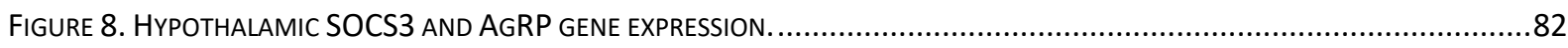

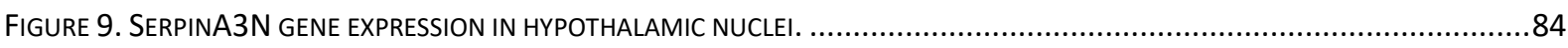

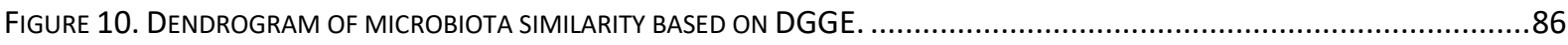

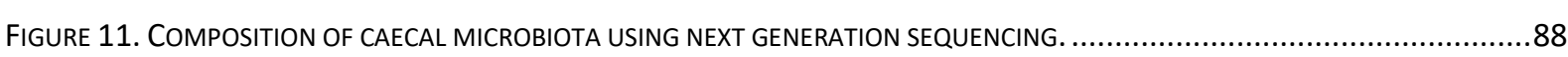

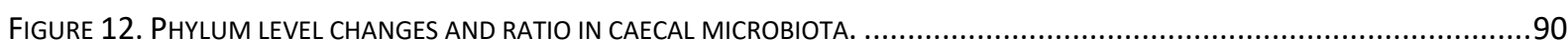

FIGURE 13. EFFECT OF DIET AND GENOTYPE ON RAREFACTION CURVES OF SPECIES RICHNESS...........................................92

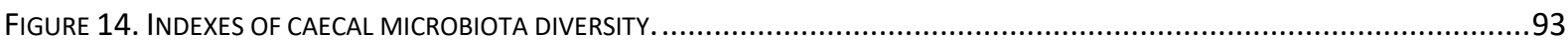

FIGURE 15. COMPOSITION OF TYPICAL MOUSE CHOW AND REFINED SEMI-PURIFIED DIETS. ............................................111

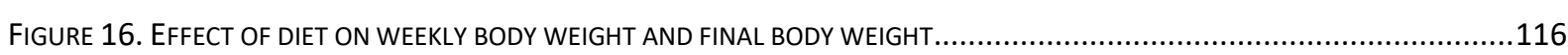

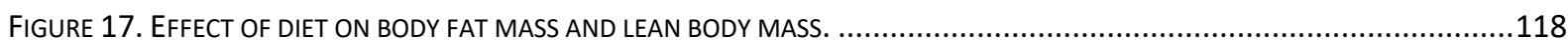

FIGURE 18. EFFECT OF DIET ON BODY FAT AND PERCENTAGE LEAN BODY MASS.............................................................119

FIGURE 19. EFFECT OF DIET ON WEEKLY AND MEAN WEEKLY FOOD INTAKE AND ENERGY INTAKE. ......................................121

FIGURE 20. EFFECT OF DIET ON ACCUMULATED AND TOTAL FOOD AND ENERGY INTAKE.....................................................122

FIGURE 21. EFFECT OF DIET ON INTRAPERITONEAL GLUCOSE TOLERANCE TEST RESPONSE. .................................................124

FIGURE 22. EFFECT OF DIET ON TOTAL, SMALL INTESTINE, CAECUM, AND COLON LENGTH.............................................126

FIGURE 23. EFFECTS OF DIET SMALL INTESTINE, CAECUM, AND COLON WEIGHT. ......................................................127

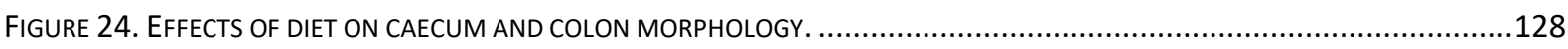

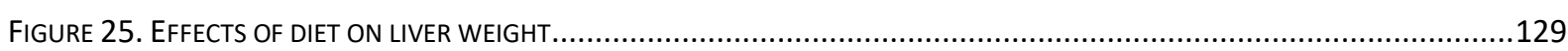

FIGURE 26. NON-METRIC MULTIDIMENSIONAL SCALING (NMDS) PLOT OF MOUSE LUMINAL GUT AND FAECAL MICROBIOTA..........131

FIGURE 27. EFFECT OF DIET ON SIMILARITY AND PHYLUM COMPOSITION OF THE MICROBIOTA. .........................................132

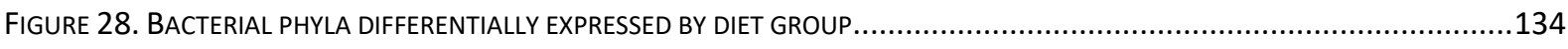

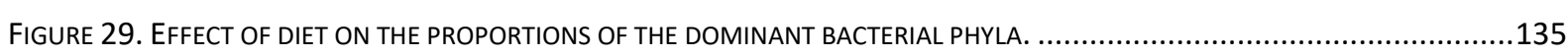

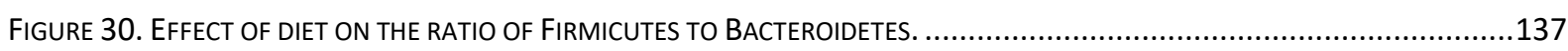

FIGURE 31. EFFECT OF DIET ON MOUSE MICROBIOTA RICHNESS AND MICROBIOTA DIVERSITY. ...........................................139

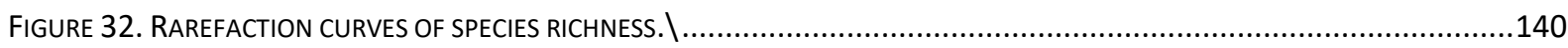

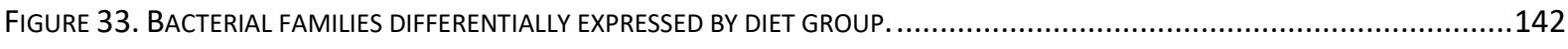

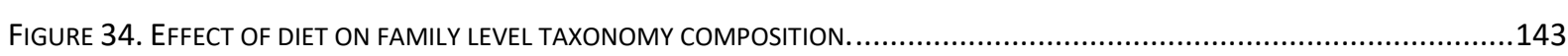

FIGURE 35. TIME COURSE OF FAMILY LEVEL COMPARISON OF FAECAL MICROBIOTA. ......................................................145

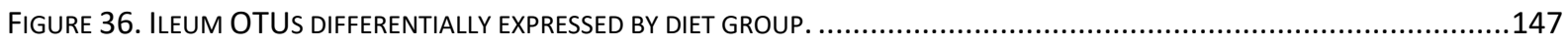


FiguRE 37. CAECUM OTUS DIFFERENTIALLY EXPRESSED BY DIET GROUP.

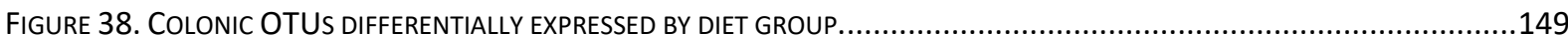

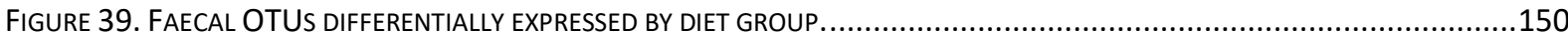

FIGURE 40. SPECIES SHOWING LARGEST CHANGES IN PROPORTIONAL ABUNDANCE DUE TO DIET. .........................................152

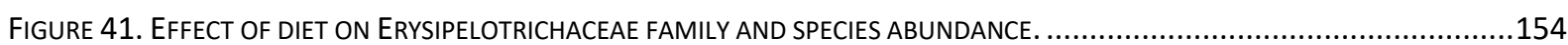

FIGURE 42. EFFECT OF DIET ON LACTOBACILLACEAE FAMILY AND SPECIES ABUNDANCE. ................................................156

FIGURE 43. EFFECT OF DIET ON BIFIDOBACTERIACEAE FAMILY AND SPECIES ABUNDANCE..............................................157

FIGURE 44. EFFECT OF DIET ON PROTEOBACTERIA PHYLUM AND SPECIES ABUNDANCE. ................................................159

FIGURE 45. EFFECT OF DIET ON AKKERMANSIA MUCINIPHILA AND MUCISPIRILLUM SCHAEDLERI..........................................161

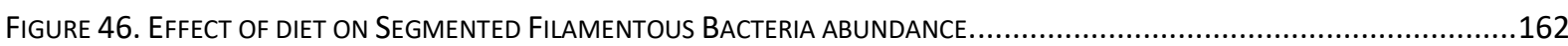

FIGURE 47. EFFECT OF DIET ON CONCENTRATION AND TOTAL CAECAL SHORT-CHAIN FATTY ACIDS. ......................................164

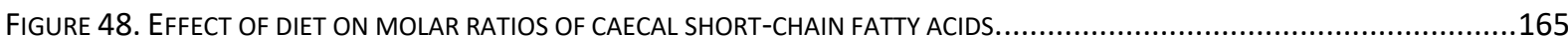

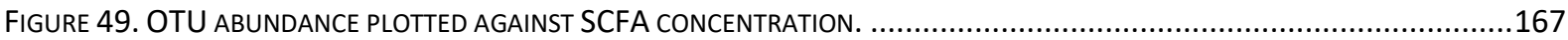

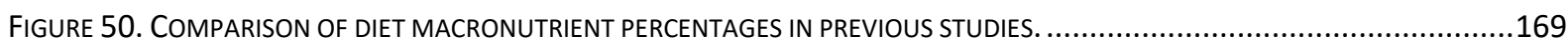

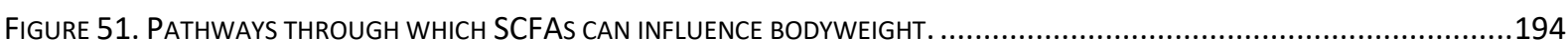

FIGURE 52. EFFECTS OF INULIN ON WEEKLY FOOD INTAKE AND ENERGY INTAKE. ..........................................................197

FIGURE 53. EFFECT OF INULIN ON OVERALL MEAN WEEKLY FOOD AND ENERGY INTAKE................................................198

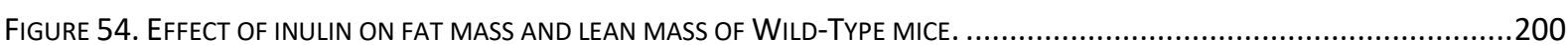

FIGURE 55. EFFECTS OF INULIN ON PERCENTAGE BODY FAT AND LEAN MASS OF WILD-TYPE MICE. .......................................201

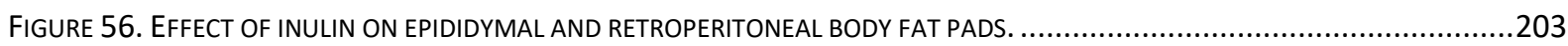

FIGURE 57. EFFECTS OF INULIN ON BODY WEIGHT GAIN AND FINAL BODY WEIGHT GAIN. ..............................................205

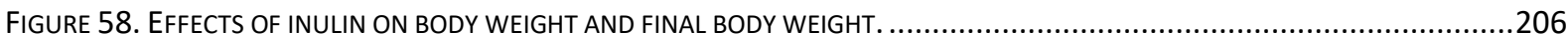

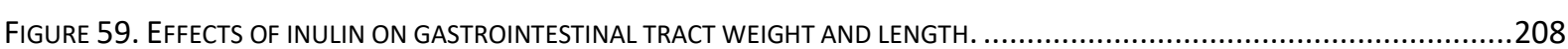

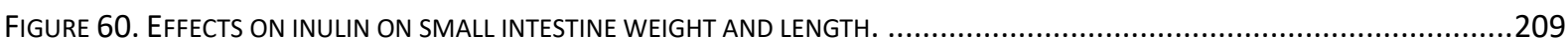

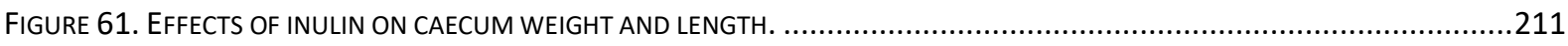

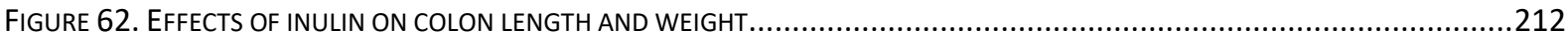

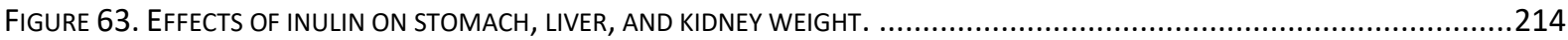

FIGURE 64. PHOTOGRAPHS OF CAECUM AND COLON MORPHOLOGY CHANGES IN RESPONSE TO INULIN..................................215

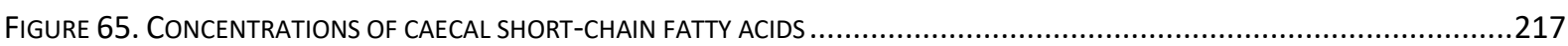

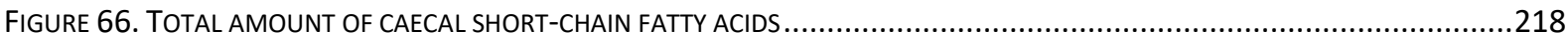

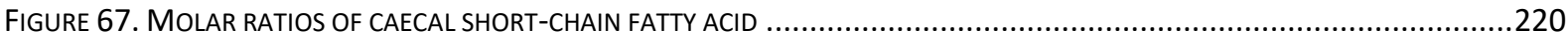

Figure 68. CAECAL CONCENTRATIONS OF ISO-ButYRATE, ISO-VALERATE, AND VALERATE. ..............................................22

FIGURE 69. WILD-TYPE CAECAL AND SERUM ACETATE CONCENTRATIONS AND CORRELATIONS ............................................223 


\section{LIST OF TABLES}

TABLE 1. ChOW, LOW-FAt, AND HIGH-FAT Diet COMPOSITION. .34

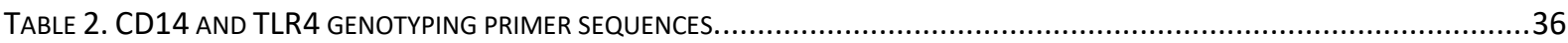

TABLE 3. LOW-FAT, HIGH-FAT+C, AND HIGH-FAT+IN DIET COMPOSITION.............................................................42

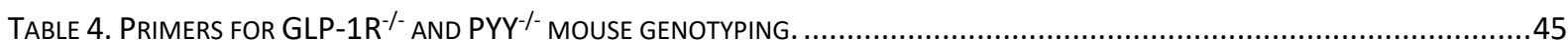

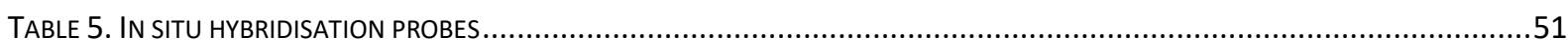

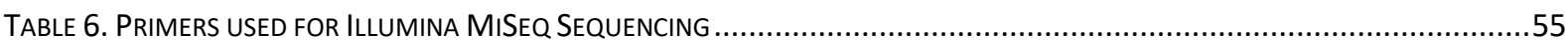

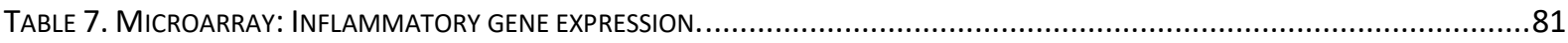

TABLE 8. MICROARRAY: GENE EXPRESSION CHANGES INVESTIGATED USING IN SITU HYBRIDISATION........................................81

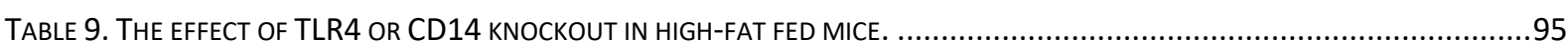

TABLE 10. EFFECTS OF CHRONIC LPS INFUSION ON BODY WEIGHT, BODY FAT, AND FOOD INTAKE. .......................................99

TABLE 11. CoRRELATIONS BETWEen OTU ABUNDANCE AND SCFA CONCENTRATION. ...................................................166

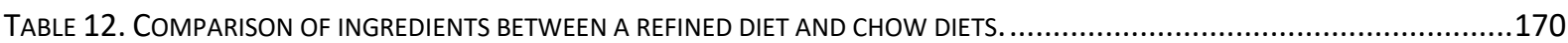

TABLE 13. HyPOthALAMIC GENES UP-REgULATEd IN HIGH-FAT DIET FED WILD-TYPE MICE .............................................2274

TABLE 14. HyPOthALAMIC GENES DOWN-REgULATED IN HIGH-FAT DIET FED WILD-TYPE MICE .........................................278

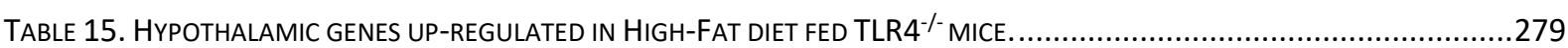

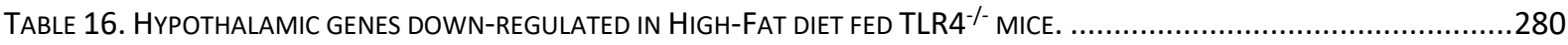




\section{LIST OF ABBREVIATIONS}

AgRP - Agouti-related peptide

CD14 - Cluster of differentiation 14

DGGE - Denaturing gradient gel electrophoresis

FFA2 - Free fatty acid receptor 2

FFA3 - Free fatty acid receptor 3

GLP-1 - Glucagon-like peptide-1

GLP-1R - Glucagon-like peptide-1 receptor

High-Fat - High-Fat diet (D12492)

High-Fat+C - High-Fat diet (D12451) with added cellulose

High-Fat+In - High-Fat diet (D12451) with added inulin (Orafti ${ }^{\circledast}$ Synergyl)

Low-Fat - Low-Fat mouse control diet (D12450J)

MYD88 - Myeloid Differentiation Primary Response 88

POMC - Pro-opiomelanocortin

PYY - Peptide YY

rRNA - Ribosomal ribonucleic acid

RELMß - Resistin-like molecule beta

SerpinA3N - Serine peptidase inhibitor, clade A, member 3N

SOCS3 - Suppressor of cytokine signalling 3

TLR2 - Toll-like receptor 2

TLR4 - Toll-like receptor 4

TLR5 - Toll-like receptor 5 


\section{CHAPTER 1.}

\section{INTRODUCTION}




\section{INTRODUCTION}

Mammals have complex microbial communities associated with them, composed primarily of bacteria, but also including archaea, fungi, and viruses (Ley et al, 2008). These microorganisms essentially coat all the skin and mucosal surfaces of the host, with the largest populations residing within the gastrointestinal tract. The interrelationship between diets, the gut flora (often called microbiota), and human health has been appreciated for over a century. As an early proponent Metchnikoff (1908) proposed that the putrefaction by microbes in the colon was responsible for aging and senility, with supplementation of bacteria rich fermented milk products as a method to avoid putrefaction (Metchnikoff, 1908). Research over the past century has concentrated primarily on the role of microbes in human health and animal disease, through the fermentative role of the intestinal microbiota in the digestion of dietary fibre (Macfarlane \& Macfarlane, 2012). More recently however, through advances in molecular biology and DNA sequencing technologies to investigate diverse microbial communities, research into the microbiota has rapidly expanded uncovering evidence of wide ranging impacts on host physiology (Walker, 2016). Over the past decade, accumulating evidence also suggests that modern diets, lifestyles, and medical care are shaping the human microbiota in novel and potentially detrimental ways to human health (Cho \& Blaser, 2012).

Simultaneously, the continued rise in rates of obesity and obesity associated health complications such as diabetes, heart disease and stroke has become one of the most prominent global public health issues. The increasing prevalence of obesity has been attributed to a predominantly sedentary lifestyle and unhealthy eating behaviours. However, the order of events that leads to the loss of energy homeostasis in obese individuals is less certain and is likely to result from a complex interplay of environmental and predisposing genetic factors. While the causes of this obesity epidemic are multifactorial, evidence has emerged that implicate an interrelationship between diet and the gastrointestinal microbiota in the aetiology and development of obesity. 


\subsection{RECENT KEY FINDINGS}

Current interest in the potential role of the intestinal microbiota in the modulation of energy homeostasis began with a publication reporting that germ-free mice, which lack an intestinal microbiota, were significantly leaner than conventionally raised mice (Backhed et al (2004). This occurred even though the germ-free mice consumed a greater amount of food. Colonisation of the gastrointestinal tract of germ-free mice with microbiota from conventionally raised mice caused a decrease in food intake yet, simultaneously, an increase in body fat (Backhed et al, 2004). A subsequent study, using leptin-deficient genetically obese ob/ob mice, identified substantial differences in the composition of the microbiota relative to their wild-type siblings at the phylum level, with most notably an increased proportion of Firmicutes and decreased proportion of Bacteroidetes as the dominant bacterial phyla in the obese mice (Ley et al (2005). Further studies on the same ob/ob mice reported that the obese mouse microbiota was enriched with bacteria of the Firmicutes phylum and with genes involved in the breakdown of complex polysaccharides, potentially enhancing energy harvest from the diet (Turnbaugh et al (2006). The pivotal finding from this study was the transplantation of microbiota from lean or obese adult donor mice into germ-free recipient mice. Fourteen days after initial the colonisation, germ-free mice with microbiota from obese mice reportedly had increased their body fat compared to germfree mice colonised with lean mouse microbiota (Turnbaugh et al, 2006). This evidence implied that alterations in the microbiota of obese mice were not only associated with obesity, but also could play a causal role in increasing body weight. This conclusion was supported using a different approach, where germ-free and conventional mice were fed a high-fat/high sugar 'Western diet' commonly used to induce obesity in mice (Backhed et al (2007). While conventional mice became obese and glucose intolerant, germ-free mice were seemingly protected against these conditions, indicating that intestinal bacteria were required for the development of obesity and associated metabolic complications (Backhed et al, 2007). Furthermore, this high-fat/high-sugar 'Western diet' resulted in increased relative abundance of the Firmicutes phyla and concomitant decrease in Bacteroidetes phyla, mirroring the changes seen in genetically obese mice (Turnbaugh et al, 2008). These diets induced changes in the mouse microbiota composition that were partially reversed after restriction of food intake. When germ- 
free mice were colonised with microbiota from lean, or diet induced obese mice, those receiving 'Western diet' associated microbiota increased their body fat more than mice colonised with microbiota from lean mice fed a rodent chow diet (Turnbaugh et al, 2008). Using humanised mice, which are germ-free mice colonised with a human microbiota, it was shown that when fed a high-fat/high-sugar 'Western diet' they exhibited similar alterations in the microbiota composition compared to obese or high fat fed conventional mice (Turnbaugh et al, 2009b). Namely germ-free mice colonised with humanised mouse microbiota from 'Western diet' fed mice increased body fat more than those colonised with microbiota from chow fed humanised mice (Turnbaugh et al, 2009b). This suggested that these effects were not limited to mouse microbiota. Nonetheless, one study that questions the simplicity of this interpretation involves the use of RELMß knockout mice, which are resistant to obesity (Hildebrandt et al, 2009). In this study it was demonstrated that RELMß knockout mice fed a high-fat diet displayed similar changes in the microbiota to high fat fed conventional mice; these were not accompanied by obesity, suggesting that microbiota composition changes were due to altered diet composition, rather than being either a cause or consequence of obesity (Hildebrandt et al, 2009).

Since 2004 the field has rapidly expanded with rodent studies providing examples of apparently causal relationships between the intestinal microbiota and obesity and have also highlighted potential mechanisms such as microbe-modulation of immune responses, potentially contributing to the development of obesity (Patterson et al, 2016). This has resulted in intense research into this area although the precise role of gut bacteria in the pathophysiology of obesity, both in terms of its development and effects, remains obscure.

\subsection{THE HUMAN GUT MICROBIOTA}

Vast numbers of anaerobic bacteria dominate the gut microbiota together with archaea, fungi, viruses, and unicellular eukaryotes that inhabit the human body. These communities of microorganisms that live in close association with their hosts have been referred to as the microbiota (Clemente et al, 2012). Approximately 500-1000 species of bacteria can be found in the human microbiota with each individual harbouring at least 160 such species (Qin et al, 2010). Interestingly, although over 50 bacterial phyla have 
been described, the microbiota is dominated by only two, the Firmicutes and Bacteroidetes, with smaller contributions from the phyla Proteobacteria, Verrucomicrobia, and Actinobacteria (Huttenhower et al, 2012; Schloss \& Handelsman, 2004).

The intestinal microbiota is not homogenous throughout the gut. The number of bacterial cells follows a gradient starting at approximately $10^{1}$ to $10^{3}$ cells per gram in the stomach and duodenum contents, and increasing up to $10^{11}$ to $10^{12}$ cells per gram in the caecum and colon (Sekirov et al, 2010). Another gradient is present from the lumen to the epithelium of the gut, with large numbers of bacteria in the lumen, but few adhering to the mucus or epithelium of the colon (Sekirov et al, 2010). Most species reside in the lumen, while a few well-adapted species of bacteria can adhere to, and reside within, the mucus layer close to the tissue (Swidsinski et al, 2005). Bacterial cells in the gut have been thought to vastly outnumber the cells of their host. Estimates have put the number of cells in the adult human body at approximately $10^{13}$, whereas it is estimated than the human body contains at least $10^{14}$ microbial cells, meaning that bacterial cells outnumber human host cells by a ratio of 10:1. (Luckey, 1972; Savage, 1977). However, this historical estimate has recently been critically reviewed and reanalysed with new estimates of the numbers of both bacterial and host cells in the human body estimated at a more equal 1:1 ratio (Sender et al, 2016). Furthermore, at each defecation event, there is the potential for the ratio to move towards favouring human cells over bacteria (Sender et al, 2016).

The combined genomes of these bacterial populations contain more than 5 million different genes, outnumbering the genetic material of their host by two orders of magnitude (Gill et al, 2006). This extensive battery of genes provides for a diversity of metabolic and biochemical activities to complement the physiology of their host. This includes the ability to synthesise vitamins, facilitate the metabolism of an array of otherwise indigestible dietary polysaccharides, as a requirement for the normal differentiation and development of the host immune system, and to confer protection against colonisation of pathogens (Smith et al, 2007).

Until recently the inhabitants of the gut have remained something of a mystery, but this has now changed and the study of the intestinal microbiota has expanded hugely in 
recent years. This is due largely to developments in molecular biology and the culture free analysis of the mammalian intestinal microbes, which have proved difficult to grow due to their anaerobic growth requirements. Molecular profiling has enabled interrogation of bacterial communities inhabiting the distal gut, thereby enabling fundamental advances in our understanding of the complex communities inhabiting the human colon (Walker, 2016). However, much is still to be investigated, especially with regards to the fungi, viruses, and protozoa where research has lagged behind that of the bacterial microbiota (Huffnagle \& Noverr, 2013; Minot et al, 2011; Parfrey et al, 2011).

\subsection{THE HUMAN GUT MICROBIOTA IN OBESITY}

While there is evidence for a potential causal link between the gut microbiota and development of obesity in mouse models, the evidence for such a linkage from human studies has proven contradictory. In a pioneering study, twelve obese individuals were shown to have reduced proportions of Bacteroidetes and increased Firmicutes, in comparison to faecal samples taken from two lean individuals (Ley et al (2006). Furthermore, the ratio of Firmicutes to Bacteroidetes decreased in the obese individuals following weight loss interventions (Ley et al, 2006). Comparison of lean and obese twins showed a reduction of bacterial species diversity in obese twins, associated with a decreased proportion of Bacteroidetes and increased Actinobacteria in the faecal microbiota (Turnbaugh et al, 2009a). However, efforts to replicate these findings in other studies have failed to confirm these associations. The proportions of Firmicutes and Bacteroidetes did not differ in the microbiota of lean and obese subjects when determined by fluorescent in situ hybridization (Duncan et al, 2008). In a larger study of 98 healthy subjects, Schwiertz et al (2010) showed that the Bacteroidetes phyla was increased in overweight and obese subjects, contrasting with previous reports. A recent reanalysis of the data sets of 10 previous studies could not identify differences in Bacteroidetes and Firmicutes phyla in obese individuals, but did report a small reduction in bacterial diversity (Sze \& Schloss, 2016). The authors also state that the relationships between the faecal microbial communities and obesity were relatively weak and that previous studies were underpowered to identify differences, with the large degree of interpersonal variations (Sze \& Schloss, 2016). 
Human subjects have variable individual microbiota, due to the stochastic nature of their acquisition, and uncontrolled diet and lifestyles. For these reasons animal models have formed a large part of the research investigating interactions between the intestinal microbiota and obesity. Using genetically identical mice fed the same controlled diets has the potential of limiting variability and confounding factors in microbiota research.

\subsection{MOUSE MICROBIOTA}

The intestinal microbiota of laboratory mice is similar to human microbiota in their composition of the bacterial phyla, dominated by Firmicutes and Bacteroidetes, with smaller proportions of Actinobacteria, Proteobacteria, and Verrucomicrobia (Ley et al, 2005). Although $85 \%$ of the sequences found in the mouse microbiota represented genera that have not been detected in humans, considerable similarities were observed between mouse and human colonic microbiotas at the phylum level (Ley et al, 2005). Despite these similarities at a phylum level, the microbiota of humans and mice differ in the Families and Genera of bacteria. The relationship between the mammalian host and its microbiota has been shaped by evolution, resulting in host specific microbiota interactions (Frese et al, 2013), and this limits the ability to extrapolate findings to humans from conventionally-raised mice.

A solution to this problem is the use of humanised mice, germ-free mice with a complex microbial community, obtained from donated human faecal microbiota (Gootenberg \& Turnbaugh, 2011). Recent high-profile research investigating microbiota obesity interactions have used this approach to investigate changes in the human gut microbiota under controlled conditions (Ridaura et al, 2013; Turnbaugh et al, 2009b). However, there are potential limitations to this approach which have yet to be fully resolved (Arrieta et al, 2016). The efficiency of colonisation by human gut microbiota has been questioned by Chung et al (2012), with key taxa of the human gut microbiota failing to colonize the gut of the mouse, or to bloom in way that is not representative of the human gut. Additionally, humanised mice fail to develop normal immune responses after colonisation, suggesting that normal immune maturation is dependent on host-specific gut microbiota interactions (Chung et al, 2012). Significant differences have also been observed in the metabolome of humanised mice compared to conventional mice (Marcobal et al, 2013). Reciprocal microbiota transfer between mice and zebrafish 
suggests that the host has a strong effect in shaping the composition of the gut microbiota (Rawls et al, 2006). As examples, the endogenous glycans that provide a substrate for the growth of many microbial species differ between mice and humans (Koropatkin et al, 2012). It is likely that human adapted microbes may not be as adept at utilizing mouse host glycans (Frese et al, 2013). Most studies have not investigated in detail whether human microbiota transplantation into mice generated humanized recipients that fully represented the microbiota of their donors.

\subsection{THE MOUSE MICROBIOTA IN OBESITY RESEARCH}

Several different mouse models of obesity are used for investigating the links between the gut microbiota and obesity. Among these are the genetically obese ob/ob mice, which lack the ability to produce a functional form of the adipose hormone leptin and without which results in obesity (Wang et al, 2014). These ob/ob mice can be used as models of obesity when fed a standard mouse chow diet and have been utilised in a number of studies to investigate the changes that occur in the composition of the microbiota relative to mice lacking the ob mutation (Ley et al (2005); Everard et al (2013). In addition to the use of genetically obese mice and rats as models of obesity, diet induced obese rodent models have also been more commonly used to study the role of microbiota in energy balance and obesity (Tschop \& Heiman, 2001). High-fat diets containing a large proportion of calories as fat are now commonly used in the study of obesity in rodents (Buettner et al, 2007; Tschop \& Heiman, 2001). Dietary fat has been found to be a key component for inducing obesity in susceptible strains of mice, particularly in the C57BL/6 strain, and this has led to the C57BL/6 strain of mice fed a high-fat diet becoming the most popular model of diet-induced obesity (Surwit et al, 1995). High-fat diets are typically compared against a low-fat control diet (high in plant polysaccharides), and this diet combination been commonly used in mouse microbiota research (Turnbaugh et al, 2008).

\subsection{GERM-FREE MICE}

The germ-free mouse has become a cornerstone of microbiota research, providing a means to isolate the effect of donor human or mouse microbiota from the confounding influence of the host microbiota (Zhao, 2013). Germ-free mice and rats have been reported to be leaner than their conventional counterparts (Backhed et al, 2004; 
Levenson, 1978). Germ-free rats were also observed to eat more food and excrete more energy in their faeces (Wostman et al, 1983). The first germ-free animal was created over a century ago, and by the latter half of the $20^{\text {th }}$ century the practice was advanced enough for germ-free animals to be widely used in nutritional studies (Wostmann, 1981). Germ-free mice are raised and bred under sterile conditions in the absence of the normal microbes found in and around the body. A normal mouse microbiota can be introduced and established into a germ-free mouse via the introduction of a faecal inoculum from a donor mouse, a process known as conventionalisation (Khoury et al, 1969). Germ-free mice can also be inoculated with a small number of defined bacteria and maintained under sterile conditions (Schaedler et al, 1965). Both germ-free mice and those with a defined microbiota are known as gnotobiotic, meaning that all the microorganisms present in the mouse are either known or excluded.

The observation by Backhed et al (2004) that germ-free mice are leaner than their conventional counterparts and the development of microbiota transfer (Turnbaugh et al, 2006), has resulted in the widespread use of germ-free mice. Compared to germ-free C57BL/6 mice, conventionally raised C57BL/ 6 counterparts were about $42 \%$ fatter, and have ca. $47 \%$ more epididymal fat when fed a standard rodent chow (Turnbaugh et al, 2006). This was despite the conventional mice consuming $29 \%$ less food than the germfree mice. Fourteen days after colonisation with an unfractionated microbiota harvested from the caecum of adult conventional mice, conventionalised germ-free mice had increased their total body fat by $57 \%$ and their epididymal fat by $61 \%$ (Turnbaugh et al, 2006). This increase in body fat was accompanied by a corresponding $27 \%$ reduction in food intake. These changes were not limited to male C57BL/6 mice, as similar changes were seen in female C57BL/6 and also male NMRI mice (Backhed et al, 2004). These findings suggested that the microbiota could have a large influence on host metabolism. However, it should be noted that not all studies have supported this interpretation across species, with a study by Swartz et al (2013) finding no difference in body weight or body fat in germ-free Fischer 344 rats, as compared to their conventionally raised controls.

In addition to body fat and food intake changes, germ-free mice have several other physiological differences that were changed with conventionalisation (Backhed et al, 2007). These included an increased metabolic rate, increased leptin, elevated fasting 
glucose and insulin levels, increased monosaccharide uptake from the gut, a doubling of the density of capillaries underlying the small intestinal villus epithelium, and increased triglyceride production in the liver (Backhed et al, 2004).

\subsection{GERM-FREE MICE AND OBESITY}

The findings that germ-free C57BL/6 mice were protected against the obesity that develops after consuming a 'Western-style' diet rich in fat and sugar suggested that the microbiota was an essential driver in obesity development (Backhed et al, 2007). In this study, adult germ-free C57BL/6 male mice were either conventionalised or maintained in their germ-free state and then fed a 'Western diet', with $41 \%$ of calories in the form of fat and $41 \%$ as readily-digestible carbohydrates including sucrose. Conventionalised animals on the 'Western diet' gained significantly more body weight than their germfree counterparts, with germ-free not significantly different than the weight gain observed in germ-free mice that had been maintained on the standard chow. However, this resistance to diet-induced obesity in germ-free mice was shown subsequently to be diet-specific. When germ-free $\mathrm{C} 3 \mathrm{H}$ mice were fed a high-fat diet based on coconut oil, instead of the lard and sucrose as used by Backhed et al (2004), the germ-free mice gained more weight and became fatter than their conventional counterparts (Fleissner et al (2010). This suggests that both diet and mouse strain can potentially influence the effects of the germ-free state.

This may be accounted for by the fact that germ-free mice have important physiological impairments relative to conventional mice. In this regard, the colonisation of mice with a microbiota has been shown to be instrumental in the normal development of several aspects of mouse physiology (Backhed et al, 2007). The intestinal villi of germ-free mice have a reduced capillary network density, potentially reducing nutrient absorption (Stappenbeck et al, 2002). The intestinal villi of germ-free mice are also thinner and the epithelium shows slower renewal rates (Banasaz et al, 2002). Germ-free rats also show reduced numbers of enteroendocrine cells in the intestinal epithelium (Uribe et al, 1994). The total surface area of germ-free mice is also reduced (Gordon \& Brucknerkardoss, 1961). Conventionalisation of germ-free mice with microbiota harvested from a conventional mouse increases energy harvest from the fermentation of dietary polysaccharides and increases glucose uptake in the small intestine (Backhed et 
al, 2004; Wolin, 1981). Backhed et al (2007) also reported no difference in the energy content of the faeces of germ-free mice. This has been questioned based on the bomb calorimetry used, which may not suitable for comparing energy excretion of germ-free and conventional mice due to the high content of undigested dietary fibre in the faeces of germ-free mice (Rabot et al, 2010). Measuring faecal lipids found significantly greater excretion of lipids in high-fat diet fed germ-free mice, indicating an inability to efficiently absorb lipids from the diet and accounting for the reduced body weight gain in the germ-free mice (Rabot et al (2010).

\subsection{MICROBIAL TRANSPLANTATION}

While many associations which show changes in gut microbiota composition and health outcomes have been reported, proving that there is a causal relationship beyond simple correlation has been challenging (Zhao, 2013). The transfer of whole microbiota, or isolated bacterial strains, into germ-free mice has formed the basis of assigning causality in microbiota research. Turnbaugh et al (2006) was the first to use microbiota transplantation to show that the obese phenotype of genetically obese ob/ob mice could be transferred to germ-free mice. Colonization of germ-free mice with an 'obese microbiota' resulted in a $47 \%$ increase in body fat, versus $27 \%$ when colonization with a 'lean microbiota' (Turnbaugh et al, 2006). However, this was limited to two weeks after colonisation and details of how the transfer was carried out were not provided. This was later repeated using germ-free mice colonised with a human donor microbiota (Turnbaugh et al, 2009b). These humanised mice were fed either a high-fat, high-sugar

'Western diet', or a standard low-fat, plant polysaccharide-rich diet. After 8 weeks of feeding these diets the microbiota of mice fed either the 'Western diet' or low-fat diet was transplanted into germ-free mice. The colonisation of mice with microbiota from obese 'Western diet'-fed humanized donors resulted in a gain of $68.5 \%$ of total body fat compared to $34.5 \%$ in mice colonized with the humanized microbiota from low-fat diet fed donors (Turnbaugh et al, 2009b).

It was also reported that TLR5 knockout mice developed obesity and metabolic syndrome due to knockout induced microbiota dysbiosis (Vijay-Kumar et al, 2010). This TLR5 mouse microbiota was reported to transmit the obese and metabolic phenotype to germ-free mice through microbiota transfer. Transfer of microbiota from lean or 
obese rats into mice also reportedly transmitted the obese phenotype to the mice (Duca et al, 2014). However, this paper has recently been retracted due to in inclusion of fabricated images (Duca et al, 2016). To limit the influence of genetics on microbiota composition used in transplant experiments, the microbiota from identical twins, but discordant for obesity, have been used in a recent microbiota transfer study (Ridaura et al, 2013). It was found that on transplantation of microbiota from the donor twins into germ-free mice, the phenotype of the recipient mice followed that of the donor; namely, increased total body and fat mass were transmissible with faecal communities from the obese twin. Much of the research so far has used germ-free mice as recipients although, as previously described, germ-free mice are not physiologically equivalent to conventional mice (Backhed et al, 2007). An alternative approach is to deplete the microbiota of conventional mice using antibiotics to recreate some of the physiological effects of the germ-free state (Reikvam et al, 2011). The transfer of obese microbiota from $\mathrm{ob} / \mathrm{ob}$ mice into either antibiotic-depleted C57BL/6 mice, either at weaning or as adults, was found to have no effect on body weight or body fat over a 10 week study period (Ellekilde et al, 2014). More research is needed to show if there are consistent differences between germ-free and antibiotic-treated conventional mice in the effects of transferring the obesity phenotype.

Perhaps of more relevance to humans is a recent case study of a female subject who gained weight after receiving a faecal transplant from an overweight relative to treat a Clostridium difficile infection (Alang \& Kelly, 2015). This raises the question of whether transfers of lean donor microbiota could transfer the lean phenotype in humans. A pilot study has attempted to test this by infusing lean donor microbiota into 9 obese subjects and assessing this against 9 obese subjects receiving an infusion of their own microbiota as controls (Vrieze et al, 2012). After 6 weeks, a small reduction in insulin resistance was observed in subjects receiving the lean microbiota however, there was no effect on body weight. Thus, at present, while transplantation studies in mice suggest that microbiota can have a causative role in increasing body weight, further studies are required in the area of human microbiota transplants. 


\subsection{THE ROLE OF INDIVIDUAL BACTERIAL SPECIES IN OBESITY DEVELOPMENT}

In recent years, the focus has shifted from broad phylum level changes in composition of the microbial ecosystem to more of a focus on the role of individual bacterial species in obesity.

\subsubsection{AKKERMANSIA}

Akkermansia muciniphila is a common intestinal bacterium, of the phylum Verrucomicrobia, inhabiting a specialised niche as a mucus degrader in the mucus layer lining the gut of both rodents and humans at all stages of life (Collado et al, 2007; Derrien et al, 2008; Derrien et al, 2004). Evidence has emerged in recent years of a range of beneficial effects of $A$. muciniphila colonisation including improved intestinal integrity and modulation of mucosal immune responses (Collado et al, 2007; Derrien et al, 2011). Other roles that $A$. muciniphila plays in regulating host function have been reviewed recently (Derrien et al, 2016). Increasing $A$. muciniphila in young NOD mice reduced diabetes incidence and, the increased $A$. muciniphila in adult mice resulting from metformin treatment improved glucose homeostasis (Hansen et al, 2012; Shin et al, 2014). The proportion of $A$. muciniphila decreased with increased obesity in high-fat diet fed mice (Schneeberger et al, 2015). Additionally, A. muciniphila increased with higher levels of fermentable fibre in the diet of rats (Van den Abbeele et al, 2011). There are also recent reports that supplementation of mice with $A$. muciniphila reduces body weight gain and prevents the thinning of the mucosal layer in high-fat diet fed mice (Everard et al, 2013). Rather than bacteria driving weight gain, this suggests that a lack of certain bacteria such as $A$. muciniphila may be a causal factor in obesity development.

\subsubsection{CHRISTENSENELLA MINUTA}

Christensenella minuta is a recently characterised bacterium inhabiting the human gut and representing a distinct branch of the order Clostridiales (Morotomi et al, 2012). Comparing the microbiotas across over a thousand samples from twins, both identical and fraternal, the family Christensenellaceae was identified as the most highly inherited bacterial group. Furthermore, increased abundance of Christensenellaceae was found in individuals with a lower body mass index (Goodrich et al, 2014). Transfer of a microbiota 
from an obese individual, supplemented with $C$. minuta, into germ-free mice reduced weight gain compared to those receiving the same microbiota lacking in C. minuta (Goodrich et al, 2014). As this was one of the most highly inherited bacterial groups it suggests that host genetics could shape the composition of the microbiota and, through that, influence host metabolism.

\subsubsection{CLOSTRIDIUM RAMOSUM}

An increase in the proportion of bacteria within the class Erysipelotrichi has previously been observed in mouse models of obesity (Turnbaugh et al, 2008; Turnbaugh et al, 2009b) and in obese adolescent subjects (Ferrer et al, 2013). Clostridium ramosum, a member of the Erysipelotrichi, was recently linked to symptoms of metabolic syndrome in women with type 2 diabetes (Karlsson et al, 2013). Woting et al (2014) investigated the potential role of $C$. ramosum in obesity development using gnotobiotic mice. Germfree mice were colonised with a simplified human intestinal (SIHUMI) consisting of eight bacterial species, with or without the addition of $C$. ramosum, and fed a high-fat or a low-fat diet. For mice fed the high-fat diet, those colonised with $C$. ramosum gained significantly more body weight and body fat over 4 weeks, and showed a higher food efficiency than mice without $C$. ramosum. This was associated with increased glucose transporter and fatty acid translocase 2 expression in jejunal mucosa of $C$. ramosum colonised mice suggesting increased absorption of nutrients. In contrast, C. ramosum showed no effect on mice fed the low-fat diet indicating that any obesogenic effect was restricted to the high-fat diet intervention (Woting et al, 2014). In contrast to $A$. muciniphila and C. minuta, this is a potential bacterium contributing directly to obesity via increased nutrient absorption. How these different bacteria with contrasting effects on energy balance and body weight might interact in a complex human or mouse microbiota is still to be investigated.

\subsection{MECHANISMS THOUGH WHICH THE GUT MICROBIOTA CAN MODULATE OBESITY}

Several mechanisms have been proposed to explain the observed influence of the gut microbiota on changes in body weight. To clarify the role of the gut microbiota in obesity two questions need to be answered. Does the microbiota influence obesity directly, or 
via bacterial components or metabolic products, and if so what are the specific bacterialhost signalling mechanisms that lead to obesity.

\subsubsection{IMMUNOMODULATION OF THE HOST IMMUNE SYSTEM}

As well as having an important role in influencing metabolic functions in nutrient digestion, absorption, and metabolism the gut microbiota also influence the host through interactions with the immune system.

\subsubsection{TOLL-LIKE RECEPTOR 5 (TLR5)}

Bacterial flagella are polymers approximately $15 \mathrm{~nm}$ in diameter and as much as $15 \mu \mathrm{m}$ in length, and used by bacteria such as E. coli to make them motile by rotating the flagella that lie on their surfaces. Flagella are composed of subunits of a protein called flagellin, which is the natural ligand for the Toll-like receptor 5 (TLR5), expressed in cells of the intestinal lamina propria. Deletion of TLR5 in mice alters host susceptibility to Salmonella infection (Uematsu et al, 2006). TLR5 knockout mice have been reported to suffer from spontaneous colitis and intestinal inflammation due to the loss of TLR5 function (Vijay-Kumar et al, 2007). While the minority of these TLR5-/mice with severe colitis suffered weight loss, the larger proportion exhibited broadly elevated inflammation and, by 4 weeks of age, these mice had body masses that were, on average, $15 \%$ higher than their wild-type littermates. Rederiving these mice using embryo transfer into mice from the Jackson Laboratory attenuated the severity of colitis in TLR $^{-1-}$ mice resulting in a uniform mild intestinal inflammation, obesity, and other features of the metabolic syndrome (Vijay-Kumar et al, 2010). This obesity and metabolic syndrome phenotype was transferable to germ-free mice after inoculation with TLR5 $^{-1-}$ intestinal contents. Similar outcomes were seen again later with mild inflammation increasing body weight in TLR5 ${ }^{-/-}$mice (Carvalho et al, 2012).

However, the reproducibility of these findings has been questioned. TLR5 ${ }^{-1-}$ from the same original source and maintained at two different institutions did not suffer from intestinal inflammation, metabolic abnormalities, or demonstrate increased body weight and fat (Letran et al, 2011). The composition of the microbiota of TLR5 ${ }^{-/}$mice were reported to differ between two different institutions, with each colony harbouring distinct and distinguishable intestinal bacterial populations (Ubeda et al, 2012). The 
intestinal microbiota of TLR5 ${ }^{-/-}$mice used by Vijay-Kumar et al (2010) was found to be distinctly different from TLR5 ${ }^{--}$mice obtained from other mouse colonies (Ubeda et al, 2012). Therefore, the institution in which the mice are bred, not the gene knockout, seems to shape the intestinal microbiota (Ubeda et al, 2012). Overall, the evidence suggests that the loss of TLR5 can result in obesity and metabolic complications, but has been shown only to occur in one specific situation and is not applicable to mice in general.

\subsubsection{TOLL-LIKE RECEPTOR 2 (TLR2)}

The Toll-like receptor 2 is another receptor of the innate immune system implicated in the link between immune regulation and metabolism. As a membrane surface receptor, TLR2 recognizes many bacterial, fungal, viral, and certain endogenous substances. The gut represents a large pool of antigens potentially able to stimulate this receptor; one such molecule is lipoteichoic acid, a major component of Gram-positive bacteria. The loss of TLR2 has been reported to protect against increases in adiposity and metabolic complications resulting from high-fat died feeding (Himes \& Smith, 2010), although, in another study, the absence of TLR2 in TLR2 ${ }^{--}$mice protected against high-fat diet induced inflammation but did not prevent the development of obesity (Davis et al, 2011). In contrast to these results, Takeuchi et al (1999) reported that loss of TLR2 function resulted in obesity and exacerbated the effects of diet induced obesity (Shechter et al, 2013). In a later study, TLR2 ${ }^{-/-}$mice suffered subclinical inflammation, increased insulin resistance, glucose intolerance, and late onset obesity (Caricilli et al, 2011). Thus, these conflicting results make it difficult to draw any firm conclusions about the role of TLR2 in diet induced obesity in mice.

\subsubsection{TOLL-LIKE RECEPTOR 4 (TLR4)}

Lipopolysaccharide (LPS) is an important structural component of the outer membrane of Gram-negative bacteria. It is one of the most studied immunostimulatory molecules produced by bacteria and, in cases of severe infection of the blood, results in sepsis (Beutler \& Poltorak, 2001). The structure of LPS is composed of three parts, lipid A, a core oligosaccharide, and an $\mathrm{O}$ side chain, with the Lipid A being the part of the molecule that binds to TLR4 (Raetz \& Whitfield, 2002). In addition to TLR4, LPS induced stimulation of cellular responses also involve interactions with several proteins, 
including lipopolysaccharide binding protein (LBP), CD14, and myeloid differentiation factor 2 (MD2) (Gioannini \& Weiss, 2007). LBP is a soluble circulating protein that binds to LPS directly and facilitates the binding of LPS and CD14 (Wright et al, 1989). CD14 is a cell surface protein anchored in the plasma membrane which facilitates the transfer of LPS to the TLR4 receptor complex and facilitates LPS recognition. CD14 forms an essential co-receptor for TLR4 that is involved in binding the LPS molecule to the receptor and, in the absence of CD14, signalling through TLR4 is lost (Wright et al, 1990). MD2 forms a heterodimer with the TLR4receptor that recognizes a common pattern in LPS molecules. Activation of the TLR4 receptor complex results in signalling through two separate intracellular signalling pathways via the downstream adaptors proteins MyD88 and the MyD88 independent TRIF pathway (Lord et al, 1990; O'Neill \& Bowie, 2007). Both signalling pathways can result in the subsequent translocation of the transcription factor NF- $\kappa B$ from the cytoplasm to the nucleus which, in addition to other immune related genes, controls the expression of proinflammatory cytokines and results in inflammatory responses within the cell ( $\mathrm{Lu}$ et al, 2008). CD14 also acts as an important co-receptor for TLR2 and the loss of CD14 attenuates responses to infection involving TLR2 (Janot et al, 2008; Raby et al, 2013; von Meyenburg et al, 2004).

In addition to LPS, saturated fatty acids can also act as ligands for TLR4 activation. There has been some controversy over the activation of TLR4 directly by saturated fatty acids, with the suggestion that in vitro experimental procedures may have been potentially confounded by contamination with LPS (Erridge \& Samani, 2009). More recent studies have shown that saturated fatty acids can activate TLR4 through a Fetuin A dependent mechanism, providing a potential route by which saturated fatty acids can stimulate TLR4 inflammatory responses (Trepanowski et al, 2015). Additionally, other endogenous ligands have been proposed to interact with TLR4, either directly or indirectly. These include heat-shock proteins, hyaluronic acid, and $\beta$-defensin 2 (Biragyn et al, 2002; Ohashi et al, 2000; Termeer et al, 2002). The relevance of these to the relationship between TLR4 and obesity remains to be determined.

\subsubsection{EVIDENCE LINKING TLR4 SIGNALLING TO OBESITY}

The first study to propose a link between TLR4 signalling, body weight, and adiposity included three different knockout mice strains (Johnson et al, 2004). These included 
the $\mathrm{CD} 14^{-/-}$mice and two different strains of TLR4 $4^{-/-}$mice. This study reported that mice harbouring mutations in either TLR4 or CD14 had an "ideal" body composition, increased bone mineral content, density, size, and decreased body fat. The observation by Cani et al (2007a) that feeding of a high-fat diet to mice resulted in chronically increased levels of circulating LPS derived from the gut, termed metabolic endotoxemia, suggested a potential link between diet-induced microbiota changes and systemic inflammation through the TLR4. In mice lacking a functional CDI4 gene, the effect of the high-fat diet on body weight was reported to be attenuated (Cani et al, 2007a). As a confirmation of the causal role of LPS in this response, LPS was chronically infused into mice with the aid of an implanted subcutaneous minipump to replicate the circulating levels seen with high-fat diet feeding. Chronic infusion of LPS for 4 weeks was reported to increase body fat and replicate some of the negative effects seen with high-fat diet feeding (Cani et al, 2007a). This appeared to show a causal link through which bacteria in the gut could influence body weight and adiposity. Increased LPS absorption may occur through decreased expression of tight-junction proteins in the intestinal epithelium, increasing the leakiness of the gut, or via incorporation of LPS into chylomicrons and absorbed along with dietary fatty acids (Ghoshal et al, 2009; Kim et al, 2012). In support of this, $\mathrm{C} 3 \mathrm{H} / \mathrm{HeJ}$ mice lacking a functional TLR4 gene, due to a natural point mutation, were reported to be protected from high-fat diet induced obesity with decreased body weight and body fat compared to control mice with a functional TLR4 (Tsukumo et al, 2007). However, not all studies involving mice with nonfunctional TLR4 have reported protective effects against obesity. Suganami et al (2007) fed $\mathrm{C} 3 \mathrm{H} / \mathrm{HeJ}$ mice and control mice a high-fat diet or a low-fat control diet for 16 weeks and reported no significant differences in body weight on either diet between mice with or without functional TLR4. Similarly, Shi et al (2006) found that, in female C57BL/6 mice lacking TLR4, while the loss of TLR4 protected against high-fat diet induced insulin resistance, these female mice became significantly more obese than control mice with a functioning TLR4.

While there is conflicting evidence in the literature the signalling of gut derived LPS through the TLR4 remains the most promising mechanism through which gut bacteria can modulate body weight via the host immune system. More work will be needed to 
resolve the conflicting evidence in the literature and further research is needed to confirm this mechanism of action.

\subsubsection{KNOCKOUT TLR4 AND CD14 MICE USED IN RESEARCH}

Evidence from research on TLR4 receptor signalling is complicated by the use of a number of different knockout strains with different origins. The CD14 ${ }^{-/}$mouse B6.129SCd14tmlFrm originated in 2000 and was created using a targeting vector to disrupt the entire CD14 coding region and back crossed to a C57BL/6J mouse background (Moore et al, 2000). The TLR4 ${ }^{-/-}$mouse strain $\mathrm{C} 3 \mathrm{H} / \mathrm{HeJ}$ originated when some mice of the control strain $\mathrm{C} 3 \mathrm{H}$ were found to have a defective response to bacterial LPS (Sultzer, 1972). Recently this was identified as a spontaneous single nucleotide missense mutation in the TLR4 gene rendering the protein non-functional (Poltorak et al, 1998). The $\mathrm{C} 3 \mathrm{H} / \mathrm{HeJ}$ mouse is a strain of the $\mathrm{C} 3 \mathrm{H}$ mouse lacking a functional TLR4 that has been back-crossed into BALB/c to introduce the $\mathrm{C} 3 \mathrm{H} / \mathrm{HeJ}$ TLR4 mutation into the BALB/c mouse strain. The $\mathrm{TLR} 4^{-/-}$mouse $\mathrm{C} 57 \mathrm{Bl} / 10 \mathrm{ScNCr}$ originated as a spontaneous deletion in mouse strain C57BL/10ScCr (Coutinho \& Meo, 1978). This deletion removed 74723 bp of DNA removing the entire TLR4 gene, no other known genes are thought to be effected (Poltorak et al, 2000). C57Bl/10ScNCr were later backcrossed with C57BL/6J mice making this mutation available in the more commonly used strain. Another TLR4 knockout mice has been produced using a targeting vector to replace a 2.54-kbp genomic fragment of the TLR4 gene (Hoshino et al, 1999).

\subsection{FASTING-INDUCED ADIPOSE FACTOR}

Fasting-induced adipose factor (FIAF), a protein produced by the adipocytes, the liver, and the intestine, has been proposed as another mechanism through which microbiota can influence body weight. FIAF is a secreted protein acting as an inhibitor of lipoprotein lipase. The secretion of intestinal derived FIAF was reported to be suppressed in germfree mice after conventionalisation with a mouse microbiota (Backhed et al, 2004). By suppressing FIAF, conventionalisation resulted in increased lipoprotein lipase activity in adipose tissue, enhancing the storage of triglycerides (Backhed et al, 2004). Additionally, germ-free knockout mice lacking FIAF were not protected from dietinduced obesity (Backhed et al, 2007). However, the role of FIAF has been questioned 
by later work and the relevance of FIAF in interactions between the microbiota and its host remains unresolved (Fleissner et al, 2010).

\subsection{INCREASED ENERGY HARVEST}

An important role of the gut microbiota in the caecum and colon is the extraction and salvage of energy from food that escapes host digestion in the small intestine. Through fermentation, the bacteria in the gut produce as a by-product short-chain fatty acids that are absorbed and used for energy, salvaging some of the energy that would otherwise be lost in the faeces (Cummings et al, 1995). Increased extraction of energy has been proposed as a potential effect of the gut microbiota in obesity (Turnbaugh et al, 2006). Genetically obese ob/ob mice were observed to have a higher concentration of short-chain fatty acids in their caecal contents and lower gross energy content in their faeces than their lean heterozygous littermates (Turnbaugh et al, 2006). This was interpreted as evidence that the obese microbiota has an increased capacity to harvest energy from the diet. In another study, the joint colonisation of germ-free mice with a bacterium, Bacteroides thetaiotaomicron, an adaptive forager of dietary polysaccharide, and an archaeon Methanobrevibacter smithii, could extract more energy from the diet and increase adiposity more than B thetaiotaomicron alone, contributing to increased body weight (Samuel \& Gordon, 2006). Increased concentrations of short-chain fatty acids have also been observed in overweight and obese human subjects, as compared to lean individuals, although the numbers of subjects involved were small (Schwiertz et al, 2010). This mechanism is difficult to test in humans as, SCFA concentrations in the colon are the result of the dynamic interplay between production and absorption and therefore faecal SCFA concentrations are not an accurate reflection of their production in the gut (Cummings et al, 1987; den Besten et al, 2013b). The evidence for increased energy harvest in mice has not been confirmed by later research (Tremaroli et al, 2010). While Murphy et al (2010) confirmed the decreased energy content of faeces and increased caecal short-chain fatty acids in ob/ob mice at 7 weeks of age, this did not persist with time. More importantly these effects were not seen in wild-type mice with diet-induced obesity resulting from a high-fat diet. 


\subsection{INTESTINAL FERMENTATION AND GUT PEPTIDE HORMONES}

While increased energy harvest of short-chain fatty acids resulting from fermentable dietary fibre in the colon has been proposed to increase body weight, a larger body of evidence suggests that increased fermentation in the colon is protective against obesity. Rising levels of obesity are associated with 'Westernised diets', which have become more refined and thereby lack the dietary fibre that was common in low-energy plant foods (Frost et al, 2014a). This lack of dietary fibre potentially disrupts feedback mechanisms in the gut involved in regulating energy intake.

\subsubsection{FERMENTATION IN THE DISTAL INTESTINE}

SCFAs produced through bacterial fermentation in the caecum and colon account for an estimated 5-10\% of human energy needs (McNeil, 1984). The contribution of SCFAs to energy requirements in mice is uncertain, although rats have been shown to ferment dietary fibre in a manner similar to human subjects (Nyman et al, 1986). Some dietary carbohydrates resist digestion in the small intestine and reach the caecum and colon largely intact where the resident bacteria ferment these as an energy substrate. This bacterial fermentation generates millimolar concentrations of SCFAs, with the principle acids produced being acetate, propionate, and butyrate in a molar ratio of approximately 57:22:21 in the human colon (Cummings et al, 1987). SCFAs produced in the colon are efficiently absorbed by the colonocytes, while the remaining $5 \%$ are secreted in the faeces (den Besten et al, 2013b). While much of the butyrate is consumed by colonocytes as an energy source, SCFA concentrations reach micromolar levels within the portal vein with propionate being utilised by the liver (Bugaut \& Bentejac, 1993; Cummings et al, 1987). In the liver, acetate can be converted into fat and cholesterol, while propionate is used to synthesise glucose (den Besten et al, 2013a).

Not limited to energy supply, SCFAs have a range of direct beneficial effects in the gut. Increased SCFA concentrations reduces luminal $\mathrm{pH}$, inhibiting the growth of pathogenic bacteria, and increasing calcium absorption (Macfarlane \& Macfarlane, 2012). Acetate production is a key factor in the ability of Bifidobacteria to inhibit pathogenic bacteria in the gut (Fukuda et al, 2011). As well as fuelling the epithelium, butyrate stimulates production of mucin, which is involved in protecting the epithelium (Jung et al, 2015) and improving the integrity tight-junctions (Peng et al, 2009). Thus, the colonic 
production of SCFA seems to have an important role in the maintenance of gut barrier function.

\subsubsection{SCFA RECEPTORS}

As well as serving as energy substrates for the host, colonic SCFAs can also influence host adiposity by acting as signalling molecules. SCFA receptors are present in the colon expressed by enteroendocrine L-cells as G protein-coupled receptors FFA2 and FFA3, previously known as GPR41 and GPR43 respectively (Stoddart et al, 2008). The same enteroendocrine L-cells that express the SCFA receptors FFA2 and FFA3 also secrete the gut peptide hormones PYY and GLP-1, two hormones with pleotropic roles that include effects on satiation (Spreckley \& Murphy, 2015). Stimulation of L-cells by infusion of SCFAs into the colon stimulates the secretion of the enteroendocrine hormones GLP-1 and PYY from L-cells (Spreckley \& Murphy, 2015). This provides a potential mechanistic link between dietary fibre, increased bacterial fermentation, and benefits to host satiety mediated by FFA2 and FFA3 (Ulven, 2012).

\subsubsection{GUT PEPTIDE HORMONES}

L-cells in the epithelium of the lower intestine secrete the gut peptide hormones PYY and GLP-1 which diffuse into the lamina propria and enter the systemic circulation via the hepatic portal vein (Spreckley \& Murphy, 2015). In addition to other functions the GLP-1 and PYY co-expressing L-cells are considered to play a role in the regulation of energy homeostasis. GLP-1 mediates its effects through the GLP-1 receptor while PYY is found in the circulation in two main forms, as a full-length peptide, PYYl-36, and in its truncated form, PYY3-36. The full-length PYY acts on Y family receptors Yl, 2, and 5, while the PYY3-36 is selective for the Y2 receptor (Grandt et al, 1994). Both hormones have a range of overlapping physiological effects (Spreckley \& Murphy, 2015). GLP-1 is known as an incretin, increasing glucose stimulated insulin release through its action on pancreatic islet cells (Vilsboll \& Holst, 2004). There is evidence that PYY can also promote glucose tolerance via increased GLP-1 secretion (Chandarana et al, 2013). GLP1 has also been found to have a tropic effect on pancreatic beta-cells, maintaining insulin secretin in aging rats (Wang et al, 1997). GLP-1 and PYY also activate the 'ileal break', an inhibitory feedback mechanism that slows intestinal motility and the transit of food 
through the gut in order to increase nutrient digestion and absorption (Lloyd et al, 1996; Nauck et al, 2011; Pironi et al, 1993).

\subsubsection{DIETARY FIBRE, GLP-1 AND PYY, AND BODY WEIGHT}

Increased fermentation of dietary fibre in the caecum and colon has been shown to result in increased secretion of GLP-1 and PYY from enteroendocrine L-cells. One method of increasing fermentation is through addition of specific types of fermentable fibre into the diet. Rats fed a diet enriched with inulin type fructans, also known as oligofructose, for three weeks increased both GLP-1 and its precursor proglucagon within the proximal colon mucosa (Cani et al, 2004). The feeding of oligofructose was later shown to reduce body weight gain in mice, as well as improving other measures of metabolic health (Cani et al, 2006). This effect was reported to be lost in mice lacking the GLP-1 receptor, suggesting that this gut peptide hormone is an important mediator of the effect. However, the duration of the study was short, at only four weeks, and numbers of mice used were low. Oligofructose increased PYY and GLP-1 levels in Sprague-Dawley rats (Zhou et al, 2008), and oligofructose feeding for 4 weeks was reported to increase GLP1 production in the proximal colon and L-cell differentiation in Wistar rats, suggesting that dietary fibre not only stimulates hormone secretion from L-cells but also increases the number of L-cells capable of secreting GLP-1 and PYY (Cani et al, 2007b). Oligofructose supplementation also increased plasma GLP-1 and reduced food intake in genetically obese ob/ob mice (Cani et al, 2009). Similar effects have been shown in rats with other types of fermentable dietary fibre. Comparison of inulin, pectin, and betaglucan fibres supplemented into the diet of Sprague Dawley rats reported similar effects reducing body weight and food intake (Adam et al, 2014). In contrast, the addition of minimally fermented cellulose did not result in changes in food intake or body weight. Pectin was later shown to have a dose response effect, with increased percentage in the diet resulting in large reductions in body weight, body fat, and increases in circulating GLP-1 and PYY (Adam et al, 2015a). The addition of either inulin or beta-glucan to the diet of mice resulted in significantly lower cumulative body weight gain compared with high fat controls (Arora et al, 2012). Energy intake was lower in beta-glucan than inulin fed mice, while inulin fed mice showed a greater effect on total body fat content suggesting that the effect of different dietary fibres may not be identical. Intervention 
studies on human subjects are less numerous. One study by Parnell \& Reimer (2009), fed overweight subjects 21 grams of oligofructose per day or a placebo for 12 weeks, and resulted in a small reduction in body weight and blood glucose, associated with increases in PYY secretion but not GLP-1. In a more recent randomised control trial the addition of 30 grams per day of inulin or cellulose to the diet of overweight, prediabetic subjects resulted in greater body weight loss in subjects supplemented with inulin (Guess et al, 2015). Inulin supplementation also resulted in lower liver and soleus muscle fat at 18 weeks compared to those consuming the cellulose.

\subsection{BRAIN REGULATION OF ENERGY HOMEOSTASIS}

Typically, adiposity and body weight remain remarkably constant in response to varying daily food intake and energy expenditure. Brain centres, including the hypothalamus, brainstem, and reward centres, are involved in regulating this control of energy homeostasis. (Wynne et al, 2005). The hypothalamus is a key brain centre for body homeostasis, including the maintenance of bodyweight and adiposity. Specialised neurons of the hypothalamus are involved in the control of food intake and energy expenditure through the integration of various peripheral hormonal and neuronal signals of satiety and nutrient status (Berthoud, 2002), and through the sensing of circulating nutrients (Blouet \& Schwartz, 2010; Lopez et al, 2007). The balance of activity of key hypothalamic neurons plays an important role in the maintenance of body weight over time (Schwartz et al, 2000). The hypothalamus also has a role in the partitioning and utilisation of nutrients in the periphery, regulating glucose homeostasis and in lipid metabolism (Dieguez et al, 2011; Koch et al, 2010).

\subsubsection{HYPOTHALAMIC REGULATION OF ENERGY HOMEOSTASIS}

Anatomically, the hypothalamus is composed of a number of nuclei (neuronal clusters) with important roles in relation to energy homeostasis. One of the most important of these is the arcuate nucleus, which is composed of groups of neurons within the mediobasal hypothalamus straddling either side of the third ventricle. While other nuclei within the hypothalamus are located inside the blood brain barrier, the arcuate nucleus lies partially outside, allowing it to be exposed to hormones and nutrients from the circulation and also the cerebrospinal fluid present in the third ventricle (Williams, 2012). Two sets of neurons in the arcuate nucleus have been characterised with 
important and opposing actions in energy balance regulation. The first of these are termed orexigenic neurons, as they express the neuropeptides AgRP and NPY, which stimulate food intake and suppress energy expenditure. The second group are the anorexigenic neurons, which express the neuropeptides POMC and CART that inhibit the appetite and increase energy expenditure. Both sets of neurons respond to leptin signalling, with leptin activating the anorexigenic POMC/CART neurons and inhibiting the orexigenic AgRP/NPY neurons. Maintaining a balanced activity between these hypothalamic neurons has been considered important for the maintenance of body weight over time (Schwartz et al, 2000). Leptin and insulin are two important anorexigenic hormones that transmit signals of adipose tissue status and nutritional status from the periphery to the hypothalamus. Leptin is produced by the white adipose tissue while the pancreatic beta-cells produce insulin. Meanwhile, several other hormones such cholecystokinin, ghrelin, glucagonlike peptide-1 (GLPl), and several other nutrients also act as modulators of the activity hypothalamic neurons.

\subsubsection{HYPOTHALAMIC ACTION OF GLP-1 AND PYY}

An important feature of the arcuate nucleus is its incomplete blood-brain barrier that enables contact with circulating factors, including PYY and GLP-1. GLP-1 receptors are expressed widely in the hypothalamus, including the arcuate nucleus, where it may elicit its effects via POMC neurons expressing the GLP-1 receptor (Richards et al, 2014; Sandoval et al, 2008). Peripheral administration of a long-acting GLP-1 receptor agonist can result in weight loss through action on arcuate nucleus POMC neurons (Secher et al, 2014). PYY receptors are also found in the brain and peripheral infusion of PYY can alter neuropeptide expression in the arcuate nucleus (Batterham et al, 2002). However, the precise action of PYY on the brain remains unclear (Spreckley \& Murphy, 2015).

After release, both PYY and GLP-1 undergo enzymatic cleavage by dipeptidyl peptidase 4, which converts PYY into its Y2 receptor selective form and inactivates GLP1. Due to this enzymatic action, the half-life of GLP-1 and PYY are approximately 2 minutes and 10 minutes, respectively (Batterham \& Bloom, 2003; Holst \& Deacon, 2005). As a result of this, there is some debate as to whether amounts of active GLP-1 is able to reach receptors in the hypothalamus to affect central action as a neuropeptide (Spreckley \& Murphy, 2015). While the evidence outlined shows clear effects of fibre fermentable on 
body weight, at least in rodents, the associated rise in GLP-1 has not been reported in all studies on human subjects or in mice (Anastasovska et al, 2012; Bodinham et al, 2011). The feeding of inulin to human subjects has been reported to increase postprandial SCFAs in the circulation (Tarini \& Wolever, 2010). Evidence from studies on alcohol metabolism have shown that the SCFA acetate can cross the blood-brain barrier into the brain where it can result in neurological effects (Carmichael et al, 1991). It has recently been proposed that circulating acetate, derived from gut fermentation, can directly influence energy homeostasis in the hypothalamus to reduce appetite (Frost et al, 2014a).

\subsubsection{HYPOTHALAMIC DYSFUNCTION IN OBESITY}

Dysfunction of hypothalamic regulation of energy homeostasis has been proposed to be a key factor in obesity development (Thaler \& Schwartz, 2010). While levels of leptin and insulin are typically increased in obesity, this is associated with increased insensitivity to both hormones, leading to leptin and insulin resistance in the hypothalamus (Frederich et al, 1995; Wang et al, 2001). A reduction in the response of the hypothalamus to the anorexic effects of insulin has been reported to be reduced in rats after 10 days and 30 days of feeding of a 'Western diet' (Prada et al, 2005). Several potential processes have been proposed for this obesity-associated hypothalamic damage occurring with diet-induced obesity, mainly in rodent models. These include endoplasmic reticulum stress, reductions in synaptic and neuronal plasticity, and increased hypothalamic autophagy (Williams, 2012; Zhang et al, 2008). Hypothalamic dysfunction results in upregulation of inhibitory transcription factors which downregulate both insulin and leptin signalling including SOCS3, and appears to be of particular importance with regard to leptin signalling (Howard \& Flier, 2006; Kievit et al, 2006).

\subsubsection{HYPOTHALAMIC INFLAMMATION}

While the role of inflammation in peripheral tissues, particularly the liver and adipose tissue, in obesity has been recognised, the role of inflammation in the hypothalamus has only recently been identified. Increased inflammation in the hypothalamus was first reported in rats after prolonged high-fat diet feeding (De Souza et al, 2005). After 16 weeks of feeding a $45 \%$ fat diet, these rats had gained a significant amount of body fat 
and showed an increased expression of several inflammatory genes in the hypothalamus. Mice with a neuron-specific knockout of myD88, an adaptor protein that links stimulation of the toll-like receptors with downstream inflammatory responses, were protected from high-fat diet induced obesity and both leptin and insulin resistance (Kleinridders et al, 2009). This suggested that toll-like receptor signalling in the brain could play a key role in this inflammatory response. Activation of toll-like receptor 4 in the hypothalamus, using an infusion of saturated fatty acids, was reported to induce inflammatory responses and, additionally, that inhibition of toll-like receptor 4 signalling protected mice from diet-induced obesity (Milanski et al, 2009). Microglia, the resident macrophages in the brain, are one of the main cell types in the brain expressing TLR4 and represent the main cells in the hypothalamus responding to TLR4 activation (Lehnardt et al, 2003). Stimulation of TLR4 using LPS resulted in upregulation of SOCS3, linking inflammatory signalling with increased insulin and leptin resistance (Brown et al, 2009). This has raised the possibility that hypothermic inflammation preceding obesity may be a cause of obesity (Wisse \& Schwartz, 2009). More recently this inflammatory response has been shown to be an early response to high-fat diet feeding in rats and mice (Thaler et al, 2012). Upregulated expression of inflammatory cytokines was seen in the hypothalamus of rats after only one day of highfat diet feeding, preceding any increase in body weight. This included upregulated expression of TNF-alpha, Il-6, and Il-beta, among other genes involved in inflammatory responses, and upregulated expression of SOCS3 (Thaler et al, 2012). This initial inflammatory response was transient, reducing to baseline levels, before resuming to produce a chronic inflammatory state after 4 weeks of high-fat diet feeding in rats; results were reportedly similar in mice. These inflammatory processes were accompanied by reactive gliosis, the accumulation of activated microglia, and markers suggestive of neuron injury within the hypothalamus (Thaler et al, 2012).

LPS is the most potent ligand for TLR4 and high levels of LPS in the circulation, found in cases of severe infection, are known to result in anorexic changes in the hypothalamus (Chakravarty \& Herkenham, 2005; von Meyenburg et al, 2004). The effects of chronic low levels of LPS in the circulation have not been investigated in the context of control of energy homeostasis in the hypothalamus. Nevertheless, the increased circulating levels of LPS in high-fat diet fed obese mice reported by Cani et al (2007a) represent a 
potential link between the gut microbiota and hypothalamic dysfunction. Despite the interest in the gut microbiota as a factor influencing obesity development, little attention has been paid to the potential influence of the gut microbiota on the brain regions controlling energy homeostasis.

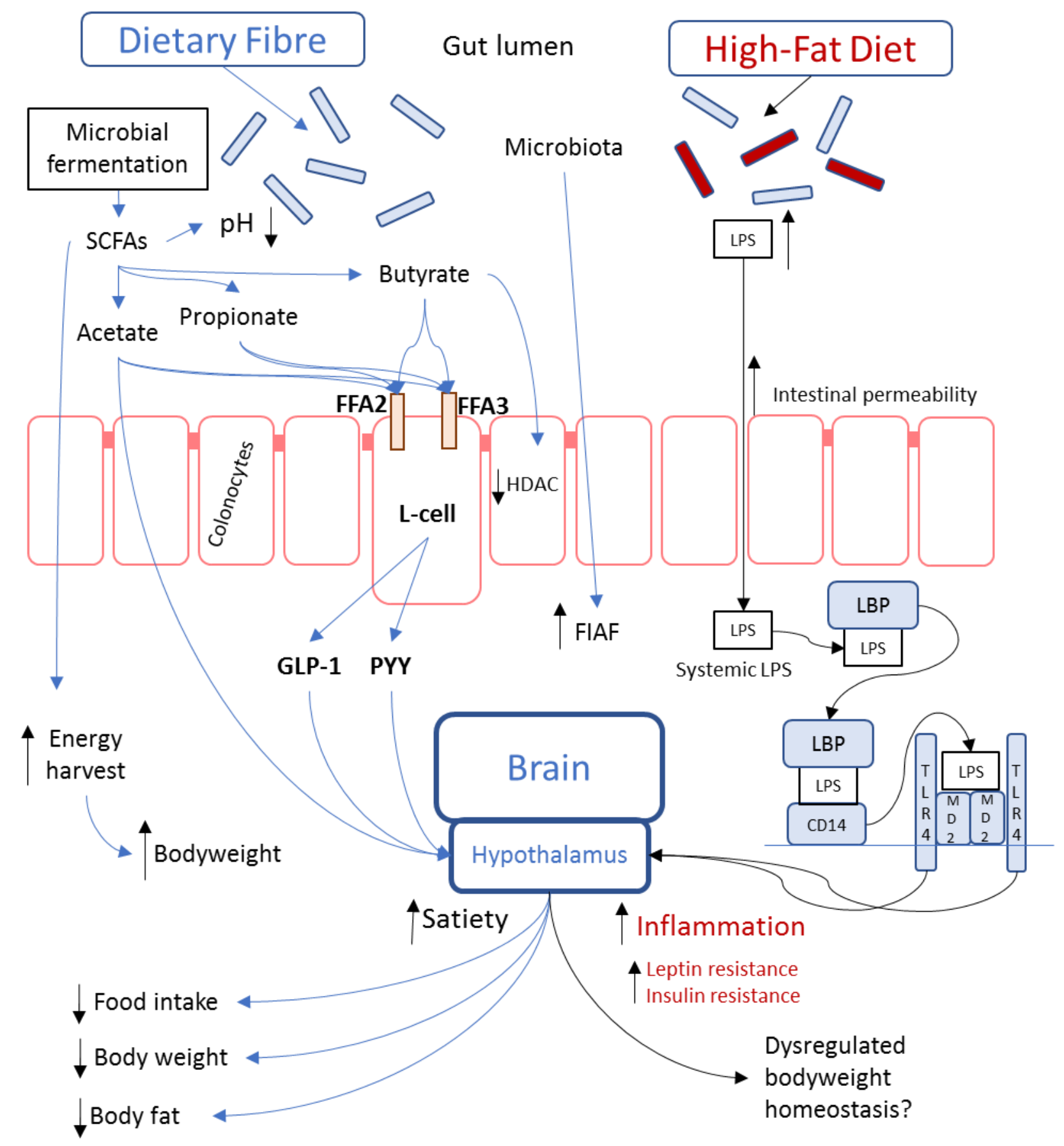

Figure 1. Proposed mechanisms linking the microbiota to body weight change. 


\section{SUMMARY}

A wide range of evidence has been put forward proposing a role for the resident gut microbiota in the development of obesity. A number of observations in mice and humans have indicated that obesity is associated with distinct changes in the composition of the intestinal microbiota and that this altered microbiota can cause increases in body fat if transferred into a germ-free mouse. However, a number of mechanisms that potentially mediate this effect have been proposed and the precise role they play is still unclear, Figure 1. Proposed mechanisms linking the microbiota to body weight change.. The presence or absence of different receptors of the innate immune system have been reported to either attenuate or exacerbate obesity in mice models. While TLR4 is has been the most studied of these, evidence as to tis role and action on obesity development is still conflicting. Despite its central role in regulating energy homeostasis, the potential effects of inflammatory antigens from the gut on energy regulation in the brain remain unexplored. Several other potential causal pathways have been suggested, some of which are contradictory, such as colonic fermentation being proposed to both increase body weight through energy harvest from the diet, and to protect from obesity development through induce greater satiety via the secretion of anorexigenic gut peptide hormones. Further research is needed to explore these areas and to clarify the potential impact of the gut microbiota in obesity. 


\section{THESIS AIMS AND HYPOTHESES}

\subsection{AIM 1}

To directly compare the absence of either TLR4, or CD14 in mice on bodyweight and body composition in response to high-fat diet feeding and to investigate the impact of diet and genotype on hypothalamic inflammation.

\subsection{HYPOTHESIS}

TLR4 knockout mice and CD14 knockout mice will show reduced susceptibility to highfat diet induced obesity relative to Wild-Type mice. TLR4 knockout and CD14 knockout mice will show reduced high-fat diet induced inflammation in the hypothalamus.

\subsection{AIM 2}

To separate out the influence of the refined nature of both High-Fat and Low-Fat diets on the gut microbiota from the obesity inducing effect of the fat in the High-Fat diet.

\subsection{HYPOTHESIS}

Gut microbiota composition associated with obesity is driven by diet composition and is unrelated to obesity. A refined Low-Fat control diet will reproduce obesity associated gut microbiota seen in the High-Fat diet in the absence of obesity.

\subsection{AIM 3}

To investigate the roles of acetate, PYY, and GLP-1 as mediators of effects of fermentable fibre on food intake and body composition of mice. mice with genetic deletions of either the PYY or GLP-1 genes, fed a high-fat diet supplemented with either cellulose or inulin.

\subsection{HYPOTHESIS}

GLP-1 and PYY are each required for dietary inulin to have its attenuating effects on food intake and body composition in mice fed a high fat diet. 


\section{CHAPTER 2.}

\section{METHODS}




\section{ANIMAL STUDY 1: THE INFLUENCE OF TLR4 AND CD14 ON DIET INDUCED OBESITY IN MICE.}

\subsection{ETHICS STATEMENT}

All animal experiments were performed under strict adherence to UK home office regulations according to the Animals (Scientific Procedures) Act, 1986, and were licensed by the UK home office under Project License PPL60/4282 and approved by the local ethics committee at the Rowett Institute of Nutrition and Health and University of Aberdeen (Approval number: SA13/03E).

\subsection{EXPERIMENTAL ANIMALS}

Male mice at 6-7 weeks of age including; 16 Toll-like receptor-4-null (TLR4 ${ }^{-/}$) mice (B6.B10ScN-Tlr4lps-del/JthJ, Stock Number 007227), and 16 Cluster of Differentiation14-null (CD14 $\left.{ }^{-/}\right)$mice (B6.129S4-Cd14tmlFrm/J, Stock Number 003726) were purchased from the Jackson Laboratory (Bar Harbor, ME, USA). Wild-type C57BL/6J (JAX ${ }^{\mathrm{TM}}$ Mice Strain, Stock Number 000664) control mice at 6-7 weeks of age were obtained from a UK based breeding colony of JAX ${ }^{\circledast}$ Mice maintained by Charles River (Charles River, UK). TLR4 ${ }^{-/-}$mice contained a spontaneous 74723 bp deletion in C57BL/10ScN mice removing the Tlr4 coding sequence and had been backcrossed to a C57BL/6J genetic background for a minimum of 7 generations. CD14 null mice had been generated using a targeting vector to disrupt the entire $\mathrm{Cd} 14$ coding region. The resulting chimeric male animals had been backcrossed to C57BL/6J females for at least 8 generations. On arrival, all mice were group housed in cages of 8-10 mice under specific pathogen free conditions within the same room in the Medical Research Facility at the University of Aberdeen. The animal room was maintained at $21 \pm 2^{\circ} \mathrm{C}$ and $55 \pm 10 \%$ relative humidity with a standard $12 \mathrm{~h}$ light, $12 \mathrm{~h}$ dark lighting regime running with lights on from 7am to 7pm. Experimental diets were the high-fat diet D12492 (60 kcal\% fat) (Research Diets, Inc.) and the control diet D12450B (10 kcal\% fat) (Research Diets, Inc.) matched for sucrose content, Table 1. Mice were offered food and water ad libitum throughout the study. 
Table 1. Chow, Low-Fat, and High-Fat diet composition.

\begin{tabular}{|c|c|c|c|c|c|c|}
\hline \multirow[t]{2}{*}{$\begin{array}{l}\text { Diet } \\
\text { Diet code }\end{array}$} & \multicolumn{2}{|c|}{$\begin{array}{c}\text { Chow diet } \\
\text { SDS* }\end{array}$} & \multicolumn{2}{|c|}{$\begin{array}{c}\text { Control diet } \\
\text { D12450J }\end{array}$} & \multicolumn{2}{|c|}{$\begin{array}{l}\text { High-Fat } \\
\text { D12492 }\end{array}$} \\
\hline & gram (\%) & kcal (\%) & gram (\%) & kcal (\%) & $\operatorname{ram}(\%)$ & kcal (\%) \\
\hline Protein & 18.4 & 22.0 & 19.2 & 20 & 26.2 & 20 \\
\hline Carbyhydrate & $57.39 * *$ & 68.9 & 67.3 & 70 & 26.3 & 20 \\
\hline Fat & 3.4 & 9.1 & 4.3 & 10 & 34.9 & 60 \\
\hline Total & 100.0 & 100.0 & & 100 & & 100 \\
\hline Kilocalories/gram MJ/kg & 11.19 & & 3.85 & & 4.4 & \\
\hline Ingredient & $\mathrm{n} / \mathrm{a} * * *$ & $n / a$ & gram & kcal & gram & kcal \\
\hline Casein, 80 Mech & - & - & 200 & 800 & 200 & 800 \\
\hline L-Cystine & - & - & 3 & 12 & 3 & 12 \\
\hline Corn Starch & - & - & 506.2 & 2024.8 & 0 & 0 \\
\hline Maltodextrin 10 & - & - & 125 & 500 & 125 & 500 \\
\hline Sucrose & - & - & 68.8 & 275.2 & 68.8 & 275.2 \\
\hline Cellulose, BW200 & $3.89 \%$ & 0 & 50 & 0 & 50 & 0 \\
\hline Total fibre & $15.06 \%$ & 0 & 50 & 0 & 50 & 0 \\
\hline Soybean oil & - & - & 25 & 225 & 25 & 225 \\
\hline Lard & - & - & 20 & 180 & 245 & 2205 \\
\hline Mineral Mix S10026 & - & - & 10 & 0 & 10 & 0 \\
\hline DiCalcium Phosphate & - & - & 13 & 0 & 13 & 0 \\
\hline Calcium Carbonate & - & - & 5.5 & 0 & 5.5 & 0 \\
\hline Potassium Citrate, 1 H2O & - & - & 16.5 & 0 & 16.5 & 0 \\
\hline Vitamin mix, V10001 & - & - & 10 & 40 & 10 & 40 \\
\hline Choline bitartrate & - & - & 2 & 0 & 2 & 0 \\
\hline FD\&C Yellow Dye \#5 & - & - & 0.04 & 0 & 0 & 0 \\
\hline FD\&C Blue Dye \#1 & - & - & 0.01 & 0 & 0.05 & 0 \\
\hline Total & - & - & 1055.05 & 4057 & 773.85 & 4057 \\
\hline
\end{tabular}

*Special Diet Services: Rat and Mouse Breeder and Grower.

**Calculated as Nitrogen Free Extract.

*** Undefined diet ingredients: Wheat, wheatfeed, barley, de-hulled extracted toasted soya, maize, macro minerals, soya oil, potato protein, hydrolised wheat gluten, full fat soya, maize gluten meal, vitamins, micro minerals, amino acids. 


\subsection{MOUSE STUDY DESIGN}

On arrival, the mice were acclimatised for 2 weeks in group housed cages of 8 or 10 mice per cage and with ad libitum commercial mouse chow (Special Diet Services, Witham, UK). Sixteen mice of each genotype, Wild-Type, CDI4 ${ }^{-/}$, and TLR4 ${ }^{-/}$, were switched to singly housed shoebox cages containing a grid floor and food intake recording started. All mice were then switched onto the Low-Fat control diet, D12450B. Wild-Type control mice not requiring food intake measurements were singly housed in solid floored cages containing sawdust bedding. For increased enrichment of the cage environment all cages contained a roof-suspended plastic mouse house, a plastic tunnel, and shredded paper for bedding and nesting. After one week on the Low-Fat control diet (D12450B) the mice of each genotype were weighed and randomised into weight matched diet groups. One half of the mice of each genotype were switched to the High-Fat diet (D12952), while the remaining mice continued the Low-Fat control diet (D12450B).

\subsection{PHYSIOLOGICAL MEASUREMENTS}

Food intake was weighed and recorded twice weekly on Monday and Thursday by weighing food added to the hopper and weighing uneaten food on the next measurement day. At the same time, spilt food was collected from the trays under the grid floor cages and weighed and deducted from the food eaten. Ad libitum feeding of the experimental diets was continued for 8 weeks. Bodyweights for all mice were recorded weekly. Whole body composition (as total fat and lean masses) was quantified at the beginning of the study and from then on, every 2 weeks by magnetic resonance imaging (MRI; EchoMRI, 2004, Echo Medical Systems, Houston, TX, USA) which measures the mass of fat and lean tissue in live animals using nuclear magnetic resonance (NMR) technology. The EchoMRI machine was calibrated using canola oil as a reference fat.

\subsection{SAMPLE COLLECTION}

The study was completed after 8 weeks of feeding the experimental diets. Mice were dispatched using cervical dislocation. Trunk blood was collected via decapitation. The brains were removed, snap frozen on dry ice and then stored at $-80^{\circ} \mathrm{C}$. The 
gastrointestinal tract was dissected out and caecum contents were collected, frozen on dry ice and then stored at $-80^{\circ} \mathrm{C}$.

\subsection{CONFIRMATION OF TLR4 ${ }^{-/-}$AND CDI4 ${ }^{-/-}$GENOTYPE}

Genotype was confirmed by PCR of DNA extracted a $10 \mathrm{mg}$ sample of ileum from a subset of half of the mice of each genotype from gut ileum using QIAamp DNA Micro Kit (QIAGEN) according to the manufactures protocol. DNA concentration was quantified using a NanoDrop ND-1000 spectrophotometer (Thermo Fisher Scientific, Wilmington, DE, USA). PCR primer sequences to differentiate wild type from TLR4 ${ }^{-/-}$and CD14 $4^{-/-}$mice are detailed in Table 2.

Table 2. CD14 and TLR4 genotyping primer sequences.

\begin{tabular}{|c|c|}
\hline Primer & Primer sequence \\
\hline \multicolumn{2}{|l|}{ CD14 } \\
\hline olMR0662 & 5'-CCGCTTCCATTGCTCAGCGG-3' \\
\hline olMR1314 & 5'-CCAAGTTTTAGCGCTGCGTAAC-3' \\
\hline olMR1315 & 5'-GCCAGCCAAGGATACATAGCC-3' \\
\hline \multicolumn{2}{|l|}{ TLR4 } \\
\hline olMR8365 & 5'-GCAAGTTTCTATATGCATTCTC-3' \\
\hline olMR8366 & 5'-CCTCCATTTCCAATAGGTAG-3' \\
\hline olMR8367 & 5'-ATATGCATGATCAACACCACAG-3' \\
\hline olMR8368 & 5'-TTTCCATTGCTGCCCTATAG-3' \\
\hline
\end{tabular}

For CD14 genotyping PCR amplification was carried out using GoTaq ${ }^{\circledast}$ G2 Hot Start Polymerase (Promega). Ileum genomic DNA were used a concentration between 70-400 ng/ $\mu$ l. Each reaction contained $4.0 \mu \mathrm{l}$ 10X Reaction Buffer, $1.6 \mu \mathrm{l} \mathrm{MgCl} 2(25 \mathrm{mM}), 2 \mu \mathrm{l}$ dNTPs $(2.5 \mathrm{mM}), 1 \mu \mathrm{l}$ primer oIMR0662 $(25 \mathrm{mM}), 1 \mu \mathrm{l}$ primer oIMRl314 $(25 \mathrm{mM}), 1 \mu \mathrm{l}$ primer oIMRl315 ( $25 \mathrm{mM}$ ), $0.1 \mu \mathrm{l}$ GoTaq HS Polymerase, and $7.7 \mu \mathrm{l} \mathrm{H2O}$. Finally, $2 \mu \mathrm{l}$ Genomic DNA was added to each reaction mixture. 
For TLR4 genotyping PCR amplification was carried out using GoTaq ${ }^{\circledast}$ G2 Hot Start Polymerase (Promega). Ileum genomic DNA were used a concentration between 70-400 $\mathrm{ng} / \mu \mathrm{l}$. Each reaction contained $4.0 \mu \mathrm{l}$ 10X Reaction Buffer, $1.6 \mu \mathrm{l} \mathrm{MgCl} 2(25 \mathrm{mM}), 2 \mu \mathrm{l}$ dNTPs $(2.5 \mathrm{mM}), 1 \mu \mathrm{l}$ primer oIMR8365 $(25 \mathrm{mM}), 1 \mu \mathrm{l}$ primer oIMR8366 $(25 \mathrm{mM}), 1 \mu \mathrm{l}$ primer oIMR8367 $(25 \mathrm{mM}), 1 \mu \mathrm{l}$ primer oIMR8368 (25 mM), $0.1 \mu \mathrm{l}$ GoTaq HS Polymerase, and $7.7 \mu \mathrm{l} \mathrm{H2O}$. Finally, $2 \mu \mathrm{l}$ Genomic DNA was added to each reaction mixture.

The PCR reaction was cycled 30 times after an initial 2 min at $95^{\circ} \mathrm{C}$, with $95^{\circ} \mathrm{C}$ for 30 sec denaturing, annealing $\left(67^{\circ} \mathrm{C}\right.$ for $\mathrm{CD} 14$ primers or $55^{\circ} \mathrm{C}$ for TLR4 primers) for 30 secs, $72{ }^{\circ} \mathrm{C}$ for $30 \mathrm{sec}$ elongation, followed by $72^{\circ} \mathrm{C}$ for 5 min extension in a T100 thermal cycler (Bio-Rad, Hertfordshire, United Kingdom). Amplification was confirmed using agarose gel electrophoresis on a $1 \%$ agarose gel containing GelRed (Cambridge Bioscience, BT-41003, Munro House, Cambridge, UK) alongside a 100 bp ladder with IX TAE as the running buffer. Gels were visualised using a Gel Dock system. The CD14 primers produced a Wild-Type band at $840 \mathrm{bp}$ and a knockout band and $600 \mathrm{bp}$. The TLR4 primers produced a Wild-Type band at $390 \mathrm{bp}$ and a knockout band and $140 \mathrm{bp}$. 


\section{MOUSE STUDY 2: A COMPARISON OF CHOW AND REFINED DIETS}

\subsection{ETHICS STATEMENT}

All animal experiments were performed under strict adherence to UK home office regulations according to the Animals (Scientific Procedures) Act, 1986, and were licensed by the UK home office under Project License PPL60/4359 and approved by the local ethics committee at the Rowett Institute of Nutrition and Health, University of Aberdeen (Approval number: 160715MD).

\subsection{EXPERIMENTAL ANIMALS}

Male C57BL/6J (JAX ${ }^{\text {тм }}$ Mice Strain, Stock Number 000664) mice were supplied by (Charles River, UK) aged 7-8 weeks after being weaned on VRFl, a GLP certificated rat and mouse breeding diet (Special Diet Services Ltd, Witham, Essex, UK). On arrival, the mice were group housed in groups of 8-10 mice in cages with sawdust bedding. The room conditions were maintained with a controlled temperature of $21 \pm 2^{\circ} \mathrm{C}$ and $55 \pm 10 \%$ relative humidity. Food and water was available ad libitum, and the lighting regime was a standard $12 \mathrm{~h}$ light, $12 \mathrm{~h}$ dark running with lights on from 7am to 7pm.

\subsection{MOUSE DIETS}

The present study used both unrefined rodent chow and refined semi-purified low-fat and high-fat rodent diets. The Chow diet (Rat and Mouse Breeder and Grower) diet is an unrefined rodent chow supplying 9\% fat, 22\% protein, and 69\% carbohydrate as calories (Special Diet Services Ltd, Witham, Essex, UK). The Low-Fat diet (D12450J) and High-Fat diet (D12492) were semi-purified, refined diets supplying 10\% fat, 20\% protein, and $70 \%$ carbohydrate and $60 \%$ fat, $20 \%$ protein, and $20 \%$ carbohydrate respectively as calories (Research Diets Inc, New Brunswick, NJ, USA), Table 1.

\subsection{STUDY DESIGN}

On arrival mice were acclimatised for 2 weeks in group housed cages (8-10 mice per cage) and maintained on Rat and Mouse Breeder and Grower diet (Special Diet Services Ltd, Witham, Essex, UK). Mice were then individually housed in shoebox cages for 
1 week of acclimatisation to individual housing fitted with grid floors for accurate food intake measurement. For enrichment cages contained a plastic tunnel, a roof suspended mouse house, and shredded paper for bedding and nesting. Mice continued to be maintained during this acclimatisation week on Rat and Mouse Breeder and Grower diet (Special Diet Services Ltd, UK). At 10 weeks, old mice were randomly allocated to one of three weight-matched groups and offered the pelleted experimental diets ad libitum for 8 weeks ( $n=16$ /group). The Chow diet group continued the Rat and Mouse Breeder and Grower pelleted chow (SDS; Special Diet Services Ltd, Witham, UK). The Low-Fat diet group were provided with diet D12450J (Research Diets Inc, New Brunswick, NJ, USA). The High-Fat diet groups were provided with D12492 (Research Diets Inc, New Brunswick, NJ, USA).

\subsection{PHYSIOLOGICAL MEASUREMENTS}

Mice body weights were recorded at baseline and then once per week thereafter. Body composition measurements of the mice were performed at the start (week 0) and thereafter once per week during the 8 weeks of the experiment by MRI. Un-anesthetized mice were physically restrained and inserted into the EchoMRI scanner. The scanner provides measurements of fat and lean tissues; fat measurement includes all the fat molecules in the mouse expressed as equivalent weight of canola oil. The lean measurement is a muscle mass equivalent of all the body parts containing water, excluding fat, bone and substances that do not contribute to the NMR signal, such as hair and claws. Food intake was recorded twice per week. Food was weighed and added to the individual mice cages twice per week, at the same time remaining food and spilled food was weighed. Average weekly food intake was calculated taking spillage into account. Energy intake was calculated using the kcal density of the diets, as kJ/gram, using the published figures provided by manufacturers of the respective mouse diets.

\subsection{INTRAPERITONEAL GLUCOSE TOLERANCE TESTS}

Intraperitoneal glucose tolerance tests (IPGTTs) were carried out to characterize the glucose tolerance of the mice following 8 weeks of diet feeding. Mice were weighed and then fasted for 5 hours from 8:00 am before the IPGTT. Glucose was prepared as $100 \mathrm{mg} / \mathrm{ml}$ in saline and then filter sterilised and stored at $-20^{\circ} \mathrm{C}$. A blood sample $(0$ mins) was taken via by a tail tip cut removing $1 \mathrm{~mm}$ of tail prior to the intraperitoneal 
(IP) glucose injection containing an amount of glucose equivalent to $1.5 \mathrm{mg} / \mathrm{g}$ body weight. Subsequent blood samples were taken from the tail vein at 15, 30, 60 and 120 mins by gently squeezing the tail to remove the scab and express a fresh spot of blood. The first spot of blood was discarded and the second spot of blood transferred directly from the tail onto an Accu-Chek ${ }^{\circledR}$ Aviva Test Strip (Roche Diagnostics, Burgess Hill, UK). Blood glucose was measured using an Accu-Chek ${ }^{\circledR}$ Aviva blood glucose monitor (Roche Diagnostics, UK). Area under the curve for individual IPGTTs were calculated also using SigmaPlot 13.0.

\subsection{PHYSICAL MEASUREMENTS AND SAMPLE COLLECTION}

Blood samples were collected using cardiac puncture. Mice were put under terminal general anaesthetic with isoflurane gas by placing in a plastic chamber until unresponsive and then were kept under aesthetic while terminal cardiac puncture was carried out. Blood samples were centrifuged at $4^{\circ} \mathrm{C}$ in a microcentrifuge (Eppendorf 5415R, Hamburg, Germany), the serum pipetted into a clean $1.5 \mathrm{ml}$ microcentrifuge tube and frozen on dry ice. Epididymal fat was dissected out, then weighed, and immediately frozen on dry ice. The entire gastrointestinal tract with liver attached was dissected out of each mouse. The liver was separated from the gastrointestinal tract, weighed, and then snap frozen on dry ice. Attached fat and connective tissue was carefully removed from the remaining intestinal tract. The length of the small intestine, the caecum, and the colon was measured in $\mathrm{cm}$. The small intestine was removed and a photograph was taken of the caecum and colon with a cm ruler for scale. The small intestine, caecum, and colon were separately weighed, first containing their luminal contents and then weighed again after the luminal contents were carefully removed. The luminal contents of the terminal ileum, the contents of the caecum, and the contents of the colon were collected and frozen on dry ice. 


\section{MOUSE STUDY 3: THE ROLE OF GLP-1 AND PYY IN MEDIATING THE EFFECTS OF FERMENTABLE FIBRE}

\subsection{ETHICS STATEMENT}

All animal experiments were performed under strict adherence to UK home office regulations according to the Animals (Scientific Procedures) Act, 1986, and were licensed by the UK home office under Project License PPL60/4282 and approved by the local ethics committee at the University of Aberdeen Rowett Institute of Nutrition and Health (Approval number: 170915MD).

\subsection{ANIMALS}

C57BL/6J control mice were supplied by Charles River at 7-8 weeks of age (Charles River, Margate, UK) where they had been weaned on pelleted VRF1 diet (Special Diet Services, UK, code: 801900) containing 13\% calories from fat, 22\% calories from protein and 65\% calories from carbohydrate. On arrival mice were maintained at the Medical Research Facility, Aberdeen, group housed in cages of 8-10, and fed pelleted Rat and Mouse Breeder and Grower diet (Special Diet Services, UK, code: 801722). $\mathrm{PYY}^{-{ }^{-}}$mice were supplied as homozygous breeding adult mice, 4 males and 4 females, by Professor Herbert Herzog, University of New South Wales, Australia (Boey et al, 2006). PYY-/- mice were bred at the Aberdeen University Medical Research Facility and weaned onto pelleted Rat and Mouse Breeder and Grower diet containing 9\% calories from fat, 22\% calories from protein and 69\% calories from carbohydrate (Special Diet Services, UK, code: 801722 ). Adult males were fed the chow diet until they were old enough to be used for the study. GLP-1R ${ }^{-/-}$mice were supplied as homozygous male mice by Dr Daniel J Drucker, University of Toronto, Canada (Scrocchi et al, 1996; Scrocchi \& Drucker, 1998). Homozygous GLP-1R ${ }^{-/-}$male mice were mated with resident female wild-type C57BL/6J mice at the Aberdeen University Medical Research Facility. Heterozygous female GLP$1 \mathrm{R}+/$ - offspring were backcrossed with the original homozygous GLP-1R ${ }^{-/-}$males to generate homozygous GLP-1R ${ }^{-/-}$offspring. Resulting male $\mathrm{GLP}^{-1 R^{-/}}$mice were fed standard murine chow diet post weaning until 8 weeks of age consisting of pelleted Rat 
and Mouse Breeder and Grower diet (Special Diet Services, UK, code: 801722). All mice were maintained with a controlled temperature of $21 \pm 2^{\circ} \mathrm{C}$ and $55 \pm 10 \%$ relative humidity, food and water was available ad libitum, and the lighting regime was a standard $12 \mathrm{~h}$ light, $12 \mathrm{~h}$ dark running with lights on from 7am to 7pm.

Table 3. Low-Fat, High-Fat $+\mathrm{C}$, and High-Fat+In diet composition

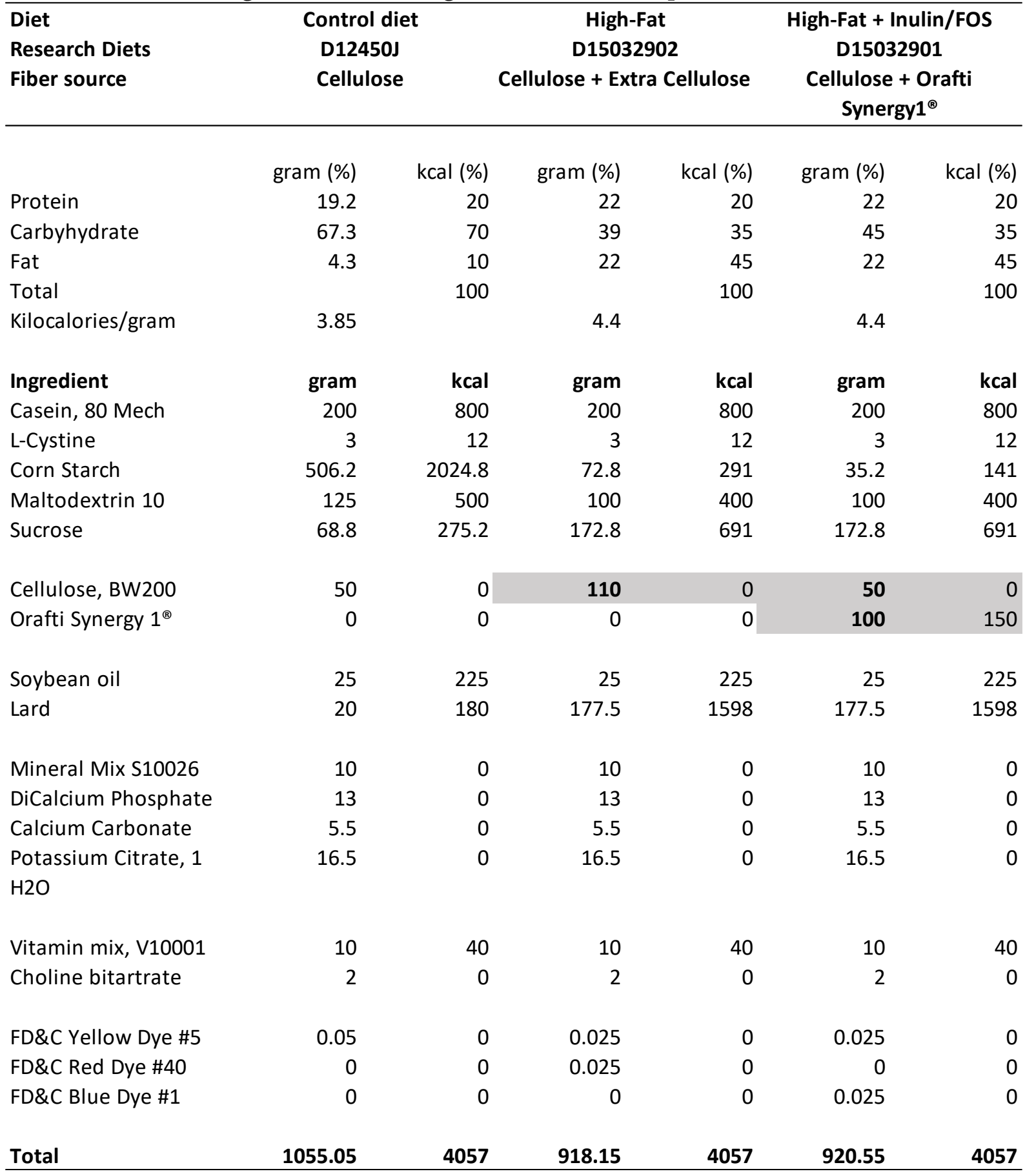




\subsection{STUDY DESIGN}

At 7-8 weeks of age male mice were allocated to single housed grid floor and acclimatised for one week with ad libitum access to the Low-Fat control diet D12450J containing $10 \%$ calories from fat, $20 \%$ calories from protein and $70 \%$ calories from carbohydrate (Research Diets, New Brunswick, NJ). Grid floor cages contained a suspended mouse house, plastic tunnel, and paper bedding for enrichment. For all mice body weight and food intakes was recorded at baseline during the acclimatisation week (week 0) and then continued throughout the study. Bodyweight was recorded once per week. Food was weighed and added to the individual mice cages twice per week, at the same time remaining food and spilled food was weighed. Average weekly food intake was calculated taking spillage into account. Energy intake was calculated using the published kcal density of the diets, as kJ/gram, determined using the published figures provided by manufacturers of the respective mouse diets. Additionally, for Wild-Type C57BL/6J mice body composition measurement was carried out at the start (week 0) and thereafter once per week during the 8 weeks of the experiment by MRI.

At 8-9 weeks of age mice were allocated to one of three experimental diets. Mice were either continued on Low-Fat control diet D12450J (Research Diets, New Brunswick, NJ), or switched to one of two high-fat diets. The first high-fat diet D15032902 (Research Diets, New Brunswick, NJ) contained 110 grams of cellulose per kg of diet. The second high-fat, highly soluble fibre diet D15032901 (Research Diets, New Brunswick, NJ) contained 50 grams of cellulose and 100 grams Orafti Synergy 1 fibre added per kg of diet. The composition of the diets used in this study are detailed in Table 3. The dietary fat was from lard and consisted of a mixture of saturated (32\%) and mono- (36\%) and poly-unsaturated (32\%) fatty acids. Orafti Synergy 1 fibre, consisting of $90 \%$ inulin and $10 \%$ fructooligosaccharides, and containing $1.5 \mathrm{kcal} / \mathrm{g}$ was added in the high-fat diet (D15032901) formula. Corn starch was reduced and cellulose increased in the control high-fat diet (D15032902) to maintain an equivalent calorie density per gram and macronutrient ratios as the control High-Fat diet. Cellulose was assumed to provide no calories. The gross energy for the control diet and high-fat diets was 3.38 and 4.4 $\mathrm{kcal} /$ gram, respectively. During these feeding protocols, the mice were maintained with a controlled temperature of $21 \pm 2^{\circ} \mathrm{C}$ and $55 \pm 10 \%$ relative humidity, food and water was 
available ad libitum, and the lighting regime was a standard $12 \mathrm{~h}$ light, $12 \mathrm{~h}$ dark running with lights on from $7 \mathrm{am}$ to $7 \mathrm{pm}$.

\subsection{PHYSICAL MEASUREMENTS AND SAMPLE COLLECTION}

Mice were placed under terminal anaesthetic with isoflurane gas in a plastic chamber until unresponsive and then maintained under aesthetic while terminal cardiac puncture was carried out. Blood was transferred to a sterile $1.5 \mathrm{ml}$ microcentrifuge tube, chilled for 30 minutes on ice, and then centrifuged in a microcentrifuge for 15 minutes at 2,000 x g (Eppendorf 5415R, Germany). Serum was removed, transferred into a sterile $1.5 \mathrm{ml}$ microcentrifuge tube, and immediately frozen on dry ice. The entire gastrointestinal tract with liver attached was dissected out of each mouse. Epididymal fat and retroperitoneal fat pads were dissected out, then weighed, and epididymal fat immediately frozen on dry ice. The liver was separated from the gastrointestinal tract, weighed, and then snap frozen on dry ice. The stomach, containing its contents, was weighed. Attached fat and connective tissue was carefully removed from the remaining intestinal tract. The length of the small intestine, the caecum, and the colon was measured in $\mathrm{cm}$. The small intestine was removed and a photograph was taken of the caecum and colon with a cm ruler for scale. The small intestine, caecum, and colon were separately weighed, first containing their luminal contents and then weighed again after the luminal contents were carefully removed. The luminal contents of the caecum were collected and frozen on dry ice. All samples were then stored at $-80^{\circ} \mathrm{C}$. 


\subsection{CONFIRMATION OF GLP-1R ${ }^{-/-}$AND PYY ${ }^{-/}$GENOTYPE}

The GLP-1R ${ }^{-/-}$and PYY ${ }^{-/-}$mice were ear clipped at the age of three weeks. Genomic DNA was extracted from the ear clips using a $\mathrm{NaOH}$ extraction method (Truett et al, 2000). The ear clip tissue was placed into a $1.5 \mathrm{ml}$ microcentrifuge tube, $75 \mu \mathrm{l}$ of $25 \mathrm{mM}$ $\mathrm{NaOH} / 0.2 \mathrm{mM}$ EDTA were added, and the tube was incubated at $98^{\circ} \mathrm{C}$ for 30 minutes. Finally, the sample was centrifuged in a microcentrifuge at $4000 \mathrm{rpm}$ for 3 minutes (Eppendorf 5415R, Germany). A $2 \mu \mathrm{l}$ aliquot of this supernatant was used for genotyping PCR.

Table 4. Primers for GLP-1R $\mathrm{R}^{-/-}$and $\mathrm{PYY}^{-/-}$mouse genotyping.

\begin{tabular}{ll}
\hline Primer & Primer sequence \\
\hline $\begin{array}{l}\text { GLP-1R } \\
\text { GLP-1R Forward }\end{array}$ & 5'-TACACAATGGGGAGCCCCTA-3' \\
GLP-1R Reverse & 5'-AAGTCATGGGATGTGTCTGGA-3' \\
Neo-A Forward & 5'-CTTGGGTGGAGAGGCTATTC-3' \\
Neo-B Reverse & 5'-AGGTGAGATGACAGGAGATC-3' \\
& \\
PYY & \\
PYY 1 Forward & 5'-AAACAGATTATTGAGACGTGG-3' \\
PYY2 Reverse & 5'-GTCGCTGTCGTCTGTGAAGAGC-3' \\
PYY3 Reverse & 5'-CAACGGGTTCTTCTGTTAGTCC-3' \\
\hline GLP-1R primer sequences provided by Dr. Daniel Drucker (Scrocchi et al, 1996). \\
PYY primers sequences provided by Professor Herbert Herzog (Boey et al, 2008).
\end{tabular}

\subsubsection{PYY $^{-/-}$GENOTYPING}

For GLP-1R $\mathrm{R}^{-/-}$mouse genotyping the PCR reaction mix contained, $10 \mu \mathrm{l}$ XX GoTaq Flexi Buffer, $2 \mu \mathrm{l}$ of $\mathrm{MgCl}_{2}(25 \mathrm{mM}), 4 \mu \mathrm{l}$ of dNTPs $(2 \mathrm{mM}), 1 \mu \mathrm{l}$ of Primer GLP-1R-F $(20 \mu \mathrm{M}), 1$ $\mu \mathrm{l}$ of Primer GLP-1R-R $(20 \mu \mathrm{M}), 1 \mu \mathrm{l}$ of Primer Neo-A $(20 \mu \mathrm{M}), 1 \mu \mathrm{l}$ of Primer Neo-B $(20 \mu \mathrm{M}), 0.25 \mu \mathrm{l}$ GoTaq HS Polymerase, and finally made up to a total volume of $48 \mu \mathrm{l}$ with water. Then $2 \mu \mathrm{l}$ of DNA was added to the reaction mixture. Primer sequences can be found in Table 4 . After an initial 30 seconds at $94^{\circ} \mathrm{C}$ the reaction was cycled 30 times, with $94^{\circ} \mathrm{C}$ for 30 seconds denaturing, $62^{\circ} \mathrm{C}$ for 30 seconds annealing, and $72^{\circ} \mathrm{C}$ for 30 seconds elongation, followed by $72^{\circ} \mathrm{C}$ for 5 min extension. Amplification was confirmed using agarose gel electrophoresis. For wild-type mice a band would be produced at 437 bp and for a GLP-1R knockout mouse a band would be produced at $280 \mathrm{bp}$. 


\subsubsection{GLP-1R $^{-/-}$GENOTYPE}

For $\mathrm{PYY}^{-/-}$mouse genotyping the PCR reaction mix contained, $10 \mu \mathrm{l} 5 \mathrm{X}$ GoTaq Flexi Buffer, $2 \mu \mathrm{l}$ of $\mathrm{MgCl}_{2}(25 \mathrm{mM}), 4 \mu \mathrm{l}$ of dNTPs (2mM), $1 \mu \mathrm{l}$ of Primer PYYl $(20 \mu \mathrm{M}), 1 \mu \mathrm{l}$ of Primer PYY2 $(20 \mu \mathrm{M}), 1 \mu \mathrm{l}$ of PYY3 $(20 \mu \mathrm{M}), 0.25 \mu \mathrm{l}$ GoTaq HS Polymerase, and finally made up to a total volume of $48 \mu \mathrm{l}$ with water. Then $2 \mu \mathrm{l}$ of DNA was added to the reaction mixture. Primer sequences can be found in Table 4. After an initial 2 minutes at $94^{\circ} \mathrm{C}$ the reaction was cycled 30 times, with $94^{\circ} \mathrm{C}$ for 30 seconds denaturing, $61^{\circ} \mathrm{C}$ for 30 secs, and $72^{\circ} \mathrm{C}$ for 30 seconds elongation, followed by $72^{\circ} \mathrm{C}$ for 5 min extension. Amplification was confirmed using agarose gel electrophoresis on a $1 \%$ agarose gel containing GelRed (Cambridge Bioscience, BT-41003, Munro House, Cambridge, UK) alongside a 100 bp ladder with $1 \mathrm{X}$ TAE as the running buffer. Gels were visualised using a Gel Dock system. For wild-type mice a band would be produced at 450 bp and for a PYY knockout mouse a band would be produced at $250 \mathrm{bp}$. 


\section{GENERAL METHODS SECTION}

\subsection{LIPOPOLYSACCHARIDE BINDING PROTEIN ELISA}

Lipopolysaccharide binding protein (LBP) serum levels were assayed by ELISA kit (Enzo Life Sciences, Switzerland) according to the manufacturer's instructions. Serum samples were diluted 1 in 800 with kit Dilution Buffer. To the precoated 96 well ELISA plate 100 $\mu \mathrm{l}$ of each sample were added in duplicate. Additionally, $100 \mu \mathrm{l}$ of standards $(50,25,12.5$, $6.25,3.12 \mathrm{ng} / \mathrm{ml}$ ) were added in duplicate into corresponding wells of the precoated plate. This was incubated for one hour at room temperature while shaken. The wells were then washed 3 times with Wash Buffer. Next $100 \mu \mathrm{l}$ of detecting antibody were added to each well and incubated at room temperature for 1 hour while shaken. The wells were then washed $3 \mathrm{x}$ with Wash Buffer. Then $100 \mu \mathrm{l}$ of Substrate solutions were added to each well and incubated for $15 \mathrm{~min}$ in the dark at room temperature. To stop the reaction, $100 \mu \mathrm{l}$ of Stopping Solution were added to each well. The plate was gently tapped to mix. The absorbance was read at $450 \mathrm{~nm}$ (reference wave length 620). The mean of optical density (OD) of standard duplicates, reference serum and the samples were calculated. A standard curve was plotted using the OD means of standards and the LBP concentration. The LBP concentration was calculated from the mean OD of samples from the standard curve and multiplied by the dilution factor.

\subsection{MICROARRAY ANALYSIS}

\subsubsection{RNA EXTRACTION FROM BRAIN TISSUE}

A hypothalamic block was dissected from each brain. First, frozen brains were thawed from $-80{ }^{\circ} \mathrm{C}$ to $-20{ }^{\circ} \mathrm{C}$ and then hypothalamic tissue weighing roughly $30 \mathrm{mg}$ was dissected with coronal cuts at stereotaxic coordinates $0.38 \mathrm{~mm}$ and $-2.28 \mathrm{~mm}$ [18]. The hypothalamus was removed from the remaining brain tissue by making an axial cut at the top of the third ventricle and sagittal cuts at the lateral margins of the optic tracts. The frozen tissue blocks were transferred into Lysis Buffer (QIAGEN, Hilden, Germany) containing $\beta$-mercaptoethanol, and then lysed and homogenised with zirconia beads (BioSpec Products, Bartlesville, OK, USA) as recommended in the QIAGEN RNeasy Mini Kit. Total RNA was isolated from the tissue lysate using a QIAGEN RNeasy Mini Kit (QIAGEN, Hilden, Germany) with an on-column DNase digestion treatment following 
the manufacturer's protocol. The RNA concentration and purity of isolated RNA was determined with a NanoDrop ND-1000 spectrophotometer (Thermo Fisher Scientific, Wilmington, DE, USA) with the spectral absorption at 260 and $280 \mathrm{~nm}$. RNA integrity was assessed with an Agilent Bioanalyzer 2100 (Agilent Technologies, Santa Clara, CA, USA) and using their RNA 6000 Nano chips. Samples with an RNA Integrity Number (RIN) of 9.8 or higher and $28 \mathrm{~S} / 18 \mathrm{~S}$ ratio of 1.8 or higher were used for microarray analysis. Prior to microarray analysis RNA samples were stored at $-80^{\circ} \mathrm{C}$.

\subsubsection{MICROARRAY}

The labelling and hybridisation of the RNA samples for the microarray was carried out by the Genomics Laboratories at the Rowett Institute of Nutrition and Health. For labelling samples for gene expression microarray and to generate fluorescent cRNA the Low Input Quick Amp Labelling Kit, two-colour (Agilent Technologies, Santa Clara, CA, USA) was used. Initially to generate cDNA, $1.5 \mu \mathrm{l}$ of each RNA sample with a concentration of $67.5 \mathrm{ng} / \mu \mathrm{l}$ was used with the addition of $2 \mu \mathrm{l}$ RNA spike-in control (RNA Spike-In Kit, two-colour, Agilent, Santa Clara, CA, USA). The synthesis and amplification of cRNA was completed using a T7 RNA Polymerase Blend, concurrently amplifying the target material and incorporating cyanine 3-CTP or cyanine 5-CTP. A two-dye microarray was used for the study and a dye-swap design between the high-fat and low-fat fed groups was used to nullify the dye bias. RNeasy Mini Kit was used to purify the labelled and amplified cRNA samples (QIAGEN, Hilden, Germany). The Gene Expression Hybridisation Kit (Agilent, Santa Clara, CA, USA) was used to prepare samples for hybridisation. Cyanine 3-labelled and cyanine 5-labelled sample pairs were added into the hybridisation mix. Samples were applied to $4 \mathrm{x} 44 \mathrm{~K}$ mouse microarray slides containing 43,604 probes per array (Agilent, Santa Clara, CA, USA) and hybridised for $17 \mathrm{~h}$ at $65^{\circ} \mathrm{C}$. A total of two microarray slides were used with 4 arrays, one slide for wild type and one for TLR4 ${ }^{-/}$hypothalamic gene expression. After hybridisation, the microarray slides were washed with the Gene Expression Wash Buffer Kit (Agilent, Santa Clara, CA, USA). A SureScan microarray scanner (Agilent, Santa Clara, CA, USA) was used for scanning of the microarray slides at the University of Dundee. The microarray analysis was carried out by Gill Campbell, Genomics, Rowett Institute of Nutrition and Health, University of Aberdeen. 


\subsubsection{MICROARRAY DATA PROCESSING}

The raw intensities of the Agilent microarrays were analysed within statistical programming environment $\mathrm{R}$ (version 3.0.2) using the Bioconductor library limma (version 3.18.0) (Smyth, 2005). The data were transformed to a log2-scale and loess normalisation was performed to remove intensity depending dye-bias (Smyth \& Speed, 2003). Transcripts with the same Agilent ID (represented by identical oligos) were averaged within each array. A linear model was fitted to the data that included diet (HF, $\mathrm{LF})$ and dye $(\mathrm{Cy} 3, \mathrm{Cy} 5)$ as factor. The limma package conducts moderated F-tests, where information is borrowed from other genes for the estimation of gene-specific residual variance [21]. We recorded $\log 2$-foldchanges $(\log 2(\mathrm{HF} / \mathrm{LF}))$ and $\mathrm{p}$-values for each gene. Genes with an expression fold change of less than +1.25 or -1.25 and a P-value $<0.05$ were considered as significantly different. The microarray experiment was conducted and data recorded according to the MIAME guidelines and the results data will be lodged with a public access repository such as the Gene Expression Omnibus (http://www.ncbi.nlm.nih.gov/geo/). Microarray data processing was carried out by Claus Mayer Biomathematics \& Statistics Scotland, Rowett Institute of Nutrition and Health, University of Aberdeen.

\subsection{IN SITU HYBRIDISATION}

\subsubsection{BRAIN TISSUE SECTIONING}

Brains stored at $-80^{\circ} \mathrm{C}$ were allowed to acclimatise to $-20^{\circ} \mathrm{C}$ for 30 minutes. Coronal brain sections through the hypothalamus were cut at a thickness of $10 \mu \mathrm{m}$ at $-20^{\circ} \mathrm{C}$ using a cryostat (Leica Biosystems, Milton Keynes, UK) and collected onto sets of 24 slides for each animal. Cut sections of brain tissue were thaw-mounted onto poly-L-Lysine coated slides (Thermo Scientific, UK), allowed to dry and then stored at $-80^{\circ} \mathrm{C}$. Sections from the hypothalamus included the arcuate nucleus and the paraventricular nucleus and were cut from the stereotaxic coordinates $0.38 \mathrm{~mm}$ to $-2.28 \mathrm{~mm}$ (Franklin \& Paxinos, 1997). The section collection started on slide 1 placing each sequentially cut section on successive slides numbered to slide 12 , before returning to slide 1 and repeating the process until each slide had 5 sections, and such that each slide had the same representation of the brain region. The process was repeated through slide numbers 12-24, Slides numbered 1-12 contained the ARC region whilst slides 12-24 contained 
the PVN region. The slides were labelled with the experiment number, slide number 1 to 24 , and the animal number. The slides were stored at $-70^{\circ} \mathrm{C}$.

\subsubsection{PREPARATION OF RNA RIBOPROBES}

Representative slides from each animal were hybridised with a cross-species radiolabelled rodent-specific antisense riboprobe complementary to partial fragments of agouti-related peptide (AgRP) (Mercer et al, 2000), suppressor of cytokine siganalling-3 (SOCS3) (Tups et al, 2004), and serpin family A member 3N (SerpinA3N) generated from cDNA, and were either mouse-specific or mouse and hamster-specific, Table 5. Plasmids were isolated from glycerol stocks for riboprobe synthesis by taking a stabbing of the glycerol stock of E. coli bacteria containing the recombinant plasmid and inoculating this into Luria-Bertani broth cultures of $5 \mathrm{ml}$, containing antibiotic appropriate for the plasmid (ampicillin or kanamycin) at a concentration of $50 \mathrm{mg} / \mathrm{ml}$. Broth cultures were incubated overnight in a $37^{\circ} \mathrm{C}$ with shaking in an orbital incubator at 200rpm. Recombinant plasmids were isolated from the broth cultures using the Wizard plus SV Miniprep DNA purification system (Promega, UK). Template DNA for Riboprobe synthesis was amplified from the isolated plasmid DNA by PCR with M13 forward and reverse primers and GoTaq hot start polymerase (Promega, UK). Each recombinant plasmid and amplified DNA was run on an agarose gel against a $100 \mathrm{bp}$ DNA marker using electrophoresis. DNA bands were visualised on a UV transilluminator to confirm the insert was of the correct size (UVIpro, UK). The amplified DNA was then purified using the QIAquick PCR purification kit (QIAGEN, UK) and DNA quantified by NanoDrop ND-1000 spectrophotometer (Thermo Fisher Scientific, Wilmington, DE, USA).

\subsubsection{PROBE SYNTHESIS}

RNA riboprobes for in-situ hybridisation were synthesised using the following transcription reaction. Briefly, $1 \mu \mathrm{l}$ of DNA template (100 ng of DNA), $5 \mu \mathrm{l}$ of $5 \mathrm{X}$ Buffer, $10 \mu \mathrm{l}$ of purified RNase-free water, $3 \mu \mathrm{dNTP} 10 \mathrm{mM}$ (dCPT, dGPT, dATP), $1 \mu \mathrm{l}$ of Ribolock, and $4 \mu \mathrm{l}$ of ${ }^{35} \mathrm{~S}$-Uridine 5'tri-phosphate for labelling were added into a sterile $1.5 \mathrm{ml}$ microcentrifuge tube. The synthesis was initiated with the addition of $1 \mu \mathrm{l}$ of the appropriate DNA dependent RNA polymerase, Table 5. The reaction mixture was incubated for $75 \mathrm{~min}$ at $37^{\circ} \mathrm{C}$, then $2 \mu$ of DNAse 1 was added and incubated for a further 
$30 \mathrm{~min}$ at $37^{\circ} \mathrm{C}$ in a water bath. The synthesised probe was then purified using a Probe Quant G50 microcolumn (GE Healthcare, UK). The column was prepared by briefly vortexing the column, snapping off the seal from the base of the column, turning the cap 90 degrees, and centrifuging for 1 minute at $3000 \mathrm{rpm}$ with a $2 \mathrm{ml}$ collection tube to collect waste. The reaction mixtures were made up to $50 \mu$ l with DEPC treated water, and this was applied directly onto the column, which was then centrifuged for 2 minutes at $3000 \mathrm{rpm}$ in a microcentrifuge (Eppendorf $5415 \mathrm{R}$, Germany) to remove unincorporated label within the column matrix and the purified probe was collected in the eluate. The specific activity of the purified radiolabelled probe was determined using $5 \mu \mathrm{l}$ of a 1 in 100 dilution in water and counted in duplicate in a $\beta$-counter (Beckman Coulter Ltd, UK).

Table 5. In situ hybridisation probes

\begin{tabular}{|c|c|c|c|c|}
\hline Probe & Insert & Primer Sequence & $\begin{array}{l}\text { RNA } \\
\text { Pol* }\end{array}$ & Source \\
\hline SerpinA3N & $\begin{array}{l}543 \text { bp } \\
\text { cDNA } \\
\text { mouse }\end{array}$ & $\begin{array}{l}\text { F: 5'-CTACGCGGGCAAGAGGA-3' } \\
\text { R: 5'-AAGGGGGCAATTTCAGTTT-3' }\end{array}$ & $\mathrm{T7}$ & $\begin{array}{l}\text { Gift from Prof. } \\
\text { Lynda Williams }\end{array}$ \\
\hline SOCS3 & $\begin{array}{l}465 \text { bp } \\
\text { cDNA } \\
\text { Siberian } \\
\text { hamster }\end{array}$ & $\begin{array}{l}\text { F: 5'-ACACCAGCCTGCGCCTCAAGACCT-3' } \\
\text { R: 5'-TCGCCCCCAGAATAGATGTAGTAA-3' }\end{array}$ & $\mathrm{T7}$ & $\begin{array}{l}\text { (Tups et al., } \\
\text { 2004) }\end{array}$ \\
\hline AgRP & $\begin{array}{l}229 \text { bp } \\
\text { cDNA } \\
\text { Siberian } \\
\text { hamster }\end{array}$ & $\begin{array}{l}\text { F: 5'-TGTTCCCAGAGTTCCCAGGTC-3' } \\
\text { R: 5'-GCATTGAAGAAGCGGCAGTAG CAC-3' }\end{array}$ & $\mathrm{T7}$ & $\begin{array}{l}\text { (Mercer et al., } \\
2000 \text { ) }\end{array}$ \\
\hline
\end{tabular}

*DNA dependent RNA polymerase

\subsubsection{HYBRIDISATION}

Hypothalamic mRNA expression was quantified by in-situ hybridisation using established methods (Mercer et al, 1997; Simmons et al, 1989). Before use all solutions 
were made up and then autoclaved, and all equipment was either baked at $200^{\circ} \mathrm{C}$ or autoclaved to ensure RNase-free conditions. The Poly L-lysine slides with coronal brain sections were first fixed in $4 \%$ paraformaldehyde for 20 minutes while on ice. The slides were then washed for 2 x 5 minutes with $0.1 \mathrm{M}$ phosphate buffer (PB). Sections were then immersed for 2 minutes in $0.1 \mathrm{M}$ phosphate buffer. The same $0.1 \mathrm{M}$ phosphate buffer was then acetylated by adding it to $1.5 \mathrm{ml}$ of $0.25 \%$ acetic anhydride (SigmaAldrich, St. Louis, Missouri, United States), and immersing the slides in this for 10 minutes. The slides were then washed for $2 \times 2$ minutes with $0.1 \mathrm{M} \mathrm{PB}$. The sections where then dehydrated in a series of ethanol washes, 50\%, 70\%, 95\%, and 100\% ethanol, for 3 minutes each, before drying in a vacuum desiccator for 60 minutes.

The ${ }^{35}$ S-labelled antisense riboprobe was prepared in formamide with the addition of 0.3 $\mathrm{M} \mathrm{NaCl}, 10 \mathrm{mM}$ DTT, 10\% dextran sulphate, 0.05\% tRNA, 50X Denhardt's Solution, 1 $\mathrm{mM}$ EDTA, and an adjusted volume of probe to ensure 10,000 counts $/ \mathrm{min} / \mu \mathrm{l}$ was applied as $70 \mu \mathrm{l}$ onto each slide. A glass coverslip was placed over the sections and sealed with DPX mounting medium, and then incubated in a hybridisation oven at $58^{\circ} \mathrm{C}$ overnight. After hybridisation, the slides were soaked in $4 \mathrm{x}$ standard saline citrate (SSC), and the DPX mounting media and coverslips were removed from the slides. Sections were then washed four times in $4 \mathrm{x}$ SSC for 5 minutes per wash. To remove any unhybridized probe the sections were then incubated in a water bath for 30 minutes at $37^{\circ} \mathrm{C}$ in an RNase A solution. Sections were then washed two times in $2 \mathrm{x}$ SCC for 5 minutes, for 10 minutes in $1 \mathrm{x}$ SCC, and for 10 minutes in $0.5 \mathrm{x}$ SCC. A final wash was carried out in $\mathrm{lx} \mathrm{SSC}$ for 30 minutes at $60^{\circ} \mathrm{C}$ to remove any un-hybridised probe. The sections where then dehydrated in a series of ethanol washes, $50 \%, 70 \%$, and $95 \%$ ethanol for 3 minutes each, then in 100\% ethanol for 1 minute, before being allowed to air dry. 


\subsubsection{IMAGE ANALYSIS}

Hybridised slides were exposed to an autoradiographic film (BioMax MR; Kodak, USA) for between 3 to 28 days depending on the strength of the radioactive signal. Autoradiographic films were scanned at 1200 d.p.i. analysed and quantified using Image-Pro Premier software version 9.2 (Media Cybernics UK, Marlow, UK), which computes the integrated optical density of the signal relative to a $14 \mathrm{C}$ autoradiographic microscale (Amersham, Pharmacia Biotech UK Ltd, Little Chalfont, UK).

\subsection{DNA EXTRACTION FROM INTESTINAL CONTENTS}

DNA was extracted from intestinal contents (terminal ileum, caecum, and colon), and faecal pellets. DNA was extracted using a FastDNA ${ }^{\circledR}$ SPIN Kit for Feces (MP Biomedicals 116570200, MP Biomedicals SARL, Illkirch, France) and processed according to the manufacturer's instructions. Due to the small size of the mouse samples available for analysis, samples used for extraction were smaller in weight than the recommended 500 $\mathrm{g}$ of faeces and varied between individual mice. Briefly, the samples were combined in a Lysing Matrix E tube with $825 \mu$ of Sodium Phosphate Buffer, and $275 \mu$ l of PLS solution. These were shaken, vortexed for 15 secs, then centrifuged at 13,200 x g for $5 \mathrm{~min}$ (Eppendorf 5415R, Hamburg, Germany), and the supernatant discarded. Then the sample was resuspended in $978 \mu$ of Sodium Phosphate Buffer and $122 \mu \mathrm{l}$ of MT Buffer by briefly vortexing. These resuspended samples were homogenised using a FastPrep ${ }^{\circledR} 24$ (MP Biomedicals SARL, Illkirch, France) set at $6.0 \mathrm{~m} / \mathrm{s}$ for 40 seconds and centrifuged for 15 minutes (Eppendorf 5415R, Hamburg, Germany). The supernatant was decanted into a $2.0 \mathrm{ml}$ microcentrifuge tube with $250 \mu \mathrm{l}$ of PPS solution added, shaken vigorously, incubated at $4^{\circ} \mathrm{C}$ for 10 minutes, and centrifuged at 3,200 x g for 2 minutes (Eppendorf $5415 \mathrm{R}$, Hamburg, Germany). Sample supernatant was decanted into a $15 \mathrm{ml}$ conical tube with $1 \mathrm{ml}$ of Binding Matrix Solution, shaken gently to mix, placed on a shaker/rocker for $5 \mathrm{~min}$, and centrifuged at 13,200 x g for 2 minutes (Eppendorf $5415 \mathrm{R}$, Hamburg, Germany). The supernatant was discarded and the pellet gently resuspended with $1 \mathrm{ml}$ of Wash Buffer \#l. First, $600 \mu \mathrm{l}$ of sample was transferred to a SPIN Filter tube and centrifuged at 13,200 x g for 1 min (Eppendorf $5415 \mathrm{R}$, Hamburg, Germany), the flow through discarded and the remaining sample centrifuged as before and flow through discarded. Next $500 \mu \mathrm{l}$ of Wash Buffer \#2 was added to the SPIN Filter and the pellet 
resuspended using gentle pipetting. This was centrifuged at 13,200 x g for 2 min (Eppendorf 5415 R, Hamburg, Germany) and the flow-through discarded. To remove residual ethanol the SPIN Filter tube was then centrifuged again for 2 min (Eppendorf 5415 R, Hamburg, Germany). The SPIN Filter was transferred to a clean $1.9 \mathrm{ml}$ Catch Tube, $60 \mu$ of TED added, and the pellet resuspended by flicking the tube. To elute purified DNA samples were centrifuged at 13,200 x g for 2 min (Eppendorf $5415 \mathrm{R}$, Germany). Eluted DNA concentration and purity was assessed using a NanoDrop ND1000 spectrophotometer (Thermo Fisher Scientific, Wilmington, DE, USA). Samples were transferred to $1.5 \mathrm{ml}$ maximum recovery microcentrifuge tubes for short-term storage at $4^{\circ} \mathrm{C}$. Each extraction was confirmed by running a $2 \mu \mathrm{l}$ volume (combined with $3 \mu \mathrm{l}$ of $6 \mathrm{X}$ Gel Loading Dye) on a 1\% agarose gel containing GelRed (Cambridge Bioscience, BT-41003, Munro House, Cambridge, UK) for 45 - 60 minutes alongside a 100 bp ladder with IX TAE as the running buffer. Gels were visualised using a Gel Dock system. Successfully extracted bacterial genomic DNA form a band near the top of the gel, higher than the top band of the ladder $(1,517 \mathrm{bp})$ due to the large product size.

\subsection{DENATURING GRADIENT GEL ELECTROPHORESIS}

The protocol used was based on that of Muyzer et al (1993) with some modifications. Samples were amplified by PCR using the following primer sequences targeting the variable region 3 (V3) of the $16 \mathrm{~S}$ bacterial rRNA sequence: Primer 1, 5'-C CTACGGGAGGCAGCAG-3' and Primer 2, 5'-CGCCCGCCGCGCGCGGCGGGCGGGGCG GGGGCACGGGGGGCCTACGGGAGGCAGCAG-3'. Primer two contains an additional 40-nucleotide GC-rich sequence (GC clamp). A 30 minutes extension was included at the end of each PCR to reduce double band formation (Janse et al, 2004). DGGE was performed using the Cipher DGGEK-2001 system (C.B.S. Scientific, Del Mar, CA, USA). PCR samples were applied directly onto a $30 \%$ to $70 \%$ gradient $8 \%$ (wt/vol) polyacrylamide gel in lx TAE (20 mM Tris acetate [pH 7.4], $10 \mathrm{mM}$ sodium acetate, 0.5 mM Na-EDTA) with gradient formed with $8 \%$ (wt/vol) acrylamide stock solutions and which contained 0 or $100 \%$ denaturant (7 M uric acid and 40\% [vol/vol] formamide. Silicone grease was applied to the spacers and glass plates to reduce to avoid edge effects (Brinkhoff \& van Hannen, 2001). Electrophoresis was performed at a constant voltage of $85 \mathrm{~V}$ and a temperature of $60^{\circ} \mathrm{C}$ for a period of 16 hours. After electrophoresis, the gels 
were incubated for $30 \mathrm{~min}$ in SYBR Gold stain, and photographed with UV transillumination with gel dock. The final DGGE fingerprints were analysed using FPQuest 5.10 software (Bio-Rad, Hercules, CA). To compensate for differences between gels, the DGGE patterns were aligned and normalised using a reference ladder lane. After normalisation, the bands were defined for each sample using appropriate densitometric curves. The position tolerance used in the band comparison was $1.00 \%$. Similarity between DGGE profiles was determined by calculating the Dice correlation with fuzzy logic. Sample profile clustering was carried out using the unweighted pair-group method using arithmetic average (UPGMA).

\subsection{PCR AMPLIFICATION FOR ILLUMINA SEQUENCING}

DNA extracted from mouse intestinal samples was used as a template for PCR amplification of the V1-V2 variable regions of the bacterial 16S gene using barcoded primers MiSeq-27F and MiSeq-338R. These primers contain adaptors for downstream Illumina MiSeq sequencing. Each sample was PCR amplified with a reverse primer containing a unique (12 base) barcode.

Table 6. Primers used for Illumina MiSeq Sequencing

\begin{tabular}{ll}
\hline Primer & Primer sequence \\
\hline MiSeq-27F & $\begin{array}{l}\text { 5'-AATGATACGGCGACCACCGAGATCTACACTATGGTAATTCCAGGTTYGAT } \\
\text { YMTGGCTCAG-3' }\end{array}$ \\
MiSeq-338R & $\begin{array}{l}\text { 5'-CAAGCAGAAGACGGCATACGAGAT-barcode-AGTCAGTCAGAAGCTGCCTC } \\
\text { CCGTAGGAGT-3' }\end{array}$ \\
\hline
\end{tabular}

PCR amplification was carried out using New England BioLabs Q5 High-Fidelity DNA Polymerase. Each reaction mix contained 5x Q5 Reaction Buffer $(5 \mu \mathrm{l}), 10 \mathrm{mM}$ dNTPs $(0.5 \mu \mathrm{l}), 10 \mu \mathrm{M}$ 27F Primer (1.25 $\mu \mathrm{l}), 10 \mu \mathrm{M}$ R Primer (1.25 $\mu \mathrm{l})$, Q5 High-Fidelity DNA Polymerase $(0.25 \mu \mathrm{l})$, DNA template $(1 \mu \mathrm{l})$, and New England BioLabs sterile, nucleotide water $(15.75 \mu \mathrm{l})$ to a final volume of $25 \mu \mathrm{l}$. PCR reactions were set up in quadruplicate for each DNA sample. Thermocycling conditions for the PCR amplification were: 2 minutes at $98^{\circ} \mathrm{C}$, then 20 cycles of 30 seconds at $98^{\circ} \mathrm{C}, 30$ seconds at $50{ }^{\circ} \mathrm{C}, 90$ seconds at $72{ }^{\circ} \mathrm{C}$; finally, a 5 minute extension at $72{ }^{\circ} \mathrm{C}$ then a holding temperature of $4{ }^{\circ} \mathrm{C}$.

Following amplification, the quadruplicate PCR reactions were pooled into a single sterile $1.5 \mathrm{ml}$ microcentrifuge tube. Successful amplification was confirmed by running a 
$5 \mu \mathrm{l}$ sample of each pooled PCR reaction from each sample (with $2 \mu \mathrm{l}$ of $6 \mathrm{x}$ Gel Loading Dye) on a 1\% agarose gel containing GelRed (Cambridge Bioscience, BT-41003, Munro House, Cambridge, UK) for 45 - 60 minutes alongside a 100 bp ladder with IX TAE as the running buffer. Gels were visualised using a Gel Dock system (UVItec Limited, Cambridge, United Kingdom). For the V1-V2 primer set of the 16S rRNA gene the product formed a band of around 350-400 bp.

Ethanol precipitation was used to clean-up the pooled PCR samples and concentrate the samples to a $30 \mu$ l volume. Briefly, 0.3 volumes of $1 \mathrm{M} \mathrm{NaCl}$ (relative to each sample) and two volumes of cold $100 \%$ ethanol (relative to the combined sample $+\mathrm{NaCl}$ ) were added to each sample, mixed by inversion and placed at $-20{ }^{\circ} \mathrm{C}$ overnight. The following day each sample was centrifuged at 13,600 x g for 20 minutes (Eppendorf $5415 \mathrm{R}$, Germany). The supernatant was removed gently, and the pellet washed with $600 \mu \mathrm{l}$ of cold $70 \%$ ethanol and then centrifuged again at 13,600 x g for 15 minutes (Eppendorf 5415R, Germany). The supernatant was removed gently and the tubed left to air dry until all the ethanol was evaporated and the pellet appeared opaque/white. The pellet was resuspended by adding $30 \mu \mathrm{l}$ of TE buffer and leaving overnight at $4{ }^{\circ} \mathrm{C}$. Samples were stored at $-20{ }^{\circ} \mathrm{C}$ before quantification. The pooled PCR amplicons were quantified using a Qubit dsDNA HS Assay Kit (Invitrogen, CA, USA, Q32854). Briefly, a Working Solution was prepared equal to $199 \mu$ l of Qubit HS buffer mixed with $1 \mu$ l of Qubit HS Reagent for each sample to be quantified. Two Qubit tubes were labelled HSl and HS2 and $10 \mu \mathrm{l}$ of Qubit HS Standard $1(0 \mathrm{ng} / \mu \mathrm{l})$ and Qubit HS Standard $2(10 \mathrm{ng} / \mu \mathrm{l})$ added in to the tubes respectively. A $190 \mu \mathrm{l}$ of Working Solution was added to each tube, vortexed for 5 seconds and incubated at room temperature for 2 minutes. The dsDNA HS setting was selected on the Qubit 2.0 Fluorometer (Invitrogen, CA, USA) and a new calibration using the two Standard Tubes was used to create a standard curve. Next $1 \mu \mathrm{l}$ of sample was added into a Qubit Assay Tube (Invitrogen, CA, USA, Q32856) together with $199 \mu \mathrm{l}$ of Working Solution, vortexed for 5 seconds, incubated at room temperature for 2 minutes, and then DNA concentration determined using the Qubit 2.0 Fluorometer (Invitrogen, CA, USA). All sample concentrations were determined using the same Working Solution. After the 2 minute incubation at room temperature the concentration of each sample was measured in $\mathrm{ng} / \mu \mathrm{l}$. 


\subsubsection{PREPARING AN EQUIMOLAR MIX FOR SEQUENCING}

An equimolar mix was prepared for sequencing using equimolar concentrations of DNA from each sample. The amount of each sample to be added was calculated using the following formula: Sample volume $(\mu \mathrm{l})=$ DNA Conc. of maximum sample $(\mathrm{ng} / \mu \mathrm{l}) / \mathrm{DNA}$ conc. of sample $(\mathrm{ng} / \mu \mathrm{l})$. All samples were above the minimum accepted concentration of $3 \mathrm{ng} / \mu \mathrm{l}$. The Equimolar mix was then split into 2 equal volumes in $1.5 \mathrm{ml}$ microcentrifuge tubes. One tube was stored at $-20^{\circ} \mathrm{C}$ as a backup while the other was prepared for sequencing.

\subsubsection{SAMPLE CLEAN-UP VIA GEL PURIFICATION}

The Equimolar mix was cleaned using gel purification to remove primer dimers using a Wizard $^{\circledR}$ SV Gel and PCR Clean-Up System (Promega, A9281, Madison, WI, USA). Briefly, the equimolar mix library was run out on a $1 \%$ agarose gel, stained with GelRed (Cambridge Bioscience, BT-41003, Munro House, Cambridge, UK), in TAE buffer. Gel containing the band between $\sim 300-400$ bp was excised from the gel using a sterile scalpel and placed into pre-weighed $1.5 \mathrm{ml}$ microcentrifuge tubes. The tubes were weighed to determine the weight of the gel slices. Into each tube was added $10 \mu \mathrm{l}$ of Membrane Binding Solution per $10 \mathrm{mg}$ of gel slice. The mix was vortexed to mix and incubated in a hot block at $65^{\circ} \mathrm{C}$ for 10 minutes or until the gel was fully dissolved. A maximum of $350 \mathrm{mg}$ of sample in $350 \mu \mathrm{l}$ of binding solution was pipetted into the SV Mini-column assembly (Filter Column plus Collection tube) and left to bind for 1 minute. This was then centrifuged for 1 minute at 16,000 x g (Eppendorf 5415R, Hamburg, Germany). The liquid was discarded, then $700 \mu \mathrm{l}$ of Membrane Wash Solution added to the column and the centrifuged for 1 minutes at 16,000 x g (Eppendorf 5415R, Hamburg, Germany). The flow through was discarded and this was repeated with $500 \mu \mathrm{l}$ of Membrane Wash Solution, centrifuged for 6 minutes at 16,000 x g (Eppendorf 5415R, Hamburg, Germany), and the flow through discarded. This was repeated until the DNA in all dissolved gel samples had been bound to the same column without eluting the DNA. The total DNA was eluted into a sterile $1.5 \mathrm{ml}$ microcentrifuge tube by adding 50 $\mu \mathrm{l}$ of Nuclease Free Water to the column, incubating at room temperature for 1 minute, and then centrifuging for 1 minute at $16,000 \mathrm{xg}$. The $50 \mu \mathrm{l}$ volume was divided into two 
tubes, $25 \mu \mathrm{l}$ in each, one stored at $-20^{\circ} \mathrm{C}$ as a backup, while the other was used for sequencing.

\subsubsection{NEXT GENERATION ILLUMINA SEQUENCING}

Sequencing of the pooled PCR products was carried out on an Illumina MiSeq machine, using a $2 \times 250$ bp read length. Illumina MiSeq sequencing was carried out by the Centre for Genome Enabled Biology and Medicine, University of Aberdeen.

\subsubsection{ILLUMINA MISEQ SEQUENCE DATA ANALYSIS}

The data obtained from Illumina MiSeq sequencing was analysed using the mothur software package (Schloss et al, 2009), and based on the Mother MiSeq Standard Operating Procedure (Kozich et al, 2013). A text file was created detailing which two paired reads, the forward and reverse reads generated from Ilumina sequencing run, belonged to each sample. This included 194 different barcoded samples in total, including samples from Mouse Study 1 and 2 the results of which are detailed in Chapters 3 and 4. These comprised of 190 mouse gut contents samples plus 4 DNA extraction kit blanks processed with only water as a control. All samples from both studies were processed together. The forward and reverse reads generated from the sequencing were then assembled into paired contigs. After assembly contigs that were shorter than 280 base pairs, or longer 470 base pairs, that contained ambiguous bases, or contained homopolymeric stretches of longer than 8 bases, were removed. One representative sequence for each unique sequence was extracted from the dataset. Unique sequences were then grouped together and aligned against the SILVA reference database. The OTUs were generated at a $97 \%$ similarity cut-off level. Due to a high number of unique singleton and doubleton sequences, those represented by only 1 or 2 sequences in the whole dataset, these were removed to reduce the total number of sequences to less than 50,000 to reduce the file size to one that could be processed.

Initially the UCHIME program was used to detect and remove chimeric molecules created during PCR amplification. However, later investigation revealed that the UCHIME program had incorrectly removed the most abundant bacterial sequences (OTUl) from the dataset. Chimeras are typically not the most abundant sequences generated and this sequence aligned to the same bacterial group along its whole length 
using BLAST nucleotide search. Perseus (Quince et al, 2011) was then used as an alternative chimera removal software to detect and remove chimeric molecules created during PCR amplification. Perseus did not remove OTU1 from the dataset and was so Perseus was used for the following analysis.

The RDP reference database (release 10) (Wang et al, 2007) was used to assign taxonomic classifications to each OTU at the phylum, family, and genus level. Spurious reads, including those derived from mitochondria, chloroplasts or Eukarya were removed from the dataset. The final dataset contained a total of 4,642,375 sequences with a range between samples of 55,843-10,596 sequences.

One $\mathrm{CD}_{1} 4^{-/}$mouse in the Low-Fat diet group returned only 511 sequences and was excluded from further analysis. The lowest number of sequence read in a single sample was 10,596 sequences. All samples were then rarefied (sub-sampled) to reduce all samples to the same number of sequences of 10,596 per sample to equalise the number of reads and sequencing depth between all samples for later analysis. A representative sequence for each OTU was generated and the most abundant OTUs were curated against the BLAST database to assign species level classification where possible.

Samples from Study 1 and Study 2 were separated for further analysis. The observed richness (Sobs), the estimated (Chaol) total richness, the Shannon diversity index, the inverse Simpson diversity index, and Good's coverage were used to calculate the bacterial diversity within each sample. Rarefaction measures of species richness were generated for each sample by repeatedly re-sampling data plotting the number of OTUs detected against sequencing depth. Bray-Curtis calculator was used generate an index of dissimilarity between samples based on OTU community structure. The Bray-Curtis matrix used generated dendrograms of microbiota similarity. The TreeClimber test (Schloss \& Handelsman, 2006), which calculates whether or not clustering patterns are significantly different, was run to calculate the statistical significance of dendrogram clustering. The Bray-Curtis index of dissimilarity was also used to calculate non-metric dimensional scaling (NMDS) plots of overall microbiota dissimilarity. The significance of NMDS clustering was tested using analysis of molecular variance (AMOVA) (Martin, 2002) and homogeneity of molecular variance (HOMOVA) (Schloss, 2008). OTUs accounting for more than $0.2 \%$ of total proportional abundance were considered for 
further analysis. LEfSe (Segata et al, 2011), a metagenomics biomarker discovery program, was used to identify features of sequence data, OTUs and higher taxa, which differentiated the groups with a linear discriminant analysis (LDA) effect size of greater than 2. Metastats (White et al, 2009), a non-parametric T-test, incorporating Fisher's exact test and the false discovery rate (FDR) was used to determine whether OTUs, or higher taxa, identified as having an LDA effect size of greater than 2 were significantly differentially represented between groups. $\mathrm{P}$ values generated using Metastats were corrected with the Benjamini-Hochberg method (Benjamini \& Hochberg, 1995) to correct for the false discovery rate across multiple comparisons.

\subsection{SHORT-CHAIN FATTY ACID ANALYSIS}

The concentrations of short-chain fatty acids and other organic acids produced by bacterial fermentation in caecal contents were determined by capillary gas chromatography. Frozen caecal contents were thawed on ice and $100 \mathrm{mg}$ of each sample was weighed out. Next $200 \mu \mathrm{l}$ of distilled water were added to each $100 \mathrm{mg}$ of sample and vortexed until suspended, before $75 \mu \mathrm{l}$ of 2-ethylbutyric acid ( $5 \mathrm{mmol} / \mathrm{L}$ ) internal standard were added, and the sample vortexed to mix. To pellet the solids the sample was then centrifuged at 16,000 $\mathrm{x} g$ for 20 minutes at $4^{\circ} \mathrm{C}$ (Eppendorf 5415R, Germany). The supernatant was then removed. Samples with suspended solutes remaining were added to a Costar ${ }^{\circledast}$ Spin- $\mathrm{X}^{\circledast}$ polypropylene tube filter (Sigma-Aldrich) and centrifuged (Eppendorf 5415 R, Germany). The samples were analysed using an Agilent HP-FFAP column (Cat No. 19095F-121) (Dimensions: 10m x 0.53mm ID x 1 $\mu \mathrm{m}$ ) gas chromatograph fitted with a fused silica capillary. This method detects and provides quantitative measures of acetate, propionate, butyrate, iso-butyrate, valerate, and iso-valerate. Caecal short-chain fatty analysis extraction was carried out by Karen Garden, Rowett Institute of Nutrition and Health, University of Aberdeen. Gas chromatography analysis was carried out by Donna Henderson, Analytical Services, Rowett Institute of Nutrition and Health, University of Aberdeen.

\subsection{SERUM ACETATE ANALYSIS}

Serum samples were treated with HemogloBind (Biotech Support Group LLC, Monmouth Junction, NJ, United States) to remove haemolysis colouration from the serum and equalise the colour between samples. Briefly, in a $1.5 \mathrm{ml}$ microcentrifuge tube 
$125 \mu \mathrm{l}$ of HemogloBind was added to $125 \mu \mathrm{l}$ of serum, vortexed for 30 seconds, mixed by inversion for 10 minutes, and then centrifuged at 9,000 rpm for 2 minutes in a microcentrifuge (Eppendorf 5415R, Hamburg, Germany). Supernatant containing the haemoglobin depleted sample was removed into a $1.5 \mathrm{ml}$ microcentrifuge tube. Concentration of acetate in the serum were determined using an Acetate Colorimetric Assay Kit (Sigma-Aldrich, MAK068, St. Louis, United States). A Reaction Mix was prepared according to the manufactures protocol. This included $42 \mu \mathrm{l}$ of Acetate Assay Buffer, $2 \mu$ l of Acetate Enzyme Mix, $2 \mu \mathrm{l}$ of ATP, $2 \mu \mathrm{l}$ of Acetate Substrate Mix, and $2 \mu \mathrm{l}$ of Probe per well in a 96 well plate. Then $50 \mu \mathrm{l}$ of sample was added to wells in duplicate, and $50 \mu \mathrm{l}$ of the Reaction Mix was added to each well. Acetate standards were added in duplicate at $0,2,4,6,8$, and 10 nmole/well to generate a standard curve plus a sample blank not containing the Acetate Enzyme Mix. This was covered to protect from light, mixed using a horizontal shaker, and incubated at room temperature for 40 minutes. Absorbance was measured at $450 \mathrm{~nm}\left(\mathrm{~A}_{450}\right)$. To calculate the concentrations the background absorbance from the standard blank was subtracted from all readings. A standard curve was determined from the standard curve. The amount of acetate present in samples was determined from the standard curve. Serum acetate analysis methods were carried out by Karen Garden, Rowett Institute of Nutrition and Health, University of Aberdeen.

\subsection{COMPARISON OF RODENT DIET COMPOSITION}

Mouse or rat studies were identified through literature searches that had used both refined low-fat diets and unrefined chow diets within the same study, either alone or in combination with high-fat obesogenic diets. Nutrient composition data sheets were obtained from the companies producing the diets in the studies identified. The following factors were compared between diets: macronutrients as percentage of energy content, ingredients, and micronutrients. 


\subsection{STATISTICAL ANALYSIS.}

Unless otherwise stated group comparisons for all data were performed using SigmaPlot 13.0 (SigmaPlot 13.0 Systat Software, San Jose, CA). The normal distribution of sample data being analysed was initially tested using the Shapiro-Wilk test. Sample groups that failed the Shapiro-Wilk test were plotted and visually assessed for normality. Comparisons between normally distributed samples were analysed by One Way ANOVA followed by Tukey's post-hoc test for multiple comparisons. Comparisons between skewed samples were analysed using Kruskal-Wallis One Way ANOVA on Ranks followed by Dunn's test for multiple comparisons. P values of 0.05 or less were considered significant. 


\section{CHAPTER 3.}

\section{THE ROLE OF TLR4 AND CD14 IN HIGH-FAT DIET INDUCED OBESITY}




\section{INTRODUCTION}

Since 2004 evidence has accumulated implicating the intestinal microbiota as a novel environmental factor that may contribute to the development of obesity (Backhed et al, 2004). This has resulted in intense research into this area and associations have been found between the obese state and altered composition of the gut microbiota in both rodents and humans. The transfer of intestinal microbiota from lean and obese mice into germ-free recipient mice has been reported to transfer aspects of the obese phenotype to those mice receiving microbiota from obese mice (Turnbaugh et al, 2006). However, although this suggests a causative role for the gut microbiota in the development of obesity, details of the mechanisms that link the gut microbiota to changes in body weight and body fat remain unresolved. One potential mechanism is modulation of the immune response, as in response to high-fat diets the composition of gut microbiota changes leading to increased intestinal permeability and increased systemic exposure to bacterial lipopolysaccharide (LPS), also referred to as endotoxin, which binds to the Toll-like receptor 4 expressed on cells of the innate immune system as well as other cell types including endothelial cells and adipocytes (Khakpour et al, 2015; Rietschel et al, 1994; Vitseva et al, 2008). The resulting state of chronic low-grade inflammation has been termed 'metabolic endotoxemia' (Cani et al, 2007a). Chronic inflammation is proposed to disrupt the normal homeostatic regulation of bodyweight, although the details of this effect remain unresolved (Cani et al, 2007a).

\subsection{LPS AND METABOLIC ENDOTOXEMIA}

Lipopolysaccharide (LPS) is a highly immunogenic cell surface molecule forming the outer cell membrane of Gram-negative bacteria. LPS is continually released endogenously in the gut through the death of Gram-negative bacteria (Drewe et al, 2001). LPS translocates across the intestinal epithelium into the circulation, where it binds to chylomicrons which neutralise and detoxify it (Tomita et al, 2004; Vreugdenhil et al, 2003). Short-term feeding of a very high-fat obesogenic diet, containing $78 \%$ of energy as fat, to mice led to increased body fat and increase plasma LPS concentrations, resulting in chronic exposure to circulating LPS (Cani et al, 2007a). This increase in LPS to 2.7 times above normal levels in response to high-fat diet feeding was termed metabolic endotoxemia (Cani et al, 2007a). This was distinguished from the 10-50 times 
higher levels of LPS that can result from infections or septic shock (Mydel et al, 2006). Plasma LPS was related to the fat content of the diet, with mice fed a diet containing of $40 \%$ energy as fat resulting in only a 1.4 times increase in plasma LPS levels (Cani et al, 2007a). It was proposed that changes in the composition of the gut microbiota associated with high-fat diet-induced obesity leads to greater numbers of Gram-negative bacteria and increased permeability of the intestinal epithelium. When LPS was chronic infused subcutaneously into mice over four weeks, to mimic the LPS increases observed in very high-fat diet fed animals, this was reported to increase body weight, body fat, and insulin resistance (Cani et al, 2007a). This suggested that LPS alone can initiates obesity and insulin resistance and represented a mechanism of cause and effect between the gut microbiota and body weight change.

\subsection{TOLL-LIKE RECEPTOR 4}

LPS initiates inflammatory responses to the presence of Gram-negative bacteria through the activation of Toll-like receptor 4 (TLR4) (Beutler \& Poltorak, 2001). CD14 (cluster of differentiation 14) acts as an essential co-receptor with TLR4 in the binding of LPS (Kitchens, 2000). CD14 together with MD-2, another essential component of the TLR4 complex, lead to the binding and activation of the TLR4 receptor by the LPS molecule (Park et al, 2009). In 2004 it was first reported that mice harbouring loss of function mutations in TLR4 or in CD14 have an "ideal” body plan with stronger bones and decreased body fat (Johnson et al, 2004), suggesting a link between TLR4/CD14 and obesity. Building on their results from chronic LPS infusion into mice Cani and colleagues fed a very high-fat diet (78\%) fat to $\mathrm{CD} 14^{-/-}$mice, with the $\mathrm{CD} 14$ gene deleted (Cani et al, 2007a). Consistent with the ideal body plan idea it was reported that, in contrast to wild-type mice, $\mathrm{CD} 14^{-/-}$mice did not increase body weight or adipose tissue weights, after LPS infusion and showed attenuated effects of high-fat diet feeding on body weight. This appeared to demonstrate a causal link high-fat diet feeding, the gut microbiota, LPS, and obesity (Cani et al, 2007a).

The loss of function of either TLR4 or CD14 has been reported to be protective against body fat gain, insulin resistance, and inflammation in other studies of high-fat diet fed mice (Roncon-Albuquerque et al, 2008; Tsukumo et al, 2007) (Li et al, 2011; Orr et al, 2012; Radin et al, 2008; Saberi et al, 2009). 


\subsection{CONFLICTING RESULTS IN THE LITERATURE}

In contrast to the research outlined above, other research using the same $\mathrm{CD} 14^{-/-}$mice found no significant differences in body weight compared to wild-type control mice after four weeks of High-Fat feeding (Yin et al, 2001). Rather the CD14 ${ }^{--}$mice gained a similar amount of weight to wild-type control mice when fed a high-fat diet for 41 weeks (Young et al, 2012). Several other studies using a variety of diets and different TLR4 ${ }^{-/}$mice have also found no influence on body weight due to the loss of TLR4 function, while reporting beneficial effects on inflammation (Kim et al, 2007; Kim et al, 2012; Poggi et al, 2007; Suganami et al, 2007). These studies include the use of several different TLR4 knockouts, both experimental and natural deletions. The use of different strains of knockout mice in the literature, both of TLR4 and CD14, and the variety of different mouse diets used represent potential confounders when trying to understand the role of the TLR4 pathway in obesity development. Several these studies were not aiming to directly address the influence on body weight and this leaves how TLR4 and LPS impact body weight unresolved.

\subsection{TLR4 AND SATURATED FATTY ACIDS}

While LPS has been recognised as the most potent ligand for TLR4 activation there has been controversy as to whether TLR4 can also be activated by saturated fatty acids (Erridge \& Samani, 2009). This is relevant to research linking TLR4 activation with obesity in mice as these studies typically use obesogenic diets high in saturated fatty acids. While there was evidence to show that saturated fatty acids could produce an inflammatory response though TLR4 activation, this was brought into question since TLR4 could not be activated in vitro with pure fatty acids and raised concerns that the effects of saturated fatty acids due to contamination by LPS (Erridge \& Samani, 2009). More recent work indicates that saturated fats can activate TLR4 in vivo in the presence of the serum protein Fetuin A suggesting that either bacterial derived LPS from the gut, or dietary saturated fat could contribute to TLR4 activation in high-fat diet fed mice (Pal et al, 2012). 


\subsection{MECHANISMS LINKING LPS AND TLR4 TO BODY WEIGHT}

Irrespective of the mechanism of activation of TLR4 (LPS or fatty acid) the important question that remains in how TLR4-mediated inflammation might be linked to an increase in mouse body weight and adiposity. Research on this question has focused on influences of LPS on the endocannabinoid system and direct action upon the adipocytes (Geurts et al, 2011; Muccioli et al, 2010). An unexplored route of action for systemic LPS is through the stimulation of inflammation in the central nervous system, particularly regions of the hypothalamus involved in body weight and energy homeostasis.

\subsection{HYPOTHALAMIC INFLAMMATION}

The hypothalamus is an area of the brain with important functions in the homeostatic control of bodyweight and metabolism. Hypothalamic dysfunction resulting in changes such as the loss of central leptin and insulin sensitivity has emerged as a central focus in the development and perpetuation of obesity (Williams, 2012). The role of hypothalamic inflammation in diet induced obesity was first described for rats fed a high-fat (45\%) diet for 16 weeks (De Souza et al, 2005). The expression of 29 genes involved in immune related functions were reported to be unregulated in obese rats relative to lean low-fat diet fed rats (De Souza et al, 2005). These genes include the cytokines TNF- $\alpha$, IL-1ß, IL2, and Il-6, which are known to participate in the induction of insulin resistance in adipose tissue (Hotamisligil, 2003). The expression of the gene SOCS3 was also reported to be increased suggesting that local inflammation in the hypothalamus resulted in reduced sensitivity to both insulin and leptin signalling from the periphery (De Souza et al, 2005). This suggested a pivotal role for local inflammation in the hypothalamic areas critical for the control of energy homeostasis and in the pathophysiology of diet-induced obesity. However, it was not possible distinguish whether this inflammation was a cause or consequence. The observation that hypothalamic inflammatory signalling could be detected in both mice and rats within 1 to 3 days following the onset of high-fat diet feeding and prior to weight gain, has been interpreted to suggest that inflammation drives obesity, rather than simply being a consequence (Thaler et al, 2012). Additionally, reactive gliosis and evidence of neuron injury were evident within the first week of highfat diet feed in the hypothalamic arcuate nucleus of rats and mice. This suggested that 
obesity is associated with inflammatory induced neuronal injury in areas of the hypothalamus critical for body weight control (Thaler et al, 2012).

\subsection{HYPOTHALAMIC INFLAMMATION AND TLR4}

The activation of TLR4 has been considered an important signalling pathway in the development of inflammation in the hypothalamus in rodents in response to high-fat diets. Infusion of stearic acid, a saturated fatty acid, into the brain was reported to increase inflammation in mice (Milanski et al, 2009). Infusion of TLR4 antagonists into the brain of mice resulted in the reduction in inflammation. The knockout of MyD88, a downstream adaptor molecule for Toll like receptors, in the central nervous system protected mice from high-fat diet induced obesity and prevented the development of leptin resistance (Kleinridders et al, 2009). Regions of the hypothalamus lie outside the protection of the blood-brain barrier and have greater exposure to the systemic circulation. In conditions of bacterial infection high levels of circulating LPS directly influence the brain, resulting in suppression of food intake and sickness behaviour (Hines et al, 2013). Chronic low-level circulating LPS originating from the gut has the potential to activate TLR4 signalling in the hypothalamus resulting in inflammation and disruption of energy homeostasis. The hypothalamus offers an unexplored site through which gut derived LPS can disrupt homeostatic control of energy balance and food intake and is illustrated in Figure 2.

\subsection{TLR4 AND CD14 KNOCKOUT MICE}

To resolve the conflicting evidence within the literature the present study aimed to compare the responses to high-fat diet induced obesity of both TLR4 ${ }^{-/-}$and CD14 $4^{-/-}$mice with wild-type mice of the same mouse strain. The CD14 $4^{-/}$mice selected were developed at a targeted knockout of the CD14 gene (Moore et al, 2000). The TLR4 ${ }^{-/-}$mice selected contained a spontaneous 74723 bp deletion that completely removes the Tlr 4 coding sequence (Poltorak et al, 1998; Poltorak et al, 2000). This TLR4 ${ }^{-/}$was one of several different knockout strains previously studied. These were chosen as both strains had been backcrossed into C57BL/6J mice and were available with species and strain match wild-type control mice. The $\mathrm{CD} 14^{-/-}$mice used were a different knockout mouse to the CD14 $\%$ developed in 1996 and used previously in studies of body weight (Cani et al, 2007a; Haziot et al, 1996). 


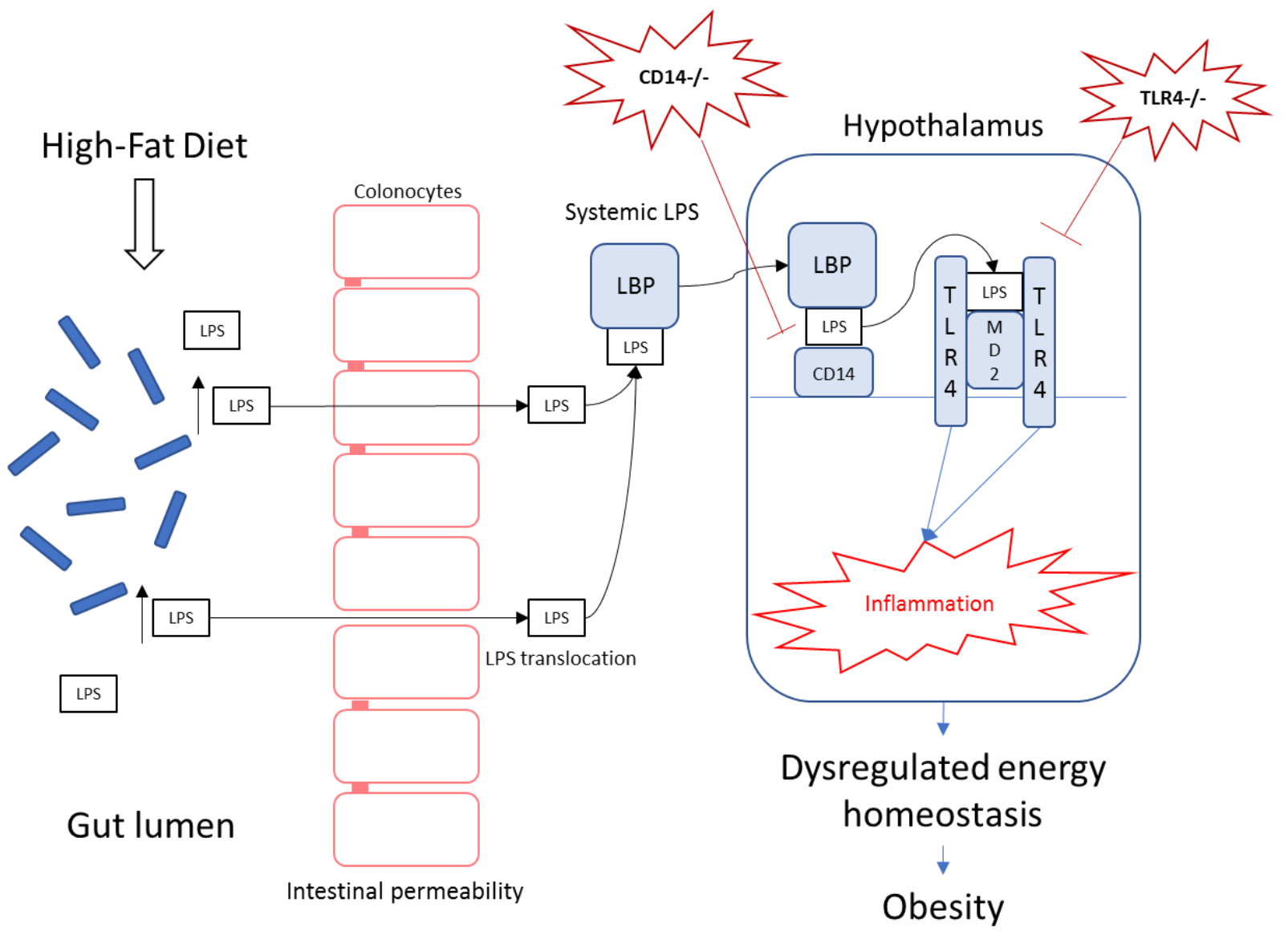

Figure 2. Pathway through which LPS from the gut can influence body weight. 


\section{AIMS}

The evidence that loss of function of TLR4 provides protection against the high-fat diet induced obesity is conflicting. The aim of this study was to directly compare the absence of either TLR4, or CD14 in mice on bodyweight and body composition in response to high-fat diet feeding and to investigate the impact of diet and genotype on hypothalamic inflammation. The first aim was to investigate the hypothesis that loss of functional TLR4 signalling would attenuate the increase in bodyweight and adiposity resulting from high-fat feeding compared to the wild-type control and observe if there were any differences in responses between TLR4 and CD14 knockout mice. To limit the confounding effects of mice from different strains or suppliers the TLR4 and CD14 knockout mice were based on the wild-type C57BL6 mice strain used as controls in the study and were purchased from the same source. Purified and standardised synthetic high-fat (60\%) and low-fat (10\%) control diets were used to limit confounding factors in the diets used. The second aim was to investigate the impact of TLR4 and CD14 knockout on gene expression in the hypothalamus. A microarray study was used to investigate the loss of TLR4 signalling on inflammatory gene expression in the hypothalamus resulting from the high fat diet feeding.

\section{HYPOTHESES}

TLR4 knockout mice and CD14 knockout mice show reduced susceptibility to high-fat diet induced obesity relative to Wild-Type mice. TLR4 knockout and CD14 knockout mice will show reduced high-fat diet induced inflammation in the hypothalamus associated with diet induced obesity. 


\section{RESULTS}

\subsection{TLR4 ${ }^{-/-}$AND CD14 ${ }^{-/-}$MICE ARE NOT PROTECTED FROM DIET- INDUCED OBESITY}

Feeding of the High-Fat diet significantly increased the body weight and body weight gain of Wild-Type, TLR4 ${ }^{-/}$, and CD14 ${ }^{-/-}$mice relative to their respective Low-Fat diet fed controls, Figure 3. The body weight of mice fed the High-Fat diet increased to a similar degree over the 8 weeks of the study, Figure 3A. At the final week, mean body weight for the Wild-Type, TLR4 ${ }^{-/}$, and $\mathrm{CD} 14^{-/-}$mice fed the High-Fat diet were not significantly different. Body weights of High-Fat fed mice was significantly higher than their respective Low-Fat diet fed controls, Figure 3B. Low-Fat fed TLR4 ${ }^{-/}$, and CD14 $4^{-/}$mice were heavier and lighter respectively than Low-Fat fed Wild-Type mice, however this difference did not reach statistical significance. To account for small differences in starting weight between groups body weight gain was calculated and normalised to a starting weight of zero, Figure 3C. High-Fat diet fed Wild-Type, TLR $4^{-/-}$, and CD14 ${ }^{-/-}$ mice gained a very similar amount of body weight during the 8 weeks of the study. LowFat diet fed $\mathrm{CD} 14^{-/-}$gained less body weight than Wild-Type mice although this did not reach statistical significance. At the final week, the mice in all High-Fat fed diet groups gained significantly more body weight than their respective Low-Fat diet fed controls, Figure 3. The mean weight gain was not significantly different between groups fed HighFat diets. Increases in bodyweight were not significantly different for TLR4 ${ }^{-/-}$and CD14 mice compared to Wild-Type mice. Neither the TLR4 nor the CD14 knockout protected mice from the High-Fat diet induced increases in body weight. 

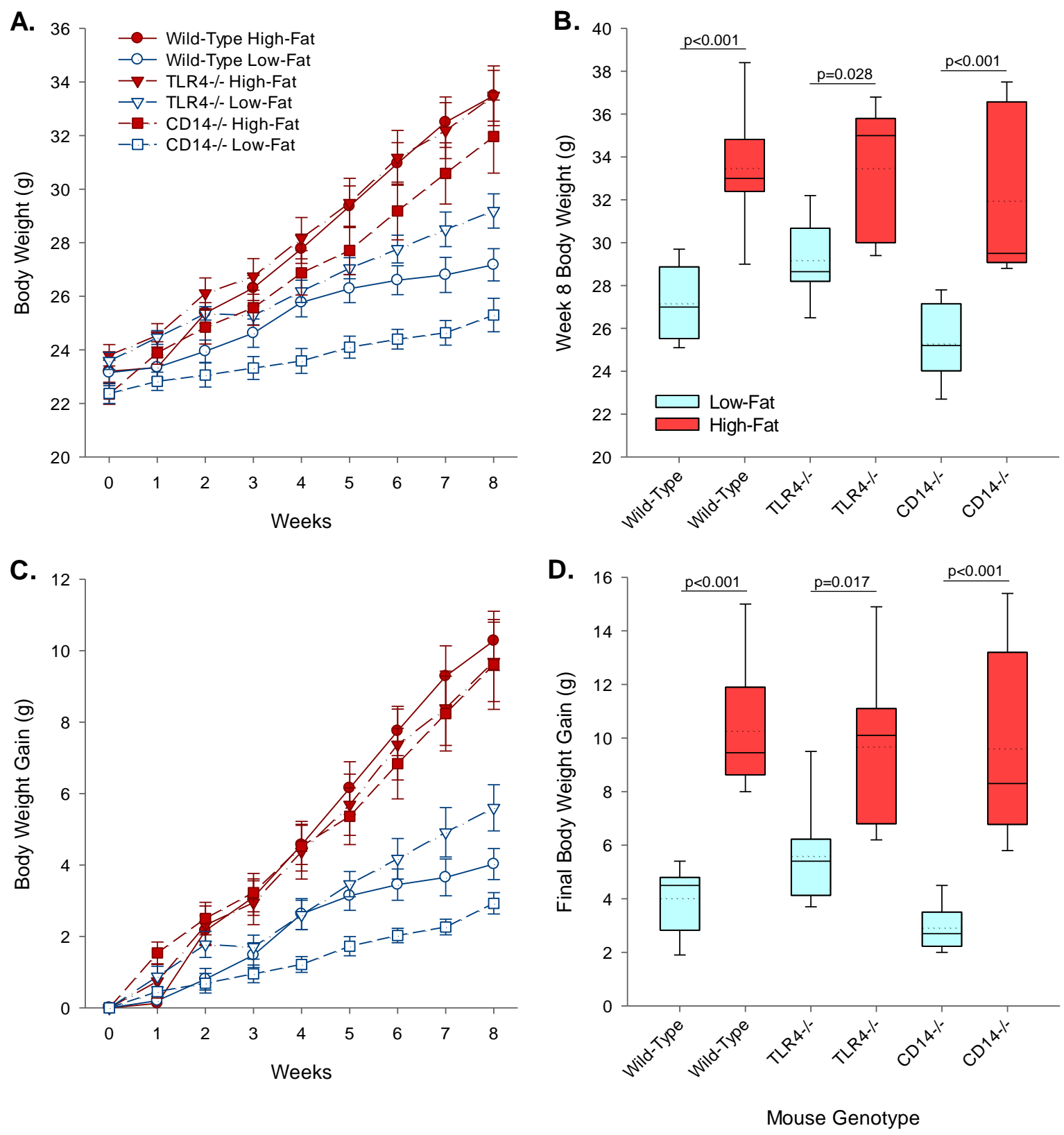

Figure 3. Effect of diet and genotype on body weight and body weight gain.

Weekly body weight (A), and the final week body weight (B), for Wild-Type, CD14 ${ }^{-/}$, and TLR4 ${ }^{-/-}$mice in response to Low-Fat and High-Fat diet feeding. Weekly body weight gain $(C)$, and the total body weight gain (D), for Wild-Type, CD14 $4^{-/-}$, and TLR4 ${ }^{-/-}$mice in response to Low-Fat and High-Fat diet feeding. Diets are represented as High-Fat (RED) and Low-Fat (BLUE). Data points represent mean \pm SEM. For Box plots the solid line = median, and dashed line = mean. Significant differences within each genotype were determined by one way ANOVA with Tukey's post hoc test. $n=8$ mice per group (TLR4 ${ }^{-}$ /- Low-Fat, $\mathrm{n}=7$ ). 


\subsection{TLR4 $^{-/-}$AND CD14 ${ }^{-/-}$MICE ARE NOT PROTECTED FROM INCREASED BODY FAT}

The percentage of body fat increased to a similar degree in High-Fat fed Wild-Type, $\mathrm{TLR}^{-/-}$, and CD14 ${ }^{-/-}$mice, Figure 4A. The body fat of Wild-Type, TLR4 ${ }^{-/-}$, and CD14 ${ }^{-/-}$ mice fed the High-Fat diet at the final week of measurement was significantly higher than the respective Low-Fat mice of the same genotype, Figure 4B. Lean body mass increased during the study in all genotypes and was not significantly different WildType, TLR4 ${ }^{-/}$, and CD14 ${ }^{-/-}$mice fed either the Low-Fat or High-Fat diets, Figure 4C. Lean body mass was not significantly different between either diet group or genotype at the final week, Figure 4D. Change in body fat mass percentage where similar to the changes in total body fat, Figure 5A. Low-Fat fed mice maintained their initial percentage of body fat. Low-Fat fed TLR4 ${ }^{-/-}$mice began the study with a higher percentage of body fat and maintained this throughout the study. At the final week of measurement, the percentage body fat of Wild-Type, TLR4 ${ }^{-/}$, and CD14 $4^{-/-}$mice fed the High-Fat diet was significantly higher than the Low-Fat mice, Figure 5B. The percentage of lean body mass decreased for Wild-Type, TLR4 $4^{-/}$, and CD14 ${ }^{-/-}$mice fed the High-Fat diet throughout the study, Figure $5 \mathrm{C}$. At the final week of percentage lean mass in Wild-Type, TLR4 $4^{-/}$, and CD14 ${ }^{-/-}$ mice fed the High-Fat diet was significantly reduced in comparison to the respective Low-Fat mice of the same genotype, Figure 5D. Increases in body weight were due to increases in body fat and not in lean mass. Increases in bodyweight were not significantly different in TLR4 ${ }^{-/-}$and CD14 mice compared to Wild-Type mice. Neither the TLR4 nor the CD14 knockout protected mice from the High-Fat diet induced increases in body fat. 
A.
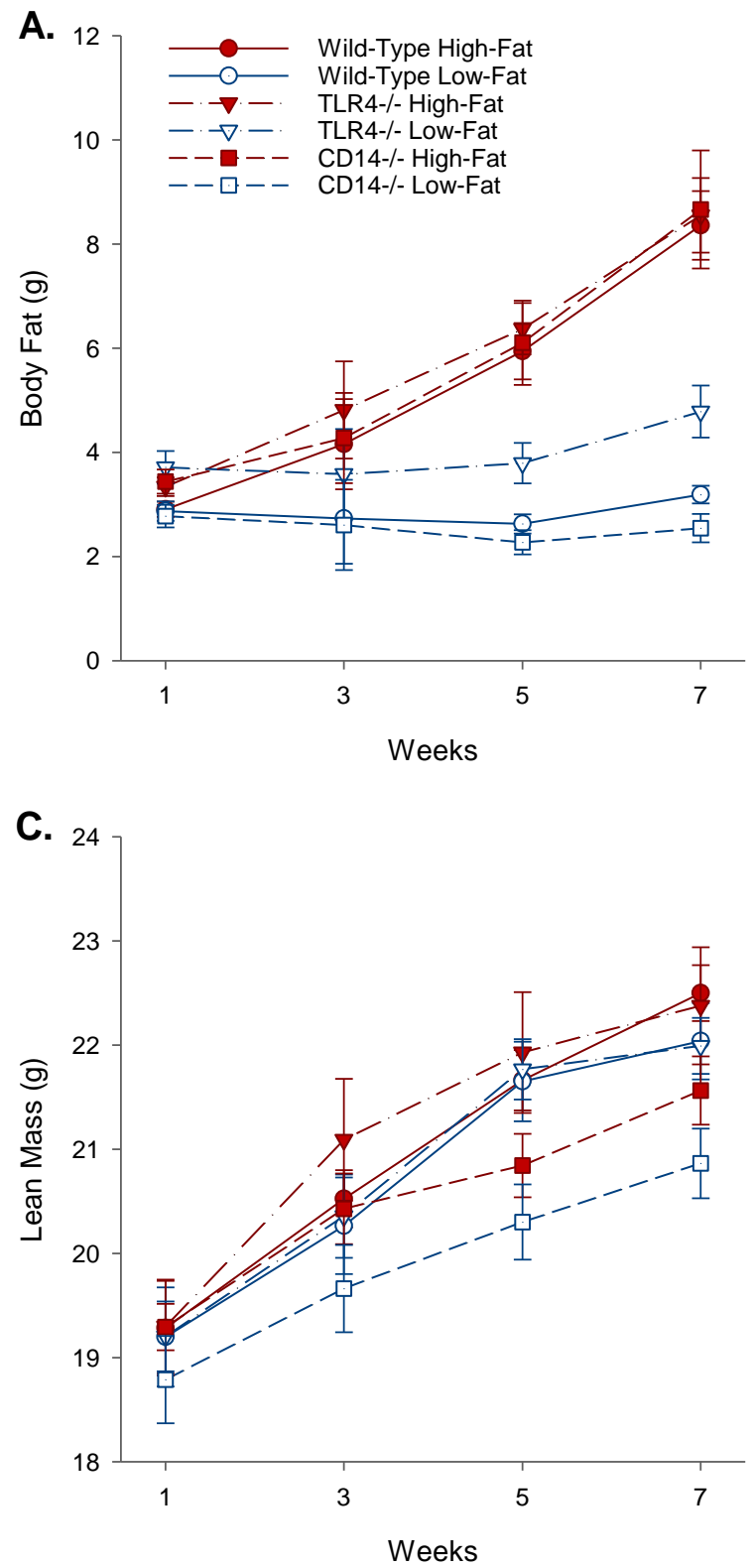

B.

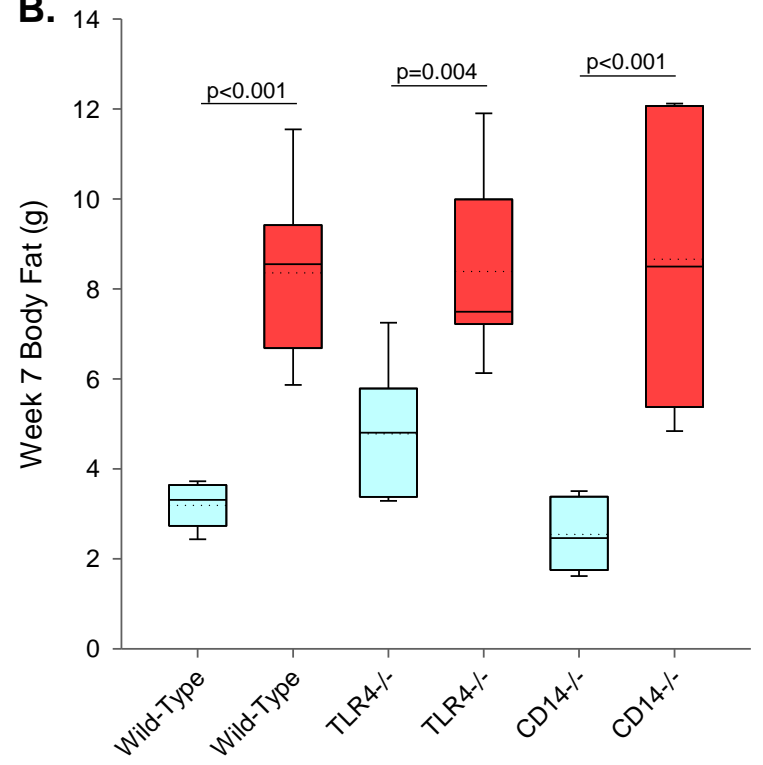

D.

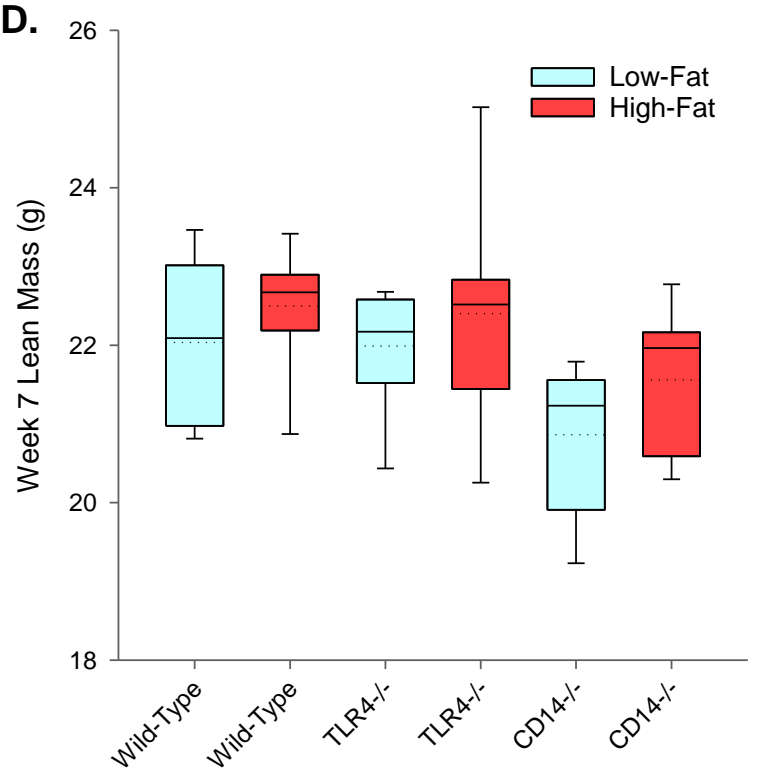

Mouse Genotype

Figure 4. Effect of diet and genotype on body fat mass and lean mass.

Weekly change in body fat mass (A), and final body fat mass (B), for Wild-Type, CD14 ${ }^{-/-}$ , and TLR $4^{-/-}$mice in response to Low-Fat and High-Fat diet feeding. Weekly change in lean body mass (C), and final week lean body mass (D), for Wild-Type, CD14 $4^{-/}$, and TLR4 ${ }^{-/-}$mice in response to Low-Fat and High-Fat diet feeding. Diets are represented as High-Fat (RED) and Low-Fat (BLUE). Body fat and lean mass was determined by EchoMRI scan. Data points represent mean \pm SEM. For Box plots the solid line $=$ median, and dashed line = mean. Significant differences within each genotype were determined by one way ANOVA with Tukey's post hoc test. $n=8$ mice per group (TLR4 ${ }^{-/-}$Low-Fat, $\mathrm{n}=7$ ). 

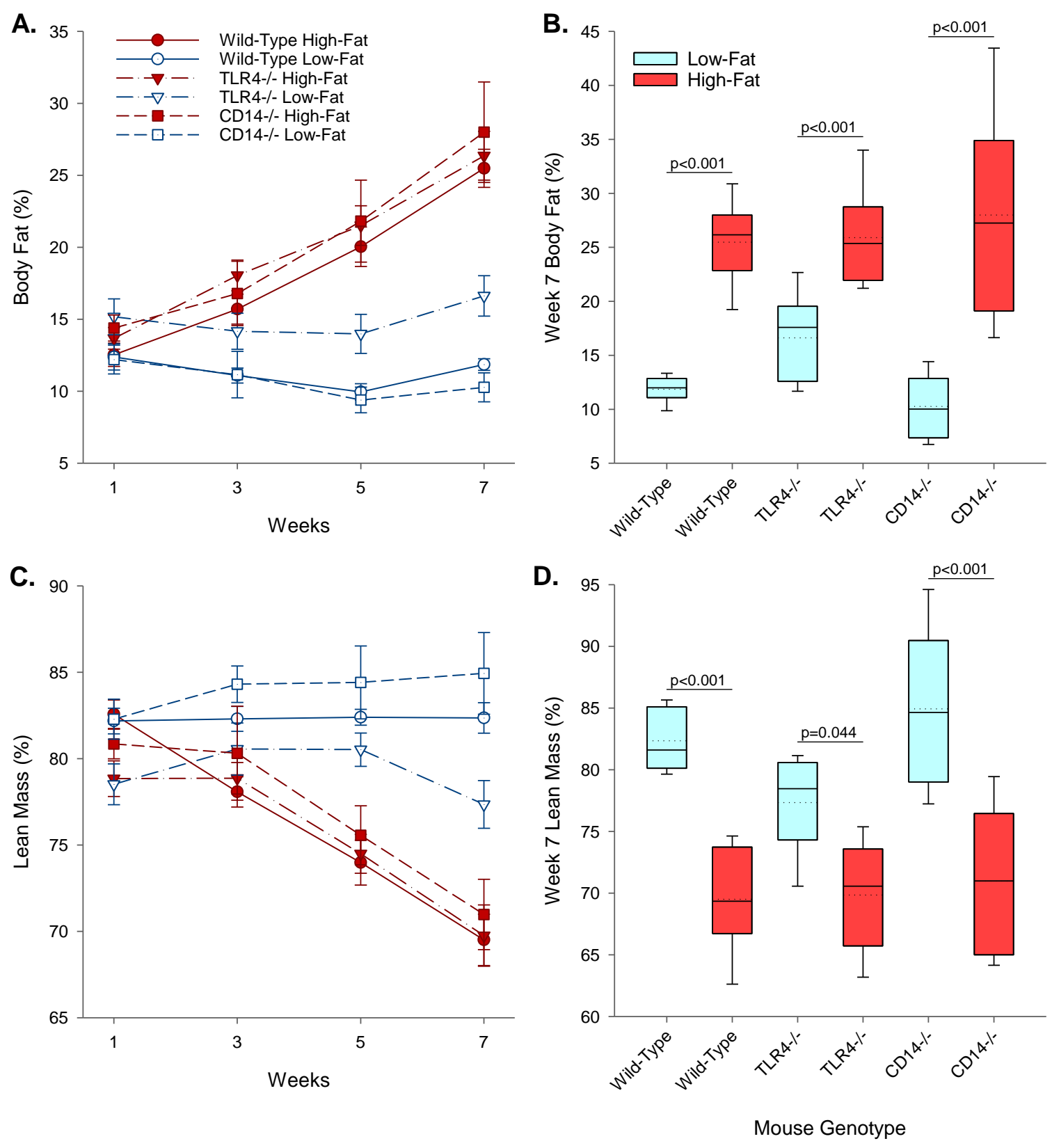

Figure 5. Effects of diet and genotype on percentage body fat and lean mass.

Percentage body fat measured biweekly (A), and final week percentage body fat (B), for Wild-Type, CD14 ${ }^{-/-}$, and TLR4 ${ }^{-/-}$mice in response to Low-Fat and High-Fat diet feeding. Percentage lean mass measured biweekly $(C)$, and final percentage lean mass (D), for Wild-Type, CD14 ${ }^{-/}$, and TLR4 ${ }^{-/-}$mice in response to Low-Fat and High-Fat diet feeding. Diets are represented as High-Fat (RED) and Low-Fat (BLUE). Body fat and lean mass was determined by EchoMRI scan. Data points represent mean \pm SEM. For Box plots the solid line $=$ median, and dashed line $=$ mean. Significant differences within each genotype were determined by one way ANOVA with Tukey's post hoc test. $n=8$ mice per group (TLR4 ${ }^{-/-}$Low-Fat, $\mathrm{n}=7$ ). 


\subsection{TLR4 $^{-/-}$AND CD14 ${ }^{-/-}$MICE DO NOT SHOW ANY DIFFERENCE IN FOOD OR ENERGY INTAKE}

Food intake decreased in mice fed the High-Fat diet relative to mice on the Low-Fat diet while energy intake increased in High-Fat diet fed mice due to the greater energy density of the diet, Figure 6A. Food intake initially decreased in all groups for the first two weeks on the experimental diets and then recovered. Mean group food intakes at each week were lower in High-Fat fed mice than the respective Low-Fat mice of the same genotype, Figure 6B. High-Fat diet feeding reduced mean food intakes non-significantly in mice of all genotypes, Figure 6. There were no significant differences in mean food intake between mouse genotypes in Low-Fat diets or between High-Fat diets. Despite the reduced food intake of High-Fat diet fed mice the energy intake of High-Fat fed mice was consistently increased compared to Low-Fat diet fed mice, Figure 6C. The High-Fat and Low-Fat diets contained $21.9 \mathrm{~kJ} / \mathrm{g}$ and $16.1 \mathrm{~kJ} / \mathrm{g}$ of energy respectively. Mean weekly energy intake was significantly higher in Wild-Type, TLR4 ${ }^{-/-}$, and CD14 $4^{-/}$mice fed the High-Fat than the respective Low-Fat mice of the same genotype, Figure 6D. Mean weekly energy intake was not significantly different between Wild-Type, TLR4 ${ }^{-/}$, and $\mathrm{CD}_{1} 4^{-/-}$mice fed the High-Fat diet or between Wild-Type, TLR4 $4^{-/}$, and CD14 ${ }^{-/-}$mice fed the Low-Fat diet. The food intake responses to the High-Fat diet in TLR4 ${ }^{-/-}$and CD14 ${ }^{-/-}$ mice were not significantly different to Wild-Type mice. Neither the TLR4 knockout nor the CD14 knockout resulted in any difference in food or energy intake compared to Wild-Type mice fed the High-Fat diet. 
A.
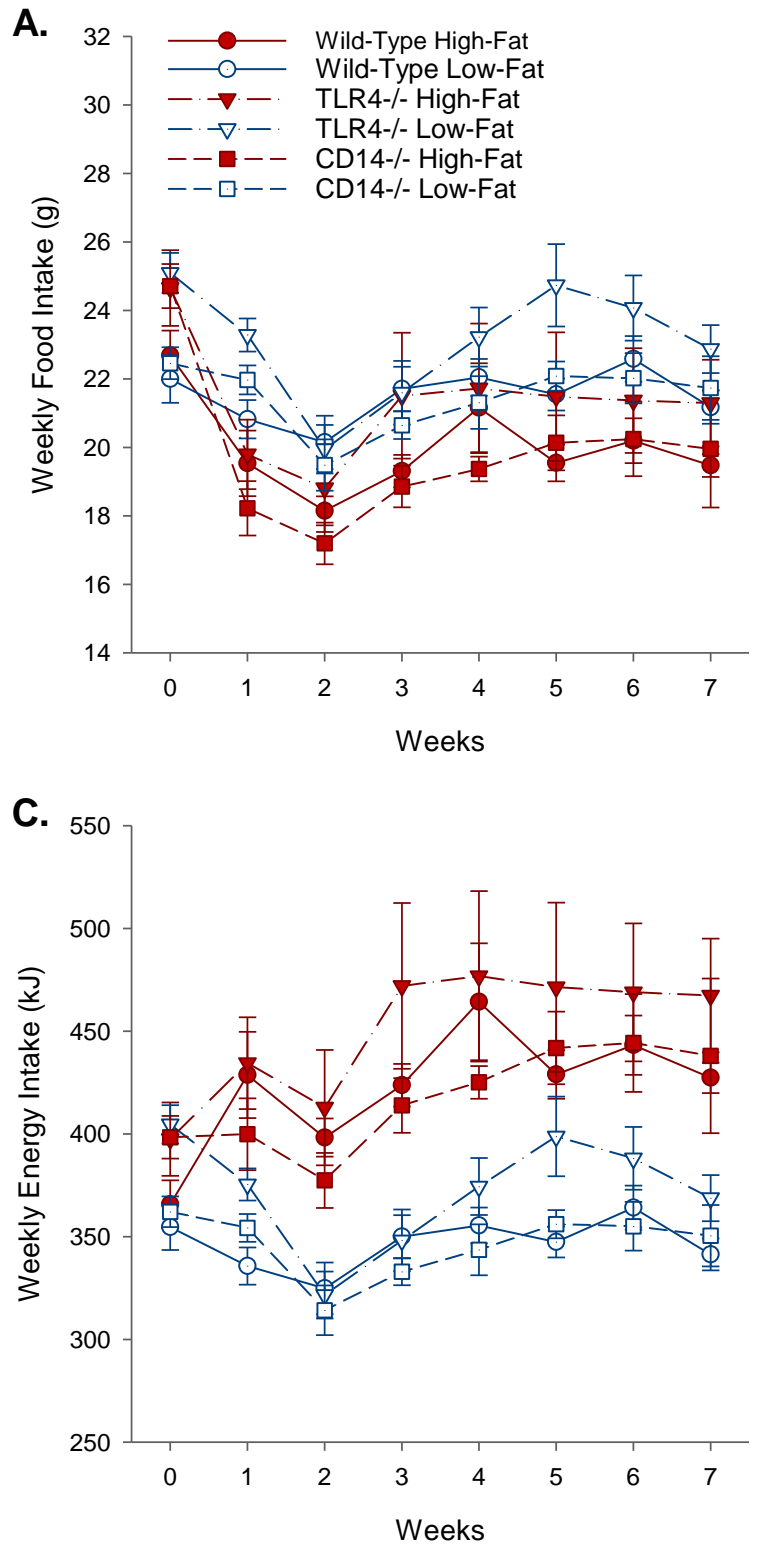

B.
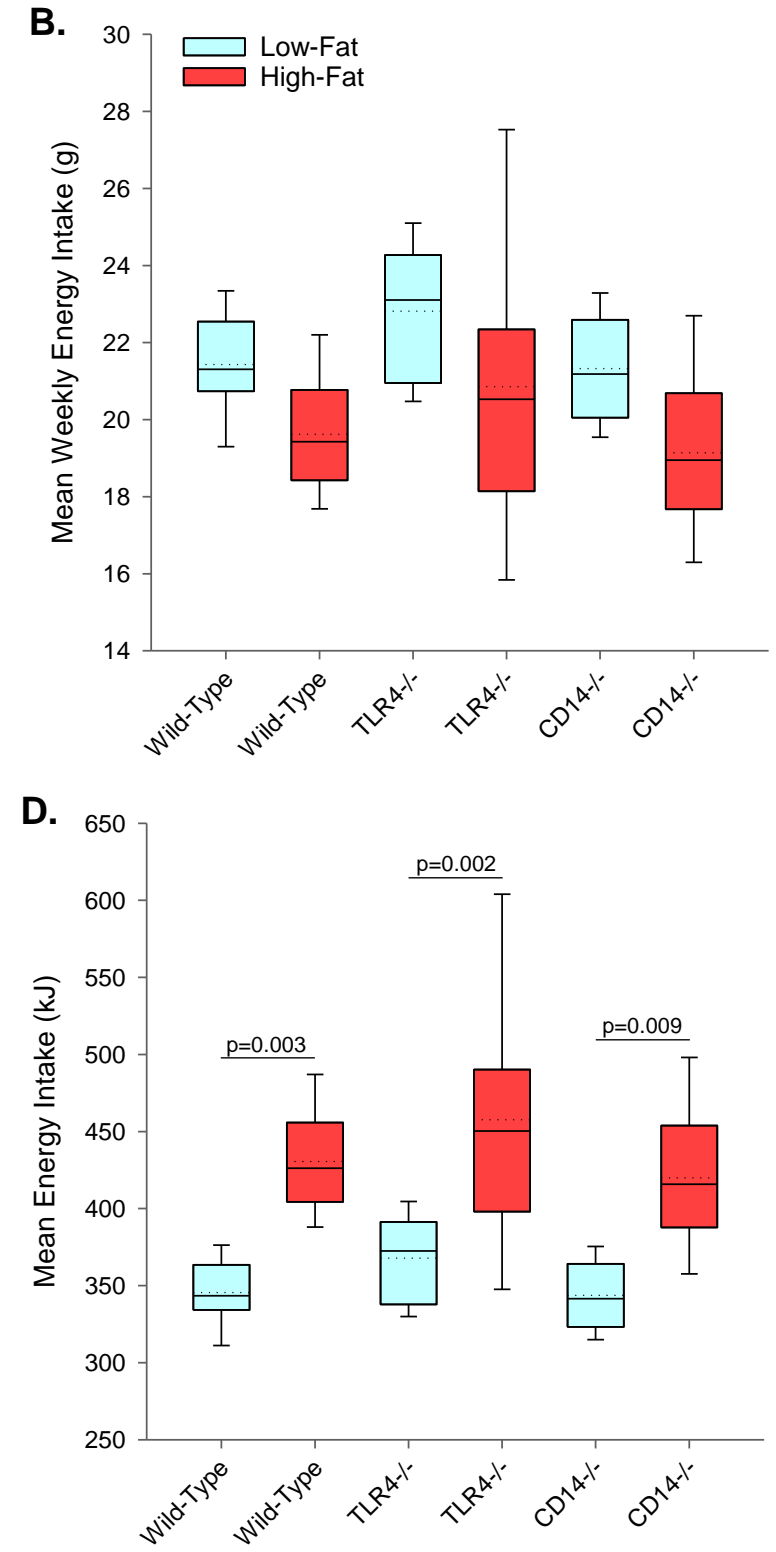

Mouse Genotype

Figure 6. Effect of diet and genotype on food and energy intake.

Weekly food intake (A), and mean weekly food intake for the whole experiment (B), for Wild-Type, CD14 ${ }^{-/-}$, and TLR4 ${ }^{-/-}$mice in response to Low-Fat and High-Fat diet feeding. Weekly energy intake $(C)$, and mean weekly energy intake for the whole experiment (D), for Wild-Type, CD14 ${ }^{-/}$, and TLR4 ${ }^{-/-}$mice in response to Low-Fat and High-Fat diet feeding. Diets are represented as High-Fat (RED) and Low-Fat (BLUE). Data points represent mean \pm SEM. For Box plots the solid line $=$ median, and dashed line $=$ mean . Significant differences within each genotype were determined by one way ANOVA with Tukey's post hoc test. $\mathrm{n}=8$ mice per group (TLR4 ${ }^{-/-}$Low-Fat, $\mathrm{n}=7$ ). 


\subsection{HIGH-FAT DIET INCREASES LIPOPOLYSACCHARIDE BINDING PROTEIN}

Lipopolysaccharide binding protein (LBP) concentrations were determined in the serum as a marker of systemic lipopolysaccharide exposure, Figure 7. High-Fat diet feeding significantly increased LPB concentration in the serum of Wild-Type mice and CDI4 $4^{-/-}$ mice relative to Low-Fat control animals of the same genotype. Serum LBP concentrations in Low-Fat fed TLR4 ${ }^{-/}$mice were higher than in Wild-Type mice or CD14 ${ }^{-/}$Low-Fat diet groups. This resulted in no significant difference in serum LPB concentration between Low-Fat and High-Fat diet fed TLR4 ${ }^{-/-}$mice. Serum LPB concentrations in Wild-Type, TLR4 ${ }^{-/}$, and $\mathrm{CD} 14^{-/}$mice were all significantly higher than in Low-Fat diet fed Wild-Type control mice. High-Fat diet feeding increases serum LPB concentrations, a marker for increased LPS exposure. 


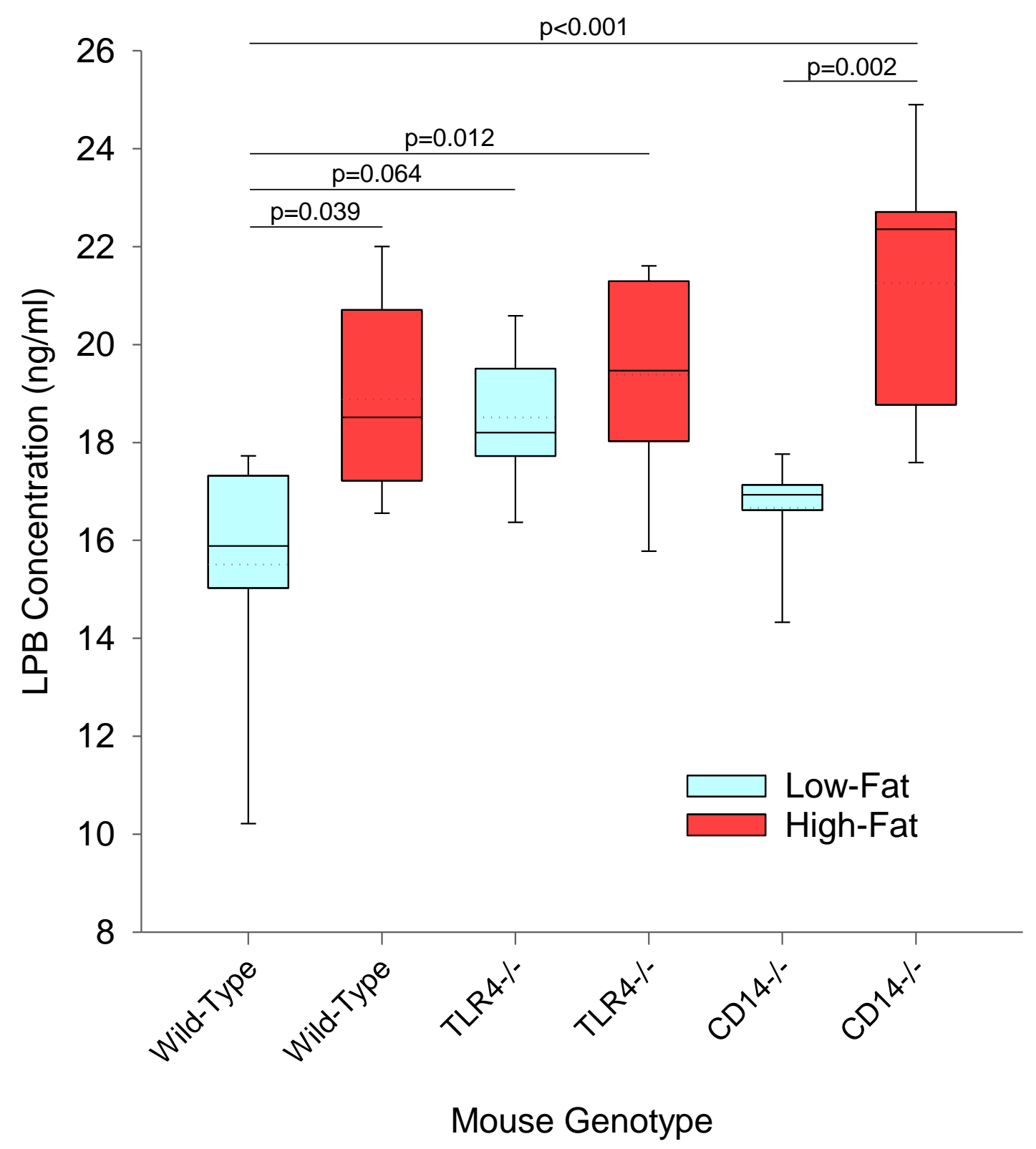

Figure 7. Effect of diet and genotype on lipopolysaccharide binding protein.

Serum lipopolysaccharide binding protein (LBP) concentration (ng/ml) for Wild-Type, $\mathrm{CD}_{14}{ }^{--}$, and TLR4 ${ }^{-/}$mice in response to Low-Fat and High-Fat diet feeding. Diets are represented as High-Fat (RED) and Low-Fat (BLUE). For Box plots the solid line = median, and dashed line $=$ mean. Significant differences within each genotype were determined by one way ANOVA with Tukey's post hoc test. $\mathrm{n}=6-8$ mice per group. 


\subsubsection{HYPOTHALAMIC INFLAMMATORY GENE EXPRESSION}

A microarray experiment was carried out to determine changes in inflammation related gene expression occurring in the hypothalamus of Wild-Type and TLR4 ${ }^{-/}$mice in response to the High-Fat diet, in comparison to Low-Fat diet fed mice. There were no significant increases in the expression of inflammation related genes previously reported to increase with High-Fat diet feeding in rats or mice (De Souza et al, 2005), Table 7. Gene expression changes were considered significant if the fold-change was greater than 1.25 , or less than -1.25 , with a significance level of $\mathrm{p}<0.01$. Using these cut-offs, no changes in inflammation related gene expression were identified from the microarray results, see Appendix 1, Table 13. Overall the degree of gene expression changes was relatively small between the Low-Fat and High-Fat diets in both Wild-Type and TLR4-/mice. Few gene expression changes were more than 1.5 fold up or down in High-Fat fed mice compared to Low-Fat fed mice. The cut-off for fold-changes was reduced to +/-1.25 to identify any small fold-changes taking place in gene expression. Hypothalamic gene expression in Wild-Type mice was found to be predominantly up-regulated in response to High-Fat diet feeding. In contrast TLR4 ${ }^{-/-}$hypothalamic gene expression was found to be predominantly down-regulated in in response to High-Fat. This evidence contrast with previous reports that inflammatory gene expression increases in mice in response to High-Fat diet feeding.

\subsubsection{HIGH-FAT DIET INDUCED CHANGE IN HYPOTHALAMIC GENE EXPRESSION}

To confirm the results of the microarray three genes were identified for follow-up investigation using in situ hybridisation, Table 8. Agouti-related protein (AgRP), suppressor of cytokine signalling 3 (SOCS3), and SerpinA3N were identified as being 


\subsection{MICROARRAY OF HYPOTHALAMIC GENE EXPRESSION}

Table 7. Microarray: Inflammatory gene expression.

\begin{tabular}{|c|c|c|c|c|c|}
\hline \multirow[b]{2}{*}{$\begin{array}{l}\text { Gene } \\
\text { Symbol }\end{array}$} & \multirow[b]{2}{*}{ Gene Name } & \multicolumn{2}{|c|}{ Wild-Type Mice } & \multicolumn{2}{|c|}{ TLR4-/- Mice } \\
\hline & & $\begin{array}{l}\text { Fold- } \\
\text { Change }\end{array}$ & P-value & $\begin{array}{l}\text { Fold- } \\
\text { Change }\end{array}$ & P-value \\
\hline Fas & Fas (TNF receptor superfamily member 6) & -1.04 & 0.48 & -1.11 & 0.10 \\
\hline $\mathrm{Ccl} 20$ & Chemokine (C-C motif) ligand 20 & 1.03 & 0.41 & 1.05 & 0.23 \\
\hline Tnfrsf8 & $\begin{array}{l}\text { Tumor necrosis factor receptor superfamily, } \\
\text { member } 8\end{array}$ & -1.04 & 0.26 & 1.03 & 0.50 \\
\hline Tgfbr1 & Transforming growth factor, beta receptor I & 1.03 & 0.45 & 1.01 & 0.80 \\
\hline Ednrb & Endothelin receptor type B & -1.07 & 0.09 & 1.01 & 0.93 \\
\hline Gpr44 & G protein-coupled receptor 44 & 1.00 & 0.90 & 1.04 & 0.60 \\
\hline Ptger2 & Prostaglandin E receptor 2 (subtype EP2) & -1.05 & 0.41 & 1.08 & 0.21 \\
\hline Tbxa2r & Thromboxane $\mathrm{A} 2$ receptor & 1.05 & 0.29 & 1.05 & 0.25 \\
\hline Tgfb3 & Transforming growth factor, beta 3 & -1.02 & 0.59 & -1.10 & 0.06 \\
\hline $\begin{array}{l}\text { Tnf- } \\
\text { alpha }\end{array}$ & Tumor necrosis factor alpha & -1.03 & 0.35 & -1.01 & 0.79 \\
\hline$\| 1 b$ & Interleukin 1 beta & 1.02 & 0.57 & -1.03 & 0.51 \\
\hline$\| 2$ & Interleukin 2 & 1.10 & 0.02 & -1.04 & 0.50 \\
\hline 116 & Interleukin 6 & -1.06 & 0.13 & 1.04 & 0.39 \\
\hline 117 & Interleukin 7 & 1.12 & 0.02 & -1.05 & 0.37 \\
\hline Jak2 & Janus kinase 2 & -1.04 & 0.23 & -1.05 & 0.31 \\
\hline Mcpt8 & Mast cell protease 8 & 1.06 & 0.30 & 1.01 & 0.90 \\
\hline Irf1 & Interferon regulatory factor 1 & 1.04 & 0.28 & -1.12 & 0.05 \\
\hline Nfkbia & $\begin{array}{l}\text { Nuclear factor of kappa light polypeptide gene } \\
\text { enhancer in B cells inhibitor, alpha }\end{array}$ & 1.08 & 0.05 & -1.07 & 0.31 \\
\hline Ikbkb & Inhibitor of kappaB kinase beta & -1.05 & 0.18 & 1.08 & 0.14 \\
\hline Ikbke & Inhibitor of kappaB kinase epsilon & 1.10 & 0.02 & 1.09 & 0.08 \\
\hline
\end{tabular}

Table 8. Microarray: Gene expression changes investigated using in situ hybridisation

\begin{tabular}{llcccc}
\hline & & \multicolumn{2}{c}{ Wild-Type Mice } & \multicolumn{2}{c}{ TLR4-/- Mice } \\
Gene & Gene Name & Fold-Change & P-value & Fold-Change & P-value \\
Symbol & & & & & \\
\hline AgRP & Agouti related protein & 1.38 & 0.0004 & 1.27 & 0.0080 \\
Socs3 & Suppressor of cytokine signalling & -1.27 & 0.0009 & -1.13 & 0.0647 \\
& 3 & & & & \\
Serpina3n & Serine (or cysteine) peptidase & -1.28 & 0.0006 & 1.02 & 0.7178 \\
& inhibitor, clade A, member 3N & & & & \\
\hline n=4 mice per group. & & & & \\
Significance $=$ p $<0.01$ & & & &
\end{tabular}




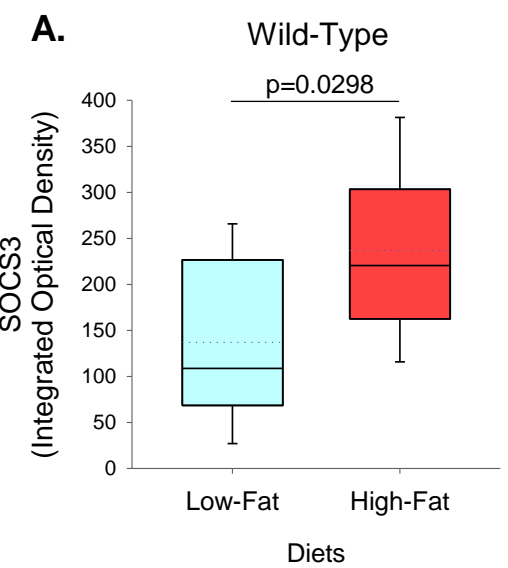

B. TLR4-/-

C.

CD14-/-
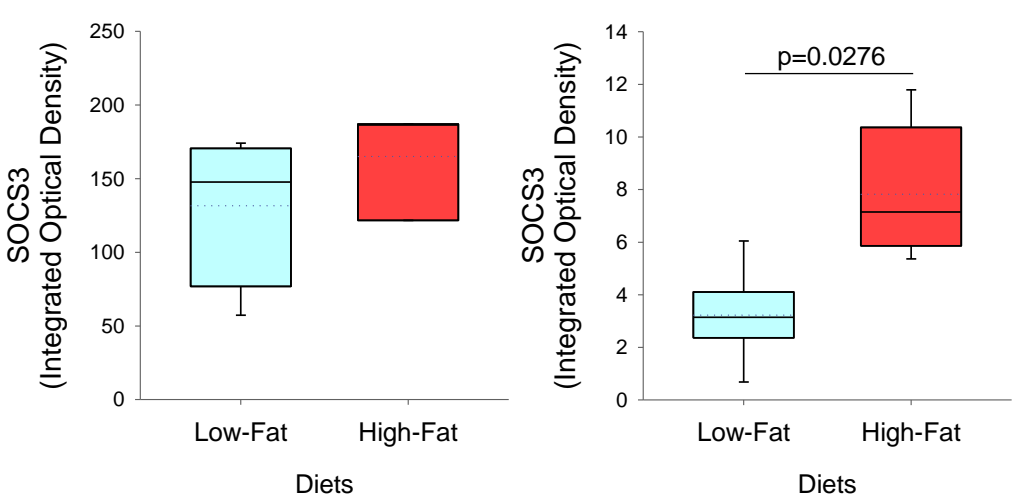

D.

E.

TLR4-/-
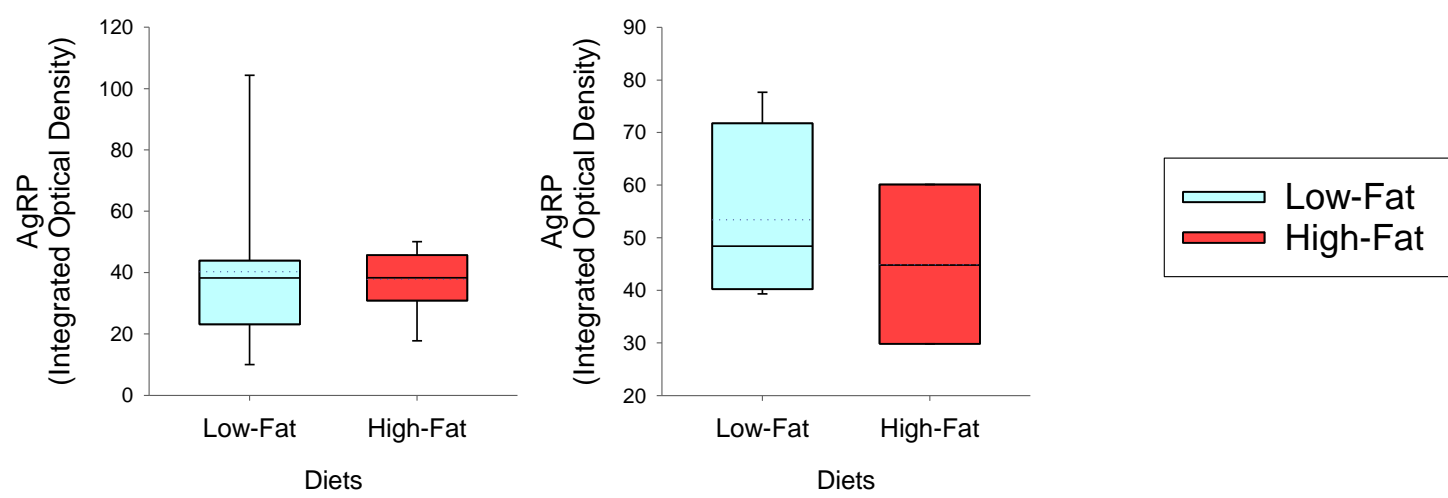

Figure 8. Hypothalamic SOCS3 and AgRP gene expression.

Hypothalamic gene expression was measured by in situ hybridisation and quantified by densitometry. Expression of the suppressor of cytokine signalling 3 (SOCS3) in the hypothalamus of Wild-Type mice (A), TLR4 ${ }^{-/-}$mice (B), and CD14 ${ }^{-/-}$mice (C), in response to Low-Fat and High-Fat diet feeding. Expression of agouti-related protein (AgRP) in the hypothalamus of Wild-Type mice (D), and TLR4 ${ }^{-/-}$mice (E), in response to Low-Fat and High-Fat diet feeding. Gene expression measured by in situ hybridisation and quantified by densitometry. For Box plots the solid line = median, and dashed line = mean. Significant differences within each genotype were determined by T-Test. $\mathrm{n}=$ LowFat/High-Fat (Wild-Type, $\mathrm{n}=9,8)$, $\left(\mathrm{TLR}_{4}{ }^{--}, \mathrm{n}=4,3\right),\left(\mathrm{CD} 14^{-/-}, \mathrm{n}=7,6\right)$ mice per group. 


\subsubsection{SOCS3 EXPRESSION IS INCREASED IN HIGH-FAT DIET FEEDING}

Microarray analysis of gene expression indicated that the expression of SOCS3 was down-regulated in response to High-Fat diet feeding, Table 8. In contrast in situ hybridisation revealed that SOCS3 was up-regulated in the hypothalamus of Wild-Type and $\mathrm{CD}_{1} 4^{-/-}$mice in response to High-Fat feeding, Figure 8A. SOCS3 expression did not increase significantly in TLR4 ${ }^{-/-}$but the small number of TLR $4^{-/-}$mice remaining for use in this assay limited the power to detect gene expression changes. The results of in situ hybridisation show that SOCS3 expression increases in response to High-Fat diet feeding and that the loss of TLR4 signalling in $\mathrm{CD}_{1} 4^{-/-}$mice does not prevent this.

\subsubsection{AGRP EXPRESSION IS NOT INCREASED IN HIGH-FAT DIET FEEDING}

Microarray analysis of gene expression indicated that the expression of AgRP was downregulated in response to High-Fat diet feeding, Table 8. In contrast the results of in situ hybridisation show that AgRP expression was not differentially changed between LowFat and High-Fat diets in either Wild-Type or TLR4 ${ }^{-/}$mice, Figure 8B. Expression in $\mathrm{CD} 14^{-/-}$mice was not investigated using in situ hybridisation. AgRP expression was not altered by diet or genotype.

\subsubsection{SERPINA3N EXPRESSION IS INCREASED IN HIGH-FAT DIET FEEDING}

Microarray analysis of gene expression indicated that the expression of SerpinA3N was down-regulated in response to High-Fat diet feeding, Table 8. In contrast in situ hybridisation revealed that SerpinA3N expression was upregulated in the arcuate nucleus, ventromedial hypothalamus, and dorsomedial hypothalamus in Wild-Type, $\mathrm{TLR}^{-/-}$, and CD14 ${ }^{-/-}$mice, Figure 9A, B, C.

These results show that gene expression changes in response to High-Fat diet feeding that were detectable using in situ hybridisation were not affected by loss of TLR4 signalling. 
Wild-Type Mice

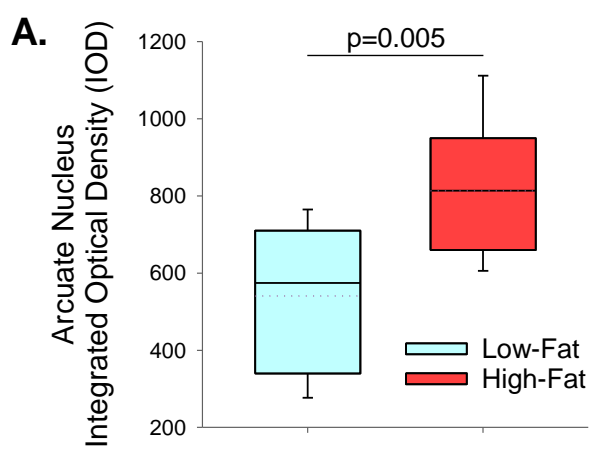

B.

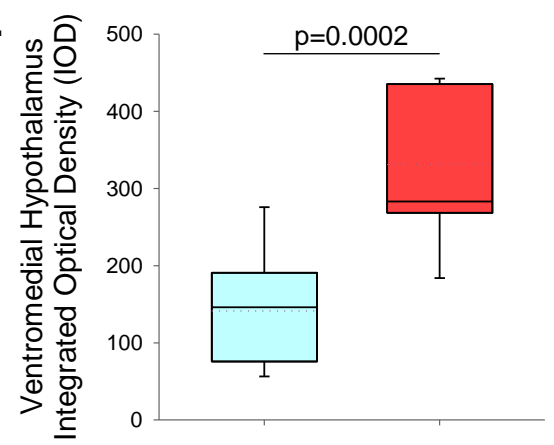

C.

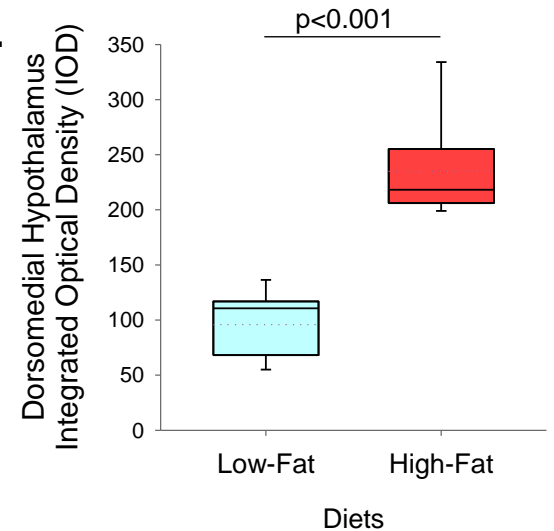

TLR4-/- Mice
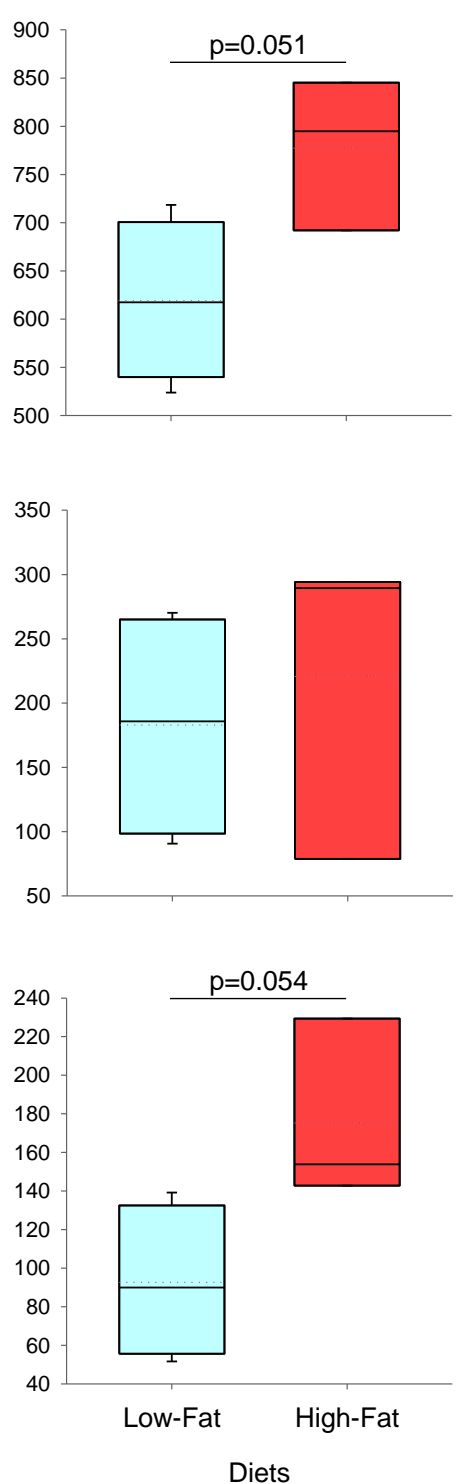

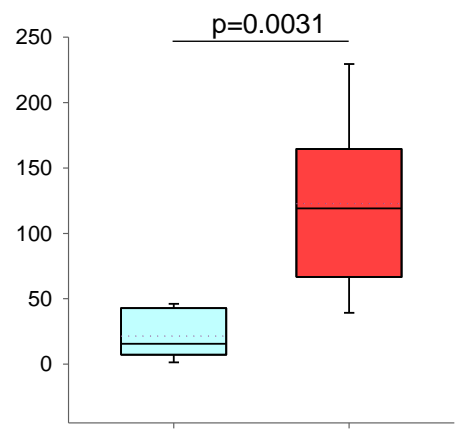

CD14-/- Mice
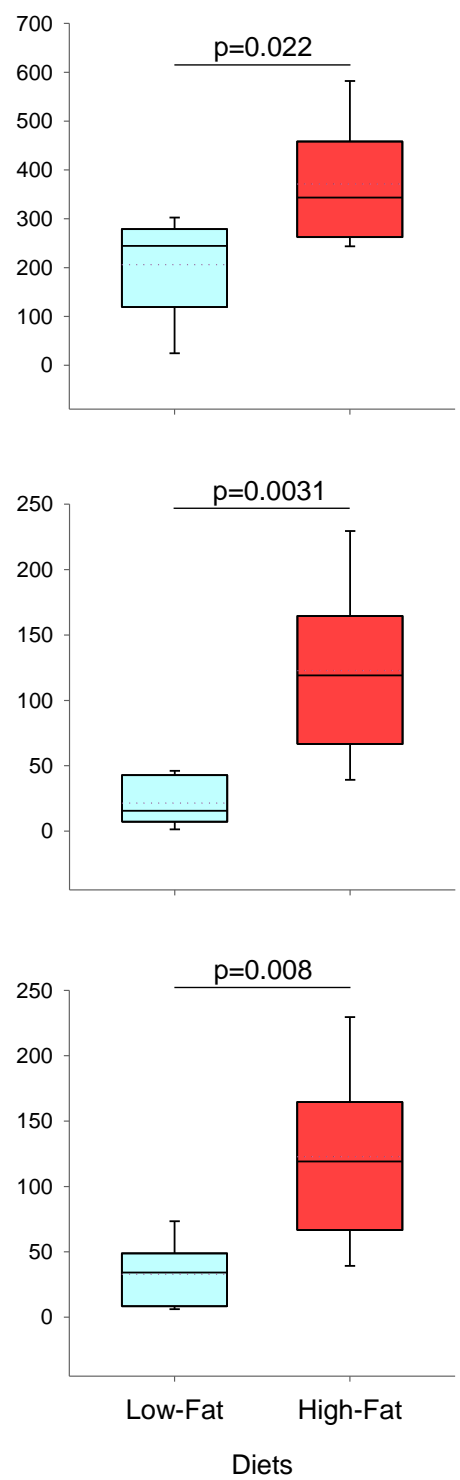

Figure 9. SerpinA3N gene expression in hypothalamic nuclei.

SerpinA3N expression in the arcuate nucleus of Wild-Type, TLR4 $4^{-/}$, and $\mathrm{CD} 14^{-/-}$mice (A), in response to Low-Fat, and High-Fat diet feeding. Expression of SerpinA3N expression in the ventromedial hypothalamus of Wild-Type, CDI4 ${ }^{-/}$, and TLR4 ${ }^{-/}$mice (B), in response to Low-Fat and High-Fat diet feeding. SerpinA3N expression in the dorsomedial hypothalamus of Wild-Type, TLR4 ${ }^{-/}$, and $\mathrm{CD}^{-/-}$mice (D), in response to Low-Fat and High-Fat diet feeding. Gene expression measured by in situ hybridisation and quantified by densitometry. For Box plots the solid line $=$ median, and dashed line $=$ mean. Significant differences within each genotype were determined by T-Test. $\mathrm{n}=$ Low-Fat/High-Fat (Wild-Type, $\mathrm{n}=9,8),\left(\mathrm{TLR}^{-/-}, \mathrm{n}=4,3\right),\left(\mathrm{CD} 14^{-/-}, \mathrm{n}=7,6\right)$ mice per group. 


\subsection{MICROBIOTA COMPOSITION}

The luminal contents were collected from the caecum at the end of the study and the effects of the TLR4 and CD14 knockouts and High-Fat diet feeding on caecal microbiota composition was investigated. Initially denaturing gradient gel electrophoresis (DGGE) was used investigate the similarity in overall composition of the microbiota. Later a more in-depth analysis of microbiota composition was carried using next generation sequencing.

\subsection{COMPARISON OF CAECAL MICROBIOTA USING DGGE}

When compared using DGGE analysis the caecal microbiota of mice was shown to be influenced both by diet and by genotype, Figure 10. The microbiota of Wild-Type mice fed Low-Fat and High-Fat diets clustered into two separate groups however the distance between the diets groups was relatively small. Overall the intensity of the gel bands did not change greatly between Low-Fat and High-Fat diets indicating relatively small shifts in the microbiota due to High-Fat diet feeding. In contrast the distance between the clustering microbiota of TLR4 ${ }^{-/-}$and $\mathrm{CD} 14^{-/-}$fed Low-Fat and High-Fat diets was considerably greater. This was largely due to a single band that dominated the gel of most of the Low-Fat diet fed TLR4 ${ }^{-/-}$and $\mathrm{CD} 14^{-/-}$mice and was absent from High-Fat diet fed mice. The microbiota of $\mathrm{TLR} 4^{-/-}$and $\mathrm{CD} 14^{-/-}$mice in both diet groups clustered distinctly from all the Wild-Type mice. DGGE analysis indicated that High-Fat diet feeding resulted in relatively small changes in composition of caecal microbiota of WildType mice. The TLR4 ${ }^{-/-}$and $\mathrm{CD}_{4} 4^{-/-}$mice contained a distinct microbiota from WildType mice. 


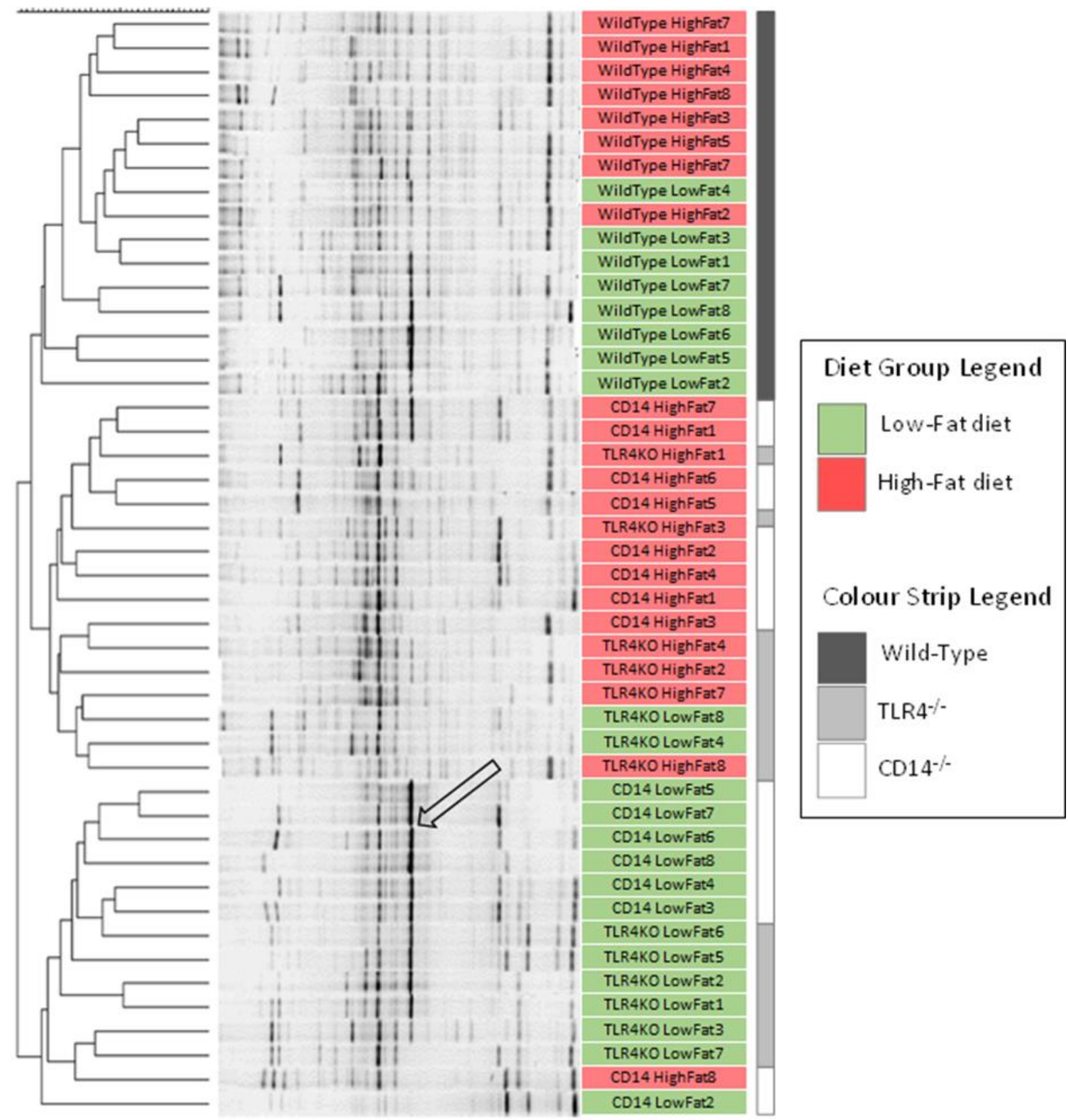

Figure 10. Dendrogram of microbiota similarity based on DGGE.

Comparison of microbiota communities determined by denaturing gradient gel electrophoresis (DGGE). DGGE analysis was based on amplification of V3 16S rRNA. DGGE gels were analysed and compared using FPQuest (version 5.10) and clustering was calculated using UPGMA (Unweighted Pair Group Method using Arithmetic Averages). Each strip represents the microbiota from a single mouse caecal sample. $\mathrm{n}=8$ mice per group (TLR4 ${ }^{--}$Low-Fat, $\mathrm{n}=7$ ). The arrow indicates a prominent band in the Low-Fat diet mice corresponding to a prominent unclassified family within the Firmicutes phylum identified using next generation sequencing. 


\subsection{NEXT GENERATION SEQUENCING OF CAECAL MICROBIOTA}

Ilumina Miseq Next Generation sequencing of the V1-V2 variable region of the $16 \mathrm{~S}$ ribosomal RNA was used to provide a more in-depth analysis of the composition of the caecal microbiota. Sequences were subsampled and capped at 10,596 sequences per sample.

\subsubsection{GENOTYPE INFLUENCES PHYLUM AND FAMILY COMPOSITION}

Presented as a dendrograms representing a Bray-Curtis dissimilarity index the microbiota of the Wild-Type mice clustered distinctly from the TLR4 ${ }^{-/-}$and CD14 ${ }^{-/-}$mice, Figure 11. Wild-Type mice fed Low-Fat and High-Fat diets clustered closely together and contained roughly equal proportions of the Firmicutes and Bacteroidetes phylum. Relatively little change was visible in the proportion of the bacterial Families between the Low-Fat and High-Fat diet groups. In contrast the microbiota of the TLR4 ${ }^{-/-}$and $\mathrm{CD}_{1} 4^{-/-}$mice was composed almost entirely of Firmicutes in both diets. A large shift in the composition of bacterial Families within the microbiota of both TLR4 ${ }^{-/-}$and CD14 $4^{-/-}$ mice between the High-Fat and Low-Fat diets. No clear distinction was observed between $\mathrm{TLR} 4^{-/-}$and $\mathrm{CD} 14^{-/-}$mice within each diet group. The TLR4 ${ }^{-/-}$and $\mathrm{CD} 14^{-/-}$mice contained a different microbiota composition that was altered to a greater degree in response to diet compared to the Wild-Type mice. The clustering of mice based on the Ilumina Miseq Next Generation sequencing was similar to the dendrogram produced using DGGE analysis, Figure 10. The microbiota of Low-Fat diet fed TLR4 ${ }^{-/-}$and CD14 ${ }^{-/-}$ mice was dominated by an unclassified Family belonging to the Firmicutes phylum, Figure 11. This Family was composed of a single operational taxonomic unit (OTU), a proxy for a bacterial species. This particular OTU was greatly reduced in High-Fat diet fed mice resulting in the increased proportions of other families of bacteria in these mice. This OUT is indicated in the DGGE results by an arrow, Figure 10. 

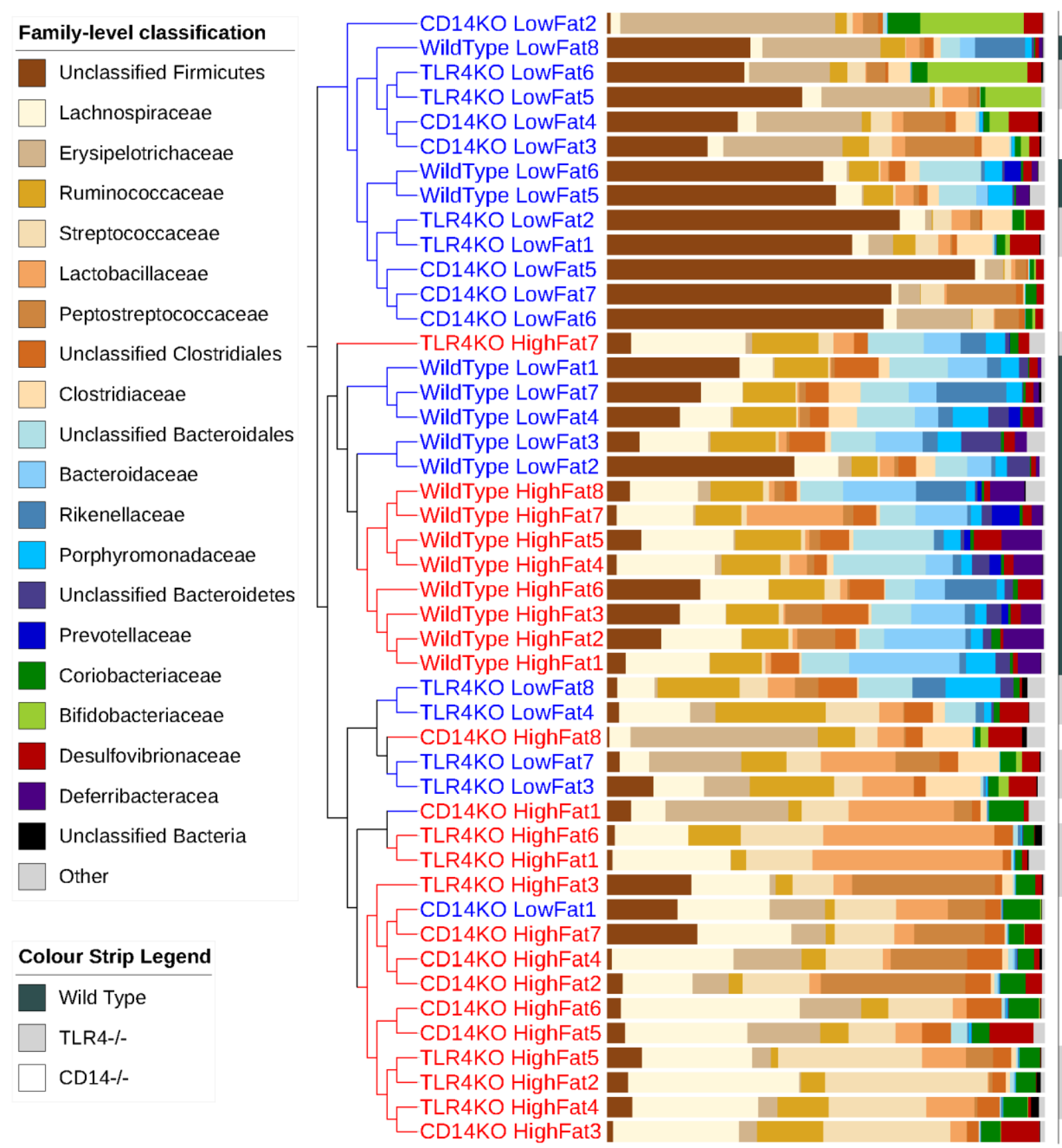

Figure 11. Composition of caecal microbiota using next generation sequencing.

The dendrogram represents clustering of caecal microbiota based on the Bray-Curtis dissimilarity matrix of OTUs sharing 97\% identity in Low-Fat and High-fat diet fed Wild-Type, TLR4 ${ }^{-/}$, and CD14 ${ }^{-/-}$mice. Families included in the Firmicutes phyla are shown as browns, the Bacteroidetes as blues, Actinobacteria as greens, Proteobacteria as reds, and other families as black. Each stacked bar indicates the proportional abundance of the bacterial phyla in the microbiota of a single mouse. Bray-Curtis dissimilarity matrix generated using the Mothur package. Dendrograms and phylum level proportions were visualised using ITOL. TLR4KO $=\mathrm{TLR}^{-/-}$, and CD14KO $=\mathrm{CD} 14^{-}$ $/$ mice. $\mathrm{n}=8$ mice per group (TLR $4^{-/-}$and $\mathrm{CD} 14^{-/-}$Low-Fat, $\mathrm{n}=7$ ). 


\subsubsection{GENOTYPE INFLUENCES PHYLUM LEVEL ABUNDANCE AND FIRMICUTES TO BACTEROIDETES RATIO}

Directly comparing the proportional abundance of each bacterial phylum between groups confirmed that significant differences were present between the Wild-Type and $\mathrm{TLR}^{-/-}$and $\mathrm{CD} 14^{-/-}$mice. The proportion of Firmicutes was lower and the proportion of Bacteroidetes was higher in Wild-Type mice, Figure 12A, B. The Firmicutes and Bacteroidetes were not different between High-Fat and Low-Fat diets within each genotype. The proportion of Proteobacteria was not different between any groups, Figure 12C. The proportion of Actinobacteria was lower in Wild-Type than TLR4 ${ }^{-/}$and CD14 $4^{-/}$mice, and lower in Low-Fat fed than High-Fat fed Wild-Type mice, Figure 12D. The phylum Deferribacteres was not detected in any TLR4 ${ }^{-/-}$and CD14 ${ }^{-/-}$mice. The proportion of Deferribacteres increased in High-Fat fed Wild-Type mice compared to Low-Fat Wild-Type mice, Figure 12E. The Firmicutes to Bacteroidetes ratio was higher in TLR4 ${ }^{-/}$and $\mathrm{CD}_{4}{ }^{-/-}$mice but did not change between Low-Fat and High-Fat diets, Figure $12 \mathrm{~F}$. At the phylum level TLR $4^{-/-}$and $\mathrm{CD} 14^{-/-}$mice strains contained significantly different microbiota composition to Wild-Type mice. Apart from minor changes in the Actinobacteria and Deferribacteres phyla diet did not influence the microbiota composition at the phylum level. 

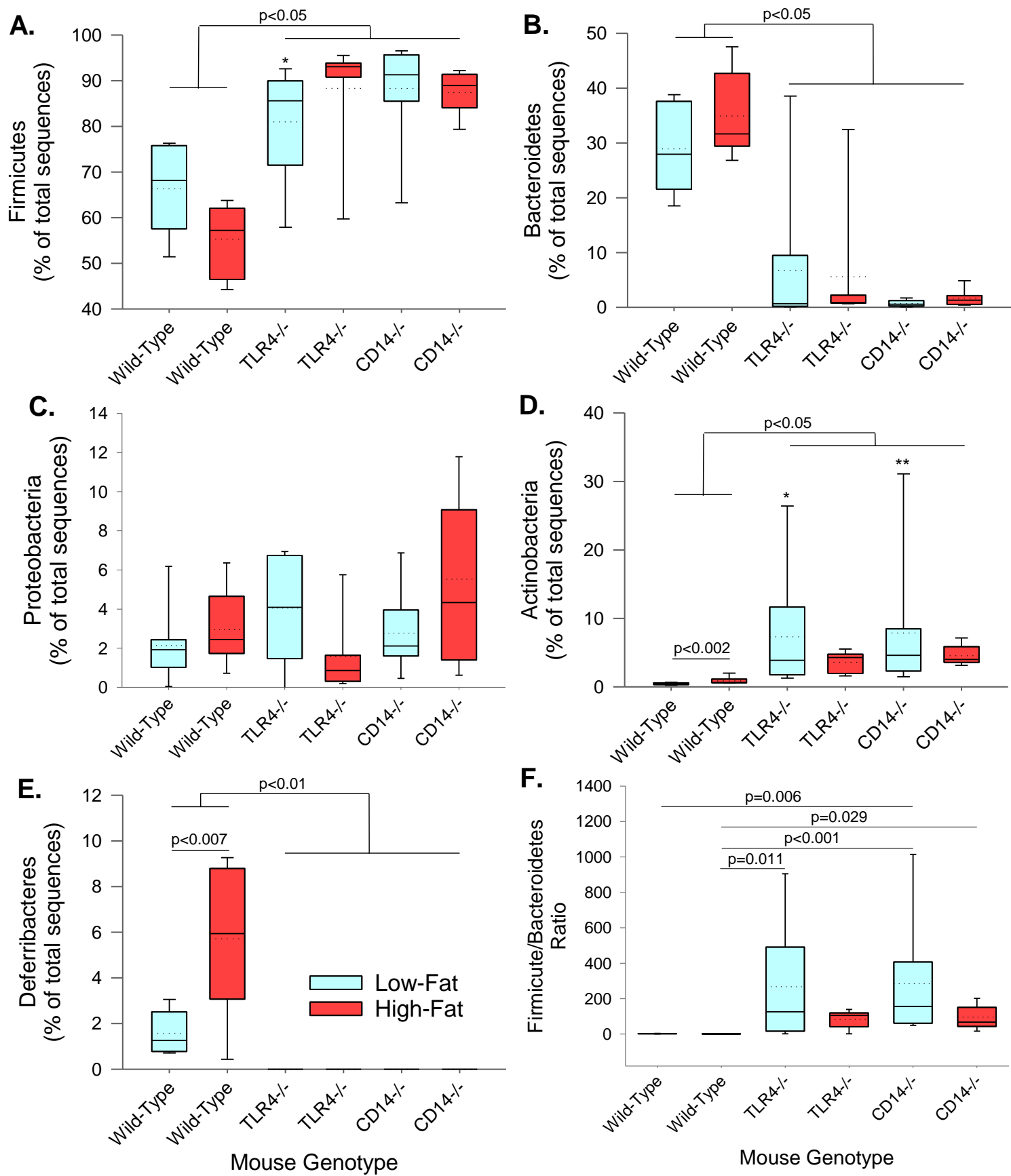

Figure 12. Phylum level changes and ratio in caecal microbiota.

The proportional abundance of phyla Firmicutes (A), Bacteroidetes (B), Proteobacteria (C), Actinobacteria (D), and Deferribacteres (E) in the caecal microbiota of Wild-Type, $\mathrm{TLR}^{-/-}$, and $\mathrm{CD} 14^{-/-}$mice in response to Low-Fat and High-Fat diet feeding. The ratio of the Firmicutes to Bacteroidetes (F) of Wild-Type, TLR4 ${ }^{-/-}$, and CD14 $4^{-/-}$mice in response to Low-Fat and High-Fat diet feeding. For box plots the solid line = median, and dashed line $=$ mean. Statistical significance was determined by Metastats function of the Mothur program with Benjamini and Hochberg correction. $\mathrm{n}=8$ mice per group (TLR4 ${ }^{-/}$and CD14 ${ }^{-/-}$Low-Fat, n=7). ( ${ }^{*}$ not sig. different to Wild-Type Low-Fat, ${ }^{* *}$ not sig, different to Wild-Type High-Fat). 


\subsubsection{REFRACTION CURVES OF MICROBIOTA RICHNESS}

Rarefaction curves were similar between the different diets indicating there was not any general difference in species richness between the High-Fat and Low-Fat diet groups within each mouse genotype, Figure 13. As a group the rarefaction curves for Wild-Type mice, Figure 13A, reached a higher level than for TLR4 $4^{-/-}$and $\mathrm{CD} 14^{-/-}$mice indicating that Wild-Type mice contained a greater species richness, Figure 13B, C. The plateauing of the rarefaction curves showed that the number of sequences generated was enough to include the majority of the OTUs present in the original samples.

\subsubsection{GENOTYPE AND NOT DIET INFLUENCES MICROBIOTA DIVERSITY}

The number of observed species (Sobs) and estimated species number (Chao) were lower in TLR4 ${ }^{-/}$and $\mathrm{CD} 14^{-/-}$mice compared to Wild-Type mice, Figure 14A, B. The microbiota diversity of the caecum was calculated by the Shannon and Inverse Simpson indexes, measures of species evenness of the community. Shannon and Inverse Simpson indexes tended to be decreased in the caecal microbiota of TLR4 ${ }^{-/-}$and CD14 ${ }^{-/-}$mice compared to Wild-Type mice, Figure 14C, D. Excluding the difference in Shannon diversity index between Low-Fat and High-Fat fed CD14 ${ }^{-/-}$mice there were no significant differences between the Low-Fat and High-Fat diet groups in any of the measures of microbiota diversity. All differences in microbiota diversity were between Wild-Type mice and TLR4 ${ }^{-/-}$and CD14 $4^{-/-}$mice. 

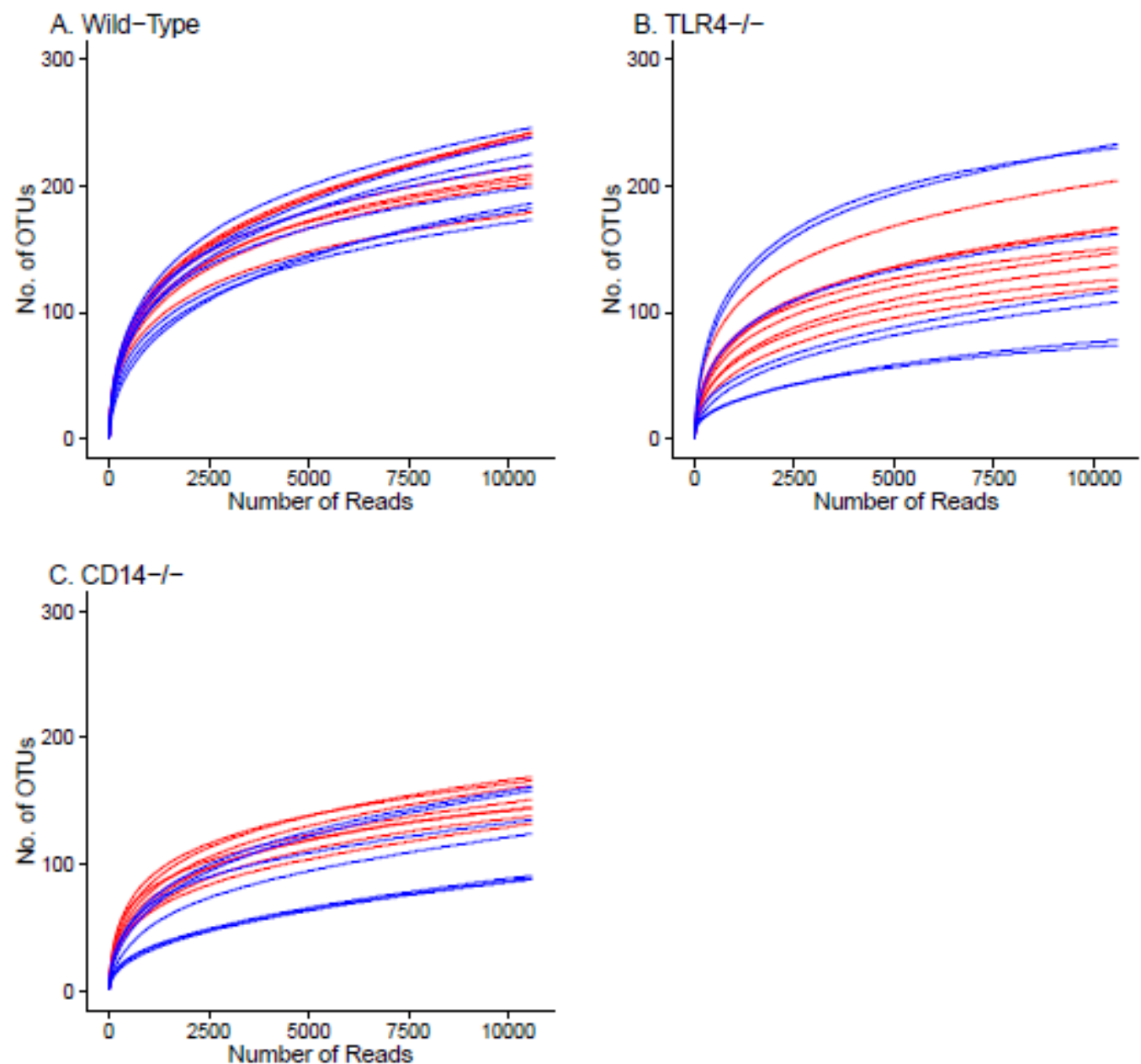

Figure 13. Effect of diet and genotype on rarefaction curves of species richness.

Rarefaction curves for Wild-Type mice (A), TLR4 $4^{-/-}$mice (B), and CD14 ${ }^{-/-}$mice (C). The Low-Fat diet fed mice are represented by Blue Lines, and High-Fat diet fed mice by Red Lines. Rarefaction curves display a plot of the number of species as a function of the number of repeated random samples. Individual lines represent the microbiota richness from one part of the gut for a single mouse. The plateauing of the curves indicate that the majority of the species (OTUs) present in each sample have been detected. $\mathrm{n}=8$ mice per group (TLR4 ${ }^{-/-}$and CD14 ${ }^{-/-}$Low-Fat, $\mathrm{n}=7$ ). 

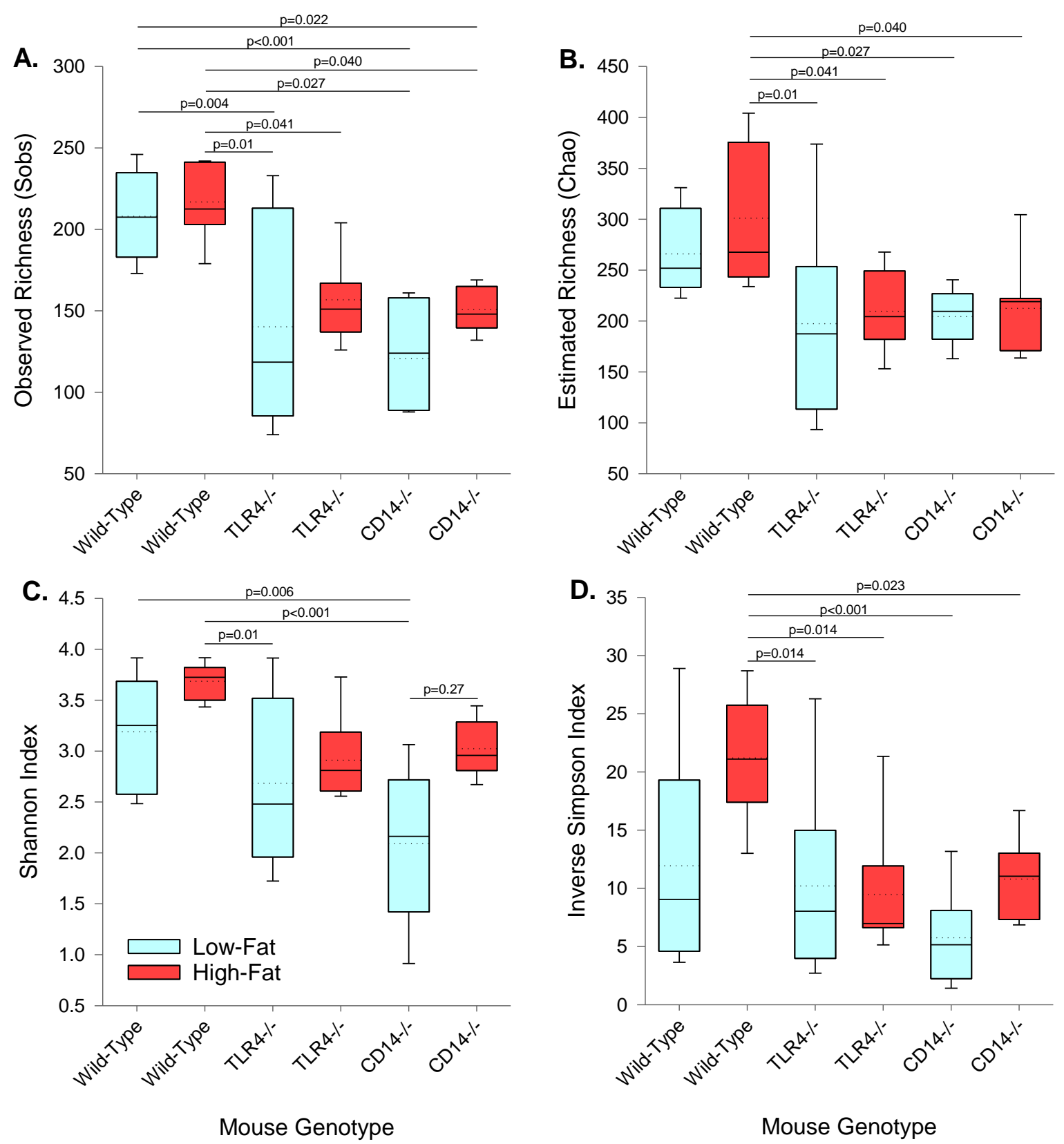

Figure 14. Indexes of caecal microbiota diversity.

Observed richness (A), and estimated richness (B), for the caecal microbiota of CD14 ${ }^{-/-}$ and TLR4 ${ }^{-/}$mice eating Low-Fat or High-Fat diets. Shannon diversity index (C), and Simpson diversity index (D), for the caecal microbiota of $\mathrm{CD} 14^{-/-}$and TLR4 ${ }^{-/-}$mice eating Low-Fat or High-Fat diets. Diversity measures calculated in Mothur. For box plots the solid line $=$ median, and dashed line $=$ mean. Statistical significance was determined by one-way ANOVA with Tukey's post hoc test. $\mathrm{n}=8$ mice per group (TLR $4^{-/-}$and CD14 ${ }^{-/-}$ Low-Fat, $\mathrm{n}=7$ ). 


\section{DISCUSSION}

In the present study neither $\mathrm{TLR} 4^{-/-}$mice nor $\mathrm{CD}^{-/-}$mice were protected against the increased body weight, body fat, and energy intake seen in Wild-Type mice resulting from High-Fat diet feeding over an 8 week period. Additionally, the High-Fat diet did not result in any changes in inflammatory gene expression in the hypothalamus of either Wild-Type or TLR4 ${ }^{-/-}$mice.

\subsection{TLR4 $^{-/-}$AND CD14 ${ }^{-/-}$MICE WERE NOT RESISTANT TO OBESITY}

\subsubsection{CD14 ${ }^{-/-} \mathrm{MICE}$}

The lack of protection of $\mathrm{CD}^{-/-}$mice fed the High-Fat diet from either body weight gain, body fat gain, or increased in energy intake resulted in an in-depth re-examination of the literature. The $\mathrm{CD} 14^{-/-}$mice used in the present study were originally believed to be the same as used by Cani et al (2007a) supplied by Jackson Laboratory (Moore et al, 2000). It was later discovered that the $\mathrm{CD}^{-/-}$mouse used by Cani et al (2007a) were an older CD14 $4^{-/-}$mouse model developed by Haziot et al (1996). The same CD14 ${ }^{-/-}$ knockout crossed with ob/ob mice did not reduce body weight compared to ob/ob mice but did reduce visceral fat (Cani et al, 2008). Roncon-Albuquerque et al (2008) reported that $\mathrm{CD} 14^{-/-}$fed a $35 \%$ fat diet weighed the same as control mice with a slightly lower of gonadal body fat. Yin et al (2001) used the same CD14 $4^{-/-}$mice as Cani and colleagues (Cani et al, 2007a) and reported no differences in bodyweight after 4 weeks on a 35\% fat liquid diet compared to Wild-Type mice. However, the CD14 ${ }^{-/-}$mice were backcrossed into BALB/c mice (Montgomery et al, 2013).

The Jackson Laboratory later switched to supplying CD14 $4^{-/}$mice developed by Moore et al (2000) and used in the present study. Young et al (2012) reported that these CD14 ${ }^{-/-}$ mice showed no difference in body weight, lean mass, or body fat when fed a $60 \%$ fat diet. In summary, one study has reported that $\mathrm{CD} 14^{-/-}$mice are protected from High-Fat diet induced body weight gain and increases in food intake. Three other studies reported reductions in body fat. The results of the present study agree with Young et al (2012), showing $\mathrm{CD} 14^{-/-}$mice does not protect against obesity. 
Table 9. The effect of TLR4 or CD14 knockout in high-fat fed mice.

\begin{tabular}{|c|c|c|c|c|c|c|}
\hline Study & Genotype & $\begin{array}{c}\% \text { Fat in } \\
\text { diet }\end{array}$ & Fat source & $\begin{array}{c}\text { Weight } \\
\text { gain }\end{array}$ & $\begin{array}{c}\text { Total } \\
\text { body fat }\end{array}$ & $\begin{array}{l}\text { Food } \\
\text { intake }\end{array}$ \\
\hline Yin 2001 & Cd14-/- & 35 & Corn oil & $=$ & ND & ND \\
\hline Cani 2007 & Cd14-/- & 78 & Lard & $\downarrow$ & $\downarrow$ & $\downarrow$ \\
\hline Cani 2008 & Cd14-/- ob/ob-/- & 78 & Lard & $=$ & $\downarrow$ & ND \\
\hline Roncon 2008 & Cd14-/- & 35 & Lard & $=$ & $\downarrow$ & $=$ \\
\hline Young 2012 & Cd14-/- & 60 & Shortening & $=$ & $=$ & $=$ \\
\hline Poggi 2007 & $\mathrm{C} 3 \mathrm{H} / \mathrm{HeJ}$ & 45 & Milkfat & $=$ & $\uparrow$ & $\uparrow$ \\
\hline Suganami 2007 & $\mathrm{C} 3 \mathrm{H} / \mathrm{HeJ}$ & 60 & Lard & $=$ & $=$ & ND \\
\hline Tsukumo 2007* & $\mathrm{C} 3 \mathrm{H} / \mathrm{HeJ}$ & 55 & ND & $\downarrow$ & $\downarrow$ & $=$ \\
\hline Shi $2006 * *$ & C57BL/6 Male & 58 & Lard & $=$ & $=$ & $=$ \\
\hline Shi 2006** & C57BL/6 Female & 58 & Lard & $\uparrow$ & $\uparrow$ & $\uparrow$ \\
\hline $\operatorname{Kim} 2007^{* *}$ & $\mathrm{C} 57 \mathrm{BL} / 6$ & 60 & Lard & $=$ & $=$ & ND \\
\hline Orr 2012** & C57BL/6 & 45 & Lard & $\downarrow$ & $=$ & $\downarrow$ \\
\hline Pierre 2013** & $\mathrm{C} 57 \mathrm{BL} / 6$ & 45 & Lard & $\downarrow$ & $\downarrow$ & $\uparrow$ \\
\hline Radin 2007 & $\mathrm{C} 57 \mathrm{BL} / 10 \mathrm{SC}$ & 45 & Lard & $\downarrow$ & $\downarrow$ & $\downarrow$ \\
\hline Davis 2008 & C57BL/10Sc & 60 & Lard or Soy & $\downarrow$ & $\downarrow$ & $=$ \\
\hline Saberi 2009 & $\mathrm{C} 57 \mathrm{BL} / 10 \mathrm{SC}$ & 60 & Lard & $\downarrow$ & $\downarrow$ & $=$ \\
\hline Li 2011 & $\mathrm{C} 57 \mathrm{BL} / 10 \mathrm{SC}$ & 32 & Lard & $\downarrow$ & ND & $=$ \\
\hline Kim 2012 & $\mathrm{C} 57 \mathrm{BL} / 10 \mathrm{SC}$ & 60 & Lard & $=$ & $\downarrow$ & ND \\
\hline Vijay-Kumar 2011 & $\mathrm{C} 57 \mathrm{BL} / 6$ & 60 & Shortening & $\uparrow$ & $\uparrow$ & $=$ \\
\hline Ding 2012 & C57BL/6 LDLR-/- & 35.5 & Lard & $=$ & $=$ & $=$ \\
\hline
\end{tabular}

CD14-/-: Mice with a gene knockout of the CD14 gene.

$\mathrm{C} 3 \mathrm{H} / \mathrm{HeJ}$ : Mice carrying a spontaneous single nucleotide loss-of-function mutation in the TLR4 gene.

10SCN: Mice carrying a sponataneous deletion of the TLR4 gene.

TLR4-/-: Mice with a knockout of the TLR4-/-

*Study retracted

**TLR4-/- mice developed by Hoshino et al (1999)

\subsubsection{TLR4 ${ }^{-/-}$MICE}

Comparison with the effects of TLR4 knockouts reported in the literature is complicated by the diversity of knockout mouse strains used, as outlined in Table 9 . C3H/HeJ mice carry a non-functional TLR4 as a result of a spontaneous point mutation in the TLR4 gene (Hoshino et al, 1999). The $\mathrm{C} 3 \mathrm{H} / \mathrm{HeOuJ}$ substrain used as a wild-type control are genetically very similar, although not identical (Tokuda et al, 2009). When fed a pelleted high-fat diet $\mathrm{C} 3 \mathrm{H} / \mathrm{HeJ}$ mice became fatter while consuming less food that $\mathrm{C} 3 \mathrm{H} / \mathrm{HeOuJ}$ controls, suggesting greater food efficiency (Poggi et al, 2007). Feeding a high-fat diet 
for 16 weeks no difference in body weight, or body fat between $\mathrm{C} 3 \mathrm{H} / \mathrm{HeJ}$ and $\mathrm{C} 3 \mathrm{H} / \mathrm{HeOuJ}$ mice (Suganami et al, 2007). A study by Tsukumo et al (2007) was previously considered food evidence that $\mathrm{C} 3 \mathrm{H} / \mathrm{HeJ}$ mice were protected against obesity, but this has study recently been retracted (Tsukumo et al, 2016).

Feeding a high-fat diet to a TLR4-/- mouse produced by (Hoshino et al, 1999) through targeted gene removal had no effect of the body weight in male TLR4 ${ }^{-/-}$mice while female mice became significantly heavier, fatter, and ate more food than wild-type controls (Shi et al, 2006). The same TLR4 ${ }^{-/-}$mice showed no difference in body weight or body fat in response to a 60\% high-fat diet (Kim et al, 2007). A slight reduction in body weight and body fat gain was reported by Orr et al (2012) in TLR4 ${ }^{-/}$mice. In contrast to the previous studies Pierre et al (2013) reported that the same TLR4 ${ }^{-/}$mice were completely protected from high-fat diet induced obesity. In double knockout mice, combining TLR4 ${ }^{-/}$and the LDL ${ }^{-/-}$(Low-Density Lipoprotein Receptor), body weight was not different between knockouts and controls on either low-fat or high-fat diets (Ding et al, 2012). The effects of this TLR4 knockout are highly inconsistent between studies.

The C57BL/10Sc mouse strain carries a different spontaneous 74723 bp deletion that removes the complete sequence of the Tlr4 gene (Poltorak et al, 2000; Vogel et al, 1979). A study by (Radin et al, 2008) reported C57BL/10Sc mice gained less weight and body fat on a 45\% high-fat diet. C57BL/10Sc mice were reported completely protected from obesity although further details were not provided (Saberi et al, 2009). The C57BL/10Sc mice were reportedly completely protected against obesity when fed a lower 35\% fat diet (Li et al, 2011). The C57BL/10Sc mice were reportedly protected against body weight and body fat increase on a diet high in saturated fat palmitate, but not a diet high in unsaturated fats (Davis et al, 2008). In contrast, Vijay-Kumar et al (2011) found the same C57BL/10Sc mice, crossed into the C57BL/6 strain of mice, gained significantly more weight and body fat than control mice on a $60 \%$ fat diet.

The present study used a TLR4 ${ }^{-/-}$mouse carrying the C57BL/10Sc mutation back-crossed into C57BL/ 6 mice. No protection from body weight, or body fat, was seen in comparison to Wild-Type C57BL/6 mice. The C57BL/10Sc mice was the TLR4 ${ }^{-/}$strain most consistently protected against body weight and body fat gain in previous studies. However, when backcrossed into C57BL/6 mice it appears to have no influence. 
In summary, several different knockout mice have been used to investigate the TLR4 signalling pathway, and divergent effects on body weight and body fat have been reported. The results of the present study showing no reduction in weight gain or body fat, and no effect on food intake in TLR4 $4^{-/-}$or $\mathrm{CD}^{-/-}$mice with High-Fat diet feeding agrees with some but not all earlier investigations, Table 9. Critical examination of the available literature emphasises the important differences that exist between earlier studies regarding the strain and sex of the mice used, the knockout model, i.e. CD14 $4^{-/ \text {, }}$ $\mathrm{C} 3 \mathrm{H} / \mathrm{HeJ}, \mathrm{C} 57 \mathrm{BL} / 10 \mathrm{ScN}$, or TLR4 ${ }^{-/-}$mice, and the type of high-fat diet used. No clear pattern was discernible to explain the conflicting results with regards to weight gain and body fat. In the context of the literature the present study suggests that while TLR4 deficiency may confer protection against weight gain and obesity in certain circumstances it is by no means universal. The present study adds strong evidence that TLR4 or CD14 is not required for diet induced obesity development.

\subsection{SERUM LIPOPOLYSACCHARIDE BINDING PROTEIN}

Lipopolysaccharide binding protein (LBP) was increased in all groups of high-fat diet fed mice relative to the low-fat fed Wild-Type mice. Serum LBP was higher in TLR4 ${ }^{-/}$mice fed the Low-Fat diet compared to other groups meaning the difference in serum LBP levels between High-Fat and Low-Fat was not significant. LBP is a serum protein produced in the liver in response to LPS exposure and is considered a biomarker for LPS exposure (Gonzalez-Quintela et al, 2013; Moreno-Navarrete et al, 2012; Stehle et al, 2012). LPS is rapidly cleared from the blood. In contrast LBP remains in the circulation with a serum half-life of 12-24 hours representing a biomarker of LPS exposure over time (Behrendt et al, 2004). This increased LBP indicates that there was greater exposure to LPS was in mice fed the High-Fat diet in comparison to those fed the Low-Fat diet. The increase in LBP in Wild-Type mice in response to High-Fat diet feeding was not associated with any differences in body weight, body fat, or food intake compared to $\mathrm{TLR}^{-/-}$or $\mathrm{CD} 14^{-/-}$mice despite these mice being unable to respond to the associated LPS. 


\subsection{LPS INFUSION EFFECTS ON BODY WEIGHT, FOOD INTAKE, AND INFLAMMATION}

Inconsistent effects of TLR4 and CD14 knockouts on body weight and body fat in mice, question the role for LPS in influencing body weight or obesity. The chronic infusion of LPS into the mouse was reported to cause an increase in body weight and body fat showing that LPS can be a causal factor in body weight change (Cani et al, 2007a). Considering the results of the present study an extensive searching of the published literature revealed several new studies that have since used similar infusions of LPS not directly focused on body weight, see Table 10. These new studies were only found by searching for the use of osmotic mini-pump used to infuse LPS (Alzet Model 2004; Alza, Palo Alto, CA).

\subsubsection{MOUSE STUDIES OF CHRONIC LPS INFUSION}

A study over a 6 week period reported no significant effects of LPS infusion at 300 $\mu \mathrm{g} / \mathrm{kg} /$ day on body weight or fat in either chow fed or high-fat diet fed mice (Geurts et al, 2013), Table 10. In another study infusion of LPS at $300 \mu \mathrm{g} / \mathrm{kg} /$ day initially caused a $10 \%$ drop in body weight, which recovered after 4 weeks, resulting in no change in body weight of food intake despite increased inflammatory gene expression in adipose tissue (Nohr et al, 2016). Infusion $300 \mu \mathrm{g} / \mathrm{kg} /$ day of LPS for 4 weeks increased adipose tissue fibrosis in mice but did not influence body weight (Vila et al, 2014). A lower dose of LPS infusion of $5 \mu \mathrm{g} /$ day did not influence body weight or visceral fat (Liang et al, 2014). While infusion of two different low doses of LPS at $24 \mathrm{pg}$ or $24 \mathrm{ng} / \mathrm{kg} /$ day increased the expression of inflammatory cytokines in adipose and liver tissues it had not effect on body weight (Iwashita et al, 2013). Only one study replicated the findings of (Cani et al, 2007a), showing a modest increase in body weight and epididymal fat (Ahn et al, 2015).

Thus, the weight of evidence from mice studies consistently shows that LPS exposure has no influence on body weight or food intake. 
Table 10. Effects of chronic LPS infusion on body weight, body fat, and food intake.

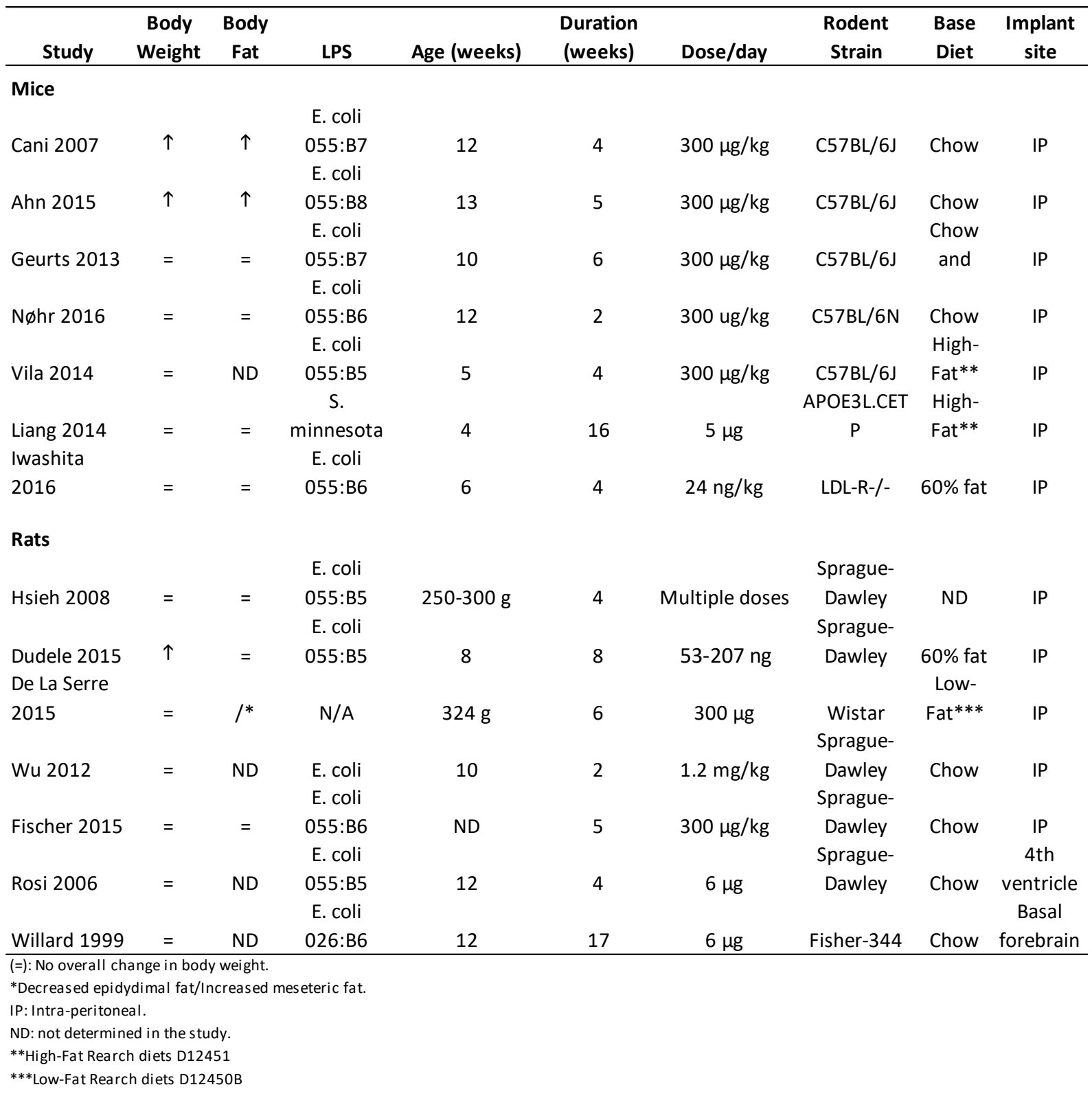

\subsubsection{RAT STUDIES OF CHRONIC LPS INFUSION}

Seven studies were identified involving chronic infusion of LPS into rats, Table 10. The infusion of LPS at 0.3 or 0.6 or $1.2 \mu \mathrm{g} / \mathrm{kg} /$ day into the portal vein of rats resulted in no effect on body weight (Hsieh et al, 2008). Infusion of LPS into rats over 8 weeks at a dose of $53 \mu \mathrm{g} /$ day or $207 \mu \mathrm{g} /$ day increased body weight in rats with reductions in visceral and mesenteric fat, suggesting an increase in lean mass (Dudele et al, 2015). Six weeks of infusion of LPS at $300 \mu \mathrm{g} /$ day increased food intake in rats but not body weight, with a decrease in epididymal fat, and increase in mesenteric fat (de La Serre et al, 2015). The infusion of LPS at $1.2 \mathrm{mg} / \mathrm{kg}$ for 2 weeks resulted in no change in body weight or food intake (Wu et al, 2012). In another study the infusion of LPS at 75 or $300 \mu \mathrm{g} / \mathrm{kg} /$ day 
caused an initial transient decrease in bodyweight, however after 4 weeks there were no effect on bodyweight, food intake, or insulin resistance (Fischer et al, 2015). The infusion of $6 \mu \mathrm{g} /$ day of LPS into the $4^{\text {th }}$ ventricle of rats produced microglia activation and markers of chronic neuroinflammation but had no effect on body weight (Rosi et al, 2006). Chronically infusing LPS in four different doses between 4 and $120 \mu \mathrm{g} /$ day into the basal forebrain of rats for up to 17 weeks had no effect on normal growth or body weight (Willard et al, 1999). The evidence from rat studies is also consistent with LPS exposure having no influence on body weight or food intake.

\subsubsection{HUMAN STUDIES OF CHRONIC LPS EXPOSURE}

In a population study on 200 men endotoxemia was found to increase with energy intake (Amar et al, 2008). It was reported that although overfeeding 18 healthy men for 8 weeks resulted in significant weight gain, it did not result in increased fasting LPS levels in the circulation (Laugerette et al, 2014). In contrast feeding 8 healthy subjects a Western-style diet for four weeks resulted in a 71\% increase in plasma LPS levels, while a prudent-style diet reduced LPS levels by 31\% (Pendyala et al, 2012).

Mice and rats are less responsive to LPS than humans (Warren et al, 2010). The lethal doses of LPS can range up to a 10,000-fold between species with mice typically highly resilient to LPS. Doses of LPS that are used in rodent studies in vivo to induce severe illness responses are about 1000-10,000 times the dosage needed to induce severe disease with septic shock in humans (Warren et al, 2010). The possibility that the results of experiments using LPS in mice might not apply to humans has not been frequently acknowledged (Munford, 2010).

Taken together, the current literature on the chronic LPS exposure, equivalent to metabolic endotoxemia, consistently shows that LPS does not have an impact on body weight or obesity. This strongly implies that any influence that the loss of TLR4 or CD14 have on body weight or body fat are unlikely to be due to low concentrations of LPS derived from the bacteria in the gut. 


\subsection{HIGH FAT DIET DOES NOT INCREASE INFLAMMATORY GENE EXPRESSION}

\subsubsection{MICROARRAY OF HYPOTHALAMIC INFLAMMATORY GENE EXPRESSION}

High-Fat diet induced obesity in rodents has been associated with increased expression of genes involved in to inflammatory responses in the hypothalamus (De Souza et al, 2005; Thaler et al, 2012). This inflammation has been linked to hypothalamic resistance to insulin and leptin signalling potentially contributing to energy imbalance (Thaler \& Schwartz, 2010). Hypothalamic gene expression of Wild-Type and TLR4 ${ }^{-/}$mice was investigated using an Agilent whole mouse genome microarray on total hypothalamic RNA to determine whether the TLR4 knockout was preventing hypothalamic inflammation. Unexpectedly, no changes in inflammatory gene expression were identified using the microarray in either Wild-Type or TLR4 ${ }^{-/-}$mice and none of the inflammatory genes reported to be upregulated in rats by De Souza et al (2005) in response to high-fat diet induced obesity were significantly changed, including TNF $\alpha$, Il-1 $\beta$, and Il-6. Of the genes reported by Thaler et al (2012) to be upregulated in rats in response to high-fat diet induced obesity, only SOCS3 was to be significantly changed in the present study. The study by De Souza et al (2005) measured hypothalamic gene expression after 16 weeks of high-fat diet feeding in Wistar rats. In comparison Thaler et al (2012) reported in rats and mice an initial transient inflammatory response in the hypothalamus in response to high-fat diet feeding in the first three days, chronic inflammation then returns after 4 weeks. In the present study, hypothalamic gene expression was measured after 8 weeks of High-Fat diet feed in mice while the mice were gaining weight, but had not yet become severely obese. From the results of present study it can be concluded that after 8 weeks of High-Fat diet feeding the previously reported changes in inflammatory genes were not detected.

\subsubsection{GENOTYPE RELATED CHANGES IN GENE EXPRESSION}

The degree of fold change of hypothalamic gene expression was small in response to High-Fat diet feeding relative to mice fed the Low-Fat. There were only three genes with a fold change greater than $+/-1.5$ fold in Wild-Type mice and only 21 genes were greater 
than 1.5 fold in TLR4 ${ }^{-/-}$mice. When the cut off for fold-change was reduced to $+/-1.25$ in the Wild-type mice, the expression of 103 genes were upregulated and 16 genes were down regulated in response to the high-fat diet. In contrast in the TLR4 ${ }^{-/-}$mice the expression of 12 genes were upregulated and 194 genes were down regulated. This indicates that the TLR4 knockout did not influence obesity development or food intake it did result in overall responses in gene expression.

Three genes were chosen for further investigation from the microarray results using in situ hybridisation. The first, AgRP was found not to change significantly when expression was determined using in situ hybridisation. The expression of two other genes, SerpinA3N and SOCS3, was found to be significantly down regulated using microarray analysis. When followed up with using in situ hybridisation using larger numbers of mice the expression of these genes was found to be upregulated. This confirmed that SerpinA3N and SOCS3 gene expression did change in response to HighFat diet feeding but indicated that the direction of fold change of gene expression in the microarray analysis was incorrectly reversed.

\subsubsection{SERPINA3N EXPRESSION INCREASED IN HIGH-FAT DIET FED MICE}

The serpinA3N gene encodes the protein Alpha 1-antichymotrypsin, an acute phase protein that is induced during inflammation and involved in the resolution of inflammation (Hsu et al, 2014). In the brain SerpinA3N has been shown to be neuroprotective in in vitro and in vivo in mouse models of neurodegenerative disease (Haile et al, 2015). SerpinA3N expression is reportedly a marker of reactive astrogliosis (Zamanian et al, 2012). Astrogliosis has been associated with damage to the hypothalamus occurring in High-Fat diet fed rodents (Dorfman \& Thaler, 2015). The results here support recent evidence that expression of serpin $\mathrm{A} 3 \mathrm{~N}$ in the hypothalamus of mice increases during High-Fat diet feeding (Sergi et al, 2015). In the present study using in situ hybridisation serpin $\mathrm{A} 3 \mathrm{~N}$ was shown to be significantly up-regulated in the hypothalamus of Wild-Type, TLR4 ${ }^{-/}$, and $\mathrm{CD}^{-/-}$mice in response to High-Fat diet feeding in both the arcuate nucleus, ventromedial hypothalamus, and dorsomedial hypothalamus. Significantly for the present study the increased serpinA $3 \mathrm{~N}$ expression 
was not attenuated in either $\mathrm{TLR} 4^{-/-}$or $\mathrm{CD}^{-/-}$mice showing that hypothalamic changes due to High-Fat diet did not require TLR4/CD14 signalling pathways.

\subsubsection{SOCS3 EXPRESSION INCREASED IN HIGH-FAT DIET FED MICE}

In the present study SOCS3 expression was increased in the hypothalamus in both WildType and $\mathrm{CD}_{1} 4^{-/-}$as determined by in situ hybridisation. In TLR4 ${ }^{-/-}$mice SOCS3 was not significantly increased, however the analysis of TLR $4^{-/-}$mice was limited by few numbers of mice brains remaining for section after the microarray analysis. Only 3 and 4 mice from High-Fat and Low-Fat diet groups respectively were available for in situ hybridisation limiting the power to detect gene expression changes between groups. However, the increased expression of SOCS3 in $\mathrm{CD}_{1} 4^{-/-}$mice indicates that this was not dependent on signalling through the TLR4 receptor.

\subsubsection{SUMMARY OF HYPOTHALAMIC INFLAMMATION RESULTS}

The in situ hybridisation results indicate that the direction of fold change in the microarray study was incorrect, despite extensive investigation of the methods used the reasons for this reversal this could not be explained. The in situ hybridisation results were taken as the more accurate results. These results show that changes in hypothalamic gene expression in response to eight weeks of High-Fat diet feeding were small in magnitude with no increased expression of inflammatory cytokines. The increased expression of SerpinA3N and SOCS3 by in situ hybridisation demonstrate that the High-Fat diet was having an influence in the hypothalamus. The High-Fat diet induced changes in hypothalamic gene expression that did occur were not prevented by the loss of TLR4.

\subsection{ANALYSIS OF CAECAL MICROBIOTA RESULTS}

Microbiota analysis revealed little difference between Low-Fat and High-Fat diets in Wild type mice in the proportional abundance of the Firmicutes and Bacteroidetes phyla or in microbiota diversity in obese Wild-Type mice contrasts with previous studies (Ley et al, 2005). Compared to Wild-Type mice the TLR4 and CD14 mice showed a significant difference in the ratio of the Firmicutes and Bacteroidetes phyla. There was also a marked lower microbiota diversity in TLR4 ${ }^{-/-}$and $\mathrm{CD} 14^{-/-}$mice, in comparison to WildType mice. There are two main factors potentially contributing to this microbiota 
difference in $\mathrm{TLR}^{-/-}$and CD14 ${ }^{-/-}$mice. The loss of TLR4 signalling could potentially influence microbiota composition as the TLR4 represents one of the main receptors through which bacteria in the gut can interact with the host. Previously, loss of TLR5, another toll-like receptor activated by bacterial flagellin, has been proposed to alter the composition of intestinal bacteria in mice (Vijay-Kumar et al, 2010), and potentially result in an unstable gut microbiota (Carvalho et al, 2012). Others have instead proposed that familial transition, rather than TLR deficiency is the major determinant of mouse intestinal microbiota (Letran et al, 2011; Ubeda et al, 2012). Ubeda et al (2012) found no difference in caecal microbiota composition in TLR4 knockout mice suggesting that the loss of TLR4 or is not contributing to an altered gut microbiota.

The TLR4 ${ }^{-/-}$and CD14 $4^{--}$mice were supplied by the Jackson Laboratory facilities at Bar Harbour, Maine, while the Wild-Type mice were supplied from a Jackson Laboratory mouse colony in the UK. Recent research suggests mouse intestinal microbiota composition of mice varies between different facilitates (Rausch et al, 2016), and even within the same animal facility the mice maintained in different rooms (Rogers et al, 2014). This suggests that the difference in microbiota between Wild-Type and knockout mice in the present study is most likely due to the origin of the mice.

Another observation was the significant changes in the Family level composition of the TLR4 and CD14 microbiota in response to high-fat diet occurring within the Firmicutes phylum. This change was identified as the reduction in a single bacterial species, all other changes in family level composition were proportionate increases in bacterial families already present. It is possible that this unidentified bacterium of the Firmicutes phylum was particularly ill suited to the High-Fat diet.

In conclusion, Wild-Type mice failed to show an obesity associated microbiota despite large increases in body fat in High-Fat fed Wild-Type mice. Change in microbiota composition is not a prerequisite for obesity development and the drastically different microbiota composition in $\mathrm{TLR}^{-/-}$and $\mathrm{CD} 14^{-/-}$mice did not lead to any different in obesity susceptibility compared to Wild-Type mice. The development of High-Fat diet induced obesity in mice can be disassociated from changes in the composition of the caecal microbiota. 


\subsection{CHOW CONTROL DIETS AS A CONFOUNDING FACTORS}

The particular diets used in the present study represent a potential explanation for both the lack of microbiota change in wild-type mice and the lack of inflammatory gene expression previously reported in the brain. In the present study both Low-Fat and HighFat diets used refined diets made from defined ingredients and were closely matched in their composition with regards to both ingredients, protein content, and fibre. In the High-Fat diet lard was substituted for cornstarch while other components remained equivalent between diets. In contrast, many of the previous research studies on both obesity associated microbiota and obesity associated inflammation have used a refined High-Fat diet, similar to the one used in the present study, matched with an undefined chow control diet (Cani et al, 2007a; Ley et al, 2005; Thaler et al, 2012). The importance of using correctly match control diets has previously been highlighted (Barnard et al, 2009; Ricci, 2013). Undefined mouse chow diets typically contain large amounts of fermentable fibre that can influence body weight in rats and mice (Blaisdell et al, 2014; Chassaing et al, 2015). Compared to the low level of non-fermentable cellulose added to refined High-Fat diets this high level of fermentable fibre in chow diets may account for the differences in microbiota composition seen in previous studies. The inclusion of high quantities of dietary fibre in mouse chow diets may also reduce inflammatory responses (Kuo, 2013). In conclusion, the use of well-controlled Low-Fat and High-Fat diets in the present study reduced confounding factors that have been present in earlier studies.

\subsection{LIMITATIONS}

There were some weaknesses in this study that were not addressed due to time constraints as more promising research areas described in the following chapters became a priority. The addition of testing of LPS levels in the blood of these mice would have more clearly shown the effect of diet on systemic LPS exposure. Additionally, the testing blood glucose responses and levels of hormones, including leptin and insulin, would have shown whether the TLR4 or CD14 knockout was having any subtle effects on High-Fat diet fed mouse metabolism. It is possible that a longer duration study beyond 8 weeks would have reviled effects of a TLR4 or CD14 knockout. However, a duration of 8 weeks was long enough to show responses reported in the previous literature. Using mice ordered from a supplier just for use in the study, rather than 
breeding the mice, prevented any follow-up testing taking place. This included testing the responses of TLR4 or CD14 knockout mice to LPS directly to confirm their lack of inflammatory responses.

\section{CONCLUSIONS}

The results of the present study do not support the hypothesis that TLR4 $4^{-/}$or CD14 $4^{-/-}$ mice are protected from obesity. In the context of the current literature, the involvement of TLR4 signalling in high-fat diet induced obesity in mice is inconsistent and the present study results add to the evidence that there TLR4 or CD14 are not required for the development of obesity in mice. This is consistent with the overall literature on the effects of chronic LPS exposure which consistently shows that LPS does not have an impact on body weight or obesity. The present study also failed to support the hypothesis that High-Fat diet induced obesity is associated with an increased hypothalamic inflammation involving increased inflammatory gene expression. Markers of neuronal damage in the hypothalamus were increased in all mice fed High-Fat diets. This suggests that effects of High-Fat diet in the brain are independent of TLR4 signalling. The evidence from analysis of caecal microbiota found High-Fat diet induced obesity could be produced in Wild-Type mice without previously reported alterations in the composition of the microbiota. In addition, the caecal microbiota of TLR4 ${ }^{-/-}$and $\mathrm{CD}^{-/-}$mice was significantly different to Wild-Type mice without and influence on obesity development. This demonstrates than the composition differences in the microbiota can be disassociated from the development of obesity in these mice. In conclusion, the present study shows that preventing inflammatory signalling through TLR4 does not influence body weight or the development of obesity. 


\section{CHAPTER 4.}

\section{A COMPARISON OF REFINED AND CHOW CONTROL DIETS}




\section{INTRODUCTION}

The study in the previous chapter the differences in the caecal gut microbiota between mice fed high-fat and low-fat diets were relatively minor. This was surprising as the High-Fat diet induced obesity has been reported to result in substantial changes in the composition of caecal microbiota compared. In this Chapter the importance of the control diet to the nature of the response and to the interpretation of the effects of HighFat diets on the composition of the gut microbiota in the diet induced obese mouse in considered.

\subsection{OBESITY IN THE ABSENCE OF AN “OBESE MICROBIOTA”}

Obesity in mice has been reported to be characterised by significant and distinct alterations in the composition of the intestinal microbiota, termed an "obese microbiota" (Ley, 2010; Ley et al, 2006; Turnbaugh et al, 2009a; Turnbaugh et al, 2006). This obese microbiota included an increased ratio of the Firmicutes to Bacteroidetes, the dominant bacterial phyla in the intestine, reductions in microbial diversity, and increases or decreases in specific bacterial families, or species.

A careful examination of the diets used in published studies of the gut microbiota and obesity research was carried out and this suggested that it was the choice of diets used in Chapter 3 that resulted in the lack of an obesity associated microbiota developing in the wild-type mice fed a High-Fat diet.

\subsection{HIGH-FAT DIET INDUCED OBESITY AND REFINED DIETS}

Obesity studies on rodents have made use of genetically obese mice and rats, diet induced obese rodent models have been suggested to be more relevant to human obesity (Tschop \& Heiman, 2001). Refined High-Fat diets are commonly used in the study of diet-induced obesity in rodents (Buettner et al, 2007; Tschop \& Heiman, 2001), yet these High-Fat rodent diets are based on the formulation of the AIN-93m rodent maintenance diet. This was developed as a nutritionally adequate purified diet that could be used to standardize studies between laboratories (Reeves et al, 1993). The recognition that fat is a key component for inducing obesity in mice, particularly in the C57BL/6 strain, led to the development of this diet and the C57BL/6 strain as a popular model of diet-induced obesity (Surwit et al, 1995). The type of high-fat rodent diet used in Chapter 3 is one of 
the most commonly used and produced. It was based on research in the 1990's and the requirement for a defined, flexible diet in a solid pellet in order to conduct food intake studies (Surwit et al, 1995; West et al, 1995). The first formulation contained 45\% kcal from fat, predominantly lard. This was later increased to $60 \%$ of kcal from fat for the popular Research Diets D12492 used in the previous study in Chapter 3.

\subsubsection{COMPOSITION OF REFINED HIGH-FAT MOUSE DIETS}

The most widely used High-Fat mouse diets are semi-synthetic refined diets composed of purified ingredients based on the AIN-93 diet (Reeves et al, 1993). The milk protein casein supplies the protein requirement with added methionine to meet sulphurcontaining amino acid needs. Carbohydrates are supplied by corn starch and sucrose, with maltodextrin added to aid pelleting. Lard and soy oil provides the fat although a range of other fats have been included in rodent High-Fat diets. The fibre is typically supplied by cellulose. Vitamin and mineral mixes specific to mice and rats are added to ensure adequate micronutrients are supplied. Each micronutrient is supplied as a separate, purified ingredient. Refined semi-purified low-fat diets composed of matched ingredients in different proportions is considered to be a more appropriate control diet for mouse studies using refined high-fat diets (Ricci, 2013). The original intent behind the development of standardised, nutritionally adequate purified diets for laboratory animals was to reduce the variation inherent in cereal-based or natural ingredient-based diets and so facilitate interpretation of results among experiments and laboratories (Reeves et al, 1993). While this move has been largely successful in the use of experimental high-fat diets, the same attention has unfortunately rarely been paid to control diets used in rodent research (Ricci, 2013). The composition of a low-fat and high-fat refined mouse diet is shown in Figure 15.

\subsection{UNCONTROLLED CHOW DIETS IN MOUSE STUDIES}

Mouse studies using refined high-fat diets often use a natural-ingredient chow control diet despite dramatic differences in the composition of these diets (Warden, 2008). A survey in 2008 of 35 papers that involved feeding mice a high-fat diet in found that only five of the studies used a well-matched control diet (Warden, 2008).

Semi-purified refined high-fat diets and matched control diets are termed OpenFormula Diets, in which the quantitative ingredient formulations and concentrations of 
all ingredients are publicly available. In contrast chow diets are typically Closed-Formula Diets. Although ingredients are listed, the quantities used are not publicly available. Variations in the ingredients used can occur if diet manufacturers use a least-cost strategy, using the least-expensive ingredients as commodity prices change without public disclosure. Another source of variation is the maintenance of consistent macronutrient composition in rodent chow diets over time as agricultural commodities are obtained from different sources. Protein, lipids, and carbohydrates in natural food ingredients change over time requiring ingredient alterations to maintain a constant macronutrient composition (Barnard et al, 2009). The majority of mouse studies using refined high-fat diets use chow diets as a control that are very different in their composition (Pellizzon, 2016; Ricci, 2013). A comparison of the composition of a typical mouse chow diet compared to refined mouse diets is shown in Figure 15.

\subsubsection{COMPOSITION OF MOUSE CHOW DIETS}

Chow diets are composed of varying quantities of whole unrefined cereal grains including wheat, barley, maize and legumes including soya beans and alfalfa together with a variety of other minor ingredients added depending on the manufacturer, such as fish protein, potato protein, wheat gluten, wheat bran, brewer's yeast, and beet pulp. Chow diets also contain undefined amounts of a variety of over compounds. Plant phytoestrogens can be found in rodent chow at levels capable of decreasing body fat in mice (Cederroth et al, 2007). Contaminating heavy metals including arsenic can be present at levels capable of significant alterations in gene expression in the liver and lungs (Kozul et al, 2008). Chow diets also include a large proportion of dietary fibre. These components are absent from refined experimental diets and have the potential to confound study outcomes. Chow diets are commonly referred to in methods as "standard chow", although there is no standardisation among the chow diets used in rodent research. The typical composition of a mouse chow diet is shown in Figure 15. 


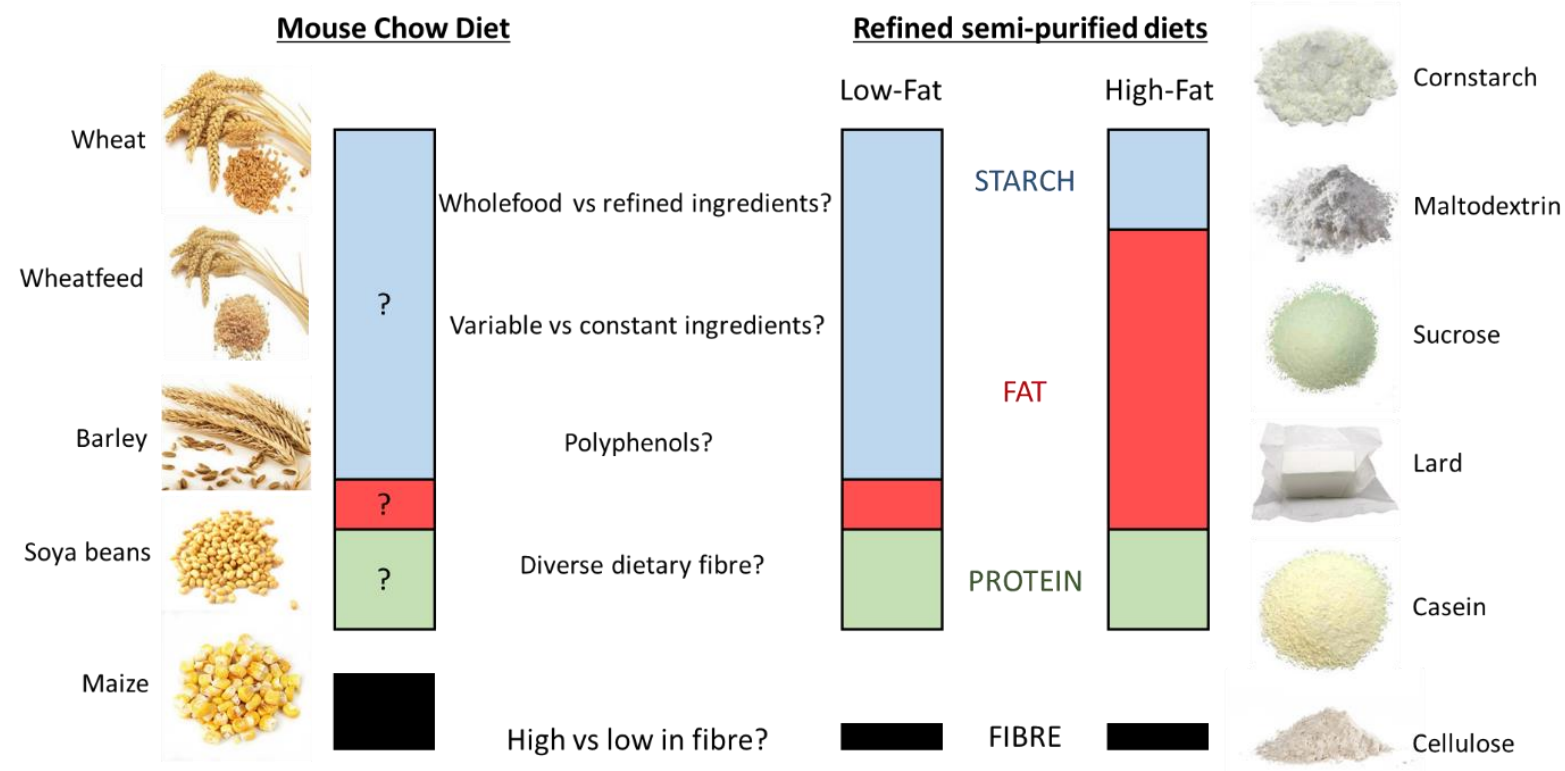

Figure 15. Composition of typical mouse chow and refined semi-purified diets.

\subsection{CONTROL DIETS AS A CONFOUNDING FACTOR}

Recent research has highlighted the potential for chow diets to be a confounding factor in mouse studies using refined high-fat diets (Benoit et al, 2013; Chassaing et al, 2015). Conclusions as to the effects of a refined $40 \%$ fat diet on various physiological measures in C57BL/6 mice varied depending on whether a refined low-fat diet or chow diet was used (Benoit et al., 2013). This study was the first to compare an unrefined chow, with both a refined low-fat, and a refined high-fat diet and to suggest that some deleterious effects of a High-Fat diet may be explained not entirely by excess fat intake, but the overall quality of ingredients in a refined diet as well. Although similar in macronutrient ratios the refined low-fat diet resulted in higher bodyweight, increased body fat, and reduced glucose tolerance, producing an intermediate phenotype between the chow and the High-Fat diet (Benoit et al, 2013). Other have also fed rodents on a refined low-fat diet and compared this to a chow diet. Feeding Long Evans rats a refined low-fat diet resulted in significantly more weight gain than those rats fed unrefined rodent chow (Blaisdell et al., 2014). In another study using Sprague Dawley rats investigating food preference the rats eating a refined low-fat diet weighed significantly more compared to rats eating chow (Apolzan \& Harris, 2012). New Zealand Obese mice were found to 
display an intermediate increase in body weight, adipose tissue and blood glucose response on a refined low-fat diet compared to either a chow diet or refined high-fat diet (Mirhashemi et al., 2011). A refined compared to chow resulted in profound differences in gene expression in both the lung and liver (Kozul et al, 2008). The control diet used has been recently shown to be vitally important in correctly determining the effect of a high-fat obesity inducing diet (Benoit et al, 2013; Chassaing et al, 2015).

\subsubsection{CHOW DIETS IN MOUSE GUT MICROBIOTA RESEARCH}

Research outlined above has important implications for research on rodents linking the gut microbiota and obesity. Numerous factors have been implicated in alterations in the gut microbiota composition. These include host genetics (Rawls et al, 2006), exercise (Cerda et al, 2016), and disruption of circadian rhythms (Voigt et al, 2014). However, in laboratory mice or rats in the same environmental conditions diet is likely to be the dominant factor shaping the composition of the gut microbiota. The composition of the microbiota can be drastically shifted within one day after a major dietary shift (Turnbaugh et al, 2009b).

The largest population of microbes in the gut are found in the caecum and colon, with smaller populations in the small intestine. The substrates available for bacterial growth in the lower intestine is predominantly dietary polysaccharides that are unavailable to host digestion, classified as dietary fibre. Rodent chow diets also contain phytoestrogens that also have the potential to modulate the intestinal microbiota composition (Clavel et al, 2005; Duenas et al, 2015). However, the dominant influence on the composition of the gut microbiota is the amounts, and types of dietary fibre present in the diet.

Rodent chow typically contains around 15\% fibre as measured by chemical analysis. Accurately determining total fibre in a diet is difficult and total fibre in rodent chow maybe closer to 20-25\% ( $20 \%$ insoluble and $~ 5 \%$ soluble) (Ricci, 2013). Analysis available for Rat and Mouse Breeder and Grower (SDS; Special Diet Services Ltd, Witham, UK) states that this diet contains $15.06 \%$ total fibre, $8.85 \%$ hemicellulose, $3.89 \%$ cellulose, $1.40 \%$ lignin, and $1.4 \%$ pectin. Hemicellulose includes a diversity of fibre types including arabinoxylan that forms a large proportion of the fibre in wheat bran and is highly fermentable by the gut microbiota (Neyrinck et al, 2011) (Ring \& Selvendran, 1980). Pectin is another fibre type present in the chow that is 
fermentable by the microbiota (Adam et al, 2015b; Chung et al, 2016). Beta-glucan is a common fibre in barley found in chow containing barley, the fermentation of which alters gut microbiota composition and host physiology (Arora et al, 2012). Lignin may be partly fermented (Niemi et al, 2013). There is a large proportion and diverse range of fermentable dietary fibre in chow diets available as microbial energy substrates.

The only fibre typically added into refined High-Fat rodent diets is purified cellulose added at $5 \%$ of the total diet and is only minimally fermented in the intestines of nonruminant animals. Other components of refined rodent diets are purified starches, sucrose, casein protein, and lipids that are absorbed in the small intestine leaving little residue to act as a substrate for microbial growth.

\subsubsection{POORLY MATCHED CHOW DIETS ARE COMMONLY USED}

A number of well cited published research studies on the mouse gut microbiota have used a refined High-Fat diet with an unrefined Chow diet as a control (Cani et al, 2007a; Cani et al, 2008; Cani et al, 2007c; Hildebrandt et al, 2009; Schneeberger et al, 2015; Shin et al, 2014; Turnbaugh et al, 2008; Turnbaugh et al, 2009b; Zhang et al, 2010). Microbiota composition changes seen in high-fat diet induced obesity may be simply an effect of the lack of fibre in the high-fat diet. Not controlling for the differences in fibre content between the diets this may lead to erroneous conclusions. 


\section{AIMS}

This study proposed to investigate the effects of the refined High-Fat and a matched refined Low-Fat diet compared to a commonly used unrefined mouse Chow diet. The High-Fat (D12492, Research Diets) and Low-Fat (D12450J, Research Diets) diets were the same as used in Chapter 3. The unrefined rodent chow chosen for this study was the Rat and Mouse Breeder and Grower (SDS, Special Diet Services). The chow was chosen due to it being already in use in the Aberdeen Medical Research Facility animal house, and it was closely matched to the refined diets in term of macronutrient ratios. This study design aimed to separate out the influence of the refined nature of both High-Fat and Low-Fat diets on the gut microbiota from the obesity inducing effect of the fat in the High-Fat diet. An additional aim was to examine the obesogenic effect of the LowFat diet compared to chow due the lack of dietary fibre.

\section{HYPOTHESIS}

Changes in the composition of the gut microbiota associated with obesity are driven by differences in diet composition unrelated to the obesogenic state. The refined nature of the obesogenic High-Fat diet drives the development of an obesity associated gut microbiota. A refined Low-Fat control diet will reproduce obesity associated gut microbiota changes in the absence of obesity. 


\section{RESULTS}

\subsection{EFFECT OF CHOW AND REFINED DIETS ON MOUSE BODY COMPOSITION AND METABOLISM}

To assess the effect of high-fat content and refined ingredients on the mouse physiology male C57BL/6J mice were single house and fed either a 60\% fat High-Fat diet, a 10\% fat matched sucrose Low-Fat diet, or Chow for 8 weeks.

\subsubsection{EFFECT OF CHOW AND REFINED DIETS ON BODY WEIGHT}

Mice fed Chow or Low-Fat diet did not significantly differ in their body weight at any point during the eight weeks of the study, see Figure 16A. Relative to the Chow and Low-Fat diets, mice fed the High-Fat diet exhibited a rapid and sustained increase in body weight, see Figure 16A. The weight of High-Fat diet fed mice was significantly heavier than the other two diets after one week and continued to increase for the remainder of the study. The final mean body weight for the High-Fat diet groups was $34.46 \pm 1.18 \mathrm{~g}$, significantly higher $(\mathrm{p}<0.001)$ than the Chow and Low-Fat diet groups at $28.340 .41 \pm \mathrm{g}$, and $27.69 \pm 0.43 \mathrm{~g}$ respectively, see Figure 16B. The final body weight shows a greater spread of body weight within the High-Fat diet group showing considerable variation in response to the High-Fat diet, see Figure 16B. 

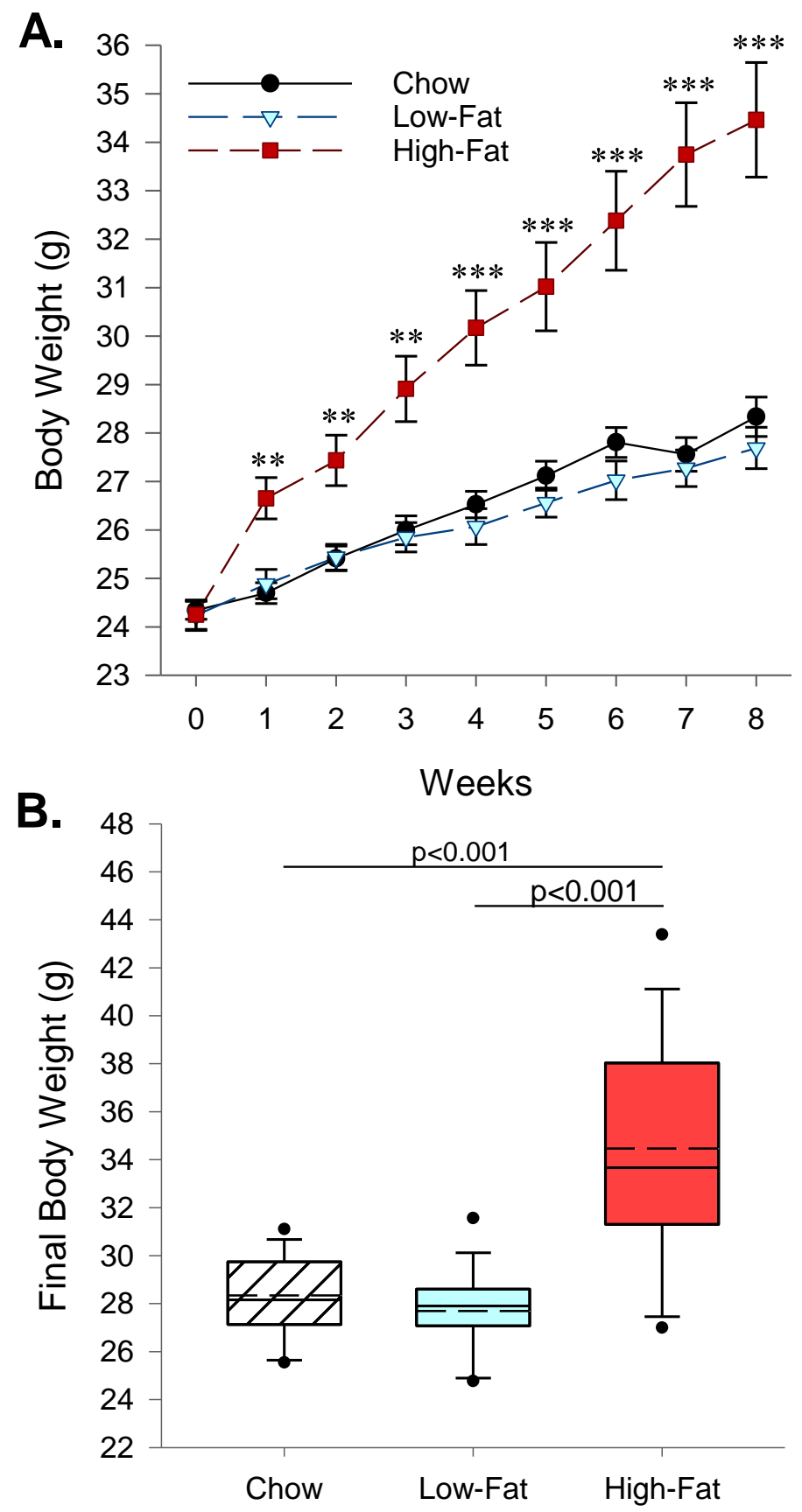

Figure 16. Effect of diet on weekly body weight and final body weight.

Weekly body weights of mice eating Chow, Low-Fat, and High-Fat diets (A). Final week body weights of mice eating Chow, Low-Fat, and High-Fat diets (B). Data represent mean \pm SEM ( $n=16$ per group). For box plots the solid line $=$ median, and dashed line $=$ mean, with outlying points plotted individually. Statistical significance was determined by one-way ANOVA with Tukey's post hoc test and presented as ${ }^{*} \mathrm{p}<0.05$, ${ }^{* *} \mathrm{p}<0.01$, ${ }^{* * *} \mathrm{p}<0.001$, or as stated. 


\subsubsection{EFFECT OF CHOW AND REFINED DIETS ON BODY COMPOSITION 2.1.1.1 BODY FAT MASS}

The body fat mass of the Low-Fat and Chow fed diet groups was equivalent at all weeks of the study, see Figure 17A. Final fat mass of the Low-Fat and Chow fed mice was $3.3 \pm$ $0.2 \mathrm{~g}$ and $3.3 \pm 0.2 \mathrm{~g}$ respectively, see Figure 17B. The fat mass of High-Fat fed mice increased rapidly and was significantly greater $(\mathrm{p}<0.001)$ than the other groups after the first week, see Figure 17A. At the final week, the fat mass of High-Fat diet mice was $10 \pm$ $0.9 \mathrm{~g}$, see Figure 17B.

\subsubsection{LEAN BODY MASS}

The lean body mass in High-Fat, Low-Fat, and Chow fed mice remained equivalent throughout the study, see Figure 17C. No significant differences were seen by the final week, see Figure 17D.

\subsubsection{PERCENTAGE BODY FAT}

The body fat percentage of mice in the Low-Fat and Chow diets groups was equivalent at all weeks of the study, see Figure 18A. The final body fat percentage of Low-Fat fed mice at $11.73 \pm 0.68 \%$ and Chow fed mice $11.44 \pm 0.49 \%$ was not different, see Figure 18B. The percentage body fat of the High-Fat diet fed mice rapidly increased from the first week and increased further during the study. High-Fat mice were significantly fatter than both Chow $(\mathrm{p}<0.001)$ and Low-fat $(\mathrm{p}<0.001)$ fed mice by week one, see Figure $18 \mathrm{~B}$. The final body fat percentage of High-Fat fed mice was $28.11 \pm 1.79 \%$.

\subsubsection{PERCENTAGE LEAN BODY MASS}

The lean body mass percentage of the Low-Fat and Chow fed diet groups was equivalent at all weeks of the study, see Figure 18C. The final lean body mass of Low-Fat and Chow fed mice was $82.0 \pm 0.8 \%$ and $81.8 \pm 0.6 \%$, see Figure 18D. A significant decrease $(\mathrm{p}<0.001)$ was seen in the lean mass percentage of High-Fat diet mice by week 1 , see Figure 18C. The final lean mass percentage of High-Fat mice was significantly reduced $(\mathrm{p}<0.001)$ at $66.7 \pm 1.7 \%$ compared to the other groups, see Figure $18 \mathrm{D}$. 

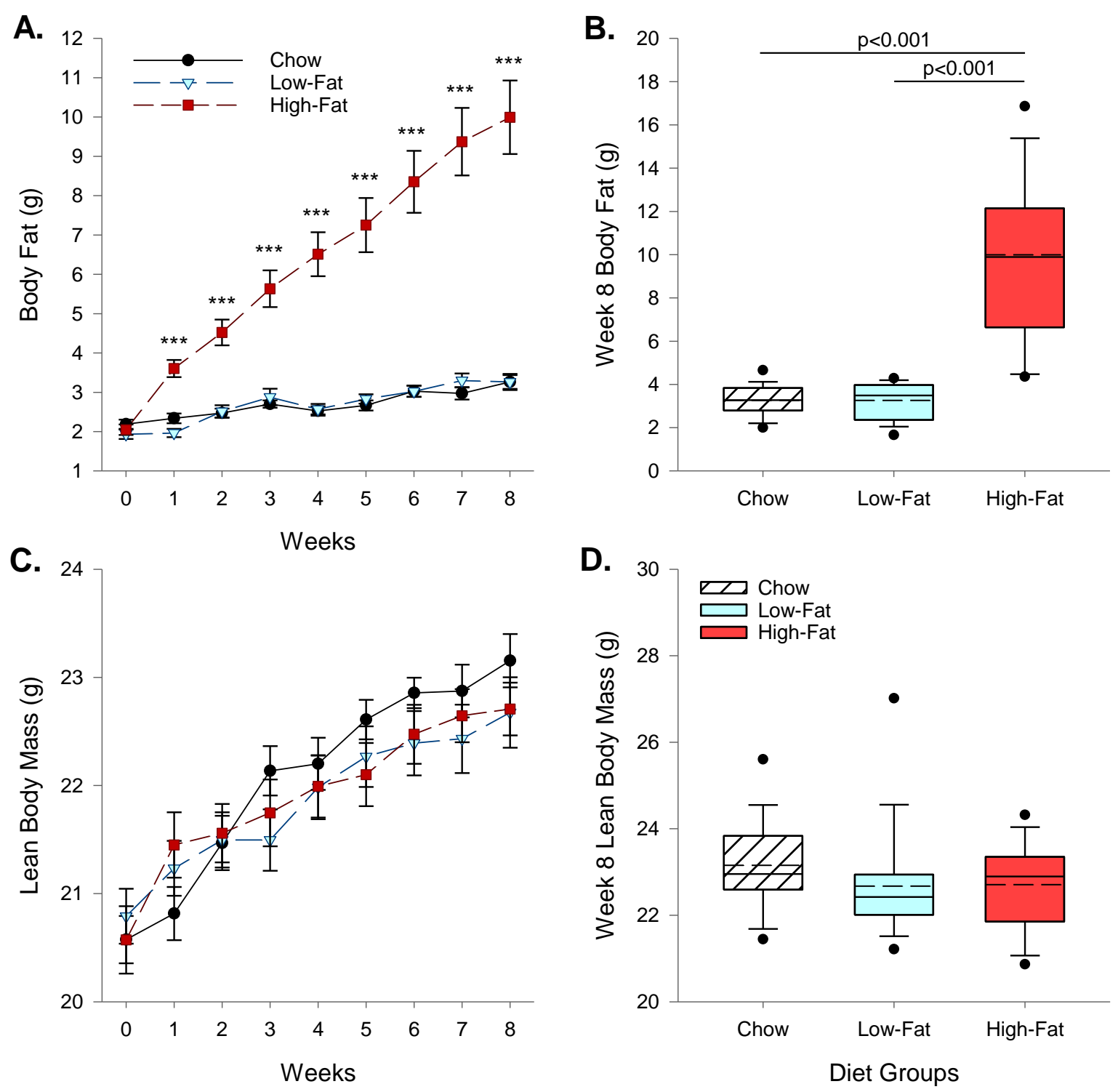

Figure 17. Effect of diet on body fat mass and lean body mass.

Weekly body fat mass of mice fed Chow, Low-Fat, and High-Fat diets (A), and body fat mass at the final week (B). Weekly lean body mass of mice eating Chow, Low-Fat, and High-Fat diets (C), and lean body mass at the final week (D). Data are presented as mean \pm SEM ( $n=16$ per group). For box plots the solid line $=$ median, and dashed line $=$ mean, with outlying points plotted individually. Statistical significance was determined by oneway ANOVA with Tukey's post hoc test and presented as ${ }^{*} \mathrm{p}<0.05,{ }^{* *} \mathrm{p}<0.01,{ }^{* * *} \mathrm{p}<0.001$, or as stated. 

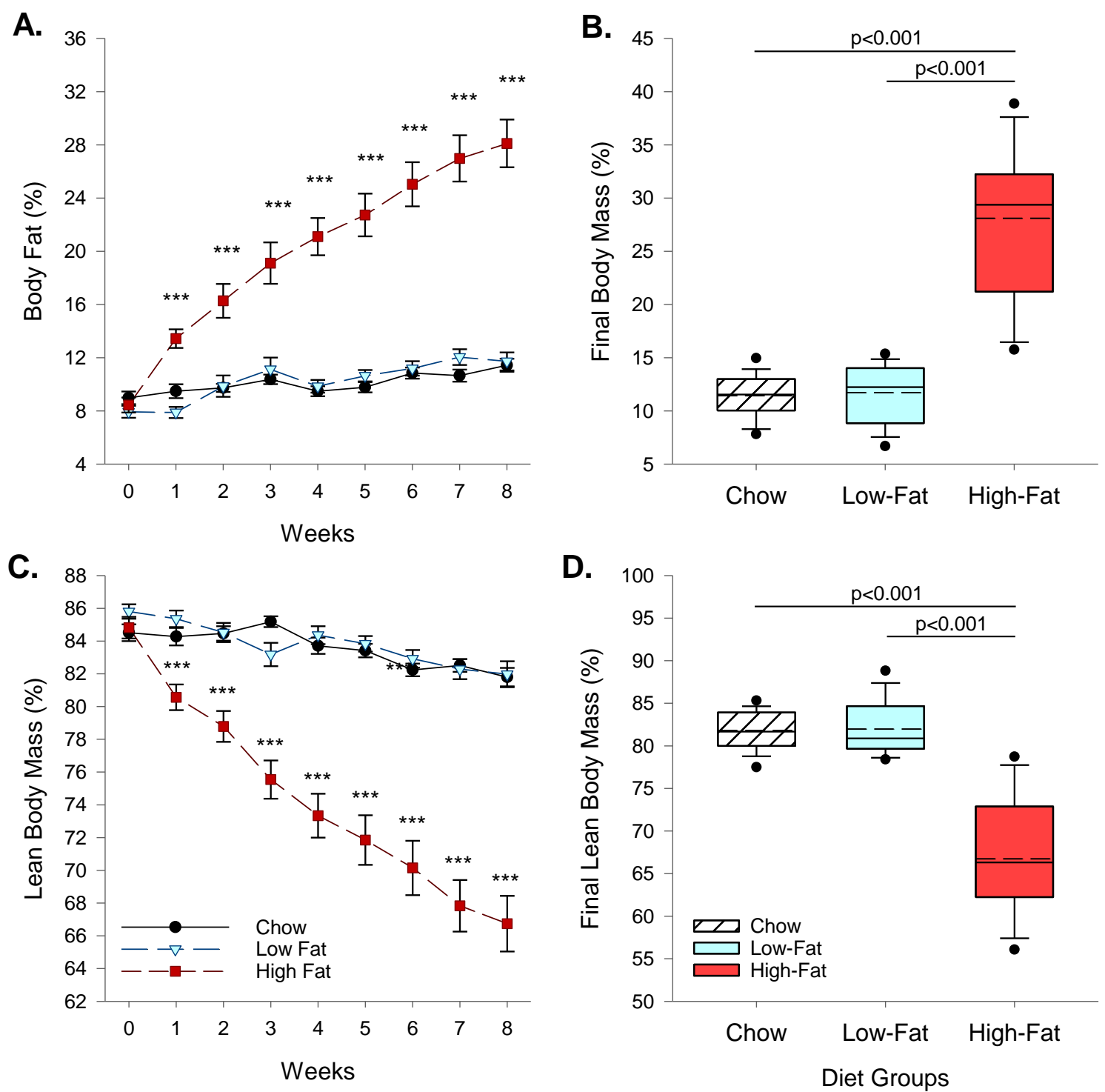

Figure 18. Effect of diet on body fat and percentage lean body mass.

Weekly body fat percentage of mice fed Chow, Low-Fat, and High-Fat diets (A), and body fat percentage at the final week (B). Weekly lean body mass percentage of mice fed Chow, Low-Fat, and High-Fat diets (C), and lean body mass percentage at the final week (D). Data are presented as mean \pm SEM ( $n=16$ per group). For box plots the solid line $=$ median, and dashed line = mean, with outlying points plotted individually. Statistical significance was determined by one-way ANOVA with Tukey's post hoc test and presented as ${ }^{*} \mathrm{p}<0.05,{ }^{* *} \mathrm{p}<0.01,{ }^{* * *} \mathrm{p}<0.001$, or as stated. 


\subsubsection{EFFECT OF CHOW AND REFINED DIETS ON FOOD AND ENERGY INTAKE}

\subsubsection{FOOD INTAKE}

Food intake was significantly reduced and energy intake significantly increased in mice fed Low-Fat and High-Fat refined diets in comparison to mice fed the Chow diet. Food intake began to diverge immediately between the three diet groups, see Figure 19A and Figure 20A. The High-Fat diet decreased food intake and this remained consistently reduced. The Low-fat diet initially resulted in fluctuating food intake for the first 3 weeks which then evened out at an intermediate intake between the Chow and High-Fat groups, see Figure 19A. Weekly food intakes remained constant from the start in the Chow group. Mean weekly food intake (grams/week) was significantly different $(\mathrm{p}<0.001)$ between the three diets groups at $26.9 \pm 0.3 \mathrm{~g}, 22.7 \pm 0.5 \mathrm{~g}$, and $19.3 \pm 0.4 \mathrm{~g}$ for the Chow, Low-Fat, and High-Fat diet groups respectively, see Figure 19C.

\subsubsection{ENERGY INTAKE}

Despite their reduced food intake the High-Fat diet group mice consumed more energy from the first week and had a higher mean weekly intake and total energy intake, see Figure 19D and Figure 20D. This was due to the higher energy density of the High-Fat diet. The Low-Fat diet group mice energy intake was significantly different from both other diet groups presenting as an intermediate energy intake between the two other diets. The Low-Fat diet contained a higher energy density than the Chow which was compensated for by a reduced food intake. 

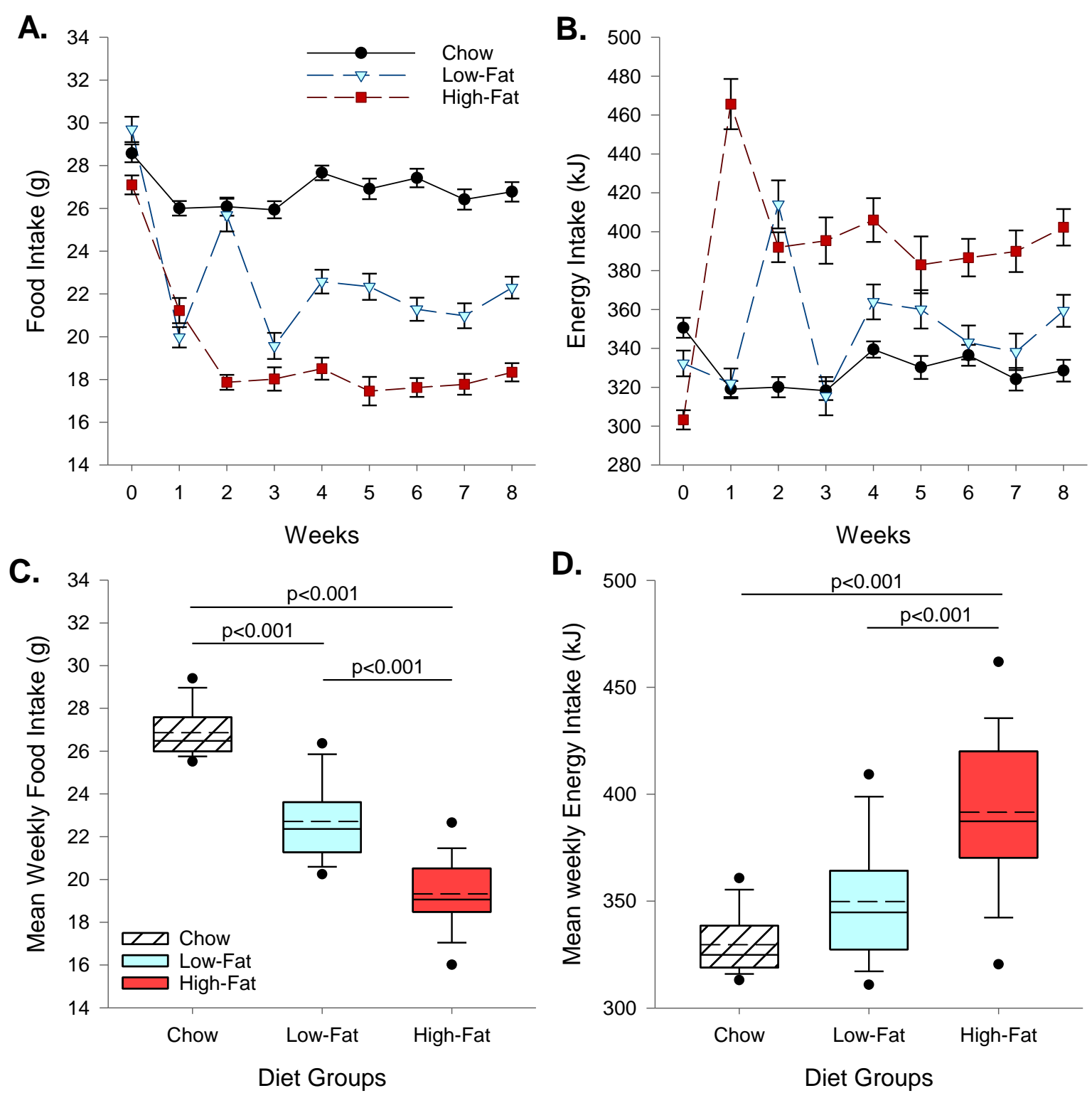

Figure 19. Effect of diet on weekly and mean weekly food intake and energy intake.

Weekly food intake (A) and energy intake (B) for Chow, Low-Fat, and High-Fat diet fed mice. Mean weekly food intake (D) and energy intake (C) averaged across the 8 weeks of the study for Chow, Low-Fat, and High-Fat diet fed mice. Low-Fat and High-Fat diet energy content determined by the Atwater system. Chow diet energy content calculated as digestible energy. Data are presented as mean \pm SEM ( $n=16$ per group). For box plots the solid line $=$ median, and dashed line $=$ mean, with outlying points plotted individually. Statistical significance was determined by one-way ANOVA with Tukey's post hoc test. 

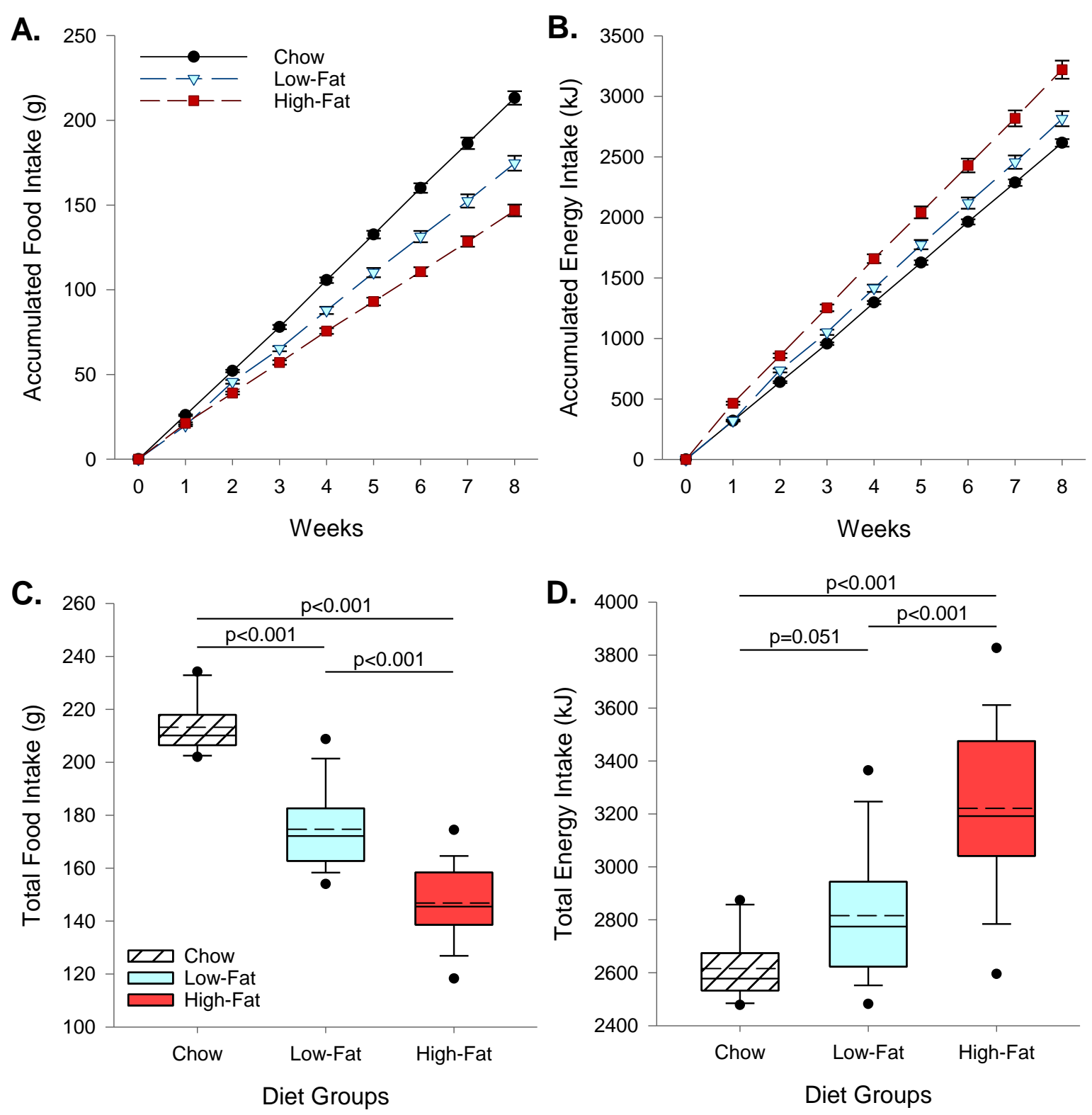

Figure 20. Effect of diet on accumulated and total food and energy intake.

Accumulated food intake (A) and accumulated energy intake (B) in Chow, Low-Fat, and High-Fat diet fed mice. Total food intake (D) and total energy intake (C) in Chow, LowFat, and High-Fat diet fed mice. Low-Fat and High-Fat diet energy content determined by the Atwater system. Chow diet energy content calculated as digestible energy. Data are presented as mean \pm SEM ( $n=16$ per group). For box plots the solid line $=$ median, and dashed line $=$ mean, with outlying points plotted individually. Statistical significance was determined by one-way ANOVA with Tukey's post hoc test. 


\subsubsection{REFINED LOW-FAT DIET DOES NOT INDUCE GLUCOSE INTOLERANCE}

In the present study mice fed the refined high-fat diet for 8 weeks had a significantly higher blood glucose level after 5 hours fasting, while there was no difference between low-fat and chow mice, Figure 21A. The intraperitoneal glucose tolerance test High-Fat fed mice showed a significantly greater rise in blood glucose, double that of mice in the refined low-fat and chow fed mice. Blood glucose in the High-Fat diet mice remained higher than the refined low-fat and chow fed mice throughout the 2 hours after glucose administration. There was no difference in the blood glucose concentration between mice refined low-fat diet and those fed the chow diet. The area under the curve of blood glucose response showed the same result, Figure 21B. There was no statistically significant difference between refined low-fat diet fed mice and chow fed mice. 

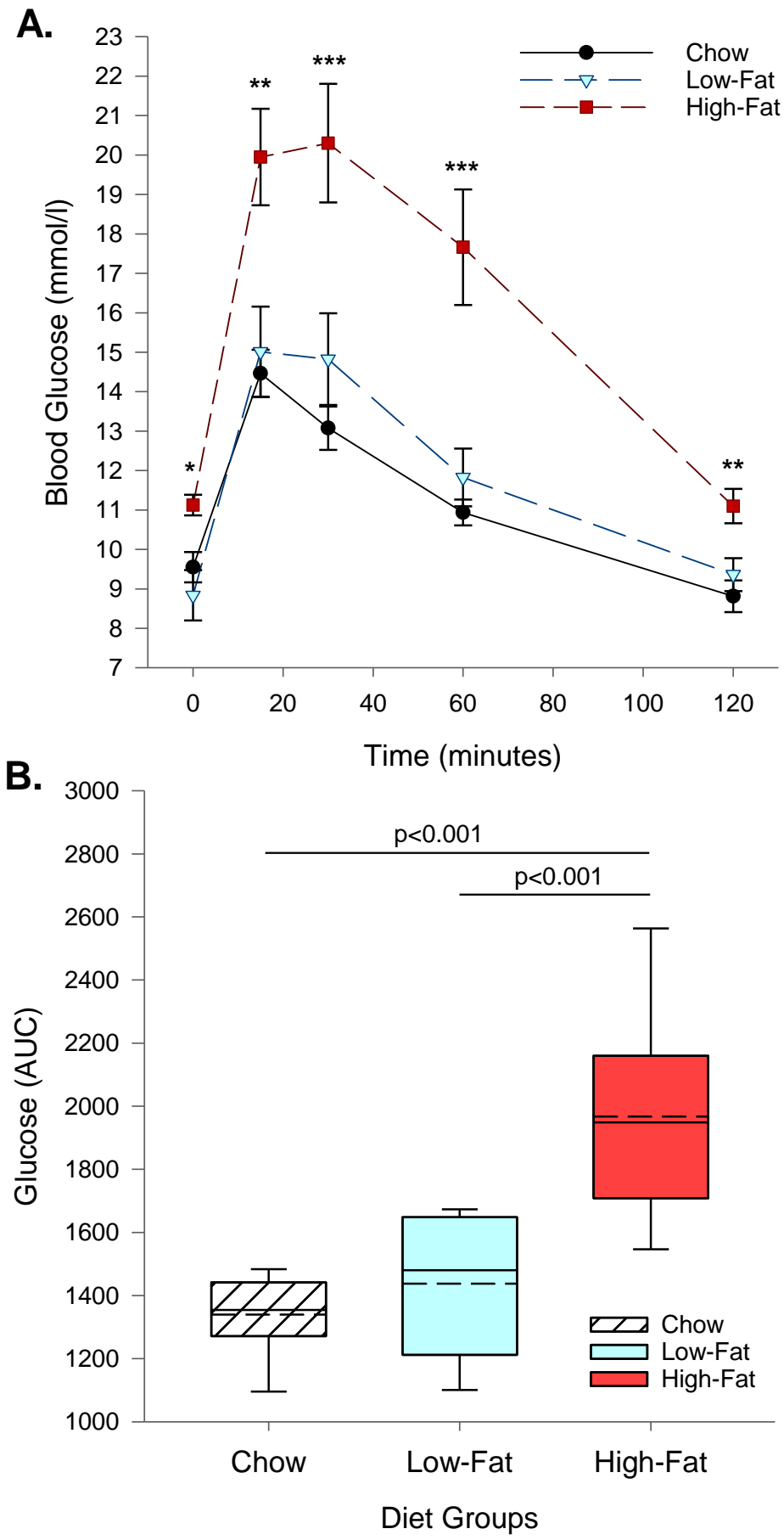

Figure 21. Effect of diet on intraperitoneal glucose tolerance test response.

Glucose response after intraperitoneal glucose tolerance test (IPGTT) different in Chow, Low-Fat, and High-Fat diet fed mice (A). Calculated area under the curve (AUC) of glucose response over 120 minutes after IPGTT in Chow, Low-Fat, and High-Fat diet fed mice (B). Data are presented as mean \pm SEM ( $n=8$ per group). For box plots the solid line $=$ median, and dashed line $=$ mean. Statistical significance was determined by oneway ANOVA with Tukey's post hoc test. ${ }^{*}=\mathrm{p}<0.05,{ }^{* *}=\mathrm{p}<0.01,{ }^{* * *}=\mathrm{p}<0.001$, or as stated. 


\subsection{EFFECT OF CHOW AND REFINED DIETS ON INTESTINAL SIZE}

\subsubsection{REFINED DIETS REDUCE INTESTINAL LENGTH}

The mean length of the gastrointestinal tract measured from the start of the duodenum to the rectum was of $45.3 \mathrm{~cm}$ in the chow fed mice and significantly shorter at $39.3 \mathrm{~cm}$ and $41.3 \mathrm{~cm}$ in refined low-fat and high-fat mice respectively, Figure 22A. Relative to the small intestine of chow fed mice (mean length of $37.8 \mathrm{~cm}$ ) those of refined low-fat and high-fat mice were each significantly shorter being 33.2 and $34.7 \mathrm{~cm}$ in respectively, Figure 22B. Similarly, the caecum lengths of mice fed both refined Low-Fat and HighFat diets were significantly shorter than chow fed mice $(1.8 \mathrm{~cm}$ cf. $2.9 \mathrm{~cm})$, Figure 22C. Colon length was also significantly reduced from $7.6 \mathrm{~cm}$ to 6.1 and $6.6 \mathrm{~cm}$ in Low-Fat and High-Fat diet mice relative to chow fed mice, refined diets during the study.

Figure 22. Effect of diet on total, small intestine, caecum, and colon length.D.

\subsubsection{EFFECTS OF CHOW AND REFINED DIETS ON INTESTINAL MASS}

Both refined Low-Fat and High-Fat diets reduces intestinal mass to a similar degree,

Figure 23. Mice fed refined diets experienced a reduction in the weight of intestinal tissue relative to the chow fed mice. This effect was evident in intestines weighed when full of contents, Figure 23A, C, D. This shows that the chow diet was bulkier and more fibrous resulting in the intestinal tract containing a greater luminal content. Small intestine weight was not significantly different between groups, Figure 23A. This became significantly different when empty, Figure 23B. Differences in weight of the caecum and colon between Chow and the High-Fat and Low-Fat groups were highly significant $(\mathrm{P}<0.001)$ when weighted full or empty, Figure 23C-F. The mice all began eating the Chow diet before introduction of the refined experimental diets. The greater intestinal mass of chow fed mice when empty of contents indicates that the tissue of the gut had reduced in length and gut tissue in the mice fed refined diets during the study. 

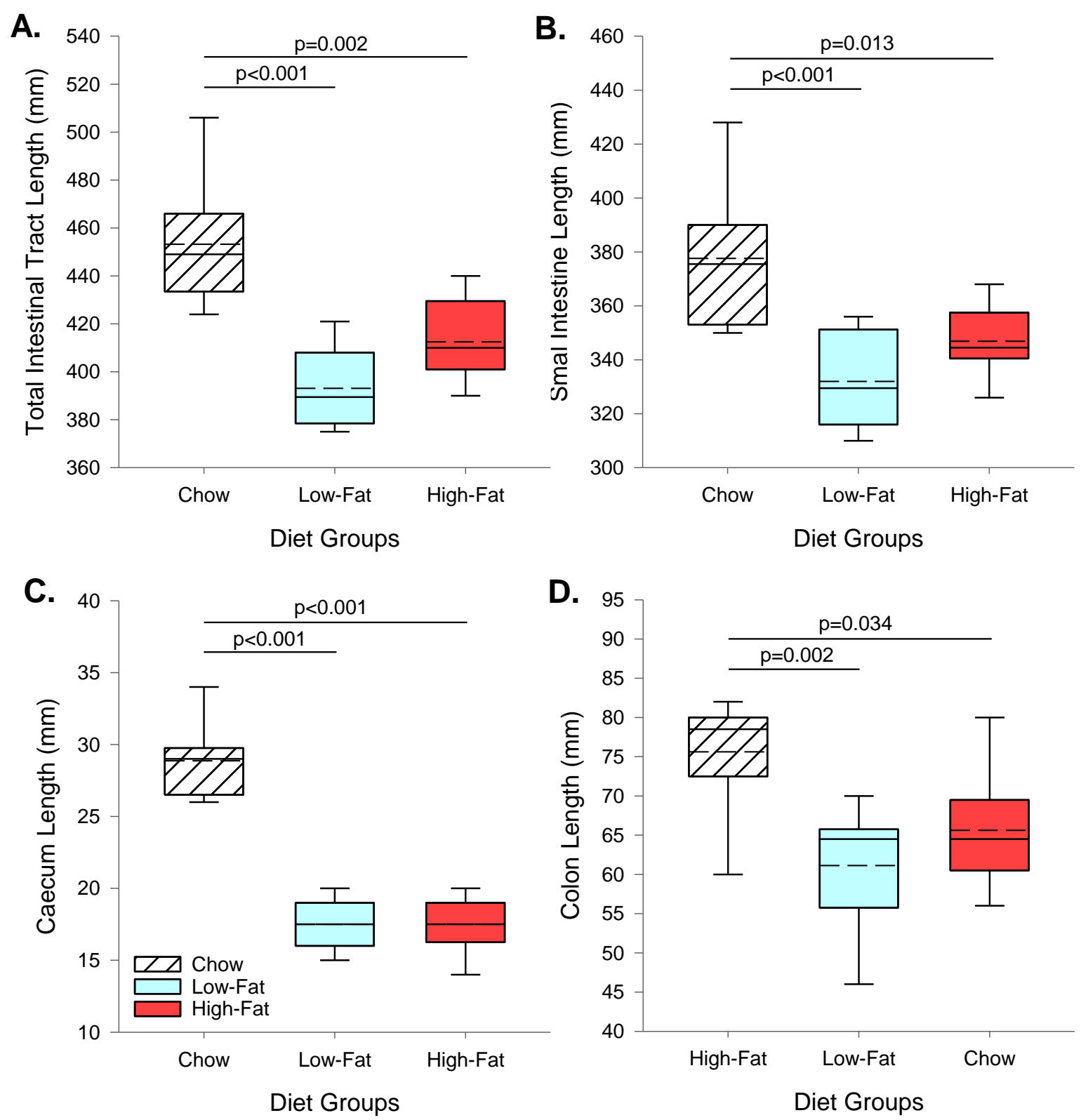

Figure 22. Effect of diet on total, small intestine, caecum, and colon length.

Total intestine length (A), small intestine length (B), caecum length (C), and colon length (D), in Chow, Low-Fat, and High-Fat diet fed mice. For box plots the solid line = median, and dashed line $=$ mean. Statistical significance was determined by one-way ANOVA with Tukey's post hoc test. $\mathrm{n}=8$ per group. 
A.

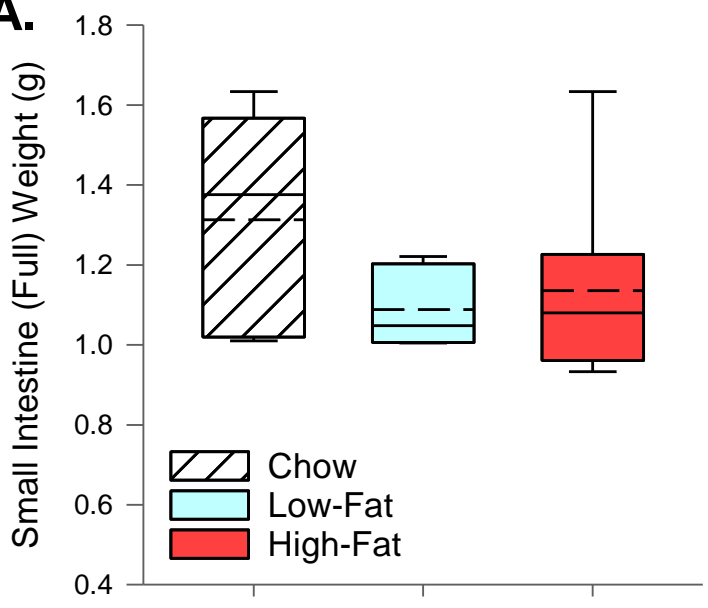

C. 0.9

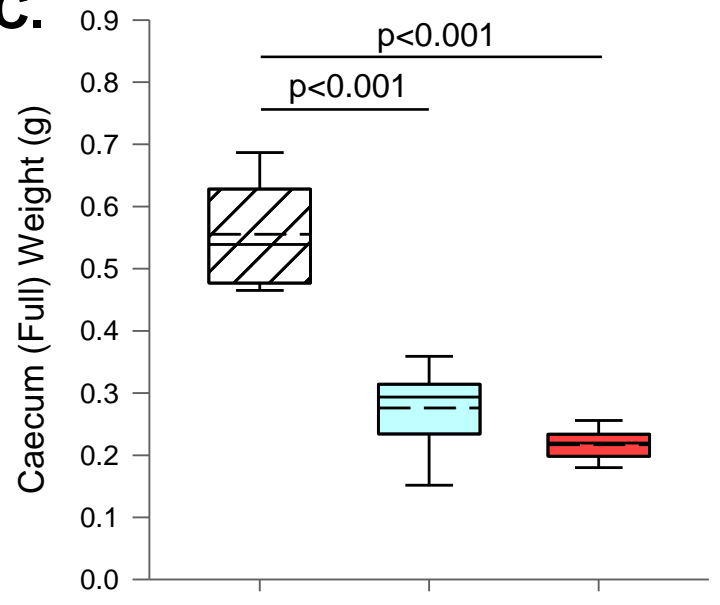

E.

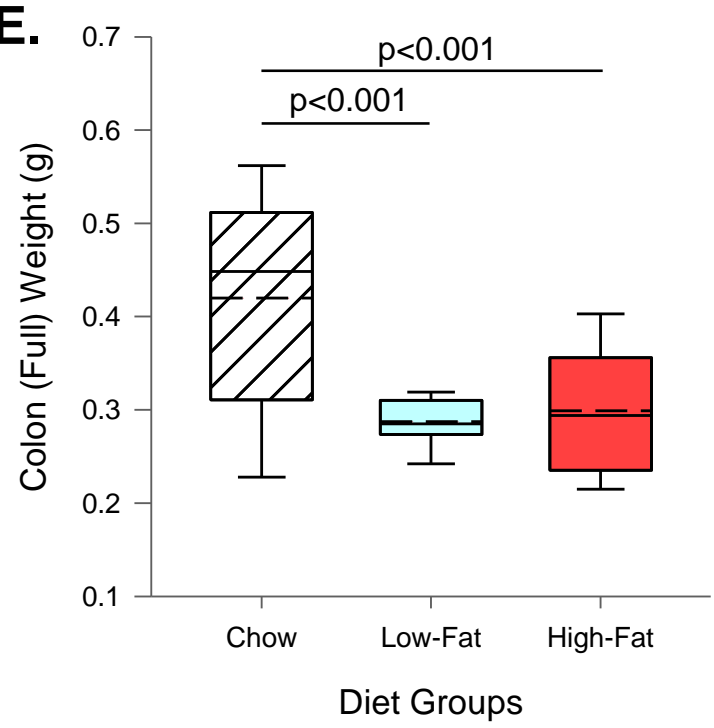

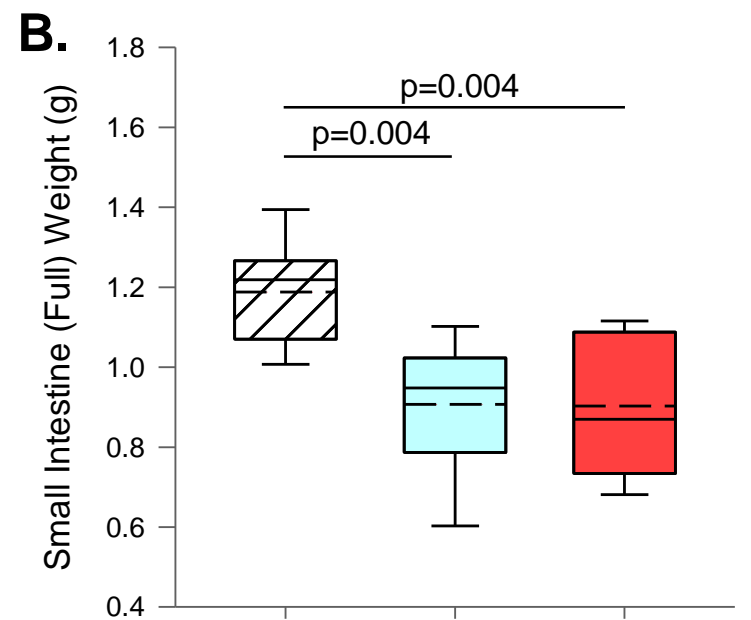

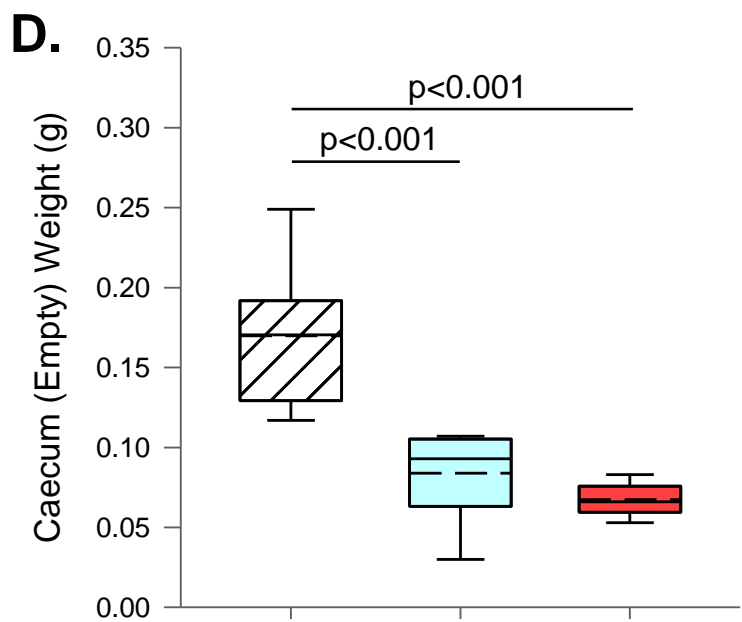

F.

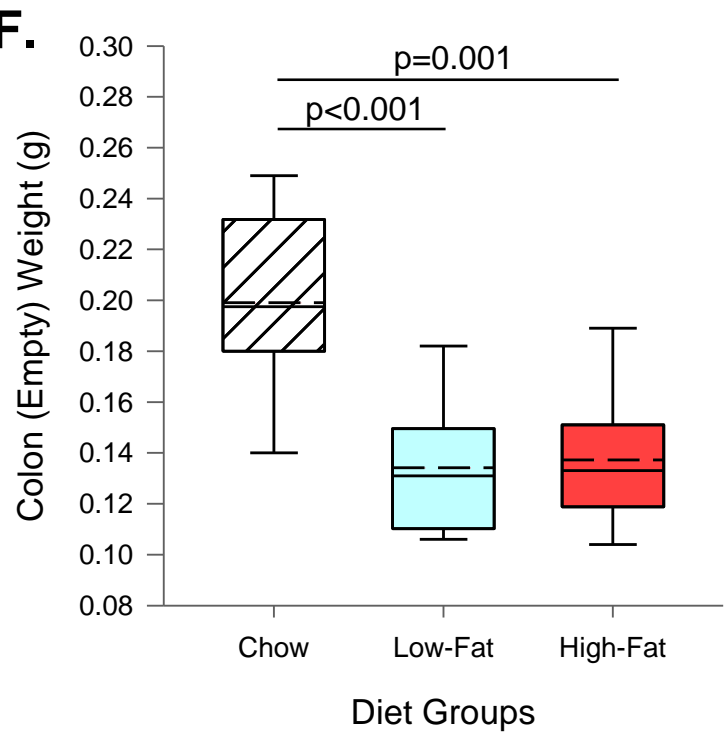

Figure 23. Effects of diet small intestine, caecum, and colon weight.

Weight of small intestine containing luminal contents (A), and when empty (B). Weight of caecum containing luminal contents (C), and when empty (D). Weight of the colon when containing luminal contents (E), and when empty (F). For box plots the solid line = median, and dashed line $=$ mean. Statistical significance was determined by one-way ANOVA with Tukey's post hoc test. $\mathrm{n}=8$ mice per group. 


\subsubsection{GROSS MORPHOLOGICAL EFFECTS OF DIET ON THE CAECUM AND COLON}

The caecum and colon of mice fed refined Low-Fat or High-Fat diets are visibly shorter and smaller than mice fed Chow diet. These photographed examples are representative of the caecum and colon of mice in each group. The differences in gross morphology are clearly visibly noticeable in the photographs of the mouse gut, see Figure 24 . The caecum is visibly longer and fuller of contents in the Chow fed mouse. In contrast the caecum of the Low-Fat or High-Fat diet fed mice appears visibly shrunken and atrophied.

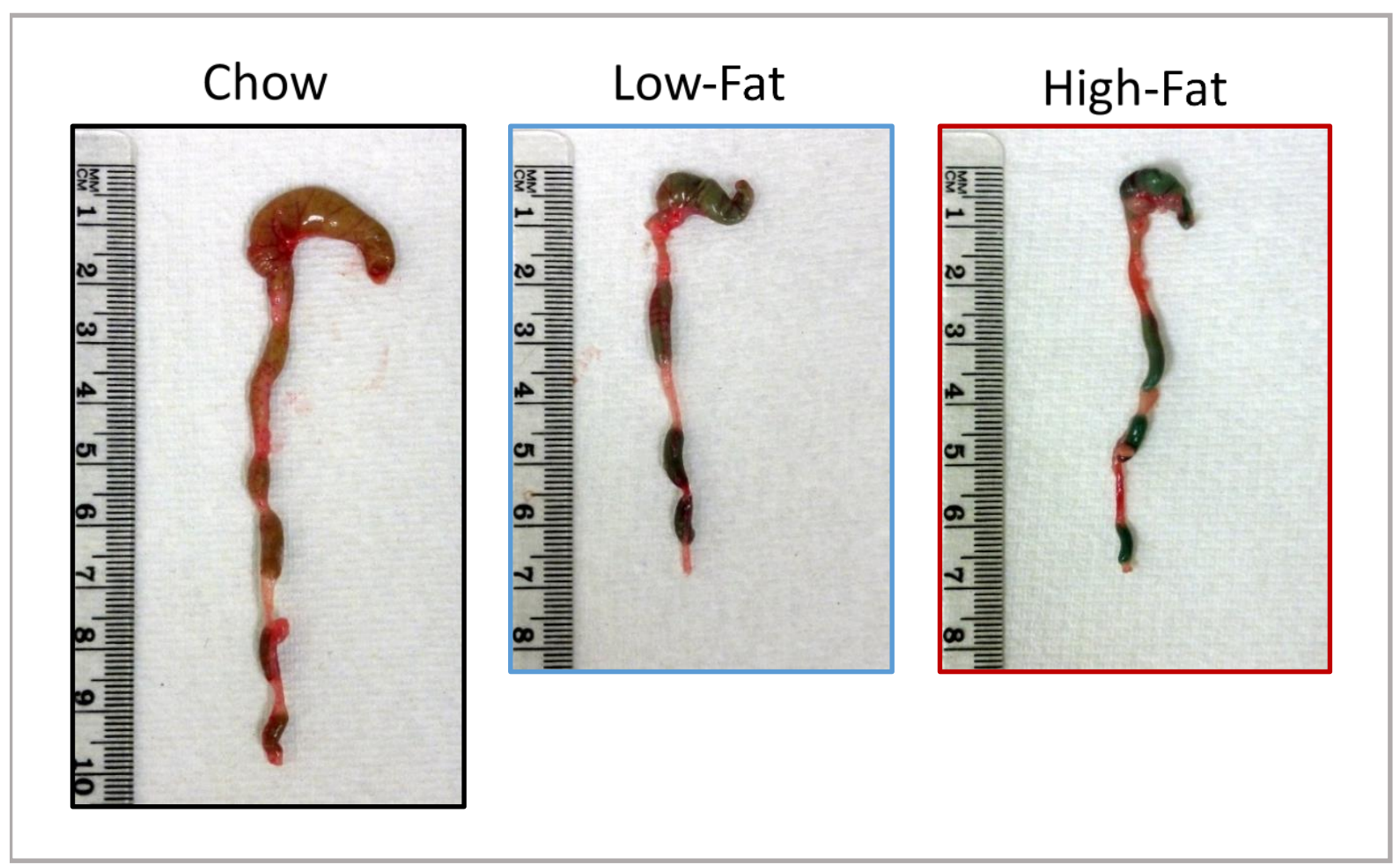

Figure 24. Effects of diet on caecum and colon morphology.

Representative photographs of the caecum and colon of Chow, Low-Fat, and High-Fat diet fed mice. A centimetre ruler is included in the photograph for scale and the scales are adjusted to be the same size in each image for comparison. 


\subsubsection{EFFECT OF DIET ON LIVER WEIGHT}

There was no difference in the weight of the liver between mice in the three groups, Figure 25.

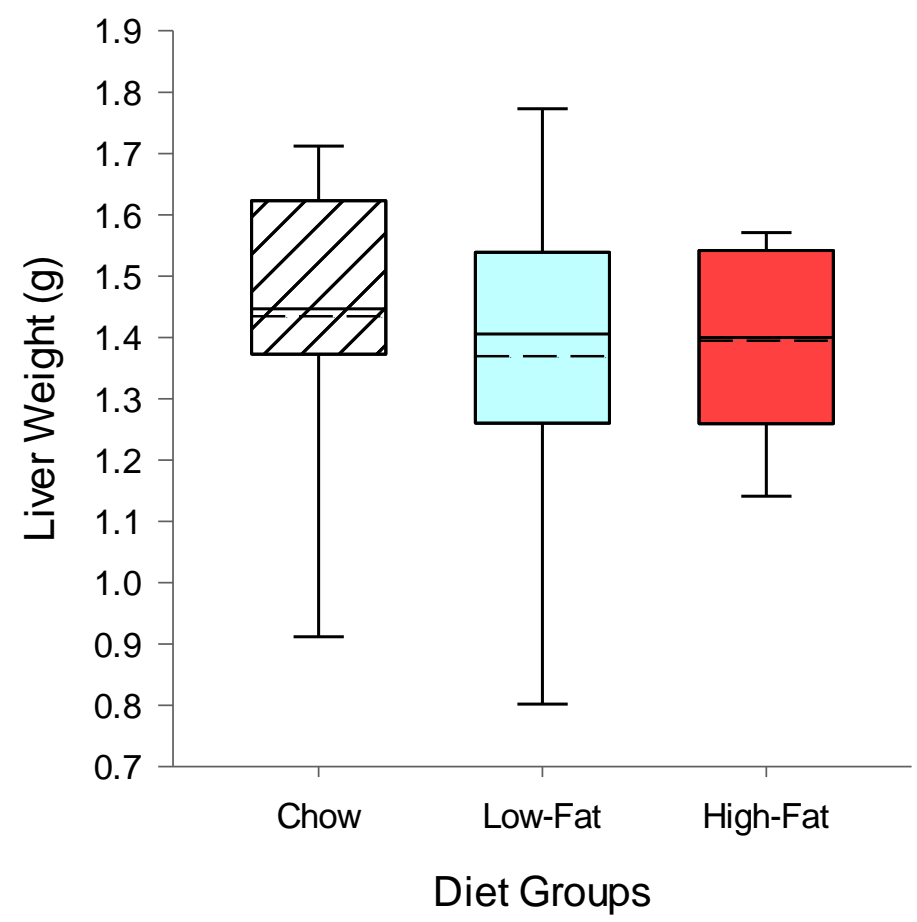

Figure 25. Effects of diet on liver weight.

Liver weight in Chow, Low-Fat, and High-Fat diet fed mice. For box plots the solid line $=$ median, and dashed line $=$ mean . Statistical significance was determined by one-way ANOVA with Tukey's post hoc test. $\mathrm{n}=8$ mice per group. 


\subsection{MICROBIOTA CHANGES IN RESPONSE TO DIETS}

The luminal gut contents were sampled from the terminal ileum, caecum and colon at the end of the study and faecal pellets collected at week 0 , week 1 and week 8 . Ilumina Miseq Next Generation sequencing was used to determine the composition of the microbiota through amplification of the V1-V2 regions of the 16S ribosomal RNA. Sequences were subsampled and capped at 10,596 sequences per sample. The composition between these sampling locations in the mouse gut and faeces was compared in the mice between the Chow diet, Low-Fat diet, and High-Fat diet.

\subsubsection{MICROBIOTA FROM CHOW FED MICE CLUSTER DISTINCTLY FROM OTHER GROUPS}

The overall similarity of the microbiota from all intestinal locations and diet groups was initially compared using two-dimensional non-metric multidimensional scaling. The microbiota of mice maintained on the chow diet clustered separately from mice fed High-Fat of Low-Fat refined diets when compared using a two dimensional NMDS ordination plot, Figure 26. The dissimilarity of the microbiota from all mice and anatomical sites was calculated using the Bray-Curtis calculator to generate an index describing the dissimilarity between the bacterial communities. This took into account the composition and abundance of all the individual OTUs in each mouse sample. The High-Fat and Low-Fat diet groups clustered more closely together with some overlap between groups for individual mice. Both groups clustered separately from the mice in the chow group at all anatomical sites of the intestine tested. The mean difference between calculated microbiota similarity was significantly different $(\mathrm{p}<0.001)$ between diet groups as determined by AMOVA, Figure 26. The variance between diet groups was not significantly different as assessed by HOMOVA, Figure 26. The clustering of data points on the NMDS plot between of the ileum, compared to the caecum, colon, and faeces within each of the diet groups indicated that the composition of the ileum microbiota was more different from that of lower intestine. The R-squared value of 0.3 , describing the amount of variation in the data set explained by the groupings, was high indicated a reduced reliability of this clustering and so any implications must be treated with caution. Overall, the Chow diet clustered distinctly as a group from the refined Low-Fat and High-Fat diets. 


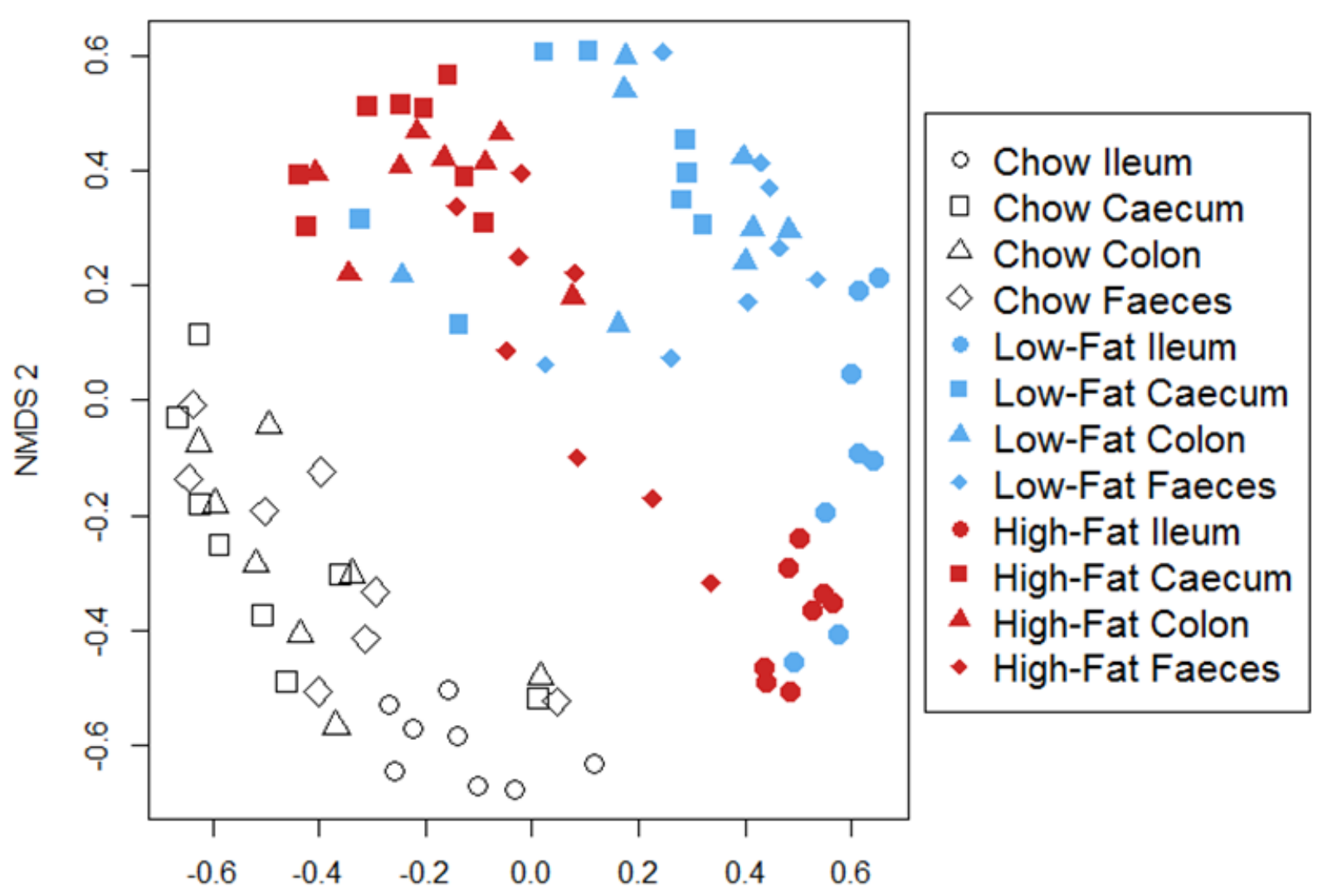

NMDS 1

\begin{tabular}{lcc} 
Significance Tests & AMOVA & HOMOVA \\
\hline Comparison & P-value & P-value \\
\hline Chow vs High-Fat & $\mathrm{P}<0.001$ & $\mathrm{~ns}$ \\
Chow vs Low-Fat & $\mathrm{P}<0.001$ & $\mathrm{~ns}$ \\
High-Fat vs Low-Fat & $\mathrm{P}<0.001$ & $\mathrm{~ns}$ \\
\hline 1. AMOVA (Nonparametric Analysis of molecular variance) \\
$\begin{array}{l}\text { 2. HOMOVA (Nonparametric Homogeneity of molecular variance) } \\
\text { ns = not significant }\end{array}$
\end{tabular}

Figure 26. Non-metric multidimensional scaling (NMDS) plot of mouse luminal gut and faecal microbiota.

NMDS plot shows a separation between microbiota of Chow (BLANK) mice and LowFat (BLUE) and High-Fat (RED) mice in the ileum $(\boldsymbol{O})$, caecum $(\boldsymbol{\square})$, colon $(\boldsymbol{\Delta})$, and faeces $(\diamond)$. Each of the points on the NMDS plot represents the bacterial microbiota from a single sample from a single mouse. Taxonomic profiles (OTU level) of samples were used to calculate a dissimilarity matrix (Bray-Curtis calculator) and this matrix was used to compute an NMDS plot in two dimensions (NMDS 1 and NMDS 2). The stress of this ordination was 0.305 and the R2 was 0.647 . Statistical significance of clustering patters between diets for each sample (ileum, caecum, colon, and faeces) were calculated by AMOVA and HOMOVA. $\mathrm{n}=8$ mice per diet group. 

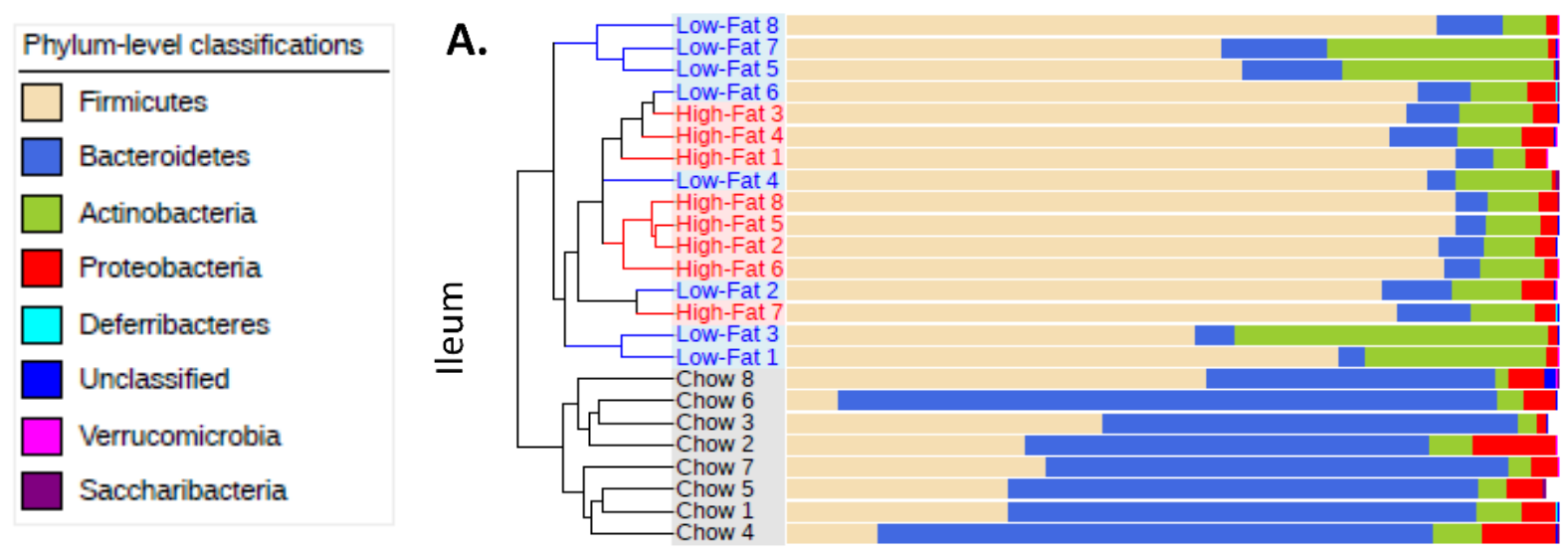

Parsimony Significance Tests

\begin{tabular}{lc}
\multicolumn{2}{c}{ lleal Microbiota } \\
\hline Comparison & P-value \\
\hline Chow vs High-Fat & $<0.0001$ \\
Chow vs Low-Fat & $<0.0001$ \\
High-Fat vs Low-Fat & 0.1279 \\
\hline & \\
\multicolumn{2}{c}{ Caecal Microbiota } \\
\hline Comparison & P-value \\
\hline Chow vs High-Fat & $<0.0001$ \\
Chow vs Low-Fat & $<0.0001$ \\
High-Fat vs Low-Fat & 0.0015 \\
\hline
\end{tabular}

B.

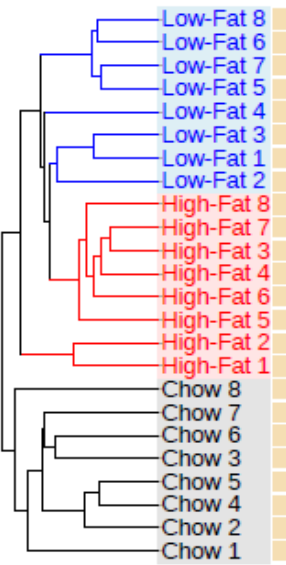

C.

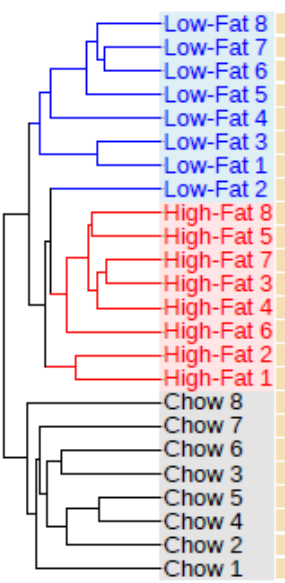

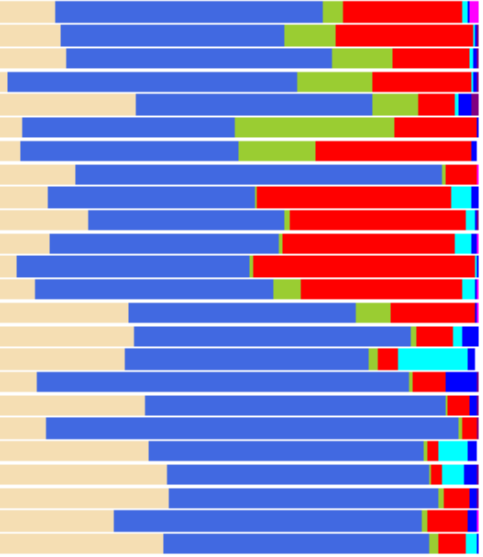

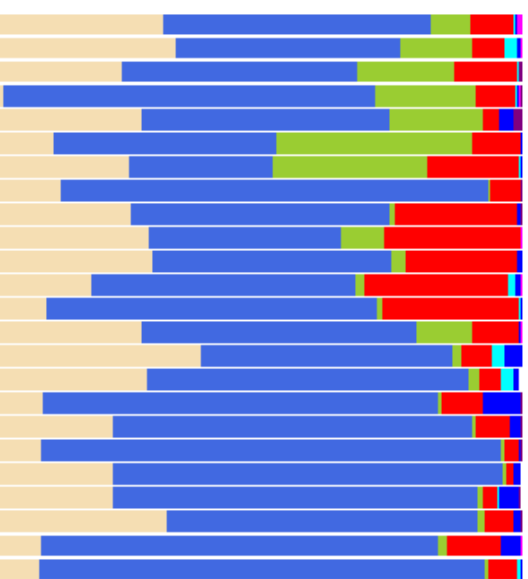

Figure 27. Effect of diet on similarity and phylum composition of the microbiota.

Similarity between individual mouse microbiota in the Ileum (A), the caecum (B), and the colon (C), in Chow, Low-Fat, and High-Fat diet fed mice. Each stacked bar indicates the proportional abundance of the bacterial phyla in the microbiota of a single mouse. Each bacterial phyla is represented as Firmicutes (BROWN), Bacteroidetes (BLUE), Actinobacteria (GREEN), and Proteobacteria (RED). The dendrograms represent clustering of samples based on the Bray-Curtis dissimilarity matrix of OTUs sharing 97\% identity. The Bray Curtis index was used to generate dendrograms trees in the Mothur package. Dendrograms and phylum level proportions were visualised using ITOL. Significance between groups was determined using the Parsimony function in the Mothur package. $\mathrm{n}=8$ mice per diet group. 


\subsubsection{REFINED DIETS ALTER PHYLUM LEVEL COMPOSITION}

Presented as a dendrograms representing a Bray-Curtis dissimilarity index the microbiota of the Chow fed mice clustered together as a distinct group from the LowFat and High-Fat diet fed mice in the ileum, caecum, and colon, Figure 27. Differences in bacterial composition at the phylum level were particularly pronounced in the ileum. The ileum microbiota of Low-Fat and High-Fat diet mice was not significantly different. The difference in the Chow diet group from both Low-Fat and High-Fat groups was highly significant $(<0.0001)$ as calculated by the Parsimony method, Figure 27A. The mouse caecal and colonic microbiota clustered separately within each diet group. This clustering was statistically significant as calculated by the Parsimony method, Figure 27B-C. High-fat and Low-Fat mice clustered into distinct groups both closer to one another than the Chow group. The proportional abundance of the bacteria phyla present was represented as stacked bars. The ileum of chow fed mice was dominated by members of the Bacteroidetes phylum, Figure 27A. Firmicutes and Bacteroidetes dominated in the Chow mice in the caecum and colon, see Figure 27B-C.

The LEfSe (linear discriminant analysis (LDA) effect size) method was used to identify bacterial phyla in the sequence data that differentiated the different diet groups with $>2$ as a cut-off (Segata et al, 2011), Figure 28. In the ileum Bacteroidetes and Proteobacteria differentiated the Chow group, while Firmicutes and Actinobacteria differentiated the Low-Fat and High-Fat groups. Metastats confirmed the Firmicutes and Actinobacteria were not significantly different between Low-Fat and High-Fat groups. In the caecum and colon Bacteroidetes differentiated the Chow group and respectively. Actinobacteria differentiated the Low-Fat group and respectively. Proteobacteria differentiated the High-Fat group and respectively. Metastats statistical analysis determined that the Proteobacteria phylum abundance was not significantly different between Low-Fat and High-Fat groups. LEfSe analysis confirms the changes in microbiota phylum level composition between groups seen in Figure 27. These high LDA values indicate that the composition changes between groups were of a large magnitude. 


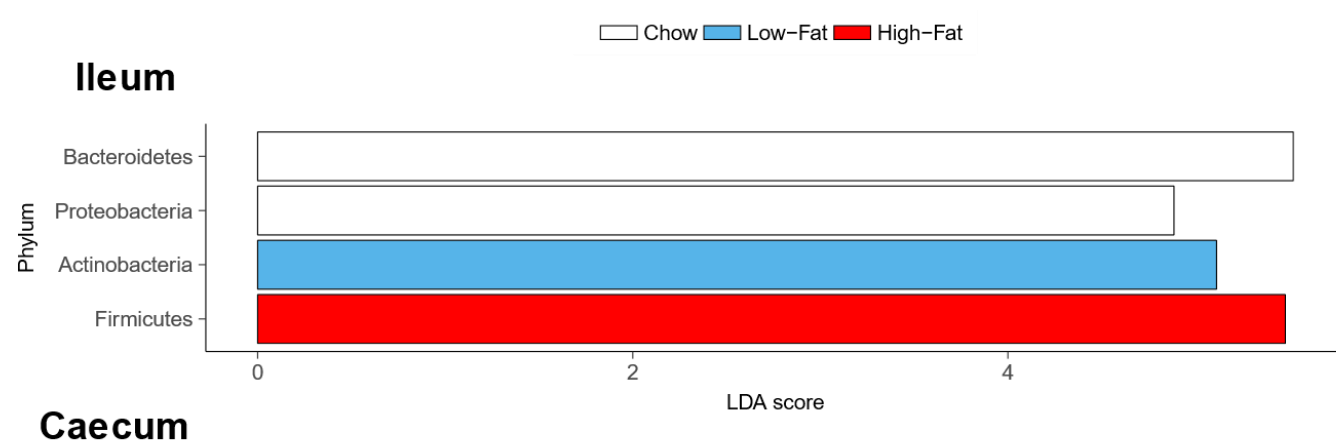

\section{Caecum}

LDA score

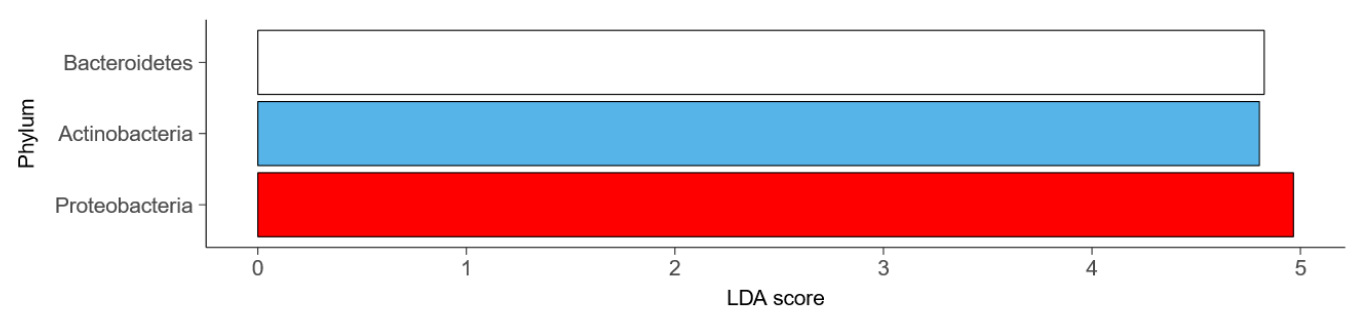

Colon

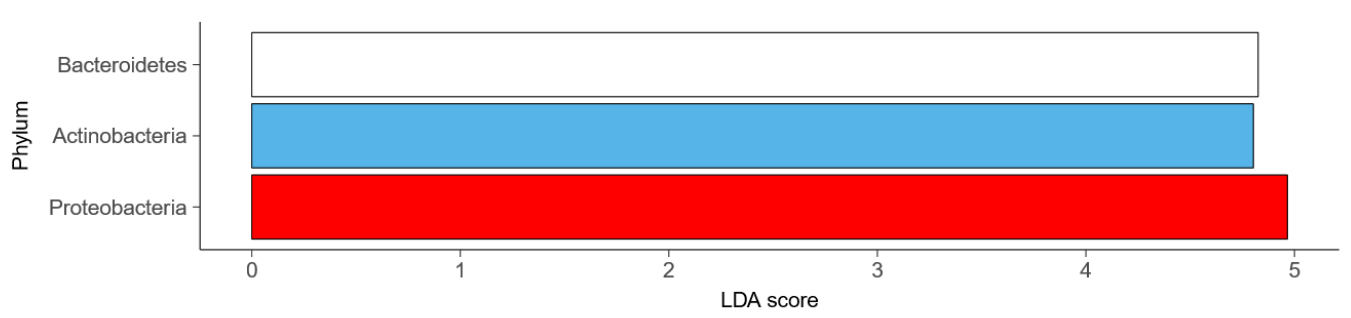

\section{Faeces Week 8}

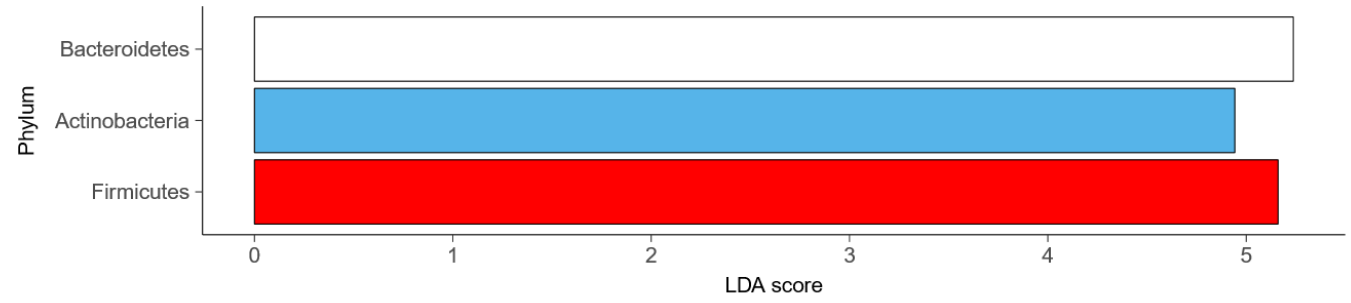

Figure 28. Bacterial phyla differentially expressed by diet group.

Bacterial phyla differentially expressed in the Chow, Low-Fat, and High-Fat diet groups by linear discriminant analysis (LDA) coupled with effect size (LEfSe) with a threshold on the logarithmic LDA score for discriminative features of 2. 
A.
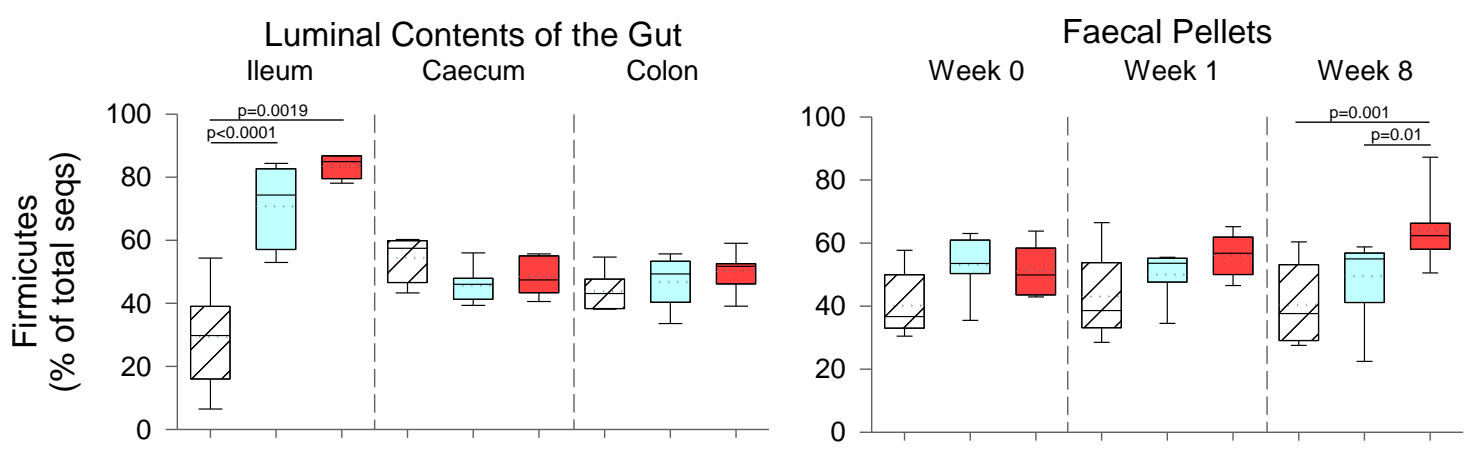

B.
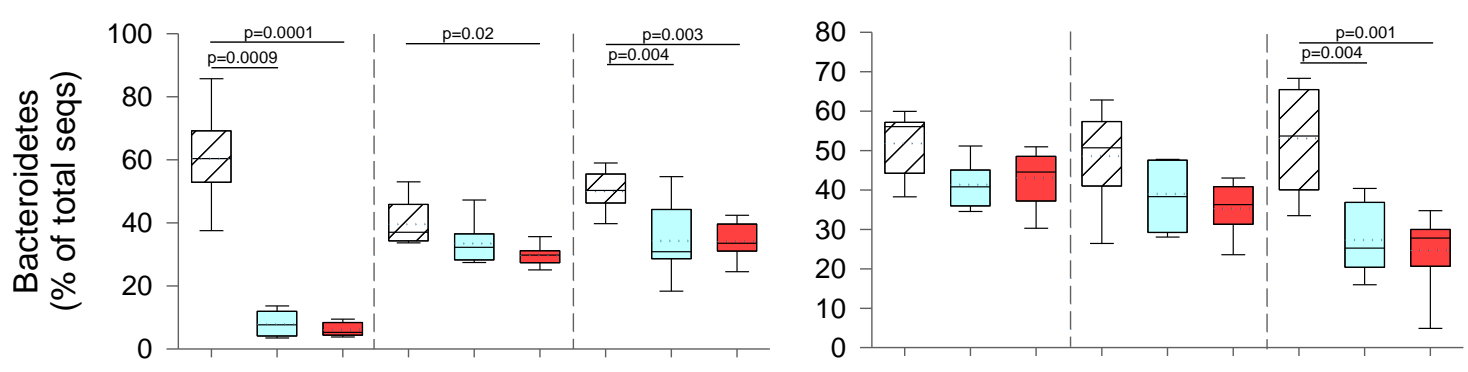

C.
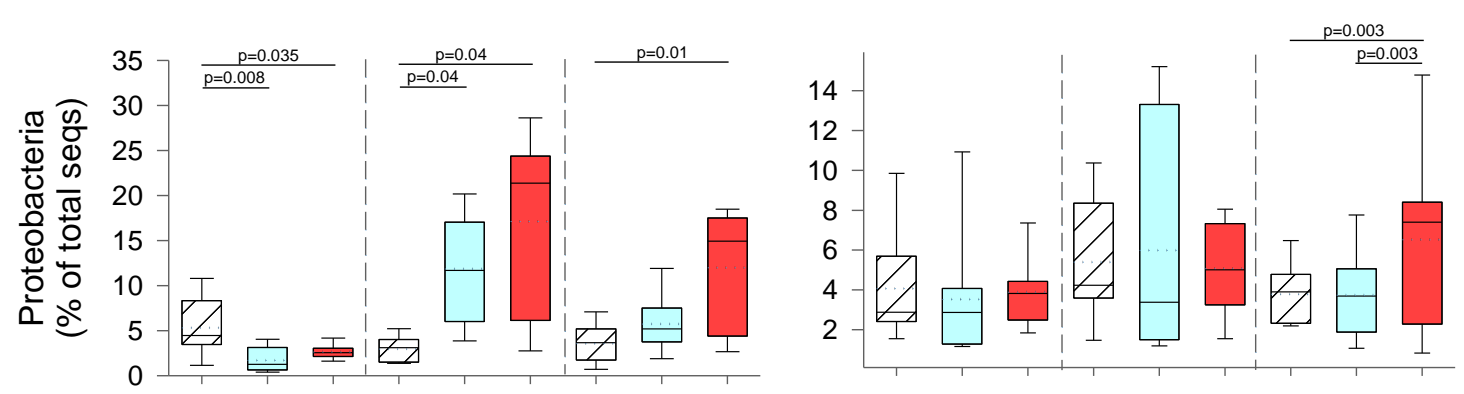

D.
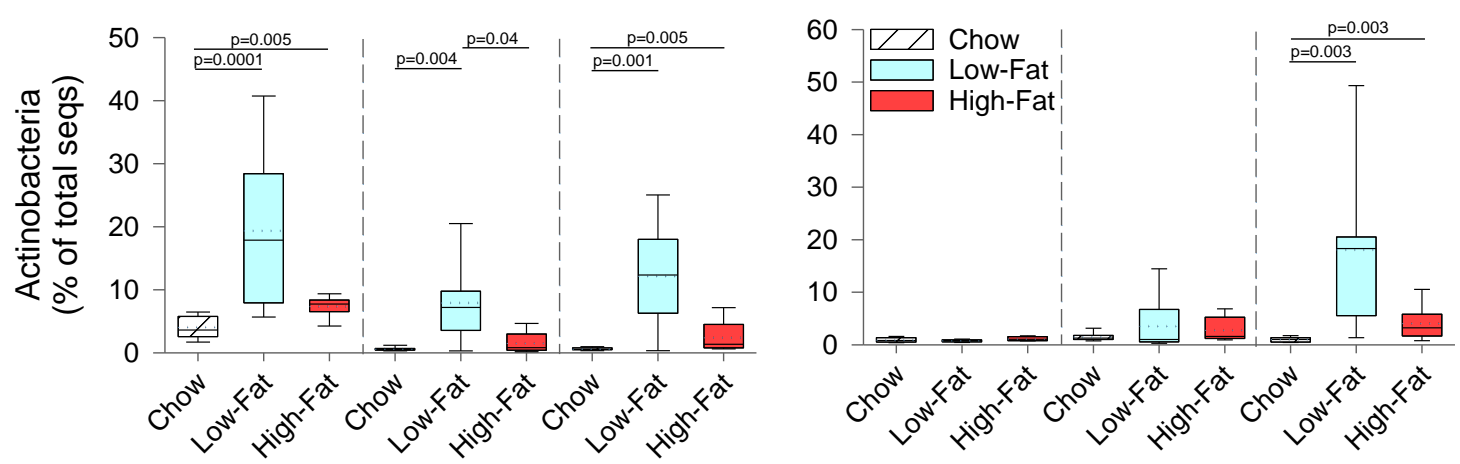

Figure 29. Effect of diet on the proportions of the dominant bacterial phyla.

The proportional abundance in Chow, Low-Fat, and High-Fat diet fed mice of Firmicutes (A), Bacteroidetes (B), Proteobacteria (C), and Actinobacteria (D), in the ileum, caecum, colon, at the end of the study and faecal pellets at week 0 , week 1 , and week 8 . Box plots represent the proportion of sequences belonging to each of the four dominant phylum as a percentage of the total sequences. The phylum changes are shown in the faecal pellets from the start of the study (Week 0), after one week (Week 1), and eight weeks (Week 8) on experimental diets (D). For box plots the solid line = median, and dashed line $=$ mean. Statistical significance was determined by Metastats in Mothur and Benjamini-Hochberg multiple testing correction. $\mathrm{n}=8$ mice per group (Low-Fat Week 1, $\mathrm{n}=7)$. 


\subsubsection{PHYLUM LEVEL CHANGES BETWEEN GROUPS AND INTESTINAL LOCATION}

Refined Low-Fat and High-Fat diets significantly changed the proportions of the dominant bacterial phyla. The proportional abundance of Firmicutes was significantly increased in the ileum of both refined diet groups while remaining similar between diets in the caecum and colon, Figure 29A. The proportion of Firmicutes in the faeces became significantly higher in the refine diets by week 8 . There was a dramatic reduction in proportion of Bacteroidetes in the ileum, with smaller reductions in the caecum and colon with reductions were seen in the faeces by the final week, Figure 29B. Contrasting changes in the proportion of Proteobacteria occurred between chow and refined diets. In the ileum Proteobacteria were significantly decreased in both Low-Fat and High-Fat diets relative to chow. In contrast Proteobacteria significantly increased in Low-Fat and High-Fat diets in the caecum, colon, and faeces by week 8, Figure 29C. Actinobacteria increased dramatically in the ileum, caecum, and colon relative to chow with large increases in the Low-Fat diet, Figure 29D. Proportions of these phyla in the faeces at the final week were consistent with the colon. Lack of significant differences in the faeces at week zero indicate later changes were due to the diets and not initial differences in microbiota. Changes in proportions of bacterial phyla were was driven by refined diets relative to the chow and these increases were independent of obesity.

\subsubsection{REFINED DIETS REDUCE FIRMICUTES/BACTEROIDETES RATIO}

Increases in the ratio of Firmicutes to Bacteroidetes has been reported as a marker of an obesity associated microbiota. The ratio between Firmicutes and Bacteroidetes increased in Low-Fat and High-Fat diets relative to the chow in the ileum, colon, and faeces, Figure 30. The Firmicutes to Bacteroidetes ratio was initially similar between groups at Week 0 and increased in Low-Fat and High-Fat groups relative to the chow by Week 8, Figure 30D. Due to decreases in the proportion of the Bacteroidetes the Firmicutes to Bacteroidetes ratio increased in the Ileum and colon (Figure 30AB), remaining similar in the caecum (Figure 30C). These results indicate that increases in the ratio of Firmicutes to Bacteroidetes were driven by refined diets relative to the chow and these increases were independent of obesity. 


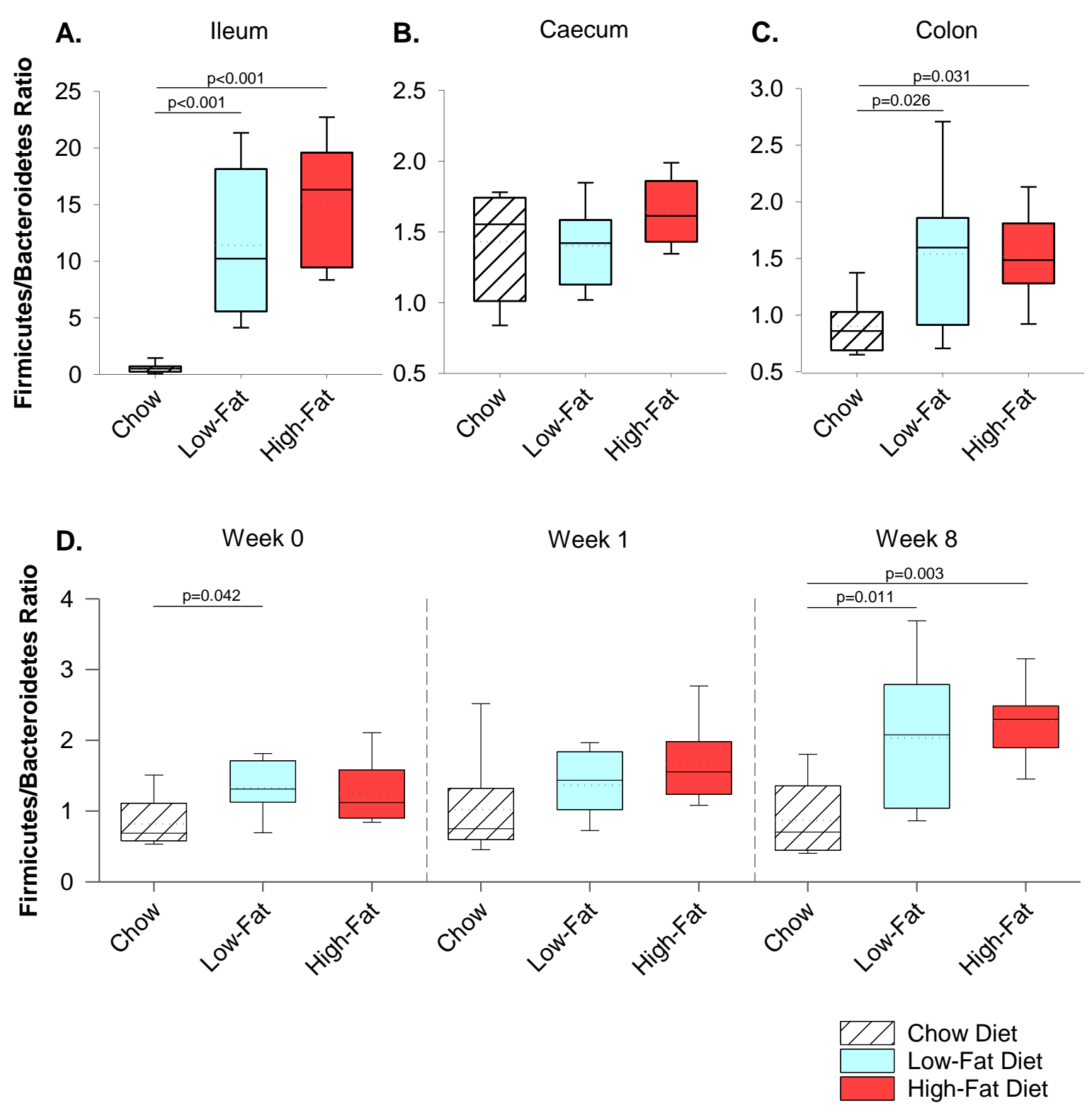

Figure 30. Effect of diet on the ratio of Firmicutes to Bacteroidetes.

The ratio of proportional abundance of the Firmicutes phylum to the Bacteroidetes phylum in the ileum (A), caecum (B), and colon (C) of mice eating Chow, Low-Fat, and High-Fat diets. The ratio of Firmicutes to Bacteroidetes in the faeces at week 0, week 1, week 8 (D). For box plots the solid line = median, and dashed line $=$ mean. Statistical significance was determined by one-way ANOVA with Tukey's post hoc test. $\mathrm{n}=8$ mice per group (Low-Fat Week 1, n=7). 


\subsubsection{SPECIES RICHNESS AND DIVERSITY BETWEEN DIET GROUPS AND INTESTINE LOCATION}

\subsubsection{DIVERSITY INDEXES}

With exception of the ileum measures of diversity and richness of the microbiota was not significantly influenced by diet, Figure 31. The observed richness (Sobs), the number of species present defined as OTUs at $97 \%$ sequence identity, was reduced in the faeces in the final week in both refined diets, only statistical significance in High-Fat mice, Figure 31A. There was also a small reduction in observed richness in the caecum and colon. An apparent increase in observed richness was found in the ileum in the HighFat diet. Similar trends were found using the Chao calculator, an estimate of species richness, Figure 31B. The microbiota diversity of the ileum measured by the Shannon and Inverse Simpson indexes represent measures of species evenness of the community. Shannon and Inverse Simpson indexes were significantly decreased in the ileum microbiota of both Low-Fat and High-Fat diet fed mice in comparison to the Chow mice $(\mathrm{p}<0.01)$, Figure 31C-D. The decrease in this index in the ileum indicate that the microbial community had become dominated by a smaller number of species.

\subsubsection{RAREFACTION CURVES}

Rarefaction curves were similar between the different diets in the ileum, caecum, colon and faeces and does not indicate any general difference in species richness between the diet groups, Figure 32. The plateauing of the curves indicates that the number of sequences generated was enough to include the majority of the OTUs present in the original samples.

Overall the diversity measures indicate that, apart from in the ileum, the three different diets did not significantly influence microbiota diversity after eight weeks of feeding. When microbiota diversity was reduced it was reduced in both refined Low-Fat and High-Fat diets. This reduction of diversity was not associated with the development of obesity. 
luminal contents of the gut

A.

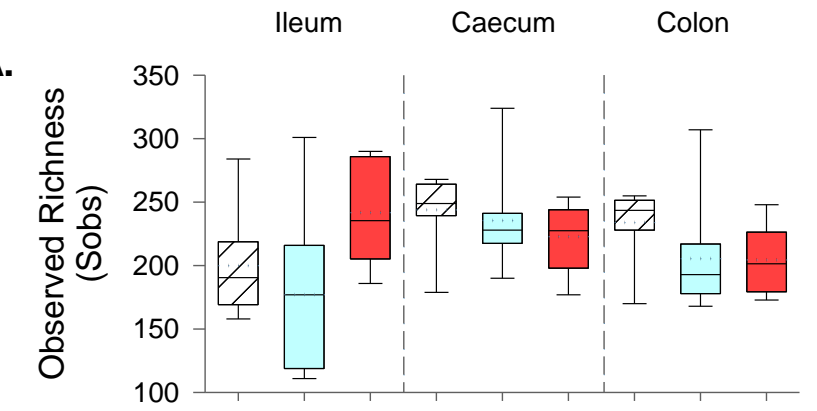

B.

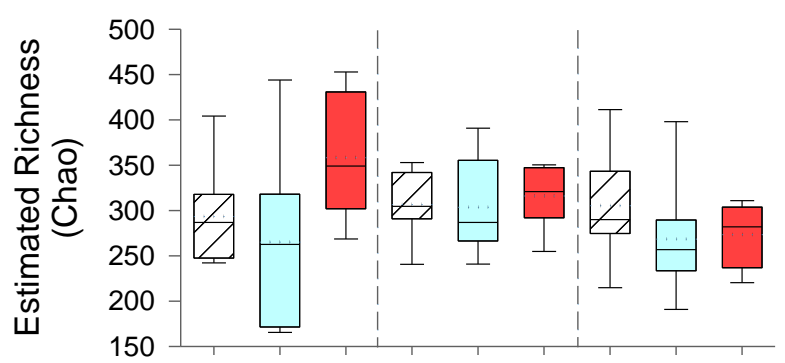

C.

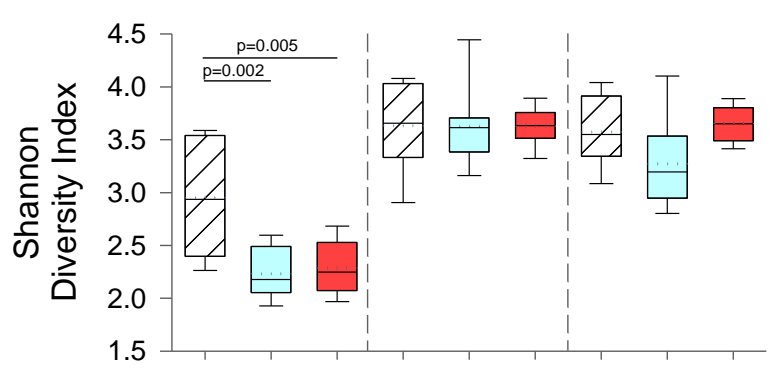

D.

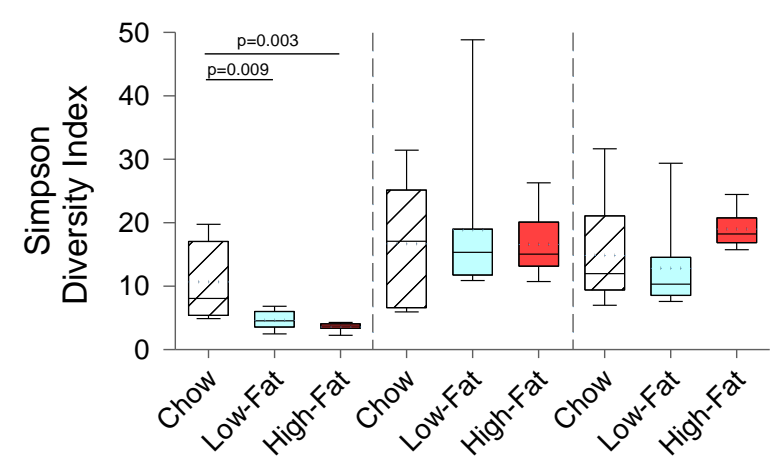

Faecal Pellets

Week $0 \quad$ Week $1 \quad$ Week 8
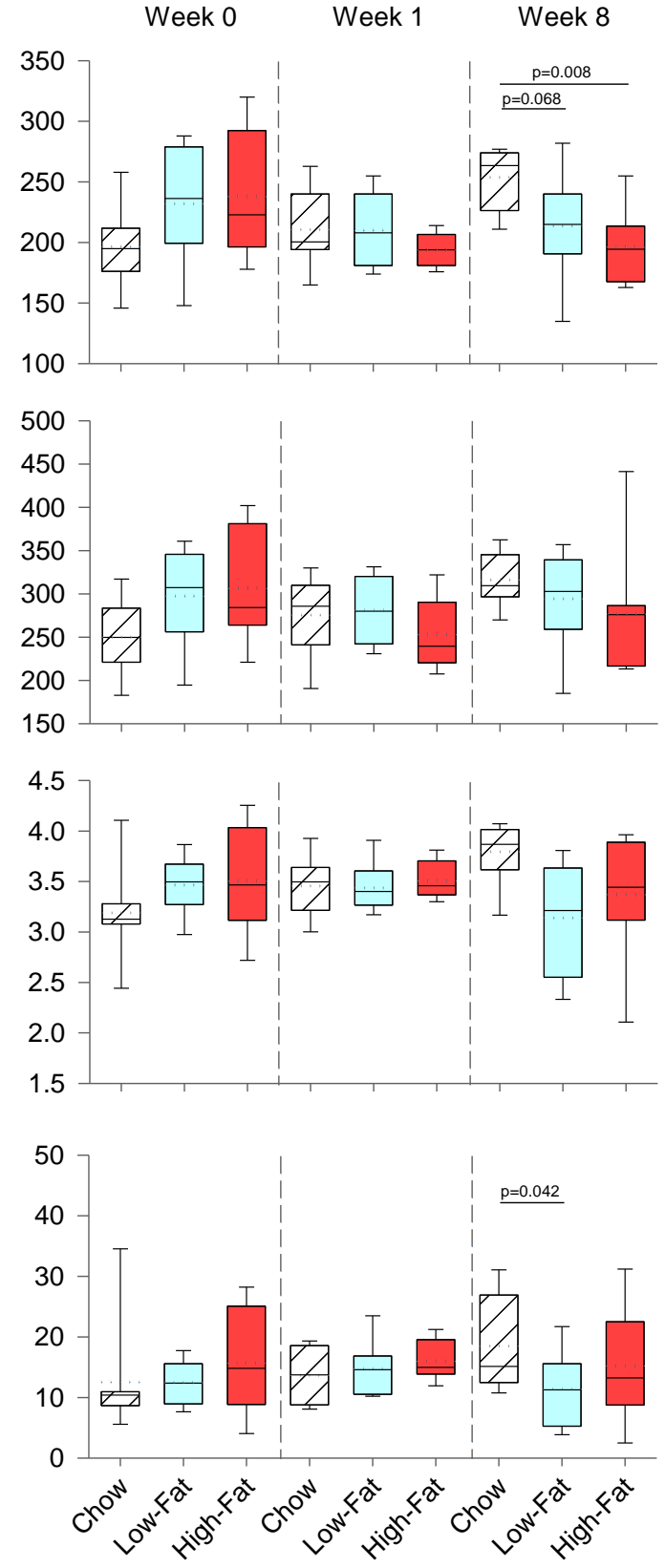

Figure 31. Effect of diet on mouse microbiota richness and microbiota diversity.

The effect of Chow, Low-Fat, and High-Fat diet on the Observed Richness (Sobs) (A), and Estimated Richness (Chao) (B), Shannon Diversity Index (C), and Inverse Simpson Diversity Index (D, in the ileum, caecum, colon, at the end of the study and faecal pellets at week 0 , week 1, and week 8. Diversity measures calculated in Mothur. For box plots the solid line $=$ median, and dashed line $=$ mean. Statistical significance was determined by one-way ANOVA with Tukey's post hoc test. $\mathrm{n}=8$ mice per group (Low-Fat Week 1, $\mathrm{n}=7$ ). 

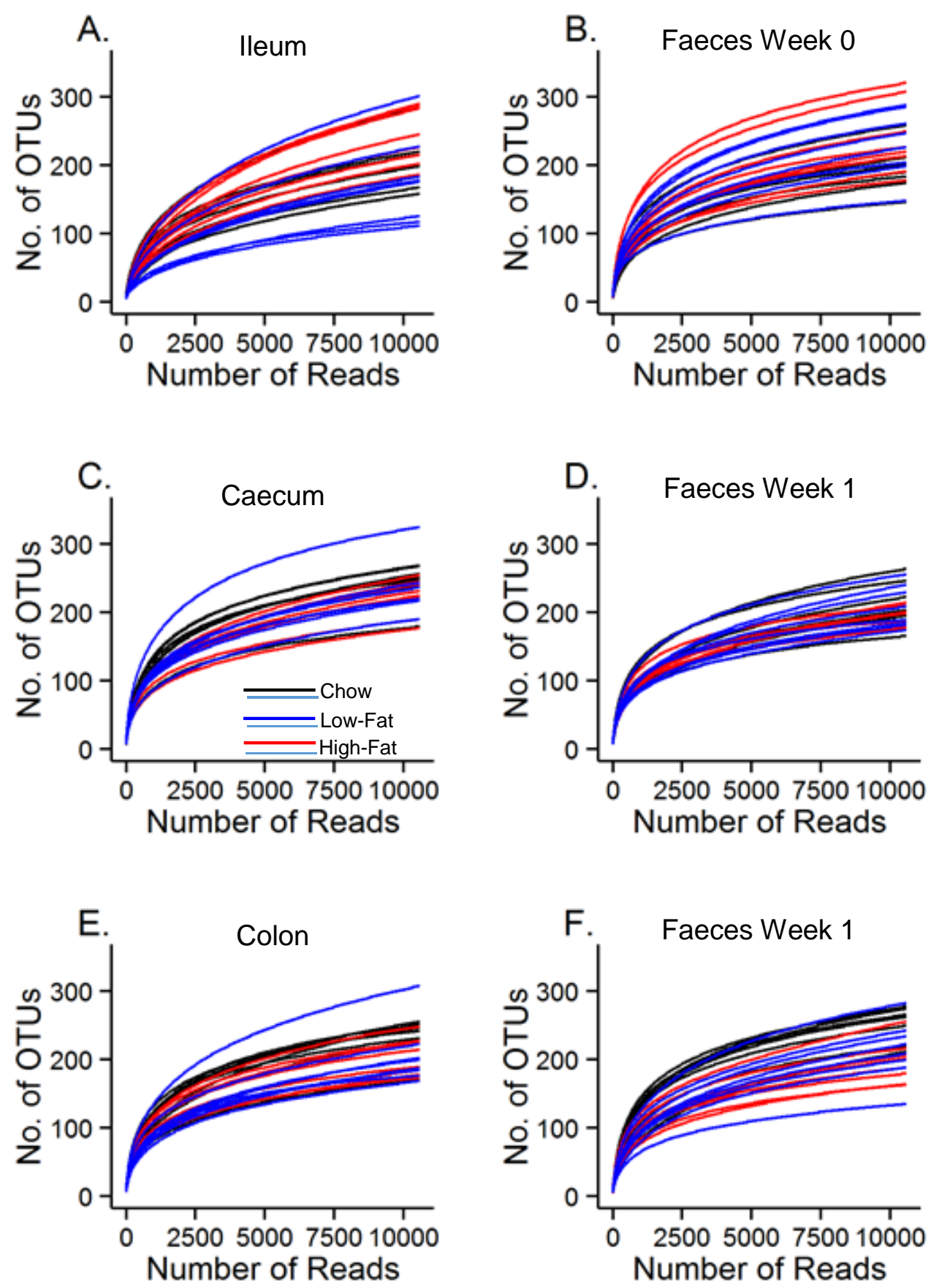

Figure 32. Rarefaction curves of species richness.।

The effect of Chow, Low-Fat, and High-Fat diet on rarefaction curves of microbiota richness in the Ileum (A), Caecum (C), Colon (C), Faeces at week 0 (B), week 1 (D), and week $8(\mathrm{~F})$, in the ileum, caecum, colon, at the end of the study and faecal pellets at week 0 , week 1 , and week 8 . The chow diet mice (Black Line), Low-Fat diet mice (Blue Line), and High-Fat diet mice (Red Line). Rarefaction curves display a plot of the number of species as a function of the number of repeated random samples. Individual lines represent the microbiota richness from one part of the gut for a single mouse. The plateauing of the curves indicate that the majority of the species (OTUs) present in each sample have been detected. 


\subsubsection{MICROBIOTA PROFILE CLUSTERING AND FAMILY LEVEL COMPOSITION}

Comparing the stacked bars displaying the proportional abundance of each bacterial family within each mouse microbiota a great deal more diversity is seen within each bacterial Phylum both between individual mice and between the different diet groups, (Figure 34). Several broad differences between the diet groups were identified in the ileum, caecum, and colon. The LEfSe (linear discriminant analysis (LDA) effect size) method was used to identify bacterial families in the sequence data that differentiate the different diet groups (Segata et al, 2011). In the ileum 27 bacterial families were differentially abundant between diet groups, 11 in Chow, 3 in Low-Fat, and 13 in HighFat, Figure 33. In the ileum, unclassified Families of Bacteroidales, Bacteroidetes, and Lactobacillaceae differentiated the Chow group with the greatest magnitude. Streptococcaceae and Peptostreptococcaceae in the Low-Fat group, and Erysipelotrichaceae and Bifidobacteriaceae in the High-Fat group. Metastats showed that these Family level differences were not significantly different between Low-Fat and High-Fat groups. In the caecum 21 bacterial families were differentially abundant between diet groups, 9 in Chow, 5 in Low-Fat, and 7 in High-Fat. In the caecum, unclassified Families of Lachnospiraceae and Bacteroidetes differentiated the Chow group with the greatest magnitude. Streptococcaceae and Peptostreptococcaceae in the Low-Fat group, and Erysipelotrichaceae and Rikenellaceae in the High-Fat group. The caecum and colon are largely similar in the Family level composition (Figure 34B-C). The change from Chow to Low-Fat or High-Fat diets resulted in a wholescale change in the family level composition of the microbiota with changes occurring of a large magnitude. 


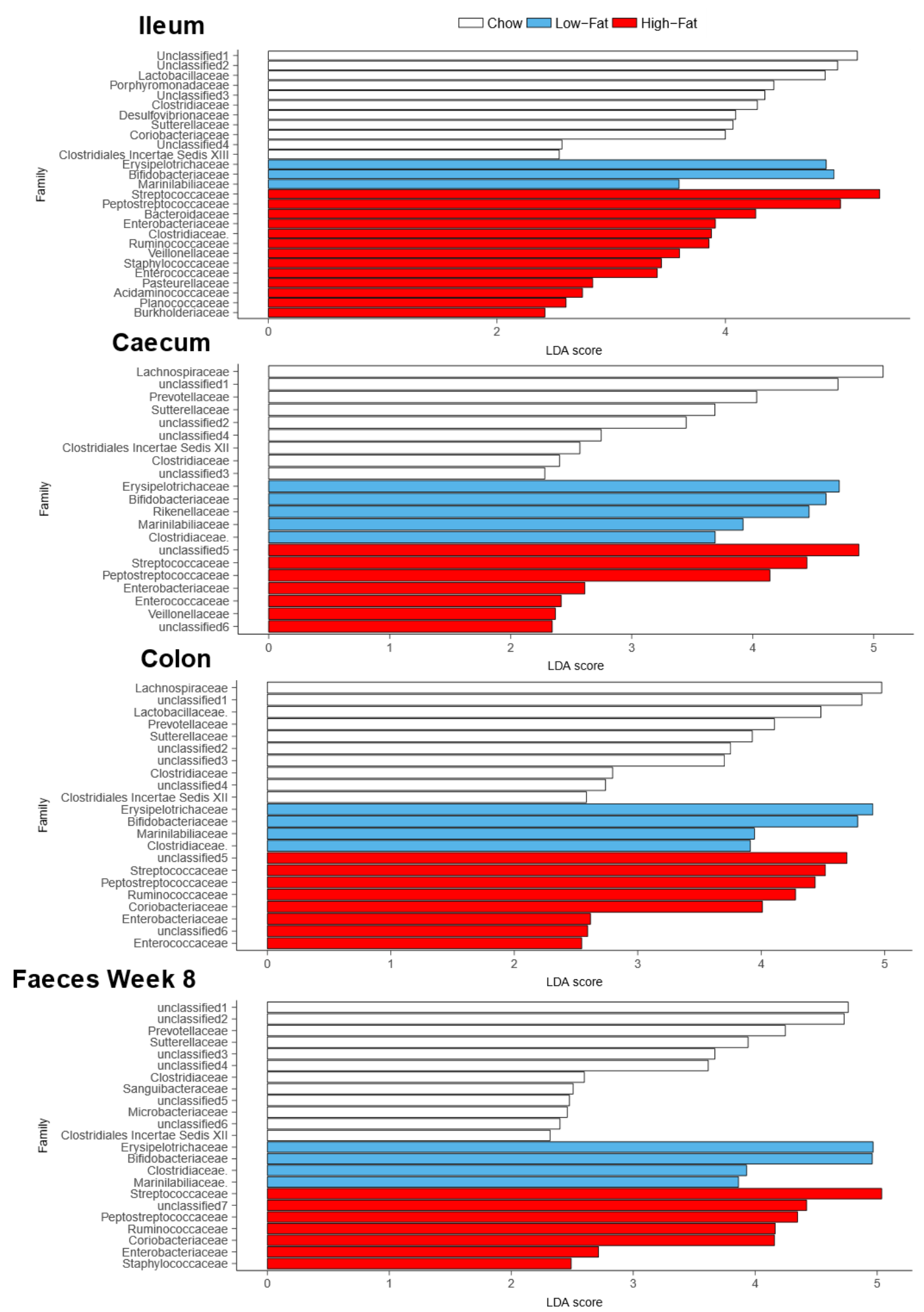

Figure 33. Bacterial families differentially expressed by diet group.

Bacterial families differentially expressed in the Chow, Low-Fat, and High-Fat diet groups by linear discriminant analysis (LDA) coupled with effect size (LEfSe) with a threshold on the logarithmic LDA score for discriminative features of 2 . 


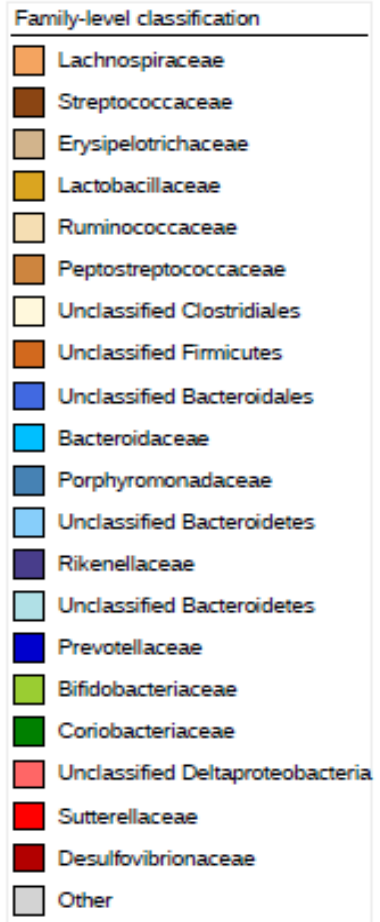

Parsimony Significance Tests

\begin{tabular}{lc}
\multicolumn{2}{c}{ lleal Microbiota } \\
\hline Comparison & P-value \\
\hline Chow vs High-Fat & $<0.0001$ \\
Chow vs Low-Fat & $<0.0001$ \\
High-Fat vs Low-Fat & 0.1279 \\
\hline
\end{tabular}

\begin{tabular}{lc}
\multicolumn{2}{c}{ Caecal Microbiota } \\
\hline Comparison & P-value \\
\hline Chow vs High-Fat & $<0.0001$ \\
Chow vs Low-Fat & $<0.0001$ \\
High-Fat vs Low-Fat & 0.0015 \\
\hline
\end{tabular}

\begin{tabular}{lc}
\multicolumn{2}{c}{ Colonic Microbiota } \\
\hline Comparison & P-value \\
\hline Chow vs High-Fat & $<0.0001$ \\
Chow vs Low-Fat & $<0.0001$ \\
High-Fat vs Low-Fat & 0.0014 \\
\hline
\end{tabular}

A.
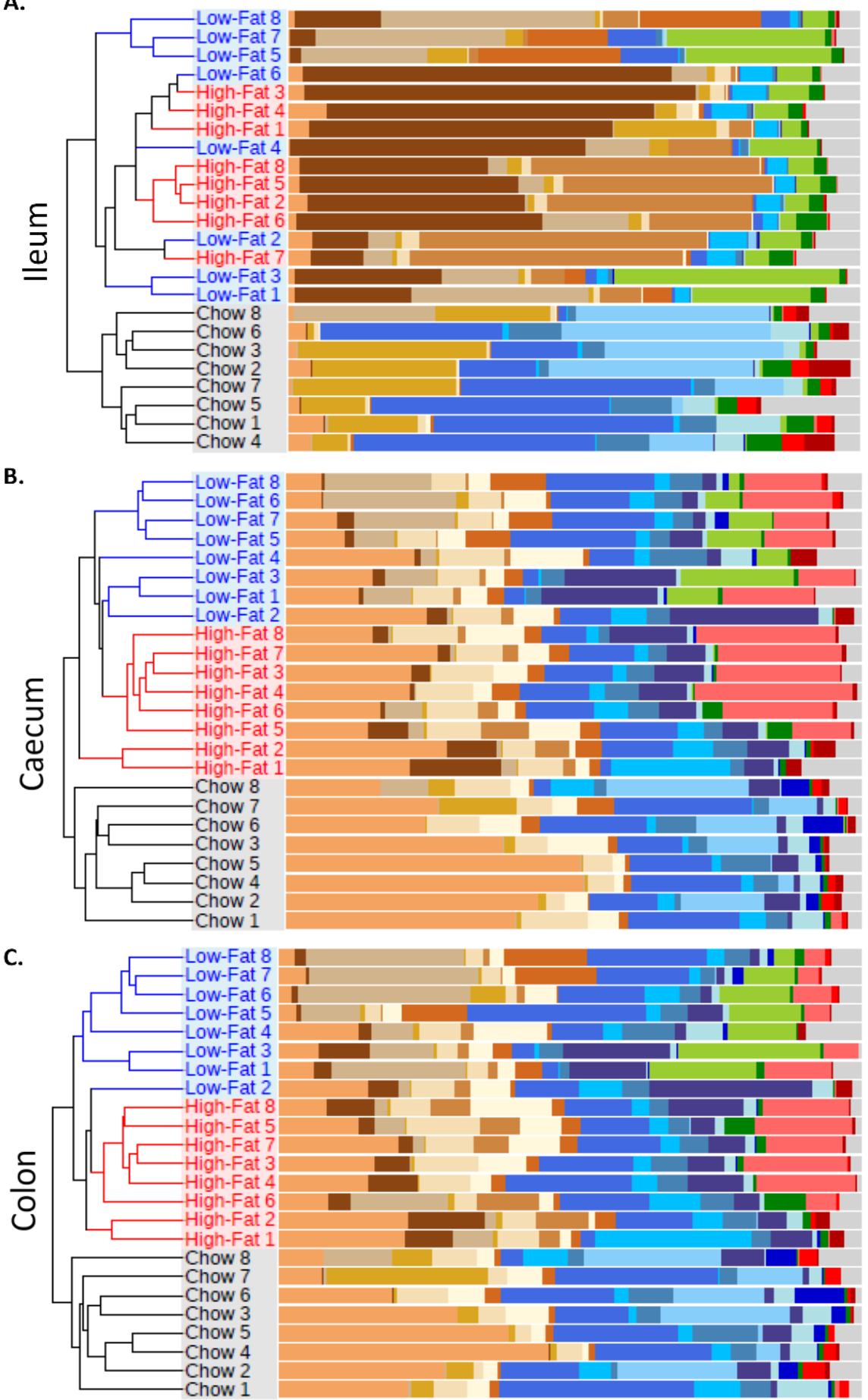

Figure 34. Effect of diet on family level taxonomy composition.

Family level comparison between microbiota in the ileum, caecum, colon, and faeces of Chow, Low-Fat, and High-fat diet fed mice. Bray-Curtis dissimilarity index clustered dendrograms are repeated from (Figure 27). The percentage of sequences from each of the 20 most abundant bacterial Families are represented as a stacked bars. Families included in the Firmicutes phyla are shown as browns, the Bacteroidetes as blues, Actinobacteria as greens, Proteobacteria as reds, and other families as grey. Dendrograms and family level proportions were visualised using ITOL. 


\subsubsection{FAECAL PELLET MICROBIOTA FAMILY LEVEL COMPOSITION}

Initially at Week zero when all mice were eating Chow there was no significant clustering in the faecal microbiota between groups, Figure 35A. Family level composition was similar with no significant difference between the three groups. By Week 8 the microbiota of mice on the three diets clustered into significantly different groups. This indicates that differences in gut microbiota composition at the end of the study were not due to any initial differences between the mice and that diet can begin to shift the microbiota after only one week. The LEfSe (linear discriminant analysis (LDA) effect size) method was used to identify bacterial families in the sequence data that differentiate the different diet groups (Segata et al, 2011). LEfSe analysis determined there were no significant differences in any bacterial families at Week 0 when all mice were eating chow, Figure 35A. After one week feeding the three different diets there were 17 bacterial families were differentially abundant between diet groups, 10 in Chow, 3 in Low-Fat, and 4 in High-Fat, Figure 35B. After eight weeks of feeding on the three different diets, there were 23 bacterial families that were differentially abundant between the diet groups, 12 in Chow, 4 in Low-Fat, and 7 in High-Fat, Figure 35. 


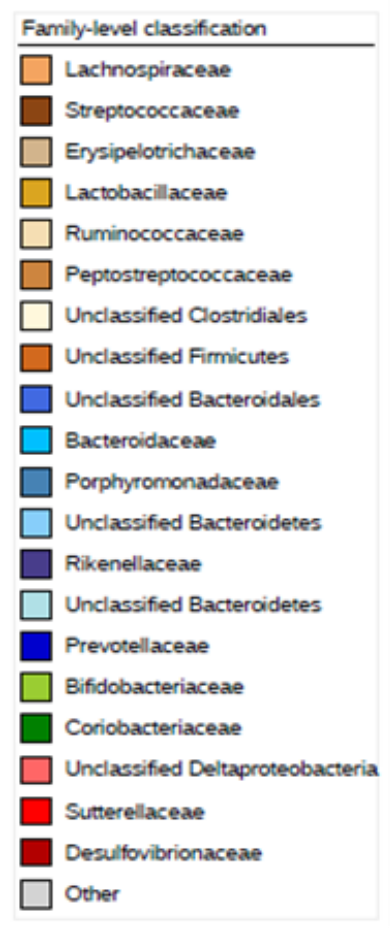

Parsimony Significance Test

Faecal Microbiota Week 0

\begin{tabular}{lc}
\hline Comparison & P-value \\
\hline Chow vs High-Fat & 0.4178 \\
Chow vs Low-Fat & 0.7953 \\
High-Fat vs Low-Fat & 0.7966 \\
\hline
\end{tabular}

Faecal Microbiota Week 1

\begin{tabular}{lc}
\hline Comparison & P-value \\
\hline Chow vs High-Fat & $\$ 0.0001$ \\
Chow vs Low-Fat & $\$ 0.0001$ \\
High-Fat vs Low-Fat & 0.0002 \\
\hline
\end{tabular}

Faecal Microbiota Week 8

\begin{tabular}{lc}
\hline Comparison & P-value \\
\hline Chow vs High-Fat & $<0.0001$ \\
Chow vs Low-Fat & $<0.0001$ \\
High-Fat vs Low-Fat & 0.0021 \\
\hline
\end{tabular}
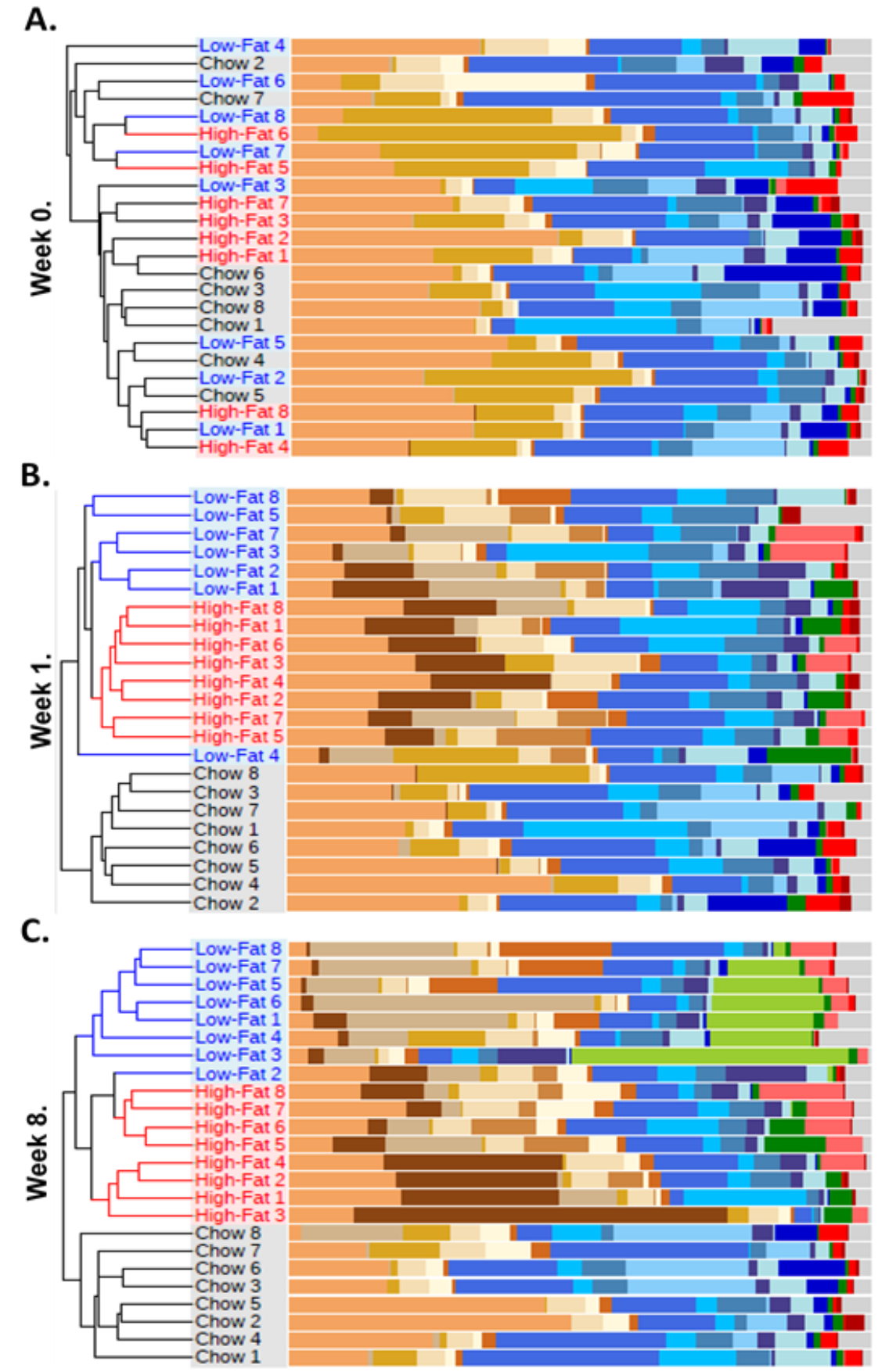

Figure 35. Time course of family level comparison of faecal microbiota.

The dendrograms represent clustering of microbiota based on the Bray-Curtis dissimilarity matrix of OTUs sharing 97\% identity at Week 0, Week 1, and Week 8 in Chow, Low-Fat, and High-fat diet fed mice. Families included in the Firmicutes phyla are shown as browns, the Bacteroidetes as blues, Actinobacteria as greens, Proteobacteria as reds, and other families as grey. Dendrograms and family level proportions were visualised using ITOL. 


\subsubsection{SPECIES LEVEL CHANGES}

The sequence data was analysed to a further level to identify significant changes in species level composition of the mouse microbiota between the Chow, Low-Fat and High-Fat diets. The Operational Taxonomic Unit (OTU) was used when analysing the 16S ribosomal RNA as a proxy for species based on the similarity threshold of $97 \%$ sequence similarity, hence forth termed species. OTUs were assigned a genus and species classification where possible, or the closest level of classification available. The LEfSe (linear discriminant analysis (LDA) effect size) method was used to identify bacterial species in the sequence data that differentiate the different diet groups (Segata et al, 2011). A large number of individual OTUs were differentially represented between three different diet groups. In the ileum, a total of 193 OTUs were differentially represented, Figure 36. In the caecum, a total of 210 OTUs were differentially represented, Figure 37. In the colon, a total of 215 OTUs were differentially represented, Figure 38. In the faeces at week 8 , a total of 221 OTUs were differentially represented, Figure 39. The proportional abundance of individual OTUs with a high LDA score and OTUs with previous associations with obesity was then analysed across the different sites of the intestine. 
A.

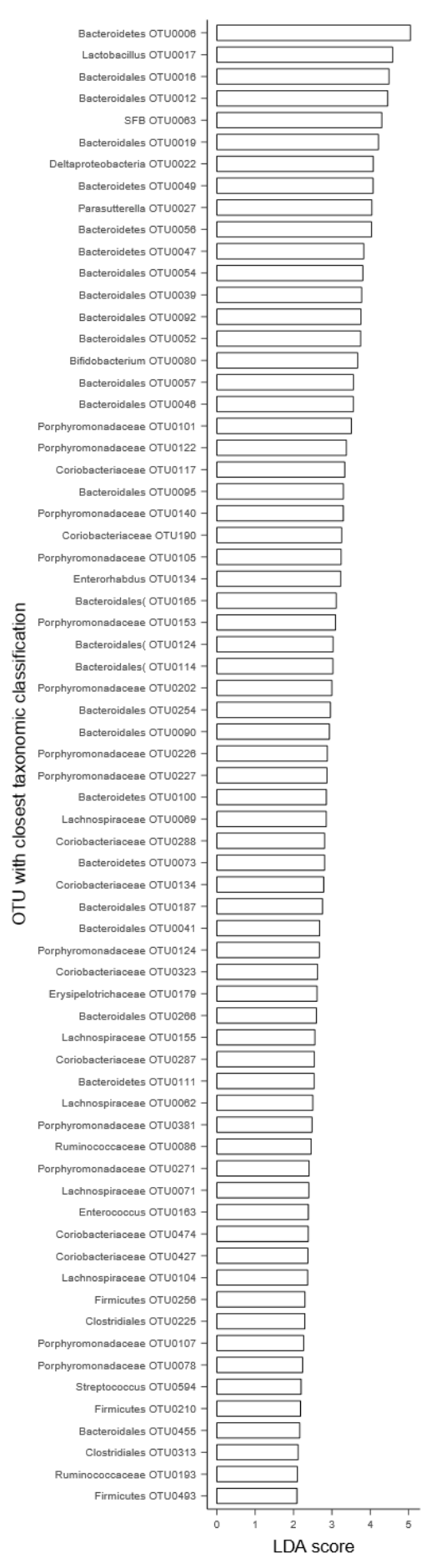

B. Low-Fat

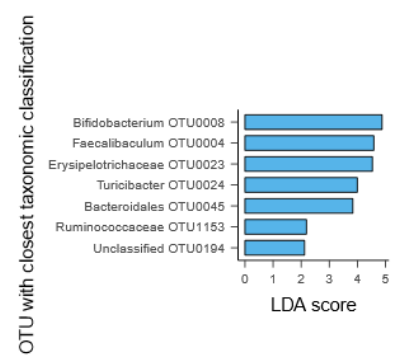

C. High-Fat

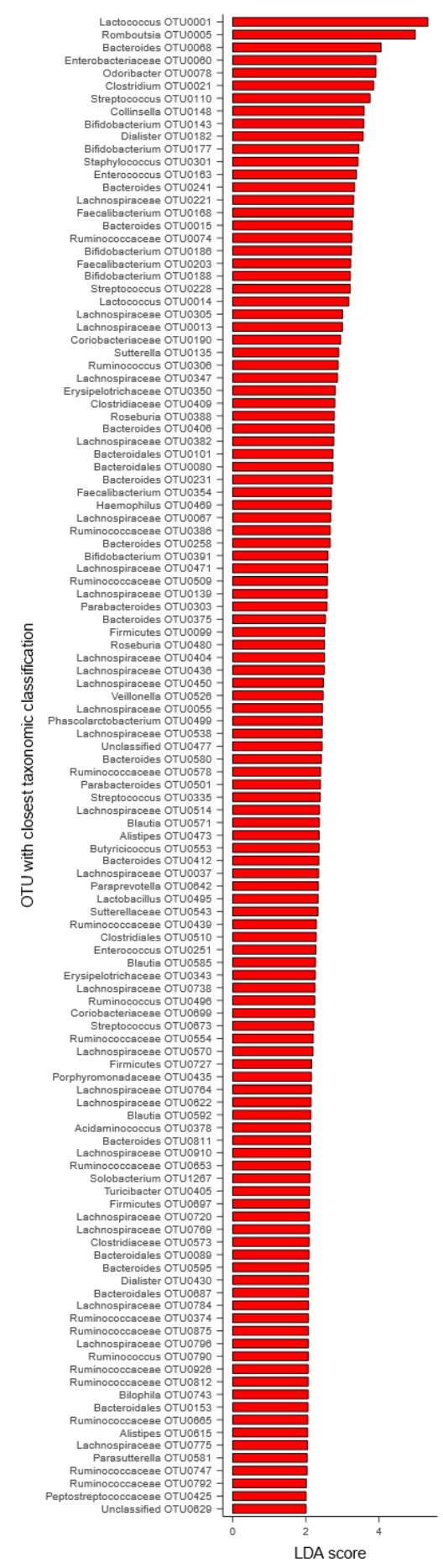

Figure 36. Ileum OTUs differentially expressed by diet group.

In the ileum 68 OTUs were differentially represented in the Chow diet group (A), 7 OTUs in the Low-Fat diet group (B), and 118 OTUs in High-Fat diet group (C) by linear discriminant analysis (LDA) coupled with effect size (LEfSe) with a threshold on the logarithmic LDA score for discriminative features of 2 . 
A.

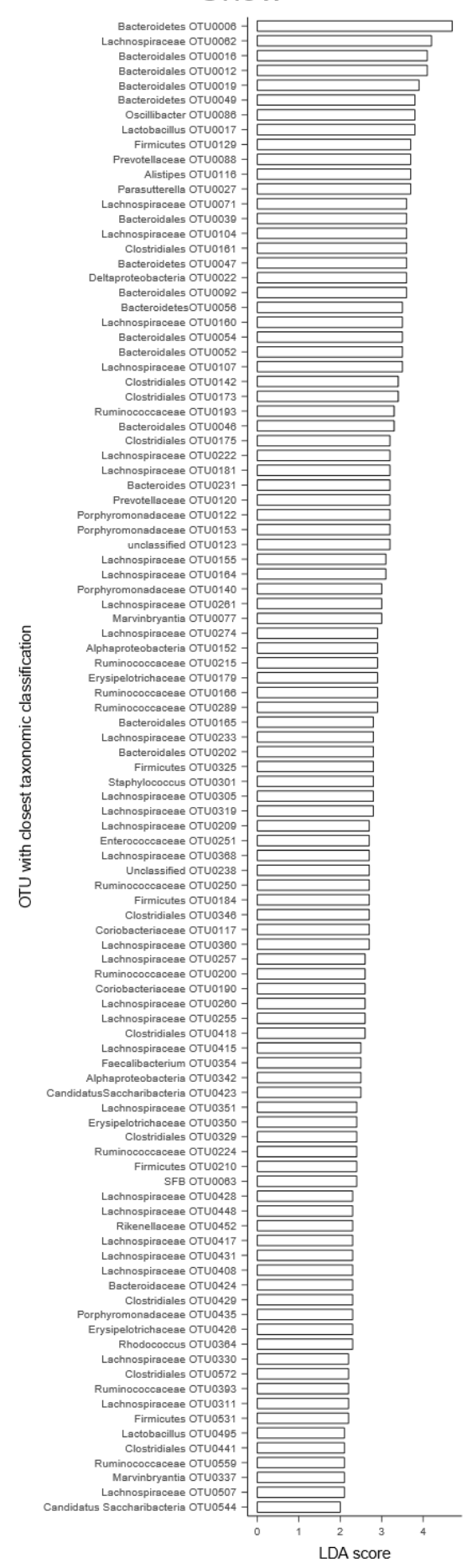

B.

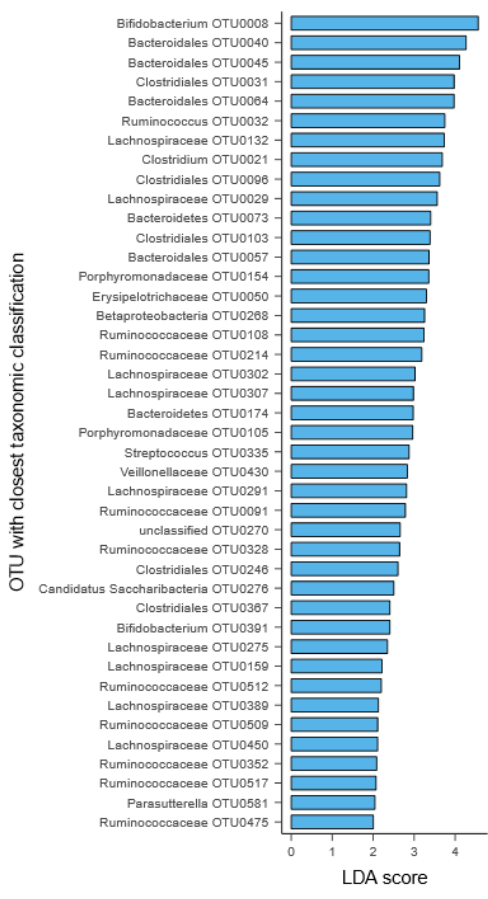

\section{High-Fat}

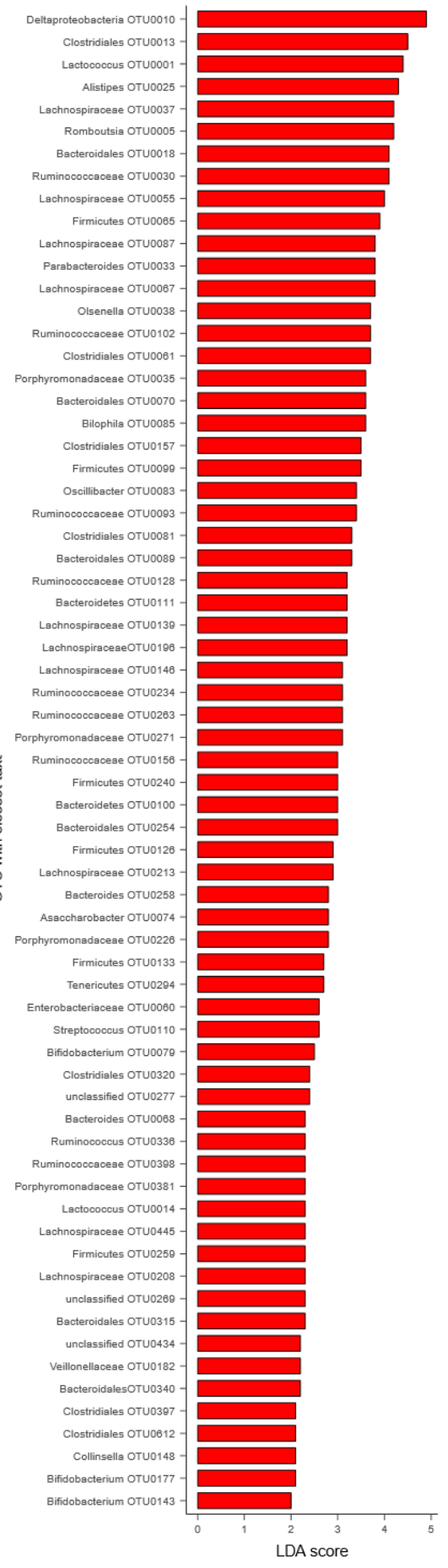

Figure 37. Caecum OTUs differentially expressed by diet group.

In the caecum 101 OTUs were differentially represented in the Chow diet group (A), 42 OTUs in the Low-Fat diet group (B), and 67 OTUs in High-Fat diet group (C) by linear discriminant analysis (LDA) coupled with effect size (LEfSe) with a threshold on the logarithmic LDA score for discriminative features of 2. 
A.

Chow

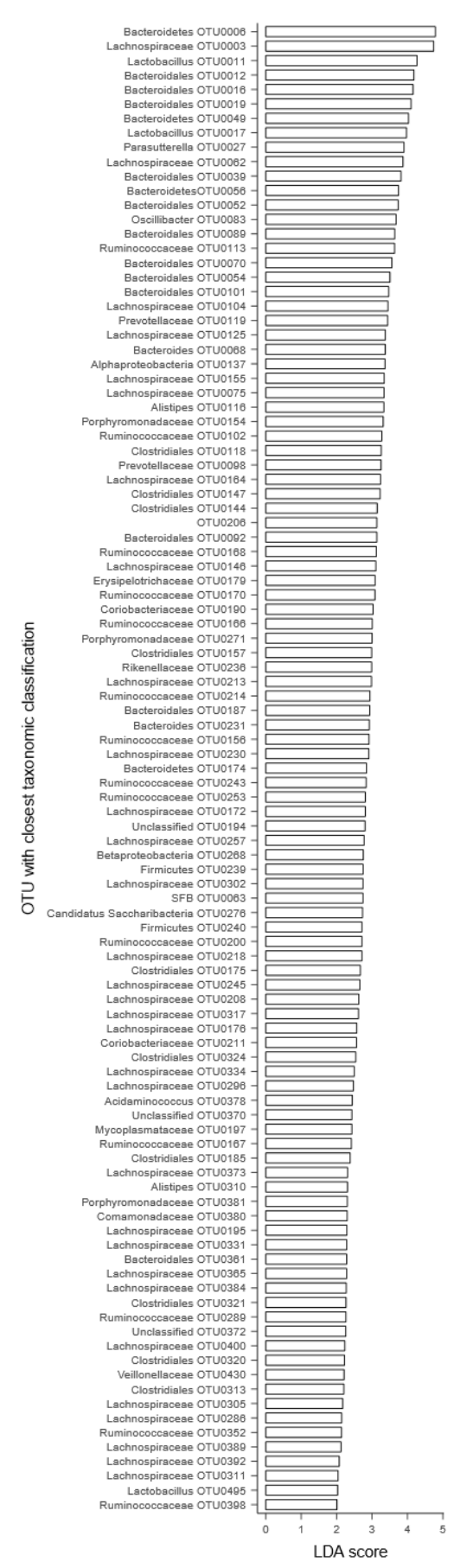

B. Low-Fat

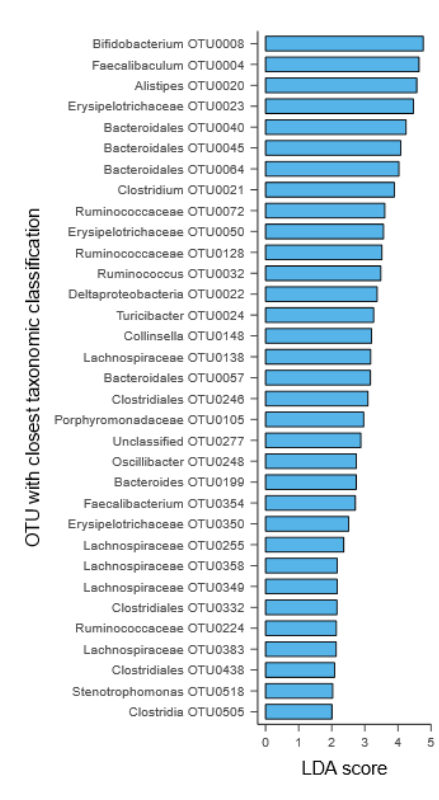

C. High-Fat

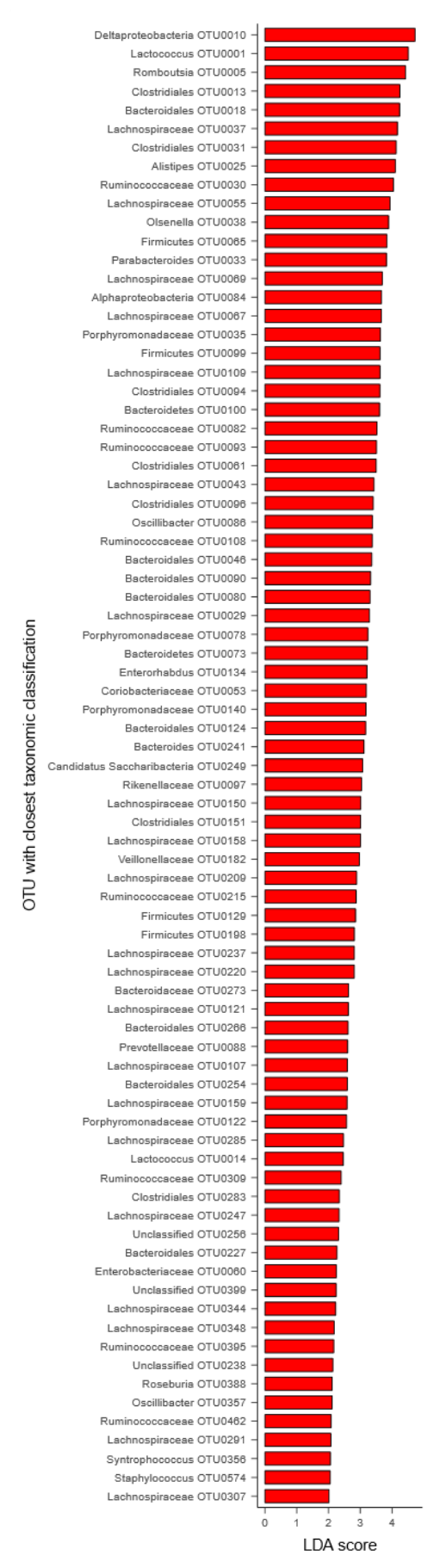

Figure 38. Colonic OTUs differentially expressed by diet group.

In the colon 103 OTUs were differentially represented in the Chow diet group (A), 33 OTUs in the Low-Fat diet group (B), and 79 OTUs in High-Fat diet group (C) by linear discriminant analysis (LDA) coupled with effect size (LEfSe) with a threshold on the logarithmic LDA score for discriminative features of 2 . 
A.

Chow

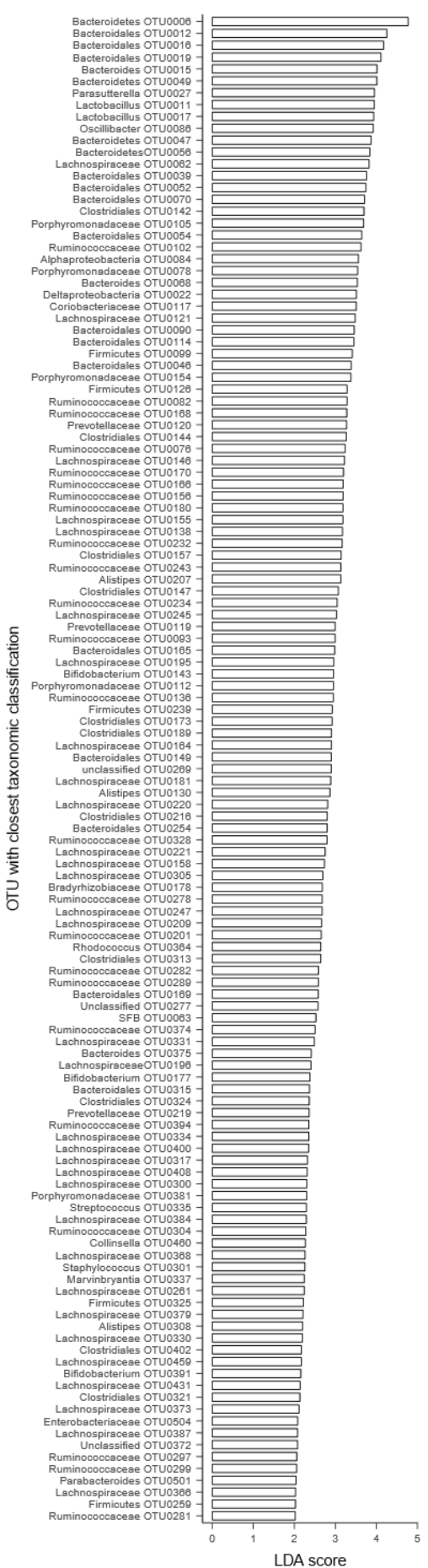

B. Low-Fat

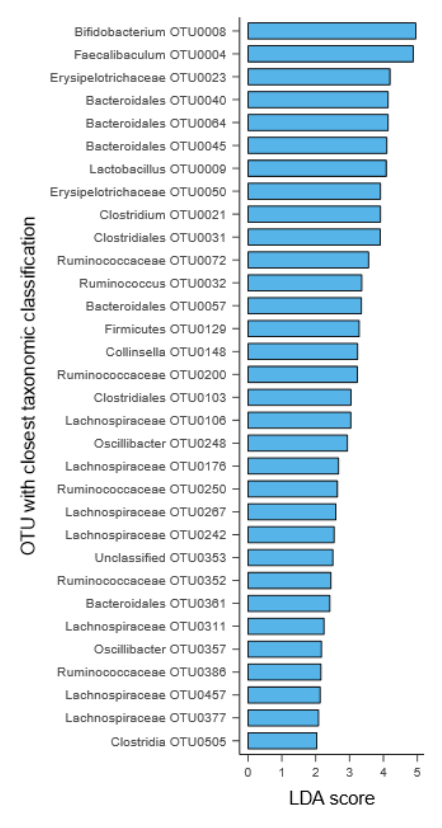

C. High-Fat

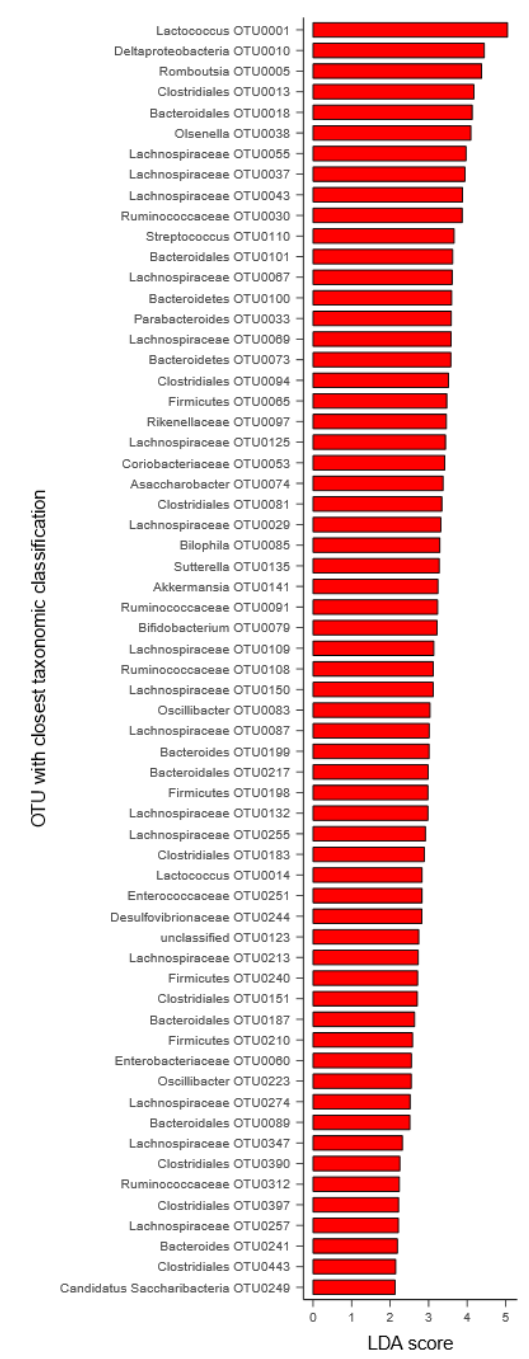

Figure 39. Faecal OTUs differentially expressed by diet group.

At week 8 in the faecal pellets 127 OTUs were differentially represented in the Chow diet group (A), 32 OTUs in the Low-Fat diet group (B), and 62 OTUs in High-Fat diet group (C) by linear discriminant analysis (LDA) coupled with effect size (LEfSe) with a threshold on the logarithmic LDA score for discriminative features of 2 . 


\subsubsection{LARGEST CHANGES IN LOW-FAT AND HIGH-FAT DIET GROUPS}

Two species represented a high proportion of the most abundant sequences in the Chow mice. The first was classified to the family level as a Lachnospiraceae OTU3, Figure 40A. This species was abundant in the caecum and colon. The second was an unknown bacterium that could not be classified beyond phylum level as a Bacteroidetes (OTU6), Figure 40B. This was common in all parts of the gut including the ileum, caecum, and colon. Both species were similarly abundant between groups at Week zero and had almost disappeared after eight weeks of feeding Low-Fat and High-Fat diets.

Two species within the Streptococcaceae and Peptostreptococcaceae families increased in Low-Fat and High-Fat diet groups and became the dominant sequences in the ileum in both diets. The first matched to Lactococcus lactis OTU1, and significantly differentiated the High-Fat group at all site including the ileum, caecum, and colon, Figure 40C. The second matched to Romboutsia ilealis OTU5, and significantly differentiated the High-Fat group at all site including the ileum, caecum, and colon, Figure 40D. Using Metastats the proportional abundance of both species was not significantly different between Low-Fat and High-Fat diet groups. Both species were close to undetectable in the Chow diet mice. 
Luminal Contents of the Gut

A.

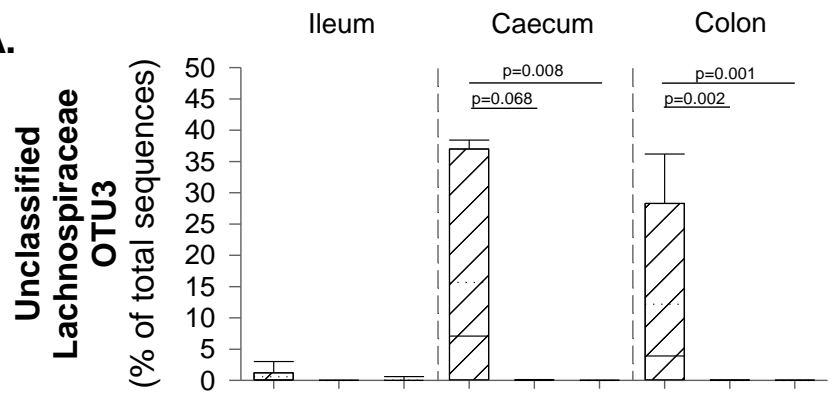

B.

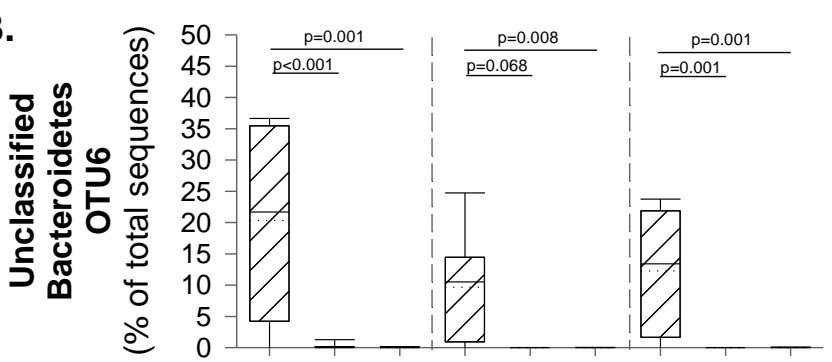

C.
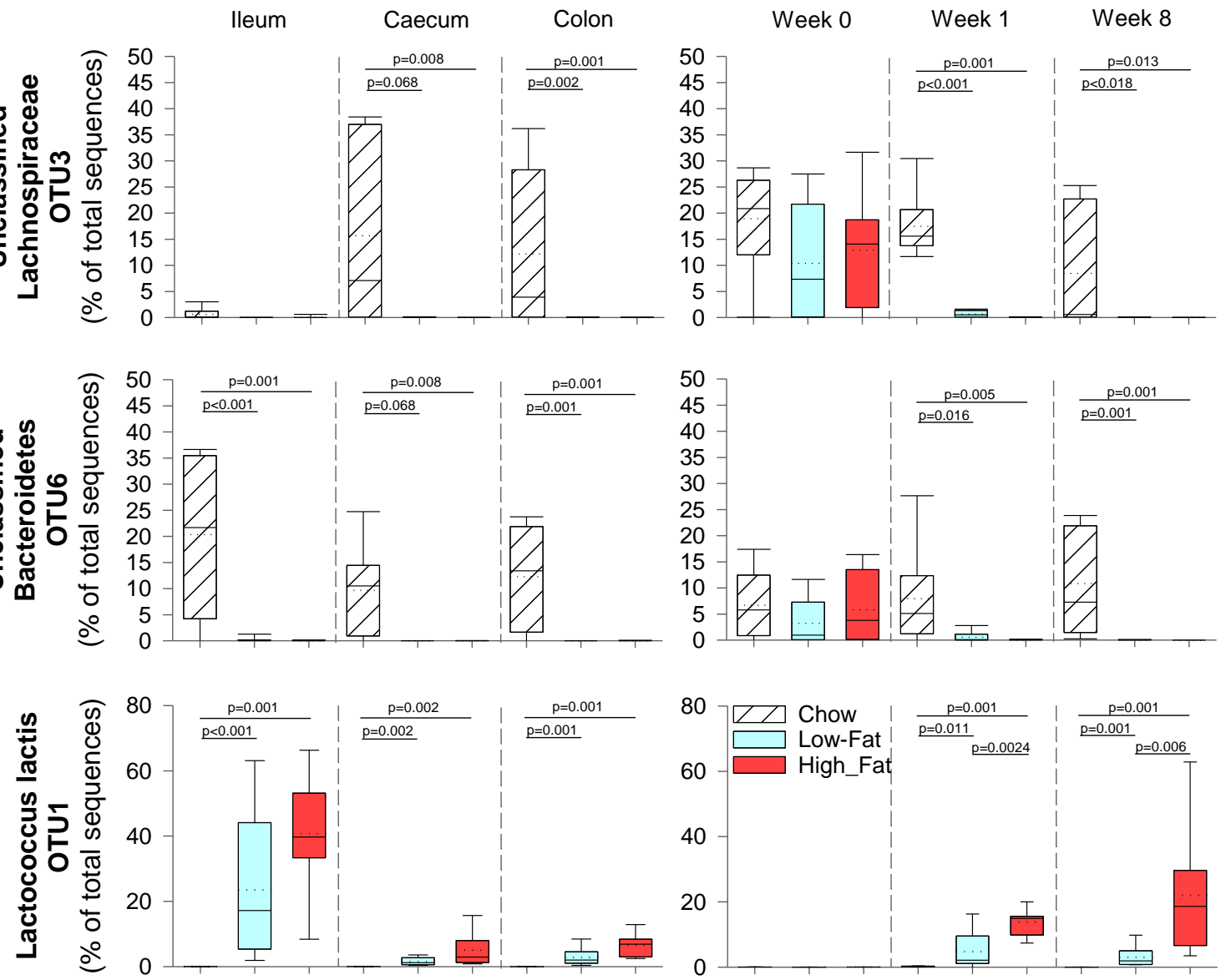

D.

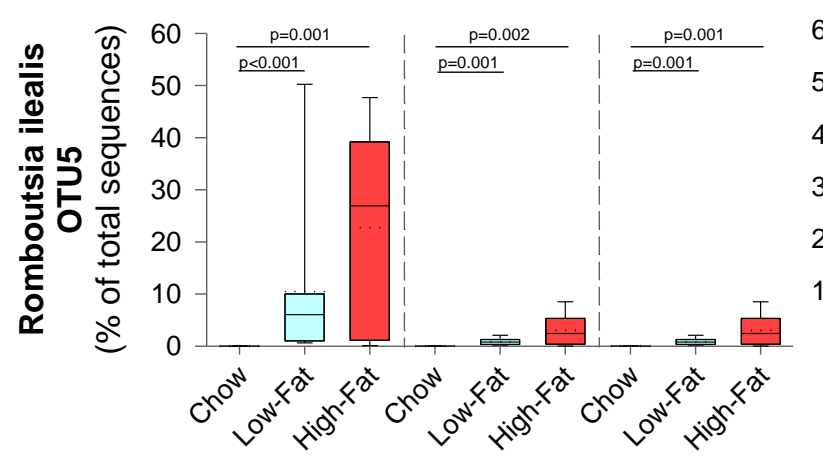

Figure 40. Species showing largest changes in proportional abundance due to diet.

Proportional abundance in Chow, Low-Fat, and High-Fat diet fed mice of an unclassified Lachnospiraceae (OTU3) (A), an unclassified Bacteroidetes (OTU6) (B), Lactococcus lactis (OTU1) (C), and Romboutsia ilealis (OTU5) (D), in the ileum, caecum, colon, at the end of the study and faecal pellets at week 0 , week 1 , and week 8 . For box plots the solid line $=$ median, and dashed line $=$ mean. Statistical significance was determined by Metastats in Mothur and Benjamini-Hochberg correction. $\mathrm{n}=8$ mice per group (Low-Fat Week 1, n=7). 


\subsubsection{SPECIES LEVEL CHANGES WITHIN THE FAMILY ERYSIPELOTRICHACEAE}

The family Erysipelotrichaceae was found to significantly differentiate the Low-Fat groups in the ileum, caecum, and colon, Figure 41A. Three species represented most of the sequences within this family. Classified as Faecalibaculum rodentium at a sequence similarity of 99\% OTU4 increased from very low levels in the Chow diet mice to be one of the dominant species in the Low-Fat diet with high LDA scores in the ileum (LDA 5.0), colon (LDA 5.1), and faeces (LDA 5.2), Figure 41B. Faecalibaculum rodentium was also increased to smaller degree in High-Fat fed mice. An unclassifiable Erysipelotrichaceae OTU23 and another classified at 97\% sequence similarity as Turicibacter sanguinis OUT24 were increased only in the Low-Fat diet group. Faecalibaculum rodentium and OTU23 were only increased by Week 8 in the faeces, Figure 41C. In contrast Turicibacter increased significantly by Week 1 in the faeces and had reduced to low levels by Week 8 suggesting a transient increase, Figure 41D. All Erysipelotrichaceae were found at very low levels of abundance at Week zero. 
Family

Luminal Contents of the Gut

A. lleum Caecum Colon

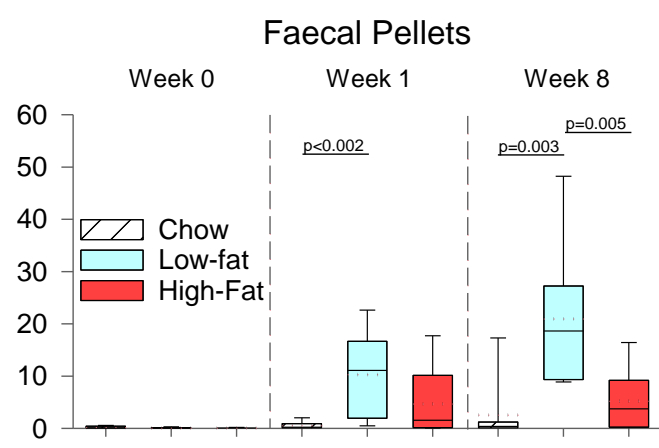

\section{Operational Taxomic Units}
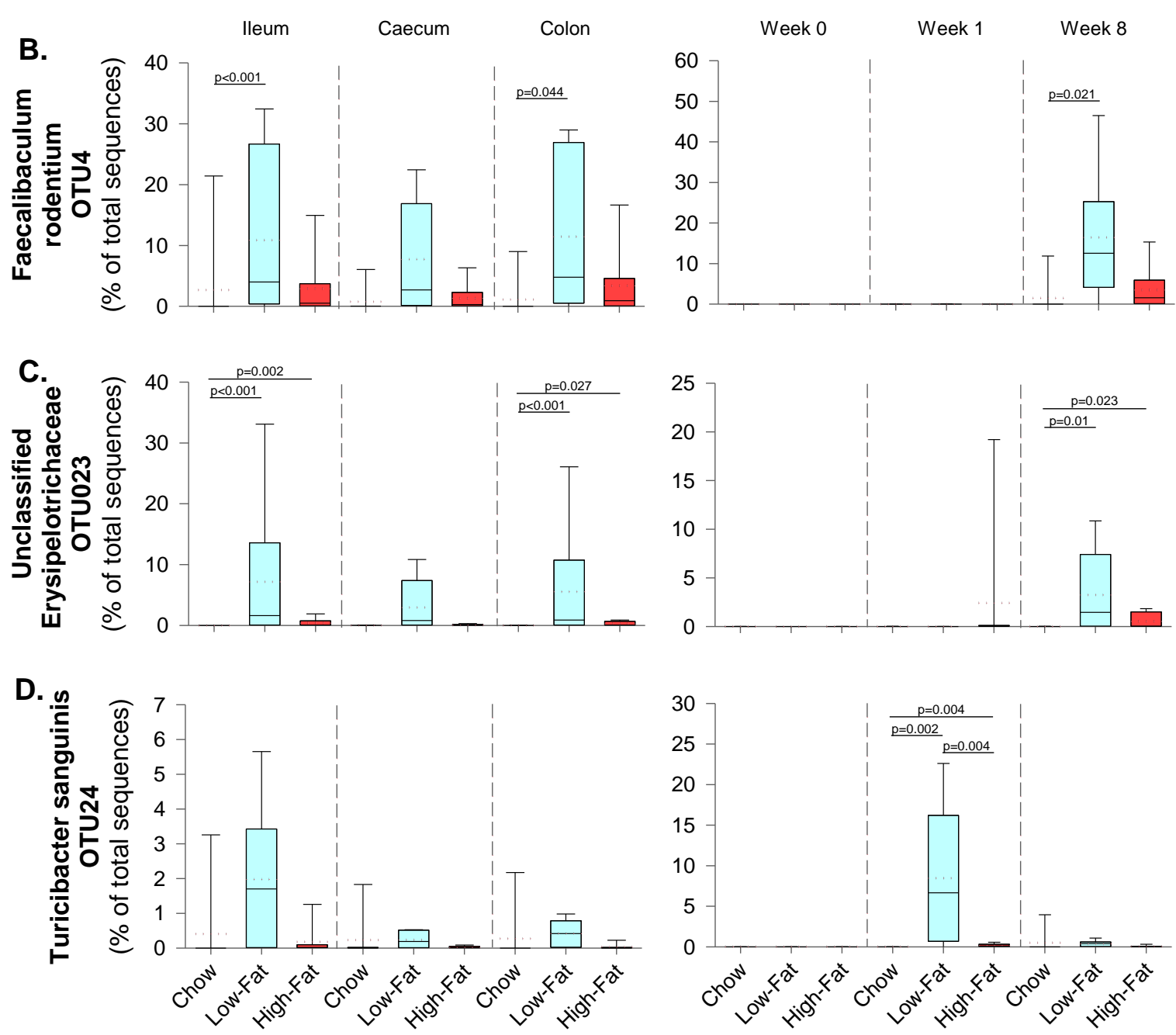

Figure 41. Effect of diet on Erysipelotrichaceae family and species abundance.

Proportional abundance in Chow, Low-Fat, and High-Fat diet fed mice of the family Erysipelotrichaceae (A), and the individual species Faecalibaculum rodentium OTU4 (B), an unclassified Erysipelotrichaceae OTU23 (C), and Turicibacter sanguinis OTU24 (D), in the ileum, caecum, colon, at the end of the study and faecal pellets at week 0 , week 1 , and week 8 . In box plots the solid line $=$ median, and dashed line $=$ mean. Statistical significance was determined by Metastats in Mothur and BenjaminiHochberg correction. $\mathrm{n}=8$ mice per group (Low-Fat Week $1, \mathrm{n}=7$ ). 


\subsubsection{LACTOBACILLACEAE FAMILY AND SPECIES LEVEL CHANGES}

The feeding of both Low-Fat and High-Fat diets was accompanied by a dramatic reduction of the Lactobacillaceae family. The Lactobacillaceae formed a substantial proportion of the bacteria in the ileum of chow fed mice and to a lesser degree in the caecum and colon, Figure 42A. Lactobacillaceae decreased to a similar extent in both Low-Fat and High-Fat diet groups. At a species level Lactobacillaceae were formed by two dominant OTUs. The first, OTU9, closely aligned to Lactobacillus murinus and remained unchanged between diet groups and increased during the study, Figure 42B. OTU17 corresponding to L. reuteri represented the majority of the Lactobacillaceae family in the Chow fed mice and became almost undetectable in Low-Fat and High-Fat diet groups, Figure 42C,

\subsubsection{BIFIDOBACTERIACEAE FAMILY AND SPECIES LEVEL CHANGES}

The proportion of Bifidobacteriaceae was very low in the Chow and High-Fat diet groups, while Bifidobacteriaceae increased dramatically in the Low-Fat diet group, Figure 43A. A level single OTU8 corresponding to Bifidobacterium pseudolongum comprised the majority of the Bifidobacteriaceae family and was virtually absent in the Chow and High-Fat diet groups. B. pseudolongum bloomed in the Low-Fat diet group, Figure 43B. This bloom was only detectable only at week 8, Figure 43B. One other species OTU79, corresponding closely to $B$. adolescents, was present in small proportions, Figure 43C. This OTUs is notable for increasing in both Low-Fat and HighFat diet groups in the Ileum. 

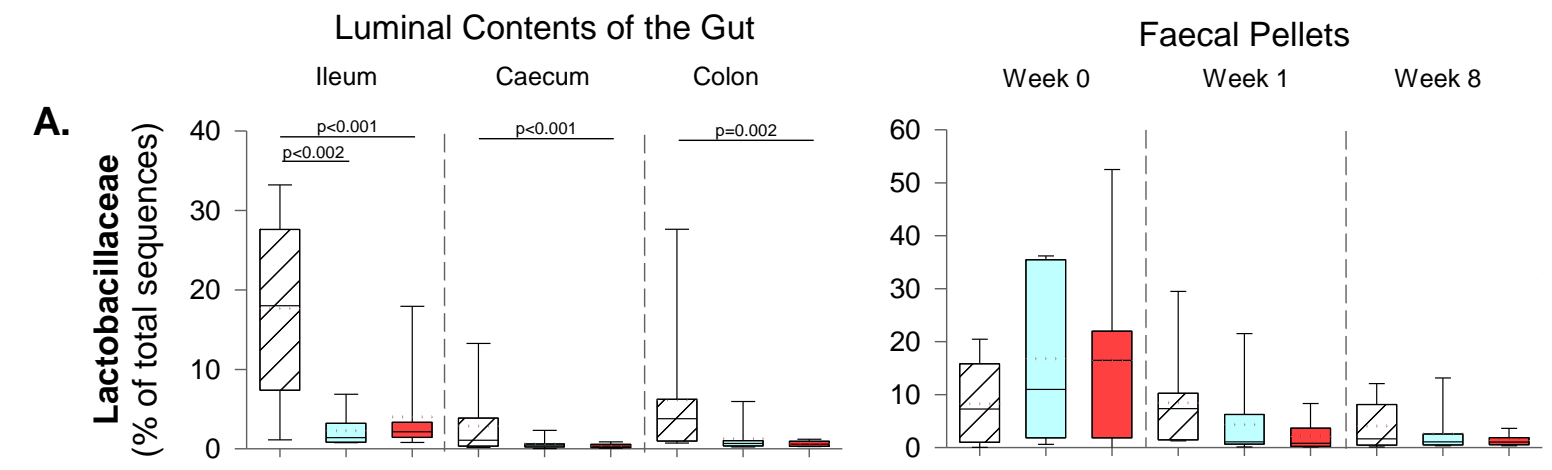

B.
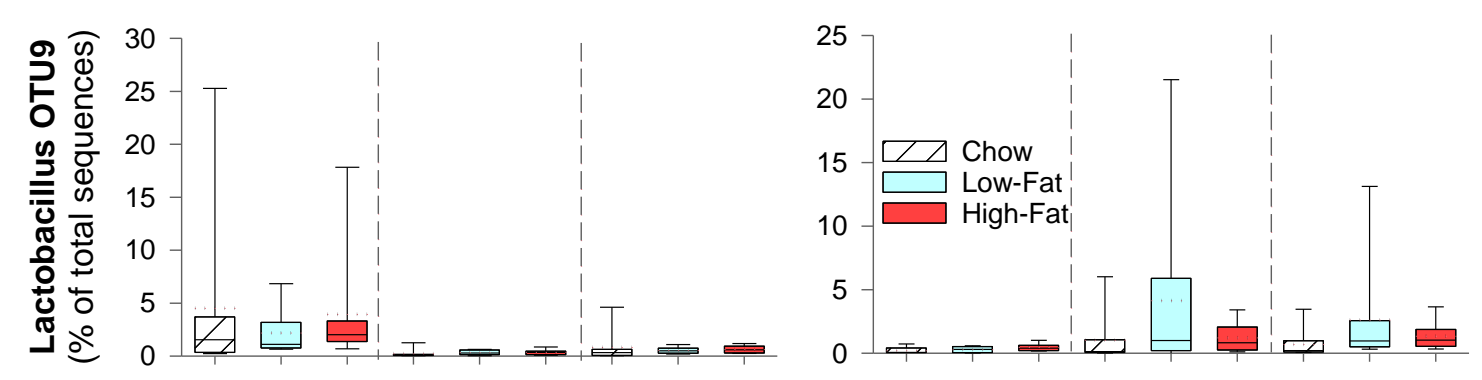

C.
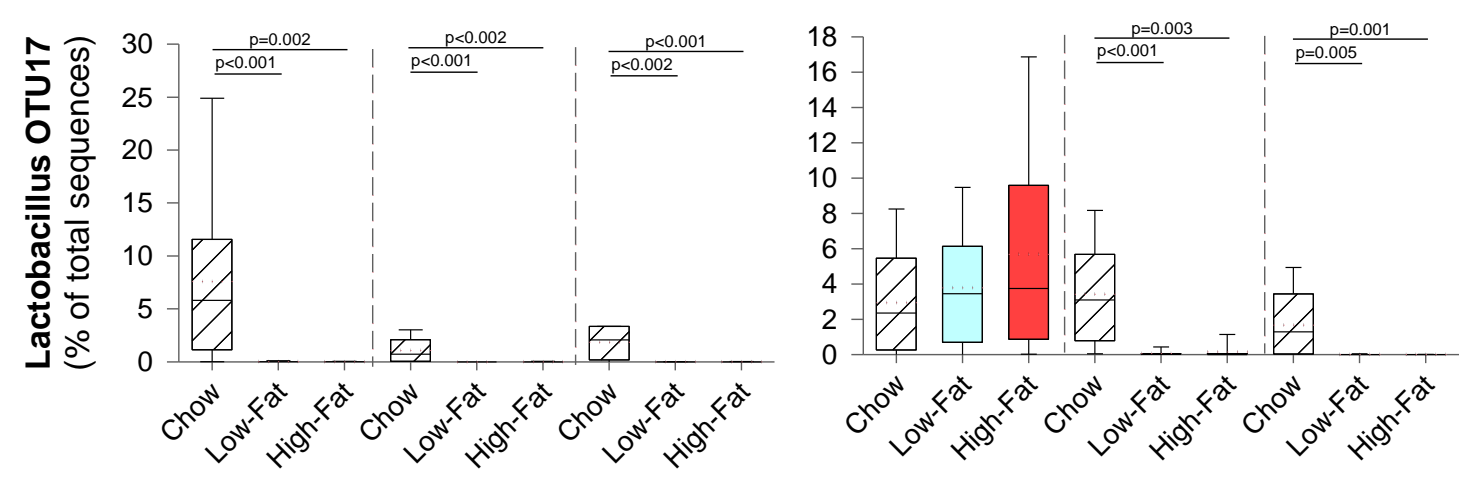

Figure 42. Effect of diet on Lactobacillaceae family and species abundance.

The proportion abundance in Chow, Low-Fat, and High-Fat diet fed mice of the family Lactobacillaceae (A), and the individual Lactobacilli species OTU9 (B), and OTU17 (C), in the ileum, caecum, colon, at the end of the study and faecal pellets at week 0 , week 1 , and week 8. In box plots the solid line = median, and dashed line $=$ mean. Statistical significance was determined by Metastats in Mothur and BenjaminiHochberg correction. $\mathrm{n}=8$ mice per group (Low-Fat Week $1, \mathrm{n}=7$ ). 

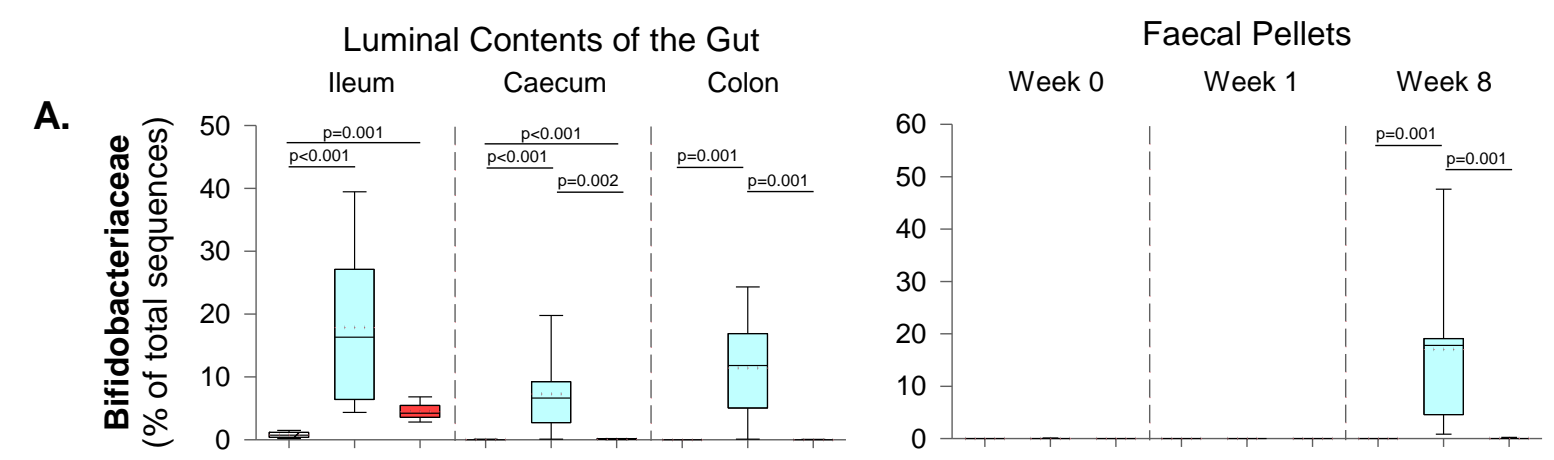

B.
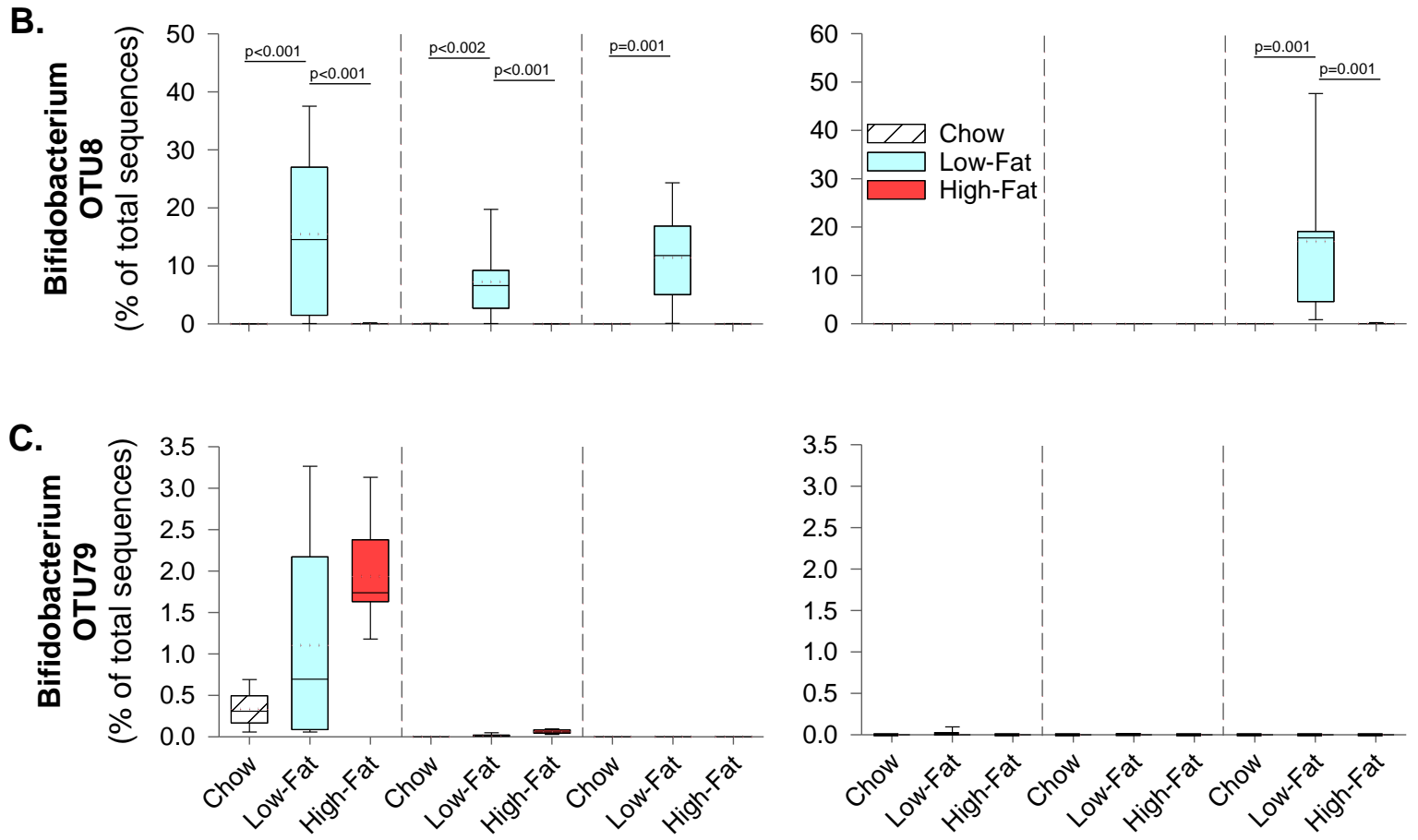

Figure 43. Effect of diet on Bifidobacteriaceae family and species abundance.

The proportion abundance in Chow, Low-Fat, and High-Fat diet fed mice of the family Bifidobacteriaceae (A), and the individual Bifidobacteria species OTU8 (B), and OUT79 (C), in the ileum, caecum, colon, at the end of the study and faecal pellets at week 0 , week 1 , and week 8 . In box plots the solid line $=$ median, and dashed line $=$ mean . Statistical significance was determined by Metastats in Mothur and BenjaminiHochberg correction. $\mathrm{n}=8$ mice per group (Low-Fat Week $1, \mathrm{n}=7$ ). 


\subsubsection{SPECIES CHANGES WITHIN THE PROTEOBACTERIA PHYLUM}

The abundance of the Proteobacteria phylum showed contrary changes between the ileum, and the caecum and colon, Figure 44. Species level analysis revealed that this was due to differing responses to the Chow and refined diets for different species. An unclassified Deltaproteobacteria (OTU10) increased in both Low-Fat and High-Fat diets from a very low initial level in the Chow fed mice. Another Deltaproteobacteria (OTU22) and a Parasutterella (OTU27) decreased in both Low-Fat and High-Fat diets relative to the Chow fed mice. Levels of all three species were similar between the Low-Fat and High-Fat diets. 
Family

Luminal Contents of the Gut

A.
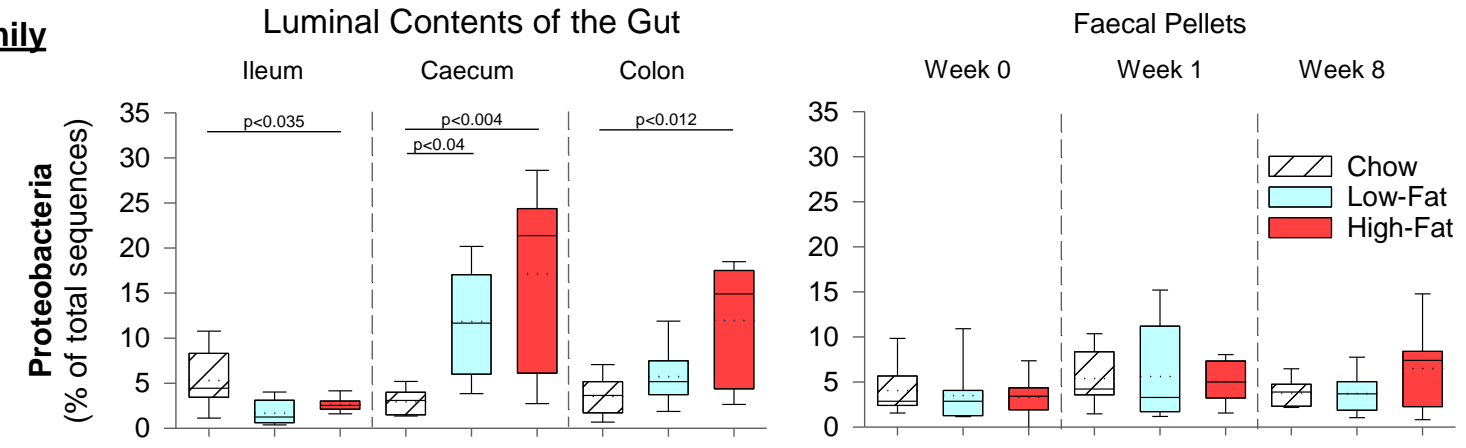

\section{Operational Taxomic Units}

B.
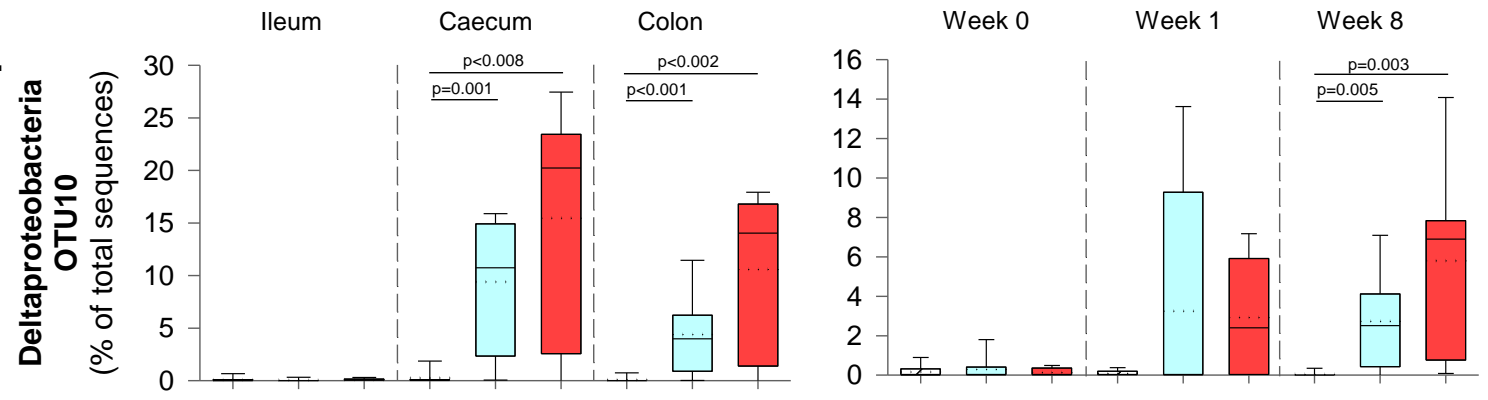

C.
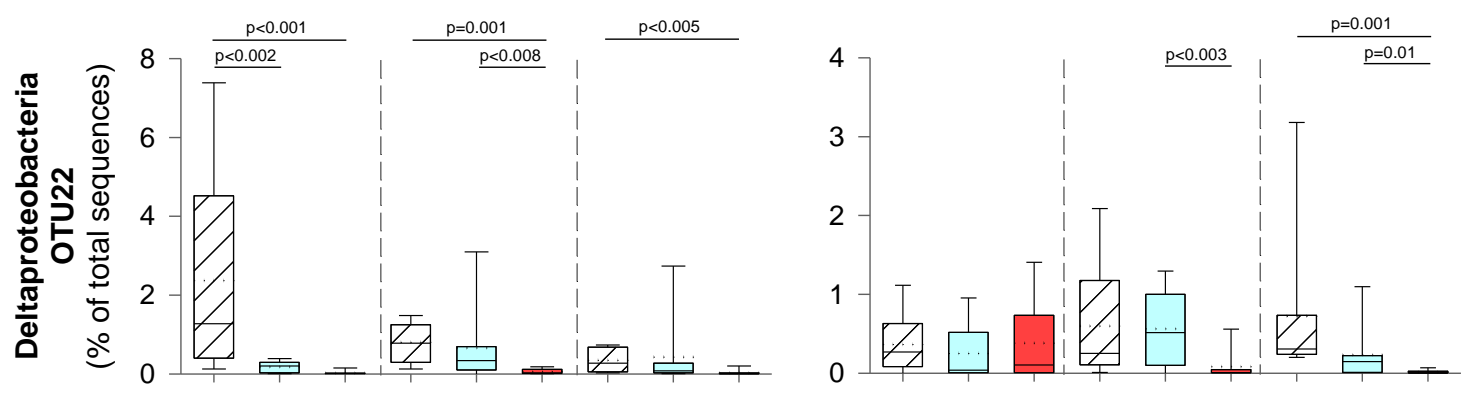

D.
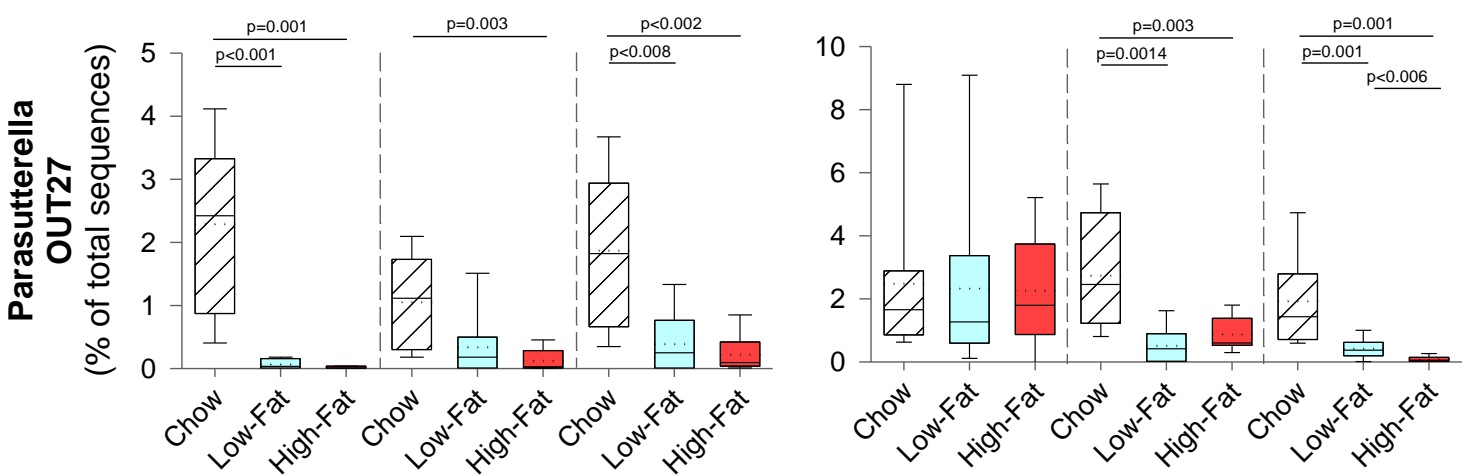

Figure 44. Effect of diet on Proteobacteria phylum and species abundance.

The proportion abundance in Chow, Low-Fat, and High-Fat diet fed mice of the Proteobacteria phylum (A), and the individual Deltaproteobacteria species OTU10 (B), OTU22 (C), and the Parasutterella OTU27 (D), in the ileum, caecum, colon, at the end of the study and faecal pellets at week 0 , week 1 , and week 8 . In box plots the solid line $=$ median, and dashed line $=$ mean . Statistical significance was determined by Metastats in Mothur and Benjamini-Hochberg correction. $\mathrm{n}=8$ mice per group (Low-Fat Week 1 , $\mathrm{n}=7$ ). 


\subsubsection{AKKERMANSIA MUCINIPHILA AND MUCISPIRILLUM SCHAEDLERI}

Akkermansia muciniphila (OTY141) was present at very low levels of abundance in the majority of mice, Figure 45A. There were no significant differences in proportional abundance of $A$. muciniphila between the different diet groups. Mucispirillum schaedleri (OTU34) was present at higher proportional abundance than A. muciniphila. M. schaedleri increased in all groups during the study from Week 0 to Week 8 . There was no significant differences in proportional abundance between diet groups. 
A.
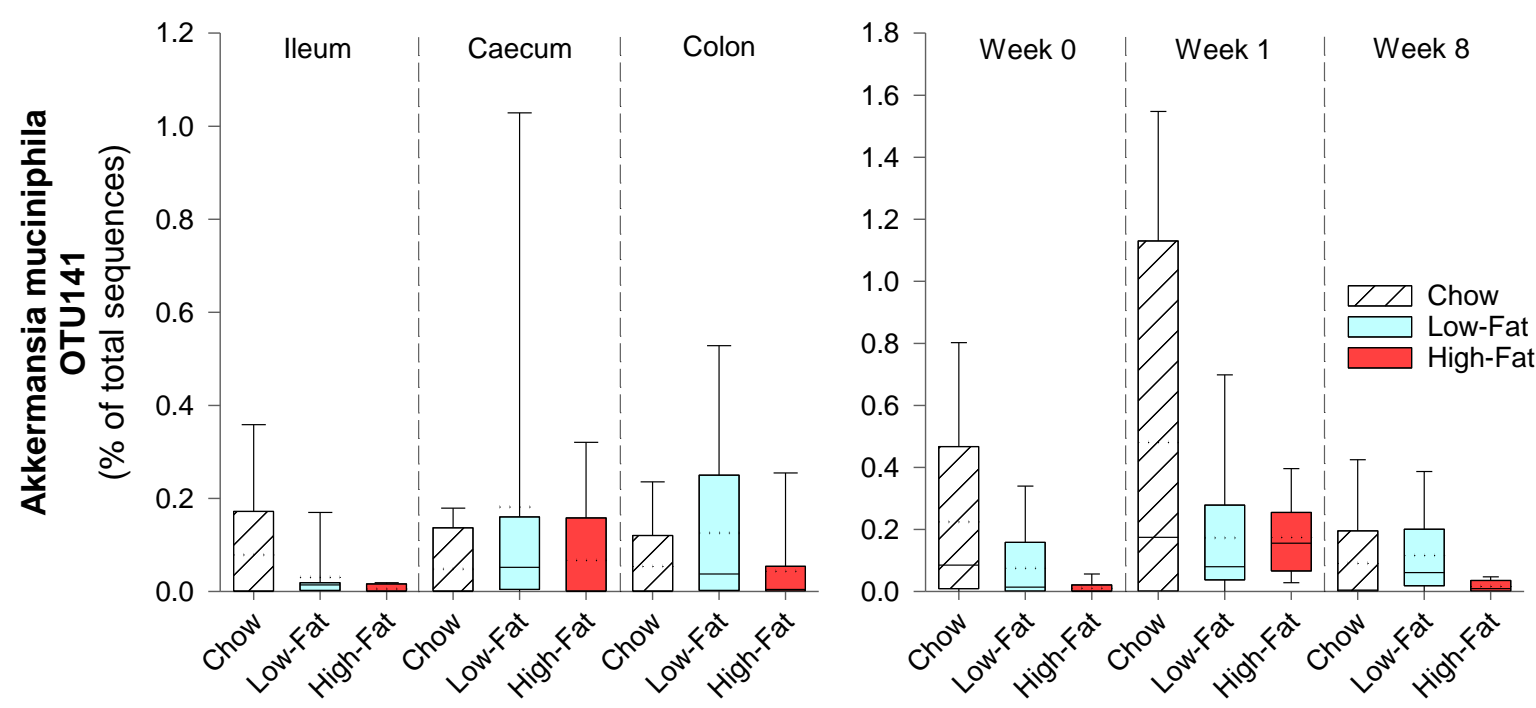

B.
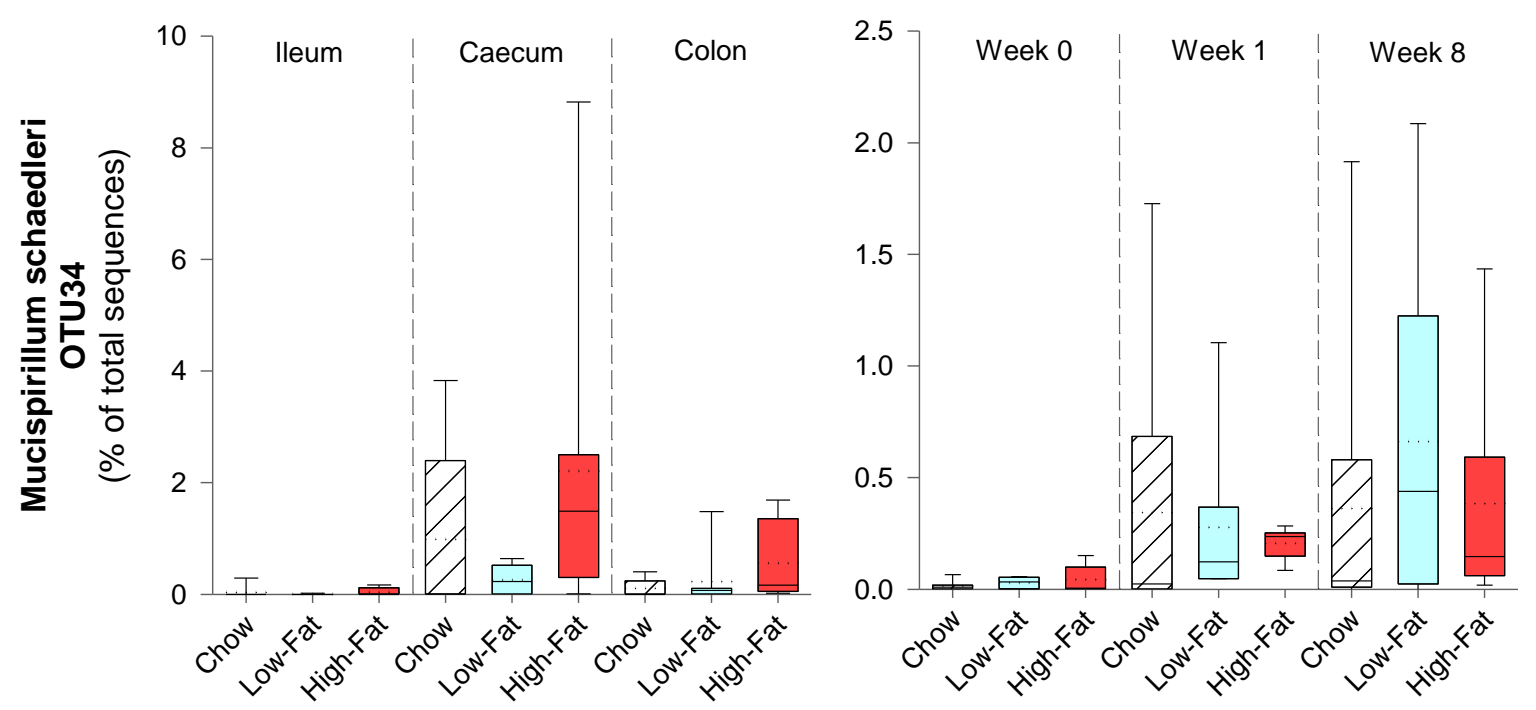

Figure 45. Effect of diet on Akkermansia muciniphila and Mucispirillum schaedleri.

The proportional abundance in Chow, Low-Fat, and High-Fat diet fed mice of Akkermansia muciniphila (A), and Mucispirillum schaedleri (C), in the ileum, caecum, colon, at the end of the study and faecal pellets at week 0 , week 1 , and week 8 . In box plots the solid line = median, and dashed line $=$ mean. Statistical significance was determined by Metastats in Mothur and Benjamini-Hochberg correction. $n=8$ mice per group (Low-Fat Week 1, n=7). 


\subsubsection{SEGMENTED FILAMENTOUS BACTERIA (SFB).}

Segmented Filamentous Bacteria were present in the ileum of Chow fed mice and almost absent from both Low-Fat and High-Fat diet groups, Figure 46. Abundance declined in all groups from Week 0 to Week 8 .
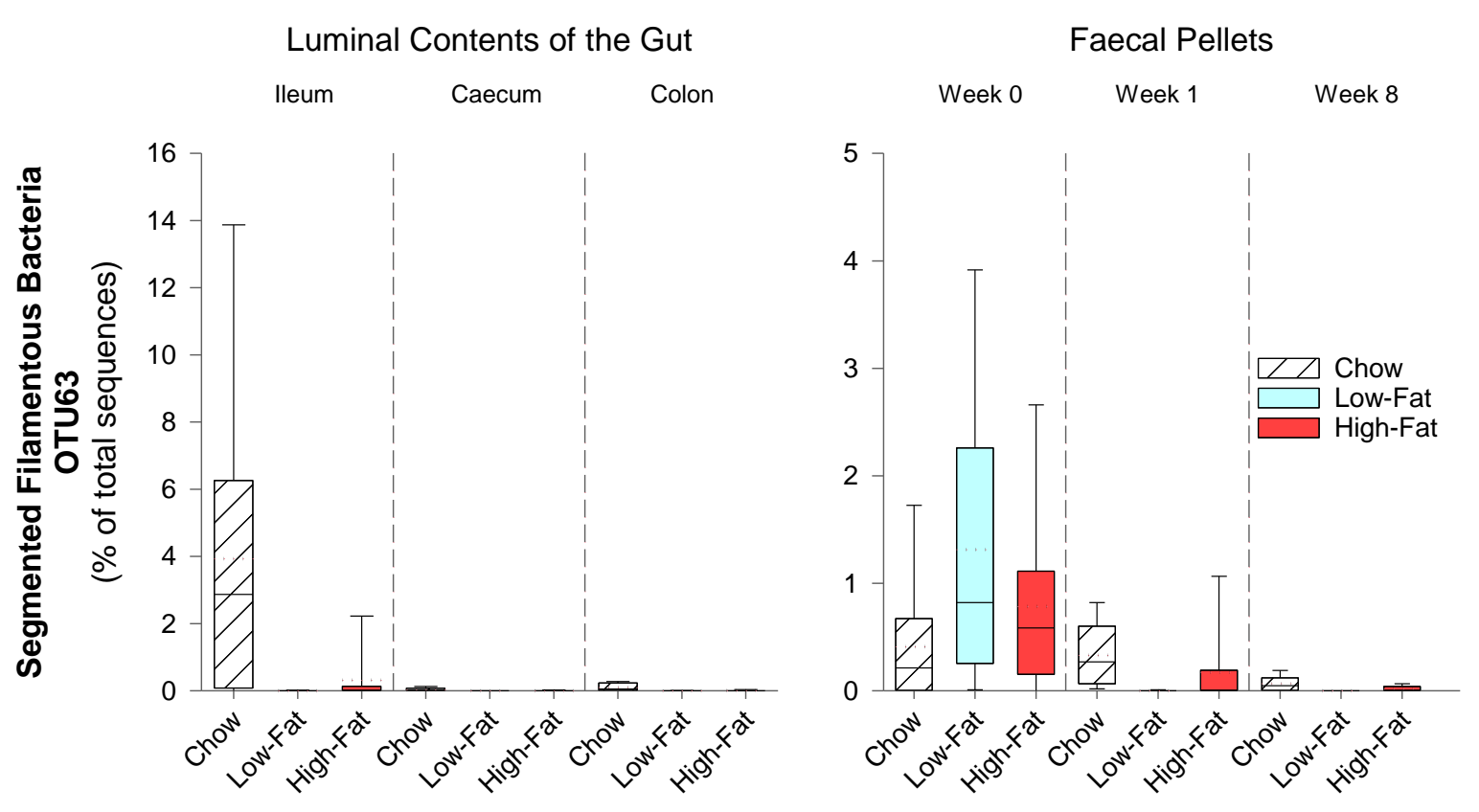

Figure 46. Effect of diet on Segmented Filamentous Bacteria abundance.

The proportional abundance in Chow, Low-Fat, and High-Fat diet fed mice of Segmented Filamentous Bacteria OTU63 in the ileum, caecum, colon, at the end of the study and faecal pellets at week 0 , week 1 , and week 8 . In box plots the solid line = median, and dashed line $=$ mean . 


\subsubsection{SHORT-CHAIN FATTY ACID ANALYSIS OF CAECAL CONTENTS}

Changes in concentration or total amounts of SCFAs were similar in both Low-Fat and High-Fat diet mice relative to Chow. The concentration of short-chain fatty acids (SCFAs) were determined in the caecal contents of mice on the three diets. Concentrations of acetate, propionate, and butyrate were all significantly reduced in the caeca of mice fed Low-Fat and High-Fat diets relative to the Chow diet, see Figure 47AC. To account for the difference in caecal size and amount of contents between the Chow and refined diets the total amount of each SCFA was calculated for the amount of caecal contents for each mouse, D-F. This increased the difference between the between the Chow and refined diets. The concentration of isobutyrate and valerate were unchanged, F-F. Isovalerate was undetectable in the caecum of Chow fed mice but was found at low concentrations in both Low-Fat and High-Fat fed mice, I. There were no differences in the concentrations or total amounts of any individual SCFAs between the Low-Fat and High-Fat diet groups. Comparing the molar ratios of acetate, propionate, and butyrate showed significant changes due to diet, with some variation between individual mice, see Figure 48. Effect of diet on molar ratios of caecal short-chain fatty acids. Figure 48E. Mean caecal ratios of Acetate: Propionate: Butyrate were 66:12:22 for Chow, 74:17:9 for Low-Fat, and 78:12:9 for High-Fat mice respectively. Both Low-Fat and High-Fat resulted in significant increases in the proportion of acetate and a decrease in butyrate, see Figure 48. Effect of diet on molar ratios of caecal short-chain fatty acids.AC. The proportion of propionate was increased in Low-Fat diet mice, Figure 48. Effect of diet on molar ratios of caecal short-chain fatty acids. Figure 48B. Combined concentrations and total amount of acetate, propionate, and butyrate were significantly reduced in Low-Fat and High-Fat diet groups compared to chow, see Figure 48. Effect of diet on molar ratios of caecal short-chain fatty acids. Figure 48F-G. 

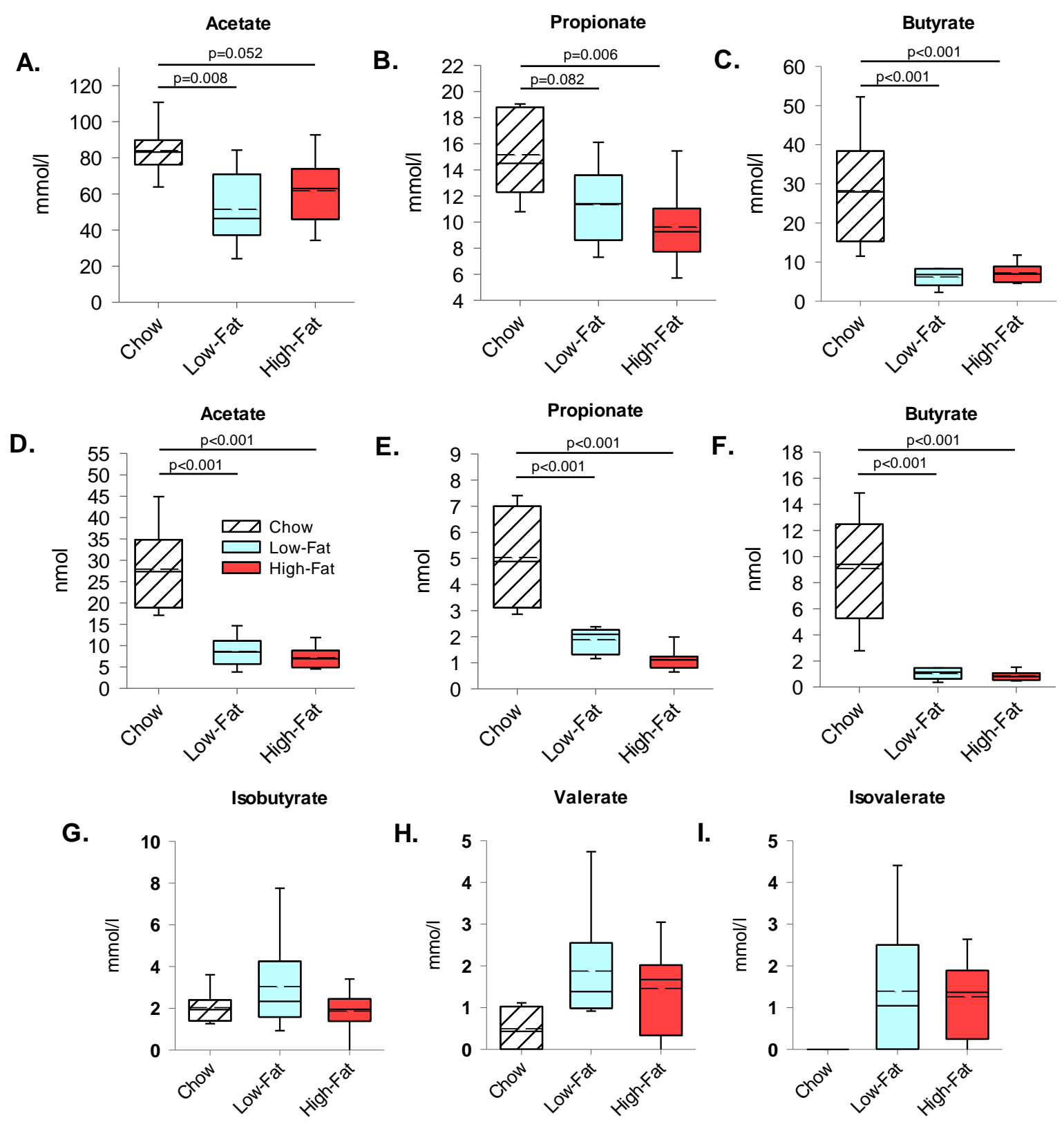

Figure 47. Effect of diet on concentration and total caecal short-chain fatty acids.

Caecal concentrations $(\mathrm{mmol} / \mathrm{l})$ of acetate $(A)$, propionate $(B)$, and butyrate $(C)$ in Chow, Low-Fat, and High-Fat diet fed mice. Total amounts (nmol) of acetate (D), propionate (E), and butyrate (F) in the caecum in Chow, Low-Fat, and High-Fat diet fed mice. Caecal concentrations isobutyrate $(G)$, valerate $(H)$, isovalerate (I) in Chow, LowFat, and High-Fat diet fed mice. In box plots the solid line = median, and dashed line = mean. Statistical significance was determined by one-way ANOVA with Tukey's post hoc test for Chow and High-Fat $n=8$, for Low-Fat $n=6$. 


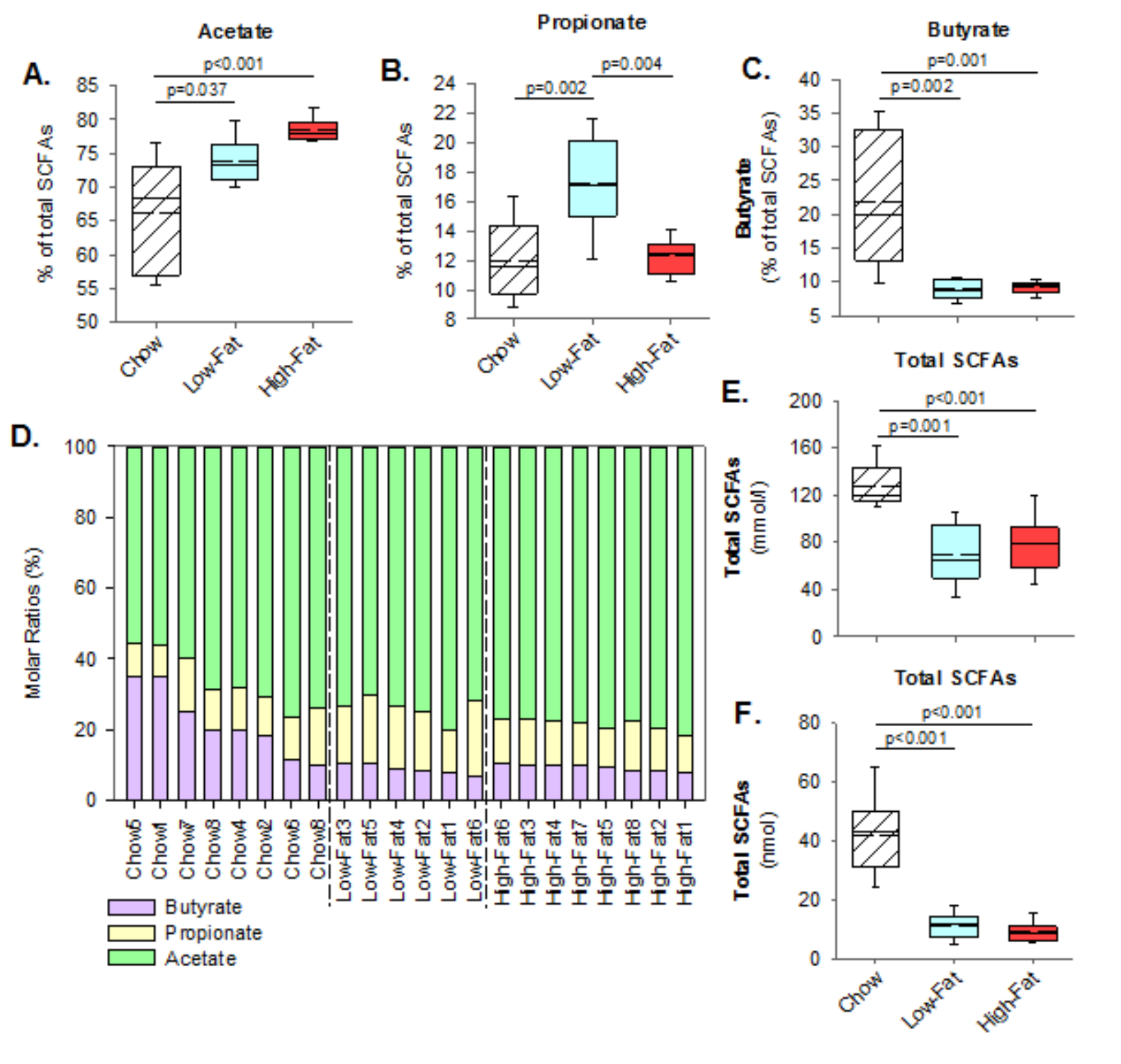

Figure 48. Effect of diet on molar ratios of caecal short-chain fatty acids.

Percentage of acetate (A), propionate (B), and butyrate (C) of total SCFAs in the caecum of Chow, Low-Fat, and High-Fat diet fed mice. Molar ratios of acetate, propionate, and butyrate for individual mice (D). Concentrations of combined SCFAs (E), and total amount of SCFAs (F), in Chow, Low-Fat, and High-Fat diet fed mice. In box plots the solid line $=$ median, and dashed line $=$ mean. Statistical significance was determined by one-way ANOVA with Tukey's post hoc test for Chow and High-Fat n=8, for Low-Fat $\mathrm{n}=6$. 


\subsection{CORRELATIONS BETWEEN MICROBIOTA COMPOSITION AND SHORT-CHAIN FATTY ACIDS}

To identify individual bacteria associated with change in SCFA concentrations the proportional abundance of individual OTUs was correlated with the concentration of individual SCFAs in the caecum using the corr.test function (Psych Package 1.7.3.21, R). A total of 209 individual OTUs were present in the caecal contents of the 24 mice in the Chow, Low-Fat and High-Fat diet groups and these were correlated against caecal concentrations of acetate, propionate, and butyrate, see Table 11. When all mice were compared together 3 OTUs correlated with acetate, 3 OTUs correlated with propionate, and 6 OTUs correlated with butyrate concentration. Individual plots of coloured by diet group are shown in A-L. These were classified as members of the families Lachnospiraceae and Ruminococcaceae, the orders Clostridiales and Bacteroidales, and one to the phylum Bacteroidetes. The OTU71 and OTU86 were correlated with acetate, propionate, and butyrate concentrations. When the OTU and SCFAs data within each diet group were correlated separately there was only one significant correlation between OTU49 and butyrate within the Chow diet group, see Table 11 and Figure 49.

Table 11. Correlations between OTU abundance and SCFA concentration.

\begin{tabular}{|c|c|c|c|c|c|c|c|c|}
\hline & \multicolumn{2}{|c|}{ All diet groups } & \multicolumn{2}{|l|}{ Chow } & \multicolumn{2}{|c|}{ Low-Fat } & \multicolumn{2}{|c|}{ High-Fat } \\
\hline OTUs & Correlation ( $r$ ) & P-value & Correlation ( $r$ ) & P-value & Correlation ( $r$ ) & P-value & Correlation (r) & P-value \\
\hline \multicolumn{9}{|l|}{ Acetate } \\
\hline OTU71 (Lachnospiraceae) & 0.75 & 0.007 & 0.07 & 1.0 & 0.48 & 1.0 & 0.64 & 0.4 \\
\hline OTU160 (Lachnospiraceae) & 0.74 & 0.01 & 0.49 & 1.0 & NA & NA & NA & NA \\
\hline OTU86 (Ruminococcaceae) & 0.69 & 0.05 & -0.67 & 0.6 & 0.47 & 1.0 & NA & NA \\
\hline \multicolumn{9}{|l|}{ Propionate } \\
\hline OTU173 (Clostridiales) & 0.71 & 0.03 & 0.31 & 1.0 & 0.20 & 1.0 & NA & NA \\
\hline OTU86 (Ruminococcaceae) & 0.71 & 0.03 & -0.52 & 1.0 & 0.16 & 1.0 & NA & NA \\
\hline OTU71 (Lachnospiraceae) & 0.69 & 0.05 & -0.10 & 1.0 & 0.22 & 1.0 & 0.7 & 0.4 \\
\hline \multicolumn{9}{|l|}{ Butyrate } \\
\hline OTU49 (Bacteroidales) & 0.84 & 0.0001 & 0.95 & 0.002 & NA & NA & 0.41 & 0.9 \\
\hline OTU86 (Ruminococcaceae) & 0.83 & 0.0002 & 0.33 & 1.0 & 0.47 & 1.0 & NA & NA \\
\hline OTU71 (Lachnospiraceae) & 0.80 & 0.0009 & 0.31 & 1.0 & 0.19 & 1.0 & 0.66 & 0.4 \\
\hline OTU142 (Bacteroidetes) & 0.71 & 0.03 & 0.55 & 1.0 & -0.18 & 1.0 & 0.13 & 1.0 \\
\hline OTU202 (Bacteroidales) & 0.71 & 0.03 & -0.19 & 1.0 & NA & NA & NA & NA \\
\hline OTU193 (Ruminococcaceae) & 0.70 & 0.04 & 0.02 & 1.0 & -0.12 & 1.0 & 0.50 & 0.8 \\
\hline
\end{tabular}




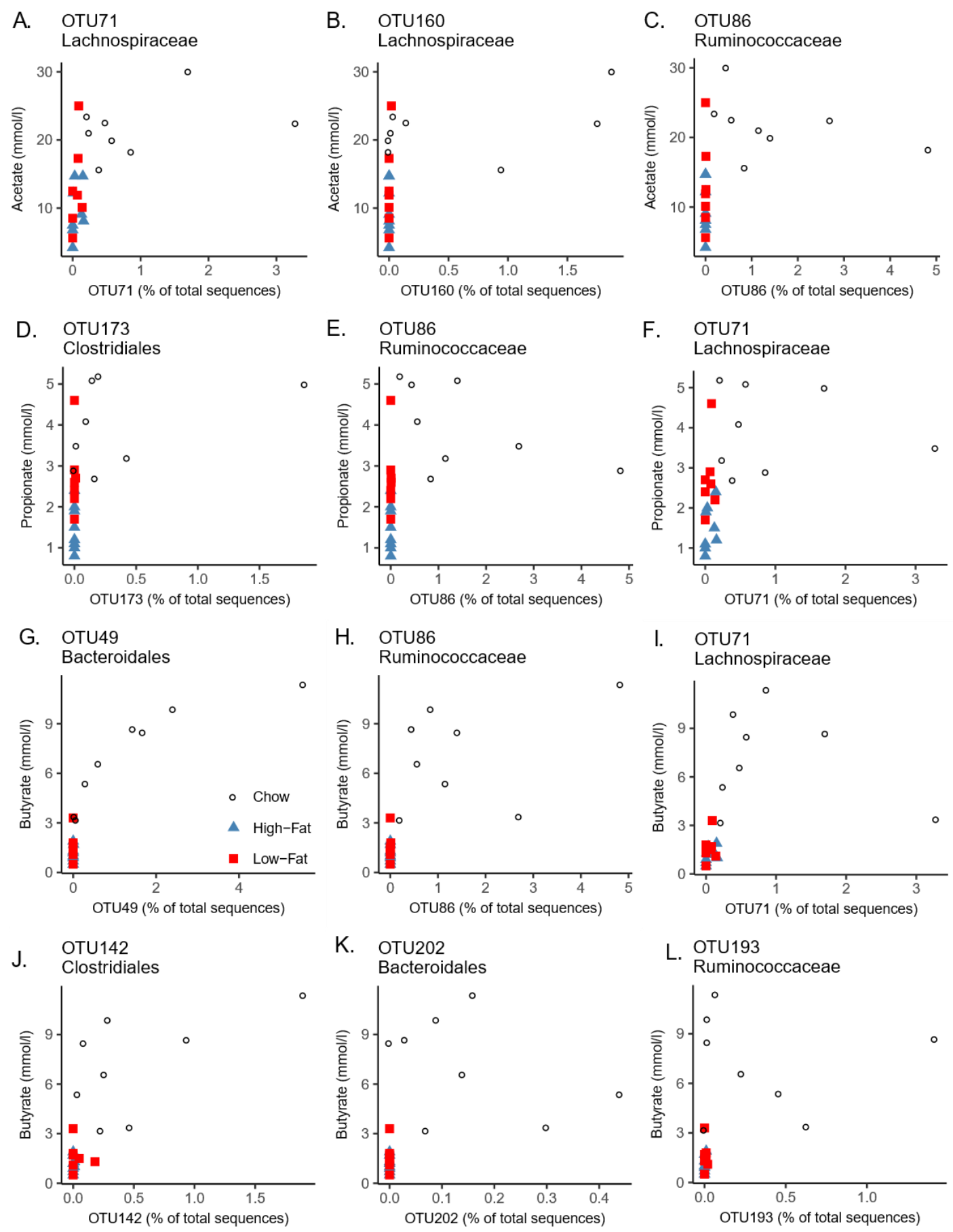

Figure 49. OTU abundance plotted against SCFA concentration.

Proportional abundance of OTUs correlating with acetate $(\mathrm{A}-\mathrm{C})$, correlating with propionate (D-F), and correlating with butyrate $(\mathrm{G}-\mathrm{L})$. 


\subsubsection{COMPARISON OF THE COMPOSITION OF DIFFERENT CHOW DIETS.}

The macronutrient composition and ingredients of commercially available refined and chow diets were compared using publicly available diet datasheets provided by the manufacturer. The macronutrient composition of diets used in previous studies, where refined and chow diets had been compared, were found to vary considerably in their macronutrient composition, see Figure 50A. While the ratio of sucrose and starch varied in refined low-fat diets and chow diets the percentage of energy from protein was consistently greater in chow diets than in refined diets, see Figure 50B. The comparison of ingredient lists used in the chow diets showed that different chow diets contain a diverse range of ingredients, see Table 12. Chow diets containing a higher percentage of protein included soybean products higher on the ingredients list. While cereal grains and legumes formed the bulk of the ingredients, the chow from LabDiets also included animal products including fish meal and whey. Rodent chow diets can contain a wide range of macronutrient percentages and differing ingredients. 
A. Present Study

1. Refined (D12492)

2. Refined (D12450J)

3. Chow (SDS B\&G)

(Chassaing et al, 2015)

4. Refined (D12492)

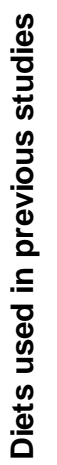

5. Refiend (D12450B)

6. Chow (LabDiet 5002)

(Blaisdell et al, 2014)

7. Refined (D12450B)

8. Chow (Labdiets 5001)

(Benoit et al, 2013)

9. Refined (SAFE HFD)

10. Refined (SAFE LFD)

11. Chow (SAFE A04)

(Apolzan \& Harris, 2012)

12. Refined (D12450B)

13. Chow (Harlan 8604)

(Mirhashemi et al, 2010)

14. Refined (D12450B)

15. Refined (Low-Sucrose)

16. Chow (ssniff@t R/M-H)

Protein (\%)

Fat $(\%)$

Sucrose (\%)

Starch (\%)

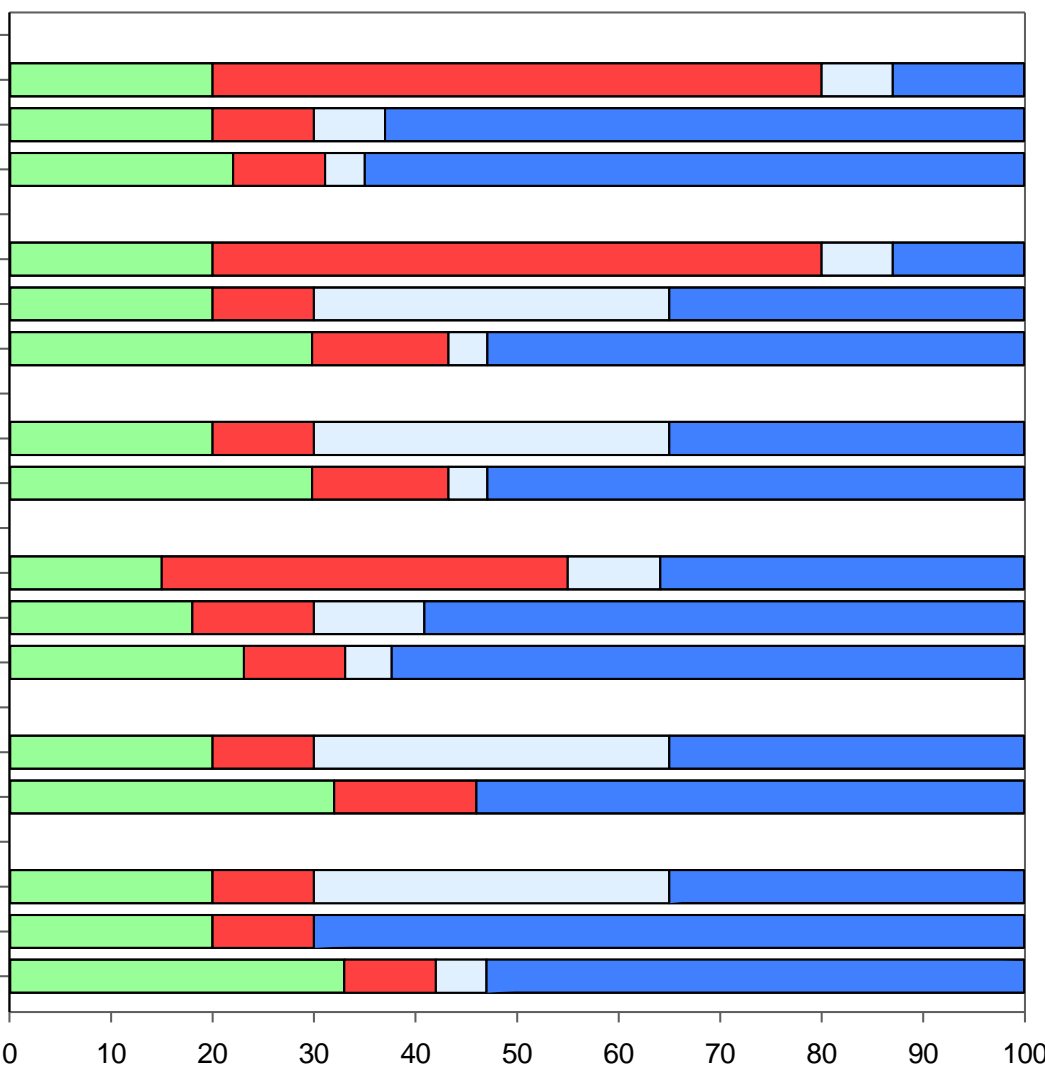

B. Macronutrients (percentage of total calories)

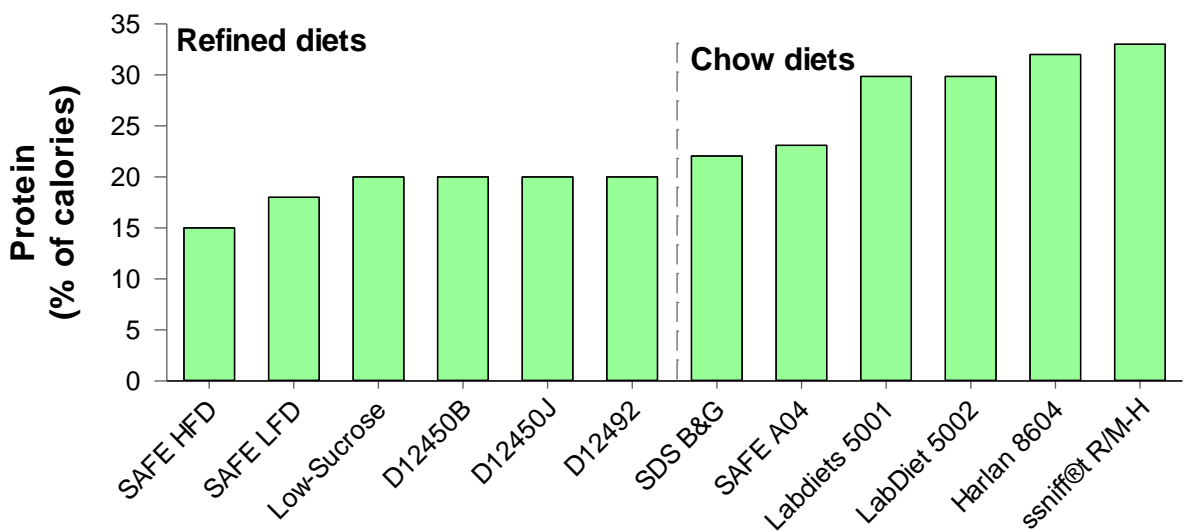

Refined and chow diets ordered by protein percentage

Figure 50. Comparison of diet macronutrient percentages in previous studies.

The relative proportion of protein, fat, sucrose, and starch as a percentage of energy in chow and refined mouse diets used in previous studies compiled from publicly available diet composition data sheets (A). Protein content of diets ordered by contribution of protein as a percentage of energy (B). 
Table 12. Comparison of ingredients between a refined diet and chow diets.

\begin{tabular}{|c|c|c|c|c|c|}
\hline \multirow{2}{*}{\begin{tabular}{|l|}
\multicolumn{1}{|c|}{ Refined } \\
Research \\
Diets D12450J
\end{tabular}} & \multicolumn{5}{|c|}{ Chow } \\
\hline & $\begin{array}{l}\text { SDS Breeder } \\
\text { and Grower }\end{array}$ & SAFE A04 & LabDiets 5001 & LabDiets 5002 & Harlan Teklad \\
\hline Corn starch & Wheat & Barley & Dehulled soyabean & Ground corn & Dehulled soybean \\
\hline Casein & Wheatfeed & Wheat & Ground œrn & Dehulled soyabean & Ground corn \\
\hline Maltodextrin & Barley & Corn & Dried beet pulp & Whole wheat & Wheat middlings \\
\hline Sucrose & $\begin{array}{l}\text { De-hulled toasted } \\
\text { Soya }\end{array}$ & Soya meal & Fish meal & Fish meal & Flaked corn \\
\hline Cellulose & Maize & Wheat bran & Ground Oats & Wheat middlings & Fish meal \\
\hline Soybean oil & Macro minerals & $\begin{array}{l}\text { Hydrolyzed fish } \\
\text { protein }\end{array}$ & Alfalfa meal & Dried beet pulp & Cane molasses \\
\hline Lard & Soya oil & $\begin{array}{l}\text { Vitamins and } \\
\text { minerals }\end{array}$ & Cane molasses & Wheat germ & Soybean oil \\
\hline Potassium citrate & Potato protein & $\begin{array}{l}\text { Dicalcuim } \\
\text { phosphate }\end{array}$ & Brewers yeast & Cane molasses & Ground wheat \\
\hline $\begin{array}{l}\text { DiCalcium } \\
\text { phosphate }\end{array}$ & $\begin{array}{l}\text { Hydrolised Wheat } \\
\text { Gluten }\end{array}$ & Calcium carbonate & Wheat germ & Brewers yeast & Dried whey \\
\hline Mineral Mix & Full fat soya & & Whey & Ground oats & $\begin{array}{l}\text { Dicalcium } \\
\text { phosphate }\end{array}$ \\
\hline Vitamin Mix & Maize gluten meal & & Lard & Soybean il & Calcium \\
\hline Calcium carbonate & Vitamins, & & Wheat middlings & Alfalfa Meal & Carbonate \\
\hline L-Cystine & Micro minerals & & Lard/bone meal & $\begin{array}{l}\text { Ground soybean } \\
\text { hulls }\end{array}$ & Brewers yeast \\
\hline Choline bitartrate & Amino acids & & Salt & Whey & Iodized Salt \\
\hline $\begin{array}{l}\text { FD\&C Yellow Dye } \\
\# 5\end{array}$ & & & Calcium carbonate & Calcium carbonate & $\begin{array}{l}\text { Vitamins and } \\
\text { Minerals }\end{array}$ \\
\hline FD\&C Blue Dye \#1 & & & $\begin{array}{l}\text { Vitamins and } \\
\text { Minerals }\end{array}$ & $\begin{array}{l}\text { Casein } \\
\text { Salt } \\
\text { Vitamins and } \\
\text { Minerals }\end{array}$ & \\
\hline
\end{tabular}

Ingredients listed in descending order of contribution by weight. 


\section{DISCUSSION}

This study shows that 8 weeks of feeding mice a refined Low-Fat diet does not induce any increase in body weight, body fatness, or glucose intolerance relative to the Chow diet. Feeding a refined High-Fat diet resulted in a rapid gain in body weight, body fat, and glucose intolerance relative to both the Chow and Low-Fat control diets. This was as expected for this well-established obesogenic diet in C57BL/6 mice and conforms to the results in wild-type mice from Chapter 1 . In contrast the effects of diet of gut morphology, gut microbiota composition, and short-chain fatty acid production were significantly changed by both the Low-Fat and High-fat diets relative to the Chow diet. This has major implications in terms of the functional relationship between gut microbiota and obesity.

\subsection{BODY WEIGHT AND BODY COMPOSITION}

The lack of any differences in body weight or body fatness, between the mice fed the Chow diet or the Low-Fat diet is a marked difference to the few previous studies that have compared refined and chow diets in rodents (Apolzan \& Harris, 2012; Benoit et al, 2013; Blaisdell et al, 2014; Chassaing et al, 2015; Mirhashemi et al, 2011; Sakamoto et al, 2012). It seems that differences in the composition of the chow and defined diets used across different studies is the most likely explanation for the difference in response seen here with previous studies.

\subsubsection{DIETARY FIBRE}

It has been proposed that the lack of soluble fibre in refined low-fat diets is responsible for increases in body weight, and body fat relative to a chow diet (Chassaing et al, 2015). Soluble fibre and bacterial fermentation of fibre to short-chain fatty acids have been shown to reduce adiposity and bodyweight in mice fed an obesity inducing high-fat diets. The SDS Rat and Mouse Breeder and Grower diet used in the present study was reported to contain $15 \%$ total dietary fibre (pectin 1.4\%, hemicellulose $8.85 \%$, cellulose 3.89, and lignin 1.4\%) consistent with other chow diets. Chow composed of wheat products will contain arabinoxylans a fermentable hemicellulose previously shown to counteract obesity in mice (Neyrinck et al., 2011). Addition of fibre to the diet of rats has similarly been shown to reduce body fat in high fat diet fed rats (Adam et al, 2015a). 
Inulin, another fermentable fibre, reduced weight gain and total body adiposity in Male C5713L/6 mice when added to high-fat diet at either 7.5\% (Anastasovska et al., 2012) or 10\% of the diet (Frost et al., 2014). A similar effect was seen for fructooligosaccharides when added at $2.5 \%$ of the diet (Nakamura et al., 2011). The Chow diet used in the present study contained about $10 \%$ of the diet as fermentable fibre compared to the LowFat containing just 5\% fibre as pure cellulose. The lack of any difference in body weight or total body fat despite this difference in dietary fibre content suggests that fibre is unlikely to be the cause of the reduced body weight seen using chow diets in previous studies.

\subsubsection{SUCROSE}

The amount of sucrose included in diets is a potential differentiating factor. The commonly used Research Diets control diet D12450B was used in all but one study and contained 35\% of energy from sucrose. In the present study, the Low-Fat diet used (D12450J, Research Diets) contained 7\% sucrose, similar to the chow diet which contained only a small amount of naturally occurring sugars. In a previous study feeding C57BL/6J mice a refined 38.5\% sucrose diet for 5 weeks did not increase bodyweight or fat relative to starch (Sakamoto et al., 2012). In another New Zealand Obese (NZO) mice fed a $34.5 \%$ sucrose diet for 21 weeks did not increase body weight relative to starch (Mirhashemi et al., 2011). Refined diets resulted in higher body weight than chow regardless of sucrose content suggesting that sucrose is not an obesogenic factor for mice fed refined diets.

\subsubsection{PHYTOESTROGENS}

Unlike refined diets, Chow diets contain phytoestrogens due to the use of soya beans as a main protein source. The content of isoflavones in laboratory rodent diets varies between different formulations and from batch to batch (Jensen \& Ritskes-Hoitinga, 2007). The addition of phytoestrogens to the diet from soy have previously can reduce body weight and adiposity in mice (Cederroth et al, 2007). The LabDiets D5001 chow has been shown to contain some of the highest levels of phytoestrogens found in rodent chow, at concentrations capable of influencing mouse physiology (Jensen \& RitskesHoitinga, 2007). The LabDiets 5001 and LabDiets 5002 chow diets also contain alfalfa a particularly rich source of phytoestrogens (Jensen \& Ritskes-Hoitinga, 2007). These 
chow diets were used in two previous studies comparing chow and refined diets (Blaisdell et al, 2014; Chassaing et al, 2015). In contrast the SDS Breeder and Grower chow used in the present study contains less soy and no alfalfa.

\subsubsection{PROTEIN}

Chow diets contain up to $30 \%$ of energy from protein while refined diets contain only $20 \%$ of energy as protein. Increasing the energy contribution from protein from $20 \%$ to $30 \%$ has been shown to significantly reduce the body weight and body fat of mice (West et al, 1995). The 10\% higher level of protein in the chow diet could account for the reduced body weight in chow fed mice relative to the low-fat diets in previous studies. The Chow diet used in the present study contained $22 \%$ protein as energy relative to the $20 \%$ protein in the Low-Fat diet. This is considered likely to account for the lack of difference in body weight or body fat between Chow and Low-Fat fed mice.

\subsubsection{SUMMARY OF CONFOUNDERS IN PREVIOUS STUDIES}

Previous studies that found body weight differences between chow and refined low-fat diets contained confounding factors such as differences in protein and potentially high levels of soy phytoestrogens (Apolzan \& Harris, 2012)(Blaisdell et al., 2014)(Chassaing et al, 2015)(Mirhashemi et al., 2011)(Sakamoto et al, 2012). In summary, the present study used Chow and Low-Fat diets that were well matched for protein content, macronutrient composition, and are unlikely to contain high phytoestrogens. The difference in fibre content was similar to those used in previous studies. This similar protein content is the most likely explanation for the lack of any obesogenic effect of the refined low-fat diet on mice in the present study relative to the chow. The present study provides evidence that refined diets are not intrinsically obesogenic if the macronutrient ratios are closely matched despite the lack of dietary fibre. The interpretation of the following results rests on there being no difference in body weight or body fat between the Chow and Low-Fat groups of mice.

\subsection{FOOD AND ENERGY INTAKE}

The reduced food consumption in Low-Fat fed mice relative to Chow is likely to be compensation for the higher energy density of the Low-Fat diet. The further reduction in High-Fat diet food intake represented a further compensation for the higher caloric 
density. The significantly higher energy intake in High-Fat diet fed mice accounted for the increased body fat and weight gain.

\subsubsection{ACCURATE ESTIMATES OF CHOW ENERGY}

Unrefined diets have a lower metabolizable energy than refined Low-Fat and High-Fat diets with mean digestibility of the gross energy in natural diets amounting to $71.4 \%$ (range 53.3-83.5\%) (Bielohuby et al, 2010). The Atwater system figures were found to be accurate for Chow diets in rats and so were used to determine the energy consumed from the Low-Fat and High-Fat diets. Digestible energy is an estimate of the energy available to the animal from digestion of a diet. However, the estimates for Chow diets have been found to be prone to significant error (Bielohuby et al, 2010). There is little published data on digestibility of Chow diets in mice and equations based on larger animals may not apply. Due to Atwater values not being accurate for Chow diets the manufacturer's calculated value for Digestible energy was used to calculate energy intake for Chow fed mice.

\subsection{GLUCOSE INTOLERANCE}

The higher glucose after intraperitoneal glucose tolerance tests in High-Fat diet mice with no differences between Chow and Low-Fat diet mice is consistent with the lack of difference in body weight or body fat between these mice in this study. The lack of any difference in glucose response between the Chow and Low-Fat is of interest regarding the changes in the gut microbiota composition and caecal short-chain fatty acids discussed later.

\subsection{GUT MORPHOLOGY}

The reduction in gut length and weight in both refined low-fat and high-fat diet groups indicate that this was not due to the fat content of the diet. This is not the first time that changes in gut morphology have been seen with decreased fibre intake. This is most likely due to the reduction in soluble fibre in the refined diet groups in comparison to the chow (Bielohuby et al, 2010). Similar effects on gut morphology to the present study have been observed in rats fed semi purified diets supplemented with the fermentable fibre pectin (Adam et al, 2015a; Adam et al, 2015b). The addition of containing raw peas to the semi purified diet of rats resulted in a marked increase in caecal tissue and 
contents (Goodlad \& Mathers, 1990). The addition of fibre can also result in increases in intestinal crypt cell production rate in both the small intestine and the colon (Goodlad et al, 1989). The proliferative effects of fibre were abolished in the germ-free rats suggesting that bacterial fermentation, not fibre itself stimulates intestinal epithelial cell proliferation in intestine (Goodlad et al, 1989). The gut shrinkage seen in the present study occurred similarly in both Low-Fat and High-Fat diets and was disassociated from the body weight, body fat, and glucose intolerance changes. Substantial morphological changes can be produced in the gut without influencing metabolism or adiposity.

\subsection{MICROBIOTA COMPOSITION}

One previous study has noted that broad phylum level changes can occur in the absence of obesity itself. Resistin-like molecule-beta (RELM $\beta)$ knockout mice display a high-fat diet induced microbiota composition while resistant to obesity development (Hildebrandt et al, 2009). Similar to the present study these included phylum level changes, an increased proportion of Firmicutes and Proteobacteria, and a reduction in Bacteroidetes (Hildebrandt et al, 2009). The present study however takes this result much further. These phylum level changes were reproduced in wild-type C57BL/6 mice with a refined low-fat diet. These changes could be determined to a species level disassociated from obesity, occurring in both Low-Fat and High-Fat refined groups relative to the Chow group.

\subsubsection{RATIO OF FIRMICUTES TO BACTEROIDETES IS NOT LINKED TO OBESITY}

The ratio between the proportions of Firmicutes and Bacteroidetes phyla has been proposed as a marker and mediator of obesity in mice and humans associated with increased energy harvest (Ley et al, 2005). An increases ratio of Firmicutes to Bacteroidetes have been reported in genetically obese ob/ob mice (Ley et al, 2005; Turnbaugh et al, 2006) and in High-Fat diet induced obese mice (Cani et al, 2007a; Everard et al, 2014; Hildebrandt et al, 2009; Turnbaugh et al, 2008). The increase is the Firmicutes to Bacteroidetes ratio has also been reported in human studies comparing obese subjects and lean subjects (Ley et al, 2006), although the opposite has also been observed (Schwiertz et al, 2010) and the evidence remains conflicting in humans. A recent reanalysis of the data from 10 previous studies failed to find significant 
associations between the Firmicutes to Bacteroidetes ratio and obesity, suggesting that previous studies were underpowered (Sze \& Schloss, 2016).

In the present study, the increased ratio of Firmicutes to Bacteroidetes in the ileum, colon, and faeces of both Low-Fat and High-Fat diet mice suggest that previous associations of an increased ratio of Firmicutes to Bacteroidetes is most likely an artefact arising from the use Chow diets. The dramatically increased ratio seen in the ileum and the absence of similar changes in the caecum suggest that sampling more than one site in the gut provided a more complete picture of the effects of this dietary intervention. Recent research found significant variations in the Firmicutes to Bacteroidetes ratio mice from animal facilities across Germany that were not associated with any differences in body weight (Rausch et al, 2016). The present study shows that changes in the ratio of Firmicutes to Bacteroidetes can be confounded by the use of chow as control diets.

\subsubsection{CHANGES IN MICROBIOTA DIVERSITY IS NOT ASSOCIATED WITH OBESITY}

It has been suggested that in High-Fat induced obesity in mice diets the accompanying loss of microbial diversity may be an important component for the development of obesity (Parks et al, 2013). The Shannon diversity index was reduced in mice made obese using a Western High-Fat diet, due to a bloom in Erysipelotrichaceae (Turnbaugh et al, 2008). In the present study, this class of bacteria only increased in the Low-Fat diet. Reduced microbial diversity has been associated with obesity in other studies both in mice and humans. However, a recent reanalysis of these datasets found that sample sizes were generally insufficient to detect the relatively small differences between lean and obese individuals and that detection of differences was confounded by large interpersonal variation (Sze \& Schloss, 2016). Recently a refined diet used for 6 weeks reduced the faecal microbial diversity of mice (Sonnenburg et al, 2016). Unfortunately, no body weight or physiological measures were reported in this study. This study used germ-free mice, humanised by implantation with human gut bacteria. The results of the present study refute the hypothesis that reduced microbiota diversity contributes to obesity as diversity was unchanged at most sites across the three diets used. Where diversity was reduced in the ileum this occurred in both Low-Fat and High-Fat refined diets relative to the Chow diet and disassociated from the development of obesity. 


\subsubsection{MICROBIOTA CHANGES ARE DISASSOCIATED FROM OBESITY}

Overall comparison of the microbiota composition between these three diet groups showed a clear effect of diet with the microbiota of each diet groups. In the ileum, the Low-Fat and High-Fat groups could not be separated by overall composition although both were very different from the Chow group. The small intestine has been little unexplored in mice with research focusing on either used stool samples or caecal contents. The limited literature on mice ileum microbiota confirms the results for the Chow fed mice in the present study with the ileum Bacteroidales and Lactobacillaceae (Gu et al, 2013). What is novel here is the large changes seen in the ileum at the phylum and family taxonomic level when switched to a refined Low-Fat or High-Fat diet. The similarity between the both Low-Fat and High-Fat diets shows that it is the refined nature of the diet inducing these changes, and not the fat content.

\subsubsection{LARGEST OTU CHANGES}

Two species, OTU3 and OTU6, were most abundant in Chow mice and formed a significant proportion of the total bacteria. The OTU3 was classified as a Lachnospiraceae, while OTU6 was classified as a member of the Bacteroidetes phylum level. These went from being two of the most common species in the chow diet almost disappearing in mice switched to refined Low-Fat and High-Fat diets. Specific roles of these unclassified species cannot be assigned however these are likely to include degrading a range of complex indigestible polysaccharides found in dietary fibre (Flint et al, 2012). The reduction of these species resulting from the refined diet is likely to be due to the absence in fermentable fibre compared to the chow.

\subsubsection{ERYSIPELOTRICHACEAE FAMILY}

The Erysipelotrichi is a class of bacteria of the phylum Firmicutes that has previously been linked to High-Fat diet induced obesity in mice. Members of the class Erysipelotrichia, belonging to the phylum Firmicutes and including the order Erysipelotrichales and the family Erysipelotrichaceae, are common members of gut microbiota of animals. An Erysipelotrichaceae was found to bloom in diet-induced obese mice (Turnbaugh et al, 2008) and the Erysipelotrichi class increased in Western-Diet fed humanised mice (Turnbaugh et al, 2009b). Increases in Erysipelotrichaceae were seen in mice fed two different High-Fat diets (Fleissner et al, 2010). It has been suggested 
that members of the Erysipelotrichaceae family could have an important in human metabolic health and obesity (Kaakoush, 2015). The family Erysipelotrichaceae was also found to be increased in obese humans subjects (Zhang et al, 2009) and in obese adolescents (Ferrer et al, 2013). However, that these associations in mice all involved comparisons between an unrefined Chow diet and a refined High-Fat diet.

In the present study, the family Erysipelotrichaceae was represented by three species. OTU4 identified in this family matched closely to a recently named and characterised bacterium Faecalibaculum rodentium (Chang et al, 2015). F. rodentium has previously been found to increase in middle-aged mice (Langille et al, 2014). The second species was an unclassified Erysipelotrichi OTU23. The third OTU24 closely matched to Turicibacter sanguinis (Bosshard et al, 2002). The Turicibacter genus has previously been found to decrease in high-fat diet-induced obese mice (Clarke et al, 2013). The same refined diets were used as in the present study and so a reduction with High-Fat feeding is consistent. The lack of literature on species level changes makes any role of these individual member of the Erysipelotrichaceae family is difficult to determine. The abundance of these three species of Erysipelotrichaceae in the present study bloomed only in the Low-Fat diet. The only ingredient difference between the refined diets was the presence of cornstarch in the Low-Fat diet suggesting that refined cornstarch may be a good energy substrate for this family of bacteria. In contrast to earlier studies the proportional abundance of Erysipelotrichaceae was unrelated to change in bodyweight and its increase in the Low-Fat diet was likely to be an artefact of the diet composition.

\subsubsection{SPECIES INCREASED IN ILEUM IN LOW-FAT AND HIGH-FAT DIET GROUPS.}

Two species with a sequence similarity to Lactococcus lactis OTU1 and Romboutsia ilealis OTU5 became dominant in the ileum of Low-Fat and High-Fat diet mice. L. lactis been previous shown to increase in High-Fat diet fed mice (Clarke et al, 2013). The ileum is a section of the gut rarely sampled in mouse microbiota studies explaining the lack of literate relating to this group. L. lactis has not been associated with negative health effects despite its widespread use of this genus in the fermentation of dairy products (Casalta \& Montel, 2008). Its dominance in the ileum suggests its growth on a dietary substrate present in the small intestine in both refined diets. Maltodextrin is the only 
starch in the High-Fat diet an ability to use this as a substrate may be responsible for the growth of this bacterium in the ileum (Gabrielsen et al, 2012).

Romboutsia ilealis has recently been isolated from the ileum of Sprague-Dawley rats (Gerritsen et al, 2014). In the present study, $R$. ilealis increased in both refined diets, particularly in the ileum. $R$. ilealis represented the only significantly member of the family Peptostreptococcaceae that have previously been found to increase with HighFat diet feeding and reduced with caloric restriction (Schulz et al, 2014; Zhang et al, 2013). $R$. ilealis has been found to increase with feeding cellulose, usually considered unfermented in the gut suggesting added cellulose contributed to increase in the refined diets. (Cox et al, 2013). R. ilealis has also been shown to increase with probiotic therapy, reduced bacterial over-growth, reduce bacterial translocation, and reduce inflammatory cytokine levels in plasma (Gerritsen et al, 2011). This limited evidence suggests beneficial role despite its associations with High-Fat diet induced obesity here.

\subsubsection{LACTOBACILLUS}

Lactobacilli have been reported reduced in High-Fat diet feeding and restored with prebiotic supplementation (Arora et al, 2012; Cani et al, 2009; Everard et al, 2014). In contrast, some human studies have reported increased proportions of Lactobacilli in human obesity (Armougom et al, 2009; Million et al, 2013; Million et al, 2012). In the present study, overall abundance of the Lactobacillaceae family was reduced by both Low-Fat and High-Fat diet feeding. Within this family Lactococcus murinus was unaffected by diet remaining at a constant level while $L$. reuteri was reduced by both Low-Fat and High-Fat diet to the point of barely being detectable.(Hemme et al, 1980). While confirming the reductions in Lactobacilli seen in previously with High-Fat feeding the present study shows that this is due to the refined nature of the diet and occurs in Low-Fat diet mice in the absence of obesity.

\subsubsection{BIFIDOBACTERIA}

The large bloom in Bifidobacterium pseudolongum OTU8 in the Low-Fat diet is considered to be due to the refined cornstarch in this diet as cornstarch is a favoured substrate for Bifidobacteria (Liu et al, 2015). In earlier studies Bifidobacteria have been reported to be reduced with High-Fat diets in mice (Cani et al, 2007a). Selective increases in Bifidobacteria through supplementing fermentable fibre in High-Fat diet fed 
mice was reported to reduce body weight gain improve intestinal permeability (Cani et al, 2007c; Cani et al, 2009). The high-fat diet used in these studies contained no cornstarch, a substrate for the growth of Bifidobacteria, while the ingredients of the chow diet used (SAFE AO4) includes corn as the third ingredient, Table 12. The lack of cornstarch in the high-fat diet could account for the reduced proportions of Bifidobacteria associated with obesity in these studies (Cani et al, 2007a; Cani et al, 2009). Changes in proportions of Bifidobacteria are induced by differences in diet composition and not to body weight changes.

\subsubsection{PROTEOBACTERIA}

Deltaproteobacteria have previously been reported to increase in mice comparing Chow and High-Fat diets (Everard et al, 2014; Hildebrandt et al, 2009; Wang et al, 2015). Proteobacteria have been proposed to be microbial signature of dysbiosis in the gut microbiota (Shin et al, 2015) and mono-association of a particular strain has been linked to obesity in mice (Fei \& Zhao, 2013). A Desulfovibrionaceae OTU22 and a Parasutterella OTU27 were found in Chow fed mice and reduced in both Low-Fat and High-Fat diets while a Deltaproteobacteria OTU10 was rare in Chow fed mice and increased dramatically in both Low-Fat and High-Fat diets. In the present study changes in Proteobacteria were shared between Low-Fat and High-Fat diets, and so not associated with obesity.

\subsubsection{AKKERMANSIA MUCINIPHILA AND MUCISPIRILLUM SCHAEDLERI}

In contrast to previous reports the proportions of Akkermansia muciniphila was not consistently different between diet groups and when present formed only very low proportions of sequences in these mice (Schneeberger et al, 2015). A. muciniphila is a common component of the human microbiota (Collado et al, 2007; Derrien et al, 2008), its abundance associated with improved metabolic health in human weight loss (Dao et al, 2015), and increased with prebiotic fibre in mice (Van den Abbeele et al, 2011).

In contrast, the proportion of Mucispirillum schaedleri a similar mucus degrading specialist inhabiting the intestinal mucus layer in mice (Robertson et al, 2005) was more abundant in mice in the present study. M. schaedleri is the sole member of the phylum Deferribacteres reportedly increased with High-Fat feeding (Clarke et al, 2013; Ravussin et al, 2012; Walker et al, 2014). However, M. schaedleri was unchanged between diets in 
the present study. The abundance of $M$. schaedleri may account for the low levels of $A$. muciniphila as both inhabit the same ecological niche in the gut mucosa.

\subsubsection{SEGMENTED FILAMENTOUS BACTERIA (SFB)}

SFB were abundant in the ileum in Chow mice however, they became almost undetectable in the ileum of Low-Fat and High-Fat diet mice. SFB live in close association with the epithelial cells and have important roles in developing and maintaining the immune system in the ileum. The loss of these bacteria in refined diets may have important implications for the immunological health in the gut.

\subsubsection{SHORT-CHAIN FATTY ACIDS}

The SCFAs results in the present study question the roles for energy harvest in mice in the context of a non-obesogenic diet. The production of SCFAs in the caecum and colon from dietary fibre has been both associated with increased development of obesity due to a greater energy harvest from the diet. Introduction of a normal mouse microbiota into germ-free mice increases body fat and energy extraction from dietary fibre that is indigestible to the host (Backhed et al, 2004). The gut microbiota was found to increase SCFA production in genetically obese ob/ob mice and enrich genes contributing to bodyweight gain (Turnbaugh et al, 2008; Turnbaugh et al, 2006). Later research has questioned this effect with evidence that changes in energy harvest in ob/ob mice is transient and that most microbiota composition changes resulted from high-fat diet feeding and not genetic obesity (Murphy et al, 2010). A recent study using rats came to the opposite conclusion finding that the microbiota of lean rats was better at extracting energy from a high-fibre diet than obese rats ( $\mathrm{Li}$ et al, 2015).

Contrarily, production of SCFAs has also reported to reduce body weight and energy intake, and to improve metabolic health. In the context of an obesity inducing high-fat diet the addition of fermentable dietary fibre and resulting increased in SCFA production has been found to decrease body weight gain and improve metabolic health (Adam et al, 2015a; Anastasovska et al, 2012; Arora et al, 2013; Cani et al, 2004; Chassaing et al, 2015; Van den Abbeele et al, 2011). The mechanisms for these effects include the SCFA stimulated increase in secretion of gut peptide hormones including GLP-1 and PYY with anorectic actions. 
In the present study, the rapid increase in body fat gain, and glucose intolerance combined with drastically reduced concentrations and amounts of SCFAs in the HighFat diet group indicates that energy harvest by the microbiota is not playing any role in obesity development in these mice. In addition, the similarly drastically reduced concentrations and amounts of SCFAs in the Low-Fat diet group without any increase in body fat suggests that microbiota produced SCFAs are not essential to maintenance of body weight in the mouse. Different diets can cause extreme changes in the production of acetate, propionate, and butyrate can occur disassociated from changes in body weight.

\subsubsection{OTU AND SHORT-CHAIN FATTY ACIDS CORRELATIONS}

Correlating the proportional abundance of individual OTUs in the caecum and concentrations of SCFAs attempted to draw links between the composition changes in the microbiota and the functional output as SCFAs, one end product of fermentations. While several individual bacterial OTUs were positively correlated with levels of SCFAs when comparing all mice. These correlations were not convincing as they largely consisted of bacterial OTUs common in the Chow and absent in Low-Fat and High-Fat diet groups, with no clear trend within those groups. When comparing within each diet group only one positive correlation between butyrate concentration and abundance of OTU49 remained significant This OTU49 could not be classified beyond the order Bacteroidales and so the functional significance of this is uncertain.

This lack of correlations may be due to the potentially numerous different bacterial species capable of fermenting dietary fibre into SCFAs within each mouse microbiota. A low level of a particular bacteria in one mouse sample could be compensated by the action of an unrelated species carrying out the same functional role. Additionally, individual SCFAs from the fermentation of complex dietary fibres, are the end stage of a fermentation process involving cross-feeding between different bacterial species (Belenguer et al, 2006; Flint et al, 2007). Such a complex relationship may confound the identification of correlations of individual bacterial species and SCFA concentrations. 


\subsubsection{WEAKNESSES AND LIMITATIONS}

A limitation of this study is that SCFA analysis was carried out only on caecum samples. Given more time the SCFA concentrations of ileum, colon, and faecal samples could also have been determined to compare the effects of the different diets throughout the gut. It would have been of particular interest in the ileum where some of the most dramatic changes in microbiota composition took place. Unfortunately, due to the small quantities of luminal contents obtained from the ileum all of the sample was required for DNA extraction for microbiota analysis, leaving no remaining sample for other analysis. With more time, RT-PCR would have been used to confirm the microbiota changes observed in the next generation sequencing data. Without such validation changes in the abundance of specific OTUs should be treated with caution as next generation sequencing results are known to produce biases in amplification. However, the large scale of the changes in microbiota composition between diets in this study means that the lack of validation does not affect the overall conclusions. With more time changes in serum leptin and insulin could have been tested for to identify if the different diets were subtly influencing metabolism even if no differences in body weight were observed. 


\section{CONCLUSIONS}

The overall results presented in this study disassociate changes in gut microbiota composition, gut morphology, and short-chain fatty acid concentrations from physiological changes in body fatness and glucose intolerance.

Changes in microbiota were unrelated to bodyweight and body fatness. Considered at the species level most changes occurred between Chow and both refined diets and were mostly likely due to the shift in energy substrates available to the bacteria. In bacterial groups, such as the Erysipelotrichaceae and Bifidobacterium previously associated with obesity in mice these only increased in the Low-Fat diet. While others such as the Proteobacteria and Lactobacilli increased, or decreased similarly in both Low-Fat and High-Fat diets relative to the Chow. These results of the present study question the links drawn between the development of obesity and changes in the gut microbiota composition when so many change can be produced through manipulation of diet in the absence of body weight change. Previous research generating diet-induced obesity in mice has been confounded by the use of refined High-Fat diets with unrefined chow diets as a control. Other studies investigating changes in the gut microbiota have used a refined High-Fat diet together with a well-matched control diet. The present study also raises questions about results drawn from these comparisons. Significant changes between a matched Low-Fat and High-Fat diet alone do not take into account the larger shifts in bacterial populations that have taken place on both diets relative to the "normal microbiota" of chow fed mice.

The refined Low-Fat diet reduced caecum and colon size and weight, negatively influencing gut health, while at the same time not influencing body weight. The refined high-fat diet increased body fat and the refined low-fat diet did not, while body diets reduce colon length and caecum weight. There is a disassociation between these two outcomes. This study provides evidence that this effect is not necessary to maintain a lean phenotype when there is no obesogenic stimulus.

Impairment in glucose tolerance has previously been associated with both changes in both microbiota composition and in short-chain fatty acid production. In the present 
study, significant changes occurred in both between the Chow and Low-Fat diets without any significant worsening of glucose tolerance.

The present study shows that large reductions can occur in SCFA production without any influence on body weight, but the changes in SCFA confirm that the functional output of the bacteria was changed. The results here contradict the claim that the microbiota of obese animals increase the energy extraction from food, with large reductions in caecal and colonic energy extraction taking place in High-Fat diet fed mice while becoming obese. Moreover, similar reductions in energy extraction were also present in Low-Fat mice, suggesting that these changes are unrelated to the development of obesity.

Comparing a chow diet with a high-fat diet produces many alterations in the gut microbiota that are due to the refined and semi-purified nature of the ingredients used to produce the high-fat diet, and the absence of components in the chow diet. Much of these alterations in the gut microbiota can be reproduced in the absence of obesity with a refined diet lacking in fat. Careful choice of mouse control diets can disassociate microbiota composition changes from physiological outcomes. 


\section{CHAPTER 5:}

\section{THE ROLE OF GLP-1 AND PYY IN MEDIATING THE EFFECTS OF FERMENTABLE FIBRE}




\section{INTRODUCTION}

The results presented in Chapter 4 indicate mice eating a chow diet high in fermentable fibre, compared to mice eating a low-fibre refined diet, did not result in any measurable effects on body weight, body fat or food intake over an eight week intervention. This appears to contradict an increasing body of evidence linking microbial fermentation of dietary fibre in the caecum and colon to reductions in body fat and food intake. However, in mouse models additional fermentable fibre has typically been added to an obesogenic diet, containing high levels of fat, or fat and sucrose. Against the background of an obesogenic diet fermentable fibre has been shown to decrease the amount of body weight and fat gained relative to the same obesogenic diet without fibre. In Chapter 4 both Chow and Low-Fat diets were low in fat and contained macronutrient ratios that are not obesogenic to rodents. This evidence suggests that for lean mice on a nonobesogenic diet, the presence, or lack of, fermentable fibre does not adversely affect body weight or food intake, at least in the short-term. In contrast evidence is growing that dietary fermentable fibre can reduce body weight and food intake, along with other benefits, in high-fat mice (Adam et al, 2015a; Adam et al, 2014; Arora et al, 2012). However, the mechanisms for these benefits are still not fully understood. This chapter investigates the mechanism through which fermentable dietary fibre exerts positive effects on food intake and adiposity in mice fed a high fat diet.

\subsection{BENEFITS OF FERMENTABLE FIBRE}

Growing appreciation of the importance of the intestinal microbiota has renewed interest in the benefits of fibre to colonic health and host metabolism (Frost et al, 2014b). Dietary fibre by definition passes through the small intestine undigested and upon reaching the caecum and colon becomes available for anaerobic fermentation by the resident intestinal microbiota (Prosky, 2000). Soluble fibre types are generally more fermentable than insoluble fibres. Common fermentable fibres include Fructans such as inulin, pectin, gums and resistant starches, whereas cellulose is a typical insoluble fibre that is only minimally fermented (Lattimer \& Haub, 2010). 


\subsection{SHORT-CHAIN FATTY ACIDS}

Some of the main beneficial effects of fermentable fibre on host physiology are due to short-chain fatty acids, which are generated through bacterial fermentation in the lower intestine (Sleeth et al, 2010). Fermentation results in the generation of acetate, propionate, and butyrate in the caecum and colon at millimolar concentrations, with molar ratios of three SCFAs being 57:22:21 in the human colon (Cummings et al, 1987). Much of the butyrate produced is consumed by the colonocytes, acetate and propionate reach micromolar concentrations within the portal vein blood, the propionate is then taken up to a large extent by the liver while a proportion of acetate reaches the wider circulation (Bugaut \& Bentejac, 1993; Cummings et al, 1987). Infusion of acetate, propionate, or butyrate into the caecum at physiologically relevant concentrations of SCFAs show that some acetate is converted into fat and cholesterol, while propionate is mainly used to synthesise glucose in the liver (den Besten et al, 2013a).

The production of SCFA effects the colonic environment in a number of way. The acid nature of SCFAs reduces the luminal $\mathrm{pH}$, inhibiting pathogenic microorganisms and increasing the absorption of some nutrients (Macfarlane \& Macfarlane, 2012). The production of acetate by bifidobacteria plays a role in the suppression of enteropathogens (Fukuda et al, 2011). The fuelling of intestinal epithelial cells by butyrate increases mucin production and improves tight junction integrity improving gut barrier function (Jung et al, 2015; Peng et al, 2009). In colonocytes SCFAs, particularly butyrate, inhibit the activity of acetylation by histone acetyltransferases and histone deacetylases (HDAC) influencing epigenetic gene expression (Cousens et al, 1979). Inhibition of HDAC has a number of consequences including reduced inflammation through the induction of regulatory T-cells and reduced epithelial cell proliferation (Donohoe et al, 2014; Furusawa et al, 2013). However, the focus here is on the consequences of SCFA activation of free fatty acid receptors.

\subsection{FREE FATTY ACID RECEPTORS.}

Metabolically short-chain fatty acids provide a source of energy, recovered from dietary fibre by microbial digestion and which otherwise would be lost as it escapes host digestion (Cummings et al, 1995). They also act as ligands for free fatty acid receptors found in enteroendocrine L-cells in the intestinal epithelium (Le Poul et al, 2003). Two 
related 7-transmembrane $\mathrm{G}$ protein coupled receptors (GPCR), free fatty acid receptors 2 and 3 (FFA2 and FFA3), previously known as GPCR43 and GPCR41, are activated by SCFAs (Brown et al, 2003). Evidence indicates that some of the beneficial effects associated with dietary fibre are mediated by FFA2 and FFA3, and that these receptors are potential targets of interest for the modulation of metabolic diseases (Ulven, 2012). These free fatty acid receptors are expressed in enteroendocrine L-cells in the intestinal epithelium where the receptors are exposed to the high concentrations of SCFAs in the intestinal lumen of the caecum and colon (Hudson et al, 2012). Stimulation of L-cells by short-chain fatty acids stimulates the production and secretion of the enteroendocrine hormones Glucagon-like peptide-1 (GLP-1) and Protein PY (PYY) (Eissele et al, 1992; Larsson et al, 1975) (Spreckley \& Murphy, 2015).

\subsection{L-CELLS}

GLP-1 and PYY-secreting L-cells are present in the intestinal epithelium and with increasing density occurring along the small intestine, caecum, and colon (Eissele et al, 1992; Larsson et al, 1975). As consequence, a two-phase release of GLP-1 and PYY occurs in response to nutrient ingestion; there is an initial rapid rise representing release from L-cells in the small intestine, and a second sustained release upon arrival of food in the distal gut (Nauck et al, 1996). Increased SCFA production in the colon has been found to increase the secretion of GLP-1 and PYY from the enteroendocrine L-cells in the colon due to stimulation of FFA receptors on the L-cell apical membrane. Infusion of propionate in the colon stimulates the secretion of GLP-1 and PYY from the colon, but this is blocked by the loss of FFA2 receptor (Psichas et al, 2015). Following secretion from the L-cells, GLP-1 and PYY undergo enzymatic cleavage by dipeptidyl peptidase IV converting GLP-1 to an inactive form, truncating PYY1-36 to the PYY3-36 form which can activate its target Y2 receptor (Holst \& Deacon, 2005; Medeiros \& Turner, 1994).

\subsection{PYY AND GLP-1 ACTIONS}

Both PYY and GLP-1 have a range of effects in several tissues and this includes several overlapping actions that can influence food intake and metabolism. GLP-1 is an incretin acting on pancreatic islet cells to increase glucose stimulated insulin release (Vilsboll \& Holst, 2004). GLP-1 also has a tropic effect on pancreatic beta-cells increasing insulin secretion and PYY has also been shown to promote glucose tolerance through increased 
GLP-1 secretion (Chandarana et al, 2013; Wang et al, 1997). Both GLP-1 and PYY play a role in the 'ileal break' decreasing gastric emptying and slowing intestinal motility, PYY through actions on the Y1 receptor (Lloyd et al, 1996; Nauck et al, 2011; Pironi et al, 1993). Exogenously administered PYY and GLP-1 each reduce food intake (Batterham et al, 2006; Ruttimann et al, 2009) and these effects may be mediated via the arcuate nucleus of the hypothalamus, an important neuronal site involved in the central integration of appetite control where peripheral signals in the circulation can gain access to appetite regulating populations of neurones due to an incomplete blood brain barrier (Spreckley \& Murphy, 2015). Hypothalamic neurones in this region of the brain contain both Y2 and GLP-1 receptors through which PYY and GLP-1 can act. However, due to the short half-life of GLP-1 it is uncertain how much circulating GLP-1 reaches the brain (Deacon et al, 1995). An alternative route of action is via vagus nerve innervation of the brain, rather than through endocrine signalling (Nishizawa et al, 1996; Spreckley \& Murphy, 2015). Additionally, GLP-1 is also produced centrally within the brain where it is thought to act as a neuropeptide (Trapp \& Richards, 2013). The fermentation of fibre in the caecum and colon has been thought to influence food intake, adiposity, and metabolism through promoting the secretion of PYY and GLP-1. However, direct experimental evidence for this mechanism is limited (Keenan et al, 2012). While increased circulating levels of GLP-1 and PYY have been reported with increased dietary fermentable fibre, this has not been observed in all studies (Anastasovska et al, 2012). As a consequence, the role of enteroendocrine hormones as the main mediators of the benefits of fibre has recently been questioned (Frost et al, 2014a).

\subsection{KNOCKOUT MICE MODELS}

A potential means of elucidating the role of GLP-1 and PYY in the effects of fermentable fibre on food intake, adiposity, and body weight is with mice unable to produce or respond to these hormones through targeted gene deletion. Limited evidence using mice with the GLP-1 receptor gene knocked out suggest that the GLP-1 receptor is required for the body fat reductions seen in response to dietary resistant starch (Zhou et al, 2015), and body weight and glucose responses to dietary fructooligosaccharides (Cani et al, 2006). There is a lack of research showing the need for PYY in the responses to fibre. 


\subsection{GLUCAGON-LIKE PEPTIDE-1 RECEPTOR KNOCKOUT MICE}

The effects of GLP-1 in mice have been studied through the deletion of the GLP-1 receptor gene (Scrocchi et al, 1996). When fed a chow diet GLP-1R ${ }^{-/-}$mice show increased glucose responses to an oral glucose challenge but do not display abnormal body weight or feeding behaviour relative to wild-type mice. In response to a high-fat diet these GLP$1 \mathrm{R}^{-/-}$mice increased their body weight relative to chow fed mice but less than wild-type mice on the same diet (Scrocchi \& Drucker, 1998). It was reported that the addition of fructooligosaccharides in a very high-fat diet reduced body weight gain and improved glucose tolerance in wild-type mice while GLP-1R $\mathrm{R}^{-/-}$mice were resistant to these effects (Cani et al, 2006). However, this study was limited in duration and numbers of mice,

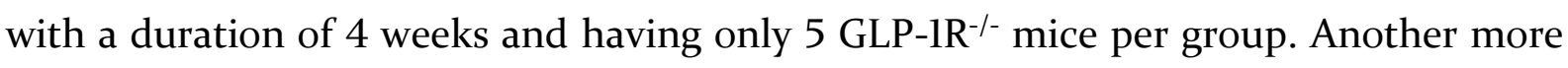
recent study compared the effects of high levels of resistant starch in the diet of wildtype and GLP-1R ${ }^{-/-}$mice (Zhou et al, 2015). Lacking the GLP-1R prevented the small reduction in body fat seen with resistant starch feeding (Zhou et al, 2015). However, the body weight differences were not significant between mice fed resistant starch or control diets after 10 weeks. The resistant starch also increased the food intake relative to the control diet in both GLP-1R $\mathrm{R}^{-/-}$and wild-type mice, making the anorectic effect of GLP-1 difficult to interpret (Zhou et al, 2015).

\subsection{PEPTIDE YY KNOCKOUT MICE}

Three different PYY knockout mice, each on a C57BL/6 background, have been developed through deletion of the PYY gene (Batterham et al, 2006; Boey et al, 2006; Schonhoff et al, 2005). The first reported that mice lacking PYY did not consume more food or become overweight when eating a chow diet (Schonhoff et al, 2005). In that study neither basal food intake, body composition, glucose homeostasis, nor dietinduced obesity were investigated. By contrast another PYY knockout mouse was reported to develop obesity, increase body fat, and increase food intake while eating a chow diet (Batterham et al, 2006). Unfortunately, this mouse strain is no long available. A third mouse PYY knockout mouse strain displayed an increased body weight in females but not in males while eating chow (Boey et al, 2006). In contrast male mice gain significantly more fat and became more glucose-intolerant compared with wildtypes when fed a high-fat diet. This last mouse knockout mouse strain 
was the only one of the three available for use in the present study (Boey et al, 2006). $\mathrm{PYY}^{-/-}$mice have been used primarily to investigate the role of PYY in the satiety inducing effects of dietary protein (Batterham et al, 2006), while the role of PYY in satiety induced by fibre fermentation in the caecum and colon has not been explored used $\mathrm{PYY}^{-/-}$mice. GLP-1R ${ }^{-/-}$and $\mathrm{PYY}^{-/-}$mice are therefore useful models for investigating the roles of GLP-1 and PYY in effects of dietary fibre on food intake and adiposity.

\subsection{ACETATE}

A potential alternative mechanism involves circulating acetate acting directly on appetite control centres in the brain (Frost et al, 2014a). Acetate represents the predominant short-chain fatty acid produced in the lower intestine as a result of microbial fermentation (Cummings et al, 1987). Unlike butyrate and propionate, which are consumed by the intestinal epithelium and the liver respectively, a proportion of acetate absorbed from the gut reaches the peripheral circulation. Up to 3\% of acetate can be taken up by the brain and is available as an energy substrate in mice after intravenous or caecal infusion (Frost et al, 2014a). It was recently reported that the feeding mice a high-fat diet containing inulin increased manganese-enhanced MRI signal in the hypothalamus, a proxy measurement of neuronal activation (Anastasovska et al, 2012). This appeared to be independent of changes in circulating GLP-1. In contrast, peripheral GLP-1 and PYY infusion reduce signal intensity in the hypothalamus (Chaudhri et al, 2006; Kuo et al, 2007). Acetate in the brain forms an important energy substrate for astrocytes in the brain and metabolising acetate has an impact on brain function (Carmichael et al, 1991; Wyss et al, 2011). The metabolism of acetate by astrocytes in the hypothalamus is proposed to influence the functioning of the homeostatic neurones (Frost et al, 2014a). Acute infusion of acetate resulted in four-fold increase of pro-opiomelanocortin (POMC) and suppressed expression of agouti-related peptide (AgRP) expression in the hypothalamus (Frost et al, 2014a). This increase in anorexigenic POMC and decrease in orexigenic AgRP is a mechanistic link between acetate and reduced food intake. Circulating acetate originating from the colon is a new mechanism for the satiety effects of dietary fibre such as inulin. 


\subsection{FRUCTOOLIGOSACCHARIDE ENRICHED INULIN DIETS}

A diverse range of types of fermentable fibre types are found in foods and of these inulin is one the most widely studied with prebiotic properties. Fructooligosaccharides and inulin are found in human diets with the most common food sources in European and North American diets being wheat, onion, banana, garlic, and leek (Moshfegh et al, 1999; van Loo et al, 1995). Initial interest in inulin was due to its "bifogenic" properties, based on its ability to selectively encourage the growth of Bifidobacteria (Meyer \& StasseWolthuis, 2009). More recently it has been shown to be a substrate for a more diverse range of bacteria (Chung et al, 2016; Ramirez-Farias et al, 2009; Scott et al, 2014). Inulin is extensively fermented in the caecum and colon producing short-chain fatty acids as a result (Roberfroid, 1999).

In this study, to maintain consistency, the mouse diets used were obtained from Research Diets and based on the D12451 high-fat diet. An inulin enriched diet (HighFat+In) was prepared using the D12451 diet composition adjusted to include 10\% fructooligosaccharide enriched inulin (Orafti ${ }^{\circledR}$ Synergyl, BENEO). The addition of fermentable fibre to mouse diets needs careful manipulation of diet composition to take account of energy content and macronutrient percentages. Simply adding a fibre like inulin to a mouse diet dilutes the other macronutrients and reduce the energy density of the diet due to the lower energy content of the fibre. To overcome this, a matched control diet (High-Fat $+\mathrm{C}$ ) was prepared with added cellulose to create a diet with the same energy density. By this means the diets used in this study maintained similar overall composition to diets used previously with dairy fat substituted with lard as the fat source (Frost et al, 2014a).

The pathways evolved between the fermentation of inulin and changes in body weight and food intake are shown in Figure 51. 


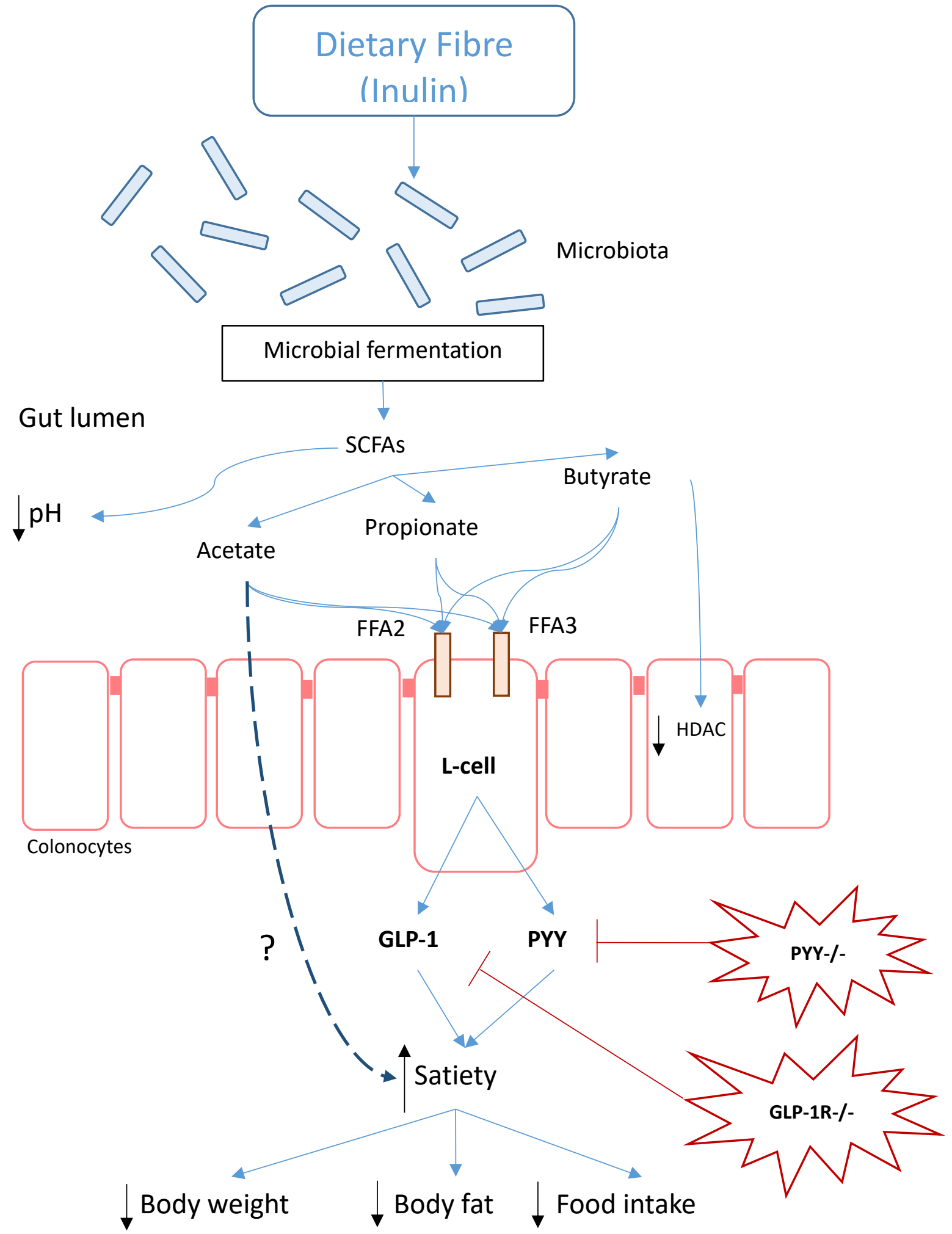

Figure 51. Pathways through which SCFAs can influence bodyweight. 


\section{AIMS}

The aim of this study was to investigate the roles of acetate, PYY, and GLP-1 as mediators of effects of fermentable fibre on food intake and body composition of mice. The study builds on the widely-held view that PYY and GLP-1 are essential to these responses, yet recent evidence suggests an alternative mechanism involving direct action of acetate on the brain (Frost et al. 2014). To test for the need of either PYY or GLP-1, wild-type mice (C57Bl/6) were fed a high-fat diet with or without inulin (as a fermentable fibre) and were compared with mice with genetic deletions of either the PYY or GLP-1 genes, fed the same diets and assessed for their food intake and metabolic responses. The effects of a high-fat diet supplemented with either cellulose or inulin on food intake, body fat, and body weight were recorded over eight weeks between Wild-Type, PYY ${ }^{-/-}$, and GLP$1 \mathrm{R}^{-/-}$mice. The rationale was that lack of GLP-1 and PYY signalling would not block the effects of acetate acting directly on the brain to control food intake and adiposity.

\section{HYPOTHESIS}

The experimental hypothesis for the study was that GLP-1 and PYY are each required for dietary inulin to have its attenuating effects on food intake and body composition in mice fed a high fat diet. 


\section{RESULTS}

\subsection{THE EFFECT OF INULIN ON FOOD AND ENERGY INTAKE}

Wild-Type mice fed the High-Fat+In diet showed a rapid and sustained reduction in in food intake, becoming significantly different from the other groups by week 4, Figure 52A. Mice fed High-Fat+C and Low-Fat diets did not differ in their food intake. Due to the higher energy density in the high-fat diets the High-Fat $+C$ fed Wild-Type mice consumed a higher level of energy with the difference becoming significant by week 4 , Figure 52B. The High-Fat+In diet fed Wild-Type mice maintained energy intake comparable to that of the Low-Fat diet fed mice Figure 52B. The overall mean weekly food intake for Wild-Type mice was significantly lower in High-Fat+In fed mice, Figure 53A, but the mean energy intake was similar to the Low-Fat fed mice, Figure 53B. A small transient reduction in weekly food intake and energy intake was observed in the $\mathrm{PYY}^{-/-}$ mice fed the High-Fat+In diet, but the reduction only reached significance at week 4, Figure $52 \mathrm{C}$, and food and energy intake converged with the High-Fat $+\mathrm{C}$ group by the end of the study Figure 52C,D. Mean weekly food intake was not different between the three diets in $\mathrm{PYY}^{-/-}$mice, Figure 53A. Mean weekly energy intake was significantly higher in both High-Fat+In and High-Fat $+\mathrm{C}$ fed mice compared to the Low-Fat group due to the higher energy density of these diets, Figure 53B. The GLP-1R ${ }^{-/-}$mice fed the High-Fat+In diet showed no change in food intake or energy intake in comparison to the High-Fat+C and Low-Fat diets, Figure 53A,B. The energy intake of GLP-1R ${ }^{-/-}$fed the High-Fat+In diet was not significantly different from the High-Fat $+\mathrm{C}$ mice during any week, Figure 52F, or for mean energy intakes, Figure 53B. High-fat diet groups consumed a higher energy intake due to the higher energy density of these diets. Overall the PYY।- and GLP-1R ${ }^{-1}$ mice show a reduced effect of inulin on food and energy intake relative to Wild-Type mice. 

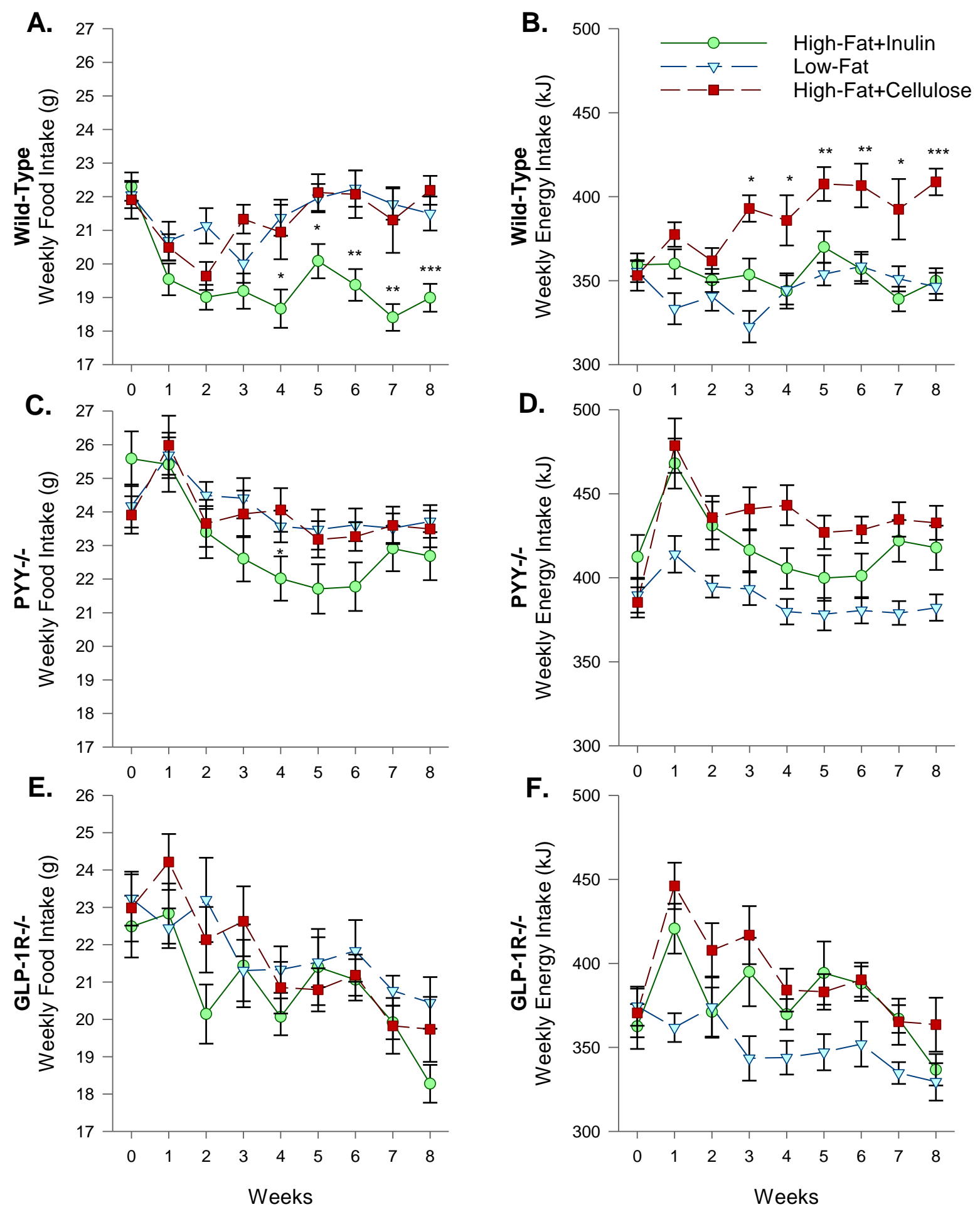

Figure 52. Effects of inulin on weekly food intake and energy intake.

Weekly food intake and energy intake for Wild-Type mice (A, B), for PYY ${ }^{-/-}$mice (C, D), and for GLP-1R ${ }^{-/-}$mice (E, F) fed Low-Fat, High-Fat+C, and High-Fat+In diets. Data represents mean \pm SEM. Significant differences within each genotype were determined by one way ANOVA with Tukey's post hoc test. $\mathrm{n}=12$ mice per group. Asterisks indicate significant difference $\left({ }^{*} \mathrm{p}<0.05 ;{ }^{* *} \mathrm{p}<0.01 ;{ }^{* *} \mathrm{p}<0.001\right)$ at each week. 

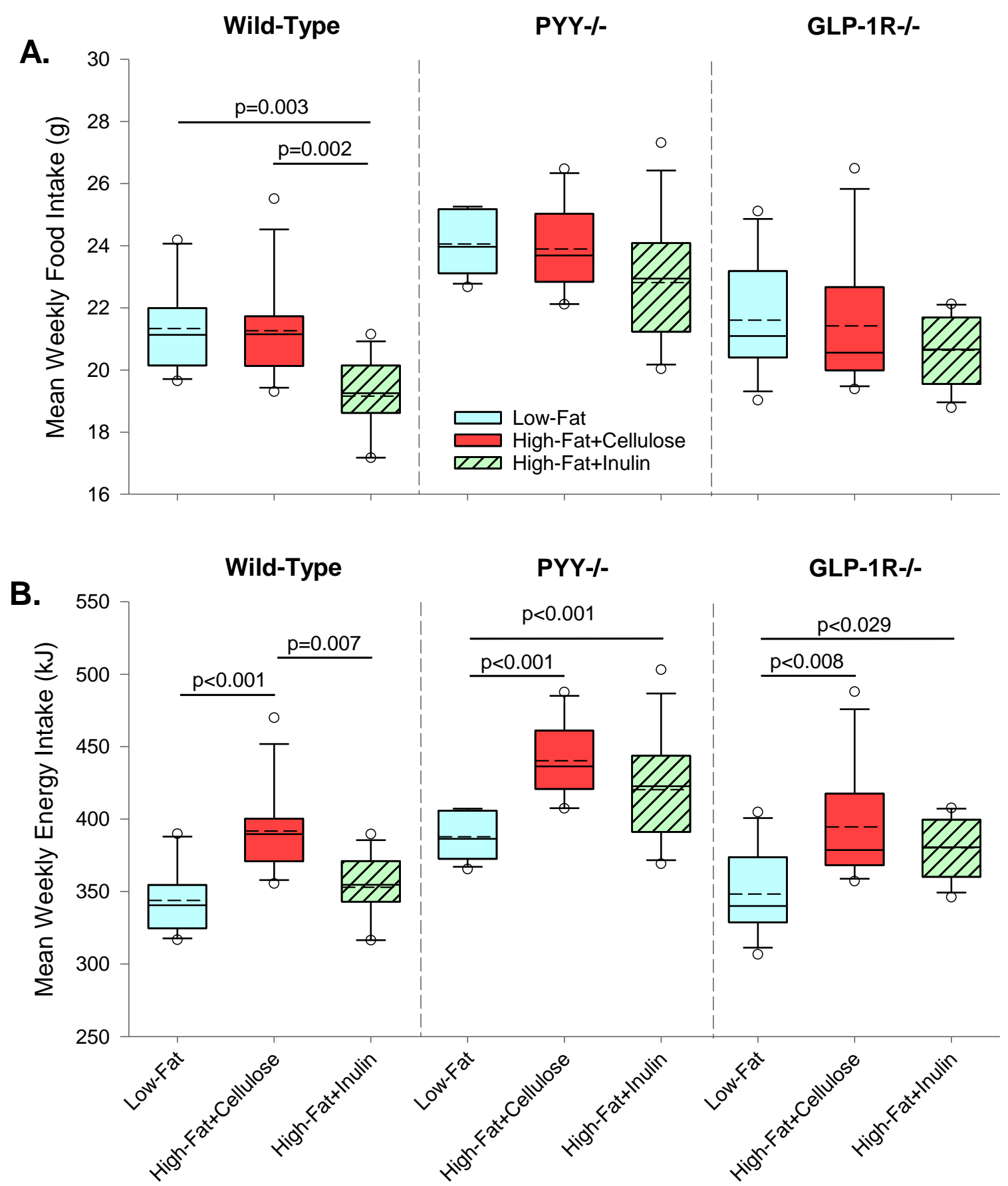

Figure 53. Effect of inulin on overall mean weekly food and energy intake.

The mean food intake (A), and mean energy intake (B) for Wild-Type, PYY ${ }^{-/-}$, and GLP$1 \mathrm{R}^{-/-}$mice fed Low-Fat, High-Fat+In, and High-Fat+C diets. For Box plots the solid line $=$ median, and dashed line = mean. Significant differences within each genotype were determined by one way ANOVA with Tukey's post hoc test. $\mathrm{n}=12$ mice per group. 


\subsection{WILD-TYPE BODY COMPOSITION}

Body fat increased in Wild-Type mice fed High-Fat $+\mathrm{C}$ and High-Fat+In diets relative to Low-Fat diet fed mice, Figure 54A. After week 5 the increase of body fat of High-Fat+In diet fed mice began to slow while the body fat of High-Fat $+C$ fed mice continued to increase, Figure 54. At the final week, High-Fat+In diet fed mice contained significantly less fat that High-Fat $+\mathrm{C}$ fed mice, Figure 54B. Both groups of High-Fat diet fed mice gained significantly more fat relative to Low-Fat diet fed mice, Figure 54B. Lean mass was equivalent between the two High-Fat groups throughout the study, Figure 54C. Lean mass initially decreased in week 1 in Low-Fat diet mice and then recovered by the end of the study. By the final week lean mass was not significantly different between diet groups, Figure 54D.

Changes in body fat percentage and percentage lean mass reflected changes in body fat, Figure 55. Body fat initially increased in both High-Fat diet fed groups, after week 5 the increase in body fat percentage slowed in mice fed the High-Fat+In diet relative to the High-Fat $+\mathrm{C}$ diet, Figure 55A. The percentage lean mass decreased in both High-Fat+In diet mice while High-Fat $+\mathrm{C}$ diet mice, Figure 55C. By the final week both body fat percentage and lean mass percentage were significantly different between High-Fat+In diet mice and other groups, Figure 55B, D. The body composition changes in Wild-Type mice indicate that all body weight changes between diet groups were due to changes in body fat and not lean mass. The presence of inulin in the diet reduced body fat and body fat percentage by the final week of the study. 

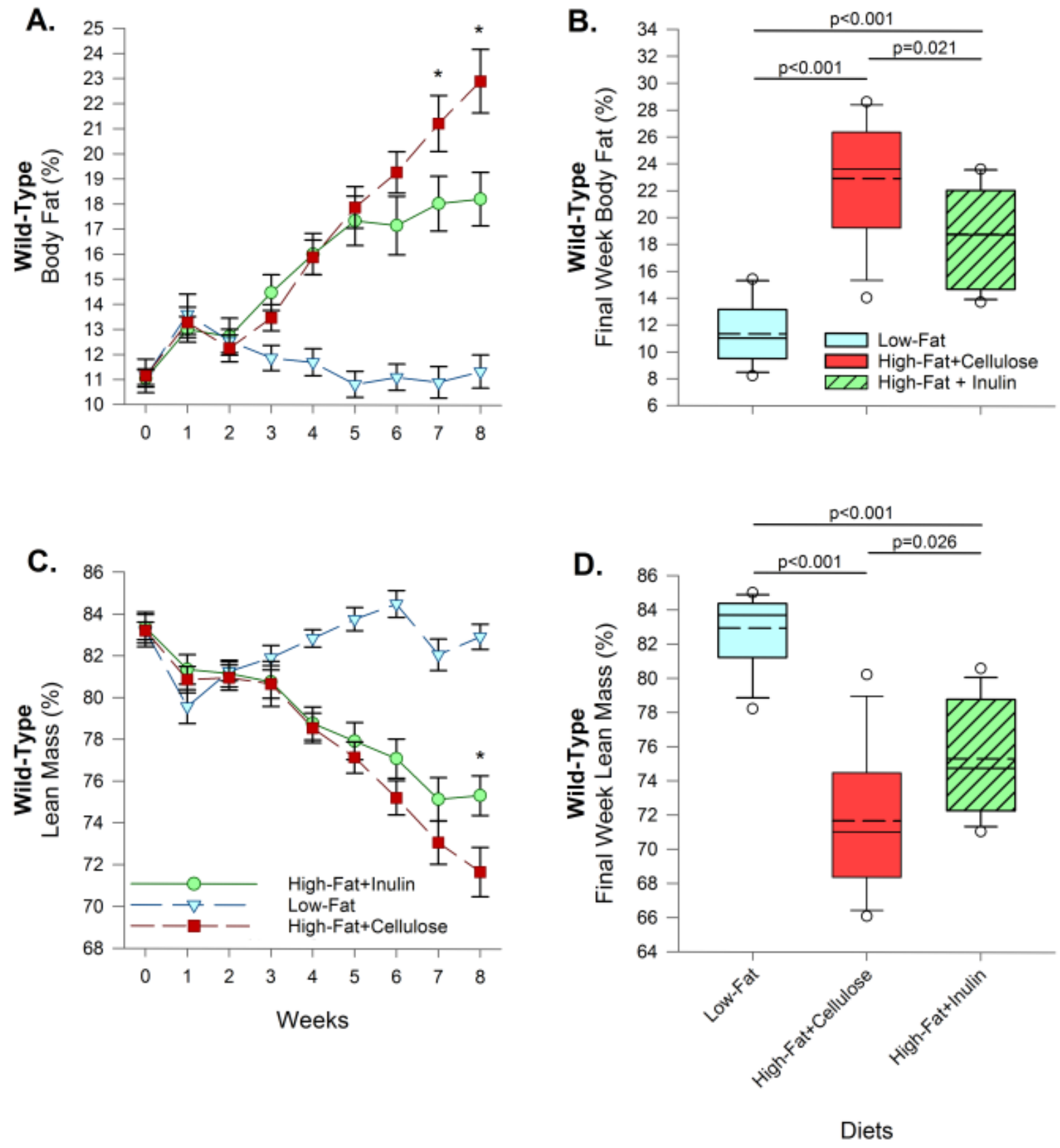

Figure 54. Effect of inulin on fat mass and lean mass of Wild-Type mice.

Weekly change in body fat mass of Wild-Type mice (A), and total body fat mass at the final week (B), for Low-Fat, High-Fat+C, and High-Fat+In fed mice. Weekly change in lean mass of Wild-Type mice (C), and total lean mass at the final week (D), for Low-Fat, High-Fat $+\mathrm{C}$, and High-Fat+In fed mice. Body fat and lean mass was determined by EchoMRI scan. Data points represent mean \pm SEM. For Box plots the solid line $=$ median, and dashed line $=$ mean. Significant differences within each genotype were determined by one way ANOVA with Tukey's post hoc test. Asterisks indicate significant differences $\left({ }^{*} \mathrm{p}<0.05 ;{ }^{* *} \mathrm{p}<0.01 ;{ }^{* * *} \mathrm{p}<0.001\right)$ at each week. $\mathrm{n}=12$ mice per group. 

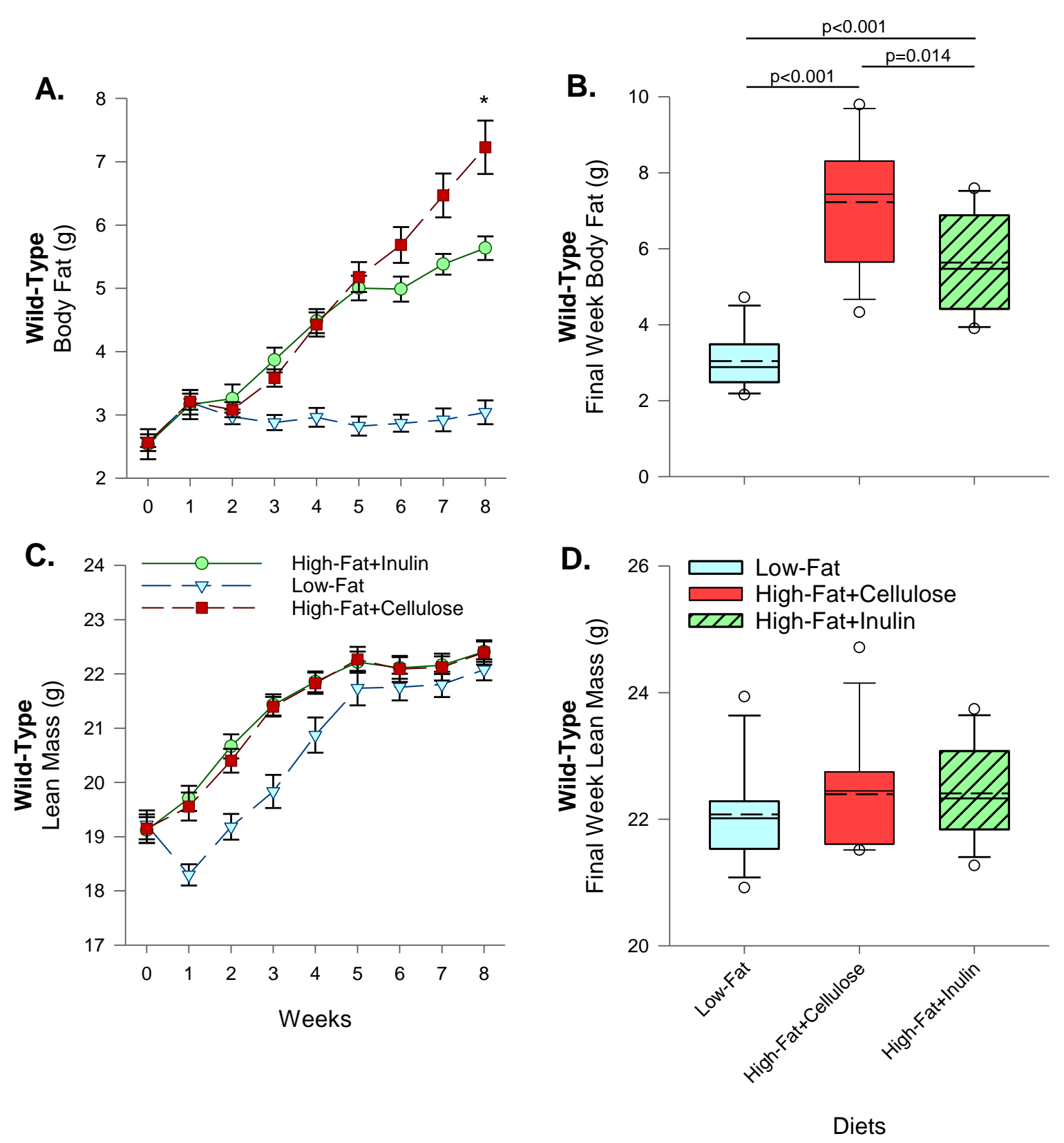

Figure 55. Effects of inulin on percentage body fat and lean mass of Wild-Type mice.

Weekly change in body fat percentage of Wild-Type mice (A), and total body fat percentage at the final week (B), for Low-Fat, High-Fat+C, and High-Fat+In fed mice. Weekly change in percentage lean of Wild-Type mice (C), and total percentage lean mass at the final week (D), for Low-Fat, High-Fat + C, and High-Fat + In fed mice. Body composition was determined by EchoMRI scan. Data points represent mean \pm SEM. For Box plots the solid line $=$ median, and dashed line $=$ mean. Significant differences within each genotype were determined by one way ANOVA with Tukey's post hoc test. Asterisks indicate significant differences $\left({ }^{*} \mathrm{p}<0.05\right.$; ${ }^{* *} \mathrm{p}<0.01$; $\left.{ }^{* * *} \mathrm{p}<0.001\right)$ at each week. $\mathrm{n}=12$ mice per group. 


\subsection{EFFECT OF INULIN ON EPIDIDYMAL AND RETROPERITONEAL FAT PADS}

Comparison of the effect of inulin feeding on body fat content between Wild-Type, PYY1-, and $\mathrm{GLP}^{-1 \mathrm{R}^{-/}}$mice was determined through dissection of the epididymal and retroperitoneal fat deposits, . A High-Fat diet significantly increased the percentage of both epididymal and retroperitoneal fat in mice of all genotypes $(\mathrm{p}<0.001)$. In WildType mice the presence of inulin in the High-Fat diet significantly reduced the

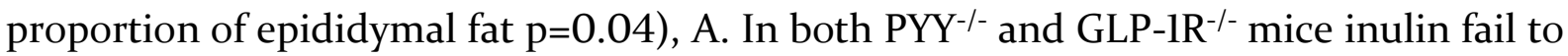
significantly reduce epididymal fat. The presence of inulin in the High-Fat diet significantly reduced retroperitoneal fat in Wild-Type mice $(\mathrm{p}=0.001)$. In $\mathrm{PYY}^{-/-}$mice inulin did not significantly reduce retroperitoneal fat, but did in $\mathrm{GLP}^{-1 \mathrm{R}^{-/}}$mice $(\mathrm{p}=0.006)$. The percentage of epididymal and retroperitoneal fat was expressed as a proportion of body weight minus the weight of the gut. Normalising for disembowelled body weight (body weight minus GI tract) corrected for the increased weight of gut and gut contents in the inulin fed mice. The changes in epididymal and retroperitoneal fat confirmed the results of Echo MRI scanning on Wild-Type mice. The loss of PYY prevented the fat reducing effect on inulin in the High-Fat diet. Loss of the GLP-IR did not prevent the fat reducing effect on inulin. 
Wild-Type

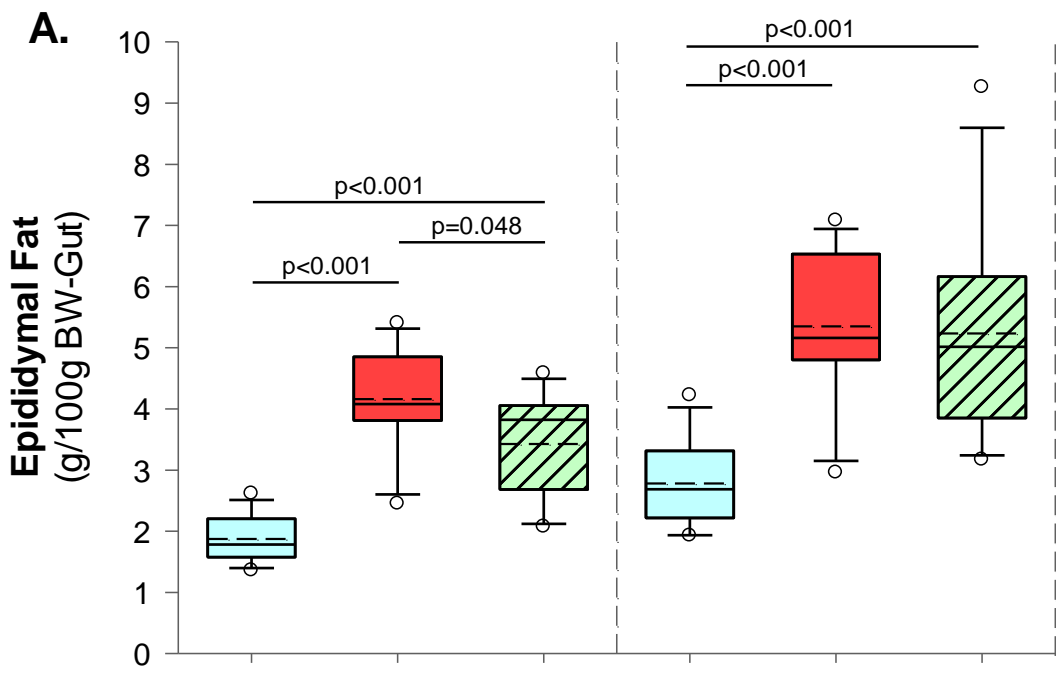

Wild-Type

PYY-/-

PYY-/-

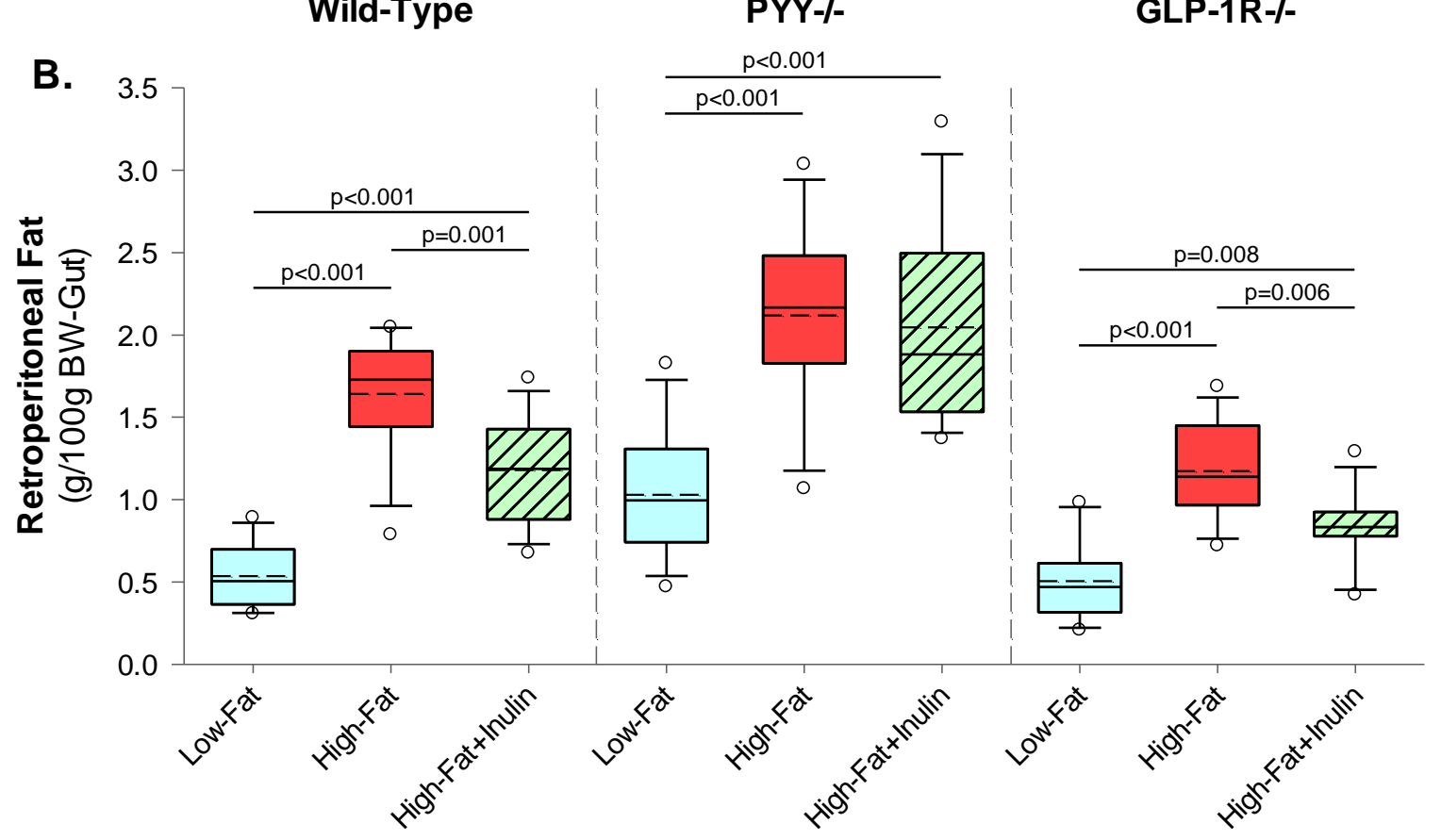

GLP-1R-/-

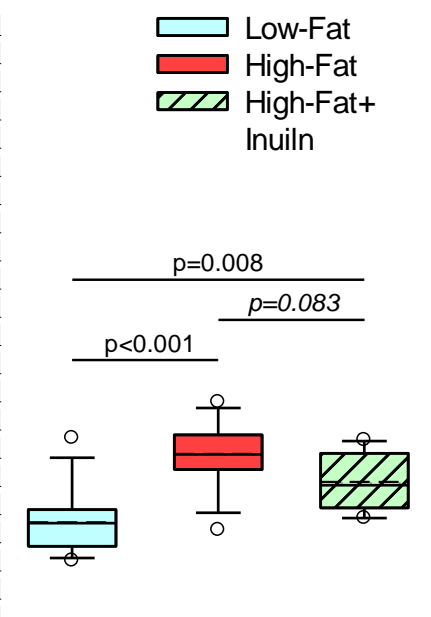

GLP-1R-/-

Figure 56. Effect of inulin on epididymal and retroperitoneal body fat pads.

Epididymal fat (A), and retroperitoneal fat (B), weight in Wild-Type, PYY-/- and GLP-1R1- mice fed Low-Fat, High-Fat+C, and High-Fat+In diets. Epididymal, and retroperitoneal fat is calculated as fat mass divided by body weight minus the gut weight at the end of study. In box plots the solid line $=$ median, and dashed line $=$ mean. Statistical significance was determined by one-way ANOVA with Tukey's post hoc test. $\mathrm{n}=12$. 


\subsection{BODY WEIGHT AND BODY WEIGHT GAIN}

In Wild-Type mice body weight gain was the same for the High-Fat+C diet and the HighFat+In diet until week 5 and then began to diverge, Figure 57A. At the final week this difference in mean body weight gain of $10.6 \mathrm{~g}$ and $9.0 \mathrm{~g}$ for High-Fat+C and High-Fat+In fed mice respectively was significantly different $(\mathrm{p}=0.023)$, Figure 57B. The body weight of both high-fat groups of Wild-Type mice increased more than the Low-Fat diet group. The $\mathrm{PYY}^{-/}$mice in all diet groups gained more weight than Wild-Type mice in the equivalent diet groups. Body weight gain was not significantly different between in PYYI- mice fed the High-Fat $+C$ diet and the High-Fat+In diets, Figure $57 \mathrm{C}$, or at the final week, Figure 57D. Body weight was also not significantly different between the High$\mathrm{Fat}+\mathrm{C}$ diet and the High-Fat+In diets at any time point, Figure 58C. The GLP-1R ${ }^{-/-}$mice gained less weight than Wild-Type mice in all respective diet groups. The body weight gain of GLP-1R ${ }^{-/-}$mice fed the High-Fat+C or High-Fat+In diets was the same between the diet groups, Figure 57E. The same was true of the body weights between these two groups, Figure 58E. The final week body weight gain was not significantly different between High-Fat+C or High-Fat+In diets, Figure 57E.

To account for the increased size and weight of the gut in response to inulin in the diet the weight of the intestinal tract was subtracted from the final body weight of the mice on dissection. The disembowelled body weight of Wild-Type mice was significantly lower in the High-Fat + In fed mice compared to those fed the High-Fat $+\mathrm{C} \operatorname{diet}(\mathrm{p}<0.001)$, Figure 58B. The disembowelled body weight of $\mathrm{PYY}^{-/-}$was not significantly different between High-Fat+In and High-Fat $+\mathrm{C}$ diet fed mice although mean weight was lower in Inulin fed mice, Figure 58D. The disembowelled body weight body weight of GLP-1R ${ }^{-/-}$ mice fed High-Fat+In was significantly lower than the High-Fat $+C$ fed mice $(p=0.039)$, Figure 58F. In GLP-1R ${ }^{-/-}$mice the increased weight of the gut was masking decreases in overall body weight. Overall weight gain and body weight reductions in response to inulin were small. Inulin only reduced body weight gain and body weight in Wild-Type mice. The High-Fat+In diet significantly decreased final body weights both Wild-Type and GLP-1R ${ }^{-/-}$mice after correction for the increased weight of the gut. 

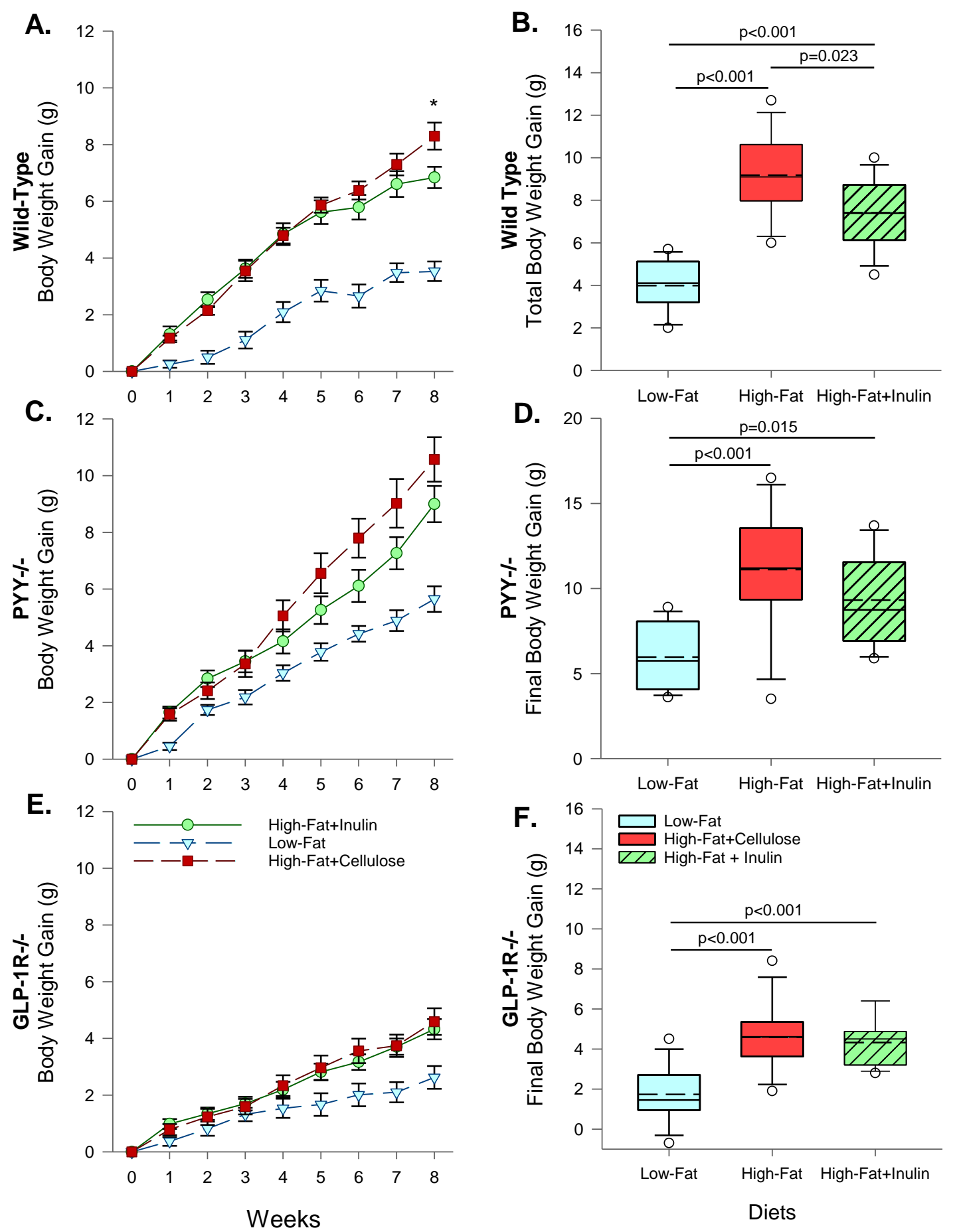

Figure 57. Effects of inulin on body weight gain and final body weight gain.

Weekly body weight gain in Wild-Type mice (A), PYY ${ }^{-/-}$mice $(\mathrm{C})$, and GLP-1R ${ }^{-/-}$mice (E) mice fed Low-Fat, High-Fat $+\mathrm{C}$, and High-Fat+In diets. Total body weight gain at the final week in Wild-Type mice (B), PYY ${ }^{-/-}$mice (D), and GLP-1R ${ }^{-/-}$mice (F) mice fed LowFat, High-Fat $+\mathrm{C}$, and High-Fat+In diets. Data points represent mean \pm SEM. For Box plots the solid line $=$ median, and dashed line $=$ mean. Significant differences within each genotype were determined by one way ANOVA with Tukey's post hoc test. Asterisks indicate significant differences $\left({ }^{*} \mathrm{p}<0.05 ;{ }^{* *} \mathrm{p}<0.01 ;{ }^{* *} \mathrm{p}<0.001\right)$ at each week. $\mathrm{n}=12$ mice per group. 

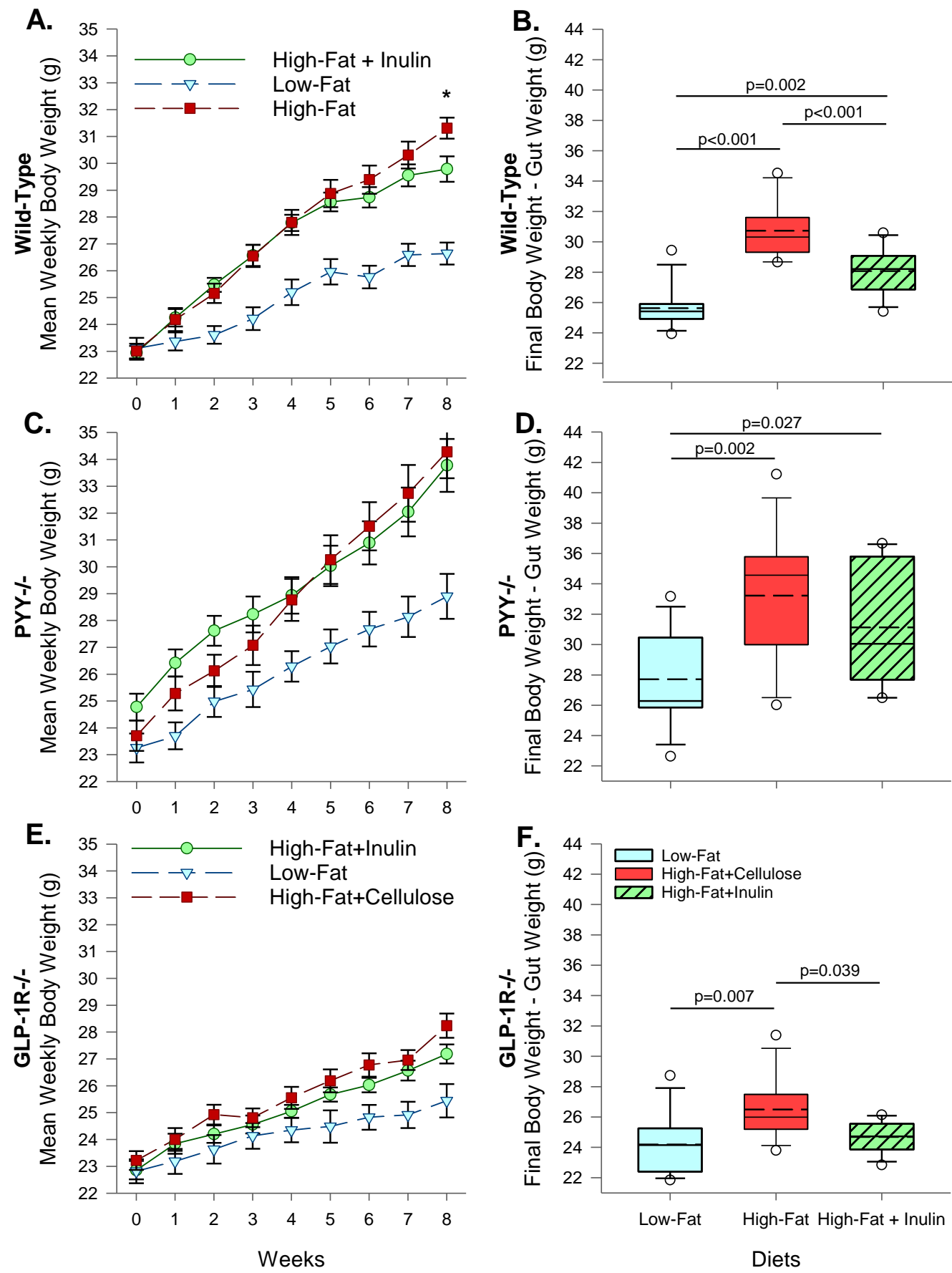

Figure 58. Effects of inulin on body weight and final body weight.

Weekly change in body weight in Wild-Type mice (A), PYY-/- mice (C), and GLP-1R ${ }^{-/-}$ mice (E) mice fed Low-Fat, High-Fat+C, and High-Fat+In diets. Body weight at the final week in Wild-Type mice (B), PYY-/- mice (D), and GLP-1R ${ }^{-/-}$mice (F) mice fed Low-Fat, High-Fat $+\mathrm{C}$, and High-Fat+In diets. Data points represent mean \pm SEM. For Box plots the solid line $=$ median, and dashed line = mean. Significant differences within each genotype were determined by one way ANOVA with Tukey's post hoc test. Asterisks indicate significant differences $\left({ }^{*} \mathrm{p}<0.05 ;{ }^{* *} \mathrm{p}<0.01 ;{ }^{* *} \mathrm{p}<0.001\right)$ at each week. $\mathrm{n}=12$ mice per group. 


\subsection{TOTAL INTESTINAL WEIGHT AND LENGTH}

The weight and length of the intestinal tract from the duodenum to the anus, minus the stomach, was weighed on dissection. The length of the gastrointestinal tract was significantly increased in mice fed the High-Fat+In diet in all genotypes of mice relative to the Low-Fat and High-Fat $+\mathrm{C}$ diets, Figure 59A. The length of the gastrointestinal tract was higher in $\mathrm{PYY}^{-/-}$fed the High-Fat+In diet than Wild-Type and GLP-1R ${ }^{-/-}$on the same diet. The weight of the gastrointestinal tract was significantly increased in mice fed the High-Fat+In diet relative to the other diets in mice of all genotypes, Figure 59B. The addition of inulin but not cellulose, to the high fat diet resulted in a longer and heavier intestinal tract. The genotype of the mice did not influence this effect.

\subsection{SMALL INTESTINE WEIGHT AND LENGTH}

When measured separately the small intestine of mice fed the High-Fat+In diet was significantly longer than for mice fed Low-Fat and High-Fat $+\mathrm{C}$ diets for each genotype Wild-Type, PYY ${ }^{-/,}$and GLP-1R ${ }^{-/-}$mice, Figure 60A. The weight of small intestine containing its contents was not significantly different between mice fed High-Fat+In and High-Fat $+\mathrm{C}$ diets, Figure 60B. The small intestine increases in length with the addition of inulin but not cellulose to the diet. The genotype of the mice did not influence this effect. 

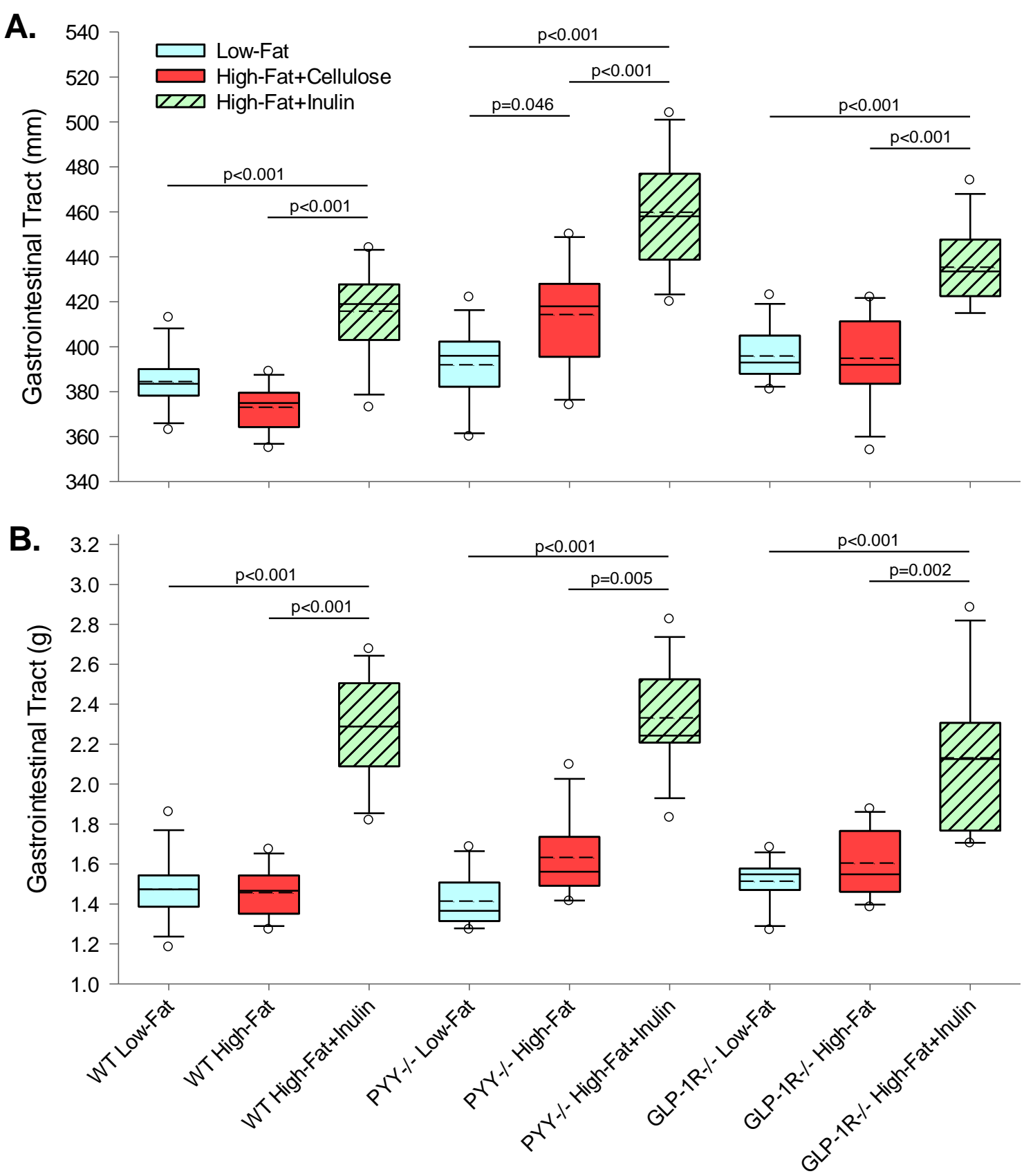

Figure 59. Effects of inulin on gastrointestinal tract weight and length.

Length of the gastrointestinal tract (A), and weight of the gastrointestinal tract (B), in Wild-Type, $\mathrm{PYY}^{-/}$, and GLP-Rl- ${ }^{-/}$mice fed Low-Fat, High-Fat+C, and High-Fat+In diets. In box plots the solid line $=$ median, and dashed line $=$ mean. In box plots the solid line $=$ median, and dashed line $=$ mean. Statistical significance was determined by one-way ANOVA with Tukey's post hoc test. $\mathrm{n}=12$. 

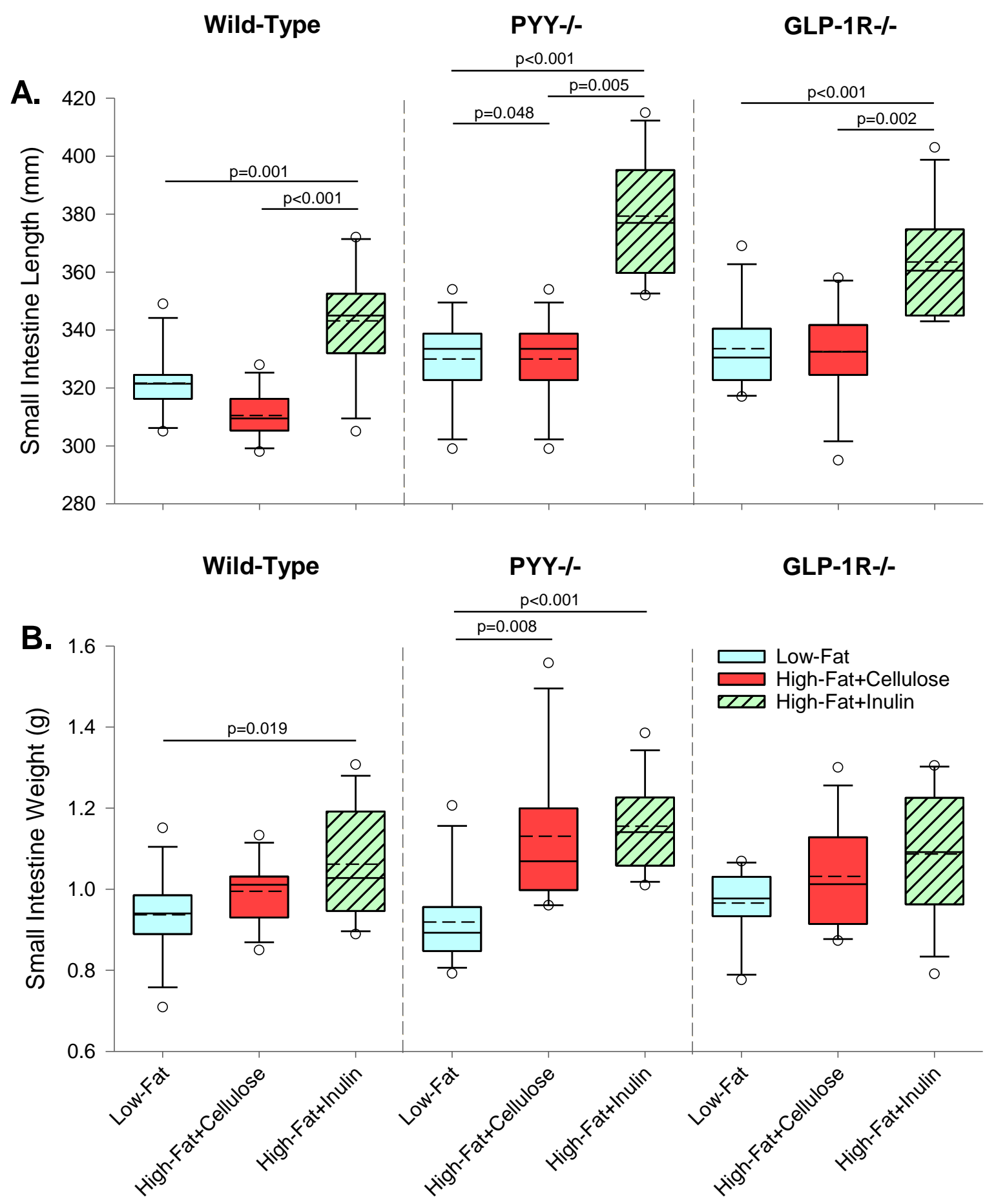

Figure 60. Effects on inulin on small intestine weight and length.

Length of the small intestine (A), and weight of the small intestine (B), in Wild-Type, $\mathrm{PYY}^{-/-}$, and GLP-Rl ${ }^{-/-}$mice fed Low-Fat, High-Fat $+\mathrm{C}$, and High-Fat+In diets. In box plots the solid line $=$ median, and dashed line $=$ mean. Statistical significance was determined by one-way ANOVA with Tukey's post hoc test. $n=12$. 


\subsection{CAECUM WEIGHT AND LENGTH}

The caecum was the part of the gut that increased in length to the greatest extent in response to the High-Fat+In diet. Due to its anatomical position the length of the caecum does not contribute to the total length of the intestinal tract in the mouse. The caecum of mice fed the High-Fat+In diet was significantly longer that mice fed Low-Fat and High-Fat $+\mathrm{C}$ diets in Wild-Type, $\mathrm{PYY}^{-/-}$, and GLP-1R ${ }^{-/-}$mice, Figure 61A. The weight of the caecum was significantly heavier while containing its contents in mice fed the High-Fat+In diet in comparison to the Low-Fat and High-Fat+C diets, Figure 61B. This difference was still significant when the caecal tissue was weighed after the luminal contents had been removed, Figure 61C. Caecal weight was not significantly different between mice fed the Low-Fat and High-Fat $+\mathrm{C}$ diets when full, Figure 61B, or when empty, Figure 61C. The size and weight of the caecum increases with the addition of inulin but not cellulose to the diet. This increase was due to both a greater weight of caecal contents and increased weight of caecum tissue. The genotype of the mice did not influence this effect.

\subsection{COLON WEIGHT AND LENGTH}

The colon length of mice fed the High-Fat+In diet was significantly longer that mice fed the Low-Fat and High-Fat+C diets in Wild-Type, PYY ${ }^{-/-}$, and GLP-1R ${ }^{-/-}$mice, Figure 62A. The caecal weight of mice fed the High-Fat+In diet was significantly heavier while containing its contents in in comparison to the Low-Fat and High-Fat $+\mathrm{C}$ diets, Figure 62B. This difference in weight between groups was still significant after the contents of the colon had been removed, Figure $62 \mathrm{C}$. The weight of the colon was not significantly different between mice fed the Low-Fat and High-Fat $+\mathrm{C}$ diets when full, $\mathrm{B}$, or when empty, $C$. The size and weight of the caecum increases with the addition of inulin but not cellulose to the diet. This increase was included both increased colonic contents and increased colon tissue. The genotype of the mice did not influence this effect. 

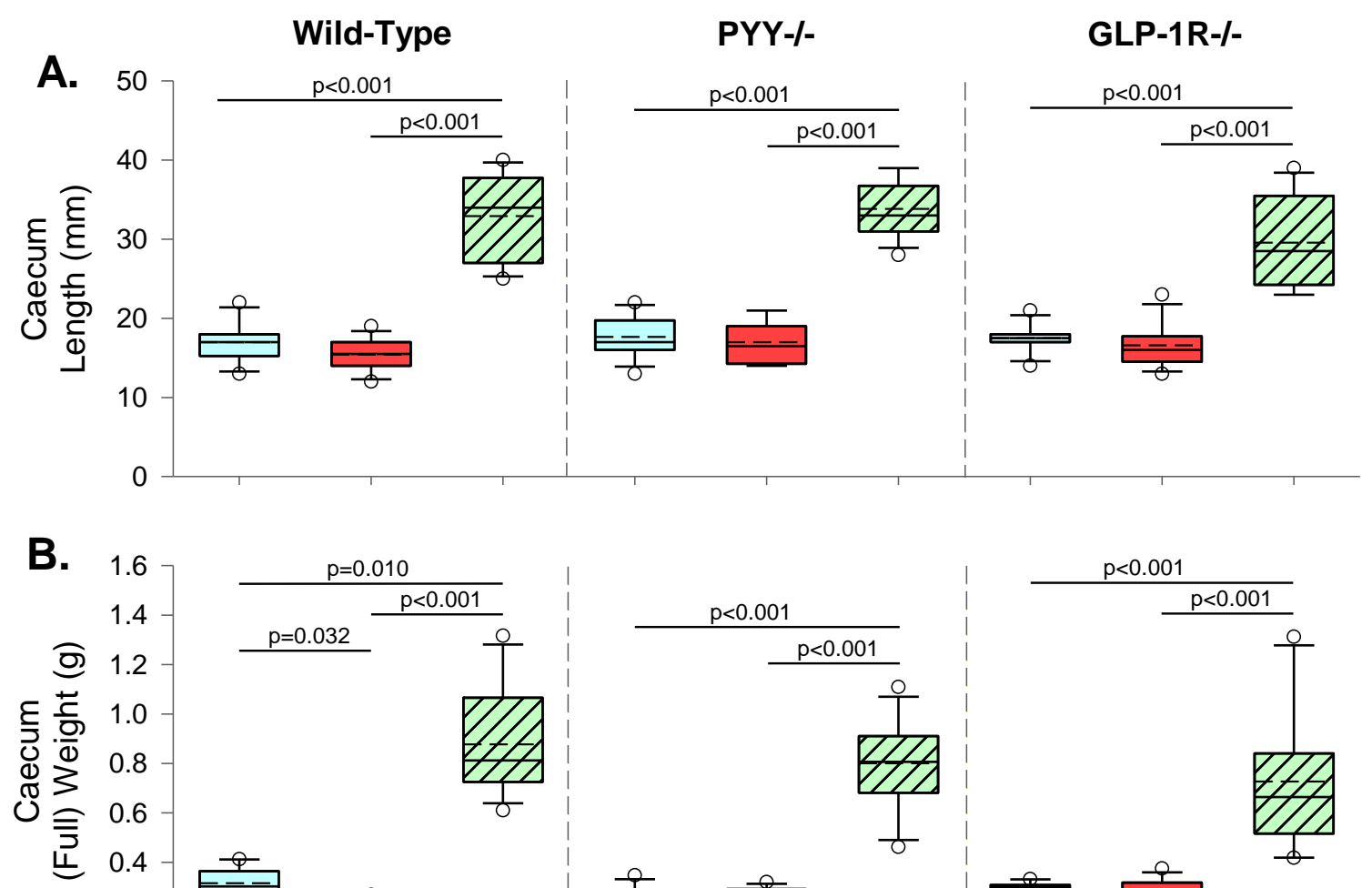

B. 1
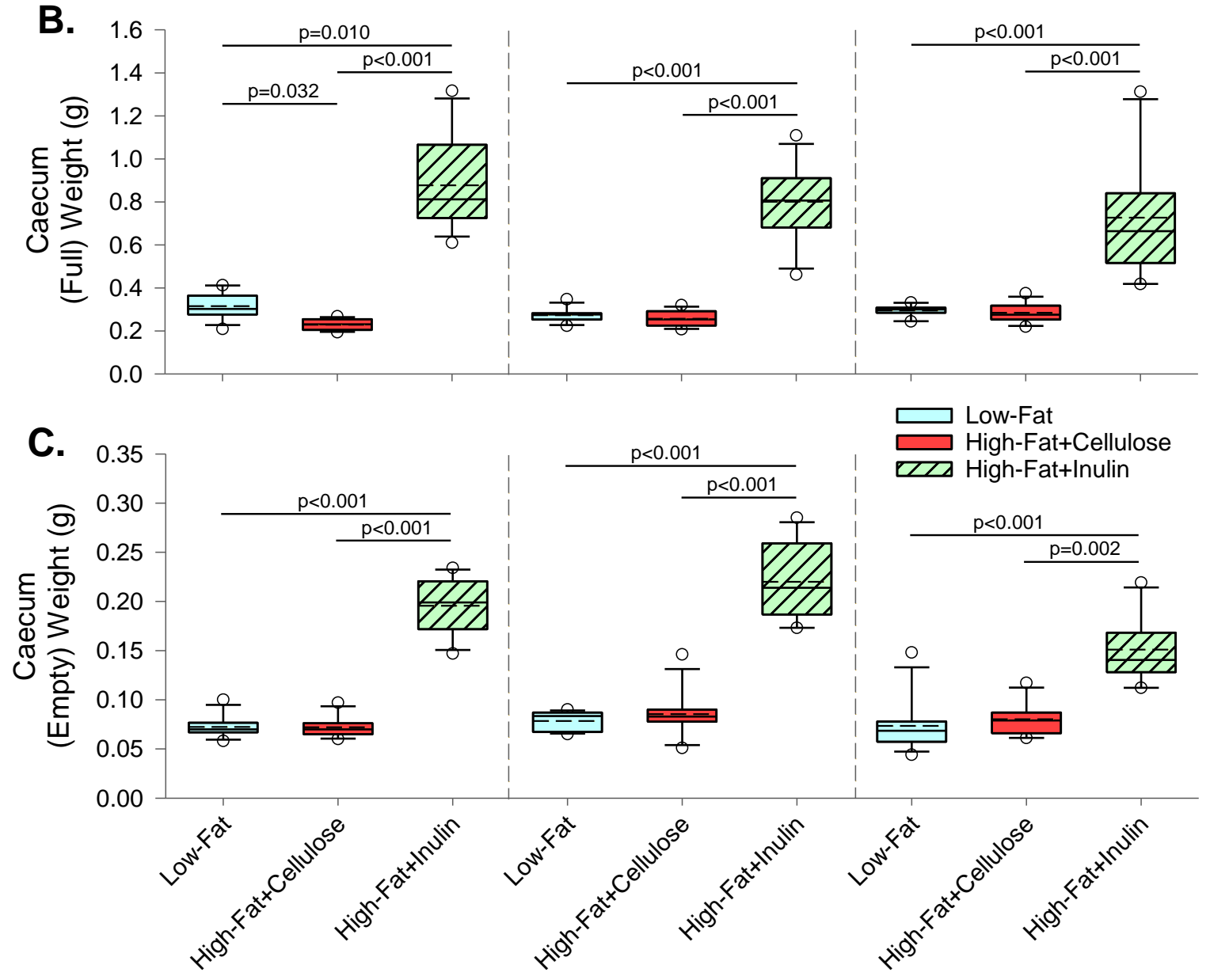

Figure 61. Effects of inulin on caecum weight and length.

Caecum length (A), caecum weight containing contents (B), and caecum weight without contents (C), in Wild-Type, $\mathrm{PYY}^{-/-}$, and GLP-Rl ${ }^{-/-}$mice fed Low-Fat, High-Fat $+\mathrm{C}$, and High-Fat+In diets. In box plots the solid line = median, and dashed line $=$ mean . Statistical significance was determined by one-way ANOVA with Tukey's post hoc test. $\mathrm{n}=12$. 

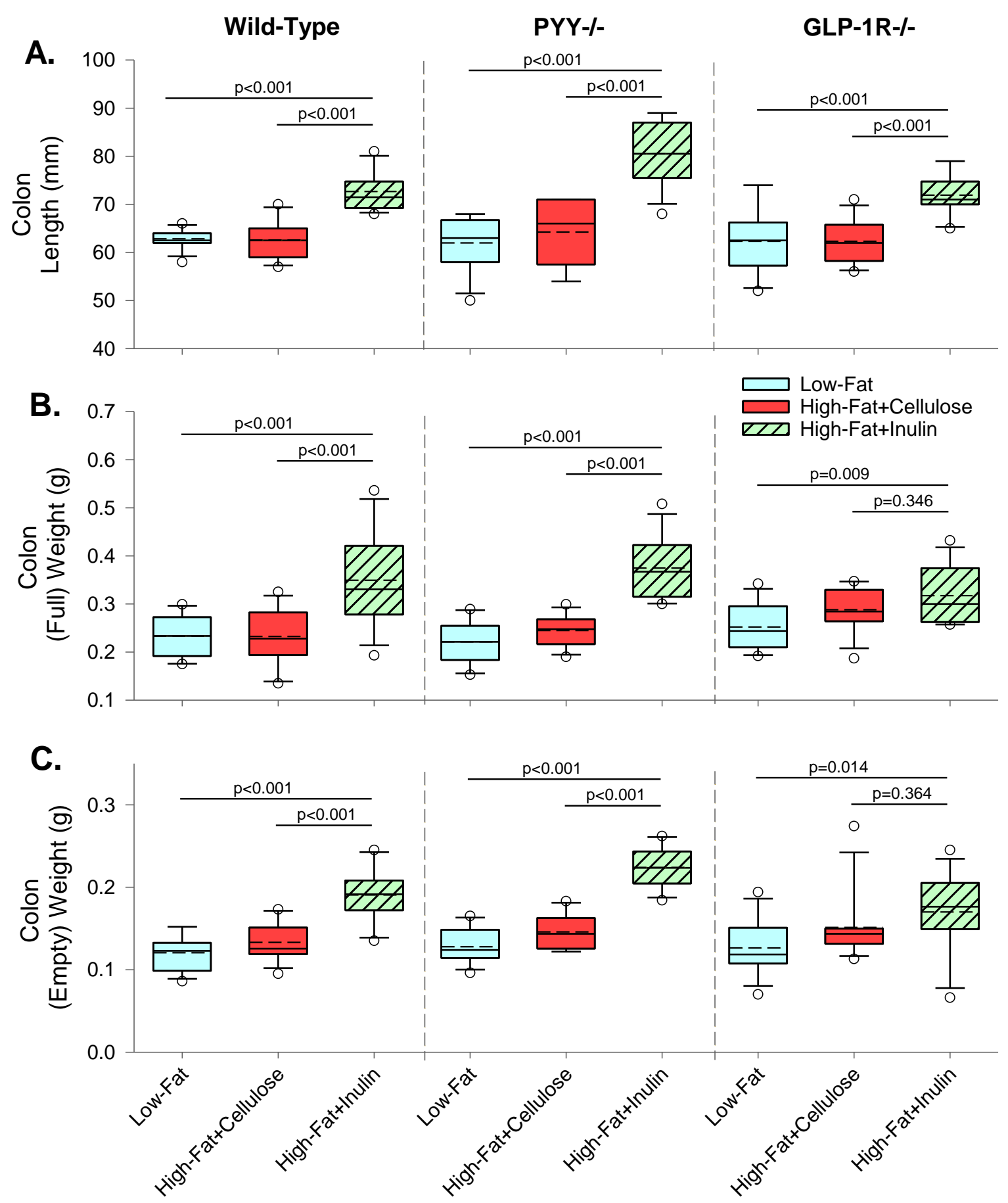

Figure 62. Effects of inulin on colon length and weight.

Colon length (A), colon weight containing contents (B), and colon weight without contents (C), in Wild-Type, $\mathrm{PYY}^{-/-}$, and GLP-R1 ${ }^{-/-}$mice fed Low-Fat, High-Fat $+\mathrm{C}$, and High-Fat+In diets. In box plots the solid line = median, and dashed line $=$ mean . Statistical significance was determined by one-way ANOVA with Tukey's post hoc test. $\mathrm{n}=12$. 


\subsection{EFFECT OF INULIN ON STOMACH, LIVER, AND KIDNEY WEIGHT}

The addition of inulin to the diet did not result in any consistent impact on the weight of the stomach, liver, or kidneys in either Wild-Type, PYY ${ }^{-/-}$, or GLP-1R ${ }^{-/-}$mice, Figure 63.

\subsection{PHOTOS OF CAECUM AND COLON}

The presence of inulin in the high-fat diet visibly increased the size of the caecum and colon in wild-type mice, PYY ${ }^{-/-}$, and GLP-1R ${ }^{-/-}$. Photographs of caecum and colon adjusted for scale clearly show the increase in size in response to the presence of inulin in the diet, Figure 64. Relative to the High-Fat+In diet, the caecum and colon of HighFat $+\mathrm{C}$ and Low-Fat diet fed mice appeared thinner and atrophied which correlated with a shortening of the colon and loss of caecal and colonic mass that was quantitated by weighing the caecum and colon, both with and without contents. 
A. $0.8 \quad$ Wild-Type

PYY-/-

GLP-1R-/-
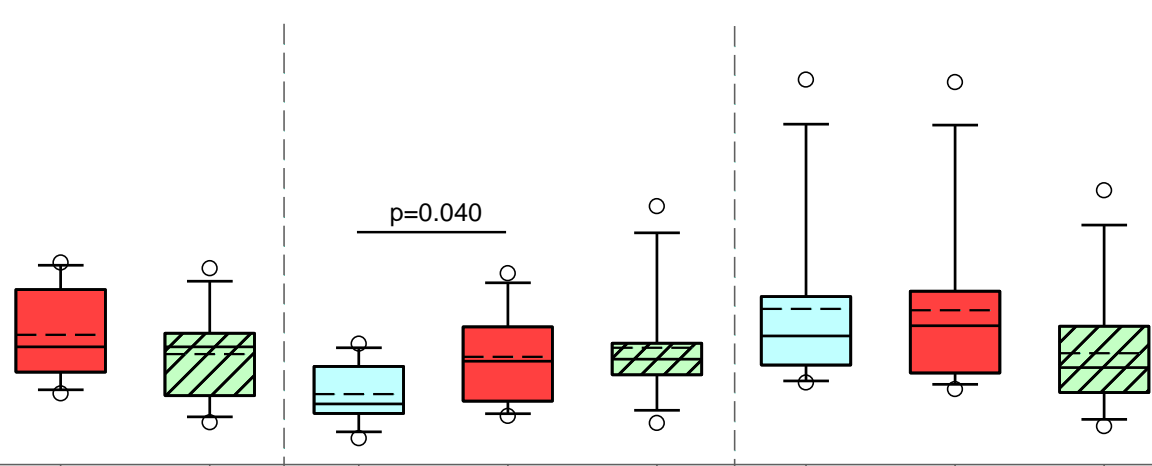

0.1

B. 1.8
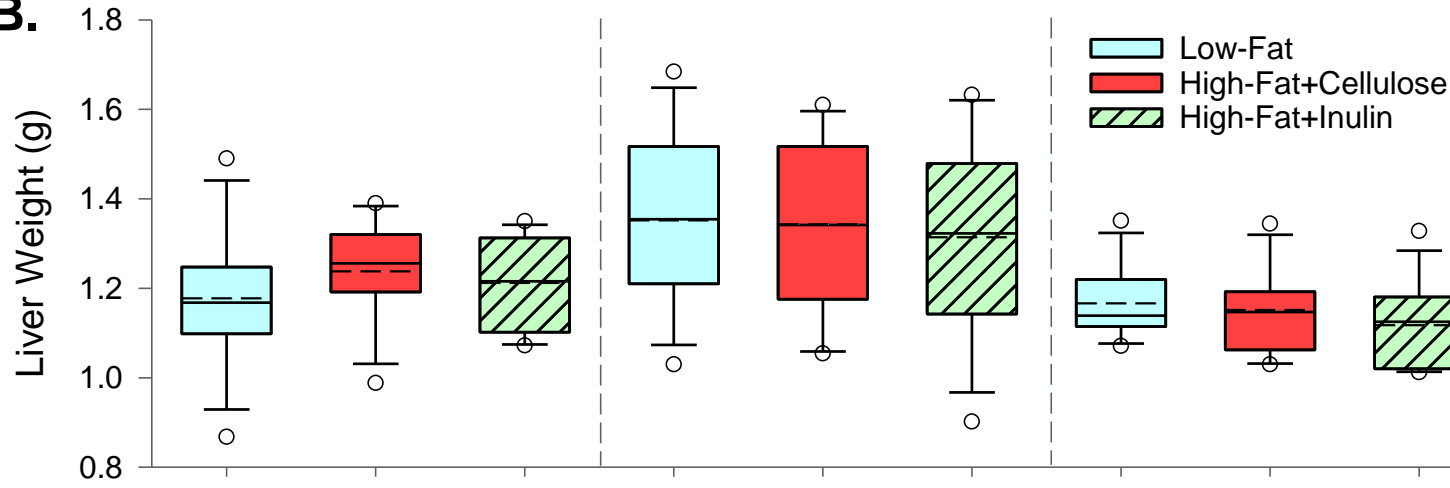

EZ] High-Fat+Inulin
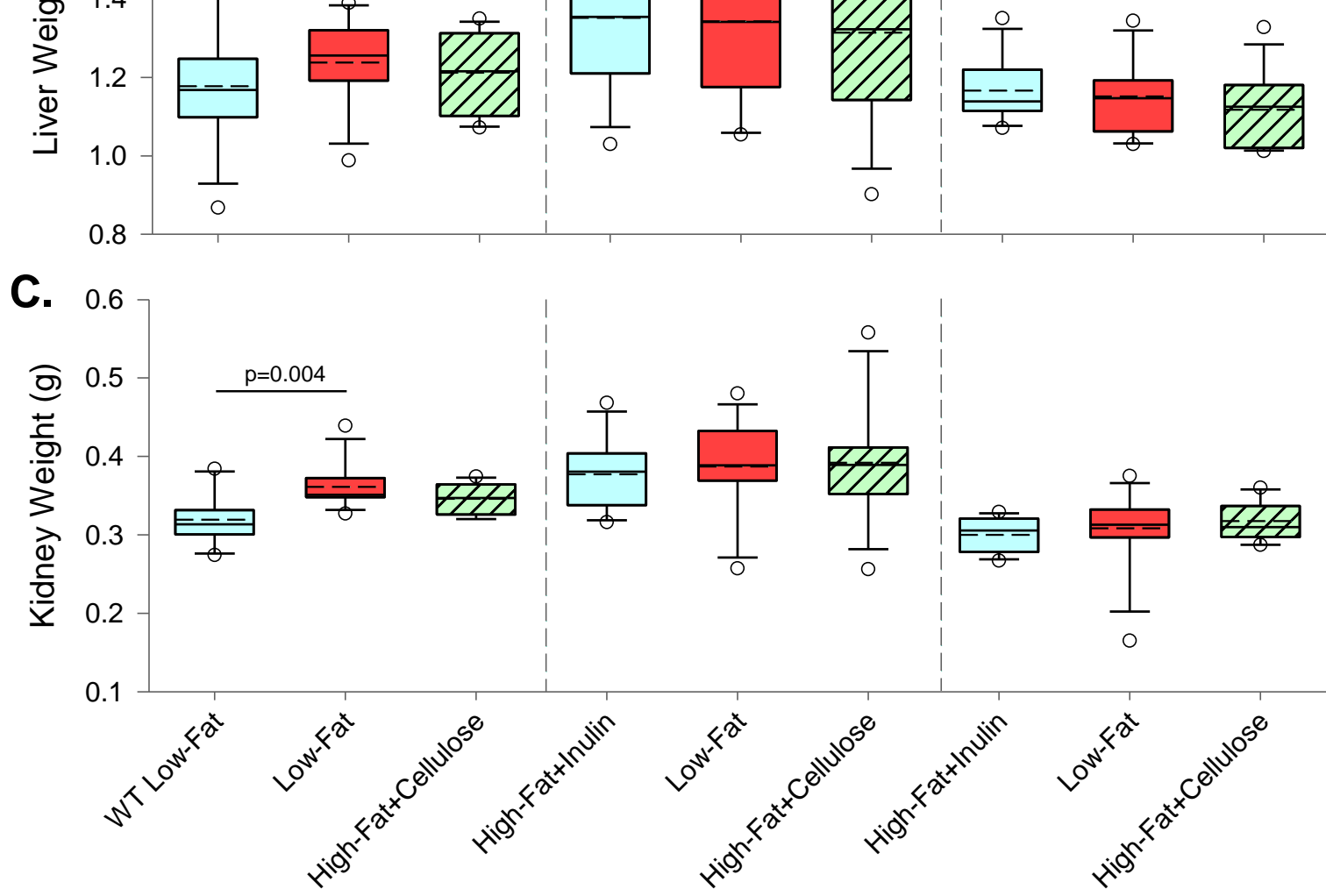

Figure 63. Effects of inulin on stomach, liver, and kidney weight.

Stomach, liver, and kidney weight in Wild-Type, PYY ${ }^{-/-}$, or GLP-1R ${ }^{-/-}$mice fed Low-Fat, High-Fat $+\mathrm{C}$, and High-Fat+In diets. In box plots the solid line = median, and dashed line $=$ mean. Statistical significance was determined by one-way ANOVA with Tukey's post hoc test. $n=12$. 
A.

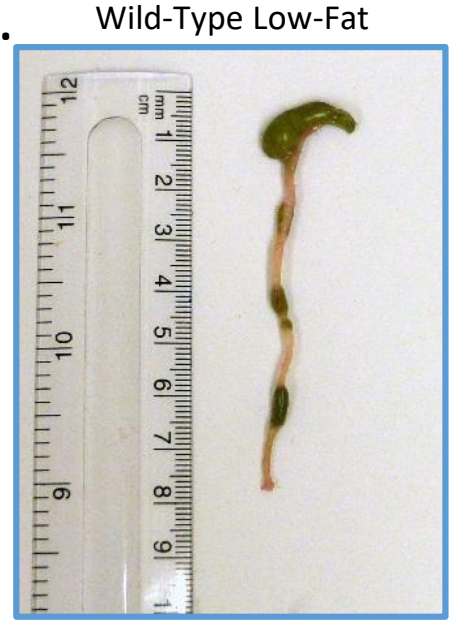

B.

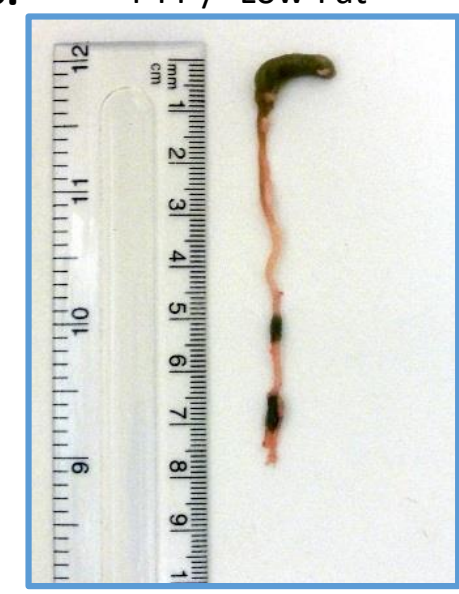

C.

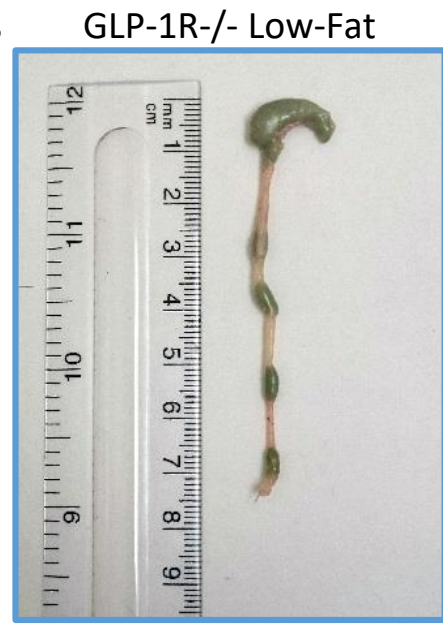

Wild-Type High-Fat

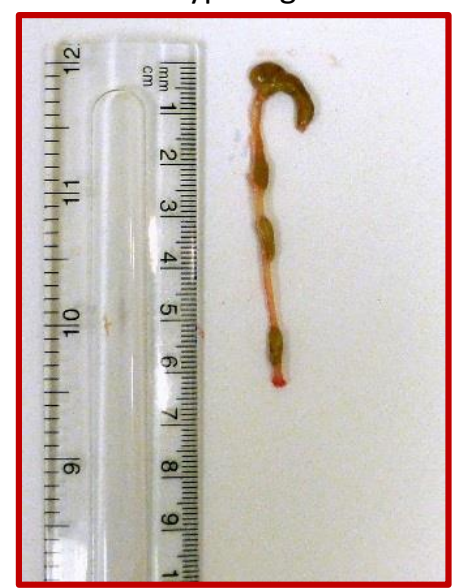

PYY-/- High-Fat

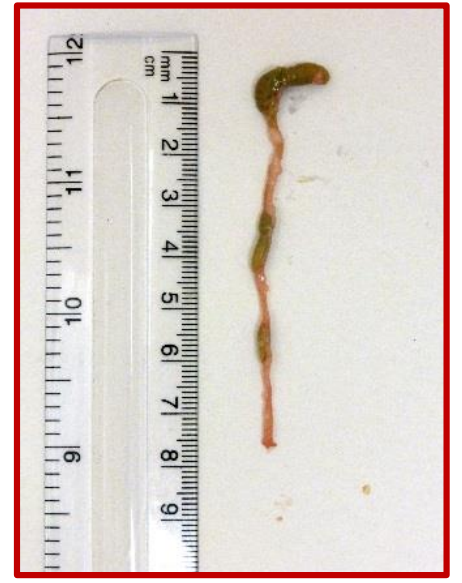

GLP-1R-/- High-Fat

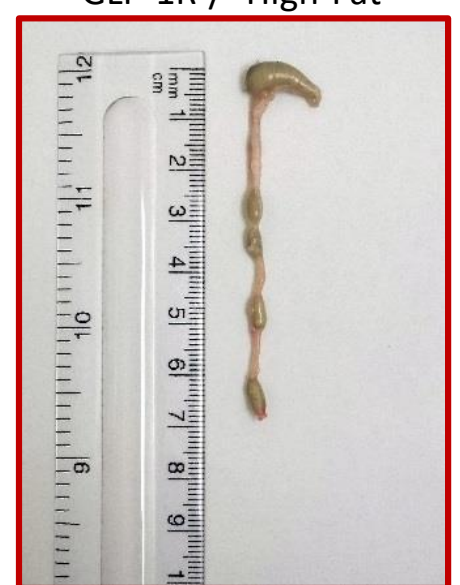

Wild-Type High-Fat + Inulin

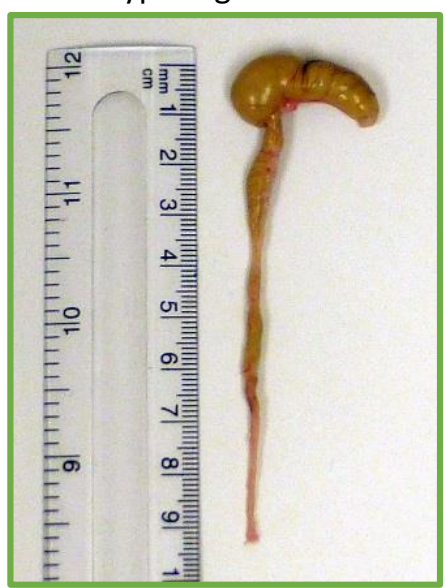

PYY-/- High-Fat + Inulin

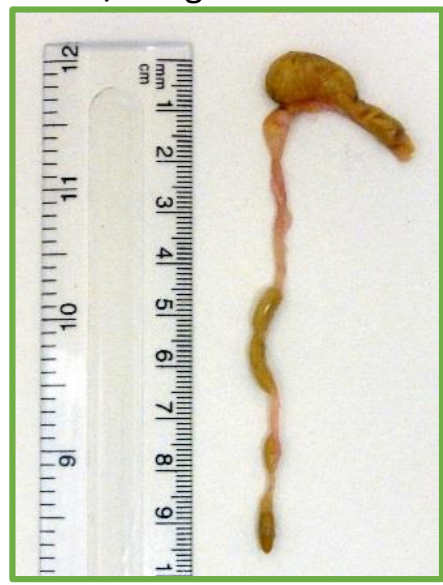

GLP-1R-/- High-Fat + Inulin

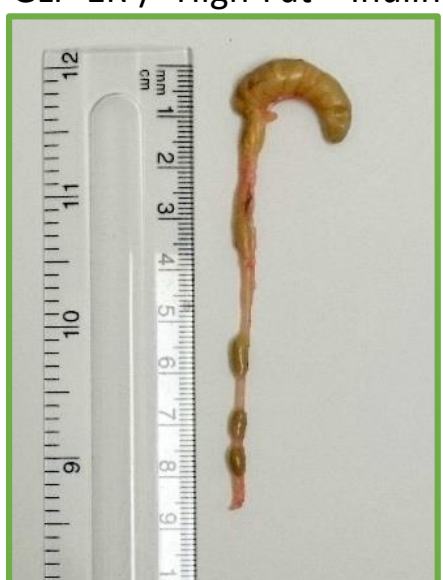

Figure 64. Photographs of caecum and colon morphology changes in response to inulin.

Representative photograph of the caecum and colon for Wild-Type mice (A), for $\mathrm{PYY}^{-/}$ mice (B), and GLP-1R ${ }^{-/-}$mice (C) fed Low-Fat, High-Fat $+\mathrm{C}$, and High-Fat+In diets. Photographs were adjusted to be of an equivalent scale. 


\subsection{EFFECTS ON INULIN ON PRODUCTION OF SHORT-CHAIN FATTY ACIDS}

The feeding of the High-Fat+In diet did not in increase concentrations of acetate in the caecum of either Wild-Type, PYY ${ }^{-/}$, or $\mathrm{GLP}^{-1 R^{-/-}}$mice, Figure 65A. Acetate concentrations were not significantly different between any diet groups. However, the variability of acetate concentrations increased in the caecum of mice fed the inulin containing diet. Propionate concentrations decreased in High-Fat $+\mathrm{C}$ diet fed mice relative to Low-Fat diet fed control mice, Figure 65B. In mice fed the High-Fat+In diet propionate concentrations were restored to the concentration found in Low-Fat diet fed mice. Changes in propionate concentrations were consistent across all genotypes of mice. Butyrate concentrations tended to increase in High-Fat+In diet fed mice of all genotypes relative to the High-Fat $+\mathrm{C}$ diet, but were only significantly different in WildType and GLP-1R ${ }^{-/-}$mice, Figure $65 \mathrm{C}$. As with acetate the concentrations of butyrate became more variable in High-Fat+In diet fed mice. Concentrations of total SCFAs were not significantly different for Wild-Type, or PYY-/-, and slightly higher in High-Fat+In fed GLP-1R ${ }^{-/-}$mice relative to the High-Fat+C diet, Figure 65.

To account for the relatively larger size of the caecum and caecal contents in HighFat+In fed mice, the total nanomoles of each SCFA was calculated per caecum of each mouse, Figure 66. While concentrations of SCFAs were not significantly increased the amount of acetate, propionate, and butyrate present in the caecum of High-Fat+In fed mice was significantly greater than other diet groups for all genotypes, Figure 66A-C. Total SCFAs in the caecum of High-Fat+In fed mice were significantly higher than HighFat $+\mathrm{C}$ and Low-Fat diet fed mice for Wild-Type, PYY ${ }^{-/,}$or GLP-1R ${ }^{-/-}$mice, Figure 66D. Feeding of inulin increases the amount of SCFAs in the caecum without greatly increasing the concentration of SCFAs. 
Mouse Genotype

A.

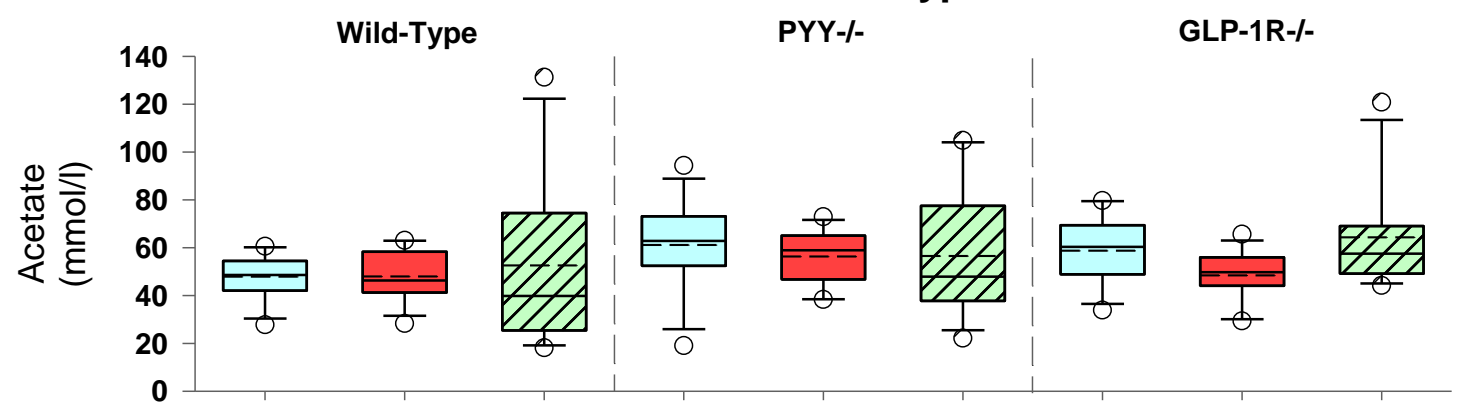

B.

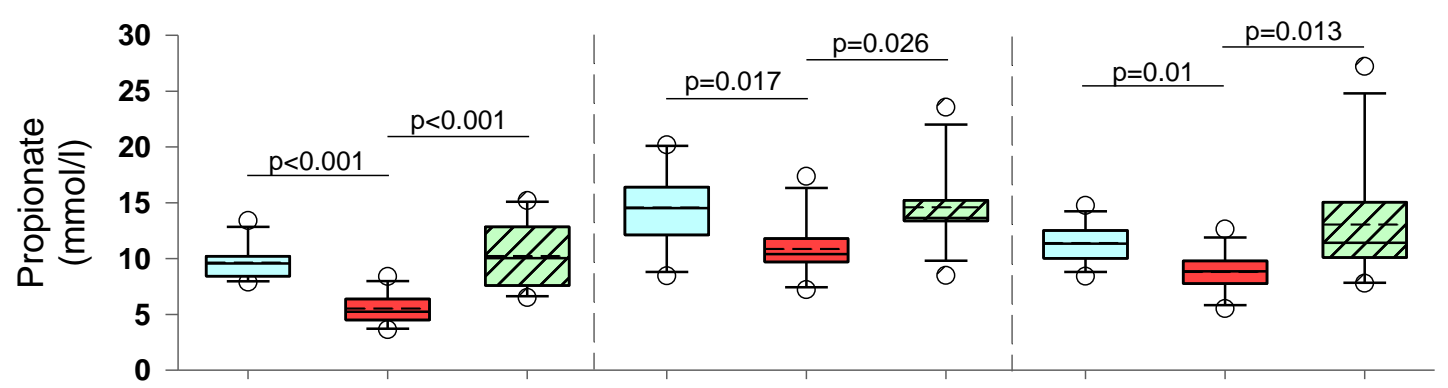

C.

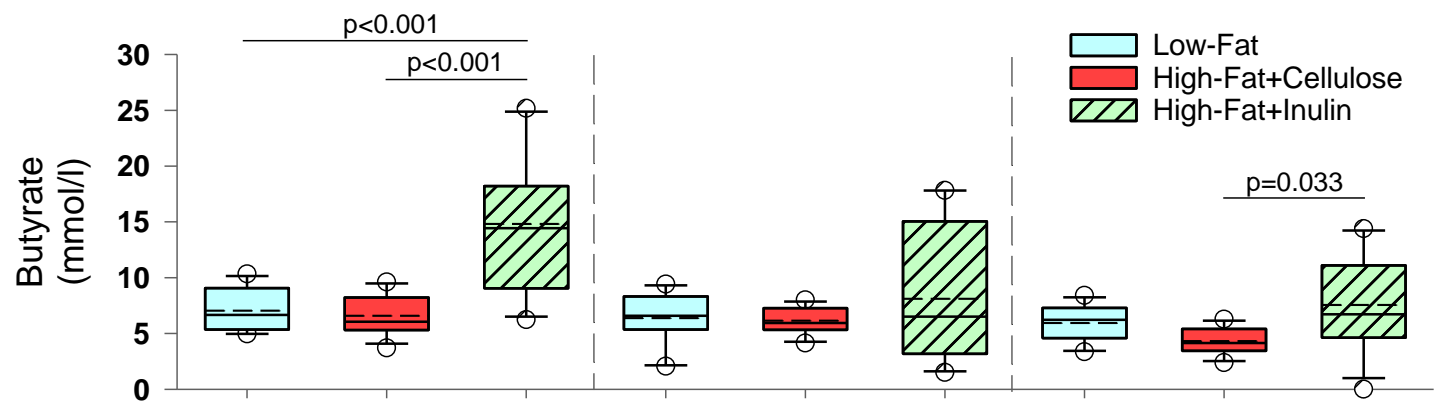

D.

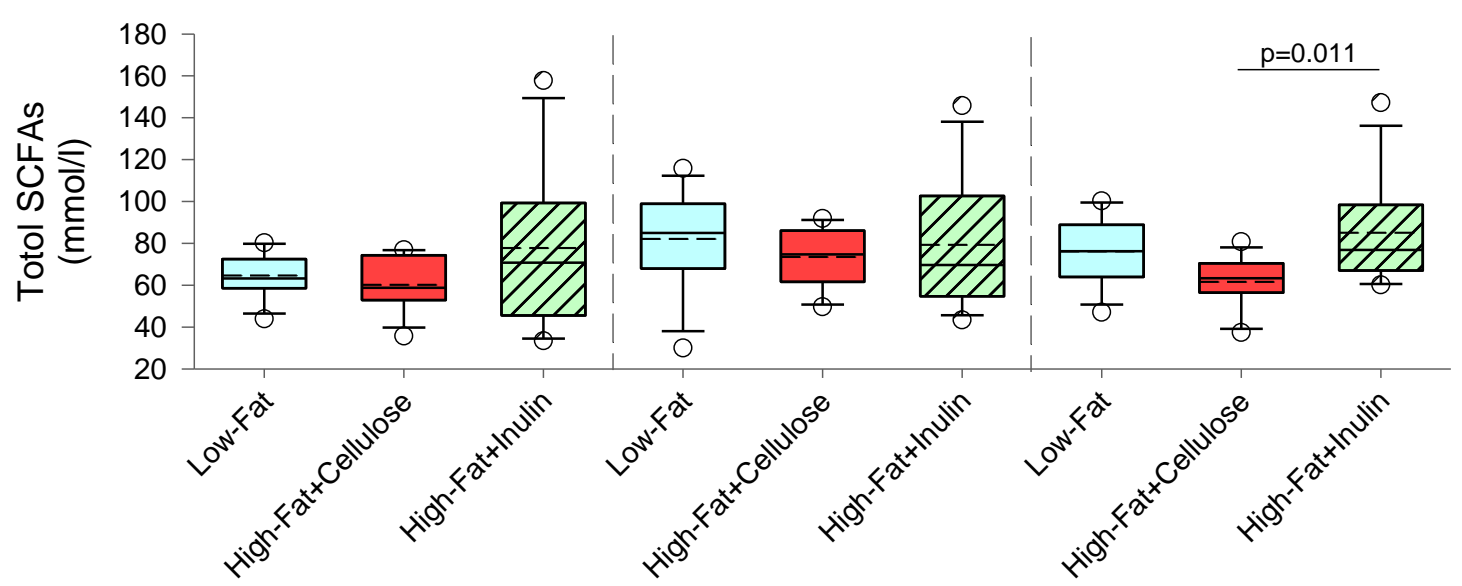

Figure 65. Concentrations of caecal short-chain fatty acids

Concentration of acetate (A), propionate (B), and butyrate (C) in the caecum of WildType, $\mathrm{PYY}^{-/}$, and GLP-1R ${ }^{-/-}$mice fed Low-Fat, High-Fat+C, and High-Fat+In diets. In box plots the solid line $=$ median, and dashed line $=$ mean. Statistical significance was determined by one-way ANOVA with Tukey's post hoc test. $\mathrm{n}=11-12$ mice per group. 
Mouse Genotype

A.

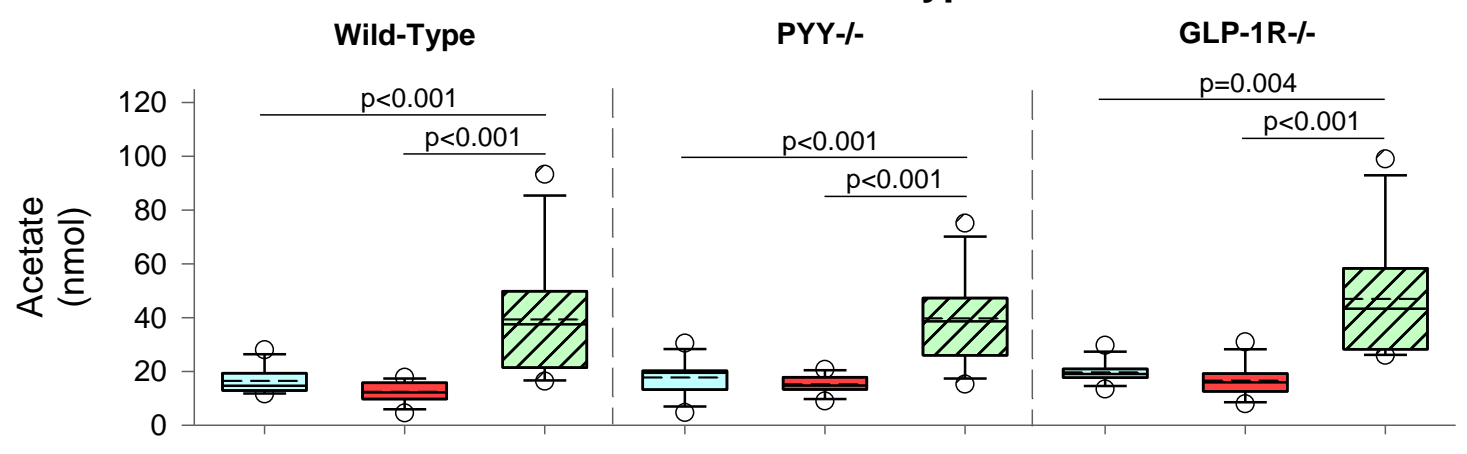

B.

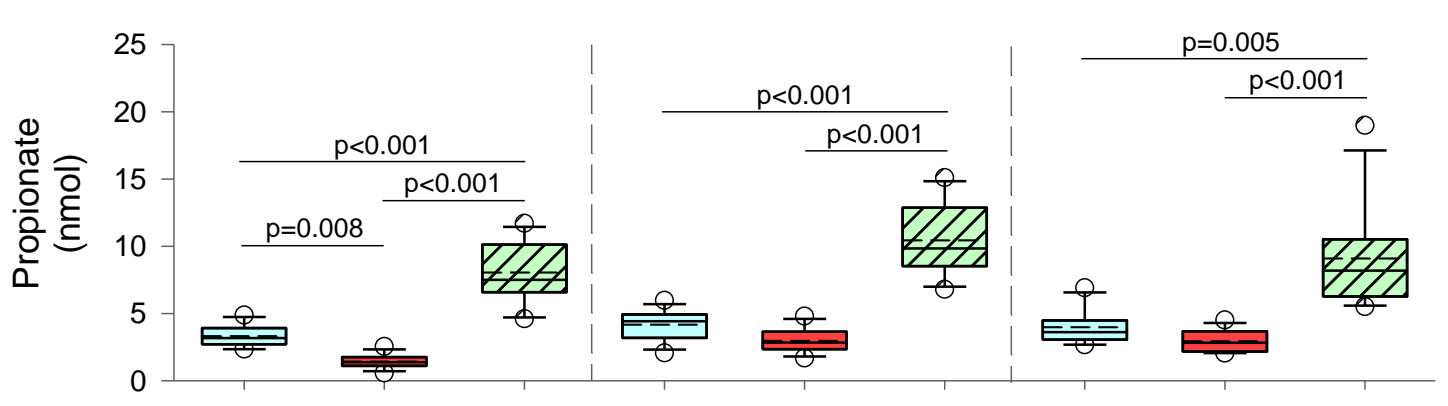

C.

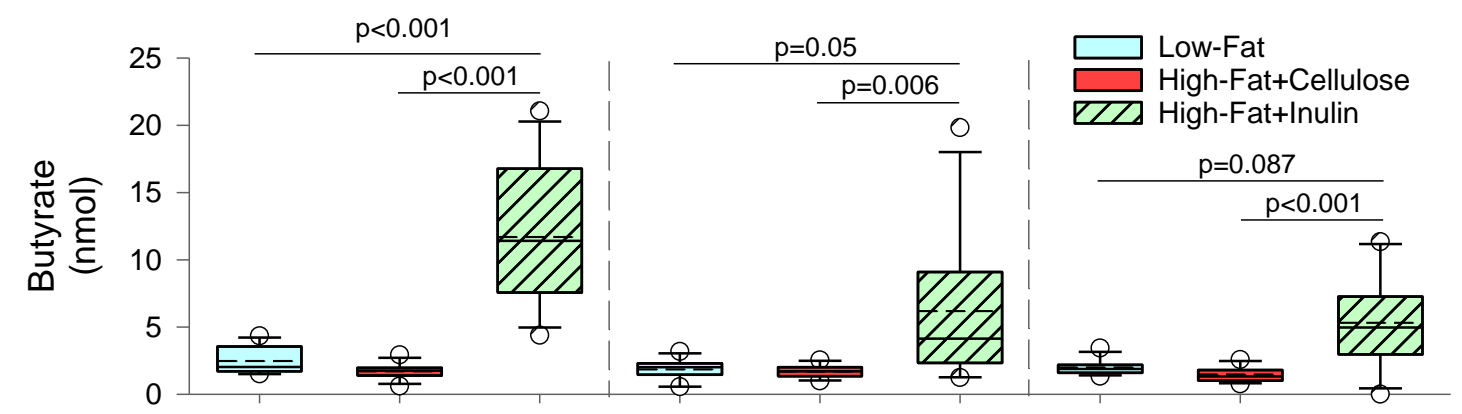

D.

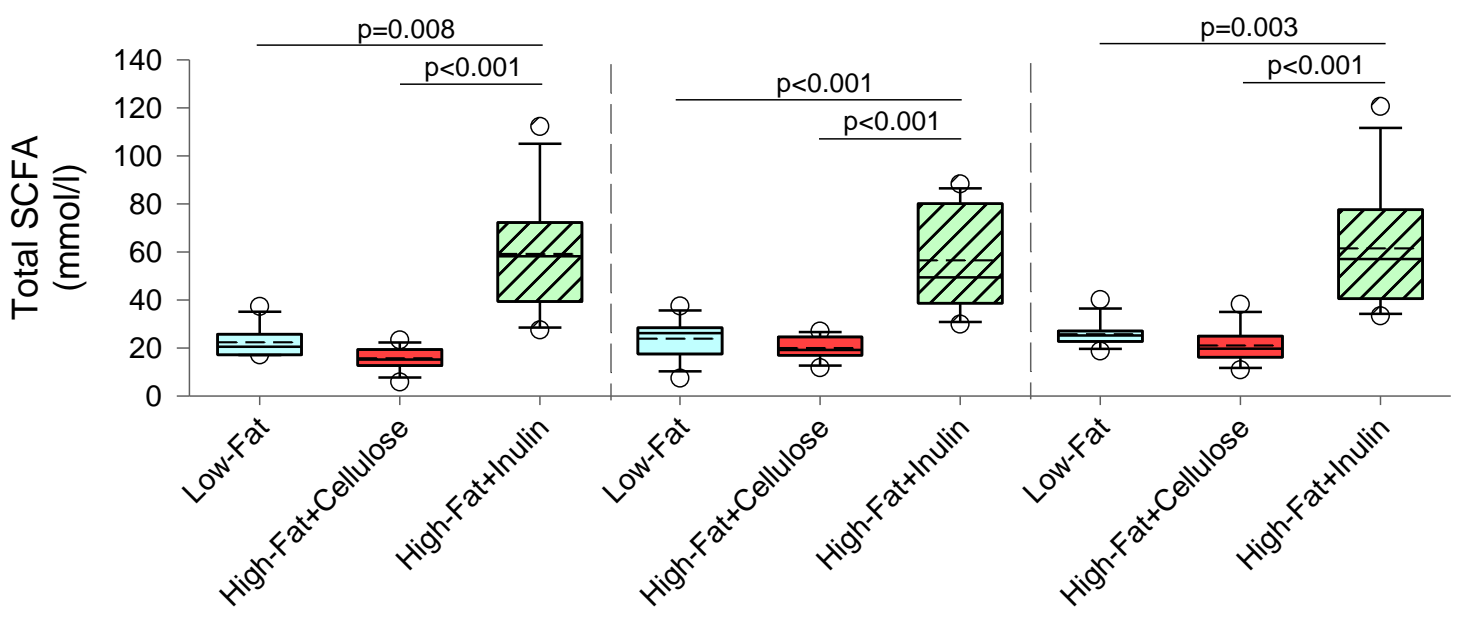

Figure 66. Total amount of caecal short-chain fatty acids

Total amounts of acetate (A), propionate (B), and butyrate (C) in the caecum of WildType, $\mathrm{PYY}^{-/-}$, and GLP-1R ${ }^{-/-}$mice fed Low-Fat, High-Fat $+\mathrm{C}$, and High-Fat+In diets. In box plots the solid line $=$ median, and dashed line $=$ mean. Statistical significance was determined by one-way ANOVA with Tukey's post hoc test. $\mathrm{n}=11-12$. 


\subsection{MOLAR RATIOS OF SHORT-CHAIN FATTY ACIDS}

Caecal molar ratios in Low-Fat and High-Fat $+C$ fed mice remained relatively consistent between mice with acetate dominating, Figure 67A. In contrast in High-Fat+In fed mice the ratios molar ratios between individual mice became more variable. In Wild-Type mice the mean caecal molar ratios of acetate: propionate: butyrate were 74:15:11 and 80:9:11 for Low-Fat and High-Fat + C respectively, Figure 67A. In High-Fat+In fed mice butyrate and propionate increased relative to acetate with a mean acetate: propionate: butyrate ratio of 64:16:21. The molar ratios also became more variable in High-Fat+In fed for PYY-/- and GLP-1R ${ }^{-/-}$mice while molar ratios did not change in GLP-1R ${ }^{-/-}$mice, Figure 67B-C. In $\mathrm{PYY}^{-/-}$mice mean molar ratios of acetate: propionate: butyrate were 74:18:8 and 77:15:8 for Low-Fat and High-Fat+C respectively with ratios in High-Fat+In fed mice being 70:20:10, Figure 67B. In GLP-1R ${ }^{-/-}$mice mean molar ratios of acetate: propionate: butyrate were 77:15:8 and 79:15:7 for Low-Fat and High-Fat+C respectively with ratios in High-Fat+In fed mice being 75:16:9, Figure 67C. Feeding of inulin tended to reduce the ratio of acetate in all mice with responses between individual mice becoming more variable.

\subsection{CAECAL CONCENTRATIONS OF ISO-BUTYRATE, ISO- VALERATE, AND VALERATE}

Caecal concentrations of iso-butyrate were not significantly changed between diets, Figure 68A. In contrast iso-Valerate and Valerate concentrations were significantly reduced in response to High-Fat+In diet feeding in Wild-Type, PYY ${ }^{-/-}$, and GLP-1R ${ }^{-/-}$ mice, Figure 68B-C. 

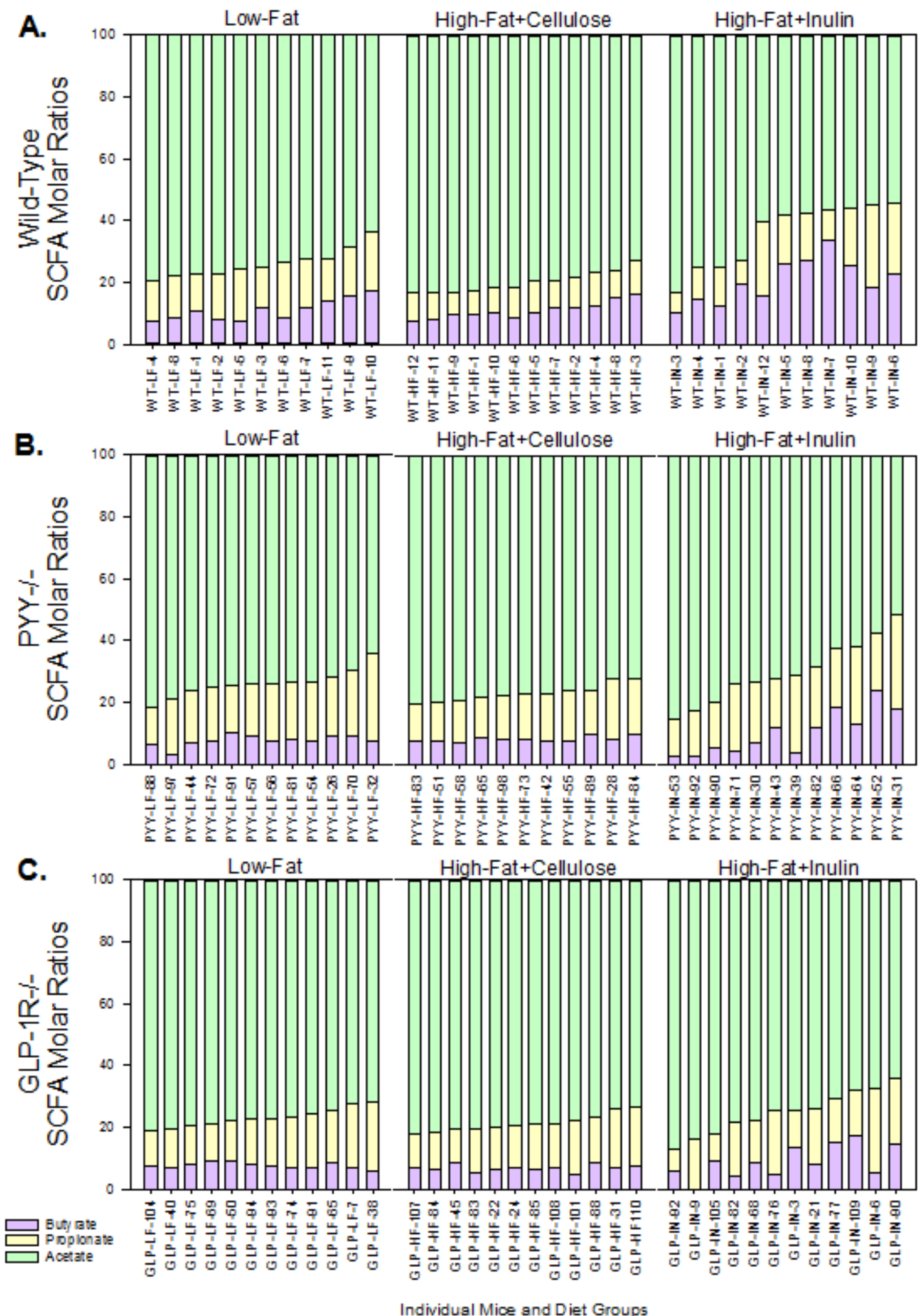

Figure 67. Molar ratios of caecal short-chain fatty acid

Molar ratios of acetate: propionate: butyrate in caecal contents of Wild-Type (A), PYY-/(B), and GLP-1R ${ }^{-/-}(C)$ mice fed Low-Fat, High-Fat+C, or High-Fat+In diets. Mice in each group ordered by decreasing ratio of acetate. Ratio of acetate (green), propionate (yellow), and butyrate (purple). 
Mouse Genotype

A.

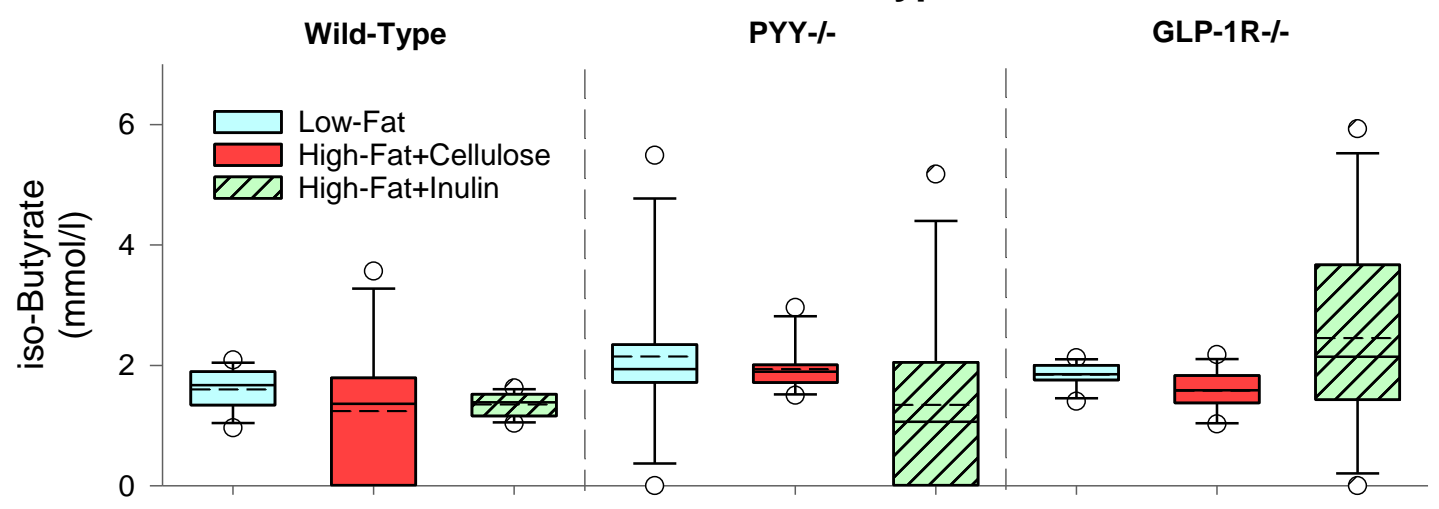

B.

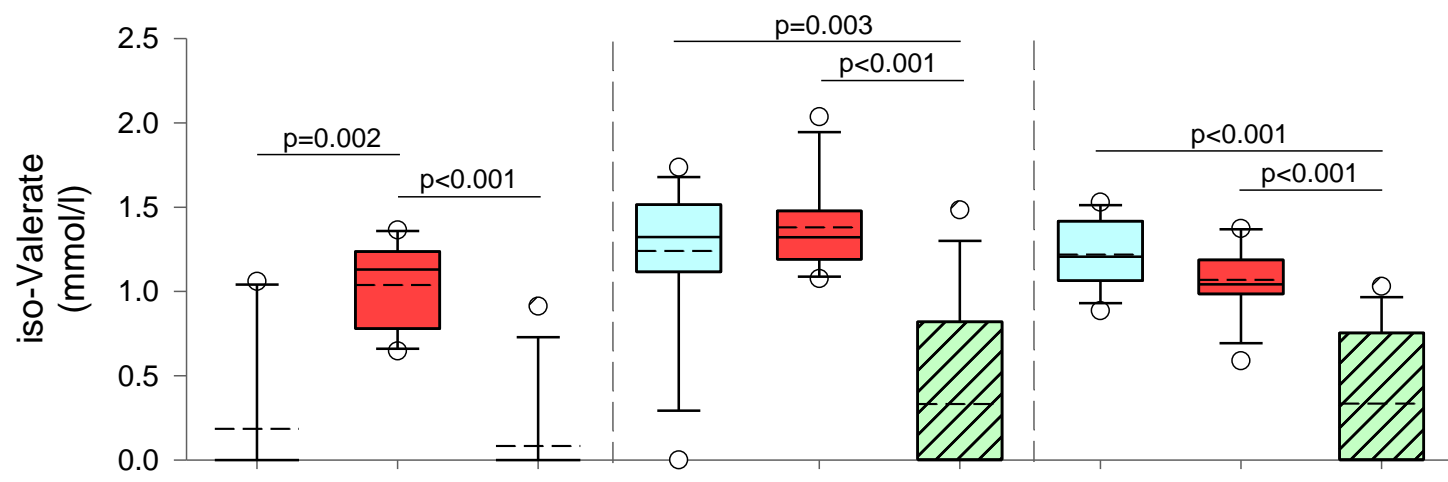

C.

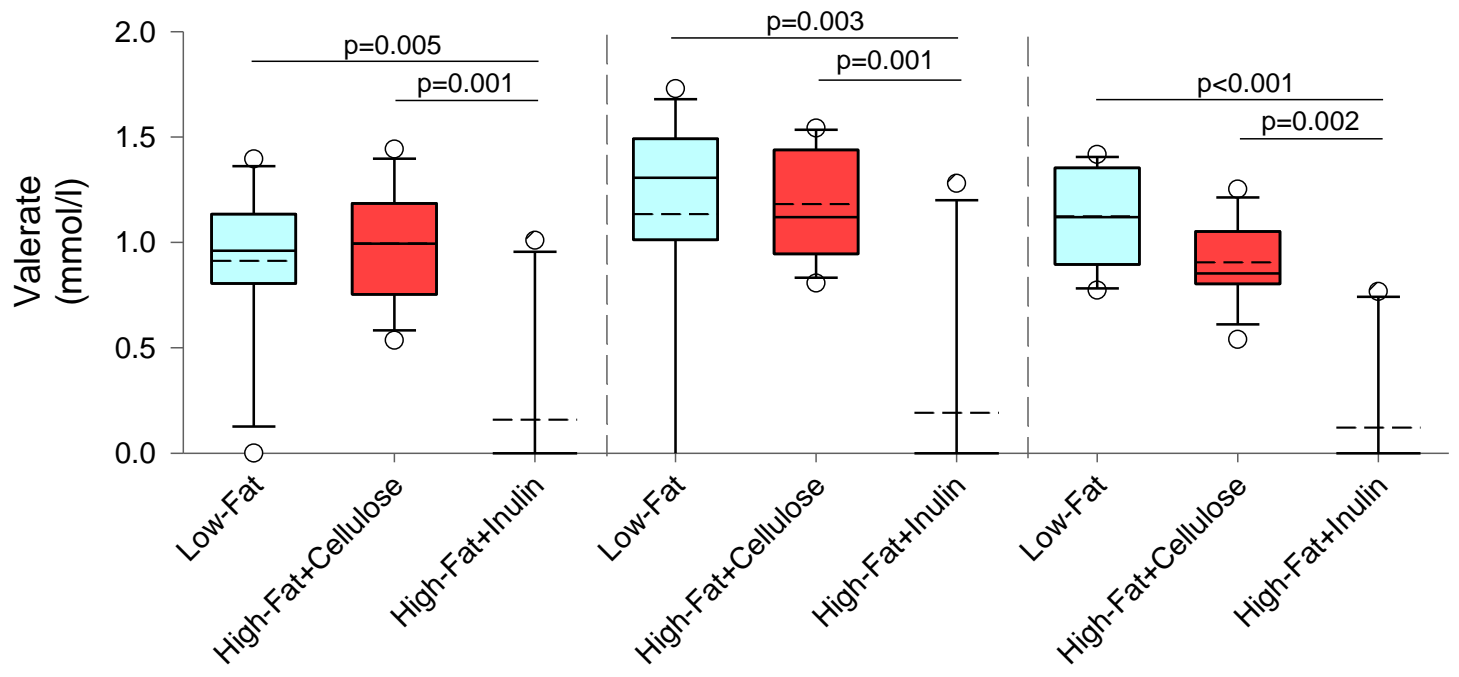

Figure 68. Caecal concentrations of iso-Butyrate, iso-Valerate, and Valerate.

Caecal concentrations of iso-butyrate (A), iso-valerate (B), and valerate in Wild-Type, $\mathrm{PYY}^{-/-}$, and GLP-1R ${ }^{-/-}$mice fed Low-Fat, High-Fat+C, and High-Fat+In diets. In box plots the solid line $=$ median, and dashed line $=$ mean. Statistical significance was determined by one-way ANOVA with Tukey's post hoc test. $n=11-12$ mice per group. 


\subsection{SERUM ACETATE CONCENTRATIONS AND CORRELATIONS}

The mean concentrations of acetate in the serum of High-Fat+In fed Wild-Type mice was not increased relative to High-Fat $+C$ fed Wild-Type mice, Figure 69B. The variation in acetate concentrations increased in High-Fat+In fed Wild-Type mice. These changes were consistent with the caecal acetate concentrations of High-Fat+In fed High-Fat+C fed Wild-Type mice, Figure 69A. In High-Fat+C fed Wild-Type mice the small variation in caecal acetate did not correlate significantly with serum acetate, Figure 69C. In HighFat+In fed Wild-Type mice caecal acetate was significantly correlated with serum acetate, Figure 69D. Serum acetate did not correlate significantly with either food intake, or percentage body fat for Wild-Type mice fed the High-Fat+In diet, Figure 69F, E. The increased range of caecal acetate concentrations in mice fed inulin was associated with similar changes in serum acetate. 
A.

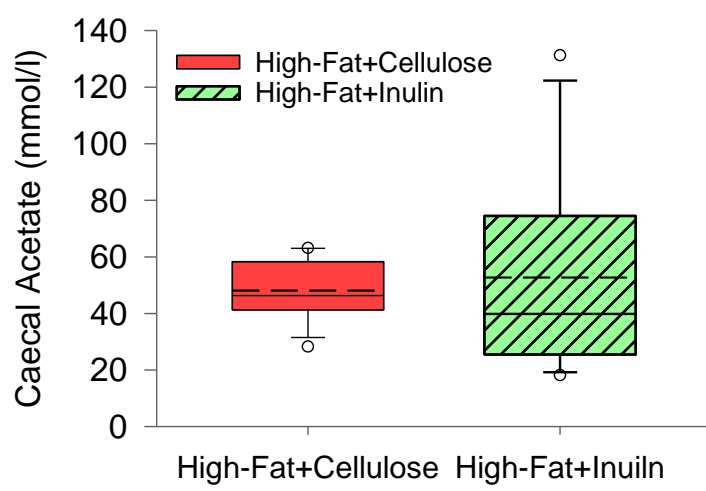

c.

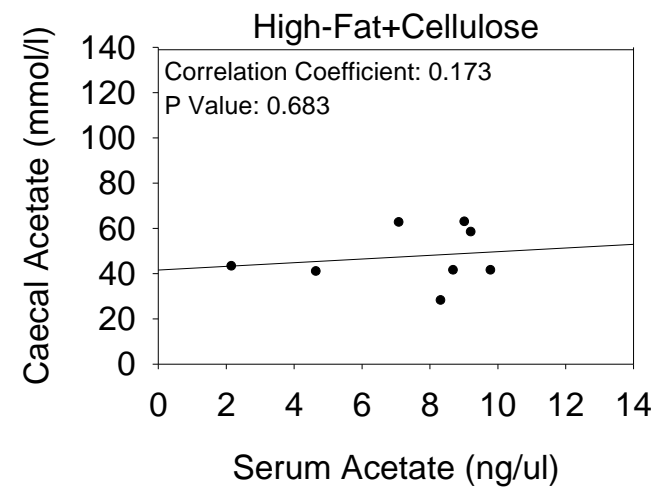

F.

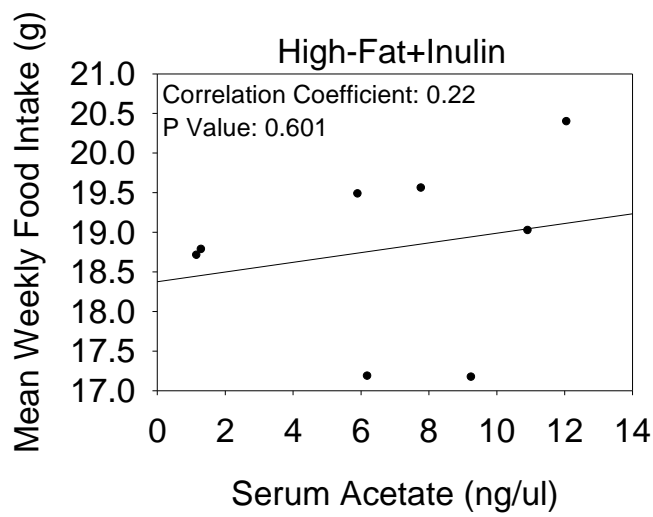

B.

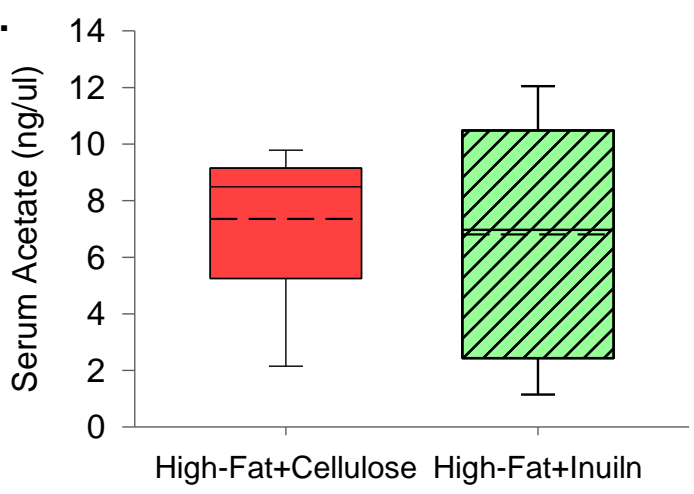

D.

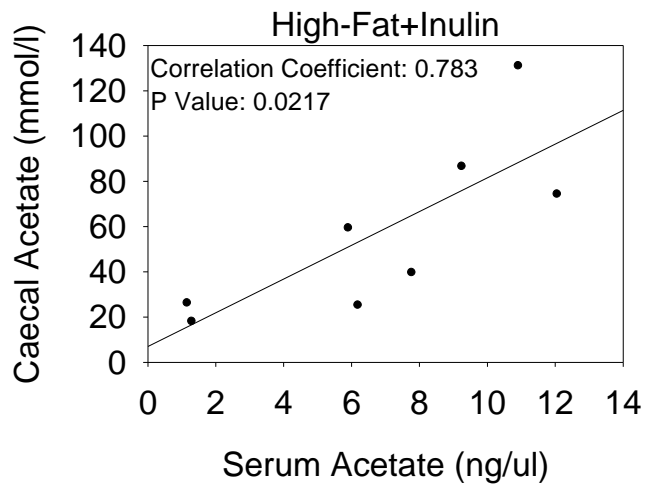

E.

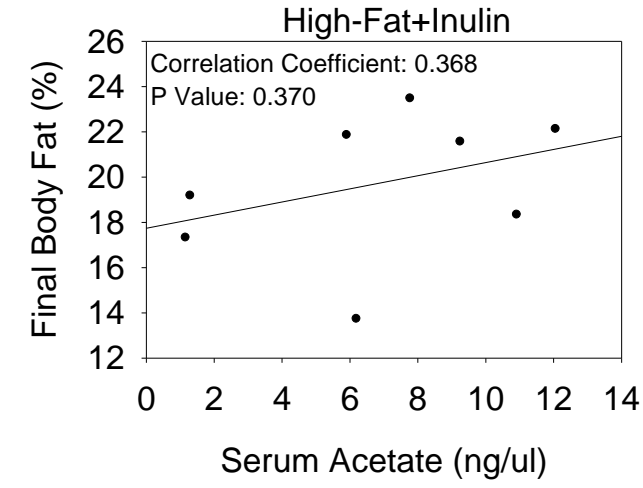

Figure 69. Wild-Type caecal and serum acetate concentrations and correlations

Caecal acetate concentrations (A), and serum acetate concentrations (B), in Wild-Type mice fed High-Fat $+C$ and High-Fat + In diets. Serum acetate correlation with caecal acetate for High-Fat $+C$ diet fed mice (C). Serum acetate correlation with caecal acetate for High-Fat+In fed mice (D). Serum acetate correlation with mean weekly food intake (F) for High-Fat+In fed mice. Serum acetate correlation with body fat percentage (E) for High-Fat+In fed mice. In box plots the solid line = median, and dashed line = mean . Statistical significance was determined by one-way ANOVA with Tukey's post hoc test. $\mathrm{n}=8$ mice per group. Correlations were determined using Pearson correlation. 


\section{DISCUSSION}

In the present study, the effects of adding fermentable fibre (inulin) relative to a nonfermentable fibre (cellulose) to a High-Fat diet were compared on food intake, body fat, body weight, intestinal morphology and caecal short-chain fatty acids in Wild-Type, $\mathrm{PYY}^{-/-}$, and GLP-1R ${ }^{-/-}$mice over eight weeks of feeding.

\subsection{FOOD INTAKE}

This reduction in food intake reduced the energy intake of High-Fat+In fed mice to a level similar to that of Low-Fat diet fed mice is consistent with previous research in C57BL/6 mice showing that the addition of 7.5\% inulin to a similar high-fat diet reduced cumulative food intake (Anastasovska et al, 2012). Smaller reductions were seen with the addition of $2.5 \%$ short-chain fructooligosaccharides to a similar high-fat diet (Nakamura et al, 2011). Reductions in energy intake due to inclusion of fibre with high fats diets have been observed in rats (Cani et al, 2004). The addition of $10 \%$ oligofructose to a zero-carbohydrate diet also reduced the energy intake of mice over 4 weeks (Cani et al, 2006). In contrast another study found that adding $10 \%$ inulin to a high-fat diet in C57BL/6 mice did not reduce food intake compared to the control high-fat diet containing cellulose (Arora et al, 2012) Additionally 5\% inulin added to a low-fat refined diet had limited effects on food intake over 4 weeks (Kuo et al, 2013). In the present study, the addition of inulin to the High-Fat diet significantly reduced food intake and normalised energy intake to that of the mice fed the Low-Fat diet.

In the present study, the loss of PYY signalling prevented the food intake reductions seen in the Wild-Type mice in response to inulin. In mice fed resistant starch, daily injections of a PYY receptor antagonist (BIIE0246) also did not significantly affect food intake (Zhou et al, 2015). However, the authors note that while BIIE0246 can block the effect of PYY on food intake for several hours it is unlikely to fully prevent the action of a daylong increase of PYY due to increased fibre fermentation in the caecum and colon. The relevance of this to the present study is uncertain as mice fed diets high in resistant starch consumed more food that control animals despite increases in serum GLP-1 and PYY (Zhou et al, 2015). The use of $\mathrm{PYY}^{-/-}$mice in the present study is a stronger indicator of the role of PYY in the effect of fermentable dietary fibre. The presence of inulin in the 
High-Fat diet had no effect on food intake or energy intake of GLP-1R ${ }^{-/}$. This confirms previous observations that mice GLP-1R ${ }^{-/-}$mice do not show reductions in energy intake in response to addition of oligofructose to a zero-carbohydrate, high-fat diet (Cani et al, 2006). However, these observed changes in control mice were small and only included $5 \mathrm{GLP}-1 \mathrm{R}^{-/-}$mice per group and fed with a duration of just 4 weeks (Cani et al, 2006). The present study included 12 mice per group and fed diets for 8 weeks giving a more robust result. These results confirm the food intake reducing effects of inulin in WildType mice. It provides strong evidence for the importance of GLP-1 and provides new evidence that PYY also plays an important role in the reduction in food intake from inulin.

\subsection{BODY COMPOSITION AND BODY FAT PADS}

Overall the presence of Inulin in the diet reduced body fat in Wild-Type and GLP-1R ${ }^{-/-}$ mice, but not in $\mathrm{PYY}^{-/}$. High-Fat+In diet mice still gained significantly more fat than Low-Fat diet fed mice despite a similar energy intake. The high fat content of this diet is likely to be a factor explaining this result. Feeding an ad libitum high-fat diet to mouse strains susceptible to obesity increases energy intake and accumulation of body fat in comparison to a high-carbohydrate control diet, (Surwit et al, 1995). While partly due to the increased energy intake associated high-fat diet feeding, there is also increased feed efficiency, with weight gain typically greater than the additional calories consumed. C57BL/6 mice eating a diet high in fat still gain more body fat when pair-fed to an equivalent energy intake low-fat diet fed mice (Petro et al, 2004). Pair-feeding C57BL/6 mice a $60 \%$-fat diet to an equal caloric intake with mice on a $10 \%$-fat diet resulted in a greater weight gain, insulin resistance, and hepatic steatosis (de Meijer et al, 2010). A single week of pair-feeding a high-fat diet can produce significantly greater increase in body fat in C57BL/6 mice before body weight differences become apparent (Williams et al, 2014). Given these findings it is predicable that the Wild-Type mice in the present study fed the High-Fat+In diet would gain more body fat despite the reductions of energy intake. In effect the addiction of Inulin to the High-Fat diet is replicating the pair-fed mouse in terms of its food and energy intake and would be unlikely to result in reductions in body fat to those seen in Low-Fat diet fed mice. 
While in Wild-Type mice both epididymal and retroperitoneal fat pads were reduced in the High-Fat+In diet relative to the High-Fat $+\mathrm{C}$ diet, only the retroperitoneal fat was reduced in the GLP-1R ${ }^{-/-}$mice. An earlier study reported that $\mathrm{GLP}-1 \mathrm{R}^{-/-}$mice did not show a reduction in body fat in response to inulin (Cani et al, 2006; Zhou et al, 2015). However, only the epididymal fat and body weight was reported (Cani et al, 2006). This study was limited in power, given that only 5 mice per group were used over 4 weeks period. In the present study, the retroperitoneal fat was significantly reduced by inulin feeding suggesting variable effect of Inulin on different fat pads in mice. In contrast the $\mathrm{PYY}^{-/-}$mice showed no reduction in either epididymal or retroperitoneal fat pads in response to Inulin feeding. This result is similar to a previous study reporting that daily injections of a PYY antagonist reduced the body fat reductions in response to a diet high in resistant starch (Zhou et al, 2015). This result is novel as this is the first time that the effects of fermentable fibre have been studied in $\mathrm{PYY}^{-/-}$mice. For the present study body fat pads were presented as percent of fat divided by disembowelled body weight and was determined by subtracting the weight of full intestinal tract (duodenum to anus) from the body weight. The disembowelled body weight was used because Inulin-fed mice contained a significantly heavier gastrointestinal tract. This method has been used previously for calculation of body fat percentages in fibre fed mice (Keenan et al, 2012; Zhou et al, 2015).

These results confirm the body fat reducing effects of Inulin in Wild-Type mice. The absence of body fat reduction in $\mathrm{PYY}^{-/-}$mice indicates that PYY has an important role in this effect. The reductions in body fat, particularly retroperitoneal fat in the GLP-1R ${ }^{-/-}$ mice suggests that GLP-1 is not essential for this effect.

\subsection{BODY WEIGHT}

Body weight responses to Inulin in the diet were small in comparison to previous studies feeding similar levels of Inulin (Anastasovska et al, 2012; Arora et al, 2012; Cani et al, 2006; Everard et al, 2013; Frost et al, 2014a). Wild-Type, PYY ${ }^{-/-}$, and GLP-1R ${ }^{-/-}$mice fed High-Fat+In or High-Fat $+\mathrm{C}$ diets were not significantly different in their body weight during the study, Figure 58. Body weight as a measure between these groups was complicated by the increased weight of the gut and gut contents in Inulin fed mice, with the gut accounting for around 1 extra gram of body weight. After subtracting the weight 
of the full gut (duodenum to anus) from the final body weight of the mice both WildType and GLP-1-/- mice fed the Inulin diet weighed significantly less than mice fed Cellulose. There was no significant difference in the $\mathrm{PYY}^{-/-}$mice fed Inulin after adjustment for weight of the gut.

The unexpected lack of body weight response to Inulin feeding in Wild-Type has several potential explanations. In the present study, the High-Fat and High-Fat+In diets were adjusted to maintain the same energy density, and macronutrient ratios between diets. Importantly this calculation included the energy supplied by inulin through caecal and colonic fermentation, considered to supply $6.3 \mathrm{~kJ} / \mathrm{g}$ (1.5 kcal/g) (Roberfroid, 1999). In contrast, some previous research has used diets in which the inulin has been added to a high-fat diet, or added to the drinking water, potentially diluting the energy density without any compensation made to the control high-fat diet (Cani et al, 2006; Everard et al, 2013; Geurts et al, 2011). Although another study using these protocols failed to have any effect on mouse body weight over 14 weeks (Cani et al, 2007c). The age of the mice at the start of the study may play a role. Wild-Type mice began to diverge in their body fat content in response to inulin after they ceased gaining lean mass. Starting the study with older mice that had finished growing may have revealed the effects of the inulin sooner. However, mice of the age used in the present study previously been used successfully (Frost et al, 2014a). The delayed reponse seen in the present study may also represent a delayed response of the gut to fully adapt to the fermentation.

\subsection{MORPHOLOGY}

The increased weight of the caecum in response to Inulin feeding indicated that both the contents of the gut and the gut tissue had increased in response to fibre fermentation. Additionally, the lack of any significant changes in gut morphology between High-Fat $+\mathrm{C}$ and Low-Fat diet confirmed that non-fermentable fibre does not contribute to these changes. This confirms previous research showing that the addition of inulin reverses the reductions in gut size and weight seen with refined mouse diets (Chassaing et al, 2015). These changes in gut morphology were consistent across WildType, PYY ${ }^{-/}$, and GLP-1R ${ }^{-/-}$mice fed the High-Fat+In diet indicating that the loss of either PYY or GLP-1 did not influence the morphological effects of the fibre. 


\subsection{CAECAL SHORT-CHAIN FATTY ACIDS}

Increased cellulose in the high-fat control diet, containing $11 \%$ cellulose, did not appear to result in any increased fermentation of the caecum of these mice. Caecum weight or length was not increased relative to the low-fat control diet containing $5 \%$ fat. This is in agreement with previous research that has considered cellulose to be a largely nonfermentable fibre in rodents. The effect of fructooligosaccharide enriched inulin on mitigating weight gain in the present study was less than previously reported (Anastasovska et al, 2012; Frost et al, 2014a). This was despite replicating closely previous research. Some differences were apparent between the present and previous studies. Compared to Frost et al 2014 the present study used a mouse diets prepared by Research Diets, Inc. The high-fat inulin containing diet contained lard as its fat source instead of dairy fat. The high-fat diet and high-fat inulin diets were more carefully matched for both energy density and macronutrient composition. Mice were C57BL/6J mice instead of C57BL/6N mice. However, there is no indication that any of these factors are responsible for the differences observed.

The lack of increased in SCFA concentrations in response to inulin feeding in the present study is at odds with previous research. Acetate concentrations did not increase in response to inulin as has been previously reported using similar levels of inulin in the diet, although other individual SCFAs were not reported (Frost et al, 2014a). This was despite the overall increase in fermentation and total acetate content of the caecum. Acetate has been reported to be increased from $12 \mathrm{mM}(12 \mathrm{mmol} / \mathrm{l})$ to $24 \mathrm{mM}$ (12 $\mathrm{mmol} / \mathrm{l}$ ) in response to similar diets containing cellulose or inulin (Frost et al, 2014a). In the present study mice consuming control diets contained mean caecal acetate concentrations of 50-60 mmol/l, above those reported previously in inulin fed mice. Similar responses to the present study have been reported although comparisons are complicated by the use of different methods to determine and present changes in SCFAs. Acetate was only increased to a small degree in response to the addition of fructooligosaccharides to a high fat diet (Everard et al, 2014). In the present study caecal propionate concentrations increased in mice fed the High-Fat+In diet relative to the High-Fat $+\mathrm{C}$ diet. However, this only restored propionate concentrations to those seen in the Low-Fat diet, and to levels similar to a previous report (Everard et al, 2014). 
Butyrate concentrations were increased by inulin in the present study. Inulin has a high osmotic potential in the caecal contents were visibly waterier in High-Fat+In fed mice. Increased water retention within the caecum is a potential contributing factor for the lack of increase in caecal acetate concentration. Incomplete fermentation in to SCFAs has previously been reported in mice fed purified fructans type fibre. Caecal succinate has previously been reported to increase in response to the addition of FructoOligosaccharides to refined mouse diets (Goto et al, 2010). Succinate is an intermediate product in the pathway to propionate production and incomplete fermentation resulting in more succinate could account for the small increases in propionate concentrations in the present study (Macfarlane \& Macfarlane, 2003). The fermentation of dietary fibre such as inulin requires cooperation and cross-feeding between different bacterial species (den Besten et al, 2013b). Individual mice carry similar, but not identical microbiota and mice originating from different rooms within the same animal facility can contain functionally diverse microbiota (Rogers et al, 2014). In this present study mice were singly house to ensure accurate food intake measurements, this may have exacerbated differences in the ability to fully ferment the inulin contained in the diet by preventing the sharing of bacteria between different mice.

While concentrations changed relatively little in response to inulin feeding the total amount of SCFAs in the caecum was significantly increased due to the increased size and contents of the caecum in High-Fat+In fed mice. The increased quantity of all SCFAs in the caecum is likely to be responsible for the morphological changes in gut with increasing weight and length. The implications of increased total amounts SCFAs with a lack of increased concentrations are unexplored in the literature.

\subsection{SERUM ACETATE}

In Wild-Type mice serum acetate did not increase in High-Fat+In fed mice relative to High-Fat $+C$ fed mice. However, the range of serum acetate concentrations increased in Inulin fed mice compared to those fed the Cellulose containing diet. This mirrored the changes in acetate concentrations seen in the caecum. Serum acetate concentrations were correlated with caecal acetate concentrations in Wild-Type mice suggesting that the caecal concentration was an important determinant of acetate concentrations in the circulation. The lack of an increase in serum acetate makes it difficult to draw definitive 
conclusions about the potential role of acetate acting directly on the brain from the present study. It remains uncertain whether acetate uptake in the brain could be increased in the absence of increased serum acetate. The increased total acetate in the caecum might suggest that more acetate is being absorbed into the circulation, although the lack of change in serum SCFA concentrations might not be consistent with such a conclusion. Serum and caecal acetate concentrations depend on the dynamic turnover of acetate production and absorption and in the present study was acetate was determined at a single time point. It is unknown whether changes in levels of acetate and other SCFAs occurred earlier on in the experiment.

\subsection{WEAKNESSES AND LIMITATIONS OF THIS STUDY}

The lack of measurements of circulating PYY and GLP-1 in this study mean that it cannot be shown whether the lack of either PYY or GLP-1 influences the action of the other. Additionally, other cells types beyond the colonic L-cells exposed to increased SCFAs also secrete PYY and GLP-1 including L-cells in the small intestine and neurones in the brainstem secrete GLP-1 within the brain. The use of whole body gene knockouts will also remove these sources of hormone secretion, potentially complication the interpretation of the results.

The duration of this animal study was set at 8 weeks based on the design and results of previous literature. However, the mice in this present study did not respond as quickly to the addition of fibre as expected limiting the effects seen on body weight and body fat between diet groups. A longer duration of experimental diet feeding beyond 8 weeks would potentially have produced more robust results. The lack of increased SCFA concentrations may not have stimulated FFA2 and FFA3 enough to increase GLP1 and PYY from intestinal L-cells. The lack of increase in total caecal SCFA concentrations in the present study potentially explain the delayed responses in body weight and body fat to the High-Fat+In diet. Due to time constraints changes in the gut cell due to inulin fibre fermentation could not be investigated. Inulin can promote L-cell differentiation in the colon resulting in an increased L-cell density (Cani et al, 2007b). Increased L-cell density due to both L-cell proliferation and expansion of caecum and colon provide more cells to secrete GLP-1 and PYY with the same concentration of SCFAs in the gut. 
The time scale of this expansion in mice remains uncertain but may account for the delay in bodyweight changes until after 4 weeks seen in Wild-Type mice.

Another limitation is the lack of microbiota composition analysis to investigate the effects on the bacterial populations in mice fed the diet inulin containing. The microbiota composition could not be analysed due to insufficient time after the animal study had finished and the end of the PhD project.

\subsection{COMPARISON OF PYY ${ }^{-/-}$AND GLP-1R ${ }^{-/-}$MICE}

The PYY ${ }^{-/-}$mice and GLP-1R ${ }^{-/-}$mice differed to Wild-Type mice in their body weight and food intake in both Low-Fat and High-Fat diets. PYY-/- ate more food and gained more weight than Wild-Type mice on the same respective diets. In contrast GLP-1R ${ }^{-/-}$mice were leaner and ate less food than Wild-Type mice on the same respective diets. The constitutional loss of these hormones influences food intake and body composition in contrasting ways. PYY and GLP-1 are anorectic hormones and so their loss would be expected to increase food intake. This was the case with $\mathrm{PYY}^{-/-}$mice that started the study heavier and with a higher food intake. As PYY is involved in acute protein induced satiety in the small intestine the loss of PYY could represent an inability to manage normal food intake. In contrast the loss of GLP-1 signalling did not lead to any increase in food intake. GLP-1 has wide range of functions and is expressed in various tissues including the brain where it is involved in food intake regulation. This suggests that normal secretion of GLP-1 is not required for maintenance of body weight in mice.

\section{CONCLUSIONS}

The loss of PYY and GLP-1 signalling attenuate different aspects of the effects of increased fermentable fibre in the diet of mice. While both PYY ${ }^{-/-}$and GLP-1R ${ }^{-/-}$mice attenuated the effects of inulin on food intake this was more complete for GLP-1R ${ }^{-/-}$mice. $\mathrm{PYY}^{-/-}$mice were resistant to the body fat reducing effects of inulin while GLP-1R ${ }^{-/-}$mice did show some reduction in body fat. The lack of increased serum acetate and attenuation of the effects of fibre on food intake and body fat $\mathrm{PYY}^{-/-}$and GLP-1R $\mathrm{R}^{-/-}$mice is evidence that gut derived acetate alone is not the mechanism linking increased gut fermentation with changes in food intake and body fat. PYY and GLP-1 are pleotropic hormones with diverse actions on several different tissues in the body. This shows that 
the action of both GLP-1 and PYY from intestinal L-cells are needed for the full range of benefits from increased dietary fermentable fibre. These results support the hypothesis that PYY and GLP-1 form an important part of the mechanism through which fermentation of dietary fibre attenuates food intake and reduces adiposity. 


\section{CHAPTER 6:}

\section{DISCUSSION}




\section{INTRODUCTION}

The results presented in this thesis raise questions about several areas of research linking the gut microbiota and obesity; they show that, despite this being an area of intense research, mechanisms are still unknown and require additional research.

\subsection{TLR4 AND LPS}

The results presented here in Chapter 3 did not replicate literature reporting that TLR4/- or $\mathrm{CD} 14^{-/-}$mice were protected from diet-induced obesity; findings indicate no differences in body weight, body fat, or food intake in either strain of mice compared to wild-type controls.

The lack of any measurable change in inflammatory gene expression in the hypothalamus of High-Fat diet fed mice questions the theory of neuroendocrine inflammation as a cause in the development of obesity.

Additionally, the lack of change in the composition of the caecal microbiota in wild-type mice in response to High-Fat diet feeding questioned the concept that an obese microbiota composition of gut microflora has a key role in increasing host adiposity.

\subsection{THE IMPORTANCE OF DIETS}

The key finding from Chapter 4 is that drastic changes in gut microbial composition can occur without any associated changes in obesity or glucose intolerance. The increases in the Firmicutes to Bacteroidetes ratio, reductions in microbial diversity, and alterations in species level abundance reported in previous studies were reproduced by switching mice from a Chow diet to a refined Low-Fat diet, and were disassociated from a change in fat content of the diet.

The lack of attention to diet composition as a confounding factor in gut microbiota studies shows a need for greater understanding of the importance of diet composition in microbiota research.

A striking observation was that the largest changes in the microbiota composition were observed in the ileum of the mice, a region of the gut that has received little attention in rodent studies of obesity. 
The results do not support a role for increased energy harvest from indigestible fibre as a mechanism through which the gut microbiota can increase body weight. Mice fed Chow or refined Low-Fat diet maintained body weight despite large differences in dietary fibre and caecal fermentation. This also questions whether dietary fibre is necessary in mice to maintain body weight in the absence of an additional obesogenic stimulus, such as dietary fat.

\subsection{GLP-1 AND PYY}

The present results show that in inulin-fed mice, both GLP-1 and PYY have a role in mediating the effects of dietary fibre on food intake and body fat.

This evidence suggests that circulating acetate from the gut is not a primary mediator of the effects inulin fermentation.

\subsection{SUMMARY}

The research detailed in this thesis questions the role of TLR4 signalling in mouse body weight regulation, while adding to evidence for the importance of gut peptide hormones GLP-1 and PYY in mediating the beneficial effects of fermentable dietary fibre. It questions the interpretation of studies involving the use of undefined chow diets, and suggests that concept of an obesogenic microbiota needs to be critically re-examined.

\section{REPRODUCIBILITY}

The inability of the present study to reproduce the protective effects of TLR4 and CDI4 knockouts in diet-induced obesity should be seen in the context of other reported interactions between the gut microbiota and host body weight.

There are a number of potential factors that may account for the lack of reproducibility highlighted here and relate more generally to larger problems with replication in research involving mice (Reardon, 2016). The intestinal microbiota of laboratory mice can differ between research facilities, confirmed by the results reported in Chapter 3, where differences in the microbiota composition could be observed between mice sourced from the United Kingdom and from the United States

It has recently been proposed that mouse Chow may contribute to the lack of reproducibility of animal experiments between institutes, and that details of the diets 
used should be reported (Laukens et al, 2016). The present research adds important evidence to diets as a confounding factor in mouse research.

The potential confounding factor of Chow diets raise questions about the interpretation of microbiota research in rodents. If results are dependent on a particular diet, or mice bred in a particular institution, it questions how broadly such results can be applied to all mice, let alone to humans.

\section{IMPLICATIONS FOR AN OBESE MICROBIOTA}

The similar gut microbiota in Chow and refined Low-Fat diet fed mice, without any change in body weight, have implications for the interpretation-of previous studies, particularly studies claiming to transfer the obesity phenotype with a microbiota transfer.

\subsection{AN OBESE MICROBIOTA WITHOUT OBESITY}

The similar microbiota composition in refined High-Fat and Low-Fat diets, relative to Chow fed mice, means that a microbiota transplanted from a mouse fed the refined LowFat diet would be predicted to also increase body fat in germ-free mice relative to an obese High-Fat diet fed donor mouse.

\subsection{MICROBIOTA TRANSFER CONVENTIONAL MICE}

Germ-free mice have been the standard model for microbiota transfers. An alternative is to transplant microbiota into mice depleted of their resident microbiota using antibiotics, recreating features of the germ-free mouse (Reikvam et al, 2011; SuarezZamorano et al, 2015). The only microbiota transplantation study, from mice fed a refined High-Fat diet or Chow diet into adult mice with depleted microbiota, showed there was no difference in body weight in recipient mice (Bruce-Keller et al, 2015). Similarly, microbiota transferred from lean wild-type or obese ob/ob mice into microbiota depleted mice, using ampicillin, resulted in no difference in body weight or body fat (Ellekilde et al, 2014). This suggests that microbiota obesity transfer may only work in germ-free mice. 


\subsection{DYSBIOTIC MICROBIOTA STIMULATE FAT GAIN}

An alternative interpretation is that the conventionalisation of germ-free mice represents a rapid developmental catch-up of gut physiology that would normally occur in early infancy. A disrupted microbiota that overstimulates the growth of the gut could set up the mouse for long-term increased body weight. Altering the intestinal microbiota using antibiotics during a critical developmental window has been proposed to have lasting metabolic consequences in mice (Cox et al, 2014). Rabot et al (2016) recently reported on gut microbiota transplantation from High-Fat diet fed mice that were either susceptible or resistant to obesity, into germ-free mice; the result-showed no differences in body weight. In relation to the present study, a mouse eating a Low-Fat refined diet could be considered to have a disrupted microbiota compared to a standard Chow fed mouse, suggesting that a Low-Fat refined diet microbiota would be more fattening to a germ-free mouse than a microbiota from a Chow diet fed mouse.

\subsection{SUMMARY}

The lack of body weight response when an obese mouse microbiota is transplanted into adult conventional mice suggests that germ-free mice may not be a good model for adult obesity. The physiological abnormalities found in germ-free mice suggests that the effects of transplanting a microbiota into a germ-free mouse may share similarities with the maturation that takes place in early infant development. There is evidence that a disrupted microbiota can trigger fat gain if implanted into germ-free mice. In addition, disruption of early life microbiota using antibiotics can shape the gut in a way that may result in lasting increases in body fat. The microbiota shaped by a refined diet could be obesogenic in germ-free mice even though it is not obesogenic to adult mice.

\section{CONCLUSIONS}

The growing interest in the role of the intestinal microbiota in obesity has generated many publications, although it remains a relatively new area of research. This thesis has demonstrated the importance of replicating research to determine how applicable results are, and to continue questioning widely cited concepts in the literature. This work has questioned the role of TLR4 and LPS in diet-induced obesity in mice. The results have highlighted the importance of using well-controlled mouse diets when 
interpreting microbiota research in relation to obesity. In confirming the role of GLP-1 and PYY on the effects of fermentable fibre, it has added to our understanding of the beneficial interactions between dietary fibre, the gut microbiota, and the host. In addition, investigations into the literature suggest that the magnitude of proposed microbiota effects on obesity are likely to be small, the mechanisms remain uncertain, and the germ-free model currently used to provide evidence for causation requires better validation. Gut microbiota research is an area of study with still more questions than definite answers. 


\section{REFERENCES}


Adam, C. L., Thomson, L. M., Williams, P. A. \& Ross, A. W. (2015a) Soluble fermentable dietary fibre (Pectin) decreases caloric intake, adiposity and lipidaemia in high-fat dietinduced obese rats. PLOS One, 10(10), e0140392.

Adam, C. L., Williams, P. A., Dalby, M. J., Garden, K., Thomson, L. M., Richardson, A. J., Gratz, S. W. \& Ross, A. W. (2014) Different types of soluble fermentable dietary fibre decrease food intake, body weight gain and adiposity in young adult male rats. Nutrition and Metabolism, 11(36), 1-12.

Adam, C. L., Williams, P. A., Garden, K. E., Thomson, L. M. \& Ross, A. W. (2015b) Dosedependent effects of a soluble dietary fibre (pectin) on food intake, adiposity, gut hypertrophy and gut satiety hormone secretion in rats. PLOS One, 10(1), e0115438.

Ahn, S. Y., Sohn, S. H., Lee, S. Y., Park, H. L., Park, Y. W., Kim, H. \& Nam, J. H. (2015) The effect of lipopolysaccharide-induced obesity and its chronic inflammation on influenza virus-related pathology. Environmental Toxicology and Pharmacology, 40(3), 924-930.

Alang, N. \& Kelly, C. R. (2015) Weight gain after fecal microbiota transplantation. Open Forum Infectious Diseases, 2(1), ofv004.

Amar, J., Burcelin, R., Ruidavets, J. B., Cani, P. D., Fauvel, J., Alessi, M. C., Chamontin, B. \& Ferrieres, J. (2008) Energy intake is associated with endotoxemia in apparently healthy men. The American Journal of Clinical Nutrition, 87(5), 1219-1223.

Anastasovska, J., Arora, T., Canon, G. J. S., Parkinson, J. R. C., Touhy, K., Gibson, G. R., Nadkarni, N. A., So, P. W., Goldstone, A. P., Thomas, E. L., Hankir, M. K., Van Loo, J., Modi, N., Bell, J. D. \& Frost, G. (2012) Fermentable carbohydrate alters hypothalamic neuronal activity and protects against the obesogenic environment. Obesity, 20(5), 10161023.

Apolzan, J. W. \& Harris, R. B. S. (2012) Differential effects of chow and purified diet on the consumption of sucrose solution and lard and the development of obesity. Physiology E Behavior, 105(2), 325-331.

Armougom, F., Henry, M., Vialettes, B., Raccah, D. \& Raoult, D. (2009) Monitoring bacterial community of human gut microbiota reveals an increase in Lactobacillus in obese patients and Methanogens in anorexic patients. PLOS One, 4(9), e7125.

Arora, T., Loo, R. L., Anastasovska, J., Gibson, G. R., Tuohy, K. M., Sharma, R. K., Swann, J. R., Deaville, E. R., Sleeth, M. L., Thomas, E. L., Holmes, E., Bell, J. D. \& Frost, G. (2012) Differential effects of two fermentable carbohydrates on central appetite regulation and body composition. PLOS One, 7(8), e43263.

Arora, T., Singh, S. \& Sharma, R. K. (2013) Probiotics: Interaction with gut microbiome and antiobesity potential. Nutrition, 29(4), 591-596.

Arrieta, M. C., Walter, J. \& Finlay, B. B. (2016) Human Microbiota-Associated Mice: A Model with Challenges. Cell Host E Microbe, 19(5), 575-578. 
Backhed, F., Ding, H., Wang, T., Hooper, L. V., Koh, G. Y., Nagy, A., Semenkovich, C. F. \& Gordon, J. I. (2004) The gut microbiota as an environmental factor that regulates fat storage. Proceedings of the National Academy of Sciences, 101(44), 15718-15723.

Backhed, F., Manchester, J. K., Semenkovich, C. F. \& Gordon, J. I. (2007) Mechanisms underlying the resistance to diet-induced obesity in germ-free mice. Proceedings of the National Academy of Sciences, 104(3), 979-984.

Banasaz, M., Norin, E., Holma, R. \& Midtvedt, T. (2002) Increased enterocyte production in gnotobiotic rats mono-associated with Lactobacillus rhamnosus GG. Applied and Environmental Microbiology, 68(6), 3031-3034.

Barnard, D. E., Lewis, S. M., Teter, B. B. \& Thigpen, J. E. (2009) Open- and closedformula laboratory animal diets and their importance to research. Journal of the American Association for Laboratory Animal Science, 48(6), 709-713.

Batterham, R. L. \& Bloom, S. R. (2003) The gut hormone peptide YY regulates appetite. Annals of the New York Academy of Sciences, 994, 162-168.

Batterham, R. L., Cowley, M. A., Small, C. J., Herzog, H., Cohen, M. A., Dakin, C. L., Wren, A. M., Brynes, A. E., Low, M. J., Ghatei, M. A., Cone, R. D. \& Bloom, S. R. (2002) Gut hormone PYY(3-36) physiologically inhibits food intake. Nature, 418(6898), 650654.

Batterham, R. L., Heffron, H., Kapoor, S., Chivers, J. E., Chandarana, K., Herzog, H., Le Roux, C. W., Thomas, E. L., Bell, J. D. \& Withers, D. J. (2006) Critical role for peptide YY in protein-mediated satiation and body-weight regulation. Cell Metabolism, 4(3), 223233.

Behrendt, D., Dembinski, J., Heep, A. \& Bartmann, P. (2004) Lipopolysaccharide binding protein in preterm infants. Archives of Disease in Childhood. Fetal and Neonatal Edition, 89(6), 551-554.

Belenguer, A., Duncan, S. H., Calder, A. G., Holtrop, G., Louis, P., Lobley, G. E. \& Flint, H. J. (2006) Two routes of metabolic cross-feeding between Bifidobacterium adolescentis and butyrate-producing anaerobes from the human gut. Appl Environ Microbiol, 72(5), 3593-9.

Benjamini, Y. \& Hochberg, Y. (1995) Controlling the false discovery rate: A practical and Powerful approach to multiple testing. Journal of the Royal Statistical Society Series BMethodological, 57(1), 289-300.

Benoit, B., Plaisancie, P., Awada, M., Geloen, A., Estienne, M., Capel, F., MalpuechBrugere, C., Debard, C., Pesenti, S., Morio, B., Vidal, H., Rieusset, J. \& Michalski, M. (2013) High-fat diet action on adiposity, inflammation, and insulin sensitivity depends on the control low-fat diet. Nutrition Research, 33(11), 952-960.

Berthoud, H. R. (2002) Multiple neural systems controlling food intake and body weight. Neuroscience and Biobehavioral Reviews, 26(4), 393-428. 
Beutler, B. \& Poltorak, A. (2001) The sole gateway to endotoxin response: How LPS was identified as TLR4, and its role in innate immunity. Drug Metabolism and Disposition, 29(4), 474-478.

Bielohuby, M., Bodendorf, K., Brandstetter, H., Bidlingmaier, M. \& Kienzle, E. (2010) Predicting metabolisable energy in commercial rat diets: physiological fuel values may be misleading. British Journal of Nutrition, 103(10), 1525-1533.

Biragyn, A., Ruffini, P. A., Leifer, C. A., Klyushnenkova, E., Shakhov, A., Chertov, O., Shirakawa, A. K., Farber, J. M., Segal, D. M., Oppenheim, J. J. \& Kwak, L. W. (2002) Tolllike receptor 4 -dependent activation of dendritic cells by beta-defensin 2. Science, 298(5595), 1025-1029.

Blaisdell, A. P., Lau, Y. L. M., Telminova, E., Lim, H. C., Fan, B., Fast, C. D., Garlick, D. \& Pendergrass, D. C. (2014) Food quality and motivation: A refined low-fat diet induces obesity and impairs performance on a progressive ratio schedule of instrumental lever pressing in rats. Physiology E Behavior, 128, 220-225.

Blouet, C. \& Schwartz, G. J. (2010) Hypothalamic nutrient sensing in the control of energy homeostasis. Behavioural Brain Research, 209(1), 1-12.

Bodinham, C. L., Hitchen, K. L., Youngman, P. J., Frost, G. S. \& Robertson, M. D. (2011) Short-term effects of whole-grain wheat on appetite and food intake in healthy adults: a pilot study. British Journal of Nutrition, 106(3), 327-330.

Boey, D., Lin, S., Karl, T., Baldock, P., Lee, N., Enriquez, R., Couzens, M., Slack, K., Dallmann, R., Sainsbury, A. \& Herzog, H. (2006) Peptide YY ablation in mice leads to the development of hyperinsulinaemia and obesity. Diabetologia, 49(6), 1360-1370.

Bosshard, P. P., Zbinden, R. \& Altwegg, M. (2002) Turicibacter sanguinis gen. nov., sp. nov., a novel anaerobic, Gram-positive bacterium. International Journal of Systematic and Evolutionary Microbiology, 52(4), 1263-1266.

Brinkhoff, T. \& van Hannen, E. J. (2001) Use of silicone grease to avoid "smiling effect" in denaturing gradient gel electrophoresis. Journal of Rapid Methods and Automation in Microbiology, 9(4), 259-261.

Brown, A. J., Goldsworthy, S. M., Barnes, A. A., Eilert, M. M., Tcheang, L., Daniels, D., Muir, A. I., Wigglesworth, M. J., Kinghorn, I., Fraser, N. J., Pike, N. B., Strum, J. C., Steplewski, K. M., Murdock, P. R., Holder, J. C., Marshall, F. H., Szekeres, P. G., Wilson, S., Ignar, D. M., Foord, S. M., Wise, A. \& Dowell, S. J. (2003) The Orphan G proteincoupled receptors GPR41 and GPR43 are activated by propionate and other short chain carboxylic acids. The Journal of Biological Chemistry, 278(13), 11312-11319.

Brown, R., Imran, S. A. \& Wilkinson, M. (2009) Lipopolysaccharide (LPS) stimulates adipokine and socs 3 gene expression in mouse brain and pituitary gland in vivo, and in N-1 hypothalamic neurons in vitro. Journal of Neuroimmunology, 209, 96-103. 
Bruce-Keller, A. J., Salbaum, J. M., Luo, M., Blanchard, E. t., Taylor, C. M., Welsh, D. A. \& Berthoud, H. R. (2015) Obese-type gut microbiota induce neurobehavioral changes in the absence of obesity. Biological Psychiatry, 77(7), 607-615.

Buettner, R., Scholmerich, J. \& Bollheimer, L. C. (2007) High-fat diets: modeling the metabolic disorders of human obesity in rodents. Obesity, 15(4), 798-808.

Bugaut, M. \& Bentejac, M. (1993) Biological effects of short-chain fatty acids in nonruminant mammals. Annual Review of Nutrition, 13, 217-241.

Cani, P. D., Amar, J., Iglesias, M. A., Poggi, M., Knauf, C., Bastelica, D., Neyrinck, A. M., Fava, F., Tuohy, K. M., Chabo, C., Waget, A., Delmee, E., Cousin, B., Sulpice, T., Chamontin, B., Ferrieres, J., Tanti, J., Gibson, G. R., Casteilla, L., Delzenne, N. M., Alessi, M. C. \& Burcelin, R. (2007a) Metabolic endotoxemia initiates obesity and insulin resistance. Diabetes, 56(7), 1761-1772.

Cani, P. D., Bibiloni, R., Knauf, C., Waget, A., Neyrinck, A. M., Delzenne, N. M. \& Burcelin, R. (2008) Changes in gut microbiota control metabolic endotoxemia-induced inflammation in high-fat diet-induced obesity and diabetes in mice. Diabetes, 57(6), $1470-1481$.

Cani, P. D., Dewever, C. \& Delzenne, N. M. (2004) Inulin-type fructans modulate gastrointestinal peptides involved in appetite regulation (glucagon-like peptide-1 and ghrelin) in rats. British Journal of Nutrition, 92(3), 521-526.

Cani, P. D., Hoste, S., Guiot, Y. \& Delzenne, N. M. (2007b) Dietary non-digestible carbohydrates promote L-cell differentiation in the proximal colon of rats. British Journal of Nutrition, 98(1), 32-37.

Cani, P. D., Knauf, C., Iglesias, M. A., Drucker, D. J., Delzenne, N. M. \& Burcelin, R. (2006) Improvement of glucose tolerance and hepatic insulin sensitivity by oligofructose requires a functional glucagon-like peptide 1 receptor. Diabetes, 55(5), 1484-1490.

Cani, P. D., Neyrinck, A. M., Fava, F., Knauf, C., Burcelin, R. G., Tuohy, K. M., Gibson, G. R. \& Delzenne, N. M. (2007c) Selective increases of bifidobacteria in gut microflora improve high-fat-diet-induced diabetes in mice through a mechanism associated with endotoxaemia. Diabetologia, 50(11), 2374-2383.

Cani, P. D., Possemiers, S., Van de Wiele, T., Guiot, Y., Everard, A., Rottier, O., Geurts, L., Naslain, D., Neyrinck, A., Lambert, D. M., Muccioli, G. G. \& Delzenne, N. M. (2009) Changes in gut microbiota control inflammation in obese mice through a mechanism involving GLP-2-driven improvement of gut permeability. Gut, 58(8), 1091-1103.

Caricilli, A. M., Picardi, P. K., de Abreu, L. L., Ueno, M., Prada, P. O., Ropelle, E. R., Hirabara, S. M., Castoldi, A., Vieira, P., Camara, N. O. S., Curi, R., Carvalheira, J. B. \& Saad, M. J. A. (2011) Gut microbiota is a key modulator of insulin resistance in TLR-2 knockout mice. PLOS Biology, 9(12), el001212. 
Carmichael, F. J., Israel, Y., Crawford, M., Minhas, K., Saldivia, V., Sandrin, S., Campisi, P. \& Orrego, H. (1991) Central nervous system effects of acetate: contribution to the central effects of ethanol. The Journal of Pharmacology and Experimental Therapeutics, 259(1), 403-408.

Carvalho, F. A., Koren, O., Goodrich, J. K., Johansson, M. E., Nalbantoglu, I., Aitken, J. D., Su, Y., Chassaing, B., Walters, W. A., Gonzalez, A., Clemente, J. C., Cullender, T. C., Barnich, N., Darfeuille-Michaud, A., Vijay-Kumar, M., Knight, R., Ley, R. E. \& Gewirtz, A. T. (2012) Transient inability to manage proteobacteria promotes chronic gut inflammation in TLR5-deficient mice. Cell Host and Microbe, 12(2), 139-152.

Casalta, E. \& Montel, M. C. (2008) Safety assessment of dairy microorganisms: the Lactococcus genus. International Journal of Food Microbiology, 126(3), 271-273.

Cederroth, C. R., Vinciguerra, M., Kuehne, F., Madani, R., Doerge, D. R., Visser, T. J., Foti, M., Rohner-Jeanrenaud, F., Vassalli, J. \& Nef, S. (2007) A phytoestrogen-rich diet increases energy expenditure and decreases adiposity in mice. Environmental Health Perspectives, 115(10), 1467-1473.

Cerda, B., Perez, M., Perez-Santiago, J. D., Tornero-Aguilera, J. F., Gonzalez-Soltero, R. \& Larrosa, M. (2016) Gut microbiota modification: Another piece in the puzzle of the benefits of physical exercise in health? Frontiers in Physiology, 7(51), 1-11.

Chakravarty, S. \& Herkenham, M. (2005) Toll-like receptor 4 on nonhematopoietic cells sustains CNS inflammation during endotoxemia, independent of systemic cytokines. Journal of Neuroscience, 25(7), 1788-1796.

Chandarana, K., Gelegen, C., Irvine, E. E., Choudhury, A. I., Amouyal, C., Andreelli, F., Withers, D. J. \& Batterham, R. L. (2013) Peripheral activation of the Y2-receptor promotes secretion of GLP-1 and improves glucose tolerance. Molecular metabolism, 2(3), 142-152.

Chang, D. H., Rhee, M. S., Ahn, S., Bang, B. H., Oh, J. E., Lee, H. K. \& Kim, B. C. (2015) Faecalibaculum rodentium gen. nov., sp. nov., isolated from the faeces of a laboratory mouse. Antonie Van Leeuwenhoek, 108(6), 1309-18.

Chassaing, B., Miles-Brown, J., Pellizzon, M., Ulman, E., Ricci, M., Zhang, L., Patterson, A. D., Vijay-Kumar, M. \& Gewirtz, A. T. (2015) Lack of soluble fiber drives diet-induced adiposity in mice. American Journal of Physiology - Gastrointestinal and Liver Physiology, 309(7), 528-541.

Chaudhri, O. B., Parkinson, J. R., Kuo, Y. T., Druce, M. R., Herlihy, A. H., Bell, J. D., Dhillo, W. S., Stanley, S. A., Ghatei, M. A. \& Bloom, S. R. (2006) Differential hypothalamic neuronal activation following peripheral injection of GLP-1 and oxyntomodulin in mice detected by manganese-enhanced magnetic resonance imaging. Biochemical and Biophysical Research Communications, 350(2), 298-306.

Cho, I. \& Blaser, M. J. (2012) The human microbiome: at the interface of health and disease. Nat Rev Genet, 13(4), 260-70. 
Chung, H., Pamp, S. J., Hill, J. A., Surana, N. K., Edelman, S. M., Troy, E. B., Reading, N. C., Villablanca, E. J., Wang, S. \& Mora, J. R. (2012) Gut immune maturation depends on colonization with a host-specific microbiota. Cell, 149(7), 1578-1593.

Chung, W. S., Walker, A. W., Louis, P., Parkhill, J., Vermeiren, J., Bosscher, D., Duncan, S. H. \& Flint, H. J. (2016) Modulation of the human gut microbiota by dietary fibres occurs at the species level. BMC Biology, 14(3), 1-13.

Clarke, S. F., Murphy, E. F., O'Sullivan, O., Ross, R. P., O'Toole, P. W., Shanahan, F. \& Cotter, P. D. (2013) Targeting the microbiota to address diet-induced obesity: a time dependent challenge. PLOS One, 8(6), e65790.

Clavel, T., Fallani, M., Lepage, P., Levenez, F., Mathey, J., Rochet, V., Serezat, M., Sutren, M., Henderson, G., Bennetau-Pelissero, C., Tondu, F., Blaut, M., Dore, J. \& Coxam, V. (2005) Isoflavones and functional foods alter the dominant intestinal microbiota in postmenopausal women. Journal of Nutrition, 135(12), 2786-2792.

Clemente, J. C., Ursell, L. K., Parfrey, L. W. \& Knight, R. (2012) The impact of the gut microbiota on human health: an integrative view. Cell, 148(6), 1258-70.

Collado, M. C., Derrien, M. \& Isolauri, E. (2007) Intestinal integrity and Akkermansia muciniphila, a mucin-degrading member of the intestinal microbiota present in infants, adults, and the elderly. Applied and Environmental Microbiology, 73(23), 7767-7770.

Cousens, L. S., Gallwitz, D. \& Alberts, B. M. (1979) Different accessibilities in chromatin to histone acetylase. J Biol Chem, 254(5), 1716-23.

Coutinho, A. \& Meo, T. (1978) Genetic basis for unresponsiveness to lipopolysaccharide in C57BL/10Cr mice. Immunogenetics, 7(1), 17-24.

Cox, L. M., Cho, I., Young, S. A., Anderson, W. H., Waters, B. J., Hung, S. C., Gao, Z., Mahana, D., Bihan, M., Alekseyenko, A. V., Methe, B. A. \& Blaser, M. J. (2013) The nonfermentable dietary fiber hydroxypropyl methylcellulose modulates intestinal microbiota. FASEB Journal, 27(2), 692-702.

Cox, L. M., Yamanishi, S., Sohn, J., Alekseyenko, A. V., Leung, J. M., Cho, I., Kim, S. G., Li, H., Gao, Z., Mahana, D., Rodriguez, J. G. Z., Rogers, A. B., Robine, N., Loke, P. \& Blaser, M. J. (2014) Altering the intestinal microbiota during a critical developmental window has lasting metabolic consequences. Cell, 158(4), 705-721.

Cummings, J. H., Pomare, E. W., Branch, W. J., Naylor, C. P. E. \& Macfarlane, G. T. (1987) Short chain fatty acids in human large intestine, portal, hepatic and venous blood. Gut, 28(10), 1221-1227.

Cummings, J. H., Rombeau, J. L. \& Sakata, T. (1995) Physiological and clinical aspects of short-chain fatty acids, in Cummings, J. H., Rombeau, J. L. \& Sakata, T. (eds). Cambridge, UK: Cambridge University Press, 434-439.

Dao, M. C., Everard, A., Aron-Wisnewsky, J., Sokolovska, N., Prifti, E., Verger, E. O., Kayser, B. D., Levenez, F., Chilloux, J., Hoyles, L., Consortium, M. I.-O., Dumas, M. E., 
Rizkalla, S. W., Dore, J., Cani, P. D. \& Clement, K. (2015) Akkermansia muciniphila and improved metabolic health during a dietary intervention in obesity: relationship with gut microbiome richness and ecology. Gut, 65(3), 426-436.

Davis, J. E., Braucher, D. R., Walker-Daniels, J. \& Spurlock, M. E. (2011) Absence of Tlr2 protects against high-fat diet-induced inflammation and results in greater insulinstimulated glucose transport in cultured adipocytes. The Journal of Nutritional Biochemistry, 22(2), 136-141.

Davis, J. E., Gabler, N. K., Walker-Daniels, J. \& Spurlock, M. E. (2008) Tlr-4 deficiency selectively protects against obesity induced by diets high in saturated fat. Obesity, 16(6), 1248-1255.

de La Serre, C. B., de Lartigue, G. \& Raybould, H. E. (2015) Chronic exposure to Low dose bacterial lipopolysaccharide inhibits leptin signaling in vagal afferent neurons. Physiology and Behavior, 139, 188-194.

de Meijer, V. E., Le, H. D., Meisel, J. A., Akhavan Sharif, M. R., Pan, A., Nose, V. \& Puder, M. (2010) Dietary fat intake promotes the development of hepatic steatosis independently from excess caloric consumption in a murine model. Metabolism, 59(8), 1092-1105.

De Souza, C. T., Araujo, E. P., Bordin, S., Ashimine, R., Zollner, R. L., Boschero, A. C., Saad, M. J. A. \& Velloso, L. A. (2005) Consumption of a fat-rich diet activates a proinflammatory response and induces insulin resistance in the hypothalamus. Endocrinology, 146(10), 4192-4199.

Deacon, C. F., Nauck, M. A., Toft-Nielsen, M., Pridal, L., Willms, B. \& Holst, J. J. (1995) Both subcutaneously and intravenously administered glucagon-like peptide I are rapidly degraded from the NH2-terminus in type II diabetic patients and in healthy subjects. Diabetes, 44(9), 1126-1131.

den Besten, G., Lange, K., Havinga, R., van Dijk, T. H., Gerding, A., van Eunen, K., Muller, M., Groen, A. K., Hooiveld, G. J., Bakker, B. M. \& Reijngoud, D.-J. (2013a) Gut-derived short-chain fatty acids are vividly assimilated into host carbohydrates and lipids. American Journal of Physiology-Gastrointestinal and Liver Physiology, 305(12), 900-910.

den Besten, G., van Eunen, K., Groen, A. K., Venema, K., Reijngoud, D. J. \& Bakker, B. M. (2013b) The role of short-chain fatty acids in the interplay between diet, gut microbiota, and host energy metabolism. Journal of Lipid Research, 54(9), 2325-2340.

Derrien, M., Belzer, C. \& de Vos, W. M. (2016) Akkermansia muciniphila and its role in regulating host functions. Microbial Pathogenisis, S0882-4010(15), 30178-9.

Derrien, M., Collado, M. C., Ben-Amor, K., Salminen, S. \& de Vos, W. M. (2008) The mucin degrader Akkermansia muciniphila is an abundant resident of the human intestinal tract. Applied and Environmental Microbiology, 74(5), 1646-1648.

Derrien, M., Van Baarlen, P., Hooiveld, G., Norin, E., Muller, M. \& de Vos, W. M. (2011) Modulation of mucosal immune response, Tolerance, and proliferation in mice 
colonized by the mucin-degrader Akkermansia muciniphila. Frontiers in Microbiology, 2(166), e00166.

Derrien, M., Vaughan, E. E., Plugge, C. M. \& de Vos, W. M. (2004) Akkermansia muciniphila gen. nov., sp. nov., a human intestinal mucin-degrading bacterium. International Journal of Systematic and Evolutionary Microbiology, 54(5), 1469-1476.

Dieguez, C., Vazquez, M. J., Romero, A., Lopez, M. \& Nogueiras, R. (2011) Hypothalamic control of lipid metabolism: focus on leptin, ghrelin and melanocortins. Neuroendocrinology, 94(1), 1-11.

Ding, Y., Subramanian, S., Montes, V. N., Goodspeed, L., Wang, S., Han, C., Teresa, A. S., 3rd, Kim, J., O'Brien, K. D. \& Chait, A. (2012) Toll-like receptor 4 deficiency decreases atherosclerosis but does not protect against inflammation in obese low-density lipoprotein receptor-deficient mice. Arteriosclerosis Thrombosis and Vascular Biology, 32(7), 1596-1604.

Donohoe, D. R., Holley, D., Collins, L. B., Montgomery, S. A., Whitmore, A. C., Hillhouse, A., Curry, K. P., Renner, S. W., Greenwalt, A., Ryan, E. P., Godfrey, V., Heise, M. T., Threadgill, D. S., Han, A., Swenberg, J. A., Threadgill, D. W. \& Bultman, S. J. (2014) A gnotobiotic mouse model demonstrates that dietary fiber protects against colorectal tumorigenesis in a microbiota- and butyrate-dependent manner. Cancer Discov, 4(12), 1387-97.

Dorfman, M. D. \& Thaler, J. P. (2015) Hypothalamic inflammation and gliosis in obesity. Current Opinion in Endocrinology Diabetes and Obesity, 22(5), 325-330.

Drewe, J., Beglinger, C. \& Fricker, G. (2001) Effect of ischemia on intestinal permeability of lipopolysaccharides. European Journal of Clinical Investigation, 31(2), 138-144.

Duca, F. A., Sakar, Y., Lepage, P., Devime, F., Langelier, B., Dore, J. \& Covasa, M. (2014) Replication of obesity and associated signaling pathways through transfer of microbiota from obese-prone rats. Diabetes, 63(5), 1624-1636.

Duca, F. A., Sakar, Y., Lepage, P., Devime, F., Langelier, B., Dore, J. \& Covasa, M. (2016) Statement of retraction. replication of obesity and associated signaling pathways through transfer of microbiota from bbese-prone rats. Diabetes, 65(5), 1447.

Dudele, A., Fischer, C. W., Elfving, B., Wegener, G., Wang, T. \& Lund, S. (2015) Chronic exposure to low doses of lipopolysaccharide and high-fat feeding increases body mass without affecting glucose tolerance in female rats. Physiological Reports, 3(11), 1-12.

Duenas, M., Munoz-Gonzalez, I., Cueva, C., Jimenez-Giron, A., Sanchez-Patan, F., Santos-Buelga, C., Moreno-Arribas, M. V. \& Bartolome, B. (2015) A survey of modulation of gut microbiota by dietary polyphenols. BioMed Research International, 2015, e850902.

Duncan, S. H., Lobley, G. E., Holtrop, G., Ince, J., Johnstone, A. M., Louis, P. \& Flint, H. J. (2008) Human colonic microbiota associated with diet, obesity and weight loss. International Journal of Obesity, 32(11), 1720-1724. 
Eissele, R., Goke, R., Willemer, S., Harthus, H. P., Vermeer, H., Arnold, R. \& Goke, B. (1992) Glucagon-like peptide-1 cells in the gastrointestinal tract and pancreas of rat, pig and man. European Journal of Clinical Investigation, 22(4), 283-291.

Ellekilde, M., Selfjord, E., Larsen, C. S., Jakesevic, M., Rune, I., Tranberg, B., Vogensen, F. K., Nielsen, D. S., Bahl, M. I., Licht, T. R., Hansen, A. K. \& Hansen, C. H. F. (2014) Transfer of gut microbiota from lean and obese mice to antibiotic-treated mice. Scientific Reports, 4(5922), 1-8.

Erridge, C. \& Samani, N. J. (2009) Saturated fatty acids do not directly stimulate Tolllike receptor signaling. Arteriosclerosis, Thrombosis, and Vascular Biology, 29(11), 19441949.

Everard, A., Belzer, C., Geurts, L., Ouwerkerk, J. P., Druart, C., Bindels, L. B., Guiot, Y., Derrien, M., Muccioli, G. G., Delzenne, N. M., de Vos, W. M. \& Cani, P. D. (2013) Crosstalk between Akkermansia muciniphila and intestinal epithelium controls diet-induced obesity. Proceedings of the National Academy of Sciences of the United States of America, 110(22), 9066-9071.

Everard, A., Lazarevic, V., Gaia, N., Johansson, M., Stahlman, M., Backhed, F., Delzenne, N. M., Schrenzel, J., Francois, P. \& Cani, P. D. (2014) Microbiome of prebiotic-treated mice reveals novel targets involved in host response during obesity. The ISME Journal, 8(10), 2116-2130.

Fei, N. \& Zhao, L. (2013) An opportunistic pathogen isolated from the gut of an obese human causes obesity in germfree mice. The ISME Journal, 7(4), 880-884.

Ferrer, M., Ruiz, A., Lanza, F., Haange, S. B., Oberbach, A., Till, H., Bargiela, R., Campoy, C., Segura, M. T., Richter, M., von Bergen, M., Seifert, J. \& Suarez, A. (2013) Microbiota from the distal guts of lean and obese adolescents exhibit partial functional redundancy besides clear differences in community structure. Environmental Microbiology, 15(1), 211226.

Fischer, C. W., Liebenberg, N., Madsen, A. M., Muller, H. K., Lund, S. \& Wegener, G. (2015) Chronic lipopolysaccharide infusion fails to induce depressive-like behaviour in adult male rats. Acta Neuropsychiatrica, 27(3), 189-194.

Fleissner, C. K., Huebel, N., El-Bary, M. M. A., Loh, G., Klaus, S. \& Blaut, M. (2010) Absence of intestinal microbiota does not protect mice from diet-induced obesity. British Journal of Nutrition, 104(6), 919-929.

Flint, H. J., Duncan, S. H., Scott, K. P. \& Louis, P. (2007) Interactions and competition within the microbial community of the human colon: links between diet and health. Environ Microbiol, 9(5), 1101-11.

Flint, H. J., Scott, K. P., Duncan, S. H., Louis, P. \& Forano, E. (2012) Microbial degradation of complex carbohydrates in the gut. Gut Microbes, 3(4), 289-306.

Franklin, K. B. J. \& Paxinos, G. (1997) The Mouse Brain in Stereotaxic Coordinates. San Diego: Academic Press. 
Frederich, R. C., Hamann, A., Anderson, S., Lollmann, B., Lowell, B. B. \& Flier, J. S. (1995) Leptin levels reflect body lipid content in mice: evidence for diet-induced resistance to leptin action. Nature Medicine, 1(12), 1311-1314.

Frese, S. A., Mackenzie, D. A., Peterson, D. A., Schmaltz, R., Fangman, T., Zhou, Y., Zhang, C., Benson, A. K., Cody, L. A., Mulholland, F., Juge, N. \& Walter, J. (2013) Molecular characterization of host-specific biofilm formation in a vertebrate gut symbiont. PLOS Genetics, 9(12), el004057.

Frost, G., Sleeth, M. L., Sahuri-Arisoylu, M., Lizarbe, B., Cerdan, S., Brody, L., Anastasovska, J., Ghourab, S., Hankir, M., Zhang, S., Carling, D., Swann, J. R., Gibson, G., Viardot, A., Morrison, D., Thomas, E. L. \& Bell, J. D. (2014a) The short-chain fatty acid acetate reduces appetite via a central homeostatic mechanism. Nature Communications, 5(3611), 1-11.

Frost, G. S., Walton, G. E., Swann, J. R., Psichas, A., Costabile, A., Johnson, L. P., Sponheimer, M., Gibson, G. R. \& Barraclough, T. G. (2014b) Impacts of plant-based foods in ancestral hominin diets on the metabolism and function of gut microbiota in vitro. MBio, 5(3), e00853.

Fukuda, S., Toh, H., Hase, K., Oshima, K., Nakanishi, Y., Yoshimura, K., Tobe, T., Clarke, J. M., Topping, D. L., Suzuki, T., Taylor, T. D., Itoh, K., Kikuchi, J., Morita, H., Hattori, M. \& Ohno, H. (2011) Bifidobacteria can protect from enteropathogenic infection through production of acetate. Nature, 469(7331), 543-547.

Furusawa, Y., Obata, Y., Fukuda, S., Endo, T. A., Nakato, G., Takahashi, D., Nakanishi, Y., Uetake, C., Kato, K., Kato, T., Takahashi, M., Fukuda, N. N., Murakami, S., Miyauchi, E., Hino, S., Atarashi, K., Onawa, S., Fujimura, Y., Lockett, T., Clarke, J. M., Topping, D. L., Tomita, M., Hori, S., Ohara, O., Morita, T., Koseki, H., Kikuchi, J., Honda, K., Hase, K. \& Ohno, H. (2013) Commensal microbe-derived butyrate induces the differentiation of colonic regulatory T cells. Nature, 504(7480), 446-50.

Gabrielsen, C., Brede, D. A., Hernandez, P. E., Nes, I. F. \& Diep, D. B. (2012) The maltose $\mathrm{ABC}$ transporter in Lactococcus lactis facilitates high-level sensitivity to the circular bacteriocin garvicin ML. Antimicrobial Agents and Chemotherapy, 56(6), 2908-2915.

Gerritsen, J., Fuentes, S., Grievink, W., van Niftrik, L., Tindall, B. J., Timmerman, H. M., Rijkers, G. T. \& Smidt, H. (2014) Characterization of Romboutsia ilealis gen. nov., sp. nov., isolated from the gastro-intestinal tract of a rat, and proposal for the reclassification of five closely related members of the genus Clostridium into the genera Romboutsia gen. nov., Intestinibacter gen. nov., Terrisporobacter gen. nov. and Asaccharospora gen. nov. International Journal of Systematic and Evolutionary Microbiology, 64(5), 1600-1616.

Gerritsen, J., Timmerman, H. M., Fuentes, S., van Minnen, L. P., Panneman, H., Konstantinov, S. R., Rombouts, F. M., Gooszen, H. G., Akkermans, L. M., Smidt, H. \& Rijkers, G. T. (2011) Correlation between protection against sepsis by probiotic therapy and stimulation of a novel bacterial phylotype. Applied and Environmental Microbiology, 77(21), 7749-7756. 
Geurts, L., Lazarevic, V., Derrien, M., Everard, A., Van Roye, M., Knauf, C., Valet, P., Girard, M., Muccioli, G. G., Francois, P., de Vos, W. M., Schrenzel, J., Delzenne, N. M. \& Cani, P. D. (2011) Altered gut microbiota and endocannabinoid system tone in obese and diabetic leptin-resistant mice: impact on apelin regulation in adipose tissue. Frontiers in Microbiology, 2(149), 1-5.

Geurts, L., Muccioli, G. G., Delzenne, N. M. \& Cani, P. D. (2013) Chronic endocannabinoid system stimulation induces muscle macrophage and lipid accumulation in type 2 diabetic mice independently of metabolic endotoxaemia. PLOS One, 8(2), e55963.

Ghoshal, S., Witta, J., Zhong, J., de Villiers, W. \& Eckhardt, E. (2009) Chylomicrons promote intestinal absorption of lipopolysaccharides. Journal of Lipid Research, 50(1), 90-97.

Gill, S. R., Pop, M., Deboy, R. T., Eckburg, P. B., Turnbaugh, P. J., Samuel, B. S., Gordon, J. I., Relman, D. A., Fraser-Liggett, C. M. \& Nelson, K. E. (2006) Metagenomic analysis of the human distal gut microbiome. Science, 312(5778), 1355-1359.

Gioannini, T. L. \& Weiss, J. P. (2007) Regulation of interactions of Gram-negative bacterial endotoxins with mammalian cells. Immunological Research, 39(3), 249-260.

Gonzalez-Quintela, A., Alonso, M., Campos, J., Vizcaino, L., Loidi, L. \& Gude, F. (2013) Determinants of serum concentrations of lipopolysaccharide-binding protein (LBP) in the adult population: The role of obesity. PLOS One, 8(1), e54600.

Goodlad, J. S. \& Mathers, J. C. (1990) Large bowel fermentation in rats given diets containing raw peas (Pisum sativum). British Journal of Nutrition, 64(2), 569-587.

Goodlad, R. A., Ratcliffe, B., Fordham, J. P. \& Wright, N. A. (1989) Does dietary fibre stimulate intestinal epithelial cell proliferation in germ free rats? Gut, 30(6), 820-825.

Goodrich, J. K., Waters, J. L., Poole, A. C., Sutter, J. L., Koren, O., Blekhman, R., Beaumont, M., Van Treuren, W., Knight, R., Bell, J. T., Spector, T. D., Clark, A. G. \& Ley, R. E. (2014) Human genetics shape the gut microbiome. Cell, 159(4), 789-799.

Gootenberg, D. B. \& Turnbaugh, P. J. (2011) Companion animals symposium: Humanized animal models of the microbiome. Journal of Animal Science, 89(5), 15311537.

Gordon, H. A. \& Brucknerkardoss, E. (1961) Effect of normal microbial flora on intestinal surface area. American Journal of Physiology, 201(1), 255-279.

Goto, H., Takemura, N., Ogasawara, T., Sasajima, N., Watanabe, J., Ito, H., Morita, T. \& Sonoyama, K. (2010) Effects of fructo-oligosaccharide on DSS-induced colitis differ in mice fed nonpurified and purified diets. Journal of Nutrition, 140(12), 2121-2127.

Grandt, D., Schimiczek, M., Struk, K., Shively, J., Eysselein, V. E., Goebell, H. \& Reeve, J. R., Jr. (1994) Characterization of two forms of peptide YY, PYY(1-36) and PYY(3-36), in the rabbit. Peptides, 15(5), 815-820. 
Gu, S. H., Chen, D. D., Zhang, J. N., Lv, X. M., Wang, K., Duan, L. P., Nie, Y. \& Wu, X. L. (2013) Bacterial community mapping of the mouse gastrointestinal tract. PLOS One, 8(10), e74957.

Guess, N. D., Dornhorst, A., Oliver, N., Bell, J. D., Thomas, E. L. \& Frost, G. S. (2015) A randomized controlled trial: the effect of inulin on weight management and ectopic fat in subjects with prediabetes. Nutrition Metabolism, 12(36), 1-10.

Haile, Y., Carmine-Simmen, K., Olechowski, C., Kerr, B., Bleackley, R. C. \& Giuliani, F. (2015) Granzyme B-inhibitor serpina3n induces neuroprotection in vitro and in vivo. Journal of Neuroinflammation, 12(157), 1-10.

Hansen, C. H., Krych, L., Nielsen, D. S., Vogensen, F. K., Hansen, L. H., Sorensen, S. J., Buschard, K. \& Hansen, A. K. (2012) Early life treatment with vancomycin propagates Akkermansia muciniphila and reduces diabetes incidence in the NOD mouse. Diabetologia, 55(8), 2285-2294.

Haziot, A., Ferrero, E., Kontgen, F., Hijiya, N., Yamamoto, S., Silver, J., Stewart, C. L. \& Goyert, S. M. (1996) Resistance to endotoxin shock and reduced dissemination of gramnegative bacteria in CD14-deficient mice. Immunity, 4(4), 407-414.

Hemme, D., Raibaud, P., Ducluzeau, R., Galpin, J. V., Sicard, P. \& Van Heijenoort, J. (1980) ["Lactobacillus murinus" n. sp., a new species of the autochtoneous dominant flora of the digestive tract of rat and mouse (author's transl)]. Annals of Microbiology, 131(3), 297-308.

Hildebrandt, M. A., Hoffmann, C., Sherrill-Mix, S. A., Keilbaugh, S. A., Hamady, M., Chen, Y.-Y., Knight, R., Ahima, R. S., Bushman, F. \& Wu, G. D. (2009) High-fat diet determines the composition of the murine gut microbiome independently of obesity. Gastroenterology, 137(5), 1716-1724.

Himes, R. W. \& Smith, C. W. (2010) Tlr2 is critical for diet-induced metabolic syndrome in a murine model. FASEB Journal, 24(3), 731-739.

Hines, D. J., Choi, H. B., Hines, R. M., Phillips, A. G. \& MacVicar, B. A. (2013) Prevention of LPS-induced microglia activation, cytokine production and sickness behavior with TLR4 receptor interfering peptides. PLOS One, 8(3), e60388.

Holst, J. J. \& Deacon, C. F. (2005) Glucagon-like peptide-1 mediates the therapeutic actions of DPP-IV inhibitors. Diabetologia, 48(4), 612-5.

Hoshino, K., Takeuchi, O., Kawai, T., Sanjo, H., Ogawa, T., Takeda, Y., Takeda, K. \& Akira, S. (1999) Cutting edge: Toll-like receptor 4 (TLR4)-deficient mice are hyporesponsive to lipopolysaccharide: Evidence for TLR4 as the Lps gene product. Journal of Immunology, 162(7), 3749-3752.

Hotamisligil, G. S. (2003) Inflammatory pathways and insulin action. International Journal of Obesity and Related Metabolic Disorders, 27(3), 53-55. 
Howard, J. K. \& Flier, J. S. (2006) Attenuation of leptin and insulin signaling by SOCS proteins. Trends in Endocrinology and Metabolism, 17(9), 365-371.

Hsieh, P. S., Chan, J. Y. H., Shyu, J. F., Chen, Y. T. \& Loh, C. H. (2008) Mild portal endotoxaemia induces subacute hepatic inflammation and pancreatic beta-cell dysfunction in rats. European Journal of Clinical Investigation, 38(9), 640-648.

Hsu, I., Parkinson, L. G., Shen, Y., Toro, A., Brown, T., Zhao, H., Bleackley, R. C. \& Granville, D. J. (2014) Serpina3n accelerates tissue repair in a diabetic mouse model of delayed wound healing. Cell Death and Disease, 5, el458.

Hudson, B. D., Tikhonova, I. G., Pandey, S. K., Ulven, T. \& Milligan, G. (2012) Extracellular ionic locks determine variation in constitutive activity and ligand potency between species orthologs of the free fatty acid receptors FFA2 and FFA3. The Journal of Biological Chemistry, 287(49), 41195-41209.

Huffnagle, G. B. \& Noverr, M. C. (2013) The emerging world of the fungal microbiome. Trends in Microbiology, 21(7), 334-341.

Huttenhower, C., Gevers, D., Knight, R., Abubucker, S., Badger, J. H., Chinwalla, A. T., Creasy, H. H., Earl, A. M., FitzGerald, M. G., Fulton, R. S., Giglio, M. G., HallsworthPepin, K., Lobos, E. A., Madupu, R., Magrini, V., Martin, J. C., Mitreva, M., Muzny, D. M., Sodergren, E. J., Versalovic, J., Wollam, A. M., Worley, K. C., Wortman, J. R., Young, S. K., Zeng, Q. D., Aagaard, K. M., Abolude, O. O., Allen-Vercoe, E., Alm, E. J., Alvarado, L., Andersen, G. L., Anderson, S., Appelbaum, E., Arachchi, H. M., Armitage, G., Arze, C. A., Ayvaz, T., Baker, C. C., Begg, L., Belachew, T., Bhonagiri, V., Bihan, M., Blaser, M. J., Bloom, T., Bonazzi, V., Brooks, J. P., Buck, G. A., Buhay, C. J., Busam, D. A., Campbell, J. L., Canon, S. R., Cantarel, B. L., Chain, P. S. G., Chen, I. M. A., Chen, L., Chhibba, S., Chu, K., Ciulla, D. M., Clemente, J. C., Clifton, S. W., Conlan, S., Crabtree, J., Cutting, M. A., Davidovics, N. J., Davis, C. C., DeSantis, T. Z., Deal, C., Delehaunty, K. D., Dewhirst, F. E., Deych, E., Ding, Y., Dooling, D. J., Dugan, S. P., Dunne, W. M., Durkin, A. S., Edgar, R. C., Erlich, R. L., Farmer, C. N., Farrell, R. M., Faust, K., Feldgarden, M., Felix, V. M., Fisher, S., Fodor, A. A., Forney, L. J., Foster, L., Di Francesco, V., Friedman, J., Friedrich, D. C., Fronick, C. C., Fulton, L. L., Gao, H. Y., Garcia, N., Giannoukos, G., Giblin, C., Giovanni, M. Y., Goldberg, J. M., Goll, J., Gonzalez, A., Griggs, A., et al (2012) Structure, function and diversity of the healthy human microbiome. Nature, 486(7402), 207-214.

Iwashita, M., Nakatsu, Y., Sakoda, H., Fujishiro, M., Kushiyama, A., Fukushima, T., Kumamoto, S., Shinjo, T., Kamata, H. \& Nishimura, F. (2013) Valsartan restores inflammatory response by macrophages in adipose and hepatic tissues of LPS-infused mice. Adipocyte, 2(1), 28-32.

Janot, L., Secher, T., Torres, D., Maillet, I., Pfeilschifter, J., Quesniaux, V. F., Landmann, R., Ryffel, B. \& Erard, F. (2008) CD14 works with toll-like receptor 2 to contribute to recognition and control of Listeria monocytogenes infection. The Journal of Infectious Diseases, 198(1), 115-124.

Janse, I., Bok, J. \& Zwart, G. (2004) A simple remedy against artifactual double bands in denaturing gradient gel electrophoresis. Journal of Microbiological Methods, 57(2), 279281. 
Jensen, M. N. \& Ritskes-Hoitinga, M. (2007) How isoflavone levels in common rodent diets can interfere with the value of animal models and with experimental results. Laboratory animals, 41(1), 1-18.

Johnson, G. B., Riggs, B. L. \& Platt, J. L. (2004) A genetic basis for the "Adonis" phenotype of low adiposity and strong bones. FASEB Journal, 18(11), 1282-1284.

Jung, T. H., Park, J. H., Jeon, W. M. \& Han, K. S. (2015) Butyrate modulates bacterial adherence on LS174T human colorectal cells by stimulating mucin secretion and MAPK signaling pathway. Nutrition Research and Practice, 9(4), 343-349.

Kaakoush, N. O. (2015) Insights into the role of Erysipelotrichaceae in the human host. Frontiers in Cellular and Infection Microbiology, 5(84), e00084.

Karlsson, F. H., Tremaroli, V., Nookaew, I., Bergstrom, G., Behre, C. J., Fagerberg, B., Nielsen, J. \& Backhed, F. (2013) Gut metagenome in European women with normal, impaired and diabetic glucose control. Nature, 498(7452), 99-103.

Keenan, M. J., Zhou, J., Raggio, A. M., McCutcheon, K. L., Senevirathne, R., Goldsmith, F., Janes, M., Tulley, R. T., Shen, L., Vidrine, K., Williams, C., Charrier, J. A., Ye, J. \& Martin, R. J. (2012) Mechanisms by which resistant starch produces gut hormones and reduces body fat. 6000 Broken Sound Parkway NW, Suite 300, Boca Raton, FL, 334872742, USA: CRC Press, Taylor \& Francis Group.

Khakpour, S., Wilhelmsen, K. \& Hellman, J. (2015) Vascular endothelial cell Toll-like receptor pathways in sepsis. Innate Immun, 21(8), 827-46.

Khoury, K. A., Floch, M. H. \& Hersh, T. (1969) Small intestinal mucosal cell proliferation and bacterial flora in conventionalization of germfree mouse. Journal of Experimental Medicine, 130(3), 659-666.

Kievit, P., Howard, J. K., Badman, M. K., Balthasar, N., Coppari, R., Mori, H., Lee, C. E., Elmquist, J. K., Yoshimura, A. \& Flier, J. S. (2006) Enhanced leptin sensitivity and improved glucose homeostasis in mice lacking suppressor of cytokine signaling-3 in POMC-expressing cells. Cell Metabolism, 4(2), 123-132.

Kim, F., Pham, M., Luttrell, I., Bannerman, D. D., Tupper, J., Thaler, J., Hawn, T. R., Raines, E. W. \& Schwartz, M. W. (2007) Toll-like receptor-4 mediates vascular inflammation and insulin resistance in diet-induced obesity. Circulation research, 100(11), 1589-1596.

Kim, K. A., Gu, W., Lee, I. A., Joh, E. H. \& Kim, D. H. (2012) High fat diet-induced gut microbiota exacerbates inflammation and obesity in mice via the TLR4 signaling pathway. PLOS One, 7(10), 1-11.

Kitchens, R. L. (2000) Role of CD14 in cellular recognition of bacterial lipopolysaccharides. Chemical Immunology, 74, 61-82.

Kleinridders, A., Schenten, D., Koenner, A. C., Belgardt, B. F., Mauer, J., Okamura, T., Wunderlich, F. T., Medzhitov, R. \& Bruening, J. C. (2009) MyD88 signaling in the CNS 
is required for development of fatty acid-induced leptin resistance and diet-induced obesity. Cell Metabolism, 10(4), 249-259.

Koch, C., Augustine, R. A., Steger, J., Ganjam, G. K., Benzler, J., Pracht, C., Lowe, C., Schwartz, M. W., Shepherd, P. R., Anderson, G. M., Grattan, D. R. \& Tups, A. (2010) Leptin rapidly improves glucose homeostasis in obese mice by increasing hypothalamic insulin sensitivity. The Journal of Neuroscience, 30(48), 16180-16187.

Koropatkin, N. M., Cameron, E. A. \& Martens, E. C. (2012) How glycan metabolism shapes the human gut microbiota. Nature Reviews Microbiology, 10(5), 323-335.

Kozich, J. J., Westcott, S. L., Baxter, N. T., Highlander, S. K. \& Schloss, P. D. (2013) Development of a dual-index sequencing strategy and curation pipeline for analyzing amplicon sequence data on the MiSeq Illumina sequencing platform. Applied and Environmental Microbiology, 79(17), 5112-5120.

Kozul, C. D., Nomikos, A. P., Hampton, T. H., Warnke, L. A., Gosse, J. A., Davey, J. C., Thorpe, J. E., Jackson, B. P., Ihnat, M. A. \& Hamilton, J. W. (2008) Laboratory diet profoundly alters gene expression and confounds genomic analysis in mouse liver and lung. Chemico-Biological Interactions, 173(2), 129-140.

Kuo, S. M. (2013) The interplay between fiber and the intestinal microbiome in the inflammatory response. Advances in Nutrition, 4(1), 16-28.

Kuo, S. M., Merhige, P. M. \& Hagey, L. R. (2013) The effect of dietary prebiotics and probiotics on body weight, large intestine indices, and fecal bile acid profile in wild type and IL10-/- mice. PLOS One, 8(3), 1-10.

Kuo, Y. T., Parkinson, J. R., Chaudhri, O. B., Herlihy, A. H., So, P. W., Dhillo, W. S., Small, C. J., Bloom, S. R. \& Bell, J. D. (2007) The temporal sequence of gut peptide CNS interactions tracked in vivo by magnetic resonance imaging. The Journal of Neuroscience, 27(45), 12341-12348.

Langille, M. G., Meehan, C. J., Koenig, J. E., Dhanani, A. S., Rose, R. A., Howlett, S. E. \& Beiko, R. G. (2014) Microbial shifts in the aging mouse gut. Microbiome, 2(50), 1-12.

Larsson, L. I., Holst, J., Hakanson, R. \& Sundler, F. (1975) Distribution and properties of glucagon immunoreactivity in the digestive tract of various mammals: an immunohistochemical and immunochemical study. Histochemistry, 44(4), 281-290.

Lattimer, J. M. \& Haub, M. D. (2010) Effects of dietary fiber and its components on metabolic health. Nutrients, 2(12), 1266-89.

Laugerette, F., Alligier, M., Bastard, J. P., Drai, J., Chanseaume, E., Lambert-Porcheron, S., Laville, M., Morio, B., Vidal, H. \& Michalski, M. C. (2014) Overfeeding increases postprandial endotoxemia in men: Inflammatory outcome may depend on LPS transporters LBP and sCD14. Molecular Nutrition and Food Research, 58(7), 1513-1518. 
Laukens, D., Brinkman, B. M., Raes, J., De Vos, M. \& Vandenabeele, P. (2016) Heterogeneity of the gut microbiome in mice: guidelines for optimizing experimental design. FEMS Microbiology Reviews, 40(1), 117-132.

Le Poul, E., Loison, C., Struyf, S., Springael, J. Y., Lannoy, V., Decobecq, M. E., Brezillon, S., Dupriez, V., Vassart, G., Van Damme, J., Parmentier, M. \& Detheux, M. (2003) Functional characterization of human receptors for short chain fatty acids and their role in polymorphonuclear cell activation. J Biol Chem, 278(28), 25481-9.

Lehnardt, S., Massillon, L., Follett, P., Jensen, F. E., Ratan, R., Rosenberg, P. A., Volpe, J. J. \& Vartanian, T. (2003) Activation of innate immunity in the CNS triggers neurodegeneration through a Toll-like receptor 4-dependent pathway. Proceedings of the National Academy of Sciences of the United States of America, 100(14), 8514-8519.

Letran, S. E., Lee, S., Atif, S. M., Flores-Langarica, A., Uematsu, S., Akira, S., Cunningham, A. F. \& McSorley, S. J. (2011) TLR5-deficient mice lack basal inflammatory and metabolic defects but exhibit impaired CD4 $\mathrm{T}$ cell responses to a flagellated pathogen. Journal of Immunology, 186(9), 5406-5412.

Levenson, S. M. (1978) The influence of the indigenous microflora on mammalian metabolism and nutrition. Journal of Parenteral and Enteral Nutrition, 2(2), 78-107.

Ley, R. E. (2010) Obesity and the human microbiome. Current Opinion in Gastroenterology, 26(1), 5-11.

Ley, R. E., Backhed, F., Turnbaugh, P., Lozupone, C. A., Knight, R. D. \& Gordon, J. I. (2005) Obesity alters gut microbial ecology. Proceedings of the National Academy of Sciences of the United States of America, 102(31), 11070-11075.

Ley, R. E., Lozupone, C. A., Hamady, M., Knight, R. \& Gordon, J. I. (2008) Worlds within worlds: evolution of the vertebrate gut microbiota. Nat Rev Microbiol, 6(10), 776-88.

Ley, R. E., Turnbaugh, P. J., Klein, S. \& Gordon, J. I. (2006) Microbial ecology - Human gut microbes associated with obesity. Nature, 444(7122), 1022-1023.

Li, L., Chen, L., Hu, L., Liu, Y., Sun, H. Y., Tang, J., Hou, Y. J., Chang, Y. X., Tu, Q. Q., Feng, G. S., Shen, F., Wu, M. C. \& Wang, H. Y. (2011) Nuclear Factor high-mobility group boxl mediating the activation of Toll-like receptor 4 signaling in hepatocytes in the early stage of nonalcoholic fatty liver disease in mice. Hepatology, 54(5), 1620-1630.

Li, S., Zhang, C., Gu, Y., Chen, L., Ou, S., Wang, Y. \& Peng, X. (2015) Lean rats gained more body weight than obese ones from a high-fibre diet. British Journal of Nutrition, 114(8), 1188-1194.

Liang, W., Lindeman, J. H., Menke, A. L., Koonen, D. P., Morrison, M., Havekes, L. M., van den Hoek, A. M. \& Kleemann, R. (2014) Metabolically induced liver inflammation leads to NASH and differs from LPS- or IL-lbeta-induced chronic inflammation. Laboratroy Investigation, 94(5), 491-502. 
Liu, S., Ren, F., Zhao, L., Jiang, L., Hao, Y., Jin, J., Zhang, M., Guo, H., Lei, X., Sun, E. \& Liu, H. (2015) Starch and starch hydrolysates are favorable carbon sources for bifidobacteria in the human gut. BMC Microbiology, 15(54), 1-9.

Lloyd, K. C., Grandt, D., Aurang, K., Eysselein, V. E., Schimiczek, M. \& Reeve, J. R., Jr. (1996) Inhibitory effect of PYY on vagally stimulated acid secretion is mediated predominantly by Y1 receptors. The American Journal of Physiology, 270(1 Pt 1), 123-127.

Lopez, M., Tovar, S., Vazquez, M. J., Williams, L. M. \& Dieguez, C. (2007) Peripheral tissue-brain interactions in the regulation of food intake. The Proceedings of the Nutrition Society, 66(1), 131-155.

Lord, K. A., Hoffman-Liebermann, B. \& Liebermann, D. A. (1990) Nucleotide sequence and expression of a cDNA encoding MyD88, a novel myeloid differentiation primary response gene induced by IL6. Oncogene, 5(7), 1095-1097.

Lu, Y. C., Yeh, W. C. \& Ohashi, P. S. (2008) LPS/TLR4 signal transduction pathway. Cytokine, 42(2), 145-151.

Luckey, T. D. (1972) Introduction to intestinal microecology. The American Journal of Clinical Nutrition, 25(12), 1292-1294.

Macfarlane, G. T. \& Macfarlane, S. (2012) Bacteria, colonic fermentation, and gastrointestinal health. Journal of AOAC International, 95(1), 50-60.

Macfarlane, S. \& Macfarlane, G. T. (2003) Regulation of short-chain fatty acid production. The Proceedings of the Nutrition Society, 62(1), 67-72.

Marcobal, A., Kashyap, P. C., Nelson, T. A., Aronov, P. A., Donia, M. S., Spormann, A., Fischbach, M. A. \& Sonnenburg, J. L. (2013) A metabolomic view of how the human gut microbiota impacts the host metabolome using humanized and gnotobiotic mice. The ISME Journal, 7(10), 1933-1943.

Martin, A. P. (2002) Phylogenetic approaches for describing and comparing the diversity of microbial communities. Applied and Environmental Microbiology, 68(8), 3673-3682.

McNeil, N. I. (1984) The contribution of the large-Intestine to energy supplies in man. American Journal of Clinical Nutrition, 39(2), 338-342.

Medeiros, M. D. \& Turner, A. J. (1994) Processing and metabolism of peptide-YY: pivotal roles of dipeptidylpeptidase-IV, aminopeptidase-P, and endopeptidase-24.11. Endocrinology, 134(5), 2088-94.

Mercer, J. G., Lawrence, C. B., Moar, K. M., Atkinson, T. \& Barrett, P. (1997) Short-day weight loss and effect of food deprivation on hypothalamic NPY and CRF mRNA in Djungarian hamsters. American Journal of Physiology, 273(2), 768-776.

Mercer, J. G., Moar, K. M., Ross, A. W., Hoggard, N. \& Morgan, P. J. (2000) Photoperiod regulates arcuate nucleus POMC, AGRP, and leptin receptor mRNA in Siberian hamster 
hypothalamus. American Journal of Physiology. Regulatory, Integrative and Comparative Physiology, 278(1), 271-281.

Metchnikoff, E. (1908) The prolongation of life: optimistic studies. New York \& London: G.P Putnams's Sons.

Meyer, D. \& Stasse-Wolthuis, M. (2009) The bifidogenic effect of inulin and oligofructose and its consequences for gut health. European Journal of Clinical Nutrition, 63(11), 1277-1289.

Milanski, M., Degasperi, G., Coope, A., Morari, J., Denis, R., Cintra, D. E., Tsukumo, D. M., Anhe, G., Amaral, M. E., Takahashi, H. K., Curi, R., Oliveira, H. C., Carvalheira, J. B., Bordin, S., Saad, M. J. \& Velloso, L. A. (2009) Saturated fatty acids produce an inflammatory response predominantly through the activation of TLR4 signaling in hypothalamus: implications for the pathogenesis of obesity. Journal of Neuroscience, 29(2), 359-370.

Million, M., Angelakis, E., Maraninchi, M., Henry, M., Giorgi, R., Valero, R., Vialettes, B. \& Raoult, D. (2013) Correlation between body mass index and gut concentrations of Lactobacillus reuteri, Bifidobacterium animalis, Methanobrevibacter smithii and Escherichia coli. International Journal of Obesity, 37(11), 1460-1466.

Million, M., Maraninchi, M., Henry, M., Armougom, F., Richet, H., Carrieri, P., Valero, R., Raccah, D., Vialettes, B. \& Raoult, D. (2012) Obesity-associated gut microbiota is enriched in Lactobacillus reuteri and depleted in Bifidobacterium animalis and Methanobrevibacter smithii. International Journal of Obesity, 36(6), 817-825.

Minot, S., Sinha, R., Chen, J., Li, H., Keilbaugh, S. A., Wu, G. D., Lewis, J. D. \& Bushman, F. D. (2011) The human gut virome: inter-individual variation and dynamic response to diet. Genome Reseach, 21(10), 1616-1625.

Mirhashemi, F., Scherneck, S., Kluth, O., Kaiser, D., Vogel, H., Kluge, R., Schuermann, A., Neschen, S. \& Joost, H. G. (2011) Diet dependence of diabetes in the New Zealand Obese (NZO) mouse: total fat, but not fat quality or sucrose accelerates and aggravates diabetes. Experimental and Clinical Endocrinology E Diabetes, 119(3), 167-171.

Montgomery, M. K., Hallahan, N. L., Brown, S. H., Liu, M., Mitchell, T. W., Cooney, G. J. \& Turner, N. (2013) Mouse strain-dependent variation in obesity and glucose homeostasis in response to high-fat feeding. Diabetologia, 56(5), 1129-1139.

Moore, K. J., Andersson, L. P., Ingalls, R. R., Monks, B. G., Li, R., Arnaout, M. A., Golenbock, D. T. \& Freeman, M. W. (2000) Divergent response to LPS and bacteria in CD14-deficient murine macrophages. Journal of Immunology, 165(8), 4272-4280.

Moreno-Navarrete, J. M., Ortega, F., Serino, M., Luche, E., Waget, A., Pardo, G., Salvador, J., Ricart, W., Fruhbeck, G., Burcelin, R. \& Fernandez-Real, J. M. (2012) Circulating lipopolysaccharide-binding protein (LBP) as a marker of obesity-related insulin resistance. International Journal of Obesity, 36(11), 1442-1449. 
Morotomi, M., Nagai, F. \& Watanabe, Y. (2012) Description of Christensenella minuta gen. nov., sp nov., isolated from human faeces, which forms a distinct branch in the order Clostridiales, and proposal of Christensenellaceae fam. nov. International Journal of Systematic and Evolutionary Microbiology, 62(1), 144-149.

Moshfegh, A. J., Friday, J. E., Goldman, J. P. \& Ahuja, J. K. (1999) Presence of inulin and oligofructose in the diets of Americans. J Nutr, 129(7 Suppl), 1407s-1ls.

Muccioli, G. G., Naslain, D., Backhed, F., Reigstad, C. S., Lambert, D. M., Delzenne, N. M. \& Cani, P. D. (2010) The endocannabinoid system links gut microbiota to adipogenesis. Molecular Systems Biology, 6(392), 1-15.

Munford, R. S. (2010) Murine responses to endotoxin: another dirty little secret? The Journal of Infectious Diseases, 201(2), 175-177.

Murphy, E. F., Cotter, P. D., Healy, S., Marques, T. M., O'Sullivan, O., Fouhy, F., Clarke, S. F., O'Toole, P. W., Quigley, E. M., Stanton, C., Ross, P. R., O'Doherty, R. M. \& Shanahan, F. (2010) Composition and energy harvesting capacity of the gut microbiota: relationship to diet, obesity and time in mouse models. Gut, 59(12), 1635-1642.

Muyzer, G., Dewaal, E. C. \& Uitterlinden, A. G. (1993) Profiling of complex microbial populations by denaturing gradient gel electrophoresis analysis of polymerase chain reaction-amplified genes coding for $16 \mathrm{~S}$ rRNA. Applied and Environmental Microbiology, 59(3), 695-700.

Mydel, P., Takahashi, Y., Yumoto, H., Sztukowska, M., Kubica, M., Gibson, F. C., 3rd, Kurtz, D. M., Jr., Travis, J., Collins, L. V., Nguyen, K. A., Genco, C. A. \& Potempa, J. (2006) Roles of the host oxidative immune response and bacterial antioxidant rubrerythrin during Porphyromonas gingivalis infection. PLOS Pathology, 2(7), 712-725.

Nakamura, Y., Natsume, M., Yasuda, A., Ishizaka, M., Kawahata, K. \& Koga, J. (2011) Fructooligosaccharides suppress high-fat diet-induced fat accumulation in C57BL/6J mice. BioFactors, 0(0), 1-7.

Nauck, M. A., Kemmeries, G., Holst, J. J. \& Meier, J. J. (2011) Rapid tachyphylaxis of the glucagon-like peptide 1-induced deceleration of gastric emptying in humans. Diabetes, 60(5), 1561-1565.

Nauck, M. A., Siemsgluss, J., Orskov, C. \& Holst, J. J. (1996) Release of glucagon-like peptide 1 (GLP-1 [7-36 amide]), gastric inhibitory polypeptide (GIP) and insulin in response to oral glucose after upper and lower intestinal resections. Zeitschrift fur Gastroenterologie, 34(3), 159-166.

Neyrinck, A. M., Possemiers, S., Druart, C., van de Wiele, T., De Backer, F., Cani, P. D., Larondelle, Y. \& Delzenne, N. M. (2011) Prebiotic effects of wheat arabinoxylan related to the increase in bifidobacteria, roseburia and bacteroides/prevotella in diet-induced obese mice. PLOS One, 6(6), e20944.

Niemi, P., Aura, A. M., Maukonen, J., Smeds, A. I., Mattila, I., Niemela, K., Tamminen, T., Faulds, C. B., Buchert, J. \& Poutanen, K. (2013) Interactions of a lignin-rich fraction 
from brewer's spent grain with gut microbiota in vitro. Journal of Agricultural and Food Chemistry, 61(27), 6754-6762.

Nishizawa, M., Nakabayashi, H., Uchida, K., Nakagawa, A. \& Niijima, A. (1996) The hepatic vagal nerve is receptive to incretin hormone glucagon-like peptide-1, but not to glucose-dependent insulinotropic polypeptide, in the portal vein. J Auton Nerv Syst, 61(2), 149-54.

Nohr, M. K., Dudele, A., Poulsen, M. M., Ebbesen, L. H., Radko, Y., Christensen, L. P., Jessen, N., Richelsen, B., Lund, S. \& Pedersen, S. B. (2016) LPS-enhanced glucosestimulated insulin secretion is normalized by resveratrol. PLOS One, 11(1), 1-15.

Nyman, M., Asp, N. G., Cummings, J. \& Wiggins, H. (1986) Fermentation of dietary fiber in the intestinal tract: comparison between man and rat. British Journal of Nutrition, 55(3), 487-496.

O'Neill, L. A. \& Bowie, A. G. (2007) The family of five: TIR-domain-containing adaptors in Toll-like receptor signalling. Nat Rev Immunol, 7(5), 353-64.

Ohashi, K., Burkart, V., Flohe, S. \& Kolb, H. (2000) Cutting edge: heat shock protein 60 is a putative endogenous ligand of the toll-like receptor-4 complex. Journal of Immunology, 164(2), 558-561.

Orr, J. S., Puglisi, M. J., Ellacott, K. L., Lumeng, C. N., Wasserman, D. H. \& Hasty, A. H. (2012) Toll-like receptor 4 deficiency promotes the alternative activation of adipose tissue macrophages. Diabetes, 61(11), 2718-2727.

Pal, D., Dasgupta, S., Kundu, R., Maitra, S., Das, G., Mukhopadhyay, S., Ray, S., Majumdar, S. S. \& Bhattacharya, S. (2012) Fetuin-A acts as an endogenous ligand of TLR4 to promote lipid-induced insulin resistance. Nature Medicine, 18(8), 1279-1285.

Parfrey, L. W., Walters, W. A. \& Knight, R. (2011) Microbial eukaryotes in the human microbiome: ecology, evolution, and future directions. Frontiers in Microbiology, 2(153), $1-6$.

Park, B. S., Song, D. H., Kim, H. M., Choi, B. S., Lee, H. \& Lee, J. O. (2009) The structural basis of lipopolysaccharide recognition by the TLR4-MD-2 complex. Nature, 458(7242), 1191-5.

Parks, B. W., Nam, E., Org, E., Kostem, E., Norheim, F., Hui, S. T., Pan, C., Civelek, M., Rau, C. D., Bennett, B. J., Mehrabian, M., Ursell, L. K., He, A., Castellani, L. W., Zinker, B., Kirby, M., Drake, T. A., Drevon, C. A., Knight, R., Gargalovic, P., Kirchgessner, T., Eskin, E. \& Lusis, A. J. (2013) Genetic control of obesity and gut microbiota composition in response to high-fat, high-sucrose diet in mice. Cell Metabolism, 17(1), 141-152.

Parnell, J. A. \& Reimer, R. A. (2009) Weight loss during oligofructose supplementation is associated with decreased ghrelin and increased peptide YY in overweight and obese adults. The American Journal of Clinical Nutrition, 89(6), 1751-1759. 
Patterson, E., Ryan, P. M., Cryan, J. F., Dinan, T. G., Ross, R. P., Fitzgerald, G. F. \& Stanton, C. (2016) Gut microbiota, obesity and diabetes. Postgrad Med J, 92(1087), 286300.

Pellizzon, M. (2016) Choice of laboratory animal diet influences intestinal health. Lab Animal, 45(6), 238-239.

Pendyala, S., Walker, J. M. \& Holt, P. R. (2012) A high-fat diet is associated with endotoxemia that originates from the gut. Gastroenterology, 142(5), 1100-1101.

Peng, L., Li, Z. R., Green, R. S., Holzman, I. R. \& Lin, J. (2009) Butyrate enhances the intestinal barrier by facilitating tight junction assembly via activation of AMP-activated protein kinase in Caco-2 cell monolayers. Journal of Nutrition, 139(9), 1619-1625.

Petro, A. E., Cotter, J., Cooper, D. A., Peters, J. C., Surwit, S. J. \& Surwit, R. S. (2004) Fat, carbohydrate, and calories in the development of diabetes and obesity in the C57BL/6J mouse. Metabolism, 53(4), 454-457.

Pierre, N., Deldicque, L., Barbe, C., Naslain, D., Cani, P. D. \& Francaux, M. (2013) TollLike receptor 4 knockout mice are protected against endoplasmic reticulum stress induced by a high-fat diet. PLOS One, 8(5), 1-11.

Pironi, L., Stanghellini, V., Miglioli, M., Corinaldesi, R., De Giorgio, R., Ruggeri, E., Tosetti, C., Poggioli, G., Morselli Labate, A. M., Monetti, N. \& et al. (1993) Fat-induced ileal brake in humans: a dose-dependent phenomenon correlated to the plasma levels of peptide YY. Gastroenterology, 105(3), 733-839.

Poggi, M., Bastelica, D., Gual, P., Iglesias, M. A., Gremeaux, T., Knauf, C., Peiretti, F., Verdier, M., Juhan-Vague, I., Tanti, J. F., Burcelin, R. \& Alessi, M. C. (2007) C3H/HeJ mice carrying a toll-like receptor 4 mutation are protected against the development of insulin resistance in white adipose tissue in response to a high-fat diet. Diabetologia, 50(6), 1267-1276.

Poltorak, A., He, X. L., Smirnova, I., Liu, M. Y., Van Huffel, C., Du, X., Birdwell, D., Alejos, E., Silva, M., Galanos, C., Freudenberg, M., Ricciardi-Castagnoli, P., Layton, B. \& Beutler, B. (1998) Defective LPS signaling in C3H/HeJ and C57BL/10ScCr mice: Mutations in Tlr4 gene. Science, 282(5396), 2085-2088.

Poltorak, A., Smirnova, I., Clisch, R. \& Beutler, B. (2000) Limits of a deletion spanning Tlr4 in C57BL/10ScCr mice. Journal of Endotoxin Research, 6(1), 51-56.

Prada, P. O., Zecchin, H. G., Gasparetti, A. L., Torsoni, M. A., Ueno, M., Hirata, A. E., Corezola do Amaral, M. E., Hoer, N. F., Boschero, A. C. \& Saad, M. J. (2005) Western diet modulates insulin signaling, c-Jun N-terminal kinase activity, and insulin receptor substrate-1ser307 phosphorylation in a tissue-specific fashion. Endocrinology, 146(3), 1576-1587.

Prosky, L. (2000) What is dietary fiber? J AOAC Int, 83(4), 985-7. 
Psichas, A., Sleeth, M. L., Murphy, K. G., Brooks, L., Bewick, G. A., Hanyaloglu, A. C., Ghatei, M. A., Bloom, S. R. \& Frost, G. (2015) The short chain fatty acid propionate stimulates GLP-1 and PYY secretion via free fatty acid receptor 2 in rodents. International Journal of Obesity, 39(3), 424-429.

Qin, J., Li, R., Raes, J., Arumugam, M., Burgdorf, K. S., Manichanh, C., Nielsen, T., Pons, N., Levenez, F., Yamada, T., Mende, D. R., Li, J., Xu, J., Li, S., Li, D., Cao, J., Wang, B., Liang, H., Zheng, H., Xie, Y., Tap, J., Lepage, P., Bertalan, M., Batto, J. M., Hansen, T., Le Paslier, D., Linneberg, A., Nielsen, H. B., Pelletier, E., Renault, P., Sicheritz-Ponten, T., Turner, K., Zhu, H., Yu, C., Li, S., Jian, M., Zhou, Y., Li, Y., Zhang, X., Li, S., Qin, N., Yang, H., Wang, J., Brunak, S., Dore, J., Guarner, F., Kristiansen, K., Pedersen, O., Parkhill, J., Weissenbach, J., Meta, H. I. T. C., Bork, P., Ehrlich, S. D. \& Wang, J. (2010) A human gut microbial gene catalogue established by metagenomic sequencing. Nature, 464(7285), 59-65.

Quince, C., Lanzen, A., Davenport, R. J. \& Turnbaugh, P. J. (2011) Removing noise from pyrosequenced amplicons. BMC Bioinformatics, 12(38), 1-18.

Rabot, S., Membrez, M., Blancher, F., Berger, B., Moine, D., Krause, L., Bibiloni, R., Bruneau, A., Gerard, P., Siddharth, J., Lauber, C. L. \& Chou, C. J. (2016) High fat diet drives obesity regardless the composition of gut microbiota in mice. Scientific Reports, 6(32484), 1-11.

Rabot, S., Membrez, M., Bruneau, A., Gerard, P., Harach, T., Moser, M., Raymond, F., Mansourian, R. \& Chou, C. J. (2010) Germ-free C57BL/6J mice are resistant to high-fatdiet-induced insulin resistance and have altered cholesterol metabolism. FASEB Journal, 24(12), 4948-4959.

Raby, A. C., Holst, B., Le Bouder, E., Diaz, C., Ferran, E., Conraux, L., Guillemot, J. C., Coles, B., Kift-Morgan, A., Colmont, C. S., Szakmany, T., Ferrara, P., Hall, J. E., Topley, N. \& Labeta, M. O. (2013) Targeting the TLR co-receptor CD14 with TLR2-derived peptides modulates immune responses to pathogens. Science Translational Medicine, 5(185), 185 ra64.

Radin, M. S., Sinha, S., Bhatt, B. A., Dedousis, N. \& O'Doherty, R. M. (2008) Inhibition or deletion of the lipopolysaccharide receptor Toll-like receptor- 4 confers partial protection against lipid-induced insulin resistance in rodent skeletal muscle. Diabetologia, 51(2), 336-346.

Raetz, C. R. \& Whitfield, C. (2002) Lipopolysaccharide endotoxins. Annual Review of Biochemistry, 71, 635-700.

Ramirez-Farias, C., Slezak, K., Fuller, Z., Duncan, A., Holtrop, G. \& Louis, P. (2009) Effect of inulin on the human gut microbiota: stimulation of Bifidobacterium adolescentis and Faecalibacterium prausnitzii. British Journal of Nutrition, 101(4), 541550 .

Rausch, P., Basic, M., Batra, A., Bischoff, S. C., Blaut, M., Clavel, T., Glasner, J., Gopalakrishnan, S., Grassl, G. A., Gunther, C., Haller, D., Hirose, M., Ibrahim, S., Loh, G., Mattner, J., Nagel, S., Pabst, O., Schmidt, F., Siegmund, B., Strowig, T., Volynets, V., 
Wirtz, S., Zeissig, S., Zeissig, Y., Bleich, A. \& Baines, J. F. (2016) Analysis of factors contributing to variation in the C57BL/6J fecal microbiota across German animal facilities. International Journal of Medical Microbiology, 306(5), 343-355.

Ravussin, Y., Koren, O., Spor, A., LeDuc, C., Gutman, R., Stombaugh, J., Knight, R., Ley, R. E. \& Leibel, R. L. (2012) Responses of gut microbiota to diet composition and weight loss in lean and obese mice. Obesity, 20(4), 738-747.

Rawls, J. F., Mahowald, M. A., Ley, R. E. \& Gordon, J. I. (2006) Reciprocal gut microbiota transplants from zebrafish and mice to germ-free recipients reveal host habitat selection. Cell, 127(2), 423-433.

Reardon, S. (2016) A mouse's house may ruin experiments. Nature, 530(7590), 264-264.

Reeves, P. G., Nielsen, F. H. \& Fahey, G. C. (1993) AIN-93 purified diets for laboratory rodents: final report of the American Institute of Nutrition ad hoc writing committee on the reformulation of the AIN-76A rodent diet. Journal of Nutrition, 123(11), 1939-1951.

Reikvam, D. H., Erofeev, A., Sandvik, A., Grcic, V., Jahnsen, F. L., Gaustad, P., McCoy, K. D., Macpherson, A. J., Meza-Zepeda, L. A. \& Johansen, F. E. (2011) Depletion of murine intestinal microbiota: Effects on gut mucosa and epithelial gene expression. PLOS One, 6(3), el7996.

Ricci, M. (2013) 2013: The Importance of a Proper Control Diet.Research Diets, Inc.

Richards, P., Parker, H. E., Adriaenssens, A. E., Hodgson, J. M., Cork, S. C., Trapp, S., Gribble, F. M. \& Reimann, F. (2014) Identification and characterization of GLP-1 receptor-expressing cells using a new transgenic mouse model. Diabetes, 63(4), 12241233.

Ridaura, V. K., Faith, J. J., Rey, F. E., Cheng, J., Duncan, A. E., Kau, A. L., Griffin, N. W., Lombard, V., Henrissat, B., Bain, J. R., Muehlbauer, M. J., Ilkayeva, O., Semenkovich, C. F., Funai, K., Hayashi, D. K., Lyle, B. J., Martini, M. C., Ursell, L. K., Clemente, J. C., Van Treuren, W., Walters, W. A., Knight, R., Newgard, C. B., Heath, A. C. \& Gordon, J. I. (2013) Gut microbiota from twins discordant for obesity modulate metabolism in mice. Science, 341(6150), 1241214-1241211.

Rietschel, E. T., Kirikae, T., Schade, F. U., Mamat, U., Schmidt, G., Loppnow, H., Ulmer, A. J., Zahringer, U., Seydel, U., Di Padova, F. \& et al. (1994) Bacterial endotoxin: molecular relationships of structure to activity and function. FASEB Journal, 8(2), 217225.

Ring, S. G. \& Selvendran, R. R. (1980) Isolation and analysis of cell wall material from beeswing wheat bran (Triticum aestivum). Phytochemistry, 19(8), 1723-1730.

Roberfroid, M. B. (1999) Caloric value of inulin and oligofructose. Journal of Nutrition, 129(7 Suppl), 1436s-147s.

Robertson, B. R., O'Rourke, J. L., Neilan, B. A., Vandamme, P., On, S. L. W., Fox, J. G. \& Lee, A. (2005) Mucispirillum schaedleri gen. nov., sp nov., a spiral-shaped bacterium 
colonizing the mucus layer of the gastrointestinal tract of laboratory rodents. International Journal of Systematic and Evolutionary Microbiology, 55(3), 1199-1204.

Rogers, G. B., Kozlowska, J., Keeble, J., Metcalfe, K., Fao, M., Dowd, S. E., Mason, A. J., McGuckin, M. A. \& Bruce, K. D. (2014) Functional divergence in gastrointestinal microbiota in physically-separated genetically identical mice. Scientific Reports, 4(5437), $1-5$.

Roncon-Albuquerque, R., Jr., Moreira-Rodrigues, M., Faria, B., Ferreira, A. P., Cerqueira, C., Lourenco, A. P., Pestana, M., von Hafe, P. \& Leite-Moreira, A. F. (2008) Attenuation of the cardiovascular and metabolic complications of obesity in CD14 knockout mice. Life Sciences, 83(13-14), 502-510.

Rosi, S., Vazdarjanova, A., Ramirez-Amaya, V., Worley, P. F., Barnes, C. A. \& Wenk, G. L. (2006) Memantine protects against LPS-induced neuroinflammation, restores behaviorally-induced gene expression and spatial learning in the rat. Neuroscience, 142(4), 1303-1315.

Ruttimann, E. B., Arnold, M., Hillebrand, J. J., Geary, N. \& Langhans, W. (2009) Intrameal hepatic portal and intraperitoneal infusions of glucagon-like peptide-1 reduce spontaneous meal size in the rat via different mechanisms. Endocrinology, 150(3), 11741181.

Saberi, M., Woods, N. B., de Luca, C., Schenk, S., Lu, J. C., Bandyopadhyay, G., Verma, I. M. \& Olefsky, J. M. (2009) Hematopoietic cell-specific deletion of toll-like receptor 4 ameliorates hepatic and adipose tissue insulin resistance in high-fat-fed mice. Cell Metabolism, 10(5), 419-429.

Sakamoto, E., Seino, Y., Fukami, A., Mizutani, N., Tsunekawa, S., Ishikawa, K., Ogata, H., Uenishi, E., Kamiya, H., Hamada, Y., Sato, H., Harada, N., Toyoda, Y., Miwa, I., Nakamura, J., Inagaki, N., Oiso, Y. \& Ozaki, N. (2012) Ingestion of a moderate highsucrose diet results in glucose intolerance with reduced liver glucokinase activity and impaired glucagon-like peptide-1 secretion. Journal of Diabetes Investigation, 3(5), 432440.

Samuel, B. S. \& Gordon, J. I. (2006) A humanized gnotobiotic mouse model of hostarchaeal-bacterial mutualism. Proceedings of the National Academy of Sciences of the United States of America, 103(26), 10011-10016.

Sandoval, D. A., Bagnol, D., Woods, S. C., D'Alessio, D. A. \& Seeley, R. J. (2008) Arcuate glucagon-like peptide 1 receptors regulate glucose homeostasis but not food intake. Diabetes, 57(8), 2046-54.

Savage, D. C. (1977) Microbial ecology of the gastrointestinal tract. Annual Review of Microbiology, 31, 107-133.

Schaedler, R. W., Dubs, R. \& Costello, R. (1965) ASSOCIATION OF GERMFREE MICE WITH BACTERIA ISOLATED FROM NORMAL MICE. J Exp Med, 122, 77-82. 
Schloss, P. D. (2008) Evaluating different approaches that test whether microbial communities have the same structure. The ISME Journal, 2(3), 265-275.

Schloss, P. D. \& Handelsman, J. (2004) Status of the microbial census. Microbiology and Molecular Biology Reviews, 68(4), 686-691.

Schloss, P. D. \& Handelsman, J. (2006) Introducing TreeClimber, a test to compare microbial community structures. Applied and Environmental Microbiology, 72(4), 23792384.

Schloss, P. D., Westcott, S. L., Ryabin, T., Hall, J. R., Hartmann, M., Hollister, E. B., Lesniewski, R. A., Oakley, B. B., Parks, D. H., Robinson, C. J., Sahl, J. W., Stres, B., Thallinger, G. G., Van Horn, D. J. \& Weber, C. F. (2009) Introducing mothur: Opensource, platform-independent, community-supported software for describing and comparing microbial communities. Applied and Environmental Microbiology, 75(23), 7537-7541.

Schneeberger, M., Everard, A., Gomez-Valades, A. G., Matamoros, S., Ramirez, S., Delzenne, N. M., Gomis, R., Claret, M. \& Cani, P. D. (2015) Akkermansia muciniphila inversely correlates with the onset of inflammation, altered adipose tissue metabolism and metabolic disorders during obesity in mice. Scientific Reports, 5(16643 ), 1-14.

Schonhoff, S., Baggio, L., Ratineau, C., Ray, S. K., Lindner, J., Magnuson, M. A., Drucker, D. J. \& Leiter, A. B. (2005) Energy homeostasis and gastrointestinal endocrine differentiation do not require the anorectic hormone peptide YY. Molecular and Cellular Biology, 25(10), 4189-4199.

Schulz, M. D., Atay, C., Heringer, J., Romrig, F. K., Schwitalla, S., Aydin, B., Ziegler, P. K., Varga, J., Reindl, W., Pommerenke, C., Salinas-Riester, G., Bock, A., Alpert, C., Blaut, M., Polson, S. C., Brandl, L., Kirchner, T., Greten, F. R., Polson, S. W. \& Arkan, M. C. (2014) High-fat-diet-mediated dysbiosis promotes intestinal carcinogenesis independently of obesity. Nature, 514(7523), 508-512.

Schwartz, M. W., Woods, S. C., Porte, D., Jr., Seeley, R. J. \& Baskin, D. G. (2000) Central nervous system control of food intake. Nature, 404(6778), 661-671.

Schwiertz, A., Taras, D., Schafer, K., Beijer, S., Bos, N. A., Donus, C. \& Hardt, P. D. (2010) Microbiota and SCFA in lean and overweight healthy subjects. Obesity, 18(1), 190-195.

Scott, K. P., Martin, J. C., Duncan, S. H. \& Flint, H. J. (2014) Prebiotic stimulation of human colonic butyrate-producing bacteria and bifidobacteria, in vitro. FEMS Microbiology Ecolology, 87(1), 30-40.

Scrocchi, L. A., Brown, T. J., MacLusky, N., Brubaker, P. L., Auerbach, A. B., Joyner, A. L. \& Drucker, D. J. (1996) Glucose intolerance but normal satiety in mice with a null mutation in the glucagon-like peptide 1 receptor gene. Nature Medicine, 2(11), 1254-1258.

Scrocchi, L. A. \& Drucker, D. J. (1998) Effects of aging and a high fat diet on body weight and glucose tolerance in glucagon-like peptide-1 receptor(-/-) mice. Endocrinology, 139(7), 3127-3132. 
Secher, A., Jelsing, J., Baquero, A. F., Hecksher-Sorensen, J., Cowley, M. A., Dalboge, L. S., Hansen, G., Grove, K. L., Pyke, C., Raun, K., Schaffer, L., Tang-Christensen, M., Verma, S., Witgen, B. M., Vrang, N. \& Bjerre Knudsen, L. (2014) The arcuate nucleus mediates GLP-1 receptor agonist liraglutide-dependent weight loss. The Journal of Clinical Investigation, 124(10), 4473-4488.

Segata, N., Izard, J., Waldron, L., Gevers, D., Miropolsky, L., Garrett, W. S. \& Huttenhower, C. (2011) Metagenomic biomarker discovery and explanation. Genome Biology, 12(60), 1-18.

Sekirov, I., Russell, S. L., Antunes, L. C. M. \& Finlay, B. B. (2010) Gut microbiota in health and disease. Physiological Reviews, 90(3), 859-904.

Sender, R., Fuchs, S. \& Milo, R. (2016) Revised estimates for the number of human and bacteria cells in the body. PLOS Biology, 19(8), el002533.

Sergi, D., Morris, A. C., Drew, J. E., Campbell, F. M., Nicol, P. \& Williams, L. M. (2015) A novel hypothalamic protein regulated by high fat diet and leptin. Proceedings of the Nutrition Society, 74(3), 190-190.

Shechter, R., London, A., Kuperman, Y., Ronen, A., Rolls, A., Chen, A. \& Schwartz, M. (2013) Hypothalamic neuronal toll-like receptor 2 protects against age-induced obesity. Scientific Reports, 3(1254), 1-8.

Shi, H., Kokoeva, M. V., Inouye, K., Tzameli, I., Yin, H. \& Flier, J. S. (2006) TLR4 links innate immunity and fatty acid-induced insulin resistance. Journal of Clinical Investigation, 116(11), 3015-3025.

Shin, N.-R., Lee, J.-C., Lee, H.-Y., Kim, M.-S., Whon, T. W., Lee, M.-S. \& Bae, J.-W. (2014) An increase in the Akkermansia spp. population induced by metformin treatment improves glucose homeostasis in diet-induced obese mice. Gut, 63(5), 727-735.

Shin, N. R., Whon, T. W. \& Bae, J. W. (2015) Proteobacteria: microbial signature of dysbiosis in gut microbiota. Trends in Biotechnololgy, 33(9), 496-503.

Simmons, D., M., Arriza, J., L. \& Swanson, L., W. (1989) A complete protocol for in situ hybridization of messenger RNAs in brain and other tissues with radiolabeled singlestranded RNA probes. Journal of Histotechnology, 12(3), 169-181.

Sleeth, M. L., Thompson, E. L., Ford, H. E., Zac-Varghese, S. E. \& Frost, G. (2010) Free fatty acid receptor 2 and nutrient sensing: a proposed role for fibre, fermentable carbohydrates and short-chain fatty acids in appetite regulation. Nutrition Research Reviews, 23(1), 135-145.

Smith, K., McCoy, K. D. \& Macpherson, A. J. (2007) Use of axenic animals in studying the adaptation of mammals to their commensal intestinal microbiota. Seminars in Immunology, 19(2), 59-69.

Smyth, G. K. (2005) Limma: Linear models for microarray data. NEW YORK; 233 SPRING STREET, NEW YORK, NY 10013, UNITED STATES: SPRINGER. 
Smyth, G. K. \& Speed, T. (2003) Normalization of cDNA microarray data. Methods, 31(4), 265-273.

Sonnenburg, E. D., Smits, S. A., Tikhonov, M., Higginbottom, S. K., Wingreen, N. S. \& Sonnenburg, J. L. (2016) Diet-induced extinctions in the gut microbiota compound over generations. Nature, 529(7585), 212-208.

Spreckley, E. \& Murphy, K. G. (2015) The L-Cell in nutritional sensing and the regulation of appetite. Frontiers in Nutrition, 2(23), 1-17.

Stappenbeck, T. S., Hooper, L. V. \& Gordon, J. I. (2002) Developmental regulation of intestinal angiogenesis by indigenous microbes via Paneth cells. Proceedings of the National Academy of Sciences of the United States of America, 99(24), 15451-15455.

Stehle, J. R., Jr., Leng, X., Kitzman, D. W., Nicklas, B. J., Kritchevsky, S. B. \& High, K. P. (2012) Lipopolysaccharide-binding protein, a surrogate marker of microbial translocation, is associated with physical function in healthy older adults. The Journals of Gerontology. Series A, Biological Sciences and Medical Sciences., 67(11), 1212-1218.

Stoddart, L. A., Smith, N. J. \& Milligan, G. (2008) International Union of Pharmacology. LXXI. Free fatty acid receptors FFAl, -2, and -3: pharmacology and pathophysiological functions. Pharmacological Reviews, 60(4), 405-417.

Suarez-Zamorano, N., Fabbiano, S., Chevalier, C., Stojanovic, O., Colin, D. J., Stevanovic, A., Veyrat-Durebex, C., Tarallo, V., Rigo, D., Germain, S., Ilievska, M., Montet, X., Seimbille, Y., Hapfelmeier, S. \& Trajkovski, M. (2015) Microbiota depletion promotes browning of white adipose tissue and reduces obesity. Nature Medicine, 21(12), 14971501.

Suganami, T., Mieda, T., Itoh, M., Shimoda, Y., Kamei, Y. \& Ogawa, Y. (2007) Attenuation of obesity-induced adipose tissue inflammation in $\mathrm{C} 3 \mathrm{H} / \mathrm{HeJ}$ mice carrying a Toll-like receptor 4 mutation. Biochemical and Biophysical Research Communications, 354(1), 45-49.

Sultzer, B. M. (1972) Genetic control of host responses to endotoxin. Infection and Immunity, 5(1), 107-113.

Surwit, R. S., Feinglos, M. N., Rodin, J., Sutherland, A., Petro, A. E., Opara, E. C., Kuhn, C. M. \& Rebuffe-Scrive, M. (1995) Differential effects of fat and sucrose on the development of obesity and diabetes in C57BL/6J and A/J mice. Metabolism, 44(5), 645651.

Swartz, T. D., Sakar, Y., Duca, F. A. \& Covasa, M. (2013) Preserved adiposity in the Fischer 344 rat devoid of gut microbiota. FASEB Journal, 27(4), 1701-1710.

Swidsinski, A., Loening-Baucke, V., Lochs, H. \& Hale, L. P. (2005) Spatial organization of bacterial flora in normal and inflamed intestine: a fluorescence in situ hybridization study in mice. World Journal of Gastroenterology, 11(8), 1131-1140. 
Sze, M. \& Schloss, P. D. (2016) Looking for a signal in the noise: Revisiting obesity and the microbiome. mBio, 7(4), e01018-16.

Takeuchi, O., Hoshino, K., Kawai, T., Sanjo, H., Takada, H., Ogawa, T., Takeda, K. \& Akira, S. (1999) Differential roles of TLR2 and TLR4 in recognition of gram-negative and gram-positive bacterial cell wall components. Immunity, 11(4), 443-451.

Tarini, J. \& Wolever, T. M. (2010) The fermentable fibre inulin increases postprandial serum short-chain fatty acids and reduces free-fatty acids and ghrelin in healthy subjects. Applied Physiology, Nutrition, and Metabolism, 35(1), 9-16.

Termeer, C., Benedix, F., Sleeman, J., Fieber, C., Voith, U., Ahrens, T., Miyake, K., Freudenberg, M., Galanos, C. \& Simon, J. C. (2002) Oligosaccharides of hyaluronan activate dendritic cells via toll-like receptor 4. The Journal of Experimental Medicine, 195(1), 99-111.

Thaler, J. P. \& Schwartz, M. W. (2010) Minireview: Inflammation and obesity pathogenesis: The hypothalamus heats up. Endocrinology, 151(9), 4109-4115.

Thaler, J. P., Yi, C.-X., Schur, E. A., Guyenet, S. J., Hwang, B. H., Dietrich, M. O., Zhao, X., Sarruf, D. A., Izgur, V., Maravilla, K. R., Nguyen, H. T., Fischer, J. D., Matsen, M. E., Wisse, B. E., Morton, G. J., Horvath, T. L., Baskin, D. G., Tschoep, M. H. \& Schwartz, M. W. (2012) Obesity is associated with hypothalamic injury in rodents and humans. Journal of Clinical Investigation, 122(1), 153-162.

Tokuda, S., Beyer, B. J. \& Frankel, W. N. (2009) Genetic complexity of absence seizures in substrains of C3H mice. Genes, Brain, and Behavior, 8(3), 283-289.

Tomita, M., Ohkubo, R. \& Hayashi, M. (2004) Lipopolysaccharide transport system across colonic epithelial cells in normal and infective rat. Drug Metabolism and Pharmacokinetics, 19(1), 33-40.

Trapp, S. \& Richards, J. E. (2013) The gut hormone glucagon-like peptide-1 produced in brain: is this physiologically relevant? Current Opinion in Pharmacology, 13(6), 964-969.

Tremaroli, V., Kovatcheva-Datchary, P. \& Backhed, F. (2010) A role for the gut microbiota in energy harvesting? Gut, 59(12), 1589-1590.

Trepanowski, J. F., Mey, J. \& Varady, K. A. (2015) Fetuin-A: a novel link between obesity and related complications. International Journal of Obesity, 39(5), 734-741.

Truett, G. E., Heeger, P., Mynatt, R. L., Truett, A. A., Walker, J. A. \& Warman, M. L. (2000) Preparation of PCR-quality mouse genomic DNA with hot sodium hydroxide and tris (HotSHOT). Biotechniques, 29(1), 52-54.

Tschop, M. \& Heiman, M. L. (2001) Rodent obesity models: an overview. Experimental and Clinical Endocrinology \& Diabetes, 109(6), 307-319.

Tsukumo, D. M., Carvalho-Filho, M. A., Carvalheira, J. B., Prada, P. O., Hirabara, S. M., Schenka, A. A., Araujo, E. P., Vassallo, J., Curi, R., Velloso, L. A. \& Saad, M. J. (2016) 
Statement of Retraction. Loss-of-Function Mutation in Toll-Like Receptor 4 Prevents Diet-Induced Obesity and Insulin Resistance. Diabetes, 65(4), 1126-1127.

Tsukumo, D. M. L., Carvalho-Filho, M. A., Carvalheira, J. B. C., Prada, P. O., Hirabara, S. M., Schenka, A. A., Araujo, E. P., Vassallo, J., Curi, R., Velloso, L. A. \& Saad, M. J. A. (2007) Loss-of-function mutation in Toll-like receptor 4 prevents diet-induced obesity and insulin resistance. Diabetes, 56(8), 1986-1998.

Tups, A., Ellis, C., Moar, K. M., Logie, T. J., Adam, C. L., Mercer, J. G. \& Klingenspor, M. (2004) Photoperiodic regulation of leptin sensitivity in the Siberian hamster, Phodopus sungorus, is reflected in arcuate nucleus SOCS-3 (suppressor of cytokine signaling) gene expression. Endocrinology, 145(3), 1185-1193.

Turnbaugh, P. J., Baeckhed, F., Fulton, L. \& Gordon, J. I. (2008) Diet-induced obesity is linked to marked but reversible alterations in the mouse distal gut microbiome. Cell Host \& Microbe, 3(4), 213-223.

Turnbaugh, P. J., Hamady, M., Yatsunenko, T., Cantarel, B. L., Duncan, A., Ley, R. E., Sogin, M. L., Jones, W. J., Roe, B. A., Affourtit, J. P., Egholm, M., Henrissat, B., Heath, A. C., Knight, R. \& Gordon, J. I. (2009a) A core gut microbiome in obese and lean twins. Nature, 457(7228), e07540.

Turnbaugh, P. J., Ley, R. E., Mahowald, M. A., Magrini, V., Mardis, E. R. \& Gordon, J. I. (2006) An obesity-associated gut microbiome with increased capacity for energy harvest. Nature, 444(7122), 1027-1031.

Turnbaugh, P. J., Ridaura, V. K., Faith, J. J., Rey, F. E., Knight, R. \& Gordon, J. I. (2009b) The effect of diet on the human gut microbiome: A metagenomic analysis in humanized gnotobiotic mice. Science Translational Medicine, 1(6), 6ral4.

Ubeda, C., Lipuma, L., Gobourne, A., Viale, A., Leiner, I., Equinda, M., Khanin, R. \& Pamer, E. G. (2012) Familial transmission rather than defective innate immunity shapes the distinct intestinal microbiota of TLR-deficient mice. Journal of Experimental Medicine, 209(8), 1445-1456.

Uematsu, S., Jang, M. H., Chevrier, N., Guo, Z., Kumagai, Y., Yamamoto, M., Kato, H., Sougawa, N., Matsui, H., Kuwata, H., Hemmi, H., Coban, C., Kawai, T., Ishii, K. J., Takeuchi, O., Miyasaka, M., Takeda, K. \& Akira, S. (2006) Detection of pathogenic intestinal bacteria by Toll-like receptor 5 on intestinal CDllc+ lamina propria cells. Nature Immunology, 7(8), 868-874.

Ulven, T. (2012) Short-chain free fatty acid receptors FFA2/GPR43 and FFA3/GPR41 as new potential therapeutic targets. Frontiers in Endocrinology, 3(111), 1-9.

Uribe, A., Alam, M., Johansson, O., Midtvedt, T. \& Theodorsson, E. (1994) Microflora modulates endocrine cells in the gastrointestinal mucosa of the rat. Gastroenterology, 107(5), 1259-1269.

Van den Abbeele, P., Gerard, P., Rabot, S., Bruneau, A., El Aidy, S., Derrien, M., Kleerebezem, M., Zoetendal, E. G., Smidt, H., Verstraete, W., Van de Wiele, T. \& 
Possemiers, S. (2011) Arabinoxylans and inulin differentially modulate the mucosal and luminal gut microbiota and mucin-degradation in humanized rats. Environmental Microbiology, 13(10), 2667-2680.

van Loo, J., Coussement, P., de Leenheer, L., Hoebregs, H. \& Smits, G. (1995) On the presence of inulin and oligofructose as natural ingredients in the western diet. Crit Rev Food Sci Nutr, 35(6), 525-52.

Vijay-Kumar, M., Aitken, J. D., Carvalho, F. A., Cullender, T. C., Mwangi, S., Srinivasan, S., Sitaraman, S. V., Knight, R., Ley, R. E. \& Gewirtz, A. T. (2010) Metabolic syndrome and altered gut microbiota in mice lacking Toll-like receptor 5. Science, 328(5975), 228231.

Vijay-Kumar, M., Aitken, J. D., Carvalho, F. A., Ziegler, T. R., Gewirtz, A. T. \& Ganji, V. (2011) Loss of function mutation in toll-like receptor-4 does not offer protection against obesity and insulin resistance induced by a diet high in trans fat in mice. Journal of Inflammation, 8(2), 1-7.

Vijay-Kumar, M., Sanders, C. J., Taylor, R. T., Kumar, A., Aitken, J. D., Sitaraman, S. V., Neish, A. S., Uematsu, S., Akira, S., Williams, I. R. \& Gewirtz, A. T. (2007) Deletion of TLR5 results in spontaneous colitis in mice. Journal of Clinical Investigation, 117(12), 3909-3921.

Vila, I. K., Badin, P. M., Marques, M. A., Monbrun, L., Lefort, C., Mir, L., Louche, K., Bourlier, V., Roussel, B., Gui, P., Grober, J., Stich, V., Rossmeislova, L., Zakaroff-Girard, A., Bouloumie, A., Viguerie, N., Moro, C., Tavernier, G. \& Langin, D. (2014) Immune cell Toll-like receptor 4 mediates the development of obesity- and endotoxemia-associated adipose tissue fibrosis. Cell Reports, 7(4), 1116-1129.

Vilsboll, T. \& Holst, J. J. (2004) Incretins, insulin secretion and Type 2 diabetes mellitus. Diabetologia, 47(3), 357-366.

Vitseva, O. I., Tanriverdi, K., Tchkonia, T. T., Kirkland, J. L., McDonnell, M. E., Apovian, C. M., Freedman, J. \& Gokce, N. (2008) Inducible Toll-like receptor and NF-kappaB regulatory pathway expression in human adipose tissue. Obesity (Silver Spring), 16(5), 932-7.

Vogel, S. N., Hansen, C. T. \& Rosenstreich, D. L. (1979) Characterization of a congenitally LPS-resistant, athymic mouse strain. Journal of Immunology, 122(2), 619-622.

Voigt, R. M., Forsyth, C. B., Green, S. J., Mutlu, E., Engen, P., Vitaterna, M. H., Turek, F. W. \& Keshavarzian, A. (2014) Circadian disorganization alters intestinal microbiota. PLOS One, 9(5), e97500.

von Meyenburg, C., Hrupka, B. H., Arsenijevic, D., Schwartz, G. J., Landmann, R. \& Langhans, W. (2004) Role for CD14, TLR2, and TLR4 in bacterial product-induced anorexia. Regulatory, Integrative and Comparative Physiology, 287(2), 298-305. 
Vreugdenhil, A. C., Rousseau, C. H., Hartung, T., Greve, J. W., van 't Veer, C. \& Buurman, W. A. (2003) Lipopolysaccharide (LPS)-binding protein mediates LPS detoxification by chylomicrons. Journal of Immunolgy, 170(3), 1399-1405.

Vrieze, A., Van Nood, E., Holleman, F., Salojarvi, J., Kootte, R. S., Bartelsman, J., Dallinga-Thie, G. M., Ackermans, M. T., Serlie, M. J., Oozeer, R., Derrien, M., Druesne, A., Vlieg, J., Bloks, V. W., Groen, A. K., Heilig, H., Zoetendal, E. G., Stroes, E. S., de Vos, W. M., Hoekstra, J. B. L. \& Nieuwdorp, M. (2012) Transfer of intestinal microbiota from fean donors increases insulin sensitivity in individuals with metabolic syndrome. Gastroenterology, 143(4), 913-916.

Walker, A., Pfitzner, B., Neschen, S., Kahle, M., Harir, M., Lucio, M., Moritz, F., Tziotis, D., Witting, M., Rothballer, M., Engel, M., Schmid, M., Endesfelder, D., Klingenspor, M., Rattei, T., Castell, W. Z., de Angelis, M. H., Hartmann, A. \& Schmitt-Kopplin, P. (2014) Distinct signatures of host-microbial meta-metabolome and gut microbiome in two C57BL/6 strains under high-fat diet. The ISME Journal, 8(12), 2380-2396.

Walker, A. W. (2016) Studying the human microbiota. Advances in Experimental Medicine and Biology, 902, 5-32.

Wang, B., Chandrasekera, P. C. \& Pippin, J. J. (2014) Leptin, and leptin receptor-deficient rodent models: relevance for human type 2 diabetes. Current Diabetes Reviews, 10(2), 131-145.

Wang, J., Obici, S., Morgan, K., Barzilai, N., Feng, Z. \& Rossetti, L. (2001) Overfeeding rapidly induces leptin and insulin resistance. Diabetes, 50(12), 2786-2791.

Wang, J., Tang, H., Zhang, C., Zhao, Y., Derrien, M., Rocher, E., van-Hylckama Vlieg, J. E., Strissel, K., Zhao, L., Obin, M. \& Shen, J. (2015) Modulation of gut microbiota during probiotic-mediated attenuation of metabolic syndrome in high fat diet-fed mice. The ISME Journal, 9(1), 1-15.

Wang, Q., Garrity, G. M., Tiedje, J. M. \& Cole, J. R. (2007) Naive Bayesian classifier for rapid assignment of rRNA sequences into the new bacterial taxonomy. Applied and Environmental Microbiology, 73(16), 5261-5267.

Wang, Y., Perfetti, R., Greig, N. H., Holloway, H. W., DeOre, K. A., Montrose-Rafizadeh, C., Elahi, D. \& Egan, J. M. (1997) Glucagon-like peptide-1 can reverse the age-related decline in glucose tolerance in rats. The Journal of Clinical Investigation, 99(12), 28832889.

Warren, H. S., Fitting, C., Hoff, E., Adib-Conquy, M., Beasley-Topliffe, L., Tesini, B., Liang, X., Valentine, C., Hellman, J., Hayden, D. \& Cavaillon, J. M. (2010) Resilience to bacterial infection: difference between species could be due to proteins in serum. The Journal of Infectious Diseases, 201(2), 223-232.

West, D. B., Waguespack, J. \& McCollister, S. (1995) Dietary obesity in the mouse: interaction of strain with diet composition. The American Journal of Physiology, 268(3), 658-665. 
White, J. R., Nagarajan, N. \& Pop, M. (2009) Statistical methods for detecting differentially abundant features in clinical metagenomic samples. PLOS Computational Biology, 5(4), el000352.

Willard, L. B., Hauss-Wegrzyniak, B. \& Wenk, G. L. (1999) Pathological and biochemical consequences of acute and chronic neuroinflammation within the basal forebrain cholinergic system of rats. Neuroscience, 88(1), 193-200.

Williams, L. M. (2012) Hypothalamic dysfunction in obesity. Proceedings of the Nutrition Society, 71(4), 521-533.

Williams, L. M., Campbell, F. M., Drew, J. E., Koch, C., Hoggard, N., Rees, W. D., Kamolrat, T., Thi Ngo, H., Steffensen, I. L., Gray, S. R. \& Tups, A. (2014) The development of diet-induced obesity and glucose intolerance in C57BL/6 mice on a high-fat diet consists of distinct phases. PLOS One, 9(8), el06159.

Wisse, B. E. \& Schwartz, M. W. (2009) Does hypothalamic inflammation cause obesity? Cell Metabolism, 10(4), 241-242.

Wolin, M. J. (1981) Fermentation in the rumen and human large intestine. Science, 213(4515), 1463-1468.

Wostman, B. S., Larkin, C., Moriarty, A. \& Brucknerkardoss, E. (1983) Dietary-intake, energy-metabolism, and excretory losses of adult male germfree wistar rats. Laboratory Animal Science, 33(1), 46-50.

Wostmann, B. S. (1981) The germfree animal in nutritional studies. Annual Review of Nutrition, 1, 257-279.

Woting, A., Pfeiffer, N., Loh, G., Klaus, S. \& Blaut, M. (2014) Clostridium ramosum promotes high-fat diet-induced obesity in gnotobiotic mouse models. mBio, 5(5), e01530-14.

Wright, S. D., Ramos, R. A., Tobias, P. S., Ulevitch, R. J. \& Mathison, J. C. (1990) CD14, a receptor for complexes of lipopolysaccharide (LPS) and LPS binding protein. Science, 249(4975), 1431-1433.

Wright, S. D., Tobias, P. S., Ulevitch, R. J. \& Ramos, R. A. (1989) Lipopolysaccharide (LPS) binding protein opsonizes LPS-bearing particles for recognition by a novel receptor on macrophages. Journal of Experimental Medicine, 170(4), 1231-1241.

Wu, K. L. H., Chan, S. H. H. \& Chan, J. Y. H. (2012) Neuroinflammation and oxidative stress in rostral ventrolateral medulla contribute to neurogenic hypertension induced by systemic inflammation. Journal of Neuroinflammation, 9(212), 1-15.

Wynne, K., Stanley, S., McGowan, B. \& Bloom, S. (2005) Appetite control. Journal of Endocrinology, 184(2), 291-318. 
Wyss, M. T., Magistretti, P. J., Buck, A. \& Weber, B. (2011) Labeled acetate as a marker of astrocytic metabolism. Journal of Cerebral Blood Flow and Metabolism, 31(8), 16681674.

Yin, M., Bradford, B. U., Wheeler, M. D., Uesugi, T., Froh, M., Goyert, S. M. \& Thurman, R. G. (2001) Reduced early alcohol-induced liver injury in CD14-deficient mice. Journal of Immunology, 166(7), 4737-4742.

Young, J. L., Mora, A., Cerny, A., Czech, M. P., Woda, B., Kurt-Jones, E. A., Finberg, R. W. \& Corvera, S. (2012) CD14 deficiency impacts glucose homeostasis in mice through altered adrenal tone. PLOS One, 7(1), e29688.

Zamanian, J. L., Xu, L., Foo, L. C., Nouri, N., Zhou, L., Giffard, R. G. \& Barres, B. A. (2012) Genomic analysis of reactive astrogliosis. Journal of Neuroscience, 32(18), 6391-6410.

Zhang, C., Li, S., Yang, L., Huang, P., Li, W., Wang, S., Zhao, G., Zhang, M., Pang, X., Yan, Z., Liu, Y. \& Zhao, L. (2013) Structural modulation of gut microbiota in life-long calorie-restricted mice. Nature Communications, 4(2163), ncomms3163.

Zhang, C. H., Zhang, M. H., Wang, S. Y., Han, R. J., Cao, Y. F., Hua, W. Y., Mao, Y. J., Zhang, X. J., Pang, X. Y., Wei, C. C., Zhao, G. P., Chen, Y. \& Zhao, L. P. (2010) Interactions between gut microbiota, host genetics and diet relevant to development of metabolic syndromes in mice. The ISME Journal, 4(2), 232-241.

Zhang, H., DiBaise, J. K., Zuccolo, A., Kudrna, D., Braidotti, M., Yu, Y., Parameswaran, P., Crowell, M. D., Wing, R., Rittmann, B. E. \& Krajmalnik-Brown, R. (2009) Human gut microbiota in obesity and after gastric bypass. Proceedings of the National Academy of Sciences, 106(7), 2365-2370.

Zhang, X., Zhang, G., Zhang, H., Karin, M., Bai, H. \& Cai, D. (2008) Hypothalamic IKK beta/NF-kappa B and ER stress link overnutrition to energy imbalance and obesity. Cell, 135(1), 61-73.

Zhao, L. (2013) The gut microbiota and obesity: from correlation to causality. Nature Reviews Microbiology, 11(9), 639-647.

Zhou, J., Martin, R. J., Raggio, A. M., Shen, L., McCutcheon, K. \& Keenan, M. J. (2015) The importance of GLP-1 and PYY in resistant starch's effect on body fat in mice. Molecular Nutrition and Food Research, 59(5), 1000-1003.

Zhou, J., Martin, R. J., Tulley, R. T., Raggio, A. M., McCutcheon, K. L., Shen, L., Danna, S. C., Tripathy, S., Hegsted, M. \& Keenan, M. J. (2008) Dietary resistant starch upregulates total GLP-1 and PYY in a sustained day-long manner through fermentation in rodents. American Journal of Physiology - Endocrinology and Metabolism, 295(5), 11601166. 


\section{APPENDIX}


Wild-Type mice Low-Fat vs High-Fat Microarray gene expression changes.

Table 13. Hypothalamic genes up-regulated in High-Fat diet fed Wild-Type mice

\begin{tabular}{|c|c|c|c|}
\hline Gene Symbol & Gene Name & $\begin{array}{c}\text { Fold } \\
\text { Change }\end{array}$ & P-value \\
\hline Rnu2-10 & U2 small nuclear RNA 10 & 1.73 & 0.0000 \\
\hline Rs5-8s1 & $5.8 \mathrm{~S}$ ribosomal RNA & 1.66 & 0.0002 \\
\hline Rn18s & $18 \mathrm{~S}$ ribosomal RNA & 1.60 & 0.0000 \\
\hline Sptan1 & spectrin alpha, non-erythrocytic 1 & 1.44 & 0.0000 \\
\hline 4932412D23Rik & RIKEN cDNA 4932412D23 gene & 1.43 & 0.0000 \\
\hline 4931402H11Rik & RIKEN cDNA 4931402H11 gene & 1.42 & 0.0001 \\
\hline Rnu1b6 & U1b6 small nuclear RNA & 1.41 & 0.0012 \\
\hline Zic2 & zinc finger protein of the cerebellum 2 & 1.41 & 0.0000 \\
\hline Spint2 & serine protease inhibitor, Kunitz type 2 & 1.40 & 0.0001 \\
\hline Zbtb7a & zinc finger and BTB domain containing $7 \mathrm{a}$ & 1.40 & 0.0009 \\
\hline Gabbr2 & gamma-aminobutyric acid (GABA) B receptor, 2 & 1.40 & 0.0020 \\
\hline Al504432 & expressed sequence Al504432 & 1.39 & 0.0000 \\
\hline Stk32c & serine/threonine kinase $32 \mathrm{C}$ & 1.39 & 0.0003 \\
\hline Thrap3 & thyroid hormone receptor associated protein 3 & 1.38 & 0.0008 \\
\hline Agrp & agouti related protein & 1.38 & 0.0004 \\
\hline Al504432 & expressed sequence Al504432 & 1.38 & 0.0000 \\
\hline Dst & dystonin & 1.38 & 0.0000 \\
\hline Kif1c & kinesin family member $1 \mathrm{C}$ & 1.37 & 0.0019 \\
\hline Palm2 & paralemmin 2 & 1.37 & 0.0000 \\
\hline 2610034E01Rik & RIKEN cDNA 2610034E01 gene & 1.37 & 0.0000 \\
\hline Map2 & microtubule-associated protein 2 & 1.37 & 0.0000 \\
\hline Hist1h4d & histone cluster $1, \mathrm{H} 4 \mathrm{~d}$ & 1.37 & 0.0000 \\
\hline Ipw & $\begin{array}{l}\text { imprinted gene in the Prader-Willi syndrome } \\
\text { region }\end{array}$ & 1.36 & 0.0001 \\
\hline Cony & cyclin $Y$ & 1.36 & 0.0002 \\
\hline Klhl11 & kelch-like 11 & 1.36 & 0.0001 \\
\hline Gm17821 & DDB1 and CUL4 associated factor 5 pseudogene & 1.35 & 0.0001 \\
\hline Specc1 & $\begin{array}{l}\text { sperm antigen with calponin homology and } \\
\text { coiled-coil domains } 1\end{array}$ & 1.35 & 0.0002 \\
\hline Dock10 & dedicator of cytokinesis 10 & 1.35 & 0.0002 \\
\hline Tpr & translocated promoter region & 1.35 & $\begin{array}{r}8.5 \mathrm{E}- \\
05 \\
\end{array}$ \\
\hline Hist1h1e & histone cluster $1, \mathrm{H} 1 \mathrm{e}$ & 1.34 & 0.00149 \\
\hline
\end{tabular}

Microarray results of hypothalamic genes up-regulated in High-Fat diet fed Wild-Type mice relative to Low-Fat diet fed Wild-Type mice. 


\begin{tabular}{|c|c|c|c|}
\hline Gene Symbol & Gene Name & $\begin{array}{c}\text { Fold } \\
\text { Change }\end{array}$ & P-value \\
\hline Megf9 & multiple EGF-like-domains 9 & 1.34 & 0.0050 \\
\hline Gaa & glucosidase, alpha, acid & 1.34 & 0.0001 \\
\hline Gm14305 & predicted gene 14305 & 1.33 & 0.0001 \\
\hline $\operatorname{lgf2}$ & insulin-like growth factor 2 & 1.33 & 0.0012 \\
\hline LOC628147 & zinc finger protein 709-like & 1.33 & 0.0002 \\
\hline Taf1 & $\begin{array}{l}\text { TAF1 RNA polymerase II, TATA box binding } \\
\text { protein (TBP)-associated factor }\end{array}$ & 1.33 & 0.0002 \\
\hline 2900072G11Rik & RIKEN cDNA 2900072G11 gene & 1.33 & 0.0001 \\
\hline 1700022A21Rik & $\begin{array}{l}\text { glycerol-3-phosphate dehydrogenase 1-like } \\
\text { pseudogene }\end{array}$ & 1.33 & 0.0001 \\
\hline Smc5 & structural maintenance of chromosomes 5 & 1.33 & 0.0002 \\
\hline Copg2os2 & $\begin{array}{l}\text { coatomer protein complex, subunit gamma } 2 \text {, } \\
\text { opposite strand } 2\end{array}$ & 1.33 & 0.0001 \\
\hline Hist4h4 & histone cluster $4, \mathrm{H} 4$ & 1.33 & 0.0001 \\
\hline Wdfy3 & WD repeat and FYVE domain containing 3 & 1.32 & 0.0004 \\
\hline Hist2h4 & histone cluster $2, \mathrm{H} 4$ & 1.32 & 0.0001 \\
\hline Zfp931 & zinc finger protein 931 & 1.32 & 0.0006 \\
\hline Tug1 & taurine upregulated gene 1 & 1.32 & 0.0001 \\
\hline Impact & impact, RWD domain protein & 1.31 & 0.0002 \\
\hline Mdm4 & transformed mouse 3T3 cell double minute 4 & 1.31 & 0.0004 \\
\hline Xndr-trpc2 & Xndr-Trpc2 readthrough & 1.31 & 0.0003 \\
\hline Snhg11 & small nucleolar RNA host gene 11 & 1.31 & 0.0002 \\
\hline Akap12 & A kinase (PRKA) anchor protein (gravin) 12 & 1.31 & 0.0003 \\
\hline Hist1h4k & histone cluster $1, \mathrm{H} 4 \mathrm{k}$ & 1.31 & 0.0002 \\
\hline A230057D06Rik & RIKEN cDNA A230057D06 gene & 1.30 & 0.0001 \\
\hline Peg3 & paternally expressed 3 & 1.30 & 0.0001 \\
\hline Fgd4 & FYVE, RhoGEF and $\mathrm{PH}$ domain containing 4 & 1.30 & 0.0001 \\
\hline Gprc5b & $\begin{array}{l}\text { G protein-coupled receptor, family C, group } 5 \text {, } \\
\text { member B }\end{array}$ & 1.30 & 0.0012 \\
\hline Snora74a & small nucleolar RNA, H/ACA box 74A & 1.30 & 0.0013 \\
\hline Csnk2a1 & casein kinase 2 , alpha 1 polypeptide & 1.30 & 0.0001 \\
\hline Srrm2 & serine/arginine repetitive matrix 2 & 1.30 & 0.0003 \\
\hline Gls & glutaminase & 1.30 & 0.0001 \\
\hline Zkscan16 & zinc finger with KRAB and SCAN domains 16 & 1.29 & 0.0052 \\
\hline
\end{tabular}

Microarray results of hypothalamic genes up-regulated in High-Fat diet fed Wild-Type mice relative to Low-Fat diet fed Wild-Type mice. 


\begin{tabular}{|c|c|c|c|}
\hline Gene Symbol & Gene Name & $\begin{array}{c}\text { Fold } \\
\text { Change }\end{array}$ & P-value \\
\hline Slc8a1 & $\begin{array}{l}\text { solute carrier family } 8 \text { (sodium/calcium } \\
\text { exchanger), member } 1\end{array}$ & $\begin{array}{c}1.29369 \\
7\end{array}$ & $\begin{array}{c}0.0001 \\
7\end{array}$ \\
\hline C78339 & expressed sequence C78339 & 1.29 & 0.0005 \\
\hline D2Ertd295e & DNA segment, Chr 2, ERATO Doi 295, expressed & 1.29 & 0.0004 \\
\hline $\mathrm{C} 2 \mathrm{~cd} 3$ & C2 calcium-dependent domain containing 3 & 1.29 & 0.0006 \\
\hline Inpp4a & inositol polyphosphate-4-phosphatase, type I & 1.29 & 0.0003 \\
\hline Dido1 & death inducer-obliterator 1 & 1.29 & 0.0006 \\
\hline Lmbrd2 & LMBR1 domain containing 2 & 1.29 & 0.0002 \\
\hline Zscan26 & zinc finger and SCAN domain containing 26 & 1.29 & 0.0004 \\
\hline Gm13547 & predicted gene 13547 & 1.29 & 0.0003 \\
\hline Hist1h4k & histone cluster $1, \mathrm{H} 4 \mathrm{k}$ & 1.29 & 0.0001 \\
\hline AB041803 & cDNA sequence $\mathrm{AB} 041803$ & 1.29 & 0.0006 \\
\hline Hist1h4j & histone cluster $1, \mathrm{H} 4 \mathrm{j}$ & 1.29 & 0.0003 \\
\hline Ap2m1 & adaptor-related protein complex 2 , mu 1 subunit & 1.29 & 0.0003 \\
\hline Thra & thyroid hormone receptor alpha & 1.29 & 0.0001 \\
\hline Gm8457 & predicted gene 8457 & 1.29 & 0.0002 \\
\hline Gm14325 & predicted gene 14325 & 1.29 & 0.0003 \\
\hline Cyld & cylindromatosis (turban tumor syndrome) & 1.28 & 0.0063 \\
\hline Gpcpd1 & $\begin{array}{l}\text { glycerophosphocholine phosphodiesterase GDE1 } \\
\text { homolog (S. cerevisiae) }\end{array}$ & 1.28 & 0.0003 \\
\hline Ifi27|2a & interferon, alpha-inducible protein 27 like $2 \mathrm{~A}$ & 1.28 & 0.0045 \\
\hline Prune2 & prune homolog 2 (Drosophila) & 1.28 & 0.0054 \\
\hline LOC100504873 & zinc finger protein 14-like & 1.28 & 0.0006 \\
\hline Scn1a & sodium channel, voltage-gated, type I, alpha & 1.28 & 0.0013 \\
\hline Slc5a3 & $\begin{array}{l}\text { solute carrier family } 5 \text { (inositol transporters), } \\
\text { member } 3\end{array}$ & 1.28 & 0.0051 \\
\hline Rnpc3 & RNA-binding region (RNP1, RRM) containing 3 & 1.28 & 0.0004 \\
\hline $\begin{array}{l}\text { A130077B15Ri } \\
k\end{array}$ & RIKEN cDNA A130077B15 gene & 1.28 & 0.0002 \\
\hline Snord116 & small nucleolar RNA, C/D box 116 & 1.28 & 0.0002 \\
\hline AB041803 & cDNA sequence $A B 041803$ & 1.28 & 0.0013 \\
\hline Ddx5 & DEAD (Asp-Glu-Ala-Asp) box polypeptide 5 & 1.28 & 0.0013 \\
\hline Tcf12 & transcription factor 12 & 1.27 & 0.0011 \\
\hline Gm1070 & predicted gene 1070 & 1.27 & 0.0008 \\
\hline
\end{tabular}

Microarray results of hypothalamic genes up-regulated in High-Fat diet fed Wild-Type mice relative to Low-Fat diet fed Wild-Type mice. 


\begin{tabular}{|c|c|c|c|}
\hline Gene Symbol & Gene Name & $\begin{array}{c}\text { Fold } \\
\text { Change }\end{array}$ & P-value \\
\hline Ppp1r9a & $\begin{array}{l}\text { protein phosphatase } 1 \text {, regulatory (inhibitor) } \\
\text { subunit } 9 A\end{array}$ & 1.27 & 0.0012 \\
\hline Cntnap2 & contactin associated protein-like 2 & 1.27 & 0.0003 \\
\hline Col25a1 & collagen, type XXV, alpha 1 & 1.269534 & 0.006 \\
\hline Gm15800 & predicted gene 15800 & 1.27 & 0.0094 \\
\hline D17Wsu92e & $\begin{array}{l}\text { DNA segment, Chr 17, Wayne State University 92, } \\
\text { expressed }\end{array}$ & 1.27 & 0.0060 \\
\hline Speer8-ps1 & $\begin{array}{l}\text { spermatogenesis associated glutamate (E)-rich } \\
\text { protein } 8, \text { pseudogene } 1\end{array}$ & 1.27 & 0.0002 \\
\hline Gm11213 & predicted gene 11213 & 1.27 & 0.0005 \\
\hline Akap11 & A kinase (PRKA) anchor protein 11 & 1.27 & 0.0007 \\
\hline Gn|3| & $\begin{array}{l}\text { guanine nucleotide binding protein-like } 3 \\
\text { (nucleolar)-like }\end{array}$ & 1.26 & 0.0003 \\
\hline Thrap3 & thyroid hormone receptor associated protein 3 & 1.26 & 0.0002 \\
\hline Kansl1 & KAT8 regulatory NSL complex subunit 1 & 1.26 & 0.0015 \\
\hline Cask & $\begin{array}{l}\text { calcium/calmodulin-dependent serine protein } \\
\text { kinase (MAGUK family) }\end{array}$ & 1.26 & 0.0003 \\
\hline Gm14391 & predicted gene 14391 & 1.26 & 0.0005 \\
\hline Tnks & $\begin{array}{l}\text { tankyrase, TRF1-interacting ankyrin-related ADP- } \\
\text { ribose polymerase }\end{array}$ & 1.26 & 0.0020 \\
\hline Fam20b & family with sequence similarity 20 , member $B$ & 1.26 & 0.0002 \\
\hline Cd151 & CD151 antigen & 1.26 & 0.0009 \\
\hline Azin1 & antizyme inhibitor 1 & 1.26 & 0.0003 \\
\hline Tmem178b & transmembrane protein $178 \mathrm{~B}$ & 1.26 & 0.0003 \\
\hline Marf1 & meiosis arrest female 1 & 1.25 & 0.0012 \\
\hline Prr14l & proline rich 14-like & 1.25 & 0.0019 \\
\hline Rora & RAR-related orphan receptor alpha & 1.25 & 0.0006 \\
\hline BC049265 & cDNA sequence BC049265 & 1.25 & 0.0004 \\
\hline Gm14431 & predicted gene 14431 & 1.25 & 0.0005 \\
\hline Pick1 & protein interacting with $\mathrm{C}$ kinase 1 & 1.25 & 0.0015 \\
\hline Ppp1r16a & $\begin{array}{l}\text { protein phosphatase 1, regulatory (inhibitor) } \\
\text { subunit } 16 \mathrm{~A}\end{array}$ & 1.25 & 0.0005 \\
\hline Fmr1 & fragile $\mathrm{X}$ mental retardation syndrome 1 & 1.25 & 0.0010 \\
\hline Gm14308 & predicted gene 14308 & 1.25 & 0.0002 \\
\hline Mbp & myelin basic protein & 1.25 & 0.0010 \\
\hline Sh3kbp1 & SH3-domain kinase binding protein 1 & 1.25 & 0.0016 \\
\hline
\end{tabular}

Microarray results of hypothalamic genes up-regulated in High-Fat diet fed Wild-Type mice relative to Low-Fat diet fed Wild-Type mice. 
Table 14. Hypothalamic genes down-regulated in High-Fat diet fed Wild-Type mice

\begin{tabular}{|l|l|c|c|}
\hline Gene Symbol & Gene Name & $\begin{array}{c}\text { Fold } \\
\text { Change }\end{array}$ & P-value \\
\hline Zc3h6 & zinc finger CCCH type containing 6 & -1.25 & 0.0004 \\
\hline Cdk17 & cyclin-dependent kinase 17 & -1.25 & 0.0035 \\
\hline Slc5a7 & $\begin{array}{l}\text { solute carrier family 5 (choline transporter), } \\
\text { member 7 }\end{array}$ & -1.26 & 0.0034 \\
\hline Zfr & zinc finger RNA binding protein & -1.26 & 0.0009 \\
\hline Fndc3a & fibronectin type III domain containing 3A & -1.2646 & 0.00149 \\
\hline Socs3 & suppressor of cytokine signalling 3 & -1.27 & 0.0009 \\
\hline Casp8ap2 & caspase 8 associated protein 2 & -1.27 & 0.0003 \\
\hline Zfp37 & zinc finger protein 37 & -1.27 & 0.0012 \\
\hline Serpina3n & $\begin{array}{l}\text { serine (or cysteine) peptidase inhibitor, clade A, } \\
\text { member 3N }\end{array}$ & -1.28 & 0.0006 \\
\hline Clip4 & $\begin{array}{l}\text { CAP-GLY domain containing linker protein family, } \\
\text { member 4 }\end{array}$ & -1.28 & 0.0010 \\
\hline Slc25a16 & $\begin{array}{l}\text { solute carrier family 25 (mitochondrial carrier, } \\
\text { Graves' disease autoantigen), member 16 }\end{array}$ & -1.28 & 0.0003 \\
\hline Trim23 & tripartite motif-containing 23 & -1.28 & 0.0001 \\
\hline Eps811 & EPS8-like 1 & -1.29 & 0.0011 \\
\hline Bpifa1 & BPI fold containing family A, member 1 & -1.44 & 0.0003 \\
\hline Phox2a & paired-like homeobox 2a & -1.48 & 0.0000 \\
\hline
\end{tabular}

Microarray results of hypothalamic genes down-regulated in High-Fat diet fed Wild-

Type mice relative to Low-Fat diet fed Wild-Type mice. 
TLR4 $^{-/-}$mice Low-Fat vs High-Fat Microarray gene expression changes.

Table 15. Hypothalamic genes up-regulated in High-Fat diet fed TLR4 ${ }^{-/-}$mice.

\begin{tabular}{|l|l|c|c|}
\hline Gene Symbol & Gene Name & $\begin{array}{c}\text { Fold } \\
\text { Change }\end{array}$ & P-value \\
\hline & $\begin{array}{l}\mid \\
\text { Prpf40 pre-mRNA processing factor 40 homolog B }\end{array}$ & 1.30 & 0.0030 \\
\hline Htatsf1 & HIV TAT specific factor 1 & 1.27 & 0.0013 \\
\hline Agrp & agouti related protein & 1.27 & 0.0080 \\
\hline Stk32c & serine/threonine kinase 32C & 1.26 & 0.0021 \\
\hline Chrna4 & cholinergic receptor, nicotinic, alpha polypeptide 4 & 1.25 & 0.0034 \\
\hline Keap1 & kelch-like ECH-associated protein 1 & 1.25 & 0.0016 \\
\hline & PRP19/PSO4 pre-mRNA processing factor 19 homolog & & \\
Prpf19 & (S. cerevisiae) & 1.25 & 0.0023 \\
\hline Dlk1 & delta-like 1 homolog (Drosophila) & 1.25 & 0.0090 \\
\hline
\end{tabular}

Microarray results of hypothalamic genes up-regulated in High-Fat diet fed TLR4 ${ }^{-/}$mice relative to Low-Fat diet fed TLR4 ${ }^{-/-}$mice. 
Table 16. Hypothalamic genes down-regulated in High-Fat diet fed TLR4 ${ }^{-/}$mice.

\begin{tabular}{|c|c|c|c|}
\hline Gene Symbol & Gene Name & $\begin{array}{c}\text { Fold } \\
\text { Change }\end{array}$ & P-value \\
\hline Colec12 & collectin sub-family member 12 & -1.25 & 0.0015 \\
\hline Mapk1 & mitogen-activated protein kinase 1 & -1.25 & 0.0073 \\
\hline Mfap5 & microfibrillar associated protein 5 & -1.25 & 0.0053 \\
\hline Asgr1 & asialoglycoprotein receptor 1 & -1.25 & 0.0023 \\
\hline Den & decorin & -1.25 & 0.0019 \\
\hline Car13 & carbonic anhydrase 13 & -1.25 & 0.0013 \\
\hline Nip7 & nuclear import 7 homolog (S. cerevisiae) & -1.25 & 0.0025 \\
\hline Trabd2b & TraB domain containing $2 \mathrm{~B}$ & -1.26 & 0.0014 \\
\hline Tbr1 & T-box brain gene 1 & -1.26 & 0.0029 \\
\hline C1qtnf7 & C1q and tumor necrosis factor related protein 7 & -1.26 & 0.0009 \\
\hline Asic4 & acid-sensing (proton-gated) ion channel family member 4 & -1.26 & 0.0023 \\
\hline Pf4 & platelet factor 4 & -1.26 & 0.0012 \\
\hline Tspan8 & tetraspanin 8 & -1.26 & 0.0025 \\
\hline Slc22a8 & $\begin{array}{l}\text { solute carrier family } 22 \text { (organic anion transporter), } \\
\text { member } 8\end{array}$ & -1.26 & 0.0035 \\
\hline $\mathrm{Ccl} 2$ & chemokine (C-C motif) ligand 2 & -1.26 & 0.0073 \\
\hline Zswim6 & zinc finger SWIM-type containing 6 & -1.26 & 0.0042 \\
\hline Wasf1 & WAS protein family, member 1 & -1.26 & 0.0056 \\
\hline Phldb2 & pleckstrin homology-like domain, family B, member 2 & -1.26 & 0.0025 \\
\hline D3Bwg0562e & $\begin{array}{l}\text { DNA segment, Chr 3, Brigham \& Women's Genetics } 0562 \\
\text { expressed }\end{array}$ & -1.26 & 0.0033 \\
\hline Oas1a & 2'-5' oligoadenylate synthetase $1 \mathrm{~A}$ & -1.26 & 0.0080 \\
\hline Lum & lumican & -1.27 & 0.0058 \\
\hline Gda & guanine deaminase & -1.27 & 0.0038 \\
\hline Prrx2 & paired related homeobox 2 & -1.27 & 0.0015 \\
\hline Ankrd63 & ankyrin repeat domain 63 & -1.27 & 0.0059 \\
\hline Fam49a & family with sequence similarity 49 , member $A$ & -1.27 & 0.0021 \\
\hline Prdm8 & PR domain containing 8 & -1.27 & 0.0034 \\
\hline Alcam & activated leukocyte cell adhesion molecule & -1.27 & 0.0015 \\
\hline Mmd & monocyte to macrophage differentiation-associated & -1.27 & 0.0050 \\
\hline Samd9l & sterile alpha motif domain containing 9-like & -1.27 & 0.0023 \\
\hline Mmd & monocyte to macrophage differentiation-associated & -1.27 & 0.0056 \\
\hline
\end{tabular}

Microarray results of hypothalamic genes down-regulated in High-Fat diet fed TLR4 ${ }^{-/-}$ mice relative to Low-Fat diet fed TLR $4^{-/-}$mice. 


\begin{tabular}{|l|l|c|r|}
\hline & & $\begin{array}{c}\text { Fold } \\
\text { Change }\end{array}$ & P-value \\
\hline Gene Symbol & Gene Name & -1.27 & 0.0009 \\
\hline Itgbl1 & integrin, beta-like 1 & -1.27 & 0.0060 \\
\hline C4b & tissue inhibitor of metalloproteinase 1 & -1.27 & 0.0013 \\
\hline Asic4 & complement component 4B (Chido blood group) & -1.28 & 0.0013 \\
\hline C030009J22Rik & acid-sensing (proton-gated) ion channel family member 4 & -1.28 & 0.0028 \\
\hline Wipf3 & WAS/WASL interacting protein family, member 3 & -1.28 & 0.0066 \\
\hline Six2 & sine oculis-related homeobox 2 & -1.29 & 0.0034 \\
\hline Syne1 & spectrin repeat containing, nuclear envelope 1 & -1.29 & 0.0013 \\
\hline Ltf & lactotransferrin & -1.29 & 0.0073 \\
\hline Cdh1 & cadherin 1 & -1.29 & 0.0010 \\
\hline Sipa1I1 & signal-induced proliferation-associated 1 like 1 & -1.29 & 0.0011 \\
\hline Kcnv1 & potassium channel, subfamily V, member 1 & -1.29 & 0.0070 \\
\hline Musk & muscle, skeletal, receptor tyrosine kinase & -1.29 & 0.0027 \\
\hline F13a1 & coagulation factor XIII, A1 subunit & -1.29 & 0.0018 \\
\hline Bcl11a & B cell CLL/lymphoma 11A (zinc finger protein) & -1.29 & 0.0034 \\
\hline Fgl2 & fibrinogen-like protein 2 & -1.29 & 0.0048 \\
\hline Pla1a & phospholipase A1 member A & -1.29 & 0.0007 \\
\hline Dlx6os1 & Dlx6 opposite strand transcript 1 & -1.30 & 0.0089 \\
\hline Mrc1 & mannose receptor, C type 1 & -1.30 & 0.0006 \\
\hline Anxa2 & annexin A2 & -1.30 & 0.0013 \\
\hline Cxcl12 & chemokine (C-X-C motif) ligand 12 & -1.30 & 0.0011 \\
\hline Emp3 & epithelial membrane protein 3 & -1.30 & 0.0011 \\
\hline Hist1h4i & histone cluster 1, H4i & -1.31 & 0.0025 \\
\hline Ranbp3I & RAN binding protein 3-like & -1.31 & 0.0004 \\
\hline Tfcp211 & transcription factor CP2-like 1 & -1.31 & 0.0010 \\
\hline Lmo4 & LIM domain only 4 & -1.31 & 0.0013 \\
\hline Gas7 & growth arrest specific 7 & -1.31 & 0.0029 \\
\hline $2010300 C 02 R i k$ & RIKEN cDNA 2010300C02 gene & -1.31 & 0.0058 \\
\hline Mas1 & MAS1 oncogene & -1.31 & 0.0014 \\
\hline Cdk5rap1 & CDK5 regulatory subunit associated protein 1 & -1.31 & 0.0021 \\
\hline & & & \\
\hline
\end{tabular}

Microarray results of hypothalamic genes down-regulated in High-Fat diet fed TLR4 ${ }^{-/}$ mice relative to Low-Fat diet fed TLR4 ${ }^{-/-}$mice. 


\begin{tabular}{|l|l|c|r|}
\hline & & $\begin{array}{c}\text { Fold } \\
\text { Change }\end{array}$ & P-value \\
\hline Gene Symbol & Gene Name & -1.31 & 0.0004 \\
\hline Pitx1 & AE binding protein 1 & -1.32 & 0.0042 \\
\hline Eya2 & paired-like homeodomain transcription factor 1 & -1.32 & 0.0064 \\
\hline & eyes absent 2 homolog (Drosophila) & & \\
Cd74 & CD74 antigen (invariant polypeptide of major & -1.32 & 0.0042 \\
\hline Dapl1 & histocompatibility complex, class II antigen-associated) & -1.32 & 0.0018 \\
\hline Serping1 & death associated protein-like 1 & -1.32 & 0.0012 \\
\hline Bche & serine (or cysteine) peptidase inhibitor, clade G, 1 & -1.32 & 0.0023 \\
\hline Lmo7 & butyrylcholinesterase & -1.33 & 0.0011 \\
\hline Ppic & LIM domain only 7 & -1.33 & 0.0004 \\
\hline Ngef & peptidylprolyl isomerase C & -1.33 & 0.0014 \\
\hline Strip2 & neuronal guanine nucleotide exchange factor & -1.33 & 0.0085 \\
\hline Rgs9 & striatin interacting protein 2 & -1.33 & 0.0086 \\
\hline Crispld2 & regulator of G-protein signalling 9 & -1.33 & 0.0008 \\
\hline Rgs9 & cysteine-rich secretory protein LCCL domain containing 2 & -1.33 & 0.0086 \\
\hline Nov & regulator of G-protein signalling 9 & -1.33 & 0.0019 \\
\hline Myo5b & nephroblastoma overexpressed gene & -1.33 & 0.0089 \\
\hline Smoc2 & myosin VB & -1.34 & 0.0003 \\
\hline Sgk1 & SPARC related modular calcium binding 2 & -1.34 & 0.0029 \\
\hline Pdyn & serum/glucocorticoid regulated kinase 1 & -1.34 & 0.0058 \\
\hline Cbr3 & prodynorphin & -1.34 & 0.0066 \\
\hline Serpind1 & carbonyl reductase 3 & -1.34 & 0.0025 \\
\hline Tmem140 & serine (or cysteine) peptidase inhibitor, clade D, member 1 & -1.34 & 0.0007 \\
\hline Cyp2f2 & transmembrane protein 140 & -1.35 & 0.0002 \\
\hline Gm5089 & cytochrome P450, family 2, subfamily f, polypeptide 2 & -1.35 & 0.0043 \\
\hline Cdk5rap1 & predicted gene 5089 & -1.35 & 0.0038 \\
\hline Foxc1 & CDK5 regulatory subunit associated protein 1 & -1.36 & 0.0008 \\
\hline Crabp2 & forkhead box C1 & -1.36 & 0.0013 \\
\hline Cebpd & cellular retinoic acid binding protein II & -1.36 & 0.0003 \\
\hline Actg2 & CCAAT/enhancer binding protein (C/EBP), delta & -1.36 & 0.0005 \\
\hline Serping1 & actin, gamma 2, smooth muscle, enteric & -1.36 & 0.0010 \\
\hline & serine (or cysteine) peptidase inhibitor, clade G, member 1 & & \\
\hline & M & - & \\
\hline
\end{tabular}

Microarray results of hypothalamic genes down-regulated in High-Fat diet fed TLR4 ${ }^{-/}$ mice relative to Low-Fat diet fed TLR4 ${ }^{-/-}$mice. 


\begin{tabular}{|c|c|c|c|}
\hline Gene Symbol & Gene Name & $\begin{array}{c}\text { Fold } \\
\text { Change }\end{array}$ & P-value \\
\hline Asgr1 & asialoglycoprotein receptor 1 & -1.37 & 0.0003 \\
\hline Kalrn & kalirin, RhoGEF kinase & -1.37 & 0.0045 \\
\hline $\mathrm{H} 2-\mathrm{Aa}$ & histocompatibility 2 , class II antigen A, alpha & -1.37 & 0.0014 \\
\hline Islr & $\begin{array}{l}\text { immunoglobulin superfamily containing leucine-rich } \\
\text { repeat }\end{array}$ & -1.37 & 0.0003 \\
\hline Cnksr2 & connector enhancer of kinase suppressor of Ras 2 & -1.38 & 0.0002 \\
\hline Rasgrf1 & $\begin{array}{l}\text { RAS protein-specific guanine nucleotide-releasing factor } \\
1\end{array}$ & -1.38 & 0.0053 \\
\hline Nr4a1 & nuclear receptor subfamily 4 , group $A$, member 1 & -1.38 & 0.0057 \\
\hline Cfh & complement component factor $\mathrm{h}$ & -1.38 & 0.0001 \\
\hline Pde10a & phosphodiesterase $10 \mathrm{~A}$ & -1.38 & 0.0055 \\
\hline Cnn1 & calponin 1 & -1.38 & 0.0004 \\
\hline Gypc & glycophorin C & -1.38 & 0.0002 \\
\hline Fn1 & fibronectin 1 & -1.39 & 0.0010 \\
\hline Adamts2 & $\begin{array}{l}\text { a disintegrin-like and metallopeptidase (reprolysin type) } \\
\text { with thrombospondin type } 1 \text { motif, } 2\end{array}$ & -1.39 & 0.0002 \\
\hline Slc6a12 & $\begin{array}{l}\text { solute carrier family } 6 \text { (neurotransmitter transporter, } \\
\text { betaine/GABA), member } 12\end{array}$ & -1.40 & 0.0001 \\
\hline Slc6a13 & $\begin{array}{l}\text { solute carrier family } 6 \text { (neurotransmitter transporter, } \\
\text { GABA), member } 13\end{array}$ & -1.41 & 0.0013 \\
\hline Ptgds & prostaglandin D2 synthase (brain) & -1.41 & 0.0001 \\
\hline Meis2 & Meis homeobox 2 & -1.41 & 0.0077 \\
\hline Mgp & matrix Gla protein & -1.41 & 0.0006 \\
\hline Omd & osteomodulin & -1.42 & 0.0004 \\
\hline Pde10a & phosphodiesterase $10 \mathrm{~A}$ & -1.42 & 0.0011 \\
\hline Baiap2 & $\begin{array}{l}\text { brain-specific angiogenesis inhibitor 1-associated } \\
\text { protein } 2\end{array}$ & -1.43 & 0.0065 \\
\hline $\mathrm{H} 2-\mathrm{Ab} 1$ & histocompatibility 2 , class II antigen $\mathrm{A}$, beta 1 & -1.43 & 0.0068 \\
\hline Fmod & fibromodulin & -1.43 & 0.0006 \\
\hline Igfbp6 & insulin-like growth factor binding protein 6 & -1.44 & 0.0001 \\
\hline Wnt6 & wingless-related MMTV integration site 6 & -1.45 & 0.0001 \\
\hline $\mathrm{H} 2-\mathrm{Ab} 1$ & histocompatibility 2 , class II antigen A, beta 1 & -1.45 & 0.0027 \\
\hline Tesc & tescalcin & -1.46 & 0.0020 \\
\hline Gjb2 & gap junction protein, beta 2 & -1.46 & 0.0002 \\
\hline Col6a1 & collagen, type $\mathrm{VI}$, alpha 1 & -1.47 & 0.0073 \\
\hline Gpr182 & G protein-coupled receptor 182 & -1.47 & 0.0004 \\
\hline
\end{tabular}

Microarray results of hypothalamic genes down-regulated in High-Fat diet fed TLR4-/mice relative to Low-Fat diet fed TLR4 ${ }^{-/-}$mice. 


\begin{tabular}{|c|c|c|c|}
\hline Gene Symbol & Gene Name & $\begin{array}{c}\text { Fold } \\
\text { Change }\end{array}$ & P-value \\
\hline Slc13a4 & $\begin{array}{l}\text { solute carrier family } 13 \text { (sodium/sulfate symporters), } \\
\text { member } 4\end{array}$ & -1.47 & 0.0012 \\
\hline Rarb & retinoic acid receptor, beta & -1.48 & 0.0086 \\
\hline Mctp1 & multiple $\mathrm{C} 2$ domains, transmembrane 1 & -1.48 & 0.0015 \\
\hline Slc22a6 & $\begin{array}{l}\text { solute carrier family } 22 \text { (organic anion transporter), } \\
\text { member } 6\end{array}$ & -1.48 & 0.0000 \\
\hline Efemp1 & $\begin{array}{l}\text { epidermal growth factor-containing fibulin-like } \\
\text { extracellular matrix protein } 1\end{array}$ & -1.48 & 0.0000 \\
\hline Lyz1 & lysozyme 1 & -1.49 & 0.0006 \\
\hline Sphk1 & sphingosine kinase 1 & -1.49 & 0.0000 \\
\hline Mpzl2 & myelin protein zero-like 2 & -1.50 & 0.0001 \\
\hline Cnksr2 & connector enhancer of kinase suppressor of Ras 2 & -1.50 & 0.0019 \\
\hline Nupr1 & nuclear protein transcription regulator 1 & -1.50 & 0.0000 \\
\hline Nphs2 & nephrosis 2, podocin & -1.51 & 0.0001 \\
\hline Ifi27|2a & interferon, alpha-inducible protein 27 like $2 \mathrm{~A}$ & -1.51 & 0.0000 \\
\hline Al835086 & expressed sequence Al835086 & -1.52 & 0.0070 \\
\hline Icam5 & intercellular adhesion molecule 5 , telencephalin & -1.52 & 0.0090 \\
\hline Prg4 & $\begin{array}{l}\text { proteoglycan } 4 \text { (megakaryocyte stimulating factor, } \\
\text { articular superficial zone protein) }\end{array}$ & -1.58 & 0.0000 \\
\hline Aldh1a2 & aldehyde dehydrogenase family 1 , subfamily $A 2$ & -1.58 & 0.0000 \\
\hline Foxc2 & forkhead box C2 & -1.59 & 0.0000 \\
\hline Thbd & thrombomodulin & -1.60 & 0.0000 \\
\hline Penk & preproenkephalin & -1.62 & 0.0073 \\
\hline Ddn & dendrin & -1.65 & 0.0059 \\
\hline Egr4 & early growth response 4 & -1.66 & 0.0014 \\
\hline Foxg1 & forkhead box G1 & -1.67 & 0.0096 \\
\hline Cga & glycoprotein hormones, alpha subunit & -1.73 & 0.0004 \\
\hline Ogn & osteoglycin & -1.73 & 0.0000 \\
\hline Serpina9 & $\begin{array}{l}\text { serine (or cysteine) peptidase inhibitor, clade A (alpha-1 } \\
\text { antiproteinase, antitrypsin), member } 9\end{array}$ & -1.76 & 0.0090 \\
\hline Kcnh3 & $\begin{array}{l}\text { potassium voltage-gated channel, subfamily } \mathrm{H} \text { (eag- } \\
\text { related), member } 3\end{array}$ & -1.77 & 0.0040 \\
\hline Itpka & inositol 1,4,5-trisphosphate 3-kinase A & -1.79 & 0.0048 \\
\hline Egr2 & early growth response 2 & -1.85 & 0.0005 \\
\hline Adora2a & adenosine $\mathrm{A} 2 \mathrm{a}$ receptor & -2.12 & 0.0012 \\
\hline S100a8 & S100 calcium binding protein A8 (calgranulin A) & -2.13 & 0.0001 \\
\hline Bpifa1 & BPI fold containing family $A$, member 1 & -2.34 & 0.0000 \\
\hline S100a9 & S100 calcium binding protein A9 (calgranulin B) & -2.47 & 0.0011 \\
\hline
\end{tabular}

Microarray results of hypothalamic genes down-regulated in High-Fat diet fed TLR4 ${ }^{-/}$ mice relative to Low-Fat diet fed TLR4 $4^{-/-}$mice. 